\title{
NMR Spectroscopic studies of calmodulin plasticity in calcium signalling
}

\author{
Ph.D. Thesis
}

\author{
In partial fulfillment of the requirements \\ for the degree "Doctor of Philosophy (Ph.D.)" \\ in the Molecular Biology Program \\ at the Georg August University Göttingen, \\ Faculty of Biology
}

submitted by

Fernando Rodríguez Castañeda

born in

Guatemala City 
Herewith I declare, that this thesis represents my original work and all the concepts and material that I did not create have been cited.

Göttingen, 29 of September of 2007, 


\section{Aknowledgements}

The work presented here has been accomplished in the NMR-Based Structural Biology Department of the Max-Planck Institute for Biophysical Chemistry within the frame of the International Molecular Biology Program, Max-Planck Research Schools and University of Göttingen. I acknowledge these Institutions and people involved in its creation and management.

I thank my Ph.D. supervisor and Director of the NMR-Based Structural Biology Department, Prof. Christian Griesinger for his guidance and trust in the scientific endeavours I have undertaken.

I would like to acknowledge Dr. Teresa Carlomagno for her contribution in the NMR studies of calmodulin interaction with Munc13. Dr. Laurent Verdier, Dr. Christophe Fàres and Dr. Vinesh Vijayan for introduction to the use of the NMR spectrometer and their assistance when problems were encountered.

I acknowledge Dr. Andrei Leonov who provided the paramagnetic tags used in this work and also for giving me advices how to use them.

I acknowledge Dr. Steffan Becker and Karin Giller for their contribution in various aspects of the sample preparation in this work.

I acknowledge Dr. Volker Klaukien, Kerstin Overkamp and Gerhard Wolff for peptide synthesis and HPLC purification.

I acknowledge Dr. Pierre Montaville for stimulating scientific discussions and practical advices on the sample preparation.

I acknowledge Dr. Nicolas Coudeville and Jegannath Korukottu for their assistance in the structure calculation part of this work

I acknowledge Pinar Karpinar for her help in Dynamic Light Scattering measurements.

I acknowledge Prof. Thomas Jovin, Director of the Department of Molecular Biology at the Max-Planck Insitute for Biophysical Chemistry for giving me the opportunity to do some fluorescence studies.

I acknowledge Prof. Nils Brose, Director of the Molecular Neurobiology Department at the Max-Planck Institute for Experimental Medicine for giving me the opportunity to collaborate on the interaction of calmodulin and Munc13. I also thank Dr. Harald Junge, Dr.Olaf Jahn, Kalina Dimova and Noa Lippstein who are also part of this joint effort.

I would like to thank all the members of the NMR-Structural Biology Department for the collegial working atmosphere.

I dedicate this work to my family who gave me their unconditional support during these five years abroad. I thank my friends in Göttingen who always gave me a helping hand when I needed it. 


\section{List of publications}

Rodríguez-Castañeda, F., Haberz, P., Leonov, A., Griesinger, C. (2006) Paramagnetic tagging of diamagnetic proteins for solution NMR. Magnetic Resonance in Chemistry. 44, S10-S16.

Haberz, P., Rodríguez-Castañeda, F., Junker, J., Becker, S., Leonov, A., Griesinger, C. (2006) Two new chiral EDTA-Based metal chelates for weak alignment of proteins in solution. Organic Letters. 8, 1275-1278.

Leonov, A., Voigt, B., Rodríguez-Castañeda, F., Sakhaii, P., Griesinger, C. (2005) Convenient syntheses of multifunctional EDTA-Based chiral metal chelates substituted with an S-mesylcysteine. Chemistry- a European Journal. 11, 1-8.

Rodríguez-Castañeda, F., Brose, N., Carlomagno, T., Griesinger, C. Resonance assignment of the Calmodulin-Munc13-1 peptide complex. J. Biomol. Assignments. To be submitted. 


\section{Abstract}

The present work focused both in structural and dynamic studies on the ubiquitous $\mathrm{Ca}^{2+}$ signaling protein calmodulin. Calmodulin has been extensively studied both for its biological importance in the regulation of its interaction partners which are key regulators in various processes like protein phosphorylation, dephosphorylation and regulation of gene transcription and as a two-domain model protein, binding the signaling calcium ion in a cooperative fashion. Pioneering high-resolution structural studies both by X-ray crystallography (Babu et al., 1988) and NMR (Ikura et al., 1992; Barbato et al., 1992) done on calmodulin revealed that its two domains are linked by a flexible linker giving a large degree of conformational freedom. Thus, its two domains can adopt various orientations to recognize and activate its targets. Upon calcium binding, the EF-hand motifs in CaM undergo a large conformational change exposing hydrophobic side-chains to the surface, which engage in hydrophobic interactions with its targets. Among this hydrophobic side chains, methionines play a prominent role in CaM plastic interactions (Siivari et al., 1995).

The dynamic part of the investigation made use of anisotropic NMR restraints that sample protein motions up to the submillisecond time-scale. In solution-state NMR, anisotropic interactions average to zero due to molecular tumbling. For this reason, these anisotropic interactions are observable (and measurable) just under special sample conditions. In this work, the use of lanthanide-binding EDTA-based paramagnetic tags attached to cysteine mutants in CaM served this purpose; since the unpaired electron in the paramagnetic lanthanide ion provides strong magnetic susceptibility anisotropy, aligning the macromolecule in solution. Using this methodology, it was possible to establish a difference in the CaM dynamics in three different activated sates. First, the paramagnetic alignment of the CaMS17C mutant established a difference in the dynamic behavior of apoCaM and holoCaM on the basis of pseudocontact shifts measured in the linker region of CaM. Second, the measurement of residual dipolar couplings from the paramagnetic alignment of the CaMT146C mutant in the free state and in complex to the C20W peptide belonging to the plasma membrane $\mathrm{Ca}^{2+}$-pump, established a difference in the dynamics between these two activated states. Bertini et al. 2004, reported a reduced alignment for the C-terminal domain of CaM (around 10\% of the alignment was retained) mutant by direct lanthanide binding to the metal binding site in the $\mathrm{N}$-terminal domain of holoCaM. In this work, a consistent reduced alignment in three independent measurements of $\sim 25 \%$ in the $\mathrm{N}$-terminal domain of $\mathrm{CaM}$ in complex with the C20W peptide is reported. In contrast, for the holoCaM case, a residual alignment of the N-terminal domain of CaM could not be measured because of the weak alignment impaired by the paramagnetic tag (up to $8 \mathrm{~Hz}$ at $900 \mathrm{MHz}$ ) yielding rDC within the error range of the measurements.

The structural part of the investigation focused in the interaction of CaM with the diacylglycerol-binding protein Munc13-1, an essential protein involved in the priming process of vesicles in neurotransmitter release. Junge et al., 2004 found that CaM binds to a conserved region in Munc13-1 and regulates neurotransmitter release in response to residual calcium signals. The complete sequential resonance assignment and determination of the NMR solution structure of the CaM/Munc13-1 (458-492) peptide complex is reported. The ${ }^{1} \mathrm{H},{ }^{15} \mathrm{~N}$, and ${ }^{13} \mathrm{C}$ resonance assignment list has been deposited to the biological magnetic resonance data bank (BMRB): deposition number 15470. The structure describes a new binding motif for CaM, where CaM interacts with Munc13-1 in a bipartite mode. The C-terminal domain of CaM interacts with the N-terminal amphiphilic $\alpha$-helix (1-5-8) hydrophobic motif of the Munc13-1 peptide and the N-terminal domain of CaM builds a hydrophobic interaction with a LW motif at the C-terminus of the peptide. Other singular properties of this protein-peptide complex include residual interdomain dynamics in the submillisecond time scale probed by 
paramagnetically-derived residual dipolar couplings; and monomer-dimer equilibrium to a (2:2) complex favored at larger salt concentrations.

Electrophisiology studies done on primary neuron cultures of the CaM insensitive (W464R) and the phorbol ester insensitive (H567K) mutants (Junge et al., 2004; Rhee et al., 2002) of Munc13-1 have revealed striking similarities in their vesicle priming properties. This motivated the study of the interaction of CaM with a fragment of Munc13-1 containing both the CaM-binding and the diacylglycerol/phorbol ester- $\mathrm{C}_{1}$ binding domains of Munc13-1. The ${ }^{15} \mathrm{~N}$-labeled NMR sample for this protein-protein complex could be prepared using a coexpression approach and allowed its spectroscopic investigation. Although the sequential backbone resonance assignment for this CaM/Munc13-1(447-631) protein complex was not undertaken, the similarity to the HSQC of the CaM/Munc13-1 (458-492) peptide complex allowed the description of several novel properties of this protein-protein interaction. First, the monomer(1:1)-dimer (2:2) equilibrium described for the CaM/Munc13-1(458-492) peptide complex is also described in this larger complex, but with an increased binding affinity. Therefore, the monomeric and dimeric complex species could be separated by sizeexclusion chromatography and studied independently. The analysis of the HSQC spectra of the monomeric complex species of the wild type, W489A and W588A mutant complexes suggest that the $\mathrm{N}$-terminal domain of CaM switches between two hydrophobic motifs in Munc13-1: the LW motif revealed in the NMR structure and a second motif within the $\mathrm{C}_{1}$ domain of Munc13-1. The rigorous proof of a direct interaction between the $\mathrm{N}$-terminal domain of $\mathrm{CaM}$ and the $\mathrm{C}_{1}$ domain of Munc13- 1 is not provided, but ongoing studies is addressing this possibility that would give a structural correlate to the physiological studies mentioned before.

The studies on the dimeric (2:2) CaM/Munc13-1(447-631) protein complex also suggest a conformational exchange equilibrium mediated by the $\mathrm{N}$-terminal domain of $\mathrm{CaM}$ and the $C_{1}$ domain of Munc13-1. Moreover, there is preliminary evidence that the $C_{1}$ domain agonist PDBu might activate Munc13-1 by shifting the equilibrium towards the monomeric state of the complex, possibly relieving an auto-inhibited state. The homodimerization of the $\mathrm{C}_{2} \mathrm{~A}$ domain of Munc13-1 has been described in the studies by Lu et al., 2006. For this reason, further studies on the relationship of the oligomerization state of Munc13-1 and its priming activity are highly encouraged to better understand how the variable $\mathrm{N}$-terminal region of Munc13 proteins with its numerous interaction partners like RIM1 and Rab3A (Dubulova et al., 2005) remodels the highly conserved C-terminal MUN catalytic domain in this family of proteins to fine-tune the priming of vesicles in the active zone of neurons and more importantly how these different protein-protein interactions shape the short-term synaptic plasticity processes in the brain. 


\section{Zusammenfassung}

Dieser Arbeit befasst sich mit den strukturellen und dynamischen Aspekten der Kalzium Signal Transduktion von Calmodulin (CaM). Dieses Protein ist intensiv erforscht worden, da es eine Vielzahl von Interaktionspartnern reguliert und in Schlüsselprozessen, wie Proteinphosphorylierung, -dephosphorylierung und Transkription involviert ist. Darüber hinaus ist ein Model eines Zwei-Domänen-Proteins, welches Kalzium kooperativ bindet. Die strukturelle Pionierarbeit, sowohl kristallographisch (Babu et al., 1988), als auch NMRspektroskopisch (Ikura et al., 1992; Barbato et al., 1992) hat gezeigt, dass beide Calmodulindomänen durch einen flexibler Linker verbunden sind, was ein hohes Maß an konformativer Freiheit zulässt. Bedingt dadurch können beide Domänen unterschiedliche Orientierungen einnehmen, um ihre Interaktionspartner zu erkennen und zu aktivieren. Nach der Kalziumbindung erfahren die EF-Hand-Motive eine umfangreiche Konformationsänderung, wodurch hydrophobe Seitenketten auf der Oberfläche präsentiert werden und eine hydrophobe Wechselwirkung mit ihren Partnern eingehen. Hierbei spielen besonders Methioninseitenketten eine wichtige Rolle (Siivari et al., 1995).

Der Teil, der sich mit der Dynamik von CaM befasst, nutzt anisotrope NMR Bedingungen, um Bewegungsvorgänge im bis zum Millisekundenbereich „abzutasten“. In Lösung belaufen sich solche anisotropen Interaktionen, bedingt durch die Molekularbewegung, im Durchschnitt auf Null. Daher lassen sie sich nur unter speziellen Probenbedingungen beobachten (und messen). Im Rahmen dieser Arbeit wurden Lanthanoidkomplexierte, auf EDTA-basierende, paramagnetische Tags verwendet, die über Cysteinseitenketten an CaM gebunden sind. Das allein stehende ungepaarte Elektron des paramagnetischen Lanthanoid-Ions bewirkt eine starke anisotropische magnetische Suszeptibilität, die das Makromolekül im Magnetfeld ausrichtet. Mit dieser Methode war es möglich, Unterschiede zwischen drei aktivierten Zuständen von CaM zu untersuchen (apoCaM, holoCaM und CaM-C20W-Peptid-Komplex). Dabei zeigte die paramagnetische Anordnung der CaM-S17C Mutante einen Unterschied im dynamischen Verhalten zwischen apoCaM und holoCaM anhand von, in der Linker-Region gemessener, Pseudokontaktverschiebungen. Durch die Messung dipolarer Kopplungen der CaM-T146C Mutante im freien und komplexierten Zustand mit dem Peptid (C20W) der membrangebundenen $\mathrm{Ca}^{2+}$-ATPase-Pumpe, konnten ebenfalls Unterschiede im dynamischen Verhalten beobachtet werden. Bertini et al. (2004) beschrieb eine verbliebene Ausrichtung der C-terminalen Domäne von rund 10\%, wenn ein Lanthanoid-Ion direkt N-terminal von holoCaM gebunden wird. In dieser Arbeit konnte, übereinstimmend in drei unabhängigen Messungen, eine verbliebene N-terminale Ausrichtung von 25\% im Komplex mit C20W beobachtet werden. Im Gegensatz dazu wurde für den N-Terminus von holoCaM keine verbliebene Orientierung gemessen, da das Alignment des paramagnetischen tags (bis zu $8 \mathrm{~Hz}$ dipolare Kopplungen am $900 \mathrm{MHz}$ Spektrometer) zu schwach ist und die Ergebnisse in der Grössenordnung des Fehlerbereiches liegen.

Der strukturelle Teil der Arbeit befasst sich mit der Interaktion von CaM mit dem Diacylglycerol-bindinden Protein Munc13-1, welches eine wichtige Rolle hinsichtlich Neurotransmitterfreisetzung spielt. Junge et al., (2004) fand heraus, dass CaM an eine konservierte Region von Munc13-1 bindet und als Antwort auf Kalziumsignale die Freisetzung solcher Neurotransmitter reguliert. Die vollständige sequenzielle Zuordnung der chemischen Verschiebungen und die gelöste NMR-Struktur des CaM/Munc13-1 (458-492) Peptid Komplexes wird in dieser Arbeit beschrieben. Die ${ }^{1} \mathrm{H},{ }^{15} \mathrm{~N}$, und ${ }^{13} \mathrm{C}$ chemischen Verschiebungen wurden auf der Biological Magnetic Resonance Data Bank (BMRB) unter der Nummer 15470 abgelegt. Die Struktur beschreibt ein neues bindendes Motiv für CaM, in dem es mit Munc13-1 in zweiteiliger Weise interagiert. Die C-terminale Domäne vom CaM wechselwirkt hierbei mit dem $\mathrm{N}$-terminalen, amphiphilen und $\alpha$-helikalen (1-5-8) Motiv des 
Munc13-1 Peptids und der CaM-N-terminalen Domäne, so dass eine hydrophobe Interaktion durch eine LW Motiv am C-terminus des Peptids aufgebaut wird. Zu den weiteren Eigenschaften dieses Protein-Peptid Komplexes zählt eine verbliebene Inter-DomänenDynamik im sub-Millisekundenbereich, die mittels paramagnetischer dipolarer Kopplungen untersucht wurde, sowie die Verschiebung eines Monomer-Dimer Gleichgewichtes in Richtung eines 2:2 Komplexes, unter Verwendung höherer Salzkonzentrationen.

Electrophysiologische Studien an primären Neuronalkulturen von Munc13-1 Mutanten, die eine Unempfindlichkeit gegenüber CaM (W464R) und Phorbolester (H567K) aufweisen, zeigten eine vergleichbare Eigenschaft hinsichtlich der Auflösung von Neurotransmittervesikeln (Junge et al., 2004; Rhee et al., 2002). Dies wiederum regte die Studie der Interaktion zwischen CaM und einem Fragment von Munc13-1 an, welches beide, die CaM- und Diacylglycerol/Phorbolester- $\mathrm{C}_{1}$ bindende Domäne enthält. Die ${ }^{15} \mathrm{~N}$ markierte NMR Probe zur Untersuchung dieses Protein-Protein Komplexes, konnte mittels einer CoExpression hergestellt und anschließend untersucht werden. Die Ähnlichkeiten zwischen den HSQC-Spektren des CaM/Munc13-1(458-492) Komplexes und des CaM/Munc13-1(447-631) Proteinkomplexes ermöglichten die Beschreibung einiger neuer Eigenschaften dieses ProteinProtein Komplexes, obwohl keine sequentielle Zuordnung des CaM/Munc13-1(447-631) Komplexes vorgenommen wurde. Erstens, das für den CaM/Munc13-1(458-492) Komplex bereits beschriebene Monomer (1:1)- Dimer (2:2)-Gleichgewicht, ist für den größeren Komplex ebenfalls zu beobachten, jedoch mit einer erhöhten Bindungs-Affinität. Daher war es möglich, das Monomer vom Dimer mittels Größenausschlusschromatographie zu trennen, um jede Spezies unabhängig voneinander untersuchen zu können. Die HSQC-Analyse des monomeren Komplexes des Wildtyps, sowie der W489A- und W588A-Mutanten weist darauf hin, dass die N-terminale Domäne von CaM zwischen zwei hydrophoben Motiven in Munc13-1 wechselt: das LW Motiv, gezeigt in der NMR Struktur, und ein zweites Motiv, innerhalb der $\mathrm{C}_{1}$-Domäne von Munc13-1. Der entscheidende Beweis für die Interaktion zwischen der N-terminalen Domäne vom CaM und der $\mathrm{C}_{1}$-Domäne vom Munc13-1 konnte nicht erbracht werden, aber laufende Studien untersuchen diese Möglichkeit, die eine Brücke zwischen die strukturellen und den genannten physiologischen Studien schlagen würde. Die Untersuchungen des CaM/Munc13-1(447-631)-Dimer-(2:2) Komplexes legen einen konformativen Gleichgewichtsaustausch zwischen der N-terminalen Domäne vom CaM und der $\mathrm{C}_{1}$-Domäne von Munc13-1 nahe. Außerdem gibt es Hinweise darauf, dass der $\mathrm{C}_{1}{ }^{-}$ Domänen-Agonist PDBu von Munc13-1 eine Verschiebung des Gleichgewichts in Richtung des Monomers bewirkt, möglicherweise bedingt durch eine reduzierte Autoinhibition. Die Homodimerisierung der $\mathrm{C}_{2}$ A Domäne von Munc13-1 wurde in den Studien von Lu et al. (2006) bereits beschrieben. Daher sind weitere Studien, die das Verhältnis zwischen dem Oligomerisierungszustand von Munc13-1 und der Regulation der Neurotransmitterfreisetzung untersuchen, wichtig, um besser verstehen zu können, wie die hoch konservierte C-terminale MUN-Domäne von Interaktionspartnern, wie RIM1 und Rab3A, von der variablen Nterminalen Region der Munc13-Proteine verändern wird. Dies führt zu einer Feinabstimmung der Neurotransmitterfreisetzung im aktiven Bereich der Neuronen. Darüber hinaus gilt es zu klären, wie diese verschiedenen Protein-Protein-Interaktionen die kurzzeitig plastischen Prozesse im Gehirn bewirken könnten. 


\section{Table of Contents}

Page

I. INTRODUCTION....................................................... 1

1. Signal transduction and second messengers............................... 1

2. Synapses and calcium signaling in neurons.............................. 2

3. Synaptic vesicles.................................................. 3

4. Brain modulator protein: calmodulin..................................... 4

5. The EF-hand superfamily of proteins: calmodulin plasticity.................. 5

6. Regulated exocytosis............................................... 10

7. Presynaptic plasticity............................................... 13

8. Active Zones and the presynaptic plasma membrane......................... 15

9. Allosteric modulation of the presynaptic $\mathrm{Ca}^{2+}$ sensor for vesicle fusion.......... 16

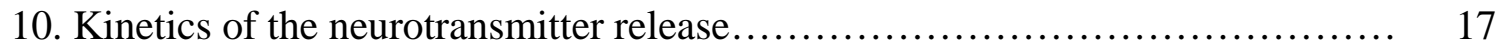

11. $\mathrm{C}_{1}$ domain as membrane localization module in Munc13-1................... 17

12. Priming activity of Munc13 proteins.................................... 19

13. Study of macromolecular structure with nuclear magnetic resonance........... 21

14. Study of macromolecular dynamics with nuclear magnetic resonance.......... 26

15. Paramagnetic tagging of diamagnetic proteins............................ 37

16. Calmodulin dynamics.............................................. 38

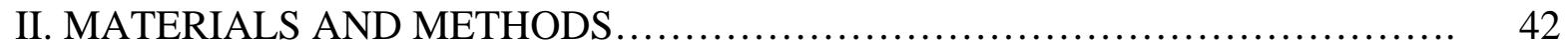

1. Polymerase chain reaction (PCR) based-cloning of the different Munc13-1 protein expression constructs............................................ 42

a. Polymerase chain reaction (PCR) .................................. 42

b. Restriction digestion of the PCR products........................... 43

c. Preparation of the plasmid DNA expression vectors for cloning............ 44

d. Ligation reaction.................................................. 44

e. Transformation of the ligated plasmid DNA........................... 45

f. Plasmid DNA amplification and purification............................ 45

g. Screening, DNA sequencing and transformation of positive clones......... 45

h. Protein over expression test in LB medium and protein solubility......... 46

2. PCR-mediated Site-directed mutagenesis................................. 47

3. Over expression of the different proteins in Minimal Medium for uniform isotope labeling ..................................................... 49 
4. Purification of the hexahistidine-tagged Munc13-1 proteins.................... 49

5. Purification of the GST-tagged Munc13-1 proteins.......................... 50

6. Purification of CaM....................................................... 50

7. Purification of the Munc13-1-185aa protein............................... 52

8. Electrospray ionization mass spectrometry (ESI-MS)...................... 52

9. NMR measurements..................................................... 52

10. Docking and structure calculation approaches for the CaM/Munc13-1(457-492) peptide complex..................................................... 54

11. Data analysis of residual dipolar couplings and pseudocontact shifts........... 56

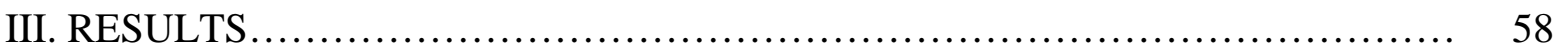

1. Cloning, overexpression and purification of CaM and Munc13-1 proteins....... 58

a. Cloning of Munc13-1(457-492) (Munc13-1-36aa).................... 58

b. Cloning of Munc13-1(567-631) C d $_{1}$ domain (Munc13-1-65aa) ............ 58

c. Cloning of Munc13-1(447-631) (Munc13-1-185aa) ..................... 59

d. Site-directed mutagenesis.......................................... 59

e. Overexpression and purification of the Munc13-1(457-492) peptide....... 59

f. Overexpression and purification of the Munc65 protein.................. 61

g. Overexpression and purification of the Munc185 protein................. 63

h. Overexpression and purification of calmodulin........................ 65

i. Biochemical separation of Munc185 from calmodulin................... 65

2. Biophysical characterization of CaM and Munc13-1 proteins.................. 67

a. NMR studies on the ${ }^{15} \mathrm{~N},{ }^{13} \mathrm{C}$ CaM/Munc13-1(459-479) peptide complex.... 67

b. NMR studies on the ${ }^{15} \mathrm{~N},{ }^{13} \mathrm{C}$ CaM/Munc13-1(459-492) peptide complex.... 68

c. NMR studies of the ${ }^{13} \mathrm{C},{ }^{15} \mathrm{~N}$ Munc13-1(457-492) peptide binding to CaM... 73

d. NMR studies of the CaM binding region and the $\mathrm{C}_{1}$ domain of Munc13-1

(Munc185).................................................... 77

e. Tryptophan $\mathrm{N} \varepsilon-\mathrm{H} \varepsilon 1$ side chain heterogeneity as evidence of a switch mechanism in the calcium coupling mechanism of CaM and Munc13-1..... 78

f. NMR studies of the $C_{1}$ domain of Munc13-1 (Munc65).................. 81

g. NMR studies of ${ }^{15} \mathrm{~N}$ CaM/ $/{ }^{15} \mathrm{~N}$ Munc13-1(185aa) complex with its agonist:

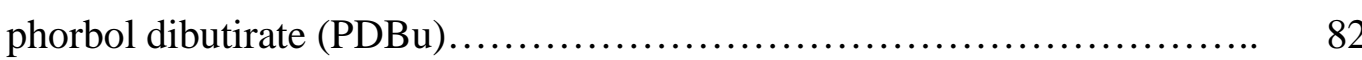

h. Sequential backbone and side-chain resonance assignment for the ${ }^{13} \mathrm{C},{ }^{15} \mathrm{~N}$ CaM/Munc13-1 (459-492) peptide complex............................

i. Long-range NMR restraints for the structure determination of the 
CaM/Munc13-1 (457-492) peptide complex

j. NMR structure determination of the CaM/Munc13-1 (457-492) peptide complex.

k. Dynamic studies of CaM in its various activated states with paramagnetic alignment

IV. DISCUSSION.

1. Dynamic studies on apoCaM

2. Dynamic studies on holoCaM.

3. Dynamic studies on the CaM/C20W peptide complex. 128

4. Structural studies on the CaM/Munc13-1 interaction. 128

a. apoCaM interaction with Munc13-1 and ubMunc13-2................... 128

b. $\left[2 \mathrm{Ca}^{2+}\right] \mathrm{CaM}$ interaction with Munc13-1.............................. 128

c. $\left[4 \mathrm{Ca}^{2+}\right] \mathrm{CaM}$ interaction with Munc13-1.............................. 129

d. Solution structure of the CaM/Munc13-1(457-492) peptide complex........ 129

e. Accuracy of the solution structure of the CaM/Munc13-1(457-492) peptide complex.

f. NMR studies on the CaM/Munc13-1(185aa) monomeric (1:1) and dimeric (2:2) complexes

V. CONCLUSIONS

VI. BIBLIOGRAPHY

VII. APENDIX

1.Magnetization pathways in the backbone nuclei of the polypeptide chain. 141

2. Pictorial view of the heteronuclear multidimensional NMR pulse sequences. 142

3. Chemical shift assignments for the CaM/Munc13-1(457-492) peptide complex... 


\section{List of Figures}

No.

1 Anatomy of a typical synapse........................................ 2

2 Molecular model of CaM X-ray crystallographic structure.................... 6

3 Model of the EF-hand motif............................................ 6

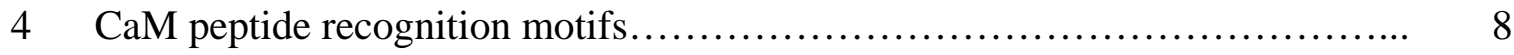

5 Different activation models for CaM binding partners...................... 9

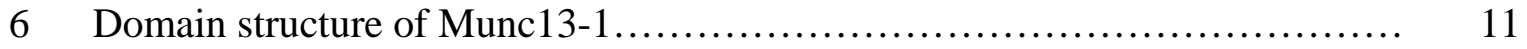

7 Neurotransmitter vesicle endo- and exocytotic cycle in a presynaptic neuron... 12

8 Flash photolysis response.......................................... 15

$9 \quad{ }^{1}$ J-Coupling pathways for the nuclei in the polypeptide backbone............... 22

10 Orientation-dependent magnetic field experienced by an amide ${ }^{15} \mathrm{~N}$ nucleus due to the directly bonded proton..................................... 32

11 Orientation of the internuclear vector and the magnetic field relative to a molecule fixed frame................................................ 35

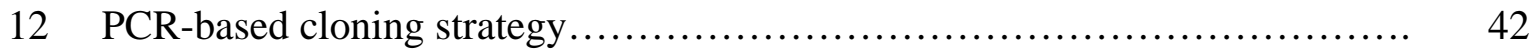

13 Pulse sequence for the ${ }^{1} \mathrm{H}$ detected heteronuclear single quantum coherence spectrum (HSQC) experiment......................................... 53

14 PCR products of the Munc13-1(457-492) DNA insert...................... 58

15 Positive clones for the pGEX2T-Munc13-1(457-492) construct.............. 58

16 PCR products of the Munc13-1 $\mathrm{C}_{1}$ domain (Munc 65aa) DNA insert.......... 59

17 PCR products of the Munc13-1 (185aa) DNA insert........................ 59

18 Over expression and purification from pGEX2T-Munc13-1(457-492)

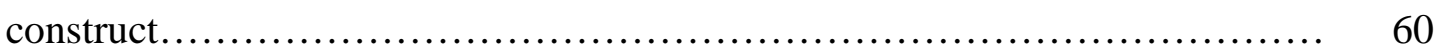

19 Munc13-1 (457-492) peptide purification.............................. 60

20 Overexpression of the MBP- Munc13-1(457-492) fusion protein............... 61

21 Overexpression and purification of the Munc65aa-(his) $)_{6}$ protein............... 62

22 Overexpression of the GST-Munc65aa fusion protein........................ 62

23 Overexpression of the pET16b-Munc185aa construct....................... 63

24 Co-expression of the pET16b-Munc185aa and pET28a CaM constructs........ 63

25 Overexpression of the CaM/Munc185aa complex from the pETDuet-1(CaMMunc185aa) construct................................................ 64

26 Purification of CaM/Munc185aa....................................... 64 
27 Purification of the CaM/GST-Munc185aa complex....................... 65

28 CaM purification...................................................... 66

29 Biochemical separation of Munc185aa..................................... 66

$30{ }^{1} \mathrm{H}_{-}{ }^{15} \mathrm{~N}$ HSQC spectrum of the uniformly labelled ${ }^{15} \mathrm{NCaM} / \mathrm{Munc} 13-1(459-479)$ peptide complex................................................ 67

31 Chemical shift perturbation plot for CaM upon binding to the Munc13-1 (459479) peptide....................................................... 68

$32{ }^{15} \mathrm{~N}-{ }^{1} \mathrm{H}$ HSQC spectra of ${ }^{15} \mathrm{~N}$ CaM and ${ }^{15} \mathrm{NCaM} /$ Munc13-1(459-492) peptide

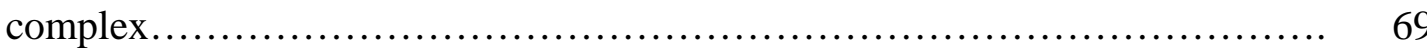

33 Chemical shift perturbation plot for the amide region in CaM upon binding to the Munc13-1 (459-492) peptide.

$34{ }^{15} \mathrm{~N}-{ }^{1} \mathrm{H}$ HSQC spectra for apoCaM and apoCaM/Munc13-1 (459-479) peptide.

$35{ }^{15} \mathrm{~N}-{ }^{1} \mathrm{H}$ spectra of apoCaM with increasing amounts of Munc13-1 (459-479) peptide.

36 Saturation binding curve for the Valine $91 \mathrm{NH}$ cross peak of ${ }^{15} \mathrm{~N}$ labelled apoCaM upon increasing amounts of Munc13-1 (459-479) peptide.

37 Residues in apoCaM affected by the binding of the Munc13-1 (459-479) and ubMunc13-2 (382-402) peptide.

$38{ }^{1} \mathrm{H}^{15}{ }^{15} \mathrm{HSQC}$ of the CaM/Munc13-1(459-492) complex at three different activated states...................................................... $\quad 72$

$39{ }^{15} \mathrm{~N}-{ }^{1} \mathrm{H}$ HSQC spectrum of the ${ }^{13} \mathrm{C},{ }^{15} \mathrm{~N}$ Munc13-1(457-492) peptide. 73

40 Strip plots of the 3D-HNN spectrum for three diagonal peaks of the ${ }^{13} \mathrm{C},{ }^{15} \mathrm{~N}$ Munc13-1(457-492) peptide.

$41{ }^{1} \mathrm{H}_{-}{ }^{15} \mathrm{~N}$ HSQC of the bound ${ }^{13} \mathrm{C}$, ${ }^{15} \mathrm{~N}$ Munc13-1(457-492) peptide to CaM......... 75

42 Chemical shift perturbation plot of the Munc13-1 (457-492) peptide upon

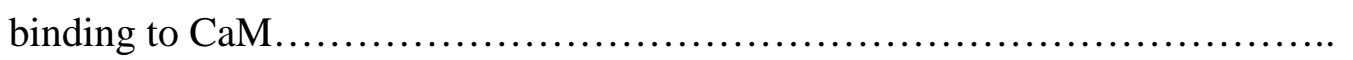

43 Chemical shift difference between the Munc13-1(457-492)/CaM and Munc131(457-492)/apoCaM peptide complexes.

$44{ }^{15} \mathrm{~N}-{ }^{1} \mathrm{H}$ HSQC spectra of the bound ${ }^{15} \mathrm{~N},{ }^{13} \mathrm{C}$ Munc13-1(457-492) peptide to CaM in $\mathrm{H}_{2} \mathrm{O}$ and $\mathrm{D}_{2} \mathrm{O}$

$45{ }^{15} \mathrm{~N}-{ }^{1} \mathrm{H}$ HSQC of the ${ }^{15} \mathrm{~N}$ CaM and ${ }^{15} \mathrm{~N} \mathrm{CaM} /{ }^{15} \mathrm{~N}$ Munc13-1(185aa) $w t$ monomeric (1:1) complex

${ }^{15} \mathrm{~N}-{ }^{1} \mathrm{H}$ HSQC spectra for the ${ }^{15} \mathrm{~N}$ CaM/ $/{ }^{15} \mathrm{~N}$ Munc13-1(185aa) wt monomeric 
(1:1) complex and dimeric (2:2) complex.

47 Intensity ratio of the amide cross-peaks between the monomeric (1:1) and dimeric (2:2) ${ }^{15} \mathrm{~N} \mathrm{CaM} /{ }^{15} \mathrm{~N}$ Munc13-1(185aa) wt complexes.

48 Residues in CaM with line-broadening upon dimerization with Munc13-1 (185aa)

$49{ }^{15} \mathrm{~N}-{ }^{1} \mathrm{H}$ HSQC spectra of the tryptophan $\mathrm{N} \varepsilon-\mathrm{H} \varepsilon 1$ side-chain region. 80

50 1D- ${ }^{1} \mathrm{H}$ spectrum for the amide region of unlabelled Munc13-1 $\mathrm{C}_{1}$ domain 81

$51{ }^{15} \mathrm{~N}-{ }^{1} \mathrm{H}$ HSQC of the $\mathrm{C}_{1}$ domain of Munc13-1... 82

$52{ }^{15} \mathrm{~N}-{ }^{1} \mathrm{H}$ HSQC spectra for the ${ }^{15} \mathrm{~N}$ CaM $/{ }^{15} \mathrm{~N}$ Munc13-1(185aa) wt monomeric (1:1) complex the ${ }^{15} \mathrm{~N}$ Munc13-1 $\mathrm{C}_{1}$ domain.

$53{ }^{15} \mathrm{~N}-{ }^{1} \mathrm{H}$ HSQC spectra of the ${ }^{15} \mathrm{~N}$ CaM $/{ }^{15} \mathrm{~N}$ Munc13-1(185aa) wt dimeric (2:2) complex in the inactive and active states.

$54{ }^{15} \mathrm{~N}-{ }^{1} \mathrm{H}$ HSQC spectra for the ${ }^{15} \mathrm{~N}$ CaM $/{ }^{15} \mathrm{~N}$ Munc13-1(185aa) W489A monomeric (1:1) complex in its inactive and active states....

55 Intensity ratio of the amide cross-peaks for the ${ }^{15} \mathrm{~N}$ CaM $/{ }^{15} \mathrm{~N}$ Munc13-1(185aa) W489A mutant in the absence and presence of its agonist PDBu.

56 Binding curve of the $\mathrm{C}_{1}$ domain agonist $\mathrm{PDBu}$ to the ${ }^{15} \mathrm{~N} \mathrm{CaM} /{ }^{15} \mathrm{~N}$ Munc131(185aa) W489A monomeric (1:1) complex.

57 Assigned ${ }^{1} \mathrm{H}^{15} \mathrm{~N}-\mathrm{HSQC}$ spectrum of the ${ }^{13} \mathrm{C},{ }^{15} \mathrm{NCaM} / \mathrm{Munc} 13-1(459-492)$ peptide complex.

58 Strips extracted from the 3D-CBCA(CO)NH and HNCACB spectra

59 Strips from the 3D-HNCO and 3D-HN(CA)CO spectra for residues F89 to D93 of CaM.

$60{ }^{13} \mathrm{C}-{ }^{1} \mathrm{H}$ HSQC spectrum of ${ }^{13} \mathrm{C},{ }^{15} \mathrm{~N}$ CaM/Munc13-1(459-492) complex............ 89

61 Strips extracted from the 3D-(H)CC(CO)NH-TOCSY spectrum................ 90

62 Strips extracted from the 3D-H(CC)(CO)NH-TOCSY spectrum................. 91

63 Strips selected from the 3D-HCCH-TOCSY spectrum to illustrate the sidechain assignment of Valine108 in CaM.

64 2D-HBCB(Caro) spectra for the aromatic side chain proton assignments ( $\mathrm{H} \varepsilon$ and $\mathrm{H} \delta)$

65 3D-HMBC spectrum for methionine $\varepsilon-\mathrm{CH}_{3}$ assignment.

66 Selected amide cross peaks of the 3D- ${ }^{15} \mathrm{~N}$ (edited) NOESY-HSQC for ${ }^{13} \mathrm{C},{ }^{15} \mathrm{~N}$ CaM/Munc13-1(459-492) peptide complex.

67 Selected aliphatic cross peaks of the $3 \mathrm{D}-{ }^{13} \mathrm{C}$ (edited) NOESY-HSQC for ${ }^{13} \mathrm{C},{ }^{15} \mathrm{~N}$ 
CaM/Munc13-1(459-492) peptide complex

68 Selected aliphatic cross-peaks of CaM that show intermolecular NOEs to the Munc13-1(459-492) peptide in the 3D- $\left({ }^{13} \mathrm{C}\right.$-filtered)-NOESY-HSQC.

$69{ }^{15} \mathrm{~N}-{ }^{1} \mathrm{H}$ IPAP-HSQC spectra for ${ }^{15} \mathrm{~N}$ CaM/Munc13-1(459-492) complex with bacteriophage Pf1 as cosolvent and in isotropic conditions

70 Correlation plots for the measured $\mathrm{NH}$ residual dipolar couplings of the ${ }^{15} \mathrm{~N}$ CaM/Munc13-1(459-492) peptide complex aligned sterically with Pf1.

71 Paramagnetic alignment of the ${ }^{15} \mathrm{~N}$ CaM/Munc13-1(459-492) peptide complex with an EDTA-based lanthanide binding tag.

72 Secondary chemical shift for the $\mathrm{H} \alpha$ resonance of CaM in complex with the Munc13-1(459-492) peptide.

73 Secondary chemical shift for the H $\alpha$ resonance of the ${ }^{13} \mathrm{C},{ }^{15} \mathrm{~N}$ Munc13-1(457492) bound to CaM. 102

74 Number of NOE restraints per amino acid residue in CaM and the Munc131(457-492) peptide 102

75 Contact map for the CaM/Munc13-1(457-492) peptide complex. 103

76 Survey of the sequential and medium range ${ }^{1} \mathrm{H}-{ }^{1} \mathrm{H}$ NOEs along the primary sequence of CaM and the Munc13-1 peptide (457-492).

77 Different models for the backbone conformation of the Munc13-1(457-492) peptide. 105

78 Model of the CaM/Munc13-1 docked structure based on intermolecular NOEs and $\mathrm{NH}$ residual dipolar couplings 106

79 CaM/Munc13-1(457-492) peptide complex calculated with CYANA using the full-length CaM and peptide restraints

80 CaM/Munc13-1 (457-492) peptide complex calculated with the C-terminal domain distance constraints for CaM.

81 Ribbon and surface representation of the C-terminal domain of CaM bound to the N-terminal amphiphilic $\alpha$-helix of the Munc13-1 (457-492) peptide. 108

82 Ribbon and surface representations for the N-terminal domain of CaM bound to the C-terminal part of the Munc13-1 (457-492) peptide.

83 Ramachandran plot of the ensemble of 20 structures of the CaM/Munc131(457-492) peptide complex 109

84 Cylinder $\alpha$-helical representation of CaM for interhelical angle measurements. 
85 Overlay of C-terminal domain of CaM/Munc13-1(457-492) peptide complex with the CaM/smMLCK and CaM/CaMKII peptide complex structures

86 Overlay of N-terminal domain of the CaM/Munc13-1(457-492) peptide complex with the CaM/CaMKII peptide complex and the CaM N-terminal structures

87 Molecular structures of pk1 and pk2 paramagnetic tags...................... 112

88 Molecular structures of t43L and t43D paramagnetic tags.................... 112

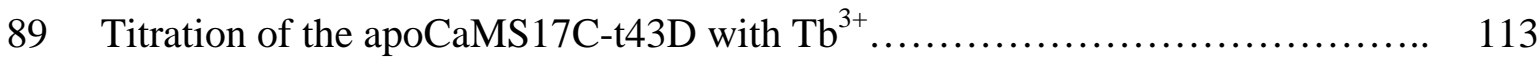

90 Correlation plot of the measured rDCs for the N-terminal domain of

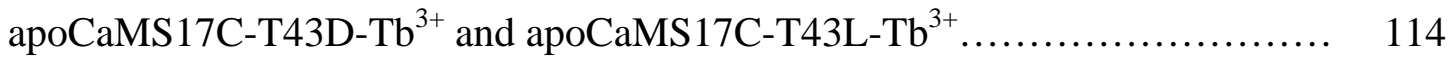

$91{ }^{15} \mathrm{~N}-{ }^{1} \mathrm{H}-\mathrm{HSQC}$ spectra for ${ }^{15} \mathrm{~N}$ apoCaMS17C-t43L and ${ }^{15} \mathrm{~N}$ apoCaM S17C-

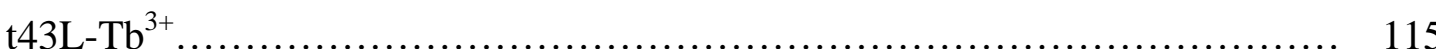

92 Correlation plot between the measured PCS for apoCaMS17C-t43D-Tb ${ }^{3+}$ and apoCaMS17C-t43L-Tb ${ }^{3+}$ against the back-calculated PCS.

93 Correlation plot between the measured PCS for apoCaMS17C-pk2-Tb ${ }^{3+}$ and the back-calculated PCS.

94 Location of the metal ion $\left(\mathrm{Tb}^{3+}\right)$ based on the PCS restraints for the N-terminal domain of apoCaMS17C-t43D, apoCaMS17C-t43L and apoCaMS17C-pk2......

95 Relationship of the measured lanthanide ion- amide distances and the observed PCS for apoCaMS17Ct43D-Tb ${ }^{3+}$ and apoCaMS17Ct43L-Tb ${ }^{3+}$

96 Histogram for the measured residual dipolar couplings of the C-terminal domain of the CaMT146C-pk2-Tb ${ }^{3+} / \mathrm{C} 20 \mathrm{~W}$ peptide complex

97 Histogram for the measured residual dipolar couplings of the $\mathrm{N}$-terminal domain of the CaMT146C-pk2-Tb ${ }^{3+} / \mathrm{C} 20 \mathrm{~W}$ peptide complex....

98 Correlation plots for the measured rDCs for the C-terminal domain and Nterminal domain of the CaMT146C-pk2- $\mathrm{Tb}^{3+} / \mathrm{C} 20 \mathrm{~W}$ peptide complex

$99{ }^{15} \mathrm{~N}-{ }^{1} \mathrm{H}$ HSQC spectra for the holoCaMT146C-t43D aligned with $\mathrm{Tb}^{3+}$ and with $\mathrm{Tm}^{3+}$

100 Correlation plot between the measured rDCs and back-calculated rDCs for the C-terminal domain of CaMT146C-pk2-Tb ${ }^{3+}$...

101 Distribution for the Axial component of the alignment tensor for the CaMT146C-pk2-Tb ${ }^{3+} / \mathrm{C} 20 \mathrm{~W}$ peptide complex....

102 Distribution for the Rhombic component of the alignment tensor for the CaMT146C-pk2-Tb ${ }^{3+} / \mathrm{C} 20 \mathrm{~W}$ peptide complex.... 
103 Mapping of the individual tensor values for the 1000 SVD calculations on a 3D

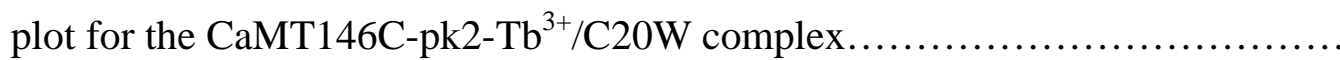

104 Distribution for the axial component (Da) of the alignment tensor for CaMT146C-pk2-Tb ${ }^{3+}$

105 Distribution for the Rhombic component of the alignment tensor for the Cterminal domain of CaMT146C-pk2-Tb ${ }^{3+}$

106 Mapping of the individual tensor values for the 1000 SVD calculations on a 3D plot for CaMT146C-pk2-Tb ${ }^{3+}$

107 Correlation plot of the measured PCS for the C-terminal domain of CaMT146C-pk2- $\mathrm{Tb}^{3+}$ and its complex with the C20W peptide against the backcalculated PCS.

108 Correlation plot between the measured distances between the lanthanide ion and each amide proton and the observed PCS for CaMT146C-pk2-Tb ${ }^{3+}$

109 Correlation plot between the measured distances between the lanthanide ion and each amide proton and the observed PCS for CaMT146C-pk2-Tb ${ }^{3+} / \mathrm{C} 20 \mathrm{~W}$ peptide complex 


\section{List of Tables}

No.

Page

1 Oligonucleotide primers for site-directed mutagenesis

2 NMR spectra acquired for the solution structure determination of the CaM/ Munc13-1(457-492) peptide complex

3 NMR experiments for the sequential backbone and side chain resonance assignment of the ${ }^{13} \mathrm{C},{ }^{15} \mathrm{~N}$ Munc13-1 (457-492) peptide.

4 NMR experiments for the sequential resonance assignment of the backbone and side chain atoms in the ${ }^{13} \mathrm{C},{ }^{15} \mathrm{~N}$ CaM/Munc13-1(459-492) peptide complex

5 Summary of the structure statistics for the NMR solution structure of the CaM/Munc13-1 (457-492) peptide complex

6 Comparison of the interhelical angles for the CaM/Munc13-1(457-492) peptide complex and previous high resolution CaM structures with different EF-hand states. ...

7 Axial (Da-HN) and Rhombic(Rhomb.) components (apoCaMS17C) of the alignment tensors and angles between them achieved for the four paramagnetic tags bound to Terbium.

8 Axial (Da-HN) and Rhombic(Rhomb.) components of the alignment tensors and angles between them achieved for the holoCaM samples aligned with Terbium....

9 Axial (Da-HN) and Rhombic(Rhomb.) components of the alignment tensors and angles between them achieved the CaMT146C/C20W complex aligned with Terbium. 


\section{INTRODUCTION}

\section{Signal transduction and second messengers}

Calcium is one of the most important signaling entities in the living cell and its intracellular levels are tightly regulated; at high concentrations calcium is also toxic for cells. Calcium is known as a "second messenger", since its concentration is regulated after a first signal arrives to the cell. Most intracellular $\mathrm{Ca}^{2+}$ ions are sequestered in the mitochondria and endoplasmic reticulum (ER) or other vesicles. Cells employ various mechanisms for regulating the concentration of free $\mathrm{Ca}^{2+}$ ions in the cytosol, which usually is kept below $0.2 \mu \mathrm{M}$. Ca ${ }^{2+}$-ATPases pump cytosolic $\mathrm{Ca}^{2+}$ ions across the plasma membrane to the cell exterior or into the lumens of the endoplasmic reticulum or other intracellular vesicles that store $\mathrm{Ca}^{2+}$ ions. (Lodish et al., 2000).

The precise spatial control of $\mathrm{Ca}^{2+}$ release from intracellular stores also plays an important role in cell physiology. Localized increases in the cytosolic level of free $\mathrm{Ca}^{2+}$ are critical to its function as a second messenger. In secretory cells, such as insulinproducing $\beta$ cells in the pancreatic islets, a rise in $\mathrm{Ca}^{2+}$ triggers the exocytosis of secretory vesicles and the release of insulin. (Lodish et al., 2000).

Other important cellular second messengers are lipidic in nature; phosphoinositides can be cleaved by the membrane associated enzyme phospholipase $\mathrm{C}$ (PLC) to generate second messengers. These cleavage reaction produce 1,2diacylglycerol (DAG), a lipophilic molecule that remains linked to the membrane, and free phosphorylated inositols, which can diffuse into the cytosol. The main pathway generates DAG and inositol 1,4,5-trisphosphate ( $\left.\mathrm{IP}_{3}\right)$. (Lodish et al., 2000).

Since $\mathrm{IP}_{3}$ is water-soluble it diffuses within the cytosol carrying a hormone signal from the cell surface to the ER surface. $\mathrm{IP}_{3}$ binds to a $\mathrm{Ca}^{2+}$-channel protein composed of four identical subunits, each containing an $\mathrm{IP}_{3}$ binding site in the large $\mathrm{N}$-terminal cytosolic domain. $\mathrm{IP}_{3}$ binding induces opening of the channel allowing $\mathrm{Ca}^{2+}$ ions to exit from the ER into the cytosol. The resulting rise in the cytosolic $\mathrm{Ca}^{2+}$ level is only transient because of the activity of the $\mathrm{Ca}^{2+}$-ATPase pumps. In addition, within a second of its generation, $\mathrm{IP}_{3}$ is hydrolyzed to inositol 1,4-bisphosphate, which does not stimulate $\mathrm{Ca}^{2+}$ release from the ER. The $\mathrm{IP}_{3}$ receptor in the ER membranes is regulated in a complex manner, which give rise to rapid oscillations in the cytosolic $\mathrm{Ca}^{2+}$ level when the $\mathrm{IP}_{3}$ pathway in cells is stimulated. (Lodish et al., 2000).

After the action of PLC on phosphatidyl-inositol phosphate, the diacylglycerol (DAG) remains associated with the membrane. The principal function of DAG is to activate a family of plasma-membrane protein kinases collectively termed protein kinase $\mathrm{C}$. In the absence of hormone stimulation, protein kinase $\mathrm{C}$ is present as a soluble cytosolic protein that is catalytically inactive. A rise in the cytosolic $\mathrm{Ca}^{2+}$ level causes protein kinase to bind to the cytosolic leaflet of the plasma membrane, where it can be activated by the membrane-associated DAG. Thus, activation of protein kinase $\mathrm{C}$ depends on both $\mathrm{Ca}^{2+}$ ions and DAG, suggesting an interaction between the two branches of the inositol-lipid signaling pathway. (Lodish et al., 2000).

The activation of protein kinase $\mathrm{C}$ in different cells results in a varied array of celluar responses, indicating that it plays a key role in many aspects of cellular growth and metabolism. In liver cells, for instance, protein kinase $\mathrm{C}$ helps regulate glycogen 
metabolism by phosphorylating glycogen synthase, yielding the inactive form of this enzyme. Protein kinase $\mathrm{C}$ also phosphorylates various transcription factors; depending on the cell type, these induce or repress synthesis of certain messenger RNAs. (Lodish et al., 2000).

\section{Synapses and calcium signaling in neurons}

Synapses generally transmit signals in only one direction: an axon terminal from the presynaptic cell sends signals that are picked up by the postsynaptic cell. There are two general types of synapse: the relatively rare electric synapse and the chemical synapse (see Figure 1). In the later type of synapse, the axon terminal of the presynaptic cell contains vesicles filled with a particular neurotransmitter. The postsynaptic cell can be a dendrite or cell body of another neuron, a muscle or gland cell. When an action potential in the presynaptic cell reaches an axon terminal, it induces a localized rise in the level of $\mathrm{Ca}^{2+}$ in the cytosol. This, in turn, causes some of the vesicles to fuse with the plasma membrane, releasing their contents in to the synaptic cleft, the narrow space between the cells. The neurotransmitters diffuse across the synaptic cleft; it takes about 0.5 milliseconds (ms) for them to bind to receptors on postsynaptic cells. (Lodish et al., 2000).

Binding of the neurotransmitter triggers changes in the ion permeability of the postsynaptic plasma membrane, which, in turn, changes the membrane's electric potential at this point. If the postsynaptic cell is a neuron, this electric disturbance may be sufficient to induce an action potential. In some cases, enzymes attached to the fibrous network connecting the cells destroy the neurotransmitter after it has functioned; in other cases, the signal is terminated when the neurotransmitter diffuses away or is transported back into the presynaptic cell. (Lodish et al., 2000).

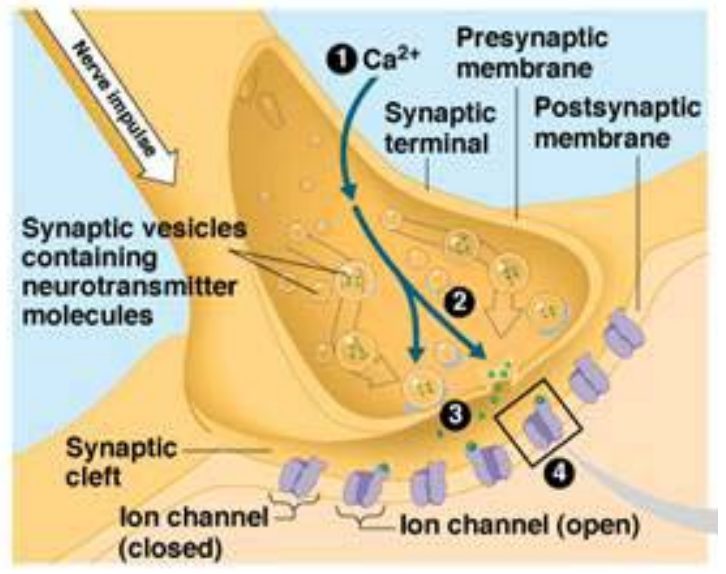

Figure 1. Anatomy of a typical chemical synapse. The inner structure of the presynaptic button is also shown (taken from FSU, 1999).

The exocytosis of neurotransmitters from synaptic vesicles involves vesicletargeting and fusion events similar to those that occur at many points in the secretory pathway. The same types of proteins- including T-SNARE and V-SNAREs, $\alpha, \beta$, and $\gamma$ SNAP proteins, and NSF- participate in both systems. However, exocytosis of neurotransmitters at chemical synapses differs from other secretory pathways in two 
critical ways: (a) Secretion is tightly coupled to arrival of the action potential at the axon terminus, and (b) synaptic vesicles are recycled locally after fusion with the plasma membrane, a process that takes less than one minute. (Lodish et al., 2000).

Depolarization of the plasma membrane cannot, by itself, cause synaptic vesicles to fuse with the plasma membrane. In order to trigger vesicle fusion, an action potential must be transduced into a chemical signal - namely, a localized rise in the cytosolic $\mathrm{Ca}^{2+}$ concentration. The transducers of the electric signals are voltage-gated $\mathrm{Ca}^{2+}$ channels localized to the region of the plasma membrane adjacent to the synaptic vesicles. The membrane depolarization due to arrival of an action potential opens these channels, permitting an influx of $\mathrm{Ca}^{2+}$ ions into the cytosol from the extracellular medium. The amount of $\mathrm{Ca}^{2+}$ that enters an axon terminal through voltage-gated $\mathrm{Ca}^{2+}$ channels is sufficient to raise the level of $\mathrm{Ca}^{2+}$ in the region of the cytosol near the synaptic vesicles from $<0.1 \mu \mathrm{M}$, characteristic of the resting state, to $1-100 \mu \mathrm{M} . \mathrm{Ca}^{2+}$ ions binds to proteins that connect the synaptic vesicle with the plasma membrane, inducing membrane fusion and thus exocytosis of the neurotransmitter. The extra $\mathrm{Ca}^{2+}$ ions are rapidly pumped out of the cell by $\mathrm{Ca}^{2+}$-ATPases, lowering the cytosolic $\mathrm{Ca}^{2+}$ level and preparing the terminal to respond again to an action potential. (Lodish et al., 2000).

Patch-clamping experiments show that voltage-gated $\mathrm{Ca}^{2+}$ channels, like voltagegated $\mathrm{Na}^{+}$channels, open transiently upon depolarization of the membrane. Two pools of neurotransmitter-filled synaptic vesicles are present in axon terminals: those "docked and primed" at the plasma membrane, which can be readily exocytosed, and those in reserve in the active zone near the plasma membrane. Each rise in $\mathrm{Ca}^{2+}$ triggers exocytosis of about 10 percent of the docked vesicles. (Lodish et al., 2000).

\section{Synaptic vesicles}

Synaptic vesicles are small abundant organelles and homogeneous in size ( 20 $\mathrm{nm}$ radius). Their only known function is to take up and release neurotransmitters. Purified vesicles have a protein:phospholipid ratio of 1:3. Many proteins that have been associated with synaptic vesicles are probably present only on a subset of vesicles or bind transiently to the vesicles, and the number of proteins that are constitutive parts of all synaptic vesicles may be comparatively small. (Südhof, 2004). They can be readily purified from the brain and their proteins isolated. The synaptic-vesicle membrane contains V-type ATPases, which generate a low intravesicular $\mathrm{pH}$, and a proton-coupled neurotransmitter antiporter, which imports neurotransmitters from the cytosol. (Lodish et al., 2000). They also contain transport proteins including intrinsic membrane proteins; proteins associated via posttranslational lipid modifications, and peripherally bound proteins. (Südhof, 2004).

The vesicles are linked together by synapsin, a fibrous phosphoprotein structurally related to other cytoskeletal proteins that bind the fibrous proteins actin and spectrin. Synapsin is localized to the cytosolic surface of all synaptic-vesicle membranes and constitutes 6 percent of vesicle proteins. Thicker filaments radiate from the plasma membrane and bind to vesicle-associated synapsin; probably these interactions keep the synaptic vesicles close to the part of the plasma membrane facing the synapse. Synapsin knockout mice, although viable, are prone to seizures; during repetitive stimulation of many neurons in such mice, the number of synaptic vesicles that fuse with the plasma 
membrane is greatly reduced. Thus synapsins are thought to recruit synaptic vesicles to the active zone. Synapsins are substrates of cAMP-dependent and $\mathrm{Ca}^{2+}$-calmodulin $(\mathrm{CaM})-$ dependent protein kinases, a rise in cytosolic $\mathrm{Ca}^{2+}$ triggers their phosphorylation, causing their release from the cytoskeleton increasing the number of vesicles available for fusion with the plasma membrane. (Lodish et al., 2000).

Rab3A is a neuron-specific GTP-binding protein similar in sequence and function to other Rab proteins that control vesicle traffic in the secretory pathway. Rab3A is located in the membrane of synaptic vesicles and appears to be essential for localization of vesicles to the active zone. Rab3A knockout mice are viable, but repetitive stimulation of certain neurons in such mice causes a reduction in the number of synaptic vesicles able to fuse with the plasma membrane. (Lodish et al., 2000).

The principal V-SNARE in synaptic vesicles is VAMP (vesicle-associated membrane protein), also called synaptobrevin. This V-SNARE binds syntaxin and SNAP25, the principal T-SNAREs in the plasma membrane of axon terminals. SNAP proteins and NSF assist in the disassociation of VAMP from T-SNAREs after vesicle fusion. (Lodish et al., 2000).

Another protein in the synaptic vesicle membrane called synaptotagmin contains four $\mathrm{Ca}^{2+}$ binding sites in its cytosolic domain. Several types of evidence support the hypothesis that synaptotagmin is the key $\mathrm{Ca}^{2+}$-sensing protein that triggers fast vesicle exocytosis. (Lodish et al., 2000).

At low $\mathrm{Ca}^{2+}$ levels found in resting cells, synaptotagmin apparently binds to a complex of the plasma membrane proteins neurexin and syntaxin. The presence of synaptotagmin blocks the binding of other essential fusion proteins to the neurexinsyntaxin complex, thereby preventing vesicle fusion. When synaptotagmin binds $\mathrm{Ca}^{2+}$ it is displaced from the complex, allowing other proteins to bind and thus initiating membrane docking and fusion. (Lodish et al., 2000)

Neurotransmitter-filled synaptic vesicles are docked at a specialized region of the presynaptic plasma membrane known as the active zone. The docked vesicles then go through a maturation process called priming to become fusion competent. Voltage-gated $\mathrm{Ca}^{2+}$ channels open during an action potential. The resulting pulse of intracellular $\mathrm{Ca}^{2+}$ triggers fusion-pore opening of ready-releasable vesicles. In most synapses, release is stimulated by $\mathrm{Ca}^{2+}$ influx through P/Q-(Cav2.1) or N-type $\mathrm{Ca}^{2+}$-channels $\left(\mathrm{Ca}_{\vee} 2.2\right)$. Even at rest, synapses have a finite but low probability of release; causing spontaneous events of exocytosis that are reflected in electrophysiological recordings as miniature postsynaptic currents. $\mathrm{Ca}^{2+}$ influx triggers at least two components of release that are probably mechanistically distinct: A fast, synchronous phasic component is induced rapidly, in as little as $50 \mu \mathrm{s}$ after a $\mathrm{Ca}^{2+}$ transient develops, and a slower asynchronous component continues for $>1 \mathrm{~s}$ as an increase in the rate of spontaneous release after the action potential. Both components of release are strictly $\mathrm{Ca}^{2+}$ dependent but change differentially upon repetitive stimulation. (Südhof, 2004).

\section{Brain modulator protein: calmodulin}

Initial biochemical studies on calmodulin $(\mathrm{CaM})$ already pointed towards a high expression level in the brain; this protein was identified as a modulator of $3^{\prime}: 5$ '-cyclic nucleotide phosphodiesterase. The modulator protein represents approximately $1 \%$ of the 
soluble protein present in the initial bovine brain homogenate supernatant. The overall yield of bovine brain modulator protein obtained was $40 \mathrm{mg} / \mathrm{kg}$ of brain $(13 \%$ from the original homogenate). The bovine brain modulator has two sets of $\mathrm{Ca}^{2+}$ - binding sites. High affinity binding $\left(k_{\mathrm{d}}=1 \times 10^{-6} \mathrm{M}\right)$ of $2 \mathrm{~mol}$ of $\mathrm{Ca}^{2+}$ bound/18,000 $\mathrm{g}$ of protein was observed. Two additional mol of $\mathrm{Ca}^{2+} / 18,000 \mathrm{~g}$ of protein bound with a dissociation constant of $8.6 \times 10^{-4} \mathrm{M}$. A number of other $\mathrm{Ca}^{2+}$ - binding proteins have been isolated from brain, the S-100 protein fraction from brain, and other small acidic $\mathrm{Ca}^{2+}$ binding proteins Wolff et al., 1972 have reported the isolation and characterization of a $\mathrm{Ca}^{2+}$ binding phosphoprotein from porcine brain, later it was known that $\mathrm{CaM}$ can be phosphorylated. The physicochemical properties of bovine brain modulator protein are very similar to those of rabbit skeletal muscle Troponin $\mathrm{C}$, which regulates myosin action in muscle contraction. This modulator protein arose early in eukaryotic evolution as a mediator of calcium second messenger function, and the interaction sites on both modulator protein and the enzymes it regulates have been highly conserved (Watterson et al., 1980).

$\mathrm{CaM}$ is expressed in high concentrations in the brain (from 10 to $100 \mu \mathrm{M}$ in different areas of the brain). The average dissociation constant $\left(k_{\mathrm{d}}\right)$ for the four $\mathrm{Ca}^{2+}$ binding sites of $\sim 15 \mu \mathrm{M}$, the physiological calcium concentrations lies between 0.1 and $10 \mu \mathrm{M}$, this apparent discrepancy was resolved when it was shown that CaM-protein complexes show heterotropic positive cooperativity for $\mathrm{Ca}^{2+}$ binding. This allows CaMregulated proteins to be activated in the presence of normal physiological concentrations of free $\mathrm{Ca}^{2+}$. (Xia and Storm, 2005).

In resting neurons, much of the $\mathrm{CaM}$ is associated with three proteins: neuromodulin, neurogranin and regulator of CaM signaling (RCS), which are thought to regulate the levels of free $\mathrm{CaM}$ or control the activity of CaM. Neuromodulin contributes to synaptic plasticity by controlling the levels of free CaM available presynaptically. (Xia and Storm, 2005)

\section{The EF-hand superfamily of proteins: calmodulin plasticity}

Each CaM molecule binds four $\mathrm{Ca}^{2+}$ ions (see Figure 2). Binding of $\mathrm{Ca}^{2+}$ causes $\mathrm{CaM}$ to undergo a conformational change that enables the $\mathrm{Ca}^{2+}-\mathrm{CaM}$ complex to bind to and activate many enzymes, such as myosin light-chain kinase (MLCK), which regulates myosin activity. Because $\mathrm{Ca}^{2+}$ binds to $\mathrm{CaM}$ in a cooperative fashion, a small change in the level of cytosolic $\mathrm{Ca}^{2+}$ leads to a large change in the level of active CaM. The $\mathrm{Ca}^{2+}$ CaM complex also activates several protein kinases that, in turn, phosphorylate transcription factors, thereby modifying their activity and regulating gene expression. (Lodish et al., 2000).

The CaM superfamily is a major class of $\mathrm{Ca}^{2+}$ sensor proteins, which collectively play a crucial role in various cellular signaling cascades through regulation of numerous target proteins in a $\mathrm{Ca}^{2+}$ dependent manner. (Ikura and Ames, 2006).

CaM contains four EF-hand motifs; with highly conserved amino acid sequences in all eukaryotes (see Figure 3). Typically, a pair of EF-hand motifs in tandem array constitutes a stable structural unit, together generating cooperativity in the binding of $\mathrm{Ca}^{2+}$ ions. Many EF-hand proteins, such as CaM and members of the neuronal calcium 
sensor family, consist of four EF-hand motifs. This results in two globular structural units in a single protein. (Ikura and Ames, 2006).

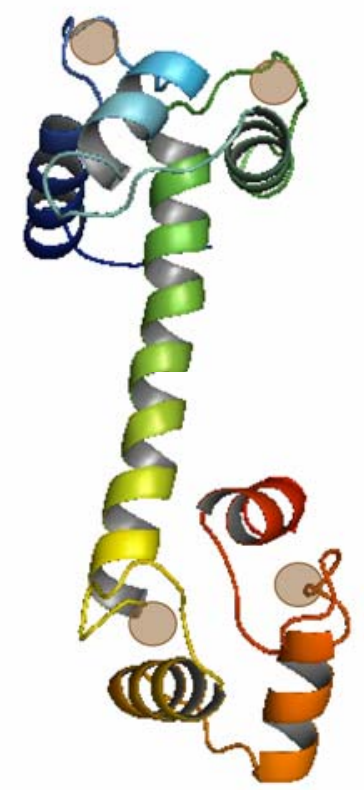

Figure 2. Molecular model of CaM X-ray crystallographic structure (Babu et al., 1988). The calcium ions are depicted as brown spheres.

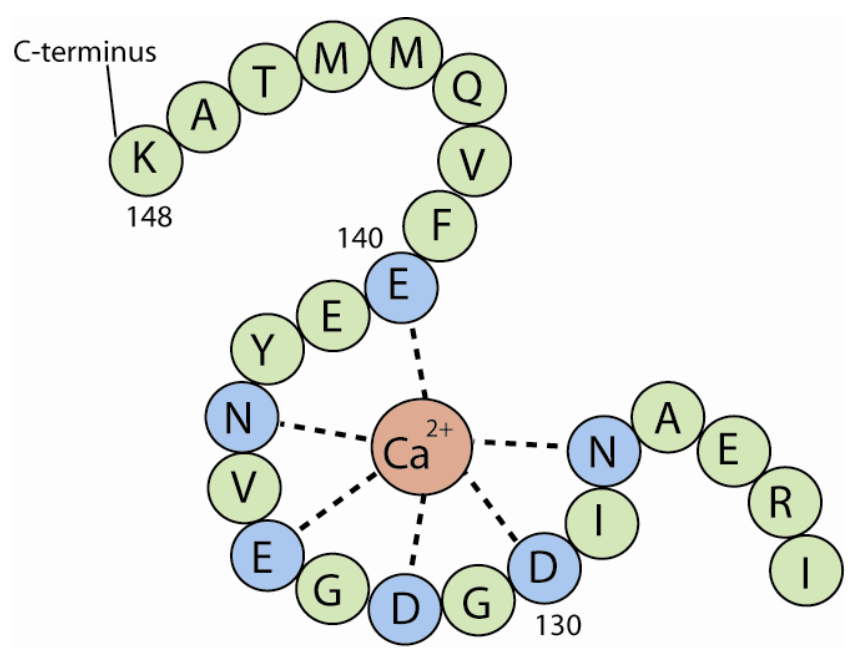

Figure 3. Model of the EF-hand motif. The coordination of a calcium ion is mediated by six amino acid residues from the loop, shown in blue (adapted from Lodish et al., 2000).

The direct interaction with $\mathrm{Ca}^{2+}$ enables these $\mathrm{Ca}^{2+}$ sensor proteins to change their conformation from the inactive state $(\mathrm{P})$ to the intermediate state $\left(\mathrm{Ca}^{2+}-\mathrm{P}^{*}\right)$, which is a prerequisite to the formation of an active conformation in complex with a target $\left(\mathrm{Ca}^{2+}\right.$ $\left.\mathrm{P}^{* *}-\mathrm{E}^{*}\right)$ required to transform the target protein from its inactive state $(\mathrm{E})$ to the active state $\left(\mathrm{E}^{*}\right)$

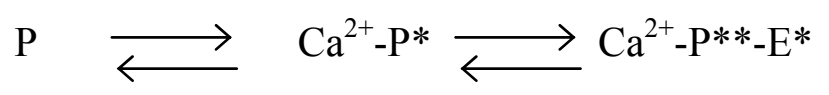


The first conformational transition is essential to the $\mathrm{Ca}^{2+}$ sensory function and is universal to all $\mathrm{Ca}^{2+}$ sensor proteins. The second conformation change plays a critical role in the activation and recognition of specific targets. (Ikura and Ames, 2006).

$\mathrm{CaM}$ regulates numerous target proteins that are functionally and structurally diverse. The first structure determined for $\mathrm{CaM}$ in complex with a target protein showed a remarkable conformational change in CaM's two EF-hand domains upon binding to a peptide derived from myosin light chain kinase (MLCK). This structure revealed that the central domain linker is highly flexible and can be bent dramatically upon binding to the target protein. The flexibility of the domain linker permits the orientation of the two domains of $\mathrm{CaM}$ to change independently to accommodate the structural nature of the target protein. In addition, two hydrophobic anchoring residues from the smooth muscle MLCK peptide (Trp-800 and Leu-813) bind simultaneously to the hydrophobic pocket in $\mathrm{N}$ - and $\mathrm{C}$-terminal domains, which is extremely rich in methionine residues. (Ikura and Ames, 2006).

$\mathrm{CaM}$ can adopt largely different, global conformations depending on the structural entity that $\mathrm{CaM}$ binds. In addition to plasticity of the protein fold, the amino acid side chains that interact with target proteins, in particular the methionine residues are remarkably flexible. The protein conformational plasticity of $\mathrm{CaM}$ emerged as a means of achieving functional diversity rather than employing the more traditional approach of genetic polymorphism. (Ikura and Ames, 2006).

Among the numerous $\mathrm{CaM}$ target proteins, $\mathrm{CaM}$-dependent serine/theronine kinases are the substrate family best characterized with respect to their structural and functional CaM interaction mechanisms. Previous studies on CaM kinase II, CaM kinase kinase, and myosin light chain kinases showed that $\mathrm{CaM}$ could assume at least three different conformations by virtue of a flexible linker connecting two globular domains responsible for binding both $\mathrm{Ca}^{2+}$ ions and its target proteins. These interactions all involve short helices (approximately 20 residues) formed by the CaM binding domain of the kinases. From these complexes, defined $\mathrm{CaM}$ recruitment motifs currently fall into three groups $\left(1-10,1-14\right.$, and $\left.1-16^{-}\right)$, which are distinguished by their spacing of bulky hydrophobic and basic amino acid residues (see Figure 4). The binding mode of these different synthetic peptides was resolved when the atomic resolution structures of three CaM-peptide complexes from smooth muscle myosin light chain kinase (smMLCK) (Meador et al., 1992), skeletal muscle myosin light chain kinase (skMLCK) (Ikura et al., 1992) and CaM-dependent kinase II $\alpha$ (Meador et al., 1993).

Another CaM binding motif is the so-called "IQ motif", corresponding to an IQxxxRGxxxR consensus sequence, which appears in tandem repeats and bind multiple $\mathrm{CaM}$ molecules in a predominantly $\mathrm{Ca}^{2+}$ independent manner. (Hoeflich and Ikura, 2002).

CaM-dependent serine/theronine protein kinases and the phophatase calcineurin are all known to use a similar mechanism underlying CaM-dependent activation, namely autoinhibitory domain (AID) displacement. In these cases, the CaM binding site is often adjacent or within an autoinhibitory domain of the enzyme which contains a pseudosubstrate region. CaM binding to the target induces a conformational rearrangement that displaces the pseudosubstrate inhibitory domain and allows for full enzyme activity (see Figure 5). (Hoeflich and Ikura, 2002). 


\section{1-16- Motif \\ 1516 \\ CaMKK VRVIPRLDTLILVKAMGHRKRFGNPFR}

\section{1-5-10 Motif $1 \quad 5 \quad 10$ \\ CaMKII ARRKLKGAILTTMLATRNFS \\ CaMKI AKSKWKQAFNATAVVRHM}

\section{1-5-8-14 Motif \\ skMLCK(M13) \\ Ca ${ }^{2+}$-pump(C20W) QILWFRGLNRIQTQIK \\ $\mathrm{Ca}^{2+}$-pump(C24W) QILWFRGLNRIQTQIRVVNAFSSS}

Figure 4. CaM peptide recognition motifs. The hydrophobic anchors of the amphiphilic $\alpha$-helix are highlighted in red

The crystal structure of Bacillus anthracis adenylyl cyclase (Drum et al., 2002), the edema factor (EF) of this pathogenic bacterium revealed a new CaM interaction mode. The EF activation involves active site remodeling. This process involves rearrangement of key switches to create the active site from an area that is totally solvent exposed in the CaM-free state. CaM trapping by EF may be a key factor in regulating its selective activity in the host cells. EF might also be more stable in the CaM-bound state as compared to the $\mathrm{CaM}$-free state, thereby increasing resistance to proteolytic turnover and lengthening its half-life time in cells. (Hoeflich and Ikura, 2002).

In addition to the release of autoinhibitory domains and active site remodeling, a third mechanism is now known for $\mathrm{CaM}$ action: $\mathrm{CaM}$-induced dimerization of membrane proteins as a system underlying ion channel activation. (Hoeflich and Ikura, 2002).

Small conductance $\mathrm{Ca}^{2+}$-activated potassium (SK) channels play a fundamental role in regulating neuronal excitability. SK channels use $\mathrm{K}^{+}$as their charge carrier, but are gated solely by intracellular $\mathrm{Ca}^{2+}$ ions at the sub-micromolar level, such as occur during an action potential in many neurons. (Hoeflich and Ikura, 2002).

A unique feature of SK channel $\alpha$-subunits is constitutive association with CaM. This interaction is maintained both in the presence or absence of $\mathrm{Ca}^{2+}$. However, channel gating also requires a $\mathrm{Ca}^{2+}$-dependent interaction. Schumacher and colleagues (2001) have shown from the crystal of the $\mathrm{Ca}^{2+}$-loaded CaM/SK2 complex that the complex presents as two CaM molecules tightly sandwiching two $\mathrm{K}^{+}$channel domains. This is the first observation of a 2:2 CaM-target complex and the active tetrameric form of the channel would likely require two sets of 2:2 dimers. CaM uses a modular strategy in which the carboxy-terminal EF hands mediate tethering to the channel and the aminoterminal EF hands are responsible for $\mathrm{Ca}^{2+}$-induced dimerization leading to channel gating and direct coupling between changes in intracellular $\mathrm{Ca}^{2+}$ concentrations and altered membrane potential. In this 2:2 arrangement, CaM adopts a very elongated structure thereby facilitating interaction of single CaM molecule with $3 \alpha$ helices. (Hoeflich and Ikura, 2002).

$\mathrm{CaM}$ has been also shown to play a prominent role in modulating the hallmark feedback inhibition or CDI ( $\mathrm{Ca}^{2+}$-dependent inactivation) of P/Q- and L-type voltage- 
dependent $\mathrm{Ca}^{2+}$ channels, and cyclic nucleotide-gated channels of the visual and olfactory systems. Fallon and collaborators (2005) solved the crystal structure of CaM bound to the hydrophobic IQ domain of the cardiac $\mathrm{Ca}_{\mathrm{V}} 1.2$ calcium channel. The structure shows that parallel binding of CaM to the IQ domain is governed by hydrophobic interactions and it represents the conformation that $\mathrm{CaM}$ assumes in calcium dependent facilitation. In the case of L-type channels, $\mathrm{Ca}^{2+}$-sensitive CaM association occurs via a standard IQ motif in the pore-forming $\alpha_{1}$ subunit of the channel. As in the case with SK channels, binding of $\mathrm{Ca}^{2+}$ to only a single CaM lobe is sufficient to trigger gating, in this channel system, it is the $\mathrm{C}$-terminal $\mathrm{CaM}$ domain which binds the $\mathrm{Ca}^{2+}$ ions. The picture that emerges is one in which $\mathrm{CaM}$ interacts in a bipartite manner with ion channels: one CaM module mediating constitutive binding and the other transmitting $\mathrm{Ca}^{2+}$ dependence. (Hoeflich and Ikura, 2002).
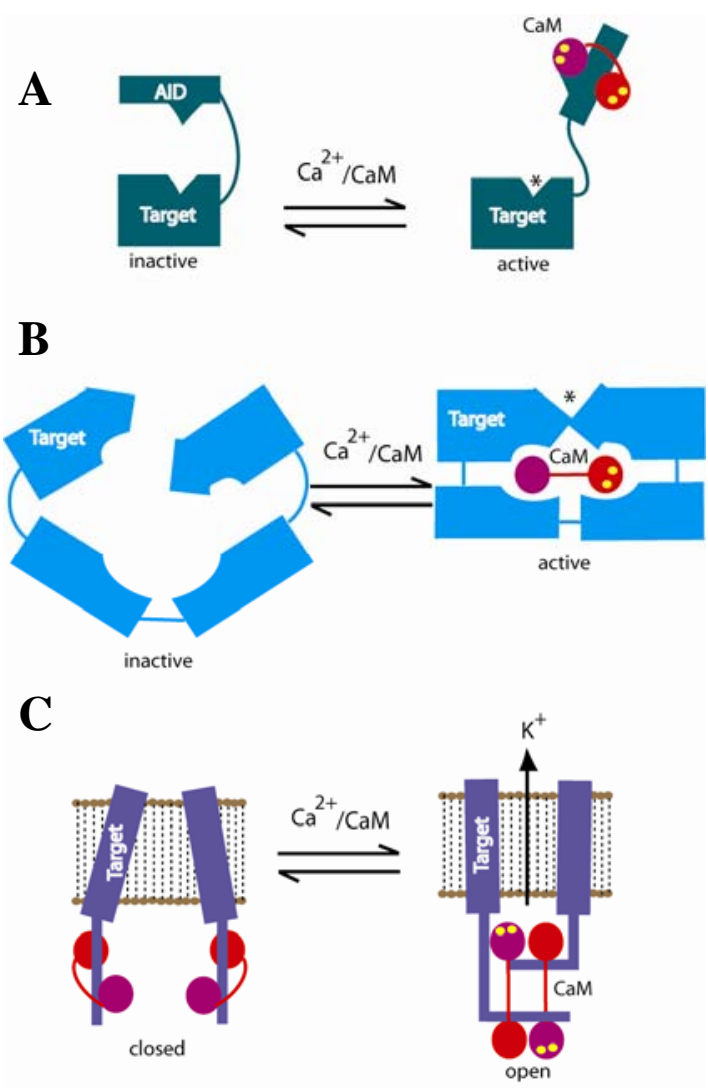

Figure 5. Different activation models for CaM binding partners. A. Relieving autoinhibition. B. Active site remodeling. C. Dimerization. Calcium ions are depicted in yellow and active sites by asterisks (adapted from Hoeflich and Ikura, 2002).

Most physiological relevant $\mathrm{CaM}$ targets are proteins, but $\mathrm{CaM}$ also binds to a number of synthetic peptides corresponding to $\mathrm{CaM}$ binding domains, to peptide hormones and toxins, as well as to small drug like molecules. (Vetter and Leclerc, 2003).

The $\mathrm{CaM} / \mathrm{CaMKII} \alpha$ complex is of ellipsoidal shape and much more compact (50 x 30 x $30 \AA$ ) than $\mathrm{Ca}^{2+} / \mathrm{CaM}$ without the peptide (maximal length of $65 \AA$ ). The central linker region is unwound and allows the C-and $\mathrm{N}$-terminal lobes to bend by $100^{\circ}$ and to rotate by $120^{\circ}$ relative to their orientation seen in the $\mathrm{Ca}^{2+} / \mathrm{CaM}$ crystal structure. The 
peptide is bound in an $\alpha$-helical conformation and is engulfed by $\mathrm{Ca}^{2+} / \mathrm{CaM}$ into a hydrophobic channel formed by bringing the $\mathrm{C}$ - and $\mathrm{N}$-terminal lobes close together. (Vetter and Leclerc, 2003).

Matsubara and collaborators (2004) solved the crystal structure of $\mathrm{Ca}^{2+}-\mathrm{CaM}$ bound to a myristoylated peptide derived from the N-terminal domain of CAP-23/NAP22. The binding of CAP-23/NAP-22 to CaM is dependent on myristoylation; only the myristoylated recombinant protein binds to $\mathrm{CaM}$.

Yap and collaborators (2003) solved the solution structure of CaM in complex with a peptide from the plant glutamate decarboxylase (GAD). The interaction is atypical, since the $\mathrm{C}$-terminal domain of $\mathrm{CaM}$ of GAD interacts simultaneously with two GAD peptides.

The domain orientation of CaM in the CaM-GADp complex is affected by the large dimeric peptide volume. This volume is significantly larger $\left(5600 \AA^{3}\right)$ than the peptide volumes in the CaM crystal complexes with smMLCKp $\left(1765 \AA^{3}\right)$, CaMdependent protein kinase I peptide $\left(2413 \AA^{3}\right), \mathrm{Ca}^{2+} / \mathrm{CaM}$-dependent protein kinase II peptide $\left(1611 \AA^{3}\right)$ and $\mathrm{Ca}^{2+} / \mathrm{CaM}$-dependent protein kinase kinase peptide $\left(2275 \AA^{3}\right)$. In accommodating the larger GADp volume, $\mathrm{CaM}$ does not wrap around the peptides completely. Backbone $\left\{{ }^{1} \mathrm{H}\right\}-{ }^{15} \mathrm{~N}$ NOE relaxation measurements indicate that in complex with GADp, the interdomain linker exhibits some flexibility but is more rigid compared to $\mathrm{Ca}^{2+}$-loaded CaM. (Yap et al., 2003).

\section{Regulated exocytosis}

Fusion of a transport vesicle with its target membranes is a fundamental process essential to cellular organization and function of all eukaryotic cells. Several protein families involved in fusion are conserved from yeast to human, and are shared not only by constitutive and regulated exocytosis but also by various intracellular membrane fusion events. The conserved protein families include soluble N-ethyl-maleimidesensitive factor attachment protein receptors (SNAREs), ATPase N-ethylmaleimidesensitive factor (NSF), Munc18/Sec1, RabGTPases, and protein components of the exocyst complex. (Li and Chin, 2003).

Compared with other forms of membrane fusion, synaptic vesicle exocytosis is much more tightly regulated both in time and space. Temporally, $\mathrm{Ca}^{2+}$-triggered secretion of neurotransmitters occurs in less than a millisecond after the arrival of an action potential. Spatially, synaptic vesicle exocytosis takes place only at the active zone, and nowhere else along the axonal membrane. Synaptic vesicle exocytosis utilizes several regulatory components, including synaptotagmin, complexin, Munc13, and RIM, which do not have homologues in yeast. ( $\mathrm{Li}$ and Chin, 2003).

The best-characterized candidate $\mathrm{Ca}^{2+}$ sensor in triggering fast neurotransmitter release is synaptotagmin, an integral membrane protein of synaptic vesicles with two $\mathrm{Ca}^{2+}$-binding $\mathrm{C}_{2}$ domains. The $\mathrm{C}_{2} \mathrm{~A}$ domain of synaptotagmin has been shown to bind three $\mathrm{Ca}^{2+}$ ions, whereas the $\mathrm{C}_{2} \mathrm{~B}$ domain binds two $\mathrm{Ca}^{2+}$ ions. Synaptotagmin binds via both $\mathrm{C}_{2}$ domains to phospholipids in a $\mathrm{Ca}^{2+}$-dependent manner with half-maximal binding $\mathrm{Ca}^{2+}$ concentrations in the range of $5-20 \mu \mathrm{M}$, which correspond very well with physiological $\mathrm{Ca}^{2+}$ levels for triggering neurotransmitter release at some synapses. ( $\mathrm{Li}$ and Chin, 2003). 
Complexin I and II are two closely related $\sim 15-\mathrm{kDa}$ cytosolic proteins originally identified based on their brain-specific expression and ability to bind neuronal SNARE complexes. Double-knockout mice lacking both complexins are perinatally lethal with a marked deficit in fast $\mathrm{Ca}^{2+}$-triggered neurotransmitter release, suggesting that complexins positively regulate a late step of synaptic vesicle exocytosis. Complexin and synaptotagmin are able to bind simultaneously to the assembled SNARE complex. (Li and Chin, 2003).

A crucial step in synaptic vesicle exocytosis is priming, which confers fusion competence to docked vesicles, enabling them to undergo rapid exocytosis upon $\mathrm{Ca}^{2+}$ influx. (Li and Chin, 2003).

Synaptic vesicle priming requires the Munc13/UNC-13 family of proteins. UNC13 was originally identified in C.elegans genetic screens for uncoordinated mutants. Munc13s are mammalian UNC-13 homologues; they are 200-kDa multidomain proteins (see Figure 6) which are encoded by three distinct genes: Munc13-1, Munc13-2, and Munc13-3. These Munc13 isoforms are differentially expressed in brain, where they are specifically localized to the active zone (Silinsky and Searl, 2003).

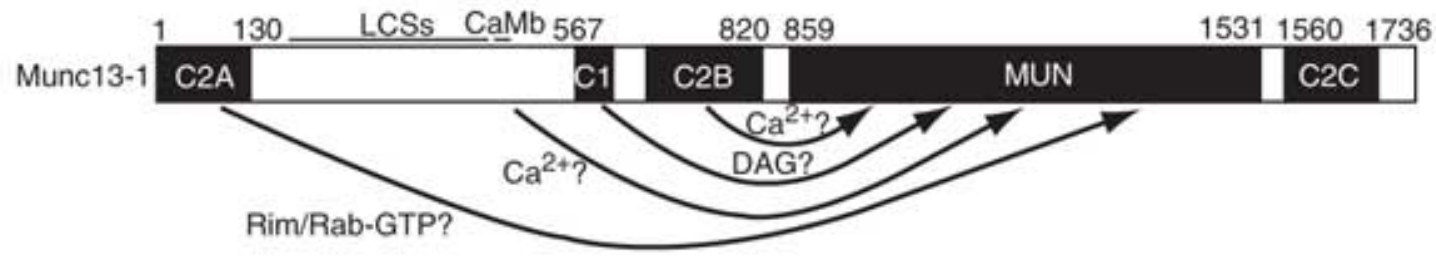

Figure 6. Domain structure of Munc13-1. Arrows illustrate the regulation of MUN domain activity by the diverse agents that mediate presynaptic plasticity processes at the $\mathrm{N}$-terminal region of Munc13-1. Low-complexity sequences (LCSs) and the CaM binding domain $(\mathrm{CaMb})$ are represented by thin lines above. (Taken from Basu et al., 2005).

Neurotransmitter release was completely eliminated in the deletion mutants of $C$. elegans and Drosophila, and this effect is independent on the nature of the neutortransmitter. When the munc13-1 isoform was deleted in mice, the glutamate release was decreased by $90 \%$. This decrease in secretion was associated with a decrease in the RRP (and thus primed vesicles) as assessed by applying sucrose and quantifying the avalanche of asynchronous release that ensues upon sucrose addition. The regions of Munc13 that bind RIM and syntaxin are both essential for neuronal function. (Silinsky and Searl, 2003).

When Munc13-1 is overexpressed in chromaffin cells, there is a threefold increase in secretion. This increase is due to a threefold larger size of both RRP and SRP and a threefold increase in the sustained component. (Rettig and Neher, 2002).

Munc13/UNC-13 proteins contain a $\mathrm{C}_{1}$ domain that binds to the endogenous second-messenger diacylglycerol (DAG) and its analogue phorbol ester, and serve as high-affinity diacylglycerol/phorbol ester receptor in brain. (Li and Chin, 2003).

DAG is one of the most important second messengers in mammalian cells. (Brose and Rosenmund, 2002). Munc13s are functional presynaptic DAG/phorbol ester receptors, which, similar to $\mathrm{PKCs}$, translocate to the plasma membrane upon phorbol 
ester binding. Studies with genetically modified mice that express mutant Munc13-1 lacking the phorbol ester-binding site instead of the wild-type Munc13-1 from the endogenous locus demonstrated that neurons expressing the mutant Munc13-1 fail to exhibit the robust increase in release in response to phorbol ester treatment. (Leenders and Sheng, 2005).

Electrophysiological analysis of Munc13-1 and Munc13-2 single- and doubleknock-out mice suggests that activity-dependent diacylglycerol-mediated Munc13 activation underlies a pre-synaptic form of short-term plasticity termed augmentation. (Brose and Rosenmund, 2002).

Munc13-1 interacts with the N-terminal coiled-coil domain of syntaxin 1 and this interaction appears to be compatible with SNARE complex assembly, suggesting that Munc13-1 bind to the open conformation of syntaxin 1. In C.elegans, UNC-13 is able to transiently interact with UNC-18 bound to the closed conformation of syntaxin and displace UNC-18 from syntaxin. Munc13 interacts with at least five other proteins, namely RIM, CaM, Doc2 (an ARF6 exchange factor msec7-1), and a brain-specific spectrin isoform $\beta$-spIII $\Sigma 1$. (Li and Chin, 2003).

RIM was originally identified in a yeast two-hybrid screen as a $180-\mathrm{kDa}$, active zone-specific protein that binds specifically to the GTP-bound form of Rab3. The RIM mutant phenotypes are much weaker than Munc13-1 and UNC-13 mutants, suggesting that RIM has a regulatory instead of an essential, executing role in synaptic vesicle exocytosis (see Figure 7). (Li and Chin, 2003).

RIM1 knockout mice exhibited a selective, $60 \%$ decrease in expression levels of Munc13-1, but not Munc13-2 or other presynaptic proteins, suggesting that RIM1 normally associates with Munc13-1. (Li and Chin, 2003). In addition to Rab3 and Munc13-1, RIM1 also interacts with SNAP-25, synaptotagmin, $\mathrm{Ca}^{2+}$ channels, RIM-BPs, cAMP-GEFII, and liprins. (Li and Chin, 2003).

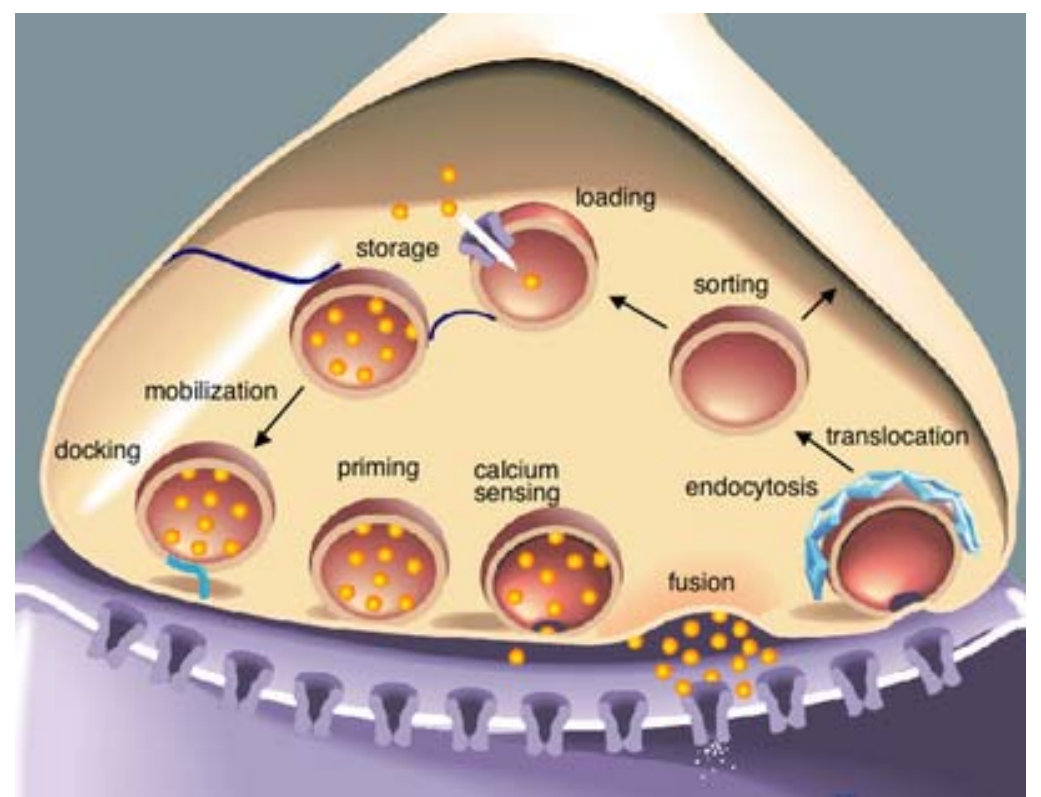

Figure 7. Neurotransmitter vesicle endo- and exocytotic cycle in a presynaptic button (taken from Richmond, 2005). 
Junge and collaborators (2004) reported that CaM binds to Munc13-1 and ubMunc13-2 in a $\mathrm{Ca}^{2+}$-dependent manner via an evolutionary conserved $\mathrm{CaM}$ recognition motif. CaM binding to Munc13 proteins caused increased priming activity and ready-releasable pool (RRP) sizes. Thus, the activation of the CaM/Munc13 complex by residual calcium represents a molecular correlate for the phenomenon of $\mathrm{Ca}$ dependent vesicle pool refilling. This mechanism controls short-term plasticity characteristics and is likely to be evolutionary conserved.

\section{Presynaptic plasticity}

Two principal points of regulating presynaptic plasticity exist: (a) the peak $\mathrm{Ca}^{2+}$ concentration produced by an action potential, i.e., the conversion of an action potential to a $\mathrm{Ca}^{2+}$ current; and (b) the release probability per given $\mathrm{Ca}^{2+}$ concentration, i.e., the conversion of $\mathrm{a} \mathrm{Ca}^{2+}$ signal to exocytosis. Both types of regulation are important. The peak $\mathrm{Ca}^{2+}$ concentration depends in principle on the shape of the action potential, the open probability of the $\mathrm{Ca}^{2+}$ channels, and the $\mathrm{Ca}^{2+}$ concentration present at the time when the $\mathrm{Ca}^{2+}$ channels open. All of these three parameters can be regulated. The release probability per peak $\mathrm{Ca}^{2+}$ concentration is highly variable and depends on two principal parameters: the number of release-ready vesicles, and the $\mathrm{Ca}^{2+}$ responsiveness of the vesicles. The release probability per $\mathrm{Ca}^{2+}$ concentration varies characteristically between different types of synapses and changes during cAMP-dependent presynaptic long-term potentiation (LTP). The number of release-ready vesicles decreases during repetitive stimulation, causing a short-term depression with a magnitude that depends on the pool size and kinetics. (Südhof, 2004).

Release of neurotransmitters and hormones is known to proceed in fractions of seconds or even milliseconds after the free $\mathrm{Ca}^{2+}$ concentration, $\left[\mathrm{Ca}^{2+}\right]$, rises. However, it is clear that vesicles have to undergo a number of steps (translocation, docking, priming) before they are ready to fuse in such a rapid manner. These steps are likely to be slower. First, a rapid burst of exocytosis, by vesicles that had been in a release-competent state at the time of the $\mathrm{Ca}^{2+}$ increase. This would be followed by a much slower, sustained response (at sustained high $\left[\mathrm{Ca}^{2+}\right]$ ) by vesicles that have to undergo preceding, ratelimiting reactions before they can fuse under those conditions. This expectation forms the basis for a possible kinetic distinction between manipulations that affect late-acting molecules and those that are important during earlier steps. (Rettig and Neher, 2002).

Manipulations of the $\mathrm{Ca}^{2+}$ sensor should alter the timing of the exocytotic burst. In contrast priming factors would primarily influence the sustained component and/or the amplitude of the exocytotic burst, that is, the number of release-competent vesicles immediately before the stimulus. Detailed kinetic analysis of data from adrenal chromaffin cells revealed that exocytotic burst, after flash-induced liberation of $\left[\mathrm{Ca}^{2+}\right]$ from "caged" $\mathrm{Ca}$, is not homogeneous. Rather, one has to assume that there are two populations of vesicles that release at largely different rates. (Rettig and Neher, 2002). Upon photolysis of caged $\mathrm{Ca}^{2+}, \mathrm{Ca}^{2+}$ triggers release at the calyx of held with a high degree of cooperativity, similar to other synapses. Release was undetectable at $\mathrm{Ca}^{2+}$ concentrations of $<1 \mu \mathrm{M}$, became measurable at $1-2 \mu \mathrm{M} \mathrm{Ca}^{2+}$, resembled the release observed during a normal action potential at $>5 \mu \mathrm{M} \mathrm{Ca}^{2+}$, and saturated at $>20 \mu \mathrm{M} \mathrm{Ca}^{2+}$. 
Thus fast release, at this synapse, is triggered by $\mathrm{Ca}^{2+}$ binding to a highly cooperative $\mathrm{Ca}^{2+}$ sensor with relatively high apparent $\mathrm{Ca}^{2+}$ affinity $\left(k_{\mathrm{d}} 5-25 \mu \mathrm{M}\right)$.

The precise $\mathrm{Ca}^{2+}$ dependence of release was fitted by different models that led to qualitatively similar but numerically distinct conclusions. In both models, the $\mathrm{Ca}^{2+}$ sensor contains $5 \mathrm{Ca}^{2+}$-binding sites. (Südhof, 2004).

As a result of the high cooperativity of the $\mathrm{Ca}^{2+}$ sensor, the relationship of the $\mathrm{Ca}^{2+}$ concentration to release is supralinear. This supralinearity restricts release to the brief time period during which the $\mathrm{Ca}^{2+}$ transient is above threshold and terminates release rapidly even though residual $\mathrm{Ca}^{2+}$ remains. These studies quantitatively described the $\mathrm{Ca}^{2+}$-binding properties of a $\mathrm{Ca}^{2+}$ sensor only for fast release but provided little information about the $\mathrm{Ca}^{2+}$ sensor for asynchronous release or the adaptations of the release machinery during repetitive stimulation. (Südhof, 2004).

The speed with which $\mathrm{Ca}^{2+}$ triggers release $(<400 \mu \mathrm{sec})$ suggests that $\mathrm{Ca}^{2+}$ binding to the $\mathrm{Ca}^{2+}$ sensor only induces fusion-pore opening and does not initiate a complex reaction cascade. Fusion pores form an aqueous connection across fusing bilayers and are likely to be at least partly lipidic. (Südhof, 2004).

Following exocytosis, synaptic vesicle membranes and protein constituents are retrieved from the plasma membrane by endocytosis, and locally recycled for future rounds of exocytosis. (Li and Chin, 2003).

In Figure 8 a flash of ultraviolet light rapidly elevated $\left[\mathrm{Ca}^{2+}\right]$ from a basal level of about $300 \mathrm{nM}$ to about $20 \mu \mathrm{M}$. As a consequence, membrane capacitance increased rapidly by about $600 \mathrm{fF}$. This increase signals the release of about 240 vesicles, because each vesicle fusing with the plasma membrane enlarges its area, contributing about 2.5 fF. (Rettig and Neher, 2002).

The capacitance increases at a rate of about $50 \mathrm{fF} / \mathrm{s}$, which corresponds to an on going rate of exocytosis of about 20 vesicles per second. These would be vesicles that undergo a priming reaction and release immediately (at high $\left[\mathrm{Ca}^{2+}\right]$ ) when they reach the release-competent state. This priming reaction is intimately linked to the initiation of SNARE complex formation. (Rettig and Neher, 2002).

A direct kinetic separation of $\mathrm{Ca}^{2+}$-dependent triggering and priming reactions, as in the case of the flash experiment, is not possible in most nerve terminals. However, in hippocampal cells, vesicle pool sizes at any given moment can be determined as the cumulative release induced by a short sucrose application. This measurement allows studying the recovery of vesicle pools after strong stimuli and estimation of the release probability by relating a given response to the pool size. In this way, vesicle recruitment or priming can be distinguished from $\mathrm{Ca}^{2+}$ triggering. (Rettig and Neher, 2002).

To describe calcium kinetics in spines correctly, a detailed knowledge of influx and efflux pathways is necessary. Several influx pathways for calcium into dendritic spines have been identified. They include voltage-gated calcium channels, NMDAreceptor and AMPA-receptor channels and calcium release from two types of intracellular stores. Less is known about calcium clearance mechanisms in spines and their functional importance. The efflux pathways influence maximum peak amplitudes of calcium increases and how long intracellular calcium stays elevated. Both are important parameters that impact on synaptic plasticity. Two main efflux pathways are described; firstly, calcium extrusion mechanisms exist in spines and dendrites, which pump calcium into the endoplasmic reticulum. It is not clear to what extent other extrusion mechanisms, 
such as plasmalemmal sodium-calcium exchange or mitochondrial calcium uptake, contribute to this process. Secondly, assuming a concentration gradient between spine head and dendrite, buffered calcium diffusion through the spine neck is an additional calcium clearance mechanism. Distal spines are more susceptible to long-term depression than proximal spines; differences in calcium kinetics found between distal and proximal spines can cause a different susceptibility to synaptic plasticity. Thus compartmentalization of small ions such as calcium might be one of the major functions of dendritic spines. (Holthoff and Tsay, 2002).

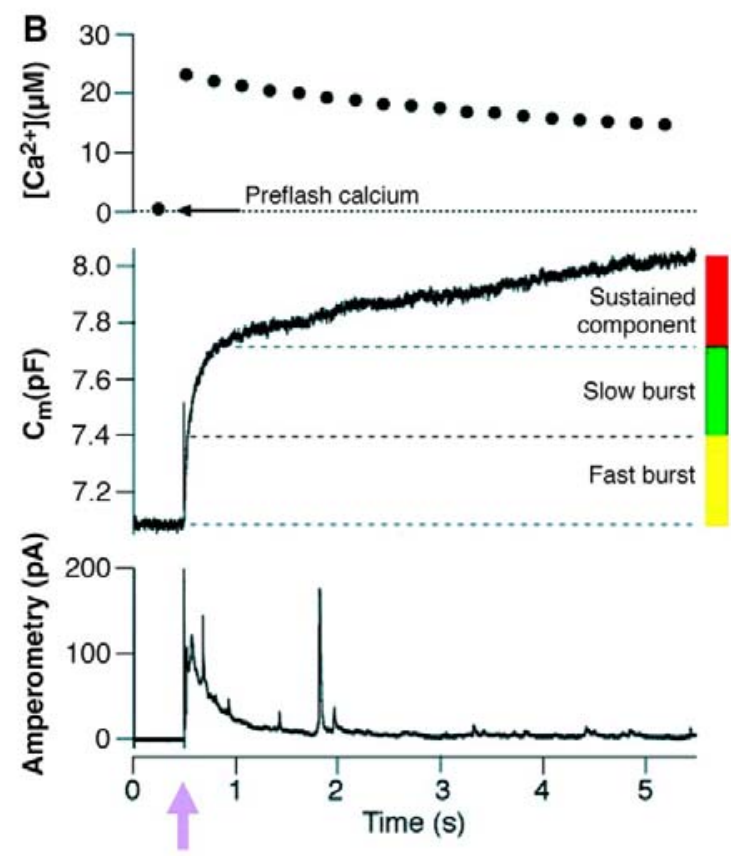

Figure 8. Flash photolysis response. When a flash causes a step increase of cytosolic $\left[\mathrm{Ca}^{2+}\right]$ from a basal level of about $300 \mathrm{nM}$ to $20 \mu \mathrm{M}$ (upper trace). Membrane capacitance (middle trace) and the amperometric signal, as measured by a carbon fiber microelectrode (lower trace), are shown. Membrane capacitance increases in proportion to the number of chromaffin granules that fuse with the plasma membrane. The color code assigns parts of the trace to the fusion of vesicles from the ready-releasable pool RRP (yellow) or slowly-releasable pool SRP (green) or to slow priming (with release immediately following) of previously unprimed vesicles (red) (Taken from Rettig and Neher, 2002).

\section{Active Zones and the presynaptic plasma membrane}

The active zone is composed of an electron-dense, biochemically insoluble material located at the presynaptic plasma membrane precisely opposite the synaptic cleft. There are six large non-membrane proteins that are each encoded by multiple genes, bind to each other, and probably form a humongous single complex at the active zone: Munc13s and RIMs are multidomain proteins that interact with each other and many other synaptic components. Piccolo and Bassoon, homologous and very large proteins, are part of the cytomatrix. ERCs(ELKS/Rab3-interacting molecule/CAST) are coiled-coil proteins whose C-terminus binds to RIMs, and RIM-BPs are SH3-domain 
proteins that also bind to RIMs. $\alpha$-liprins bind to RIMs, ERCs, and receptor protein tyrosine phosphatases. (Südhof, 2004).

\section{Allosteric modulation of the presynaptic $\mathrm{Ca}^{2+}$ sensor for vesicle fusion}

Modulation of the release probability of releasable vesicles in response to $\mathrm{Ca}^{2+}$ influx $\left(\right.$ Prob $\left._{\mathrm{Ca}}\right)$ is involved in mediating several forms of synaptic plasticity, including short-term depression, short-term augmentation, and potentiation induced by protein kinases. Activation of protein kinase $\mathrm{C}$ (PKC) enhances synaptic transmission at a variety of synapses and may underlie certain forms of synaptic plasticity. The enhancement is mediated by an increase of transmitter release. After the $\mathrm{Ca}^{2+}$ influx, transmitter release depends on two factors, the size of a pool of vesicles immediately available for release and the averaged release probability of vesicles in this pool in response to the $\mathrm{Ca}^{2+}$ influx $\left(\right.$ Prob $\left._{\mathrm{Ca}}\right)$. (Wu and $\left.\mathrm{Wu}, 2001\right)$.

Modulation of the $\operatorname{Prob}_{\mathrm{Ca}}$ is often a pathway by which synaptic strength is regulated. For example, modulation of the $\operatorname{Prob}_{\mathrm{Ca}}$ is involved in mediating short-time depression, short-term synaptic augmentation, and enhancement of transmitter release that is induced by the activation of protein kinase A. Although modulation of the Prob $\mathrm{Ca}$ plays an important role in regulating synaptic strength, its underlying mechanisms are unclear. Three potential mechanisms may modulate the Prob $_{\mathrm{Ca}}$ : (1) modulation of the affinity of the $\mathrm{Ca}^{2+}$ sensor to $\mathrm{Ca}^{2+}$, (2) modulation of the number of $\mathrm{Ca}^{2+}$ ions required to bind the $\mathrm{Ca}^{2+}$ sensor and trigger release, and (3) modulation of any step at the release machinery to $\mathrm{Ca}^{2+}$. (Wu and $\mathrm{Wu}, 2001$ ).

$\mathrm{Wu}$ and $\mathrm{Wu}$ (2001) showed that phorbol myristate acetate (PMA) increased the Prob $_{\mathrm{Ca}}$ without affecting the releasable pool or the rate of vesicle mobilization. PMA shifted the sigmoidal relationship between the capacitance jump and the $\mathrm{Ca}^{2+}$ current integral to the left. After fitting the sigmoidal curves with the Hill equation, the $\mathrm{EC}_{50}$ was reduced by approximately one-half by PMA. These results suggested that PMA increases the $P r o b_{\mathrm{Ca}}$ by increasing the apparent affinity of the release machinery to $\mathrm{Ca}^{2+}$. This was the first example showing that modulation of the release machinery downstream of the $\mathrm{Ca}^{2+}$ sensor is involved in mediating modulation of the Prob $_{\mathrm{Ca}}$ and thus the synaptic strength. The protein kinase $\mathrm{C}$ inhibitor BIS did not block PMA-induced enhancement of the capacitance jump completely in calyx-type synapses; the $83 \%$ enhancement got reduced to a $25 \%$ enhancement. It is likely that the remaining effect of PMA on the capacitance jump in the presence of BIS involves the diacylglycerol/phorbol ester binding protein Munc13-1. (Wu and Wu, 2002).

Lou and collaborators (2005) found that phorbol dibutyrate (PDBu) increases the intracellular $\mathrm{Ca}^{2+}$ sensitivity of vesicle fusion in response to sub-maximal $\mathrm{Ca}^{2+}$ stimuli. In pituitary cells, phorbol esters increase $\mathrm{Ca}^{2+}$ sensitivity by increasing the vesicle pool depletion time constants; however such an increase is not apparent in the Calyx of Held.

Synaptic vesicle fusion at resting $\left[\mathrm{Ca}^{2+}\right]_{i}$ does not appear to be constitutive. Rather it is regulated by phorbol esters as well as by $\mathrm{Ca}^{2+}$, although the $\mathrm{Ca}^{2+}$ cooperativity is low close to baseline $\left[\mathrm{Ca}^{2+}\right]_{\mathrm{i}}$. Evidence for a low $\mathrm{Ca}^{2+}$ cooperativity close to resting $\left[\mathrm{Ca}^{2+}\right]_{\mathrm{i}}$ has also been obtained at neuromuscular synapses. This low $\mathrm{Ca}^{2+}$ cooperativity is functionally relevant, because it prevents a large increase in asynchronous release activity 
during moderate elevations of residual $\left[\mathrm{Ca}^{2+}\right]_{\mathrm{i}}$, which would be expected if a steeply $\mathrm{Ca}^{2+}$ -dependent mechanism operated close to baseline $\left[\mathrm{Ca}^{2+}\right]_{\mathrm{i}}$. (Lou et al., 2005).

\section{Kinetics of the neurotransmitter release}

The $\mathrm{Ca}^{2+}$-dependent priming of vesicles occurs on the time scale of tens of seconds, and it can enlarge the size of the releasable pool by up to a factor of 5. (Rettig and Neher, 2002).

Two isoforms of Munc13 produce two distinct forms of short-term plasticity, one (Munc13-1) characterized by short-term depression at moderate stimulation frequencies and another one (Munc13-2) by a $\mathrm{Ca}^{2+}$ - and stimulation-dependent augmentation. (Rettig and Neher, 2002).

\section{1. $C_{1}$ domain as membrane localization module in Munc13-1}

Shen and collaborators (2005) solved the solution structure of Munc13-1 $\mathrm{C}_{1}$ domain; the overall conformation of the Munc13-1 $\mathrm{C}_{1}$ domain is analogous to that of the $\mathrm{PKC} \delta \mathrm{C}_{1} \mathrm{~B}$ domain, but the ligand-binding region showed striking structural differences. A conserved tryptophan residue occludes the DAG/PE-binding site in the munc13-1 $\mathrm{C}_{1}$ domain. Thus, the munc13-1 $\mathrm{C}_{1}$ domain needs to experience a considerable conformational change for ligand binding. The fact that W588 packs against the ligandbinding site in the Munc13-1 $\mathrm{C}_{1}$ domain but not in the $\mathrm{PKC} \delta \mathrm{C}_{1} \mathrm{~B}$ domain appears to arise from differential conformational preferences of the backbone of loops 1 and 2, and perhaps from slight overall structural differences between the two $\mathrm{C}_{1}$ domains, rather than from contacts between the W588 aromatic ring and specific side chains of the munc13-1 $\mathrm{C}_{1}$ domain. (Shen et al., 2005).

The occlusion of the DAG-binding site observed in the structure of the Munc13-1 $\mathrm{C}_{1}$ domain is expected to impose a kinetic barrier for ligand binding and hence decrease the binding on rate constant $\left(k_{\mathrm{on}}\right)$. (Shen et al., 2005).

Phorbol esters produce various physiological effects on cells in a variety of tissues, with one of the most commonly observed effects being the promotion of tumor growth. Phorbol esters activate protein kinase $\mathrm{C}(\mathrm{PKC})$ and after extended incubation periods, cause down regulation of the enzyme. Phorbol esters act as stable substitutes of the natural activator of PKC, diacylglycerol. (Silinsky and Searl, 2003).

Phorbol ester binding does not produce a significant conformational change within the activator binding domain. Rather, it acts to cover the polar interior of the groove and completes a contiguous hydrophobic surface over a large portion of the domain. Phorbol esters activate PKC by altering the nature of the protein surface and by stabilizing the membrane-inserted state, rather than by inducing a conformational change at the activator-binding site. (Zhang et al., 1995).

The regulatory domain of PKC contains the binding sites for phorbol esters, the $\mathrm{C}_{1}$ domains. These domains are the natural receptors for the endogenous ligand DAG, which is formed from membrane phospholipids (predominantly phosphatidylinositol bisphosphate) after the activation of phospholipase $\mathrm{C}$ (PLC) by a wide variety of biological modulators and neurotransmitters. The $\mathrm{C}_{1}$ region is a zinc-finger receptor, a common term for these regions because of their richness in cysteine residues, even 
though the domain is not structurally related to the zinc-finger proteins that bind nucleic acids. The purpose of the $\mathrm{C}_{1}$ domain is to help recruit proteins to membranes. The binding of phorbols in the $\mathrm{C}_{1}$ domain provides a hydrophobic cover allowing the complex to be inserted and anchored into membranes with little conformational change. After insertion into the membrane, the catalytic domain of PKC is activated and, with the appropriate substrates, phosphorylation ensues. (Silinsky and Searl, 2003).

There are other important receptors for phorbol esters, like Unc13, the chimaerins, DAG kinase $\gamma$, RasGRP (which activates Ras and similar small G-proteins) and protein kinase D1, an enzyme essential for targeting proteins to the cell surface. (Silinsky and Searl, 2003).

High affinity binding $\left(k_{\mathrm{d}} \sim 1 \mathrm{nM}\right)$ of phorbol esters to PKC requires phospholipids, and acidic phospholipids are the most efficient cofactors for ligand binding. However, phorbol esters have been shown to bind in the absence of phospholipids with a reduced affinity $\left(k_{\mathrm{d}} \sim 0.165 \mu \mathrm{M}\right)$ (Kazanietz et al., 1995). DAG or phorbol esters are required for the reversible recruiting of $\mathrm{PKC}$ to membranes, a process referred to as " $\mathrm{PKC}$ translocation". The conserved $\mathrm{C}_{1}$ and $\mathrm{C}_{2}$ domains in $\mathrm{PKC}$ isozymes play a key role in membrane association. (Kazanietz, 2002).

The $\mathrm{C}_{1}$ domain, a motif of 50 or 51 amino acids located in the $\mathrm{N}$-terminal regulatory region of $\mathrm{PKC}$, is the minimum domain required for phorbol ester/DAG binding. This domain is duplicated in tandem $\left(\mathrm{C}_{1} \mathrm{~A}\right.$ and $\left.\mathrm{C}_{1} \mathrm{~B}\right)$ in cPKCs, $\mathrm{nPKCs}$, and in $\mathrm{PKC} \mu / \mathrm{PKD}$. The $\mathrm{C}_{1}$ domains are rich in cysteine and possess the motif $\mathrm{HX}_{12} \mathrm{CX}_{2} \mathrm{CX}_{13 / 14} \mathrm{CX}_{2} \mathrm{CX}_{4} \mathrm{HX}_{2} \mathrm{CX}_{7} \mathrm{C}$, where $\mathrm{H}$ is histidine, $\mathrm{C}$ is cysteine, and $\mathrm{X}$ is any other amino acid. The two histidines and five of the cysteines coordinate two $\mathrm{Zn}^{2+}$ ions in each $\mathrm{C}_{1}$ domain. The mutation of any of the essential histidines or cysteines affects the structural integrity of the domain and consequently disrupts ligand binding. Ligand binding to the $\mathrm{C}_{1}$ domain leads to a large-scale conformational change in $\mathrm{PKC}$ that result in the allosteric activation of the enzyme and stimulation of its phosphotransferase activity. (Kazanietz, 2002).

In the $\mathrm{C}_{1}$ domain crystal structure of $\mathrm{PKC} \delta$, the phorbol ester binding does not produce a significant conformational change within the activator binding domain. Rather, it acts to cover the polar interior of the groove and completes a contiguous hydrophobic surface over a large portion of the domain. The long-chain lipid tails of PKC activators are probably not directly involved in binding to $\mathrm{PKC}$, but instead retain the activator within the membrane. The retention of the long-chain phorbol esters in the membrane means that the favorable free energy of protein-phorbol binding can be used to drive the insertion of the protein into the membrane. (Zhang et al., 1995).

There is experimental evidence which shows that chimaerins, Munc13s and RasGRPs redistribute in response to phorbol esters. Studies using GFP- $\beta 2$-chimaerin revealed a cytoplasmic staining in the absence of phorbol ester stimulation and a significant translocation both to the plasma membrane and to the perinucleus after phorbol ester treatment. (Kazanietz, 2002).

Evidence for phorbol ester-induced translocation of Munc13 isozymes has been reported in human embryonic kidney 293 cells transiently transfected with GFP-fused Munc13 constructs. All three Munc13 isoforms translocate to the plasma membrane in response to PMA. Phorbol esters promote a transient interaction of Munc13-1 with the calcium-binding protein DOC2. (Kazanietz, 2002). 
Both DOC2A and DOC2B translocate to the plasma membrane in the presence of $\left[\mathrm{Ca}^{2+}\right]_{\mathrm{i}}$ below $0.5 \mu \mathrm{M}$. In depolarized neurons, both DOC2 isoforms associated reversibly with the neuronal plasma membrane but with a different sensitivity. Thus both DOC2 isoforms may contribute to short-term plasticity of the nervous system. The elevation of $\left[\mathrm{Ca}^{2+}\right]_{\mathrm{i}}$, which causes the translocation of DOC 2 to the membrane, may accelerate the priming activity of Munc13. In contrast to synaptotagmin, the DOC2 proteins are optimally adapted to sense residual $\left[\mathrm{Ca}^{2+}\right]_{\mathrm{i}}$ in a timescale of seconds (Groffen et al., 2006).

The enzymatic activity of the different isoforms of the phopholipase $\mathrm{C}$ family of proteins results in the hydrolysis of phosphatidylinositol 4,5-bisphosphate, which contains mainly polyunsaturated fatty acids, to inositol 1,4,5-trisphosphate and polyunsaturated DAGs. Polyunsaturated DAG second messenger molecules are inactivated by the activity of diacylglycerol kinases (Brose and Rosenmund, 2002).

DAG- and phorbol-ester-mediated subcellular translocation of PKD1 is essential for protein transport from the trans-Golgi network to the cell surface (Brose and Rosenmund, 2002).

Studies of PKC function that rely on the use of phorbol esters as an investigative tool have to be interpreted with caution. As in the case for $\mathrm{C}_{1}$-domain-directed PKC activators, $\mathrm{C}_{1}$-domain-directed $\mathrm{PKC}$ inhibitors are non-specific pharmacological tools that bind with comparable affinity to other $\mathrm{C}_{1}$ domain proteins. Such non-specific inhibitors include one of the PKC inhibitors used most widely in the past, calphostin $\mathrm{C}$. For the functional separation of PKC-specific effects from those mediated by alternative DAG/phorbol ester receptors, some of the most useful pharmacological tools are ATPbinding site inhibitors. However, some of the most specific bisindolylmaleimide-derived PKC inhibitors are partially toxic in certain situations (Brose and Rosenmund, 2002).

\section{Priming activity of Munc13 proteins}

The control of the priming activity of the MUN domain (residues 859-1531) may be the focal point of a variety of presynaptic plasticity processes that are regulated by diverse agents such as $\mathrm{Ca}^{2+}$, DAG and Rab3 and that are believed to mediate information processing in the brain (Basu et al., 2005).

The overexpression of the Munc13-1 MUN domain rescues release in cultured excitatory and inhibitory hippocampal autaptic neurons from Munc13-1 and Munc13-2 double-knockout mice. In contrast, the rescue with the MHD1-2 fragment (residues 10451531) was unsuccessful (Basu et al., 2005).

There is still conflicting data on this point, Stevens and collaborators (2005) identified a different minimal Munc13-1 domain that is responsible for its priming activity, this region mapped to the C-terminus of Munc13-1 residues 1100-1735.

The Ready Releasable Pool size is reduced in Munc13-1 ${ }^{\mathrm{H} 567 \mathrm{~K}}$ mutant neurons, but evoked response amplitudes are unaltered, resulting in a relatively higher vesicular release probability $\left(\mathrm{P}_{\mathrm{vr}}\right)$ compared with wild-type $(w t)$ neurons. The activation of the Munc13-1 $\mathrm{C}_{1}$ domain increases the number of vesicles released only when the hypertonic stimulus is submaximal and does not cause any additional potentiation of responses during saturating osmotic challenges. Thus $\mathrm{C}_{1}$ domain activation does not affect the RRP per se but lowers the energy threshold for fusion of vesicles within the pool. The 
activation of the Munc13-1 $\mathrm{C}_{1}$ domain accelerates vesicular release in general, consistent with the model of a lowered energy barrier for fusion. The Munc13-1 ${ }^{\mathrm{H} 567 \mathrm{~K}} \mathrm{C}_{1}$ mutant mimics PDBu-mediated $\mathrm{C}_{1}$ domain activation (Basu et al., 2007).

The activation of Munc13 $\mathrm{C}_{1}$ domain causes a left shift of the sensitivity to sucrose indicating that the activation of the $\mathrm{C}_{1}$ domain lowers the energy threshold for fusion of vesicles. Thus Munc13 $\mathrm{C}_{1}$ domain activation impacts vesicular release after vesicles have been primed to form the RRP, and hence Munc13s are not only priming proteins but also play a role as post-priming modulators of neurotransmitter release. In the case of augmentation, it includes among other likely processes the $\mathrm{Ca}^{2+}$ and activitydependent stimulation of N-terminal Munc13 domains by $\mathrm{Ca}^{2+} / \mathrm{CaM}$ and by DAG (Basu et al., 2007).

The dual mode of Munc13 function equips synapses with the ability to effectively regulate vesicular release probability. In their activated state, Munc13s lower the energy barrier for vesicle fusion such that vesicles can be released at twofold to fourfold higher rates. Primed synaptic vesicles may possibly be equipped with SNARE complexes with variable fusogenic potentials depending on the associated Munc13 conformation (Basu et al., 2007).

Kalla and collaborators (2006) showed by Fluorescence Recovery After Photobleaching of Munc13-1-EYFP knock-in neurons the presence of two Munc13-1EYFP pools: one with an exchange time constant of $\sim 3$ min that represents $\sim 40 \%$ of total synaptic Munc13-1-EYFP, and a second pool, which exchanged more slowly (time constant of $\sim 80 \mathrm{~min}$ ), that represents $\sim 60 \%$ of the total Munc13-1-EYFP. The quickly exchanging Munc13-1-EYFP pool most likely represents proteins whose diffusion is hindered by transient and weak proteinaceous interactions, because its exchange rate is still much slower than expected for free diffusion. In contrast, the slowly exchanging Munc13-1-EYFP pool may represent Munc13-1-EYFP anchored to the synaptic plasma membrane, the submembranous cytoskeleton, and the active zone protein network via high-affinity proteinaceous interactions (Kalla et al., 2006).

Another imaging study done by Nofal and collaborators (2007) with the use of total internal reflection fluorescence microscopy (TIRFM), which enables the real-time visualization of vesicles, near the plasma membrane in their different molecular states, revealed that the nearly immobile vesicles with a caging diameter (CD) of $101 \mathrm{~nm}$ represent primed vesicles because the pool of vesicles displaying this type of mobility was significantly increased after PMA treatment and Munc13-1 overexpression and decreased during tetanus toxin expression. In contrast, the movement of docked but unprimed vesicles is restricted to a confined region CD $220 \mathrm{~nm}$.

$\mathrm{Lu}$ and collaborators (2006) studied the homodimerization of the $\mathrm{C}_{2} \mathrm{~A}$ domain of Munc13-1 and its heterodimerization to the zinc-finger domain of RIM1 $\alpha$. Munc13-1 may exist in solution as a homodimer that needs to be disrupted in order to bind to $\alpha$ RIMs at the active zone. The physiological role of Munc13-1 homodimerization is unclear, but it is plausible that it may play an inhibitory role by hindering $\alpha$-RIM binding or it may help to stabilize Munc13-1 in the cytoplasm, before engaging in interactions at the active zone.

The $\mathrm{C}_{2}$ domains present in UNC13 homologues could serve as a $\mathrm{Ca}^{2+}$ sensor which responds to the $\mathrm{Ca}^{2+}$ influx required for exocytosis. Therefore, the question arises as to the function of a potential second type of $\mathrm{Ca}^{2+}$ sensor provided by the binding of 
CaM to dUNC13. One possibility is that each $\mathrm{UNC13}$ protein really has only one $\mathrm{Ca}^{2+}$ sensor and that is supplied in some isoforms by the $\mathrm{C}_{2}$ domain and in others through $\mathrm{Ca}^{2+} / \mathrm{CaM}$. An alternative proposal is that some UNC13 proteins may be regulated by $\mathrm{Ca}^{2+}$ via both $\mathrm{C}_{2}$ domains and CaM. Such dual regulation may provide a mechanism for extremely rapid as well a sustained responses to highly transient increases in $\mathrm{Ca}^{2+}$. Dual binding of $\mathrm{Ca}^{2+}$ to $\mathrm{CaM}$ and $\mathrm{C}_{2}$ domain may enable UNC13 proteins to sense the $\mathrm{Ca}^{2+}$ rise within few microseconds and sustain the response for several hundred microseconds to several milliseconds. (Xu et al., 1998).

\section{Study of macromolecular structure with nuclear magnetic resonance}

All multidimensional heteronuclear NMR experiments correlate a heteronuclear resonance with a proton resonance by transfer of coherence (or polarization) between the heteronuclear (S) and proton (I) spins. Regardless of the specific protocol utilized to effect coherence transfer, the NMR experiment can start with excitation of either I or S spin polarization and must end with detection of either I or S spin magnetization. The overall sensitivity $\mathrm{S} / \mathrm{N}$ of heteronuclear correlation $\mathrm{NMR}$ experiments is proportional to $\gamma_{\text {ex }} \gamma_{\text {det }}^{3 / 2}\left[1-\exp \left(-R_{1, e x} T_{c}\right)\right]$, where $\gamma_{\text {ex }}$ and $\gamma_{\text {det }}$ are the gyro magnetic ratios of each nucleus excited at the beginning of the sequence and detected at the end of the sequence, respectively; $T_{c}$ is the recycle time of the experiment; and $R_{1, e x}$ is the spin-lattice relaxation rate constant of the excited nucleus. Therefore, indirect proton detection is used whenever possible in order to maximize sensitivity. (Cavanagh et al, 1996).

Stable isotope labeling provides coherence pathways for multi-dimensional experiments that can either be selected for or filtered out. Whenever possible it is advantageous to observe less sensitive nuclei (such as ${ }^{13} \mathrm{C}$ and ${ }^{15} \mathrm{~N}$ ) indirectly through coherences with ${ }^{1} \mathrm{H}$ (Markley and Kainosho, 1993).

The complete assignment of the NMR spectrum of a protein involves three tasks:

1) sorting of NMR peaks by amino acid type

2) linking of the peaks (sorted by amino acid type) by sequential NMR connectivities

3) alignment of these linked groups of peaks with the protein sequence

The three tasks can be carried out separately, but they usually are combined in iterative searches as the assignment proceeds. Secondary structural elements $(\alpha$-helix, $\beta$ sheet, and turns) give rise to characteristic patterns of sequence dependent NOEs; such additional information can be used to verify or extend assignments (Wühtrich, 1986).

Amino acid selective stable isotope labeling provides a powerful means of accomplishing the first task. With proteins labeled uniformly with ${ }^{13} \mathrm{C}$ and/or ${ }^{15} \mathrm{~N}$, sorting is accomplished by classifying resonances from each residue according to its spin system type. With uniform dual ${ }^{13} \mathrm{C} /{ }^{15} \mathrm{~N}$-labelling signals can be sorted cleanly for all 20 amino acids (Wühtrich, 1986).

The multinuclear spin systems of proteins labeled with ${ }^{13} \mathrm{C}$ and ${ }^{15} \mathrm{~N}$ extend from one residue to the next by virtue of ${ }^{1} \mathrm{~J}_{\mathrm{CC}}$ and ${ }^{1} \mathrm{~J}_{\mathrm{CN}}$ coupling along the peptide backbone (Figure 9). Heteronuclear 2D, 3D, and 4D experiments have been developed that exploit coupling along the peptide backbone to provide sequence-specific assignments in proteins labeled completely with ${ }^{13} \mathrm{C}$ and ${ }^{15} \mathrm{~N}$. In principle the $4 \mathrm{D}$ triple-resonance approach should provide backbone assignments with as few as two data sets. The 3D 
approach requires more data sets but may be applicable to larger proteins. There are two categories for sequential assignment in uniformly labeled proteins; the first one relies on sequential through-space (NOE) connectivities. Common types of sequential, stable isotope-assisted NOEs are: $\left({ }^{15} \mathrm{~N}-{ }^{1} \mathrm{H}\right)_{\mathrm{i}} \ldots\left({ }^{1} \mathrm{H}-{ }^{15} \mathrm{~N}\right)_{\mathrm{i}+1},\left({ }^{13} \mathrm{C}_{\alpha, \beta, \gamma^{-}}{ }^{1} \mathrm{H}\right)_{\mathrm{i}} \ldots\left({ }^{1} \mathrm{H}-{ }^{15} \mathrm{~N}\right)_{\mathrm{i}+1}$. The second one relies on sequential through-bond connectivities. These are provided by a range of 1D-4D experiments, where redundancy of connectivities or higher dimensionality is used to resolve overlap problems (Sattler et al., 1999).

Additional isotope-assisted 3D and 4D methods support side chain and NOE assignments. An approach commonly used with larger proteins is to simplify the assignment problem by collecting high dimensional (3D and 4D) NMR data. Such data have fewer redundancies in assignment pathways than 2D data so that the solution can be found without testing all possible assignment pathways against all the data (Sattler et al., 1999).

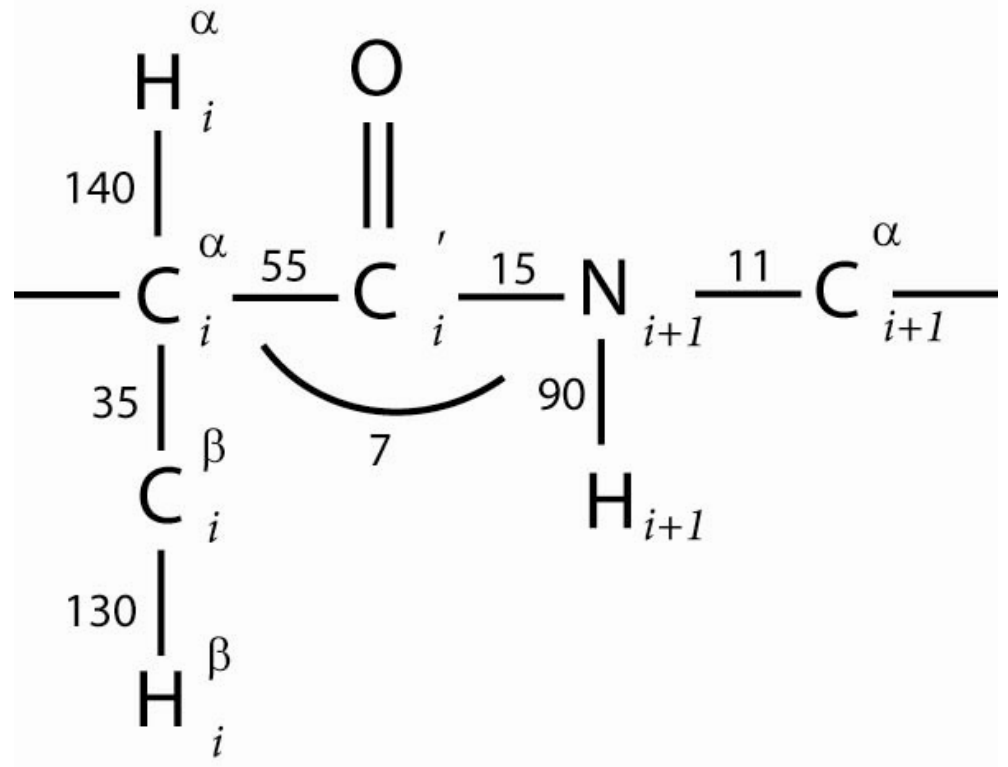

Figure 9. Schematic representation of the coupling pathways that can be exploited in making sequential assignments along the peptide backbone form through-bond coupling connectivities with proteins labeled with ${ }^{13} \mathrm{C}$ and ${ }^{15} \mathrm{~N},{ }^{1} \mathrm{~J}$ coupling constant values are in $\mathrm{Hz}$ (adapted from Sattler et al., 1999).

The introduction of heteronuclear triple resonance experiments relying exclusively on ${ }^{1} \mathrm{~J} /{ }^{2} \mathrm{~J}$ couplings (Ikura et al., 1990), which allow to order independent of conformation the resonance peaks of ${ }^{1} \mathrm{H},{ }^{15} \mathrm{~N}$ and ${ }^{13} \mathrm{C}$ in uniformly labeled proteins has yielded a large number of experiments and new assignment strategies. (Sattler et al., 1999). With the use of deuterium labeling, it has been possible to extend the study of proteins with molecular weights above 30kDa (LeMaster, 1990; Grzesiek et al., 1993).

Transverse Relaxation -Optimized Spectroscopy (TROSY) allows to study large biological macromolecules, these pulse sequences attenuate $\mathrm{T}_{2}$-relaxation at high magnetic fields by mutual cancellation of dipole/dipole and CSA relaxation mechanisms (Sattler et al., 1999).

The original assignment strategy employs the 3D experiments HNCO, HNCA, $\mathrm{HN}(\mathrm{CO}) \mathrm{CA}, \mathrm{HCACO}$ and $\mathrm{HCA}(\mathrm{CO}) \mathrm{N}$ to exclusively correlate the resonances of the 
peptide backbone $\left(\mathrm{H}^{\mathrm{N}}(i), \mathrm{N}(i), \mathrm{C}_{\alpha}(i), \mathrm{H}_{\alpha}(i), \mathrm{C}_{\alpha}(i-1), \mathrm{H}_{\alpha}(i-1), \mathrm{C}^{\prime}(i)\right.$ and $\mathrm{C}^{\prime}(i-1)$. With the inclusion of the $3 \mathrm{D}$ experiments $\mathrm{CBCA}(\mathrm{CO}) \mathrm{NH}$ and $\mathrm{HNCACB}$, the sequential assignment can be improved, since the information about the chemical shifts of the $\mathrm{C}_{\alpha}$ and $\mathrm{C}_{\beta}$ carbons is especially valuable, because they are characteristic of the different types of amino acids and can therefore help to position a sequentially connected stretch of amino acids within the known primary sequence (Sattler et al., 1999).

The NMR pulse sequences for the assignment of uniformly ${ }^{13} \mathrm{C}-,{ }^{15} \mathrm{~N}$-labelled proteins are composed of a large number of pulses that have to be applied at three or four different frequencies in order to excite ${ }^{1} \mathrm{H},{ }^{15} \mathrm{~N}$, aliphatic ${ }^{13} \mathrm{C}$ and ${ }^{13} \mathrm{C}^{\prime}$ resonances. Signal gets lost as a result of miscalibration or pulse imperfection accumulate, accurate calibration of pulses and good RF homogeneity of the probe on all frequency channels is essential. Especial techniques and the use of pulse field gradients in the design of pulse sequences have led to improved signal to noise and better artifact suppression in NMR experiments (Sattler et al., 1999).

The pulse sequence for the 3D-HNCO and 3D-HNCA experiments are identical, except for the interchange of $\mathrm{C}^{\alpha}$ and $\mathrm{C}^{\prime}$ pulses. Two types of cross peaks are observed in the HNCA experiment, the correlations $\mathrm{H}^{\mathrm{N}}(i), \mathrm{N}(i), \mathrm{C}_{\alpha}(i)$ and $\mathrm{H}^{\mathrm{N}}(i), \mathrm{N}(i), \mathrm{C}_{\alpha}(i-1)$ because both the couplings ${ }^{1} \mathrm{~J}_{\mathrm{C} \alpha(\mathrm{i}), \mathrm{N}(\mathrm{i})}=11 \mathrm{~Hz}$ and ${ }^{2} \mathrm{~J}_{\mathrm{C} \alpha(\mathrm{i}-1), \mathrm{N}(\mathrm{i})}=7 \mathrm{~Hz}$ are of similar size. In contrast, only the correlations $\mathrm{H}^{\mathrm{N}}(i), \mathrm{N}(i), \mathrm{C}^{\prime}(i-1)$ are observed in the $\mathrm{HNCO}$, since ${ }^{1} \mathrm{~J}_{\mathrm{C}^{\prime}(\mathrm{i}-1), \mathrm{N}(\mathrm{i})}=$ $15 \mathrm{~Hz}$, while ${ }^{2} \mathrm{~J}_{\mathrm{C}^{\prime}(\mathrm{i}), \mathrm{N}(\mathrm{i})} \approx 0 \mathrm{~Hz}$. The transfer pathway starts on a nucleus (the $\mathrm{H}^{\mathrm{N}}$ in these experiments) and the magnetization is detected on the same nucleus, after chemical shifts of other nuclei have been followed, this type of experiment is called "out and back". The magnetization can also be transferred from a starting nucleus to another nucleus to be detected. This type of experiment is called "transfer experiment" (Sattler et al., 1999).

The $\mathrm{HN}(\mathrm{CA}) \mathrm{CO}$ experiment correlates the chemical shifts of $\mathrm{H}^{\mathrm{N}}(i), \mathrm{N}(i)$ and $\mathrm{C}^{\prime}(i)$. In analogy to the HNCO experiment, cross peaks involving the resonances of $\mathrm{C}^{\prime}(i-$ 1) and $\mathrm{C}_{\alpha}(i-1)$ can also be observed, depending on the relaxation properties of the protein. The $\mathrm{HN}(\mathrm{CA}) \mathrm{CO}$ experiment is derived from the HNCA experiment. Starting on the amide protons, magnetization is transferred via the amide nitrogens to the $C_{\alpha}$ spins. For the following magnetization transfer between the $\mathrm{C}_{\alpha}$ and the $\mathrm{C}^{\prime}$ spins either an HMQCor an HSQC-type based experiment can be used. Analogously to the HNCA experiment two cross peaks are expected. The intraresidual peak correlating to $\mathrm{C}_{\alpha}(i)$ originates from $\mathrm{N} \rightarrow \mathrm{C}_{\alpha}$ transfer via the ${ }^{1} \mathrm{~J}_{\mathrm{C} \alpha, \mathrm{N}}$ coupling and the sequential cross peak to $\mathrm{C}_{\alpha}(i-1)$ originates from $\mathrm{N} \rightarrow \mathrm{C}_{\alpha}$ transfer via the ${ }^{2} \mathrm{~J}_{\mathrm{C} \alpha, \mathrm{N}}$ coupling (Sattler et al., 1999).

The 3D CBCA $(\mathrm{CO}) \mathrm{NH}$ experiment is a transfer experiment, which uses a relayCOSY step $\mathrm{C}_{\beta} / \mathrm{C}_{\alpha} \rightarrow \mathrm{C}_{\alpha}$ to correlate the chemical shifts $\mathrm{H}^{\mathrm{N}}(\mathrm{i}), \mathrm{N}(\mathrm{i}), \mathrm{C}_{\alpha}(\mathrm{i}-1)$ and $\mathrm{C}_{\beta}(\mathrm{i}-1)$. The magnetization is transferred from $\mathrm{H}_{\alpha, \beta}$ to the directly bound $\mathrm{C}_{\alpha, \beta}$ followed by constant time chemical shift evolution of $\mathrm{C}_{\alpha, \beta}$. After the relay-COSY step, the $\mathrm{C}_{\beta}, \mathrm{C}_{\alpha}$ coupling is refocused and the magnetization is transferred from $\mathrm{C}_{\beta} / \mathrm{C}_{\alpha}$ to the amide nitrogen in two INEPT steps, followed by constant time chemical shift evolution of the amide nitrogens $\left(t_{2}\right)$. The magnetization is detected $\left(t_{3}\right)$ after transfer to the amide protons using a INEPT sequence. The transfer amplitudes for $\mathrm{C}_{\beta}$ and $\mathrm{C}_{\alpha}$ cross peaks in the CBCA(CO)NH experiment depend on the carbon multiplicity of the carbon spins (Sattler et al., 1999).

The 3D HNCACB experiment uses an "out and back" magnetization transfer 


$$
\mathrm{H}^{\mathrm{N}} \rightarrow \mathrm{N} \rightarrow \mathrm{C}_{\alpha} \rightarrow \mathrm{C}_{\alpha, \beta}\left(t_{1}\right) \rightarrow \mathrm{C}_{\alpha} \rightarrow \mathrm{N}\left(t_{2}\right) \rightarrow \mathrm{H}^{\mathrm{N}}\left(t_{3}\right)
$$

The relay-COSY transfer $\mathrm{C}_{\beta}, \mathrm{C}_{\alpha} \rightarrow \mathrm{C}_{\alpha}$, which is used in the $\mathrm{CBCA}(\mathrm{CO}) \mathrm{NH}$ and CBCANH experiments, is efficient for proteins up to $20 \mathrm{kD}$ molecular weight. For smaller proteins with more favorable relaxation properties, it is also possible to transfer magnetization from all side-chain resonances to the $\mathrm{C}_{\alpha}$ nucleus by homonuclear $\mathrm{C}, \mathrm{C}$ TOCSY transfer and via $\mathrm{C}^{\prime}$ and $\mathrm{N}$ to the amide protons. Such experiments are less sensitive than the relay-COSY experiments, because the magnetization originating from all nuclei is distributed into the whole spin system, with only a small part being transferred to the $\mathrm{C}_{\alpha}$. In addition, it is difficult to find a mixing time, $\tau_{\mathrm{m}}$ that yields a good transfer for all aliphatic side-chain carbons of all amino acids to their $\mathrm{C}_{\alpha}$ carbon (Sattler et al., 1999).

The 3D HCCH-TOCSY experiment is used to assign aliphatic ${ }^{1} \mathrm{H},{ }^{13} \mathrm{C}$ spin systems and to link them to the sequentially assigned backbone resonances. In an $\mathrm{H}_{\mathrm{n}} \mathrm{C}(\mathrm{i})$ - ... - $\mathrm{H}_{\mathrm{m}} \mathrm{C}(j)$ - spin system a 3D H(C)CH-TOCSY experiment connects chemical shifts $\mathrm{H}(i), \mathrm{H}(j)$ and $\mathrm{C}(j)$. This connectivity information yields the complete assignment of the ${ }^{1} \mathrm{H}$ and ${ }^{13} \mathrm{C}$ resonances, because the aliphatic proton chemical shifts of a given spin system are found at the ${ }^{13} \mathrm{C}$ chemical shift of all the carbon frequencies involved in that spin system (Sattler et al., 1999).

The assignment of aromatic resonances is usually complicated because of strong coupling between ${ }^{1} \mathrm{H}$ and the ${ }^{13} \mathrm{C}$ spins within the aromatic ring system and short relaxation times. In the INEPT transfer based experiments, magnetization is transferred from $\mathrm{H}_{\beta}$ to $\mathrm{C}_{\beta}$ spins to the quaternary ${ }^{13} \mathrm{C}_{\gamma}$ nuclei using selective pulses for the aromatic $\mathrm{C}_{\gamma}$ spins, and adjusting the power level of the $\mathrm{C}_{\beta}$ pulses such that the $\mathrm{C}_{\gamma}$ spins are not affected. After carbon relay transfers to the protonated aromatic carbons, the magnetization is finally detected on the $\mathrm{H}_{\delta}$ or $\mathrm{H}_{\varepsilon}$ protons (Sattler et al., 1999).

Methionine $\mathrm{C}_{\varepsilon}$ methyl groups play an essential role in CaM binding to its numerous target sequences. For structure determination by NMR is important to obtain unambiguous assignments of these methyl groups. A pulse sequence based on ${ }^{13} \mathrm{C}^{13} \mathrm{C}$ correlation has been introduced by Bax et al., 1994. The magnetization transfer in this 3D-HMBC experiment is as follows: $\mathrm{H}_{\varepsilon} \rightarrow \mathrm{H}_{\varepsilon} \mathrm{C}_{\gamma}\left(\mathrm{MQ}, \mathrm{t}_{1}\right) \rightarrow \mathrm{H}_{\varepsilon} \rightarrow \mathrm{C}_{\varepsilon}\left(\mathrm{t}_{2}\right) \rightarrow \mathrm{H}_{\varepsilon}\left(\mathrm{t}_{3}\right)$ where MQ referes to heteronuclear $\mathrm{H}_{\varepsilon}-\mathrm{C}_{\gamma}$ multiple quantum coherence. The first part of the sequence corresponds to the original HMBC pulse scheme, but for uniformly ${ }^{13} \mathrm{C}$ enriched proteins, the de- and rephrasing delays, $\Delta$, must be adjusted to an integer multiple of $1 /{ }^{1} \mathrm{~J}_{\mathrm{C} \varepsilon \mathrm{H}}$ to minimize generation of $\mathrm{H} \varepsilon$-C $\varepsilon$ multiple-quantum coherence. This stage is followed by a constant-time HSQC experiment, where the duration of the constant time, $2 \mathrm{~T}$, is adjusted to $1 /\left(2^{1} \mathrm{~J}_{\mathrm{CC}}\right)$. This choice for $2 \mathrm{~T}$ acts as a methionine methyl filter, because the signal of all other carbons is greatly reduced by ${ }^{1} \mathrm{~J}_{\mathrm{CC}}$ dephasing (Bax et al., 1994).

In flexible and unfolded proteins, ${ }^{1} \mathrm{H}$ and ${ }^{13} \mathrm{C}$ chemical shift dispersions are very poor and this poses a serious problem in obtaining sequence specific assignments by the standard procedures. ${ }^{15} \mathrm{~N}$ chemical shift dispersions are very good even in unfolded proteins. The possibility of direct identification of $\mathrm{H}^{\mathrm{N}}$ and ${ }^{15} \mathrm{~N}$ chemical shifts of neighboring residues in the HNN spectrum suggests an easy strategy for rapid assignment of resonances along the polypeptide chain. The F1-F3 planes help to identify the ${ }^{15} \mathrm{~N}$ chemical shifts, while the F2-F3 planes enable to identify the corresponding $\mathrm{H}^{\mathrm{N}}$ chemical 
shifts. These features provide many starting points for a backbone walk. (Panchal et al., 2001).

The Nuclear Overhauser Effect (NOE) is a consequence of modulation of the dipole-dipole coupling between different nuclear spins by the Brownian motion of the molecules in solution, and the NOE intensity can be related to the distance $r$ between preirradiated and observed spin by an equation of the general form

$$
\mathrm{NOE} \propto\left\langle 1 / r^{6}\right\rangle \cdot f\left(\tau_{\mathrm{c}}\right)
$$

the second term in the equation is a function of the correlation time $\tau_{\mathrm{c}}$, which accounts for the influence of the motional averaging process on the observed NOE. Because of the low sensitivity for observation of NOEs, the accuracy of integration of line intensities is also limited by low $\mathrm{S} / \mathrm{N}$. Fundamental difficulties can arise when trying to correlate experimental NOE intensities with distances, for example, because of spin diffusion or the prevalence of intramolecular mobility in macromolecules (Wühtrich, 1986).

In polypeptides a ${ }^{1} \mathrm{H}-{ }^{1} \mathrm{H}$ bidimensional NOESY spectrum can be divided into six areas containing the following connectivities:

a. $\mathrm{NH}$; aromatics-NH; aromatics.

b. $\mathrm{NH}$; aromatics- $\alpha \mathrm{H} ; \delta \mathrm{H}$ of Pro; $\beta \mathrm{H}$ of Ser and Thr

c. NH; aromatics-aliphatic side chains

d. $\alpha \mathrm{H} ; \delta \mathrm{H}$ of Pro; $\beta \mathrm{H}$ of Ser and Thr; $\delta \mathrm{H}$ of Pro; $\beta \mathrm{H}$ of Ser and Thr

e. $\alpha \mathrm{H} ; \delta \mathrm{H}$ of Pro; $\beta \mathrm{H}$ of Ser and Thr-aliphatic side chains

f. Aliphatic side chains-aliphatic side chains

to minimize interference with the solvent resonance, the regions $\mathrm{d}$, e, and $\mathrm{f}$ are preferably studied in $\mathrm{D}_{2} \mathrm{O}$ solution. (Wühtrich, 1986)

Three interresidue ${ }^{1} \mathrm{H}-{ }^{1} \mathrm{H}$ distances in the primary polypeptide structure are defined: Sequential distances are those between backbone protons or between a backbone proton and a $\beta$ proton in residues that are nearest neighbors in the amino acid sequence, for example $d_{\alpha \mathrm{N}}(i, i+1)$ and $d_{\mathrm{NN}}(i, i+1)$. Medium-range distances are all nonsequential interresidue distances between backbone protons or between a backbone proton and a $\beta$ proton within a segment of five consecutive residues. Long-range backbone distances are between backbone protons in residues that are at least six positions apart in the sequence. (Wühtrich, 1986)

The NMR structure determination of a protein commonly involves the preparation of uniformly ${ }^{13} \mathrm{C} /{ }^{15} \mathrm{~N}$-labeled, soluble protein, the acquisition of a set of 2D and 3D NMR experiments, NMR data processing, peak picking, chemical shift assignment, NOE assignment and collection of conformational restraints, structure calculation, refinement, and validation. (Wühtrich, 1986).

In order to extract informative distance constraints from a NOESY spectrum, its cross-peaks have to be assigned, i.e. the pairs of hydrogen atoms that give rise to the observed cross-peaks need to be identified. These NOESY assignments are based on ${ }^{1} \mathrm{H}$ chemical shifts values that result from previous sequence-specific resonance assignments. (Herrmann et al., 2002).

Dipolar couplings contain unique "long-range" information not available from NOEs or J couplings, as they orient all vectors relative to a single axis system. For directly bonded pairs of atoms, the internuclear distance is accurately known, and the measured dipolar couplings provide information on the orientation of the internuclear bond vector. For a protein of unknown structure, a reasonable estimate for $A_{a}$ can be 
obtained from the range of dipolar couplings observed. The value of $A_{\mathrm{r}}$ can be determined in a stepwise iterative manner. In contrast, the orientation of the alignment tensor floats freely during structure calculations. (Tjandra and Bax, 1997).

\section{Study of macromolecular dynamics with nuclear magnetic resonance}

Dynamics reflect the molecule's successive passing through different conformational states and represents its "fourth dimension" in space and time. (Brüschweiler, 1994).

The dynamics of spin density operator $\sigma$ is governed by the Liouville-von Neumann equation

$$
(\mathrm{d} / \mathrm{d} t) \sigma(t)=-i \hat{H} \sigma(t)-\Gamma\left\{\sigma(t)-\sigma_{0}\right\}
$$

where $\hat{H}$ and $\Gamma$ describe the Hamiltonian and relaxation superoperators, respectively. Coherent coherence transfer experiments, such as COSY, TOCSY, and INEPT, on one hand exploit the properties of $\hat{H}$ giving rise to deterministic spin dynamics in scalar coupled spin-systems. On the other hand, relaxation experiments, such as NOESY, ROESY, heteronuclear NOE, $\mathrm{T}_{1}, \mathrm{~T}_{2}$, and $\mathrm{T}_{1 \rho}$ experiments, depend critically on $\Gamma$ and monitor spin dynamics induced by stochastic time modulated interactions. Deterministic spin dynamics are indispensable for the characterization of connectivities in scalar Jcoupling networks and are therefore most valuable for spin resonance assignments in complex spectra. Relaxation experiments such as NOESY and ROESY are mainly used as a source of information about averaged internuclear distances of protons for the generation of an (averaged) structure. Experiments measuring heteronuclear NOE values and $\mathrm{T}_{1^{-}}, \mathrm{T}_{2^{-}}$, and $\mathrm{T}_{1 \rho^{-}}$relaxation times allow assessing local molecular mobility as well as medium to fast time scale conformational exchange. (Brüschweiler, 1994).

Although the interplay of the deterministic part $\hat{H}$ with the stochastic part $\Gamma$ can be too complex for analytical calculations, a practical approach in the case of NOESY and ROESY experiments, give good results for the relaxation periods or mixing times, at least in an initial rate regime. (Brüschweiler, 1994).

The total spin Hamiltonian can be split into a deterministic and a stochastic part

$$
\mathrm{H}_{\mathrm{tot}}(t)=\mathrm{H}_{\mathrm{det}}+\mathrm{H}_{\text {stoch }}(t)
$$

the deterministic part of the Hamiltonian operator $\mathrm{H}_{\text {det }}$ in liquids consists of three terms

$$
\mathrm{H}_{\mathrm{det}}=\mathrm{H}_{Z}+\mathrm{H}_{J}{ }^{\text {iso }}+\mathrm{H}_{R F}
$$

$\mathrm{H}_{Z}$ is the time-averaged Zeeman term representing the coupling of the spin-induced nuclear magnetic moments with the external magnetic field $\mathbf{B}_{0}$, which defines the $z$ axis of the laboratory coordinate system

$$
\mathrm{H}_{Z}=\sum_{k=1}^{\mathrm{N}} \omega_{0 k} \mathrm{I}_{k Z}
$$

where $\omega_{0 k}$ is the Larmor frequency of spin $k$ defined as $\omega_{0 k}=-\gamma_{k} B_{0}\left(1-\sigma_{k}{ }^{\text {iso }}\right) \cdot \gamma_{k}$ is the gyromagnetic ratio of spin $k$ and $\sigma_{k}$ iso its time-averaged isotropic chemical shift. $\mathrm{I}_{k z}$ is the spin operator $I_{z}$ acting exclusively on the Liouville-subspace belonging to spin $k$. The Hamiltonian is given in multiples of $h / 2 \pi$ and has the unit rad $\cdot \mathrm{s}^{-1}$. (Brüschweiler, 1994).

The coupling of the spins to a linearly polarized radio frequency (rf) field of strength $2 \mathrm{~B}_{1}$ oscillating along an axis in the $x y$-plane of the laboratory frame with 
frequency $\omega$ and phase $\varphi$ with respect to the $x$-axis is best described in the rotating frame with frequency $\omega$ around the $z$-axis. (Brüschweiler, 1994).

where

$$
\mathrm{H}_{\mathrm{RF}} \cong-\gamma \mathrm{B}_{1}\left\{\mathrm{~F}_{x} \cos (\varphi)+\mathrm{F}_{y} \sin (\varphi)\right\},
$$

$$
\mathrm{F}_{x}=\sum_{k=1}^{\mathrm{N}} \mathrm{I}_{k x} \text { and } \mathrm{F}_{y}=\sum_{k=1}^{\mathrm{N}} \mathrm{I}_{k y}
$$

coupling

$\mathrm{H}_{J}{ }^{\text {iso }}$ represents the time-averaged, indirect, electron-mediated, scalar spin-spin

$$
\begin{aligned}
\mathrm{H}_{J}{ }^{\text {iso }}= & 2 \pi \sum_{k<l} J_{k l} \mathbf{I}_{k} \cdot \mathbf{I}_{l}=2 \pi \sum_{k<l} J_{k l}\left\{\mathrm{I}_{k x} \mathrm{I}_{l x}+\mathrm{I}_{k y} \mathrm{I}_{l y}+\mathrm{I}_{k z} \mathrm{I}_{l z}\right\} \\
= & 2 \pi \sum_{k<l} J_{k l}\left\{1 / 2\left(\mathrm{I}_{k+} \mathrm{I}_{l-}+\mathrm{I}_{k-} \mathrm{I}_{l+}\right)+\mathrm{I}_{k z} \mathrm{I}_{l z}\right\},
\end{aligned}
$$

where the shift operators $\mathrm{I}_{k+}$ are defined by $\mathrm{I}_{k \pm}=\mathrm{I}_{k x} \pm i \mathrm{I}_{k y} \cdot \mathrm{J}_{k l}$ denotes the scalar coupling constant between spin $k$ and $l$ is expressed in units of $\mathrm{Hz}$.

Of practical relevance are couplings between spins separated by less than four chemical bonds. Many multidimensional heteronuclear NMR applications exploit the sensitivity gain achieved by the transfer of magnetization via J-couplings from a spin species with higher $\gamma$, normally ${ }^{1} \mathrm{H}$, to a spin with lower $\gamma$, such as ${ }^{13} \mathrm{C}$ or ${ }^{15} \mathrm{~N}$ using the INEPT or DEPT techniques. (Brüschweiler, 1994).

The structural information carried by vicinal ${ }^{3} \mathrm{~J}$-coupling constants concerning torsion angles is obtained using Karplus relationships. They have the general form

$$
{ }^{3} \mathrm{~J}_{k l}=A \cos ^{2} \chi+B \cos \chi+C
$$

where $A, B, C$ are empirical constants and $\chi$ is the torsion angle defined by the locations of the two coupled spins together with the two intervening atoms. In the weak coupling limit, i.e., in the case where the chemical-shift difference of the spins $k$ and $l$ is much larger than their scalar coupling $\mathrm{J}_{k l}$ (which is always true in the heteronuclear case), first order perturbation theory yields a good approximation for the description of coupling effects and $\mathrm{H}_{\mathrm{J}}$ iso simplifies to

$$
\mathrm{H}_{\mathrm{J}}^{\text {iso }}=2 \pi \sum_{k<l} \mathrm{~J}_{k l} \mathrm{I}_{k z} \mathrm{I}_{l z}
$$

in this limit the effect of $\mathrm{H}_{\mathrm{J}}$ iso on the spin density matrix $\sigma$ can be calculated analytically on the basis of a few simple transformation rules summarized in the product operator formalism. Examples are:

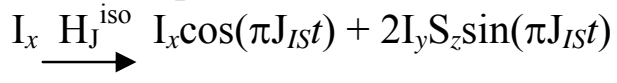

and cyclic permutations in $x, y$, and $z$. (Brüschweiler, 1994).

The stochastic part $\mathrm{H}_{\text {stoch }}(t)$ of the total Hamiltonian can be chosen to have a time average of zero. It manifests itself in the spectrum in the form of relaxation and exchange effects. Neglecting chemical shift anisotropy, the main contributions to $\mathrm{H}_{\text {stoch }}(t)$ for spin systems consisting of spin $1 / 2$ nuclei are the dipolar interaction $\mathrm{H}_{D D}(t)$ and time-dependent parts of $\mathrm{H}_{Z}$ and $\mathrm{H}_{\mathrm{J}}$ iso caused by internal molecular dynamic processes.

$$
\mathrm{H}_{\text {stoch }}(t)=\mathrm{H}_{D D}(t)+\mathrm{H}_{Z}(t)+\mathrm{H}_{J}{ }^{\text {iso }}(t)
$$

(Brüschweiler, 1994).

The time-modulation of $\mathrm{H}_{J}{ }^{\text {iso }}(t)$ can have two different origins. Intramolecular motion can cause a time-dependence in the scalar coupling constant $\mathrm{J}_{k l}$ iso; the relaxation mechanism based on this effect is called scalar relaxation of the first kind. Alternatively, 
fluctuation in $\mathrm{H}_{\mathrm{J}}{ }^{\text {iso }}(t)$ can be caused by a relaxation time of spin $S$, which takes part in the interaction $\mathrm{H}_{\mathrm{J}}^{\text {iso }}(t)$, that is much shorter than $1 / \mathrm{J}_{k l}$. In this case, the $S$ spin contribution to $\mathrm{H}_{\mathrm{J}}{ }^{\text {iso }}(t)$ can be treated like a lattice vector which decorrelates longitudinally with the time constant $T_{1}$ and transversally with $T_{2}$. The resulting relaxation mechanism is called scalar relaxation of the second kind. (Brüschweiler, 1994).

A chemical shift fluctuation $\mathrm{H}_{Z}(t)$ on a time scale much faster than the inverse of the shift fluctuation leads to line-broadening and dependence of $\mathrm{T}_{1 \rho}$ on the rf-field strength $B_{1}$.

As a consequence of the isotropy of space (composed of spin space and real space), any spin Hamiltonian $H$ transforms under a rotation, which is applied identically to spin space and real space, like a scalar. $\mathrm{H}$ can be expressed as a tensor contraction of irreducible tensor operators $\left\{\mathrm{T}_{k}{ }^{q}\right\}$ of rank $k$ in spin space and irreducibly transforming spherical harmonics $\left\{\mathrm{F}_{k}{ }^{q}\right\}$ of rank $k$ in normal space

$$
\mathrm{H}=\sum_{k} c_{k} \sum_{q=-k}(-1)^{q} \mathrm{~F}_{k}^{-q} \mathrm{~T}_{k}^{q},
$$

where $c_{k}$ is the interaction strength of the component of rank $k$. An irreducible tensor operator $\left\{\mathrm{T}_{k}^{q}\right\}$ of order $\mathrm{k}$ is defined as a set of $2 \mathrm{k}+1$ operators $\left\{\mathrm{T}_{k}^{q}\right\}, q=-k,-k+1, \ldots, k-$ $1, k$ that fulfill

$$
\left[\mathrm{J}_{z}, \mathrm{~T}_{k}^{q}\right]=q \mathrm{~T}_{k}^{q} \text { and }\left[\mathrm{J}_{ \pm}, \mathrm{T}_{k}^{q}\right]=\mathrm{T}_{k}^{q \pm 1} \sqrt{k}(k+1)-q(q \pm 1)
$$

$k=2$ for $\mathrm{H}_{D D}(t), k=1$ for $\mathrm{H}_{\mathrm{Z}}(t)$, and $k=0$ for $\mathrm{H}_{\mathrm{J}}$ iso $(t)$. The tensor operators have simple transformation properties under three-dimensional rotations $\mathrm{R}(\alpha, \beta, \gamma)$

$$
\mathrm{R}(\alpha, \beta, \gamma) \mathrm{T}_{k}^{m} \mathrm{R}^{-1}(\alpha, \beta, \gamma)=\sum_{m^{\prime}=-k} \mathrm{~T}_{k}^{m^{\prime}} D^{k}{ }_{m}{ }^{\prime}(\alpha, \beta, \gamma),
$$

where $\alpha, \beta, \gamma$ are the three Euler angles and $\mathrm{D}^{k}{ }_{m}{ }^{\prime}$ the Wigner rotation matrix elements of order $k$. The $\left\{\mathrm{T}_{k}{ }^{q}\right\}$ can be transformed easily into the interaction frame induced by the interaction dominant in $H_{\text {det. }}$ (Brüschweiler, 1994).

$\mathrm{H}_{D D}(t)$ refers to the magnetic dipole-dipole interaction of two nuclei $I$ and $S$ connected by the vector $\mathbf{r}_{I S}$ which has an orientation described by the polar angles $\Omega_{D D}{ }^{\text {lab }}$ $=\left(\theta^{\text {lab }}, \phi^{\text {lab }}\right)$ with respect to the laboratory frame

$$
\mathrm{H}_{D D}(t)=\xi\left(1 / \mathrm{r}^{3}{ }_{I S}\right) \sum_{q=-2}(-1)^{q} \mathrm{~F}_{2}^{-q}\left(\Omega_{D D}{ }^{\mathrm{lab}}(t)\right) \mathrm{T}_{2}^{q}(\mathbf{I}, \mathbf{S})
$$

The prefactor $\xi=-\sqrt{2} 4 \pi / 5\left(\mu_{0} / 4 \pi\right)(h / 2 \pi) \gamma I \gamma S$ is chosen such that the lattice functions $\mathrm{F}_{2}{ }^{q}$ are identical to the normalized second order spherical harmonics $\mathrm{Y}_{2}{ }^{q}(\theta, \phi)$ given by

$$
\begin{aligned}
& Y_{2}{ }^{0}(\theta, \phi)=\sqrt{ } 5 / 16 \pi\left(3 \cos ^{2} \theta-1\right) \\
& Y_{2}{ }^{ \pm 1}(\theta, \phi)= \pm \sqrt{ } 15 / 8 \pi \sin \theta \cos \theta e^{ \pm i \phi} \\
& Y_{2}{ }^{ \pm 2}(\theta, \phi)=\sqrt{ } 15 / 32 \pi \sin ^{2} \theta e^{ \pm i 2 \phi}
\end{aligned}
$$

The irreducible spin tensor operators $\mathrm{T}_{2}{ }^{q}(\mathbf{I}, \mathbf{S})$ are in the case of the dipole-dipole interaction $\mathrm{H}_{D D}$ given by

$$
\begin{aligned}
& \mathrm{T}_{2}^{0}(\mathbf{I}, \mathbf{S})=1 / \sqrt{ } 6\left(2 \mathrm{I}_{\mathrm{Z}} \mathrm{S}_{\mathrm{Z}^{-}}{ }^{1 / 2}\left(\mathrm{I}_{+} \mathrm{S}_{-}+\mathrm{I}_{-} \mathrm{S}_{+}\right)\right) \\
& \mathrm{T}_{2}^{ \pm 2}(\mathbf{I}, \mathbf{S})=1 / 2\left(\mathrm{I}_{ \pm} \mathrm{S}_{ \pm}\right)
\end{aligned}
$$


$\mathrm{H}_{D D}$ depends on the orientation of the molecule with respect to the external magnetic field $\mathbf{B}_{0}$ and on the distance between the interacting spins. Therefore, molecular dynamics taking place in real space is reflected in the form of time-modulated interactions in spinspace. This is the basis of any NMR relaxation experiment in solution. At a given time the values of the stochastically fluctuating Hamiltonians differ from the different spin systems, i.e., molecules, in the ensemble. This is in contrast to the deterministic Hamiltonian. Although the interaction strength of $\mathrm{H}_{D D}$ can be in the order of $10 \mathrm{KHz}$, considerably larger than the magnitude of $\mathrm{H}_{\mathrm{J}}$ iso, $\mathrm{H}_{D D}$ has within first order perturbation theory no effect on the resulting $1 \mathrm{D}$ spectrum, i.e., $\mathrm{H}_{D D}$ does not cause peak shifts and peak splittings. This is a consequence of the rapid rotational tumbling of peptides and proteins with correlation times between $10^{-10}$ and $10^{-7} \mathrm{~s}$ and the fact that $\mathrm{H}_{D D}$ is a traceless operator. However, $\mathrm{H}_{D D}$ plays a significant and for spin $1 / 2$ systems often dominant role for spin relaxation. (Brüschweiler, 1994).

The model free approach introduced by Lipari and Szabo (1981) aims at a description relying on a minimum number of parameters to circumvent over interpretation of the experimental relaxation data and relates a generalized meaning to the parameters. The parameter set consists of two correlation times reflecting the overall tumbling motion and internal time scale of intramolecular motion and an order parameter $S^{2}$ representative of the orientational restriction of the involved internuclear vector. A restriction lies in an ambiguity due to the fact, that the dipolar Hamiltonian is invariant under exchange of the two involved spins. For instance, absence of angular motion implies $S^{2}=1$, but $S^{2}=1$ does not imply absence of angular motion. The advantage of the approach is that it gives spatially resolved, direct information about the extent and time scale of motion being present. Furthermore, it provides both intuitive and quantitative insight into the interplay of measurable relaxation rates and internal motion and serves as a convenient interface between experiments and more detailed motional models. (Brüschweiler, 1994).

A type of motion which is always present for molecules in solution is the overall rotational tumbling. It is the rotational analogue to the Brownian translational diffusion and also a consequence of intermolecular collisions. For biomolecules dissolved in simple solvents the overall tumbling corresponds to a small step diffusion process. Tensor interactions which depend on the orientation of the molecule in the laboratory frame, such as $\mathrm{H}_{D D}(t)$, couple directly to the tumbling, whereas $\mathrm{H}_{Z}(t)$ and $\mathrm{H}_{\mathrm{J}}{ }^{\text {iso }}(t)$ remain unaffected by the tumbling since they depend on internal coordinates only. The overall rotational tumbling therefore decouples these two classes of interactions and as a result, relaxative interference between them cannot occur. Hence, for the first class both rotational tumbling and internal dynamics influence the resulting relaxation rates, whereas for the second class relaxation is induced by intramolecular motion only. (Brüschweiler, 1994).

For $\mathrm{H}_{D D}(t)$ in the isotropic case, using the transformation properties of spherical harmonics of order two, the following correlation function is obtained

$$
\begin{aligned}
\mathrm{C}_{\mu \nu}{ }^{\mathrm{q}}(\tau) & =\left\langle c_{\mu}(0) c_{v}(\tau) \mathrm{Y}_{2}{ }^{q}\left(\Phi_{\mu}{ }^{{ }^{\mathrm{lab}}}(0)\right) \mathrm{Y}_{2}{ }^{q_{*}}\left(\Phi_{v}{ }^{{ }^{\mathrm{lab}}}(\tau)\right)\right\rangle \\
& =\sum_{r r}\left\langle\mathrm{D}_{r q}{ }^{(2)}(\Omega(0)) \mathrm{D}_{r^{\prime}{ }^{\prime}}{ }^{(2)} *(\Omega(\tau))\right\rangle \cdot\left\langle c_{\mu}(0) c_{v}(\tau) \mathrm{Y}_{2}{ }^{r}\left(\Phi_{\mu}{ }^{\mathrm{mol}}(0)\right) \mathrm{Y}_{2}{ }^{{ }^{\prime}}{ }^{\prime} *\left(\Phi_{v}{ }^{\mathrm{mol}}(\tau)\right)\right\rangle,
\end{aligned}
$$

where $\Phi_{\mu}{ }^{\mathrm{lab}}, \Phi_{v}{ }^{\mathrm{mol}}$ denote polar angles $(\theta, \varphi)$ of the internuclear vector of interaction $\mu$ in the laboratory frame and the molecule fixed frame, respectively. $\Omega$, which is the 
argument of the Wigner matrix $\mathrm{D}_{r q}{ }^{(2)}$, represents the three Euler angles $(\alpha, \beta, \gamma)$ relating the laboratory frame to the molecular frame for different times. Rotational tumbling affects only the first factor of the previous equation and the averaging can be carried out by solving the rotational diffusion equation

$$
(d f / d \tau)=\mathrm{D} \nabla_{\text {rot }}^{2} \text { with } f(0)=\delta(\Omega(0)),
$$

where $\nabla_{\text {rot }}^{2}$ is the angular part of the Laplacian operator in spherical coordinates leading to

$$
\left\langle\mathrm{D}_{r q}{ }^{(2)}(\Omega(0)) \mathrm{D}_{r^{\prime} q}{ }^{(2)} *(\Omega(\tau))\right\rangle=(1 / 5) e^{-6 \mathrm{D} \tau \delta r, r^{\prime}},
$$

where $D$ is the rotational diffusion constant. After substitution into the correlation function equation it yields

$$
\mathrm{C}_{\mu \nu}{ }^{q}(\tau)=(1 / 5) e^{-6 D \tau} \sum_{r=-2}\left\langle c_{\mu}(0) c_{v}(\tau) \mathrm{Y}_{2}{ }^{r}\left(\Phi_{\mu}{ }^{\mathrm{mol}}(0)\right) \mathrm{Y}_{2}{ }^{r} *\left(\Phi_{v}{ }^{\mathrm{mol}}(\tau)\right)\right\rangle
$$

The correlation function $\mathrm{C}_{\mu \nu}{ }^{\mathrm{q}}(\tau)$ does not depend on the index $q$. This is a direct consequence of the isotropy of the solvent and applies also in the case of anisotropic tumbling. In the case of a rigid molecule the quantities $c_{\mu}, c_{v}, \Phi_{\mu}{ }^{\mathrm{mol}}$, and $\Phi_{v}{ }^{\mathrm{mol}}$ are time independent and the sum can be simplified by the use of the addition theorem of spherical harmonics

$$
((2 l+1) / 4 \pi) P_{k}\left(\cos \theta_{\mu \nu}\right)=\sum_{q=-k} \mathrm{Y}_{k}^{q}\left(\Phi_{\mu}\right) \mathrm{Y}_{k}^{q *}\left(\Phi_{v}\right)
$$

The equation (1) can be rewritten

(Brüschweiler, 1994).

$$
\mathrm{C}_{\mu \nu}(\tau)=(1 / 4 \pi) e^{-\tau / \tau c} c_{\mu} C_{v} P_{2}\left(\cos \theta_{\mu \nu}\right),
$$

where the rotational tumbling correlation time is defined by $\tau_{\mathrm{c}}=1 / 6 \mathrm{D}$. (Brüschweiler, 1994).

The Fourier transform of the previous equation leads to the spectral density $J_{\mu v}(\omega)$

$$
\mathrm{J}_{\mu v}(\omega)=(1 / 4 \pi) c_{\mu} c_{v} P_{2}\left(\cos \theta_{\mu \nu}\right)\left(2 \tau_{\mathrm{c}} /\left(1+\omega^{2} \tau_{\mathrm{c}}{ }^{2}\right)\right) \quad \text { (Brüschweiler, 1994). }
$$

For auto-correlation relaxation experiments (e.g., NOESY) where $\mu=v$ and thus $\theta_{\mu \mu}=0$, the structurally important information is contained in the distance dependent term $c_{\mu}{ }^{2}$ only. For cross-correlation experiments, on the other hand, additional geometric information can be obtained from the term $P_{2}\left(\cos \theta_{\mu \nu}\right)$.(Brüschweiler, 1994).

The diffusion coefficient $D$ can be related to the hydrodynamic molecular radius $\mathrm{R}_{h}$ according to the Einstein-Stokes relationship

$$
\mathrm{R}_{h}=k \mathrm{~T} / 8 \pi \eta D \text {, }
$$

where $\eta$ is the solvent viscosity. (Brüschweiler, 1994).

The decorrelation of a tensor of rank 1, e.g. a vector attached to a molecule, is obtained similarly

$$
\left\langle\cos \left(\Omega-\Omega^{\prime}\right)\right\rangle=e^{-2 D \tau}
$$

where $\Omega-\Omega^{\prime}$ is the angle between the two directions $\Omega$ and $\Omega^{\prime}$, which are adopted at times 0 and $\tau$, respectively. (Brüschweiler, 1994).

A protein in solution undergoes constant random thermal motions within a stable equilibrium structure. These motions involve displacements of individual atoms, bonds, functional groups, side-chains, local regions of the backbone, secondary structure elements, and entire folded domains. Many proteins also undergo thermally driven transitions, called conformational changes, between two or more equilibrium structures. (Falke, 2002). 
Both types of motions can play important functional roles. Random thermal motions and the average conformation can both change substantially when a protein is modified by substrate or ligand binding, docking to another macromolecule, or covalent modification (such as phosphorylation). Such changes often have important functional consequences for the tuning of binding affinities and the switching of regulatory proteins. (Falke, 2002).

Dynamics play a role in certain aspects of enzyme function, but the links between dynamics and catalysis remain unclear. In the first step of an enzyme-catalyzed reaction, substrate binding typically induces a conformational change within the enzyme, thereby enclosing the substrate in a cavity protected from solvent or places the catalytic residues near the substrate. An even more fascinating structural rearrangement may occur during the catalytic step of the reaction, when the complex moves from the ground state to the transition state. Such transient rearrangement could simply serve to accommodate the structural changes in the substrate as the transition state is reached or could actively contribute to catalysis by preferentially stabilizing the transition state. Yet, because the transition state exists only fleetingly, enzyme dynamics during transition state formation and decay have never been directly detected during an enzyme-catalyzed reaction in solution. (Falke, 2002).

Early experiments provided evidence for rotation of bulky aromatic side chains as a function of temperature within the hydrophobic core of small globular proteins. This showed that proteins were dynamic over a spectrum of time scales and complemented the static pictures of protein structure that were already emerging from X-ray diffraction. We now know that there is an intimate relation between dynamics and molecular function. Nuclear magnetic resonance (NMR) spectroscopy is uniquely suited to study many of these dynamic processes, because site-specific information can be obtained for motions that span many time scales, from rapid bond librations (picoseconds) to events that take seconds. (Mittermaier and Kay, 2006).

Many biochemical events occur on the microsecond to millisecond time scale, and it is of considerable interest to characterize the conformational transitions that are involved in such processes. However, intermediates are often formed only transiently and are populated at levels that are not amenable to traditional structural approaches. (Mittermaier and Kay, 2006).

When internal motions and molecular tumbling cause reorientation of the ${ }^{1} \mathrm{H}-{ }^{15} \mathrm{~N}$ bond vector with respect to the external magnetic field, the local magnetic field at the site of the ${ }^{15} \mathrm{~N}$ spin that derives from the directly attached ${ }^{1} \mathrm{H}$ magnetic dipole fluctuates (see Figure 10).

Although the local dipolar interaction between ${ }^{1} \mathrm{H}$ and ${ }^{15} \mathrm{~N}$ spins averages to zero because of the molecular tumbling, the time-dependent variations in the field lead a spin system that has been perturbed by radio-frequency pulses to return, or relax, to thermal equilibrium. Because the fluctuations of the local magnetic fields are sensitive to internal motions, measurement of NMR relaxation rates provides a direct avenue to extracting dynamic parameters. (Mittermaier and Kay, 2006).

A pair of basic nitrogen-spin relaxation experiments are used to probe backbone dynamics in proteins. The ${ }^{15} \mathrm{~N}$ relaxation experiments monitor either the recovery of ${ }^{15} \mathrm{~N}$ Z-magnetization to its equilibrium position $\left(\mathrm{T}_{1}\right)$ or the decay of magnetization orthogonal to the $\mathrm{Z}$ axis to its zero equilibrium value $\left(\mathrm{T}_{2}\right)$. The ${ }^{1} \mathrm{H}$ spin has a magnetogyric ratio $(\gamma)$ 
that is 10 times larger than that of ${ }^{15} \mathrm{~N}$, and the inherent sensitivity of the NMR experiment scales as $\gamma^{5 / 2}$; therefore experimental sensitivity is optimized by shuffling magnetization from an amide ${ }^{1} \mathrm{H}$ to its directly coupled ${ }^{15} \mathrm{~N}$ and then back again to ${ }^{1} \mathrm{H}$ for detection as a 2D data set. One peak is obtained for each $\left({ }^{1} \mathrm{H}-{ }^{15} \mathrm{~N}\right)$ pair in the protein, with an intensity proportional to $\exp (\tau / \mathrm{T} i)$ where $\mathrm{T} i$ is the $\mathrm{T}_{1}$ or $\mathrm{T}_{2}$ value of the particular ${ }^{15} \mathrm{~N}$ nucleus and $\tau$ is a variable relaxation delay; relaxation times are measured by recording a series of spectra and fitting the peak intensities as a function of $\tau$. The values of Ti so obtained are usually interpreted in terms of generalized order parameters that describe the amplitude of bond vector motions and time constants that indicate the time scale of the internal motions, but specific models can be used as well. (Mittermaier and Kay, 2006).
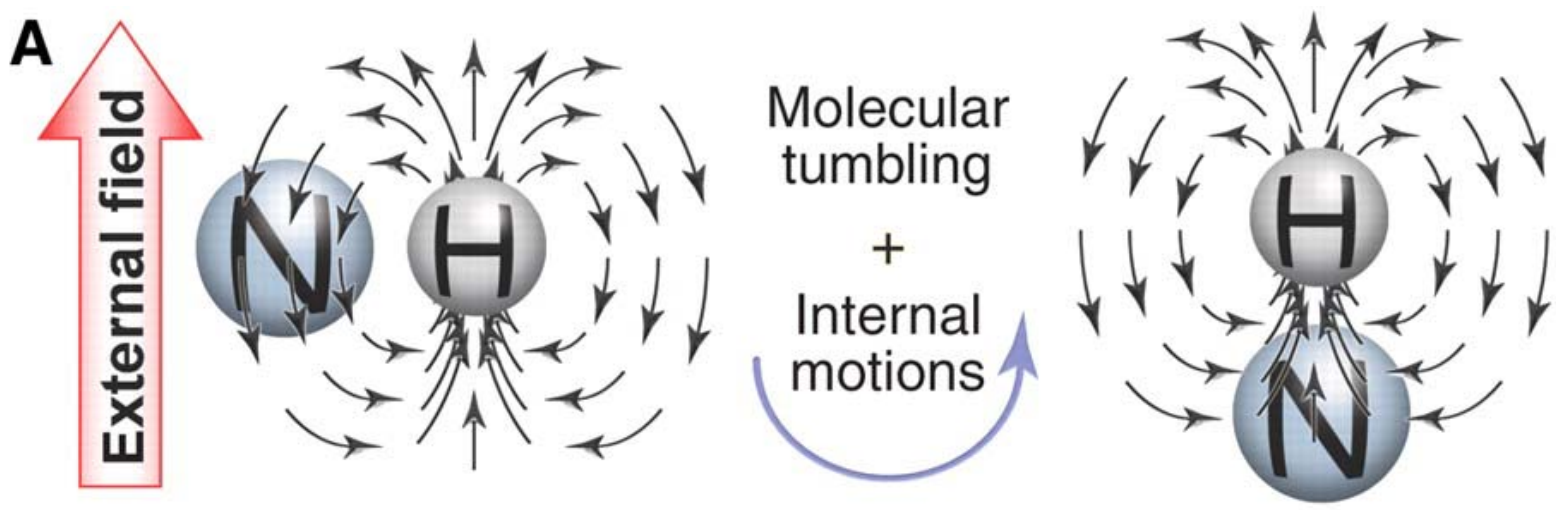

Figure 10. Orientation-dependent magnetic field experienced by an amide ${ }^{15} \mathrm{~N}$ nucleus due to the directly bonded proton (taken from Mittermaier and Kay, 2006).

When a protein is not dissolved in isotropic solution but rather in a media that leads to fractional alignment (typically about $0.1 \%$ ), the dipolar interaction does not average to zero. Instead, for a fixed orientation of the ${ }^{1} \mathrm{H}-{ }^{15} \mathrm{~N}$ spin vector, the effective magnetic field at the ${ }^{15} \mathrm{~N}$ spin is either increased or decreased depending on the ${ }^{1} \mathrm{H}$ spin state, leading to dipolar splittings in spectra. These splittings are a rich source of structural information, because they report on the orientation of bond vectors with respect to an external coordinate frame (the magnetic field). However, there is also potential for studies of biomolecular dynamics because motions that modulate local fields over a broad time regime (picosecond to millisecond) can affect dipolar splittings. (Mittermaier and Kay, 2006).

Recently, new NMR experiments have been developed to provide site-specific information about protein motions spanning a range of time scales. Some of the new applications involve large molecular complexes, where motion is likely to be critical for function. NMR methods exploiting the TROSY (transverse relaxation optimized spectroscopy) principle have emerged for both backbone positions and side chain methyl groups. (Mittermaier and Kay, 2006).

Upon placement of a molecule in a magnetic field, a magnetic dipole moment is induced which is proportional to the susceptibility, $\chi$. For diamagnetic molecules $(\chi<0)$, induced moments will oppose the field while for paramagnetic molecules $(\chi>0)$, induced moments will be along the field. These induced moments will in turn interact with the magnetic field. Molecules of non-spherical symmetry will exhibit an orientational 
dependence of this interaction due to their anisotropic distribution of electron density. Thus, the susceptibility is a tensorial quantity and the orientation dependent energy of interaction, $W$, can be written,

$$
W=1 / \mu_{0}(-1 / 2 \mathbf{B} \cdot \chi \cdot \mathbf{B}) \text {, }
$$

the size of the interaction energy $(W)$ depends on the square of the magnetic field strength $\left(\mathrm{B}_{0}{ }^{2}\right)$ and the magnetic susceptibility tensor, $\chi$. Variations in $W$ with orientation arise from the anisotropic part of the $\chi$ tensor, and if these variations are large enough compared to the thermal energy, $k \mathrm{~T}$, a measurable degree of orientational order will be induced (Tjandra and Bax, 1997).

Cooperatively aligned media are generally applicable to the widest range of systems. Moreover, the degree of solute alignment is usually higher and often tuneable. Direct magnetic field alignment, often allows independent determination of the degree of alignment, and thus, may become the method of choice for binding or dynamic studies (Tjandra and Bax, 1997).

The dipolar couplings provide direct information on the orientations of the corresponding bond vectors relative to the protein's magnetic susceptibility tensor. These constraints are therefore fundamentally different from the strictly local NOE and J coupling constraints (Tjandra and Bax, 1997).

Unfortunately, the magnetic interaction energy for an individual macromolecule is generally so weak that only in favourable systems, and with considerable effort, can these dipolar couplings be measured with sufficient accuracy. With the use of a an aqueous, dilute, liquid crystalline (LC) phase, an adjustable degree of molecular alignment can be obtained, it allows the direct measurement of ${ }^{1} \mathrm{H}_{-}{ }^{15} \mathrm{~N}$ and many other types of dipolar interactions with high accuracy (Tjandra and Bax, 1997).

In full analogy to the case of magnetic susceptibility anisotropy, where dipolar couplings define the orientation of the interaction relative to the magnetic susceptibility tensor, a molecular alignment tensor $\mathbf{A}$ is defined, which can be decomposed into an axially symmetric component $A_{a}$ and a rhombic component $A_{r}$. The magnitude and orientation of $\mathbf{A}$ is not known a priori but is determined from the measured dipolar couplings. The observed residual dipolar coupling between two nuclei, $\mathrm{P}$ and $\mathrm{Q}$, is given by

$$
\mathrm{D}_{\mathrm{PQ}}(\theta, \phi)=S\left(\mu_{0} / 4 \pi\right) \gamma_{\mathrm{P}} \gamma_{\mathrm{Q}} h\left[A_{\mathrm{a}}\left(3 \cos ^{2} \theta-1\right)+3 / 2 A_{\mathrm{r}} \sin ^{2} \theta \cos 2 \phi\right] / 4 \pi^{2} r^{3}{ }_{\mathrm{PQ}},
$$

where $S$ is the generalized order parameter for internal motion of the vector PQ, $\mu_{0}$ is the magnetic permeability of vacuum, $\gamma_{\mathrm{P}}$ and $\gamma_{\mathrm{Q}}$ are the magnetogyric ratios of $\mathrm{P}$ and $\mathrm{Q}, h$ is Planck's constant, $r_{\mathrm{PQ}}$ is the distance between $\mathrm{P}$ and $\mathrm{Q}$, and $\theta$ and $\phi$ are cylindrical coordinates describing the orientation of the $\mathrm{PQ}$ vector in the principal axis system of $\mathbf{A}$. Values of $S^{2}$ obtained from ${ }^{15} \mathrm{~N}$ or ${ }^{13} \mathrm{C}$ relaxation experiments typically range from 0.7 to 0.9 , that is, $S$ falls between 0.85 and 0.95 . Values for $A_{\mathrm{a}}$ and $A_{\mathrm{r}}$ depend on the shape of the protein and vary with the bicelle concentration, which is adjusted to yield and $A_{\mathrm{a}}$ of $\sim 10^{-3}$. (Tjandra and Bax, 1997).

For such small alignment values, only dipolar couplings between nearby nuclei give rise to observable splittings, which keeps the NMR spectrum simple. If there is also a scalar coupling $\mathrm{J}$ between the two nuclei, the observed splitting corresponds to $\mathrm{J}_{\mathrm{PQ}}+$ $\mathrm{D}_{\mathrm{PQ}}$. For one-bond ${ }^{1} \mathrm{H}-{ }^{13} \mathrm{C},{ }^{1} \mathrm{H}-{ }^{15} \mathrm{~N}$, or ${ }^{13} \mathrm{C}-{ }^{13} \mathrm{C}$ interactions, $\mathrm{J}_{\mathrm{PQ}}$ is relatively large and its sign is known. The change from the isotropic to the aligned phase then yields the magnitude and sign of $\mathrm{D}_{\mathrm{PQ}}$. For pairs of protons that are not $\mathrm{J}$ coupled, only the 
magnitude of $\mathrm{D}_{\mathrm{PQ}}$ is obtained, and additional experiments need to be developed to obtained information about the sign of this coupling. (Tjandra and Bax, 1997).

For a protein of unknown structure, a reasonable estimate for $A_{\mathrm{a}}$ can be obtained from the range of dipolar couplings observed. The value of $A_{\mathrm{r}}$ can be determined in a stepwise iterative manner. In contrast, the orientation of the alignment tensor floats freely during structure calculations. For directly bonded pairs of atoms, the internuclear distance is accurately known, and the measured dipolar coupling provides information on the orientation of the internuclear bond vector. (Tjandra and Bax, 1997).

Small changes in chemical shift between the LC and isotropic states $[\leq 0.1 \mathrm{ppm}$ for ${ }^{15} \mathrm{~N}$ and ${ }^{13} \mathrm{C} ; \leq 0.01 \mathrm{ppm}$ for $\left.{ }^{1} \mathrm{H}\right]$ result from incomplete averaging of chemical shift anisotropy (CSA) in the aligned state. In principle, it is possible to separate this CSA effect from the temperature dependence of the chemical shift, thereby providing access to the potentially important structural information contained in the CSA tensor. (Tjandra and Bax, 1997).

An arbitrary reference frame can be chosen, that is fixed within the molecule, allowing the description of the orientation of the ijth internuclear vector in terms of its time independent polar angles $\theta$ and $\phi$. Likewise, at any instant in time, the orientation of the magnetic field can be described in terms of its polar angles $\zeta(t)$ and $\xi(t)$, which are time dependent due to overall reorientation of the molecule (see Figure 11). The $\mathrm{P}_{2}(\cos \theta(t))$ term is expanded making use of the spherical harmonic addition theorem,

$$
\mathrm{P}_{2}(\cos \theta(t))=(4 \pi / 5) \sum_{q=-2} \mathrm{Y}_{2}{ }^{\mathrm{q}} *(\theta \phi) \mathrm{Y}_{2}{ }^{\mathrm{q}}(\zeta(t) \xi(t))
$$

where the Ys refer to the normalized spherical harmonics. The formula for the dipolar couplings becomes

$$
\mathrm{D}_{i j}{ }^{\text {res }}=-\left(\mu_{0} / 4 \pi\right)\left(\gamma_{i} \gamma_{j} h / 2 \pi^{2} \mathrm{r}^{3}{ }_{i j}\right)(4 \pi / 5) \sum_{q=-2}^{2} \mathrm{Y}_{2}{ }^{q_{*}}(\Phi)\left\langle\mathrm{Y}_{2}{ }^{q}(\Omega(t))\right\rangle,
$$

where $\Phi=(\theta, \phi)$ and $\Omega(t)=(\zeta(t) \xi(t))$ have been used to represent the polar angles of the internuclear vector and the magnetic field relative to the molecule fixed frame, respectively.

When combined with knowledge of the structure of the molecule or molecular fragment, measurement of dipolar couplings corresponding to at least five independent dipolar interaction vectors will allow all five averages to be determined. This procedure corresponds to a determination of Saupe's order tensor, originally formulated in a Cartesian representation:

$\mathrm{D}_{i j}{ }^{\text {res }}=-\left(\mu_{0} / 4 \pi\right)\left(\gamma_{i} \gamma_{j} h / 2 \pi^{2} r^{3}{ }_{i j}\right)(4 \pi / 5) \sum S_{k l} \cos \left(\alpha_{k l}\right) \cos \left(\alpha_{l}\right) \quad$ (Prestegard et al., 2000).

The internal geometry is represented by direction cosines describing the orientation of the internuclear vector within the molecular frame. Overall motion and orientation of the alignment frame is absorbed into the elements $S_{k l}$, which form the order tensor, a traceless, symmetric 3 X 3 matrix having just five independent elements. The Cartesian and spherical representations are related by the following linear transformations:

$S_{X z}=\sqrt{ } 3 / 8 \sqrt{ }(4 \pi / 5)\left(\left\langle\mathrm{Y}_{2}{ }^{1}(\Omega(t))\right\rangle-\left\langle\mathrm{Y}_{2}{ }^{-1}(\Omega(t))\right\rangle\right)$

$S_{y z}=i \sqrt{ } 3 / 8 \sqrt{ }(4 \pi / 5)\left(\left\langle\mathrm{Y}_{2}{ }^{-1}(\Omega(t))\right\rangle-\left\langle\mathrm{Y}_{2}{ }^{1}(\Omega(t))\right\rangle\right)$

$S_{x y}=i \sqrt{ } 3 / 8 \sqrt{ }(4 \pi / 5)\left(\left\langle\mathrm{Y}_{2}^{2}(\Omega(t))\right\rangle-\left\langle\mathrm{Y}_{2}^{-2}(\Omega(t))\right\rangle\right)$ 


$$
\begin{aligned}
& S_{x x^{-}} S_{y y}=\sqrt{ } 3 / 2 \sqrt{ }(4 \pi / 5)\left(\left\langle\mathrm{Y}_{2}^{2}(\Omega(t))\right\rangle-\left\langle\mathrm{Y}_{2}^{-2}(\Omega(t))\right\rangle\right) \\
& S_{z z}=\sqrt{ }(4 \pi / 5)\left(\left\langle\mathrm{Y}_{2}^{0}(\Omega(t))\right\rangle\right.
\end{aligned}
$$

(Prestegard et al., 2000).

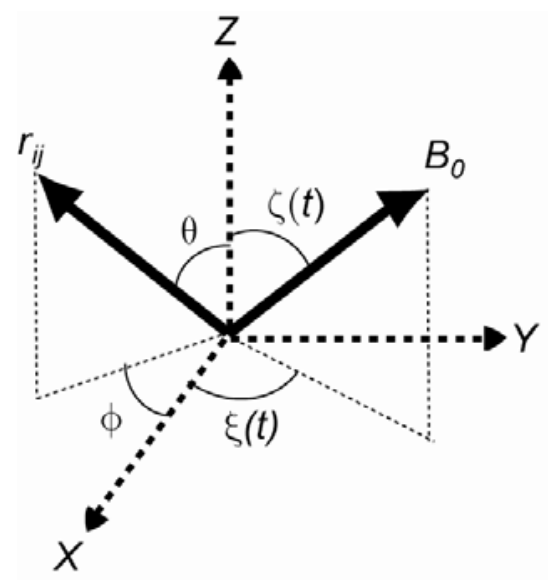

Figure 11. Orientation of the internuclear vector and the magnetic field relative to a molecule fixed frame. The time dependence of the magnetic field vector arises due to molecular reorientation (taken from Prestegard et al., 2000).

The extraction of molecular orientation information from order information contained in the above expressions is achieved by diagonalization of the order tensor. The principle values can be recast into an order parameter for the most ordered axis, $S_{z Z}$, and an asymmetry parameter, $\eta=\left(S_{x x}-S_{y y}\right) / S_{z z}$, which describes the deviation from axially symmetric ordering. The transformation leading to diagonalization also relates the orientation of the principal alignment axes to the initial molecular frame, and hence, describes the mean orientation of the molecule, or a fragment of a molecule, relative to the magnetic field. This transformation is frequently represented in terms of three Euler angles. (Prestegard et al., 2000).

Working in the principal axis frame described by the three Euler angles, only two of the averages in equation (2) remain nonzero. These two averages can also be related to the anisotropies of a generalized alignment tensor, as well as to elements of the Saupe order tensor,

$\left\langle\mathrm{Y}_{2}{ }^{0}(\Omega(t))\right\rangle=\sqrt{ }(5 / 4 \pi) S_{z z}=\sqrt{ }(5 / 4 \pi) A_{\mathrm{a}}$ $\left\langle\mathrm{Y}_{2}^{ \pm 2}(\Omega(t))\right\rangle=\sqrt{ }(5 / 24 \pi)\left(S_{z z}-S_{y y}\right)=\sqrt{ }(5 / 32 \pi) A_{\mathrm{r}}$, for cases in which alignment is achieved due to interaction between the magnetic field and the anisotropic magnetic susceptibility of the molecule, an analytical description of the magnitudes of overall alignment can be formulated, provided that solute molecules do not interact with one another. (Prestegard et al., 2000).

$$
A_{\mathrm{a}}=\Delta \chi\left[\mathrm{B}^{2} / 15 \mu_{0} k \mathrm{~T}\right], A_{\mathrm{r}}=\delta \chi\left[\mathrm{B}^{2} / 15 \mu_{0} k \mathrm{~T}\right]
$$

Both structural and motional variation affect measured residual dipolar couplings, and, in general, the effects must be separated before conclusions about either structure or internal motion can be drawn. (Prestegard et al., 2000). 
Lanthanides can impart a large paramagnetic anisotropy and, hence, greatly enhance levels of alignment. Dysprosium and Terbium are the lanthanide ions with the highest magnetic susceptibility anisotropy. (Prestegard et al., 2000).

The electronic properties of lanthanides are peculiar in that spin-orbit interactions are very large, larger in fact than ligand field effects. Spin-orbit interactions couple the orbital, L, and spin, S, angular momenta. The resulting free ion terms are characterized by J values which are vector combinations of L and S. (Bertini and Luchinat, 1996).

Ligand field effects split the $\mathrm{J}$ manifold in a way that is not easily predicted without specific calculations. However, the overall splitting is such that many of the levels are appreciably populated at room temperature. Ligand field effects remove the spherical symmetry around the metal ion and cause magnetic susceptibility anisotropy. (Bertini and Luchinat, 1996).

Magnetic susceptibility anisotropies are tensorially additive; and depending on the relative orientation of the resulting magnetic susceptibility tensors, constructive or destructive addition may result. (Prestegard et al., 2000).

A broad class of applications involves replacement of native metals in metalloproteins with paramagnetic ions of similar ionic radii. One class of proteins for which this approach has been successfully applied is the calcium binding proteins. (Prestegard et al., 2000).

For paramagnetic systems, the paramagnetic susceptibility tensor will often dominate the susceptibility, and paramagnetic contribution to the tensor can be calculated independently from the measurement of a sufficiently large number of pseudocontact shifts. Moreover, the measurement of pseudocontact shifts can provide an independent measure of order that is less sensitive to internal motions. (Prestegard et al., 2000).

One of the inherent limitations that arise in the analysis of dipolar data stems from the fact that dipolar coupling measurements do not have a one-to-one correspondence to a pair of polar angles describing the orientation of the interaction vector. A given residual dipolar coupling measurement can only restrict the orientation of the corresponding internuclear vector to two cones. Addition of data corresponding to other vectors rigidly related to the first can reduce the number of possible solutions. (Prestegard et al., 2000).

In an unpaired electron-nucleus system, the $\mathbf{I} \cdot A_{\text {dip }} \cdot \mathbf{S}$ term of the Hamiltonian describes the dipolar part of the electron-nucleus coupling. For a single unpaired electron with spin angular momentum, $\hat{\mathrm{s}}$, this term can be written as

$$
H_{\text {dip }}=\left(\mu_{0} / 4 \pi\right) h \gamma_{\mathrm{N}} \mu_{\mathrm{B}}\left\{\left(2 l_{\mathrm{N}} \cdot \mathbf{I} / \mathbf{r}_{\mathrm{N}}^{3}\right)+g_{e}\left[\left(3\left(\mathbf{r}_{\mathrm{N}} \cdot \hat{\mathbf{s}}\right)\left(\mathbf{r}_{\mathrm{N}} \cdot \mathbf{I}\right) / \mathbf{r}_{\mathrm{N}}^{5}\right)-\left(\hat{\mathbf{s}} \cdot \mathbf{I} / \mathbf{r}_{\mathrm{N}}^{3}\right]\right\}\right. \text {, }
$$

where $l_{\mathrm{N}}$ is the electron orbital angular momentum and $\mathbf{r}_{\mathrm{N}}$ is the electron-nucleus distance. The part of the Hamiltonian containing $l_{\mathrm{N}}$ accounts for the magnetic anisotropy contribution to the coupling. By substituting the metal-nucleus distance $r$ for $\mathbf{r}_{\mathrm{N}}$, the metal-centered dipolar shift (or pseudocontact shift) can be expressed in terms of the magnetic susceptibility anisotropy for any spin system $\mathrm{S}$ :

$$
\left(\Delta v / v_{0}\right)^{\text {dip }}=\left(1 / 4 \pi 2 r^{3}\right)\left[\left(3 \cos ^{2} \theta-1\right)\left(2 / 3 \chi_{z z}-1 / 3 \chi_{x x}-1 / 3 \chi_{y y}\right)+\sin ^{2} \theta \cos 2 \Omega\left(\chi_{x x}-\chi_{y y}\right)\right](3)
$$
by assuming negligible zero field splitting and with some approximations, $\chi$ and $g$ can be related by

$$
\chi=\chi_{\mathrm{M}} / N_{\mathrm{A}}=\mu_{0}\left(g^{-2} \mu_{\mathrm{B}}^{2} S(S+1) / 3 k \mathrm{~T},\right.
$$

the previous equation becomes

$\left(\Delta v / v_{0}\right)^{\text {dip }}=\left(\mu_{0} / 4 \pi\right)\left(\mu_{\mathrm{B}}^{2} S(S+1) / 18 k \operatorname{Tr}^{3}\right)\left\{\left[2 g_{z z}^{2}-\left(g_{x x}^{2}+g_{y y}^{2}\right)\right]\left(3 \cos ^{2} \theta-1\right)\right.$

$\left.+3\left(g_{x x}^{2}-g_{y y}^{2}\right) \sin ^{2} \theta \cos 2 \Omega\right\}$, 
where $\theta$ and $\Omega$ are the angles formed by $r$ with the molecular $z$ axis and by the projection of $r$ on the $x y$-plane with the molecular $x$ axis. The magnitude of the dipolar shift does not depend on any magnetic property of the particular nucleus. (Bertini and Luchinat, 1996).

In the case where the symmetry of the magnetic susceptibility tensor is axial, equations 3 and 4 take the more familiar form

$\left(\Delta v / v_{0}\right)^{\text {dip }}=\left(1 / 4 \pi 3 r^{3}\right)\left(3 \cos ^{2} \theta-1\right)\left(\chi \|-\chi_{\perp}\right)$

$\left(\Delta v / v_{0}\right)^{\operatorname{dip}}=\left(\mu_{0} / 4 \pi\right)\left(\mu_{\mathrm{B}}^{2} S(S+1) / 9 k \operatorname{Tr}^{3}\right)\left(3 \cos ^{2} \theta-1\right)\left(g^{2} \|-g_{\perp}^{2}\right) \quad$ (Bertini and Luchinat, 1996).

The pseudocontact shifts provide information for the determination of the position

of the protein atoms in the metal frame. Conversely, they also permit the determination of the position of the metal ion in the protein frame. (Bertini et al., 2002).

The pseudocontact shifts relative to one metal ion can provide only the absolute values of the coordinates of all atoms in any rigid domain, as the angular dependence is provided by quadratic trigonometric functions. Therefore, there is an intrinsic degeneracy in the problem, since eight positions for any domain are possible. Four of these solutions can be excluded as they lead to amino acid structures with the wrong chirality. The residual four-fold degeneracy can be removed, for instance, by measuring the pseudocontact shifts relative to two different metal ions, having magnetic susceptibility tensors with no principal axis in common. (Bertini et al., 2002).

Residual dipolar couplings in paramagnetic compounds, like pseudocontact shifts, can be used as structural constraints. However, they can provide information on the relative orientation of internuclear pairs only, and not on the distance of the pairs from the metal ion.

Pseudocontact shifts and residual dipolar couplings have been proved experimentally to be consistent with one another and with the NOEs. (Bertini et al., 2002).

\section{Paramagnetic tagging of diamagnetic proteins}

The use of external alignment media is widely use for structure determination and dynamic studies of proteins, however the introduction of a paramagnetic centre in a diamagnetic biomolecule induces an alignment with different biophysical properties giving access to complementary and novel dynamic information on the system. One such application is the incomplete labelling of a homodimer with a paramagnetic tag leading to two sets of signal that contain information about the relative orientation and contact surfaces of the homodimer. Another application is the study of domain motions in multidomain proteins. In the presence of interdomain motion, there is a reduction in the magnitude of the axial component of the alignment tensor of the domain which is not tagged for the two-domain model protein CaM. (Rodríguez-Castañeda et al., 2006). $\left[4 \mathrm{Ca}^{2+}\right] \mathrm{CaM}$ has ample conformational freedom in solution. Bertini and collaborators (2004) aligned paramagnetically the N-terminal domain of a CaM mutant with a lanthanide ion; they found that the distribution of the conformational space was nonuniform. Within all possible conformations, there must be some with less favourable orientations of the C-terminal domain.

Various small paramagnetic tags that are attached to proteins via cysteine sidechains have been introduced. The Ethylene-diamine-tetraacetate (EDTA) derived tags have been used by Gaponenko and collaborators (2004) for the study of homodimers. 
Ikegami and collaborators (2004) made use of EDTA derived tags which do not form new stereocenters when ligated to lanthanides. They have dissociation constants in the $10^{-18} \mathrm{M}$ range (Leonov et al., 2005) therefore can be used for the investigation of metal binding proteins. These tags have been used to study the peptidyl prolyl cis/trans isomerase from E.coli, trigger factor (Haberz et.al, 2006) and different activated states of $\mathrm{CaM}$ in this thesis.

\section{Calmodulin dynamics}

The first static picture of CaM came from its x-ray crystallographic structure (Babu et al., 1988). The structure revealed a dumbbell like shape, where the two domains are linked by a long helix. Fallon and Quiocho (2003) crystallized a closed compact structure of $\mathrm{Ca}^{2+} \mathrm{CaM}$ with a sharp bent in the helix linker and a more compact Nterminal domain.

Chou and collaborators (2001) determined the solution structure of $\mathrm{Ca}^{2+}$ ligated $\mathrm{CaM}$ from residual dipolar couplings measured in a liquid crystalline medium. The $\mathrm{C}$ terminal domain solution structure is similar to the X-ray crystal structure; however the EF hands of the N-terminal domain are considerably less open.

Various studies undertaken in solution have widened the picture of the numerous interdomain orientations of $\mathrm{Ca}^{2+}$-CaM; moreover the relative orientation of the different domains is important for their physiological function. Early small angle X-ray scattering studies in solution carried out on Troponin C and CaM (Heidorn and Trewhella, 1988) already suggested a closer distance between the domains as seen in the crystal structure by Babu et al. 1988. Kataoka and collaborators (1991) studied two CaM/peptide complexes derived from the $\mathrm{Ca}^{2+}$ pump with SAXS. The $\mathrm{C} 20 \mathrm{~W}$ peptide complex revealed an interatomic length distribution function, $\mathrm{P}(r)$, similar to that of $\mathrm{CaM}$ alone, indicating that the $\mathrm{CaM} / \mathrm{C} 20 \mathrm{~W}$ complex retained an extended, dumbbell-shaped structure. By contrast, the binding of $\mathrm{C} 24 \mathrm{~W}$ resulted in the formation of a globular structure similar to those observed with many other CaM-binding peptides, like the smooth muscle myosine light chain kinase peptide (smMLCK) for instance.

Elshorst and collaborators (1999) solved the solution structure of the CaM complex with the $\mathrm{C} 20 \mathrm{~W}$ peptide of the calcium pump. The structure revealed that this peptide binds solely to the C-terminal domain of $\mathrm{CaM}$.

As heteronuclear NMR experiments became available in isotopically enriched proteins, Barbato and collaborators (1992) showed through ${ }^{15} \mathrm{~N}$ relaxation that the residues of the central linker 78-81 of CaM had a very high degree of mobility. They argued that this mobility was responsible for the fact that, from a ${ }^{15} \mathrm{~N}$ relaxation view point, the $\mathrm{N}$ - and $\mathrm{C}$-terminal halves reorient nearly isotropically. The motional correlation times for the two halves were slightly different and compatible with the difference in size of their structured regions. These findings support the "flexible tether" hypothesis, where the central helix only serves to keep the two domains in close proximity for binding to their target. In addition, each domain within $\mathrm{CaM}$ undergoes independent diffusion at a faster rate than the overall tumbling of the protein. The interdomain motion of each domain was characterized by an order parameter and a time constant on the nanosecond time scale. (Chang et al., 2003). 
In the absence of calcium, CaM adopts a well-folded structure with numerous slowly exchanging backbone amide protons. Its dynamics could not be described adequately by a model of an isotropically tumbling protein with fast, small, amplitude internal motions. The ${ }^{15} \mathrm{~N} \mathrm{~T}_{1}$ data measured at 51 and $61 \mathrm{MHz}^{15} \mathrm{~N}$ frequency were incompatible with a model of a rigid protein tumbling anisotropically. Significantly better agreement with the measured relaxation data was obtained in a model where the individual domains have restricted mobility on a time scale of about $3 \mathrm{~ns}$, superimposed on the overall tumbling of the molecule which occurs on a time scale which is about 4 times slower. (Tjandra et al., 1995).

Substantial line broadening of many of the ${ }^{15} \mathrm{~N}$ resonances in the C-terminal domain is caused by conformational exchange, on a time scale of a few hundred microseconds. (Tjandra et al., 1995).

For apoCaM, NMR relaxation data provided strong evidence that the C-terminal domain exists in a dynamic equilibrium between its regular, closed apo- state and an open conformation that is similar to that of the $\mathrm{Ca}^{2+}$-ligated state. (Tjandra et al., 1995).

The relative orientations of the $\mathrm{N}$ - and $\mathrm{C}$-terminal domains are nearly random in solution, in addition the interhelical angles in the N-terminal domain also differ by as much as $25^{\circ}$ from what is seen in the crystalline state. Not only the hydrophobic clefts, which are lined with flexible Met residues, provide a pliable interaction surface, the relative helix orientations are also easily changed in order to optimize target binding. (Tjandra et al., 1995).

Chang and collaborators (2003) reported a significant drop in the slow order parameter from 35 to $43{ }^{\circ} \mathrm{C}$, indicating and unexpected increase in the amplitude of interdomain motions. The simplest explanation is that the flexibility of the central helix increases. They suggest that the disordered region of the central helix (residues 78-81) doubles in size on going from 35 to $43^{\circ} \mathrm{C}$ because residues $74-77$ partially "melt".

The available conformational space is quite ample and spans beyond the cone suggested by the ${ }^{15} \mathrm{~N}$ relaxation data from Chang and Tjandra (2001), which senses the motions in the nano- to picosecond scale, whereas pseudocontact shifts and residual dipolar couplings are averaged by motions spanning a time scale that extends down to milliseconds. Thus, slower motions than those affecting relaxation measurements may contribute to the sampling of conformational space in CaM. (Bertini et al., 2004).

The $\mathrm{C}$-terminal rDCs arise from averaging among very diverse orientations. Therefore, pendulum-like motions, no matter how ample, that do not imply also a rotation of the C-terminal domain about the axis of its first helix, are not sufficient in averaging the rDCs down to the small values observed. However not all C-terminal orientations are equally probable, since the rDCs don't average to zero. Electrostatic repulsion between the negatively charged domains may contribute to the different probabilities of sterically accessible conformations. (Bertini et al., 2004).

Single molecule fluorescence experiments (Johnson, 2006) have also shown that $\mathrm{CaM}$ exists in conformational sub-states. These sub-states were observed in FRET distributions with bin times up to $800 \mu \mathrm{s}$. If conformational interchange occurred on a faster time scale, conformational averaging would result in narrowing of the FRET distribution. The absence of such narrowing shows that the conformational interchange must occur on a longer time scale. 
In order to explore further the time scale of conformational interchange, singlemolecule fluorescence burst FRET trajectories were selected with durations of several milliseconds. Occasional jumps from one conformational sub-state to another occur on the millisecond time scale. Interchange on this time scale has implications for the function of CaM. The millisecond interchange of conformational sub-states of CaM may permit rapid exploration of conformational space for molecular recognition of target binding domains. (Johnson et al., 2005).

Recent advances in the field of molecular dynamics which allow longer simulations, have contributed to the understanding of CaM function in its different activated states. During the 4-ns apoCaM trajectory, the interdomain distance fluctuated in the 29-39 $\AA$ range, with an average of $33 \AA$. For the $\left[4 \mathrm{Ca}^{2+}\right]$-CaM trajectory, the interdomain distance remained between 34 and $41 \AA$. The average distance was $38 \AA$. During the CaM:smMLCK peptide complex simulation the interdomain distance falls in the 22-25 $\AA$ range, with an average value of $24 \AA$. Thus the simulation results indicate that large-scale domain motions occur in calcium-free and calcium-loaded CaM, while such motions are absent in the collapsed CaM-peptide complex. (Yang et al., 2003).

Wang and collaborators (2005) studied the dynamics of CaM and CaM/smMLCK peptide complex based on the transverse cross-correlated relaxation rates between ${ }^{13} \mathrm{CO}$ chemical shift anisotropy and the ${ }^{13} \mathrm{CO}-{ }^{13} \mathrm{C} \alpha$ dipolar interactions which are sensitive to the motion of the ${ }^{13} \mathrm{CO}-{ }^{13} \mathrm{C} \alpha$ bond vector.

In the presence of ligand, the two domains of CaM form a clam-shell around the bound peptide. Only small structural changes occur within the N- and C-terminal domains. The ligand-induced conformations are accompanied by the diminishment in the amplitude of sub-nanosecond time scale dynamics of the methyl-bearing side chains, as detected by deuterium relaxation in methyl groups (Wand, 2001). In contrast, the amplitude of sub-nanosecond time scale dynamics of the protein backbone as monitored by ${ }^{15} \mathrm{~N}$ relaxation in amide $\mathrm{NH}$ groups is not affected, except for the region bridging the two globular domains. These findings implied that peptide binding causes a major loss of conformational entropy of the side chains, but seemed to indicate that the entropy of the backbone was not affected by the binding process. However, the ${ }^{13} \mathrm{CO}-{ }^{13} \mathrm{C} \alpha$ vectors report a significant loss in dynamics upon ligand binding, suggesting that the entropy of the backbone does contribute to the binding free energy. (Wang et al., 2005).

In contrast to the view afforded by the ${ }^{15} \mathrm{~N}$ relaxation data, the ${ }^{13} \mathrm{CO}-{ }^{13} \mathrm{C} \alpha$ crosscorrelated relaxation data reveal that the backbone of un-ligated $\mathrm{CaM}$ contains residual motion, which is affected and partially quenched upon binding of the target domain. The change in backbone dynamics as sensed by the ${ }^{13} \mathrm{CO}$ environment is not sensed at all by the NH vector, despite the fact that both are part of the same peptide plane. The apparent lack of full correlation of the $\mathrm{NH}$ and ${ }^{13} \mathrm{CO}-{ }^{13} \mathrm{C} \alpha$ detectors of protein backbone motion may be explained by anisotropic local motion of the peptide planes and/or dynamic pyrimidalization of the nitrogen atom which partially decouples the motion of the $\mathrm{NH}$ vector from that of the peptide plane. (Wang et al., 2005).

CaM shows two different types of allosteric behaviour: (i) cooperativity between calcium-binding sites and (ii) cooperativity between its target- and calcium-binding sites. The allosteric coupling between calcium- and peptide-binding sites is reciprocal, with the $\mathrm{Ca}^{2+}$ binding affinity of CaM increasing 30-100-fold in the presence of smMLCKp. 
Igumenova and collaborators (2005) studied the dynamics of different CaM mutants in complex with the smMLCK peptide; they found that the backbone dynamics of these mutants in complex with $\mathrm{Ca}^{2+}$ and smMLCKp was indistinguishable from that of the wild-type complex. In contrast, for three of the mutants the dynamic properties of methyl-bearing side chains were found to be very different compared to those of the wildtype complex. Both local and long-range perturbation of the methyl dynamics exist. This behaviour does not correlate with chemical shift perturbations imposed by the mutations. Thus, the three mutants D58N, D95N, and E84K, have an altered allosteric behaviour comparable to that of the wild-type protein.

Larsson and collaborators (2001) studied the interaction of $\mathrm{CaM}$ with the dimeric basic helix-loop-helix transcription factor SEF2-1 using NMR. The stoichiometry of this interaction is one dimeric peptide binding two CaM molecules. The binding does not occur via the classical wrap-around binding mode; CaM interacts with SEF2-1mp in a more open conformation. In addition, the peptide does not form an $\alpha$-helix. In a backbone dynamics study by ${ }^{15} \mathrm{~N}$-spin relaxation, the same group reported in 2005 that all CaM residues experience internal motion with an effective correlation time of $\sim 2.5 \mathrm{~ns}$ with squared order parameter $\left(\mathrm{S}^{2}\right)$ of $\sim 0.75$. This could be attributed to motions of the $\mathrm{N}$ - and $\mathrm{C}$-terminal domains of the CaM dimer in the complex. The data were consistent with a motional model in which the two N-terminal and two $\mathrm{C}$-terminal CaM domains in the dimeric CaM:SEF2-1 mp complex undergo a small-scale wobbling motion with halfangle of $\sim 20^{\circ}$ as estimated from $\mathrm{S}_{\mathrm{s}}{ }^{2}=0.75$. However, the internal motion involving reorientation of the $\mathrm{CaM}$ monomers cannot be excluded. Residues with significant exchange broadening were found, they clustered in the CaM:SEF2-1mp binding interface, the CaM:CaM interface, and in the flexible helix connecting the $\mathrm{CaM} \mathrm{N}$ - and $\mathrm{C}$-terminal domains, and have similar exchange times $(\sim 50 \mu \mathrm{s})$, suggesting a cooperative mechanism. 


\section{MATERIALS AND METHODS}

1. Polymerase chain reaction (PCR) based-cloning of the different Munc13-1 protein expression constructs

To clone the different Munc13-1 constructs the oligonucleotide primers for the PCR were designed for directed cloning into the different expression vectors with two different restriction sites in the 5' regions. The amplification generates a target fragment whose termini carry new restriction sites that can be used for directional cloning into plasmid vectors. The purified fragment and the vector are digested with the appropriate restriction enzymes, ligated together, and transformed into E.coli (see Figure 12). (Sambrook and Russell, 2001).

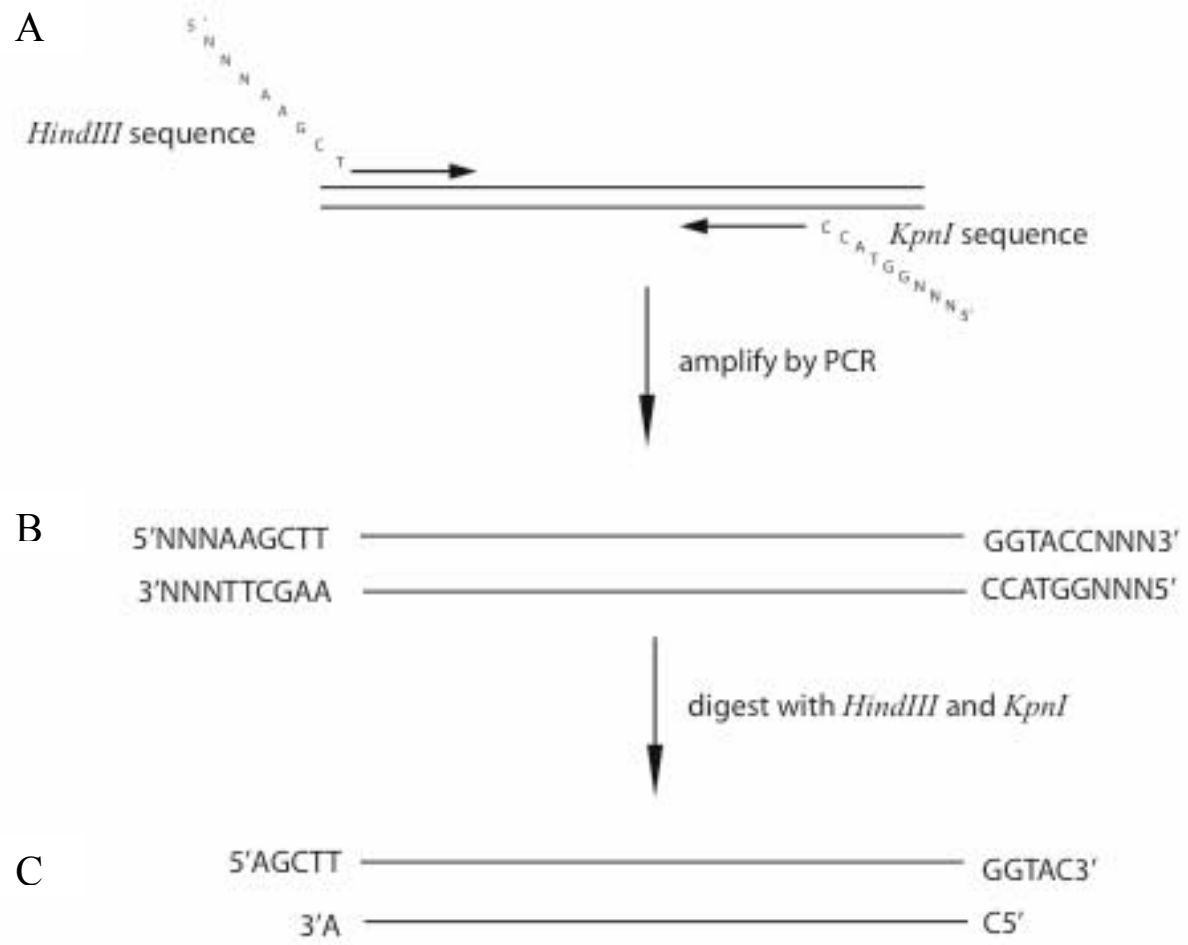

Figure12. PCR-based cloning strategy. The sense strand primer contains the sense strand of a restriction site, and the antisense primer contains the complementary sequence of a second restriction site. (A) To achieve high efficiency digestion, additional nucleotides must be included on both sides of the restriction endonuclease sequence. (B) Amplification by PCR produces a specific product with an HindIII site at the 5' end and a KpnI site at the 3' end. (C) Digestion with HindIII and KpnI produces a PCR product that can be cloned directionally. (Adapted from Sambrook and Russell, 2001).

a. Polymerase chain reaction (PCR)

Oligonucleotide primers

Munc13-1 (447-631) including the CaM binding site and the $\mathrm{C}_{1}$ domain Munc185

M13c1-1 5' GAGCATATGGGATCCGCTGAGGAGGGCCAGGAAG 3'

M13c1-2 5' GCGGGATCCCATGGCTGAGGAGGGCCAGGAAG 3'

M13c1-3 5' CGACTCGAGGAATTCTTAGTCTTCAGCGCCATGCTTAGAAC 3'

M13c1-5 5' GAGGGTACCAGATCTATGGGTCATCACCATCACCATCA 3' 
Munc13-1 (567-631) $C_{1}$ domain Munc65

\title{
M13c1-4 5' GAGCATATGGGATCCCACAACTTCGAGGTGTGGACG 3' M13c1-3 5' CGACTCGAGGAATTCTTAGTCTTCAGCGCCATGCTTAGAAC 3'
}

\author{
Munc13-1 (458-492) CaM binding site Munc36
}

\section{M1334aa-1 5' GAGGGATCCAGGGCCAAAGCCAACTGGTTG 3' M1334aa-2 5' CGAGAATTCTTAGCCTTTGAACCACAGAGACTTGGA 3' M1334aa-3 5' CGAAAGCTTTTAGCCTTTGAACCACAGAGACTTGGA 3'}

The oligonucleotide primers were obtained in a lyophilized form from Invitrogen. 400 $\mu 1$ of TE buffer (10 mM TRIS, $1 \mathrm{mM}$ EDTA, $\mathrm{pH} 8.0$ ) were added to reconstitute them. The UV spectrum was measured to verify their purity and estimate their concentration.

Plasmid DNA vectors for protein expression :

pET 16b (Novagen) with a Tobacco Etch Virus protease cleavage site introduced pGEX 2T (Pharmacia)

pET Duet-1 (Novagen)

pMAL

The cDNA of the munc13-1 gene was kindly provided by Prof. Nils Brose MaxPlanck for Experimental Medicine. For the PCR, $1 \mu 1$ of the munc13-1 cDNA (10 ng/ $\mu \mathrm{l})$ was taken as template for the amplification. The reaction was done in a total volume of $50 \mu 1$. In a $250 \mu 1$ eppendorf PCR tube, $5 \mu 1$ of the 10X PCR reaction buffer, $1 \mu 1$ of $20 \mathrm{mM}$ dNTP, $1 \mathrm{ul}$ of the $200 \mu \mathrm{M}$ oligonucleotide, sterile (Millipore)-water to reach the $50 \mu 1$ volume and $0.5 \mu 1$ of the Pfu DNA polymerase. The polymerase chain reaction was done in a PCR sprint thermal cycler (Hybaid) using the following cycle:

10 minutes initial denaturation step at $95^{\circ} \mathrm{C}$

25 cycles consisting of a $30 \mathrm{sec}$ denaturing step at $95^{\circ} \mathrm{C}$;

$30 \mathrm{sec}$ annealing step at $55^{\circ} \mathrm{C}$;

And a $30 \mathrm{sec}$ extension step at $68^{\circ} \mathrm{C}$.

A 10 minutes final extension step at $68^{\circ} \mathrm{C}$.

The PCR product was checked with a $1 \%(\mathrm{~m} / \mathrm{v})$ agarose gel electrophoresis. Briefly, $0.3 \mathrm{~g}$ of agarose were dissolved in $30 \mathrm{ml}$ of Tris-borate-EDTA buffer (TBE) and boiled in the microwave for 1 minute; when the solution cooled to approximately $40^{\circ} \mathrm{C}, 1 \mu 1$ of a $1 \%(\mathrm{~m} / \mathrm{v})$ ethidium bromide solution was added and then poured into the casting cassette. After the gel solidified it was put into the electrophoresis chamber containing the TBE buffer. The molecular weight marker and the samples, containing $4 \mathrm{ul}$ of $6 \mathrm{x}$ loading buffer, were loaded. The electrophoresis was carried out for one hour at a constant voltage of $80 \mathrm{~V}$.

After the polymerase chain reaction; the product was purified with a PCR cleaning kit previous to the reaction with the restriction enzymes.

\section{b. Restriction digestion of the PCR products}

The restriction digestion of PCR fragments may work very well with non-purified PCR fragments, however when the purpose of the generated fragments is their cloning into a vector, it is advisable to purify the PCR products, since the Taq-polymerase its active at $37^{\circ} \mathrm{C}$ and can therefore fill the cohesive ends leading to an unsuccessful cloning. An additional problem for the restriction of the PCR products is the fact that some restriction enzymes have 
very little activity when the recognition sequence is located at the end of the sequence, for this purpose it is advisable to design the primers with at least three additional bases upstream of the recognition site. In general it is possible to carry a double digest, which means to do the restriction reaction with two enzymes at the same time. For this purpose, a buffer should be used in which both enzymes possess enough activity (above 75\%) (Mülhardt, 2003).

The purified PCR products were obtained in a final volume of $28 \mu \mathrm{l}$. For cloning into the pET16b (TEV) expression vector the PCR product was digested with the NcoI and XhoI restriction enzymes (MBI Fermentas) in the 2X yellow TANGO buffer with Bovine Serum Albumin (BSA) (MBI Fermentas). The restriction digestion reaction was done in a total volume of $50 \mu 1.10 \mu \mathrm{l}$ of the 10X yellow TANGO buffer with BSA, the total amount of the PCR product, 10ul of sterile (Millipore)-water, $1 \mathrm{ul}$ of $\mathrm{NCOI}\left(10 \mathrm{U}^{1} / \mu \mathrm{l}\right)$ and $1 \mathrm{ul}$ of XhoI $(10 \mathrm{U} / \mu \mathrm{l})$ were mixed in a $1.5 \mathrm{ml}$ eppendorf tube. For cloning into the pGEX-2T expression vector, the PCR product was digested with the BamHI and EcoRI restriction enzymes (MBI Fermentas) in the $2 \mathrm{X}$ yellow TANGO buffer with BSA in the same manner as above. For cloning into the multiple cloning site-2 (MCS2) of the pET Duet-1 expression vector, the PCR product was digested with NdeI and XhoI restriction enzymes (MBI Fermentas) in the $1 \mathrm{X}$ red TANGO buffer with BSA. The restriction digestion reaction was done in a total volume of 50 $\mu 1.5 \mu \mathrm{l}$ of the $10 \mathrm{X}$ red TANGO buffer, the total amount of the PCR product, $15 \mu 1$ of sterile (Millipore)-water, $1 \mu \mathrm{l}$ of $\mathrm{NdeI}(10 \mathrm{U} / \mu \mathrm{l})$ and $1 \mathrm{ul}$ of XhoI were mixed in a $1.5 \mathrm{ml}$ eppendorf tube. All the reactions were performed overnight at $37^{\circ} \mathrm{C}$ to ensure complete digestion of the PCR products. To remove the restriction enzymes, the restricted PCR products were purified with the PCR cleaning kit mentioned above.

\section{c. Preparation of the plasmid DNA expression vectors for cloning}

Plasmids are circular double stranded DNA molecules, which can replicate in an independent fashion from the bacterial genome. The minimum composition of a plasmid consists of an origin of replication ori, a gene for the selection (usually an antibiotic resistance gene) and a cloning site, where the desired DNA is inserted into the plasmid. In addition, it is possible to introduce various sequences, for example a second selection marker or a promoter for directing the expression of the inserted gene (Mülhardt, 2003).

The cloning site contains various recognition sites for restriction enzymes, which usually are only present once in the vector. This allows to cut the vector for cloning, avoiding the fragmentation of the vector in many pieces. In theory it suffices a single recognition site to insert a DNA fragment, however most of the plasmid vectors contain ten to twenty of such recognition sites to allow flexibility in the cloning strategies, and these series of recognition sites are called multiple cloning sites (MCS).

$1 \mu \mathrm{g}$ of vector DNA was digested with the different restriction enzymes in the same manner as done for the PCR products. The only difference was that the reaction digestion was done just for 1 hour to prevent any star activity of the restriction enzymes. After the restriction digestion was complete the vector DNA was dephosphorylated by the addition of $1 \mathrm{ul}$ of Calf Intestine Phosphatase (1U/ $\mu 1)\left(M B I\right.$ Fermentas) and further incubation at $37^{\circ} \mathrm{C}$ for 1 hour. The vector plasmid DNA was loaded into a $1 \%(\mathrm{~m} / \mathrm{v})$ agarose gel, gel-extracted and purified with a gel extraction purification kit (Qiagen).

\section{d. Ligation reaction}

To ensure a favorable stoichiometric ratio between the restricted PCR product and the plasmid DNA vector for the ligation reaction to take place, it is desirable to have around 3

\footnotetext{
${ }^{1}$ The unit of the restriction enzyme is defined as the amount of enzyme needed for digesting completely $1 \mu \mathrm{g}$ of the DNA substrate in 60 minutes at the correct temperature and buffer in a total volume of $50 \mu 1$.
} 
molar excess of the insert to the vector. The ligation reaction was done in a total volume of 20 $\mu \mathrm{l}: 2 \mu \mathrm{l}$ of the $10 \mathrm{X}$ ligation buffer, $100 \mathrm{ng}$ of vector DNA, typically around 1ul, the necessary amount of insert to achieve the favorable ligation reaction conditions, sterile (Millipore)-water and $1 \mu \mathrm{l}$ of T4 DNA ligase (MBI Fermentas). The ligation reaction was done overnight at $16^{\circ} \mathrm{C}$.

\section{e. Transformation of the ligated plasmid DNA}

The classical method to make bacteria competent for transformation is carried out with calcium chloride. The chemical competent bacteria give $10^{6}$ to $10^{8}$ colonies per $\mu \mathrm{g}$ of TestDNA (Mülhardt, 2003).

The ligated plasmid DNA needs to be amplified, for that purpose, $4 \mu 1$ of the ligation reaction were added to $50 \mu \mathrm{l}$ of chemical competent Escherichia coli (XL2-Blue) cells and incubated on ice for 30 minutes. The cells were exposed to a heat-shock at $42^{\circ} \mathrm{C}$ for 45 seconds and then incubated on ice for 2 minutes. $600 \mu \mathrm{l}$ of pre-warmed LB medium at $37^{\circ} \mathrm{C}$ were added and the cells were grown for $1 \mathrm{hr}$ at $37^{\circ} \mathrm{C}$ prior to plating them on LB-agar plates containing ampicillin $(50 \mu \mathrm{g} / \mathrm{ml})$. The LB-agar plates were incubated overnight at $37^{\circ} \mathrm{C}$.

\section{f. Plasmid DNA amplification and purification}

At least two colonies for each ligation reaction were picked and used for inoculating $5 \mathrm{ml}$ of LB medium with $50 \mu \mathrm{g} / \mathrm{ml}$ ampicillin; the cultures were grown overnight at $37^{\circ} \mathrm{C}$. A glycerol stock to preserve the clones at $-80^{\circ} \mathrm{C}$ was prepared by adding $500 \mu 1$ of $50 \%(\mathrm{v} / \mathrm{v})$ glycerol to $500 \mu 1$ of culture.

To purify the plasmid DNA from the E.coli culture the alkaline lysis protocol was used with the aid of a mini-prep Plasmid DNA purification kit (Qiagen). This protocol purifies the plasmid DNA from the bacterial chromosomal DNA based on the different renaturation properties of the circular plasmid DNA and the genomic DNA; the high alkaline solution denatures the DNA and upon neutralization the plasmid DNA renatures fast, while the much larger chromosomal DNA remains in a single stranded conformation and precipitates (Mülhardt, 2003). Briefly, the cultures were centrifuged at 5,000 rpm for 10 minutes. The supernatant was discarded and the cells were resuspended with $250 \mu 1$ of RNAse containing buffer; the cells were lysed adding $250 \mu \mathrm{l}$ of lysis buffer $(0.2 \mathrm{M}$ sodium hydroxide $/ 1 \%(\mathrm{w} / \mathrm{v})$ sodium dodecyl sulphate). The lysates were neutralized with $300 \mu \mathrm{l}$ of neutralization buffer ( $3 \mathrm{M}$ potassium acetate $\mathrm{pH}$ 5.5) in order to precipitate potassium-acetate together with the protein-genomic DNA complexes. The precipitate was removed by centrifugation in a table-top centrifuge for 10 minutes at $16,000 \mathrm{~g}$. The supernatant was added to a silica column and purified washing with an ethanol containing buffer. The plasmid DNA was eluted from the silica matrix with $50 \mu$ of the elution buffer and the concentration was determined measuring the UV spectrum.

\section{g. Screening, DNA sequencing and transformation of positive clones}

To verify that the purified plasmid DNA is the desired clone; the clones were screened, for this purpose, the plasmid DNA was digested with the same restriction enzymes used for the cloning. A $1 \%(\mathrm{~m} / \mathrm{v})$ agarose gel electrophoresis was performed to visualize which clones were positive.

For the PCR-based cloning of DNA it is essential to sequence the insert DNA to confirm the $100 \%$ identity of the coding region with the original DNA source. The sequencing was done by a commercial service company. $600 \mathrm{ng}$ of the purified plasmid DNA 
and 20 pmol of the sequencing oligonucleotide primer were sent in a total volume of $7 \mu 1$ in a $250 \mu 1$ eppendorf cup.

$1 \mu \mathrm{l}$ of the purified plasmid DNA were added to $50 \mu \mathrm{l}$ of heat shock transformation competent E.coli BL21(DE3) cells and incubated for 30 minutes on ice. The cells were exposed to $42^{\circ} \mathrm{C}$ for 45 seconds and then incubated on ice for 2 minutes. $600 \mu 1$ of prewarmed $\mathrm{LB}$ medium at $37^{\circ} \mathrm{C}$ were added and the cells were grown for $1 \mathrm{hr}$ at $37^{\circ} \mathrm{C}$ prior to plating them on LB-agar plates containing ampicillin $(50 \mu \mathrm{g} / \mathrm{ml})$. The LB-agar plates were incubated overnight at $37^{\circ} \mathrm{C}$.

\section{h. Protein overexpression test in LB medium and protein solubility}

For the heterologous expression of proteins in bacteria, a common organism is E.coli, since it is an intensively studied bacterium, which yields large amounts of protein. It is common to introduce at the beginning or at the end of the cDNA an additional coding sequence, to obtain a fusion protein with special characteristics. For example the common histidine-tag (a polyhistidine sequence of 6-8 amino acids) allows an efficient purification by means of immobilized metal affinity chromatography (IMAC) columns. It is also possible to introduce a protease recognition site, e.g. Thrombin to cut the fusion tag after the purification (Mülhardt, 2003).

After the cloning succeeded, it was decisive to test the overexpression of the desired Munc13-1 construct. For this purpose, a $2 \mathrm{ml} \mathrm{LB}$ with $50 \mu \mathrm{g} / \mathrm{ml}$ ampicillin culture was grown overnight at $37^{\circ} \mathrm{C}$ and used to inoculate $100 \mathrm{ml}$ of LB medium with $50 \mu \mathrm{g} / \mathrm{ml}$ ampicillin. The culture was grown at $37^{\circ} \mathrm{C}$ for several hours; the growth was monitored measuring the absorbance at $600 \mathrm{~nm}$. The overexpression of the protein was induced by the addition of 0.5 $\mathrm{mM}$ isopropyl thio- $\beta$-galactoside (IPTG) when the cells have reached an absorbance of 0.6 . The overexpression was carried out for 4 hours at $37^{\circ} \mathrm{C}$ and then the cells are harvested by centrifugation at 5,000 g for 15 minutes. A small aliquot of the cells were directly lysed with the SDS-PAGE laemmli buffer by heating for 3 minutes at $95^{\circ} \mathrm{C}$ and the lysates were run in a protein mini-gel to compare the protein content of the cells before and after induction. Another critical issue concerns the protein solubility after the lysis, since this is essential for the purification of the protein under native conditions. After harvesting the cells; the lysis was done according to the purification protocol for the different tagged versions of the constructs. The lysis of the hexahistidine tagged proteins was done in the following buffer: $20 \mathrm{mM}$ $\mathrm{NaH}_{2} \mathrm{PO}_{4}, 150 \mathrm{mM} \mathrm{NaCl}, 1 \mathrm{mM}$ dithiotreitol (DTT), $10 \mathrm{mM}$ Imidazole, $\mathrm{pH} 8.0$ supplemented with $200 \mu \mathrm{M} \mathrm{ZnCl}_{2}, 1 \mathrm{mg} / \mathrm{ml}$ of lysozyme, $0.5 \mathrm{mM}$ Phenylmethylsulfonate fluoride (PMSF), 1 tablet of EDTA-free complete protease inhibitor. The lysis buffer of the GST-tagged proteins was done in $1 \mathrm{X}$ PBS buffer $\left(50 \mathrm{mM} \mathrm{NaH}_{2} \mathrm{PO}_{4}, 150 \mathrm{mM} \mathrm{NaCl}, \mathrm{pH}\right.$ 7.4) supplemented with 1 tablet of complete protease inhibitor, $1 \mathrm{mg} / \mathrm{ml}$ of lysozyme, $200 \mu \mathrm{M}$ of $\mathrm{ZnCl}_{2}, 0.5 \mathrm{mM}$ PMSF and $1 \mathrm{mM}$ DTT. The cells were disrupted by ultrasound sonication six times for 20 seconds with a power of $60 \%$. The cell debris was separated by centrifugation at $45,000 \mathrm{~g}$ for 45 minutes at $4^{\circ} \mathrm{C}$. The protein content of the supernatant and pellet fractions was evaluated with SDS-PAGE.

The composition of the SDS-polyacrylamide gel is the following:

$17.5 \%$ separating gel

Acrylamide solution ( $30 \%$ acrylamide, $0.8 \%$ bisacrylamide) $3 \mathrm{ml}$

1M TrisHCl, pH $8.81 .88 \mathrm{ml}$

$10 \%$ SDS $50 \mu 1$

$10 \%$ APS $50 \mu 1$

TEMED $2.5 \mu 1$

$3 \%$ stacking gel

Acrylamide solution ( $30 \%$ acrylamide, $0.8 \%$ bisacrylamide) $250 \mu 1$ 
$1 \mathrm{M}$ TrisHCl, $\mathrm{pH} 6.8313 \mu \mathrm{l}$

$10 \%$ SDS $25 \mu 1$

$10 \%$ APS $25 \mu 1$

TEMED $2 \mu 1$

dd $\mathrm{H}_{2} \mathrm{O} 1.88 \mathrm{ml}$

The electrophoresis was carried out at a constant current of $25 \mathrm{~mA}$ per mini-gel in a $1 \mathrm{X}$ Running Buffer. The composition of the 10x Running Buffer ( $\mathrm{pH} 8.3$ ) was the following:

$30 \mathrm{~g}$ Tris Base, $144 \mathrm{~g}$ glycine, $10 \mathrm{~g}$ SDS and $\mathrm{ddH}_{2} \mathrm{O}$ to 1 liter.

The protein samples needed to be denatured and reduced in order to analyze their electrophoretic properties under non-native conditions. The composition of this denaturing

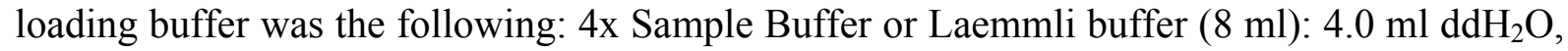
$1.0 \mathrm{ml}$ of $0.5 \mathrm{M}$ Tris- $\mathrm{HCl}, 0.8 \mathrm{ml}$ of glycerol, $1.6 \mathrm{ml}$ of $10 \%(\mathrm{w} / \mathrm{v}) \mathrm{SDS}, 0.4 \mathrm{ml}$ of $\beta-$ mercaptoethanol and $0.2 \mathrm{ml}$ of $0.05 \%(\mathrm{w} / \mathrm{v})$ bromophenol blue.

Dynamic Light Scattering (DLS) measurements after the size exclusion chromatography were also done on the CaM/Munc185 complex. For this purpose, the protein was concentrated to $100 \mu \mathrm{M}$. To remove suspended particles the protein solution was filtered through a $22 \mu \mathrm{m}$ pore size membrane and ultracentrifugation was performed at $60,000 \mathrm{~g}$ for 1 hour. The DLS measurement relies on the Brownian motion of a macromolecule. When a light beam is directed onto a solution with spherical particles the Brownian motion causes a Doppler shift when the light hits the moving particle, changing the wavelength of the incoming light. This change is related to the size of the particle. It is possible to compute the sphere size distribution and give a description of the particle's motion in the medium, measuring the diffusion coefficient of the particle and using the autocorrelation function. (Sartor, 2003)

\section{PCR-mediated Site-directed mutagenesis}

Oligonucleotide-directed mutagenesis is used to test the role of particular residues in the structure, catalytic activity, and ligand-binding capacity of a protein. In the absence of a three-dimensional structure, one relies on informed guesses concerning the structure of the protein and the contribution of individual residues to protein stability and function. A major problem is distinguishing mutations that affect local structures from those that have profound effects on the folding or stability of the entire protein (Sambrook and Russell, 2001).

A synthetic oligonucleotide encoding the desired mutation is annealed to the target region of the wild-type template DNA where it serves as a primer for intitiation of DNA synthesis in vitro. The extension of the oligonucleotide by a DNA polymerase generates a double-stranded DNA that carries the desired mutation. The mutated DNA is then inserted at the appropriate location of the target gene, and the mutant protein is expressed (Sambrook and Russell, 2001).

In vitro mutagenesis using double-stranded DNA templates and selection of mutants with DpnI: In this method, two oligonucleotides are used to prime DNA synthesis by a highfidelity polymerase on a denatured plasmid template. The two oligonucleotides both contain the desired mutation and have the same starting and ending positions on opposite strands of the plasmid DNA. In this protocol, the entire lengths of both strands of the plasmid DNA are amplified in a linear fashion during several rounds of thermal cycling, generating mutated plasmid containing staggered nicks on opposite strands. The products of the linear amplification reaction are treated with the restriction enzyme DpnI, which specifically cleaves fully methylated $\mathrm{G}^{\mathrm{Me} 6} \mathrm{ATC}$ sequences. DpnI will digest the bacterially generated DNA used as template for amplification, but it will not digest DNA synthesized during the course of the reaction in vitro. DpnI-resistant molecules, which are enriched in the desired mutants, are recovered by transforming E.coli to antibiotic resistance. Depending on the complexity of the 
mutation, and the length of the template DNA, between $15 \%$ and $80 \%$ of the transformed colonies will contain plasmids with the desired mutation (Sambrook and Russell, 2001).

Site-directed mutagenesis in the C-terminal domain of X. laevis CaM and on rat Munc13-1 was performed using the Stratagene QuikChange ${ }^{\circledR}$ site-directed mutagenesis kit. The oligonucleotide primers were ordered from Invitrogen (see Table1).

Table 1. Oligonucleotide primers for site-directed mutagenesis

\begin{tabular}{|c|c|c|}
\hline Mutation & \multicolumn{2}{|l|}{ Primers } \\
\hline \multirow{2}{*}{ CaM S101C } & $\begin{array}{l}\text { CaM17 } \\
(+) \text { strand }\end{array}$ & 5' GGAACGGCTACATCTGCGCTGCTGAATTACG 3' \\
\hline & $\begin{array}{l}\text { CaM18 } \\
(-) \text { strand }\end{array}$ & 5' CGTAATTCAGCAGCGCAGATGTAGCCGTTCCC 3' \\
\hline \multirow{2}{*}{ CaM T110C } & $\begin{array}{l}\text { CaM19 } \\
(+) \text { strand }\end{array}$ & 5' CGTCACGTCATGTGCAACCTCGGGGAGAAGTTAACAGACG 3' \\
\hline & $\begin{array}{l}\text { CaM20 } \\
\text { (-) strand }\end{array}$ & 5' CGTCTGTTAACTTCTCCCCGAGGTTGCACATGACGTGACG 3' \\
\hline \multirow{2}{*}{ CaM T146C } & $\begin{array}{l}\text { CaM21 } \\
(+) \text { strand }\end{array}$ & 5' GAGTTTGTACAAATGATGTGCGCAAAGTGAAGAGTCTAGAATCG 3' \\
\hline & $\begin{array}{l}\text { CaM22 } \\
\text { (-) strand }\end{array}$ & 5' CGATTCTAGACTCTTCACTTTGCGCACATCATTTGTACAAACTC 3' \\
\hline \multirow[t]{2}{*}{ Munc13-1 W489A } & $\begin{array}{l}\text { M13c1-8 } \\
(+) \text { strand }\end{array}$ & 5' GAGATGTCCAAGTCTCTGGCGTTCAAAGGCGGCCCT 3' \\
\hline & $\begin{array}{l}\text { M13c1-11 } \\
\text { (-) strand }\end{array}$ & 5'AGGGCCGCCTTTGAACGCCAGAGACTTGGACATCTC 3' \\
\hline \multirow[t]{2}{*}{ Munc13-1 W572Y } & $\begin{array}{l}\text { M13c1-14 } \\
(+) \text { strand }\end{array}$ & 5' CACAACTTCGAGGTGTACACGGCCACCACTCCCACC 3' \\
\hline & $\begin{array}{l}\text { M13c1-15 } \\
\text { (-) strand }\end{array}$ & 5' GGTGGGAGTGGTGGCCGTGTACACCTCGAAGTTGTG 3' \\
\hline \multirow[t]{2}{*}{ Munc13-1 W588A } & $\begin{array}{l}\text { M13c1-10 } \\
(+) \text { strand }\end{array}$ & 5' TGCGAGGGGCTGCTGGCGGGCATCGCGCGG 3' \\
\hline & $\begin{array}{l}\text { M13c1-13 } \\
\text { (-) strand }\end{array}$ & 5' CCGCGCGATGCCCGCCAGCAGCCCCTCGCA 3' \\
\hline
\end{tabular}

The oligonucleotide primers were reconstituted in $\mathrm{ddH}_{2} \mathrm{O}$ to obtain an approximate concentration of $1 \mu \mathrm{g} / \mathrm{ml}$; the $\mathrm{A}_{260}$ was measured to determine the exact concentration of each primer. The reaction mix was prepared as following (all mixing steps done on ice): $2.5 \mu 1$ of $10 \mathrm{x}$ reaction buffer, $1 \mu \mathrm{l}(39 \mathrm{ng})$ of the pET28a CaM wild type clone, $0.5 \mu \mathrm{l}$ of oligonucleotide primer, $0.5 \mu \mathrm{l}$ of dNTP (20mM each) mix, $14 \mu \mathrm{l}$ of $\mathrm{ddH}_{2} \mathrm{O}$ and $0.5 \mu \mathrm{l}$ of Pfu Turbo DNA polymerase $(2.5 \mathrm{U} / \mu \mathrm{l})$

Thermal cycling was done according to following scheme:

1) $95^{\circ} \mathrm{C} 30 \mathrm{sec}$

2) $95^{\circ} \mathrm{C} 30 \mathrm{sec}$

$55^{\circ} \mathrm{C} 1 \mathrm{~min}$

$68^{\circ} \mathrm{C} 12 \mathrm{~min}$

16 cycles

3) $68^{\circ} \mathrm{C} 10 \mathrm{~min}$ final extension

4) $4{ }^{\circ} \mathrm{C}$

The products obtained after the thermal cycling were stored at $-20^{\circ} \mathrm{C}$. The samples were thawed on ice prior digestion, then $0.5 \mu 1$ of $D p n I$ restrictase $(10 \mathrm{U} / \mu 1)$ were added and the samples were incubated at $37^{\circ} \mathrm{C}$ for $1 \mathrm{~h}$. The mixture after incubation was used to transform competent E.coli (XL2-Blue) bacteria. For each CaM and Munc13-1 mutant a $50 \mu 1$ aliquot of competent cells was thawed on ice, $1 \mu 1$ of plasmid-containing solution was added followed by $30 \mathrm{~min}$ incubation on ice. A heat pulse was applied $\left(45 \mathrm{sec}, 42^{\circ} \mathrm{C}\right)$ and the cells 
were incubated on ice for additional $2 \mathrm{~min}$. After this $0.5 \mathrm{ml}$ of LB-medium preheated at $42^{\circ} \mathrm{C}$ were added and cells were incubated at $37^{\circ} \mathrm{C}, 220 \mathrm{rpm}$ for $1 \mathrm{~h}$.

Incubation mix was shortly centrifuged, the pellet resuspended in $100 \mu 1$ of LB medium and plated onto LB plates $(70 \mu \mathrm{g} / \mathrm{ml}$ kanamycin for CaM and $50 \mu \mathrm{g} / \mathrm{ml}$ ampicillin for Munc13-1). After overnight incubation at $37^{\circ} \mathrm{C}$ two colonies from each plate were picked and grown into $3 \mathrm{ml} \mathrm{LB}$-medium containing with kanamycin or ampicillin overnight at $37^{\circ} \mathrm{C}$.

\section{Overexpression of the different proteins in Minimal Medium for uniform isotope labeling}

The expression vector was transformed into E.coli strain BL21 (DE3) and kept as glycerol stock at $-80^{\circ} \mathrm{C}$. A small amount of the glycerol stock was taken with a sterile loop and streaked into a LB agar plate containing the appropriate antibiotic $(50 \mu \mathrm{g} / \mathrm{ml}$ of ampicillin and $/$ or $70 \mu \mathrm{g} / \mathrm{ml}$ kanamycin). The plate was incubated overnight at $37^{\circ} \mathrm{C}$ to obtain single colonies. A colony was picked and was inoculated into $3 \mathrm{ml}$ of LB medium (with $50 \mu \mathrm{g} / \mathrm{ml}$ ampicillin and/or $70 \mu \mathrm{g} / \mathrm{ml}$ kanamycin); the culture was incubated at $37^{\circ} \mathrm{C}$ for at least 6 hours. $50 \mathrm{ml}$ of M9 minimal medium with trace elements (containing $50 \mu \mathrm{g} / \mathrm{ml}$ of ampicillin and/or $70 \mu \mathrm{g} / \mathrm{ml}$ of kanamycin) were prepared and inoculated with $500 \mu \mathrm{l}$ of the previous LB culture and grown overnight at $37^{\circ} \mathrm{C}$.

$1 \mathrm{~L}$ of minimal medium were prepared; the composition for $1 \mathrm{~L}$ of $\left({ }^{15} \mathrm{~N}\right.$ and/or ${ }^{13} \mathrm{C}$ labeled) M9 minimal medium was the following: $6.78 \mathrm{~g}$ of $\mathrm{Na}_{2} \mathrm{HPO}_{4}, 3 \mathrm{~g}$ of $\mathrm{KH}_{2} \mathrm{PO}_{4}, 0.5 \mathrm{~g}$ of $\mathrm{NaCl}, 2 \mathrm{mM} \mathrm{MgSO}_{4}, 0.1 \mathrm{mM} \mathrm{CaCl}_{2}, 30 \mathrm{mg}$ of thiamine hydrochloride, $4 \mathrm{~g}$ of $\left({ }^{13} \mathrm{C}\right.$-labeled or natural abundance) glucose and $1 \mathrm{~g}$ of $\left({ }^{15} \mathrm{~N}\right.$-labeled or natural abundance) ammonium chloride, $\mathrm{pH}$ 7.4. In addition, $10 \mathrm{ml}$ of the following stock solution of trace elements were added: $0.06 \mathrm{~g} / \mathrm{l}$ of $\mathrm{FeSO}_{4} \bullet 7 \mathrm{H}_{2} \mathrm{O}, 0.0115 \mathrm{~g} / 1$ of $\mathrm{MnCl}_{2} \bullet 2 \mathrm{H}_{2} \mathrm{O}, 0.008 \mathrm{~g} / 1$ of $\mathrm{CoCl}_{2} \bullet 6 \mathrm{H}_{2} \mathrm{O}, 0.007$ $\mathrm{g} / \mathrm{l}$ of $\mathrm{ZnSO}_{4} \bullet 7 \mathrm{H}_{2} \mathrm{O}, 0.003 \mathrm{~g} / 1$ of $\mathrm{CuCl}_{2} \bullet 2 \mathrm{H}_{2} \mathrm{O}, 0.0002 \mathrm{~g} / 1$ of $\mathrm{H}_{3} \mathrm{BO}_{3}, 0.0025 \mathrm{~g} / \mathrm{l}$ of $\left(\mathrm{NH}_{4}\right)_{6} \mathrm{Mo}_{7} \mathrm{O}_{2} \bullet 4 \mathrm{H}_{2} \mathrm{O}$.

The $\left({ }^{15} \mathrm{~N}\right.$ and/or ${ }^{13} \mathrm{C}$ labeled) M9 medium was inoculated with the $50 \mathrm{ml}$ overnight culture in M9 medium and the bacteria were grown at $37^{\circ} \mathrm{C}$ to an O.D. $600 \mathrm{~nm}$ of 0.6 . The expression of the protein was induced with $0.5 \mathrm{mM} \mathrm{IPTG}$ for different amount of time and at different temperatures, depending on the protein to be expressed. To harvest, the cells were centrifuged at $5,000 \mathrm{~g}$ for 15 minutes. The pellet was stored at $-80^{\circ} \mathrm{C}$ until the purification was done.

\section{Purification of the hexahistidine-tagged Munc13-1 proteins}

The 6XHis-tagged recombinant proteins can be easily purified by binding with strong affinity and high selectivity to Ni-NTA agarose. The Ni-NTA agarose is comprised of Sepharose CL-6B coupled to Ni-NTA. The agarose matrix combines nitrilotriacetic acid (NTA), a tetradentate chelating ligand, in a cross-linked $6 \%$ agarose structure. NTA binds $\mathrm{Ni}^{2+}$ ions by four coordination sites. This design allows for a high binding affinity of the fusion protein with minimal nonspecific binding. The Ni-NTA matrix allows for binding of approximately 5 to $10 \mathrm{mg}$ of 6 his-tagged proteins per milliliter of resin. Proteins bound to the Ni-NTA agarose are eluted either by competition binding imidazole, or with a low $\mathrm{pH}$ buffer. The soluble hexahistidine-tagged Munc13-1 proteins were bound for 1 hour at room temperature to $4 \mathrm{ml}$ of Ni-NTA agarose (Qiagen) previously equilibrated with the Hispurification wash buffer $\left(20 \mathrm{mM} \mathrm{NaH}{ }_{2} \mathrm{PO}_{4}, 150 \mathrm{mM} \mathrm{NaCl}, 20 \mathrm{mM}\right.$ imidazole, $\mathrm{pH}$ 8.0). The Ni-NTA-agarose was retained in a disposable $10 \mathrm{ml}$ pierce column and it was washed 5 times with $10 \mathrm{ml}$ of wash buffer. The hexahistidine tagged Munc13-1 protein was eluted 4 times with $2 \mathrm{ml}$ of elution buffer ( $20 \mathrm{mM} \mathrm{NaH}_{2} \mathrm{PO}_{4}, 150 \mathrm{mM} \mathrm{NaCl}, 300 \mathrm{mM}$ imidazole, $\mathrm{pH} \mathrm{8.0)}$. 
The Munc185-(his) 6 protein was insoluble, however when it was co expressed with $\mathrm{CaM}$ it was soluble and CaM co-purified with Munc185-(his)6. The Munc65-(his) 6 protein was soluble, but the yield after elution from the Ni-NTA agarose was rather low.

The CaM/Munc185-(his) 6 complex was dialyzed overnight against 2L of TEV cleavage buffer $(50 \mathrm{mM}$ Tris, $100 \mathrm{mM} \mathrm{NaCl}, 1 \mathrm{mM}$ DTT, $\mathrm{pH} 8.0)$ at $4^{\circ} \mathrm{C}$. To cleave the hexahistidine tag, 20 units of TEV protease were added per $1 \mathrm{mg}$ of fusion protein and incubated at room temperature for 3 hours. To remove the hexahistidine tag and the TEV protease, the cleaved protein solution was incubated for 1 hour at room temperature with $2 \mathrm{ml}$ of Ni-NTA agarose, previously equilibrated with his-wash buffer. The resin was retained with a $5 \mathrm{ml}$ Pierce column and the flow through containing the cleaved CaM/Munc185 complex was obtained. The resin was washed 3 times with $1 \mathrm{ml}$ of his-wash buffer. The different fractions were analyzed by SDS-PAGE. The CaM/Munc185 complex was further purified by size exclusion chromatography on a Superdex 75 (HI-LOAD) gel filtration column (Pharmacia). The complex containing fractions after the Ni-NTA agarose purification were dialyzed against the gel filtration buffer (20 mM HEPES, $300 \mathrm{mM} \mathrm{NaCl}, 1 \mathrm{mM}$ DTT, $\mathrm{pH}$ 8.0) and concentrated by ultrafiltration with a $10 \mathrm{kDa}$ Vivaspin membrane device prior to injection to the gel filtration column.

\section{Purification of the GST-tagged Munc13-1 proteins}

The glutathione-S-transferase (GST)-fusion protein is a common affinity purification tag, which has affinity for immobilized glutathione. Glutathione-resin based purifications require that the GST domain is soluble and properly folded. The gentle elution condition (10 $\mathrm{mM}$ reduced glutathione) avoids target protein denaturation.

The soluble GST-tagged Munc13-1 proteins were bound for 1 hour at room temperature to $2 \mathrm{ml}$ of GST-Sepharose previously equilibrated with 1X PBS. The GSTSepharose was retained in a disposable Pierce $10 \mathrm{ml}$ column and it was washed 4 times with 1X PBS. The GST-tag was cleaved overnight at room temperature on the column by the addition of 10 Units of thrombin per mg of fusion protein. The flow-through containing the Munc13-1 protein was recovered and the GST-Sepharose was washed 3 times with $1 \mathrm{ml}$ of 1X PBS. The GST-Munc185 fusion protein was insoluble; it was only soluble when coexpressed with CaM. However the hexahistidine-tagged version of the complex was preferred, since the yield was better. The GST-Munc65 fusion protein was soluble, it was purification by GST-Sepharose affinity chromatography and the N-terminal GST tagged was cleaved with thrombin. The Munc13-1 C1 domain was further purified by reverse-phase High Performance Liquid Chromatography (RP-HPLC) using a C18 (vydac) preparative column with a linear acetonitrile-water $0.1 \%(\mathrm{v} / \mathrm{v})$ trifluoroacetic acid elution gradient. The fractions containing the Munc65 protein were lyophilized and reconstituted with $0.05 \%(\mathrm{v} / \mathrm{v})$ TFA. The refolding of the Munc65 protein was achieved with the addition of a 3 molar excess of $\mathrm{ZnCl}_{2}$ and increasing the $\mathrm{pH}$ to 6.5 with a diluted sodium hydroxide solution $(0.05 \mathrm{M})$.

The GST-Munc36 fusion protein was insoluble; however the overnight co-expression with $\mathrm{CaM}$ at $20^{\circ} \mathrm{C}$ gave a soluble complex that co-purified with $\mathrm{CaM}$. The purification of the Munc13-1(457-492) peptide after the thrombin cleavage was also done by RP-HPLC under the same conditions as for the Munc65 protein. The fractions containing the Munc13-1(457492) peptide were lyophilized and dissolved in water; the $\mathrm{pH}$ was adjusted with $\mathrm{NaOH}$ to 6.5 before addition of CaM to avoid precipitation.

\section{Purification of CaM}

The purification procedure for CaM used in this work is based on early biochemical work done on $\mathrm{CaM}$; it exploits the hydrophobic properties of $\mathrm{CaM}$ in its calcium bound state. 
It has the advantage, that it is not necessary to introduce an affinity tag, which decreases the total yield. In addition, depending on the protease used in the removal of the tag, a non-native glycine may remain in the primary sequence.

The bacterial pellet was thawed on ice and resuspended in $40 \mathrm{ml}$ of lysis buffer (50mM Tris, pH 7.4, $4 \mathrm{mM} \mathrm{MgCl}_{2}, 8 \mathrm{mg}$ Lysozyme, 1 tablet of Complete TM inhibitor mix (Roche) ). The mixture was stirred for $30 \mathrm{~min}$, then $2 \mathrm{mg}$ of DNAse I were added and stirred for additional $30 \mathrm{~min}$. EDTA was added up to a final concentration of $1 \mathrm{mM}$ (from a $500 \mathrm{mM}$ stock solution, $\mathrm{pH} 8.0$ ). The mixture was treated by ultrasound sonication (8 pulses of $20 \mathrm{sec}$, TT13 tip, $60-100 \%$ intensity) to disrupt the cells. The lysed cells were centrifuged at 45,000 g for $45 \mathrm{~min}$.

After centrifugation, the supernatant was collected and EDTA added up to a final concentration of $2.5 \mathrm{mM}$. Trichloroacetic acid (TCA) was added up to final concentration of $2.84 \%$, from a $50 \%(\mathrm{w} / \mathrm{v})$ stock solution. The $\mathrm{pH}$ was adjusted to 6.0 (with $5 \mathrm{M} \mathrm{NaOH}$ ). The solution was stirred for $1 \mathrm{~h}$ and the precipitate was removed by centrifugation at $45.000 \mathrm{~g}$ for $20 \mathrm{~min}$. TCA was added to the supernatant up to a final concentration of $3.4 \%$. The solution was stirred for $1 \mathrm{~h}$ followed by centrifugation at 45,000 g for $20 \mathrm{~min}$. The pellet was collected

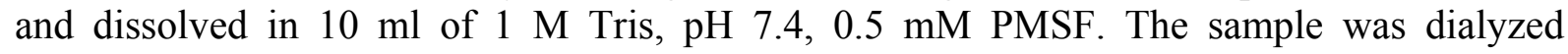
overnight against $2 \mathrm{~L}$ of dialysis buffer ( $50 \mathrm{mM}$ Tris, $\mathrm{pH} 7.4,0.5 \mathrm{mM}$ PMSF).

EDTA was added to the dialyzed sample up to a final concentration $5 \mathrm{mM}$ and $10 \mathrm{ml}$ of Phenyl-Sepharose were equilibrated with buffer A (50 mM Tris, $\mathrm{pH} 7.4,0.5 \mathrm{mM}$ PMSF, 5mM EDTA).

The dialyzed sample was incubated with the Phenyl-Sepharose for $3 \mathrm{~h}$ with stirring. The incubation mixture was passed through a glass filter; the resin was washed with $10 \mathrm{ml}$ of fresh buffer $\mathrm{A}$ and $\mathrm{CaCl}_{2}$ was added to the flow-trough up to a final concentration of $15 \mathrm{mM}$. The 15-ml Phenyl-Sepharose column was equilibrated with buffer B (50 mM Tris, $\mathrm{pH} 7.4,0.5$ $\mathrm{mM}$ PMSF, $1 \mathrm{mM} \mathrm{CaCl} 2,1.4 \mathrm{mM} \beta$-mercaptoethanol) and the flow-through after the batch purification step was loaded. The column was washed with 3 column volumes of buffer $\mathrm{B}$ and then with buffer $\mathrm{C}(50 \mathrm{mM}$ TrisHCl, pH 7.4, $0.5 \mathrm{mM}$ PMSF, $1 \mathrm{mM} \mathrm{CaCl} 2,1.4 \mathrm{mM} \beta$ mercaptoethanol, $0.5 \mathrm{M} \mathrm{NaCl}$ ) until a stable baseline was reached. The $\mathrm{CaM}$ elution was carried out using buffer D $(50 \mathrm{mM}$ TrisHCl, pH 7.4, $1.4 \mathrm{mM} \beta$-mercaptoethanol, $5 \mathrm{mM}$ EDTA), $1 \mathrm{ml}$ fractions were collected and analyzed by SDS-PAGE. In each case 5,0 $\mu 1$ of sample solution were mixed with $5.0 \mu \mathrm{l}$ of sample buffer and boiled at $95^{\circ} \mathrm{C}$ for $2 \mathrm{~min}$. Fractions containing $\mathrm{CaM}$ were combined and dialyzed against NMR-buffer (20 mM BisTris, $150 \mathrm{mM} \mathrm{KCl}, 10 \mathrm{mM} \mathrm{CaCl}_{2}$, pH 6.8)

The protein concentration after dialysis was estimated by UV-spectrophotometry $\left(\mathrm{A}_{280}=0.246,0.5 \mathrm{mg} / \mathrm{ml}\right.$ of $\left.\mathrm{CaM}\right) .50 \mu \mathrm{l}$ of this solution was taken for Electrospray Ionization Mass spectrometry (ESI-MS) analysis, for this purpose the sample was dialyzed against 20 $\mathrm{mM} \mathrm{NH}_{4} \mathrm{Ac}$ buffer, $\mathrm{pH}$ 6.5. After dialysis, the sample was concentrated using Centricon ${ }^{\mathrm{TM}}$ membranes, MWCO 10.000 until a final volume of $0.6 \mathrm{ml}(1 \mathrm{mM} \mathrm{CaM})$ was reached. Then it was washed two times with NMR-buffer ( $2 \mathrm{ml}$ of buffer each time) to remove $\beta$ mercaptoethanol and to increase the $\mathrm{Ca}^{2+}$ concentration.

For the samples used in paramagnetic alignment studies, the CaM cysteine mutant was derivatized with the paramagnetic tag (S-Methanesulfonyl-L-cysteaminyl-1-carbonic-acidethylenediamine-N,N,N',N'-tetraacetic acid, MS-EDTA). The total volume of the concentrated protein solution (in NMR buffer) was mixed with a 3 -fold molar excess of tag (1 $\mathrm{mg} / \mathrm{ml}$ solution in $\mathrm{H}_{2} \mathrm{O}$ ). The sample was left for $20 \mathrm{~min}$ at room temperature; the excess of tag was removed by washing with NMR-buffer 2 times in a centricon membrane $(10 \mathrm{kDa}$ cutoff) with $2 \mathrm{ml}$ each time and the sample was stored at $4^{\circ} \mathrm{C}$. 


\section{Purification of the Munc13-1-185aa protein}

The Munc185-(his) 6 protein could not be expressed alone in a soluble form; for this reason the approach of the co-expression with CaM chosen. However, it was of interest to study the free protein as well, for this reason a method to dissociate the complex was developed. From the primary sequence of the two proteins it was expected that they differ significantly in their isolectric point $(\mathrm{pI})$; for this reason ion exchange chromatography was the method of choice for the biochemical separation. Since the CaM/Munc13-1 interaction is calcium dependant, the complex was dialyzed against a high concentration of EDTA $(50 \mathrm{mM})$ and $\mathrm{pH} 8.8$ to remove calcium from CaM. Afterwards it was dialyzed against the cation exchange buffer ( $25 \mathrm{mM}$ sodium acetate, $25 \mathrm{mM}$ EDTA, $\mathrm{pH}$ 5.5). At this $\mathrm{pH}$, Munc 185 was positively charged and could be bound to the SP column. CaM has a pI of 4.4 and does not bind to the column at this $\mathrm{pH}$. The elution of the Munc185 protein from the column was achieved with a linear gradient with $1 \mathrm{M}$ sodium chloride as target concentration. Alternatively, the same cation exchange chromatographic method can be used under denaturing conditions to avoid precipitation of Munc185; since it aggregates in the absence of CaM. Due to its aggregaton at the concentrations needed for NMR, it was not possible to record any NMR spectra.

\section{Electrospray ionization mass spectrometry (ESI-MS)}

For the paramagnetic tagging of the different cysteine CaM mutants it was essential to verify the completeness of the reaction with the tag. For this purpose the ESI-MS spectrum of the free protein was measured as reference spectrum. After the reaction proceeded, the ESIMS spectrum of the tagged protein was recorded. The mass spectra were recorded in a WATERS ESI-Mass spectrometer in the positive mode, with a cone voltage of $30 \mathrm{eV}$. The samples had a protein concentration of $5 \mu \mathrm{M}$ and were in $20 \mathrm{mM}$ ammonium acetate buffer with $50 \%(\mathrm{v} / \mathrm{v})$ acetonitrile and $0.1 \%(\mathrm{v} / \mathrm{v})$ trifluoroacetic acid.

\section{NMR measurements}

The recordings were done in Bruker AVANCE spectrometers at ${ }^{1} \mathrm{H}$ NMR frequency of $900,800,700$ and $600 \mathrm{MHz}$. The experiments were performed at $298 \mathrm{~K}, 303 \mathrm{~K}$ or $308 \mathrm{~K}$. The spectra were processed and analyzed using the following software: XWIN-NMR, NMR-pipe (Delaglio et al., 1995), NMR Draw, Felix (Accelerys Inc.), Sparky (Goddard and Kneller, 1999) and PALES (Zweckstetter and Bax, 2000). The acquisition parameters are found in table 2 .

The most basic experiment used in biomolecular NMR is the heteronuclear single quantum coherence spectrum (HSQC), a pictorial representation of the pulse sequence is shown in Figure 13. Other pulse sequences used in this work are found in the Apendix.

A total of five NMR samples were used for the solution structure determination of the CaM/Munc13-1(457-492) peptide complex. Three samples consisted of uniformly $\left({ }^{15} \mathrm{~N}\right.$ and ${ }^{15} \mathrm{~N} /{ }^{13} \mathrm{C}$ ) labelled CaM complexed with unlabelled Munc13-1 synthetic peptide (residues 459492: The first sample $\left({ }^{13} \mathrm{C},{ }^{15} \mathrm{~N}\right)$ was dissolved in $90 \%(\mathrm{v} / \mathrm{v}) \mathrm{H}_{2} \mathrm{O}, 10 \%(\mathrm{v} / \mathrm{v}) \mathrm{D}_{2} \mathrm{O}$ and used for sequential assignment spectra involving the exchangeable $\mathrm{NH}$ group; the second one $\left({ }^{15} \mathrm{~N}\right)$ aligned by adding filamentous bacteriophage (Pf1) solution to a final concentration of 15 $\mathrm{mg} / \mathrm{ml}$. (Asla Labs). The third sample $\left({ }^{13} \mathrm{C},{ }^{15} \mathrm{~N}\right)$ was dissolved in $99 \%(\mathrm{v} / \mathrm{v}) \mathrm{D}_{2} \mathrm{O}$ for certain NMR experiments were optimal water suppression was needed. The last two samples consisted of uniformly $\left({ }^{13} \mathrm{C},{ }^{15} \mathrm{~N}\right)$ labelled Munc13-1 (457-492) peptide complexed to natural abundance CaM, dissolved in $90 \%(\mathrm{v} / \mathrm{v}) \mathrm{H}_{2} \mathrm{O}, 10 \% \mathrm{D}_{2} \mathrm{O}$ for all sequential assignment and NOESY spectra and another one with $16 \mathrm{mg} / \mathrm{ml}$ Pf1 cosolvent for rDC measurements. 


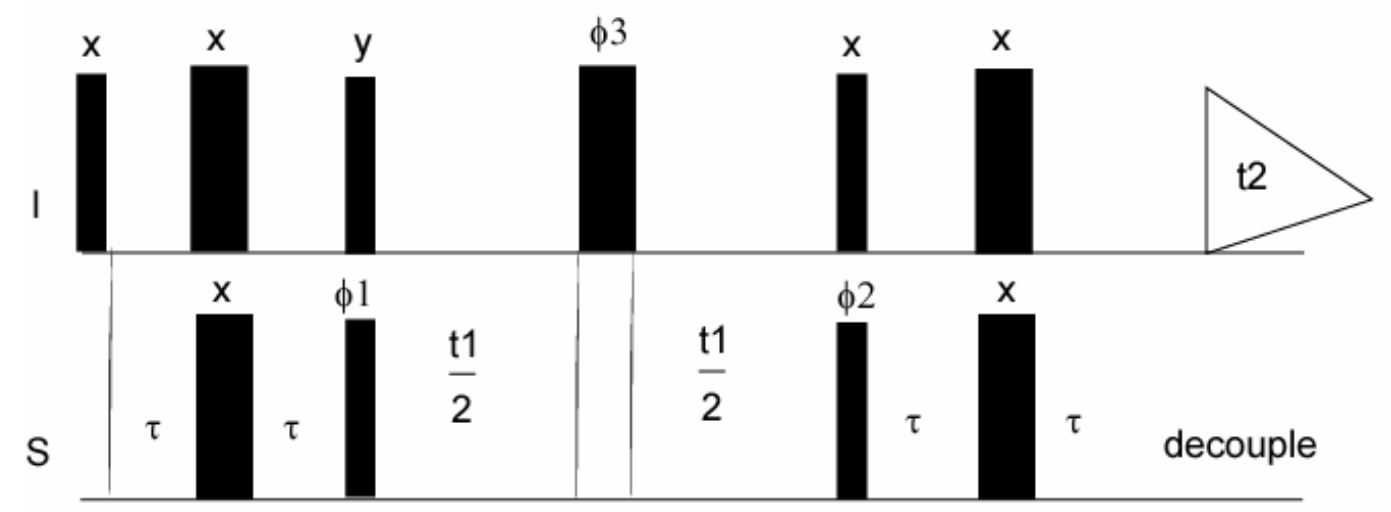

Figure 13. Pulse sequence for the ${ }^{1} \mathrm{H}$ detected heteronuclear single quantum coherence (HSQC) experiment. Thin bars represent $90^{\circ}$ pulses and thick bars represent $180^{\circ}$ pulses. The phase of each pulse is indicated above the bar. The phase cycling is $\phi 1=x,-x ; \phi 2=2(x), 2(-$ $\mathrm{x}) ; \phi 3=4(\mathrm{y}), 4(-\mathrm{y})$; and receiver $=2(\mathrm{x},-\mathrm{x},-\mathrm{x}, \mathrm{x})$ (Adapted from Cavanagh et al., 1996).

Table 2. NMR spectra acquired for the solution structure determination of the CaM/Munc13-1 (457-492) peptide complex

\begin{tabular}{|c|c|c|c|c|c|}
\hline \multirow{4}{*}{$\begin{array}{l}\text { Sample conditions } \\
{ }^{15} \mathrm{NCaM} / \mathrm{Munc} 13-1 \\
(459-492) \text { in } 90 \%\end{array}$} & \multirow{2}{*}{ Experiment } & \multirow{3}{*}{ 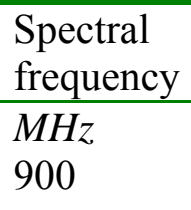 } & \multicolumn{3}{|c|}{ Complex points } \\
\hline & & & $\mathrm{D}_{1}$ & $\mathrm{D}_{2}$ & $\mathrm{D}_{3}$ \\
\hline & $2 \mathrm{D}-{ }^{1} \mathrm{H}-{ }^{15} \mathrm{~N} H S Q \mathrm{C}$ & & 2048 & 128 & - \\
\hline & & & & & \\
\hline $\begin{array}{l}{ }^{15} \mathrm{NCaM} / \text { Munc } 13-1 \\
(459-492) \text { in } 90 \%\end{array}$ & $2 \mathrm{D}-{ }^{1} \mathrm{H}-{ }^{15} \mathrm{~N}$ IPAP-HSQC & 900 & 2048 & 800 & - \\
\hline $\begin{array}{l}\mathrm{H}_{2} \mathrm{O}, 10 \% \mathrm{D}_{2} \mathrm{O} \\
\text { with } 16 \mathrm{mg} / \mathrm{ml} \mathrm{Pf1}\end{array}$ & & & & & \\
\hline \multirow{9}{*}{$\begin{array}{l}{ }^{15} \mathrm{~N},{ }^{13} \mathrm{C} \mathrm{CaM} / \mathrm{Munc} \\
13-1(459-492) \text { in } \\
90 \% \mathrm{H}_{2} \mathrm{O}, 10 \% \mathrm{D}_{2} \mathrm{O}\end{array}$} & $3 \mathrm{D}-{ }^{1} \mathrm{H}-{ }^{15} \mathrm{~N}$ NOESY-HSQC, & 900 & 2048 & 48 & 128 \\
\hline & $t_{\operatorname{mix}} 100 \mathrm{~ms}$ & & & & \\
\hline & 3D-HNCO & 700 & 2048 & 46 & 46 \\
\hline & 3D-HN(CA)CO & 600 & 1024 & 60 & 60 \\
\hline & 3D-HNCACB & 700 & 1024 & 40 & 25 \\
\hline & 3D-CBCA $(\mathrm{CO}) \mathrm{NH}$ & 600 & 1024 & 58 & 24 \\
\hline & 3D-H(CC)(CO)NH-TOCSY & 700 & 2048 & 30 & 64 \\
\hline & 3D-(H)CC(CO)NH-TOCSY & 700 & 2048 & 70 & 30 \\
\hline & $2 \mathrm{D}-{ }^{1} \mathrm{H}-{ }^{13} \mathrm{C}$ HSQC & 600 & 2048 & 128 & - \\
\hline \multirow{9}{*}{$\begin{array}{l}\text { Lyophilized, then } \\
\text { dissolved in } \mathrm{D}_{2} \mathrm{O} \\
{ }^{15} \mathrm{~N},{ }^{13} \mathrm{C} \mathrm{CaM} / \text { Munc } \\
13-1(459-492) \text { in } 99.9 \\
\% \mathrm{D}_{2} \mathrm{O}\end{array}$} & Series of $2 \mathrm{D}-{ }^{1} \mathrm{H}-{ }^{15} \mathrm{~N}$ HSQC & 600 & 2048 & 128 & - \\
\hline & $3 \mathrm{D}-{ }^{1} \mathrm{H}-{ }^{13} \mathrm{C}$ NOESY-HSQC & 700 & 2048 & 48 & 58 \\
\hline & $t_{\text {mix }} 120 \mathrm{~ms}$ & & & & \\
\hline & 3D-HCCH-TOCSY & 600 & 2048 & 128 & 64 \\
\hline & 2D- ${ }^{1} \mathrm{H}_{-}{ }^{13} \mathrm{C}$ HSQC & 700 & 2048 & 128 & - \\
\hline & $\begin{array}{l}3 \mathrm{D}-{ }^{1} \mathrm{H}-{ }^{13} \mathrm{C} \text { NOESY-HSQC } \\
\text { (aromatic region) } t_{\text {mix }} 130 \mathrm{~ms}\end{array}$ & 700 & 2048 & 48 & 40 \\
\hline & 2D-HBCB(Caro)HE & 600 & 2048 & 46 & - \\
\hline & 2D-HBCB(Caro)HG & 600 & 2048 & 46 & - \\
\hline & $3 \mathrm{D}-{ }^{1} \mathrm{H}^{-13} \mathrm{C}$ HMBC & 700 & 2048 & 48 & 120 \\
\hline
\end{tabular}




\begin{tabular}{|c|c|c|c|c|c|}
\hline \multirow{10}{*}{$\begin{array}{l}{ }^{15} \mathrm{~N},{ }^{13} \mathrm{C} \text { Munc } 13-1 \\
(457-492) / \mathrm{CaM} \text { in } \\
90 \% \mathrm{H}_{2} \mathrm{O}, 10 \% \mathrm{D}_{2} \mathrm{O}\end{array}$} & \multicolumn{5}{|l|}{ (Methionines) } \\
\hline & $2 \mathrm{D}-{ }^{1} \mathrm{H}-{ }^{15} \mathrm{~N}$ HSQC & 600 & 1024 & 128 & - \\
\hline & $\begin{array}{l}3 \mathrm{D}-{ }^{1} \mathrm{H}-{ }^{15} \mathrm{~N} \text { NOESY-HSQC } \\
\text { and } 3 \mathrm{D}-{ }^{1} \mathrm{H}-{ }^{13} \mathrm{C} \text { NOESY- } \\
\text { HSQC shared version, } t_{\text {mix }} \\
120 \mathrm{~ms}\end{array}$ & 600 & 2048 & 180 & 114 \\
\hline & 3D-HNCO & 900 & 1024 & 64 & 32 \\
\hline & 3D-HN(CA)CO & 600 & 1024 & 48 & 46 \\
\hline & 3D-HNCACB & 600 & 1024 & 50 & 100 \\
\hline & 2D-CBCA $(\mathrm{CO}) \mathrm{NH}$ & 600 & 1024 & 29 & 60 \\
\hline & 3D-H(CC)(CO)NH-TOCSY & 600 & 1024 & 26 & 50 \\
\hline & 3D-(H)CC(CO)NH-TOCSY & 600 & 1024 & 30 & 52 \\
\hline & 3D-HCCH-TOCSY & 600 & 2048 & 32 & 128 \\
\hline $\begin{array}{l}\text { Lyophilized, then } \\
\text { dissolved in } \mathrm{D}_{2} \mathrm{O}\end{array}$ & Series of $2 \mathrm{D}-{ }^{1} \mathrm{H}-{ }^{15} \mathrm{~N}$ HSQC & 800 & 2048 & 128 & - \\
\hline $\begin{array}{l}{ }^{15} \mathrm{~N}^{13} \mathrm{C} \mathrm{Munc13-1} \\
(457-492) / \mathrm{CaM}) \text { in } \\
90 \% \mathrm{H}_{2} \mathrm{O}, 10 \% \mathrm{D}_{2} \mathrm{O} \\
\text { with } 16 \mathrm{mg} / \mathrm{ml} \mathrm{Pfl}\end{array}$ & $2 \mathrm{D}-{ }^{1} \mathrm{H}-{ }^{15} \mathrm{~N}$ IPAP-HSQC & 600 & 1024 & 512 & - \\
\hline
\end{tabular}

The one-bond ${ }^{1} \mathrm{H}^{-}{ }^{15} \mathrm{~N}$ residual dipolar couplings were obtained from a Heteronuclear ${ }^{15} \mathrm{~N}-{ }^{1} \mathrm{H}$ Single Quantum Coherence 2D NMR spectrum (Refer to Figure 13). For an accurate measurement of the scalar and dipolar couplings it was essential to record at least 512 real points in the indirect dimension. A good digitization of the FID was achieved by zero filling to 8192 real plus imaginary points. The FID was Fourier transformed, the phase was corrected in the direct dimension and the FID was apodized with a sine-bell function. The frequency value from the center of the peak in the 2D spectrum was read out with the Sparky program after centering the peak. For couplings measurements In-Phase Anti-Phase (IPAP)-HSQC spectra were recorded (Ottiger et al., 1998). With this pulse sequence, two sub-spectra are generated, the first one contains only the up field component and the second the downfield component of a doublet. This type of spectrum has the advantage of reducing the overlap in comparison to the coupled HSQC spectrum.

\section{Docking and structure calculation approaches for the CaM/Munc13-1(457-492) peptide complex}

For the automatic assignment of NOESY spectra and structure calculation the combined automated NOE assignment and structure determination (CANDID) procedure introduced by Herrmann and collaborators (2002) was used. It combines features from NOAH and ARIA, such as the use of three-dimensional structure-based filters and ambiguous distance constraints, with the new concepts of network-anchoring and constraint-combination that further enable an efficient and reliable search for the correct fold in the initial cycle of $d e$ novo NMR structure determinations.

Among the guidelines for the successful performance of CANDID the input chemical shift list must contain more than $90 \%$ of the non-labile and backbone amide ${ }^{1} \mathrm{H}$ chemical shifts, the average backbone root mean square deviation (RMSD) o the mean coordinates for the structured parts of the polypeptide chain should be below $3.0 \AA$ for the bundle of conformers used to represent the structure from CANDID cycle 1 and the RMSD drift between the mean atom coordinates after the first and the last CANDID cycles calculated for 
the backbone heavy atoms of the structured part of the polypeptide chain should be smaller than $3.0 \AA$, and it should not exceed the average RMSD to the mean coordinates after cycle 1 by more than $25 \%$ (Herrmann et al., 2002).

An initial model of CaM complexed with the Munc13-1(457-492) peptide for the CANDID approach was not available. The initial trial to run CANDID using an extended structure of CaM linked with the Munc13-1(457-492) peptide failed to give a bundle of structures with a RMSD of less than $3 \AA$ in the structured regions after the first cycle; for this reason a docking approach was used to generate an initial model. Based on the information of the chemical shifts, the residual dipolar couplings and the intermolecular NOE data for the bound Munc36 the initial model of the complex was generated.

Clore (2000) showed that accurate docking can be achieved by rigid body minimization using a target function that comprises only three terms, consisting of intermolecular NOE and ${ }^{1} \mathrm{D}_{\mathrm{NH}}$ dipolar coupling restraints, supplemented by an intermolecular van der Waals repulsive potential.

The Rosetta de novo structure prediction method was used for obtaining the initial backbone conformation of the Munc13-1(457-492) peptide. Rosetta is based on a picture of protein folding in which local sequence segments rapidly alternate between different possible local structures, and folding occurs when the conformations of these local segments combine to form low energy global structures. The distribution of conformations sampled by an isolated chain segment is approximated by the distribution of conformations adopted by that sequence segment and related sequence segments in the protein structure database. Non-local interactions are optimized by a Monte Carlo search through the set of conformations that can be built from the ensemble of local structure fragments for each sequence segment. The procedure results in structures that have low free energy local and non-local interactions. (Bonneau et al., 2002).

The backbone torsion angle prediction package TALOS (Torsion Angle Likelihood Obtained from Shifts and sequence similarity. TALOS reads the experimental protein chemical shift tables and converts them to secondary chemical shifts before entering them in the database. TALOS evaluates the similarity in amino acid sequence and secondary shifts for a string of three sequential amino acids relative to all triplets of sequential residues contained in the database. The dihedral constraints for the backbone torsion angles obtained from TALOS are available immediately after completion of the resonance assignment and therefore can be used at the very early stages of structure calculation. (Cornilescu et al., 1999)

The side chains of Munc36 where added with the What if program (Vriend, 1990). The rigid body minimization protocol was done as described by Clore (2000), 500 steps of rigid body minimization with the force constants for the dipolar coupling $\left(k_{\text {dip }}\right)$, NOE-derived interproton distance $\left(k_{\mathrm{NOE}}\right)$ and quartic repulsive van der Waals repulsion $\left(k_{\mathrm{vdw}}\right)$ terms set to $0.1 \mathrm{kcal}^{*} \mathrm{~mol}^{-1} * \mathrm{~Hz}^{-2}, 0.01 \mathrm{kcal}^{*} \mathrm{~mol}^{-1} * \AA^{-2}$, and $4 \mathrm{kcal}^{*} \mathrm{~mol}^{-1} * \AA^{-4}$, respectively. This was followed by 100 cycles of rigid body minimization ( 500 steps per cycle) in which $k_{\mathrm{dip}}, k_{\mathrm{NOE}}$, and $k_{\mathrm{vdw}}$, where slowly increased from 0.1 to $0.5 \mathrm{kcal}^{*} \mathrm{~mol}^{-1} * \mathrm{~Hz}^{-2}, 0.01$ to $30 \mathrm{kcal}^{*} \mathrm{~mol}^{-1} * \AA^{-2}$, and 0.004 to $1.0 \mathrm{kcal}^{*} \mathrm{~mol}^{-1} * \AA^{-4}$, respectively. This was finally followed by 500 steps of rigid body minimization with the values of $k_{\mathrm{dip}}, k_{\mathrm{NOE}}$ and $k_{\mathrm{vdw}}$ set to $0.5 \mathrm{kcal}^{*} \mathrm{~mol}^{-1} * \mathrm{~Hz}^{-2}, 60$ $\mathrm{kcal}^{*} \mathrm{~mol}^{-1} * \AA^{-2}, 3 \mathrm{kcal}^{*} \mathrm{~mol}^{-1} * \AA^{-4}$.

For torsion angle dynamics (TAD) calculations with DYANA the molecule is represented as a tree structure consisting of a base rigid body that is fixed in space and $n$ rigid bodies, which are connected by $n$ rotatable bonds. The degrees of freedom are exclusively torsion angles, i.e. rotation about single bonds. Each rigid body is made of one or several mass points (atoms) for which the relative positions are invariable. The tree structure starts from a "base", typically at the N-terminus of the polypeptide chain, and terminates with "leaves" at the ends of the side-chains and at the C-terminus. The rigid bodies are numbered from 0 to $n$. The base has the number 0 . Each other rigid body, with a number $k \geq 1$, has a 
single nearest neighbor in the direction towards the base, which has a number $\mathrm{p}(k)<k$. The torsion angle between the rigid bodies $\mathrm{p}(k)$ and $k$ is denoted by $\theta_{k}$. The conformation of the molecule is uniquely specified by the values of all torsion angles, $\theta=\left(\theta_{1}, \ldots, \theta_{n}\right)$. All threedimensional vectors are in an inertial frame of reference that is fixed in space. For each rotatable bond, $\mathrm{e}{ }_{k}$ denotes a unit vector in the direction of the bond, and $\vec{r}_{k}$ is the position vector of its end point, which is subsequently used as the "reference point" of the rigid body $k$ in the inertial reference frame. (Güntert et al., 1997).

The DYANA standard simulated annealing protocol consists of the following steps:

(1) Perform 100 conjugate gradient minimization steps at level 3, i.e. including only distance constraints between atoms up to three residues apart along the sequence, followed by further 100 minimization steps including all constraints.

(2) Perform N/5 TAD steps at a constant high reference temperature, Thigh, where $N$ is the user-defined total number of TAD steps. The initial time-step, $\Delta t=2 \mathrm{fs}$, is adapted such as to achieve a relative accuracy of energy conservation of $\varepsilon^{\text {ref }}=0.005$.

(3) Perform 4N/5 TAD steps with reference values for temperature and the relative accuracy of energy conservation of:

$$
\begin{aligned}
& \mathrm{T}^{\mathrm{ref}}(\mathrm{s})=(1-\mathrm{s})^{4} \mathrm{~T}_{\text {high }} \\
& \varepsilon^{\mathrm{ref}}(\mathrm{s})=0.005^{*} 0.002^{s}
\end{aligned}
$$

The parameter $s$ varies linearly from 0 in the first time-step to 1 in the last time-step.

(4) Perform 200 TAD steps at zero reference temperature and $\varepsilon^{\text {ref }}=0.0001$. Initial velocities are those from the last time-step of step 3 above, and the length of the initial time-step is set to one fourth of the last time-step.

(5) Perform 1000 conjugate gradient minimization steps, including all constraints. The default values for the initial temperature, $\mathrm{T}_{\text {high }}=9600 \mathrm{~K}$. The default value for $N$ is 4000 .

Refinement of the CaM/Munc13-1 (457-492) peptide complex in explicit solvent was done in a full molecular dynamics force field including electrostatic and Lennard-Jones nonbonded potentials, since the interaction with the solvent is one way to improve the quality of the structures (Linge et al., 2003).

\section{Data analysis of residual dipolar couplings and pseudocontact shifts}

The measured $\mathrm{NH}$ residual dipolar couplings from the sterically aligned (with Pf1) or paramagnetically aligned samples of $\mathrm{CaM}$ in its different activated states need to be analyzed for each domain separately. The reason for this is precisely the flexible linker joining the two domains. The flexibility impaired by the linker allows each domain to sample numerous orientations in the time-scale sampled by dipolar couplings and a global analysis of the rDC data done without taking care of these considerations would fail.

Thus the rDC data was divided into N-terminal domain (residues 2-76) and C-terminal (residues 84-146). The N-terminal amide (residue 1) is not observable in the HSQC spectrum due to fast exchange with the solvent and the last two residues in $\mathrm{CaM}$ are too flexible and therefore disordered in different high-resolution structures.

There are high resolution NMR structures available for the N-terminal domain of apoCaM (1F70.pdb) and holoCaM (1J7O.pdb) and for the C-terminal domain of apoCaM (1F71.pdb) and holoCaM (1J7P.pdb). These structures have been refined with numerous rDC data (Chou et al., 2001 and Chou et al., 2000) and were taken as molecular models for fitting the experimental rDCs measured in this work.

The program PALES (Zweckstetter and Bax, 2000) was used for all the rDC data analysis. The primary NH rDC data was analyzed by two different routines: First, a histogram of the rDC data was generated and the principal components of the alignment tensor values $\left(d_{\mathrm{zz}}, d_{\mathrm{yy}}\right.$ and $\left.d_{\mathrm{xx}}\right)$ were obtained in this form. Alternatively, the rDC data was fitted to the simulated rDC data derived from the high resolution molecular models with a Singular Value 
Decomposition (SVD) algorithm. In addition, to the values of the principal components of the alignment tensor, this second routine provides more information on the alignment tensor: A linear correlation between the experimental rDCs and the back-calculated rDCs is obtained. If the SVD algorithm is run repeatedly, statistics about the alignment tensor values are also obtained, this permits to gain access to the precision of the obtained results.

The Pseudocontact shift data was measured in the ${ }^{1} \mathrm{H}$ dimension as the difference in the resonance values of the shifted $\mathrm{NH}$ cross peak and the reference (isotropic) $\mathrm{NH}$ cross peak. The PCS values were fitted to the back-calculated PCS derived from the coordinates of the same high-resolution molecular models of CaM used in the rDC data analysis. For this purpose, the metal position was located by a grid search minimizing the root-mean square deviation of the experimental PCS and calculated ones.

Once the position of the metal was precisely located, the distance between the metal ion and each amide was measured in the molecular model. The distances were plotted against the PCS values and a power fit was applied to the data, since there is a $r^{3}$ dependency for the PCS values on the metal-amide distance. An upper bound $r^{3}$ function was applied maximizing the angular values of the PCS expression $\left(3 \cos ^{2} \theta-1\right)$ and $\left(\sin ^{2} \theta \cos 2 \Omega\right)$. 


\section{RESULTS}

1. Cloning, overexpression and purification of CaM and Munc13-1 proteins

$$
\text { a.Cloning of Munc13-1 (457-492) (Munc13-1-36aa) }
$$

The results for the PCR amplification are shown in Figure 14. The Munc13-1(457-492) insert for the pGEX2T vector runs in the agarose gel as a double stranded DNA of around 100 base-pairs (bp); this corresponded very well to the expected length of the PCR product. The Munc13-1(457-492) insert for the pMAL vector also had an apparent molecular weight corresponding well to the expected one.

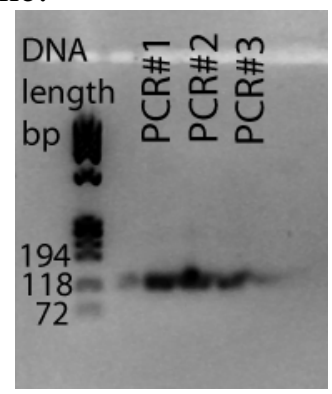

Figure 14. 2\% (w/v) agarose gel electrophoresis for the PCR product of the Munc13-1(457492) DNA insert for the pGEX2T cloning.

The agarose gel for the restriction digest of the positive clones is shown in Figure15; this confirmed that the desired insert was cloned successfully. Since the PCR based cloning, relies on the fidelity of the polymerase enzyme used it is essential to sequence the insert region of the plasmid DNA to ensure the whole sequence of the insert is correct. This is not so critical for the Pfu DNA polymerase enzyme due to its proof-reading activity, but is good practice especially for structural studies.

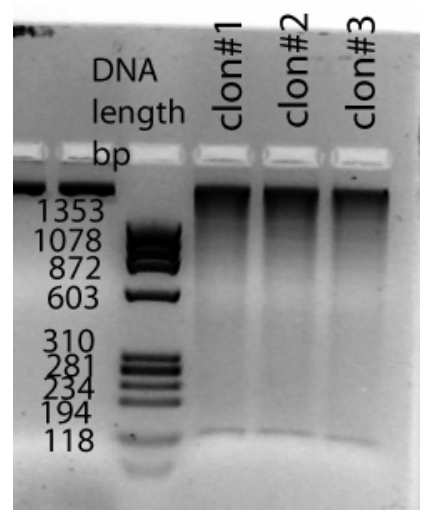

Figure 15. 1\% (w/v) agarose gel electrophoresis for three positive clones for the pGEX2TMunc13-1(457-492) construct cleaved with BaMHI and EcoRI.

\section{b. Cloning of Munc13-1 (567-631) $C_{1}$ domain (Munc13-1-65aa)}

The agarose gel electrophoresis for the PCR products of the amplification reaction is shown in Figure 16. The Munc65 insert had an apparent molecular weight corresponding to a double stranded DNA of around $260 \mathrm{bp}$; this agreed well with the expected length of the PCR product. After cloning, the positive clones were digested with the restriction enzymes NdeI and XhoI for the pET16b construct and BaMHI and EcoRI for the pGEX constructs. The agarose gel for the restriction digest of the positive clones is shown in Figure 16; this confirmed that the desired insert was cloned successfully. All the clones were positive and the first two clones were sequenced. 


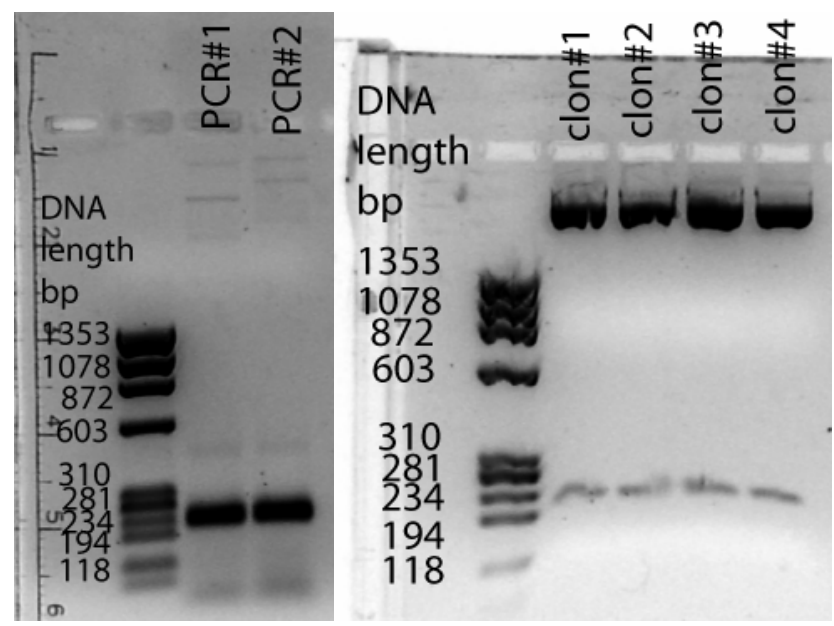

Figure 16. 1\% (w/v) agarose gel electrophoresis for the PCR products of the Munc65 insert for the cloning into pET16b, pGEX2T and pGEX2T-TEV expression vectors (left) and restriction analysis of the positive pGEX2T-TEV-Munc65 constructs (right).

\section{c. Cloning of Munc13-1 (447-631) (Munc13-1-185aa)}

The agarose gel electrophoresis for the PCR products of the amplification reaction is shown in Figure 17. The Munc185 insert had an apparent molecular weight corresponding to a double stranded DNA of around $600 \mathrm{bp}$; this agreed well with the expected length of the PCR product. The agarose gel for the restriction digest of the positive clones of the pET16bMunc185 construct is shown in Figure 17; this confirmed that the desired insert was cloned successfully. All the clones analyzed were positive. The first two clones were sequenced.

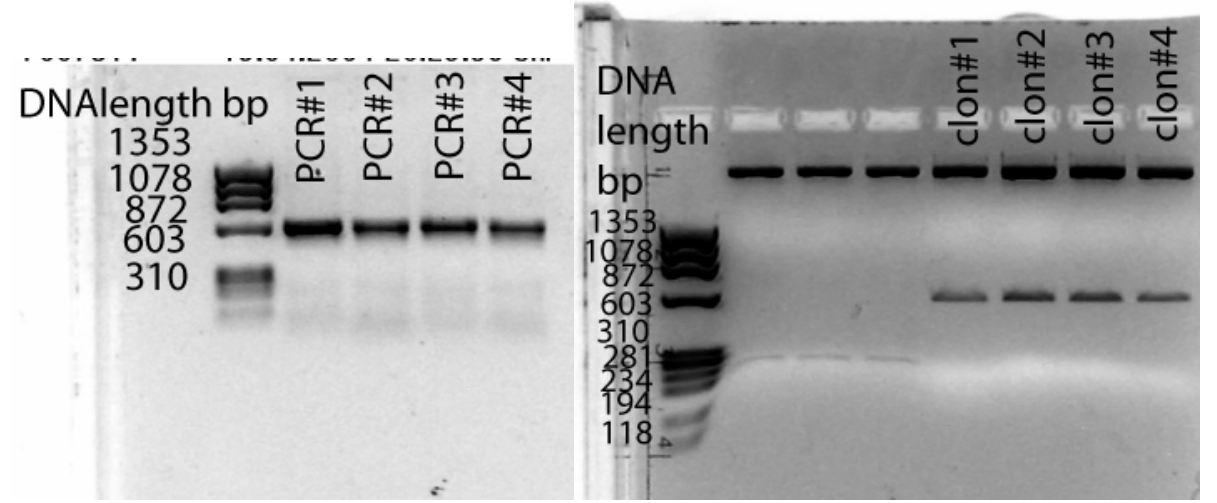

Figure 17. 1\% (w/v) agarose gel electrophoresis for the PCR products of the Munc185 insert for the cloning into pET16b, pGEX2T and pETDuet-1 expression vectors (left) and restriction analysis of the positive pET16b-Munc185 constructs (right).

\section{d. Site directed mutagenesis}

All the desired mutations for CaM and Munc13-1 were obtained with the Quick-Change kit from Stratagene. Each mutation was verified by DNA sequencing of the plasmid DNA of the expression construct.

\section{e. Overexpression and purification of the Munc13-1(457-492)}

The N-terminal GST fusion protein of Munc13-1(457-492) could be expressed in the desired amounts after the induction with $0.5 \mathrm{mM}$ IPTG as shown in the SDS-PAGE in Figure 18. Since there was a low solubility and there were several proteolytic products of the GSTMunc13-1(457-492) fusion protein, a co-expression approach with CaM was chosen to 
protect the Munc13-1(457-492) from proteolytic cleavage and to increase its solubility. In the SDS-PAGE in Figure 18 two soluble bands corresponding to CaM and GST- Munc13-1(457492 ) in the soluble fraction of the cell lysate were found.

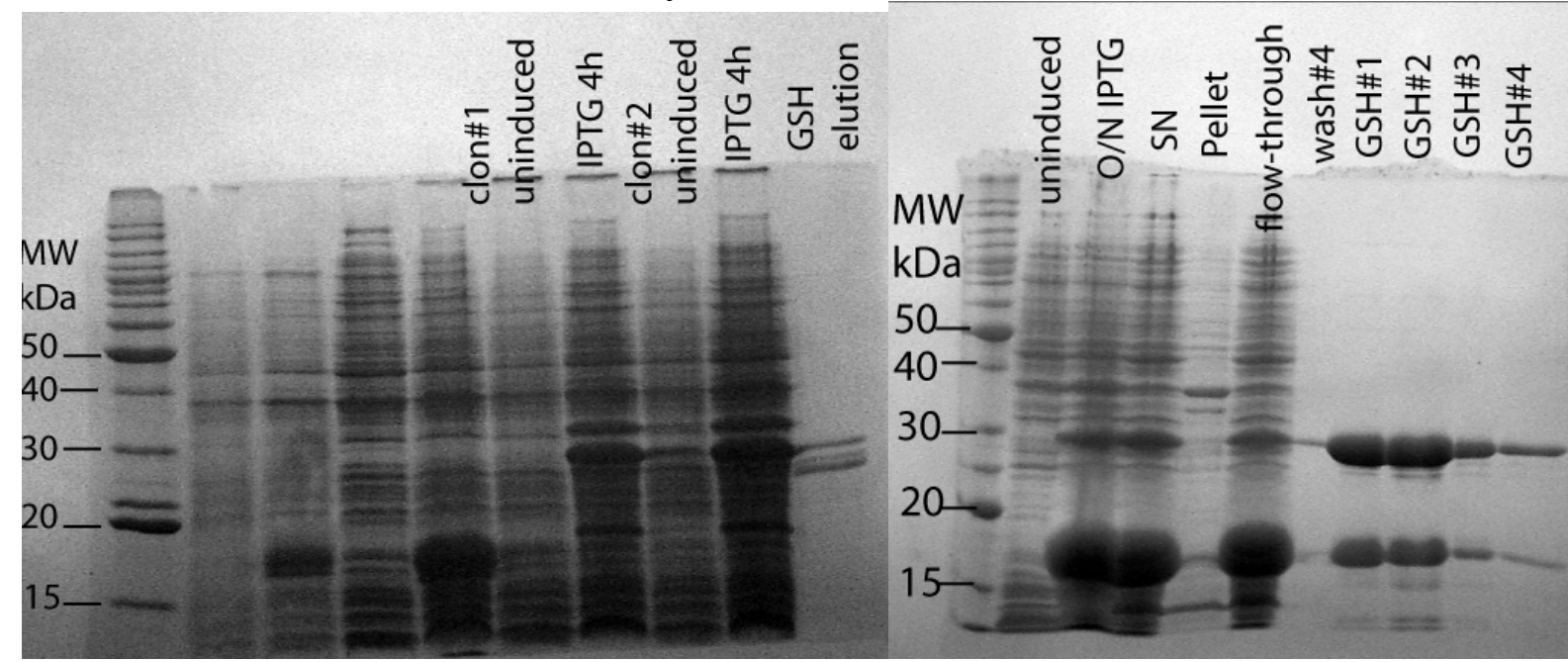

Figure 18. 12\% (v/v) polyacrylamide SDS-PAGE for the overexpression and purification analysis of the pGEX2T- Munc13-1(457-492) construct (left) and for its co-expression with CaM (right).

The GST- Munc13-1(457-492)/CaM complex was co-purified by means of its affinity tag with GST-Sepharose beads, the TEV cleavage was unsuccessful. For this reason it was necessary to sub-clone the Munc13-1(457-492) insert into the pGEX2T vector which contains a thrombin recognition site between the N-terminal fusion GST and the Munc13-1(457-492) peptide. The cleavage with thrombin was successful as can be seen in the gel in Figure 19. To separate the Munc13-1(457-492) peptide from CaM, a preparative RP-HPLC method with a C18 column (Vydac) was used. The chromatogram is shown in Figure 19. The Munc131(457-492) peptide eluted at a retention time of 22.8 minutes and CaM at a retention time of 29.8 minutes. The identity of each peak was confirmed with ESI-Mass spectrometry. The mass for ${ }^{13} \mathrm{C},{ }^{15} \mathrm{~N}$ CaM was $17588 \mathrm{Da}$ and for the ${ }^{13} \mathrm{C},{ }^{15} \mathrm{~N}$ Munc13-1 (457-492) peptide was 4407.6 Da.

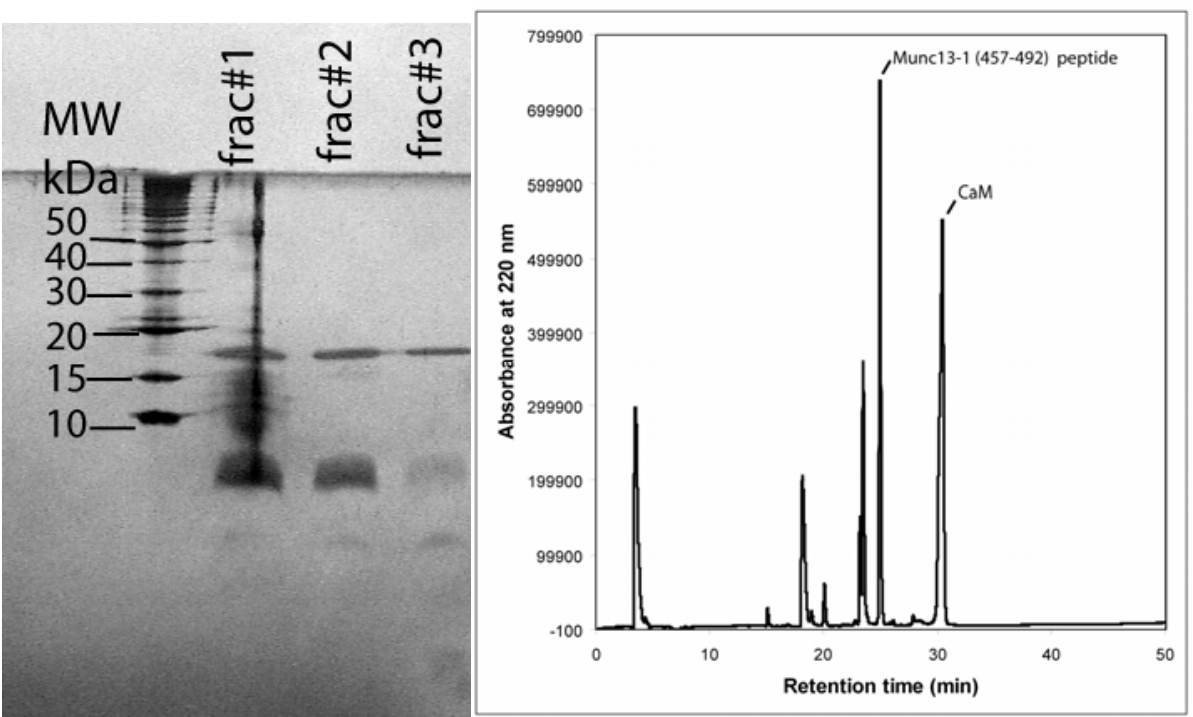

Figure 19. 17.5\% Munc13-1 (457-492) peptide purification. Polyacrylamide SDS-PAGE of the fractions from the GST-Sepharose column after cleavage with thrombin (left). On the right the chromatogram of the RP-HPLC separation of the Munc13-1(457-492) peptide from CaM is shown. 
The N-terminal Maltose-binding protein (MBP) fusion protein of Munc13-1(457-492) could be expressed in high amounts after its overnight induction with $0.5 \mathrm{mM} \mathrm{IPTG}$ at $22^{\circ} \mathrm{C}$ as shown in the SDS-PAGE in Figure 20. The fusion protein was soluble and could be purified by means of its affinity tag with amylose-Sepharose resin. The fractions after the elution with maltose were verified with SDS-PAGE (Figure 20). The TEV cleavage was successful, however the amounts of Munc13-1(457-492) peptide were not large enough and therefore the co-expression approach with CaM was chosen for the overexpression of the uniformly isotopically enriched material for the NMR experiments.

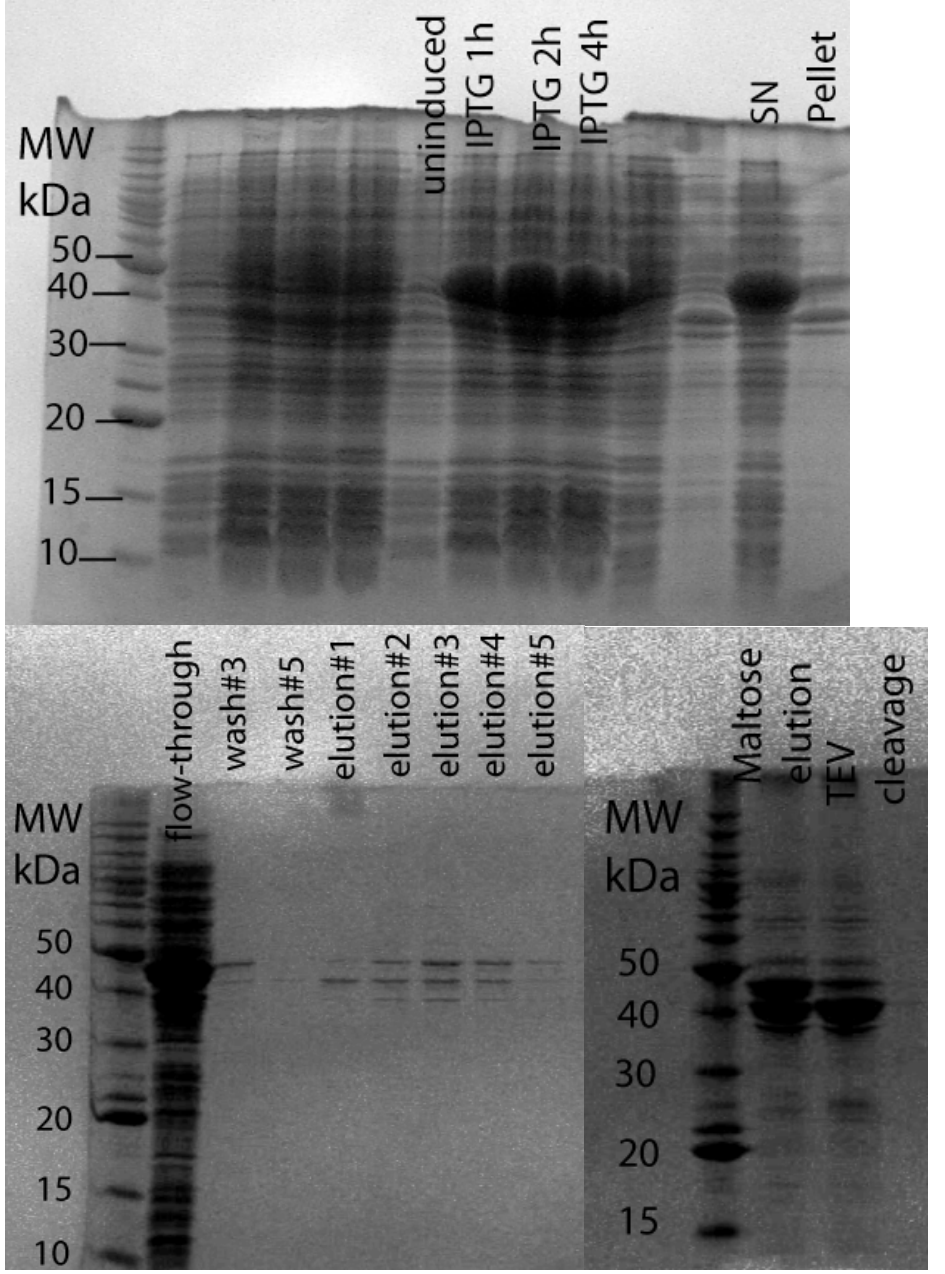

Figure 20.12\% (v/v) polyacrylamide SDS-PAGE for the overexpression of the MBPMunc13-1(457-492) fusion protein (left), the elution from the amylose-Sepharose column with maltose (right) and its cleavage with TEV protease (down-left).

\section{f. Overexpression and purification of the Munc65 protein}

The N-terminal hexahistidine fusion protein of Munc65 could not be expressed in the desired amounts; in general it is difficult to over express low molecular weight proteins in bacteria. Since these small polypeptides are more prone to proteolytic degradation. The results of the purification are shown in the SDS-PAGE in Figure 21.

The N-terminal GST fusion protein of Munc65 could be expressed in the desired amounts after the overnight induction with $0.5 \mathrm{mM}$ IPTG at $22^{\circ} \mathrm{C}$ as can be seen in the SDSPAGE shown in Figure 22. The fusion protein was highly soluble as can be seen from the soluble part of the lysate fraction in the gel. The purification was achieved by affinity chromatography with the GST-Sepharose beads; the fractions from the elution with $50 \mathrm{mM}$ reduced glutathione were analyzed by SDS-PAGE (see Figure 22). The cleavage with TEV protease was unsuccessful. For this reason, a similar approach as for the Munc36 protein was 
followed. The fusion protein was successfully cleaved with thrombin. The fractions after the cleavage are shown in Figure 22. A preparative RP-HPLC run in a C18 column (Vydac) was performed to separate the Munc65 protein from the GST tag. The chromatogram is shown in Figure 22. The Munc65 protein eluted at a retention time of 19.5 minutes and the GST tag eluted at a retention time of 29 minutes in the acetonitrile-water gradient. The identity of the proteins was confirmed with ESI-Mass spectrometry.

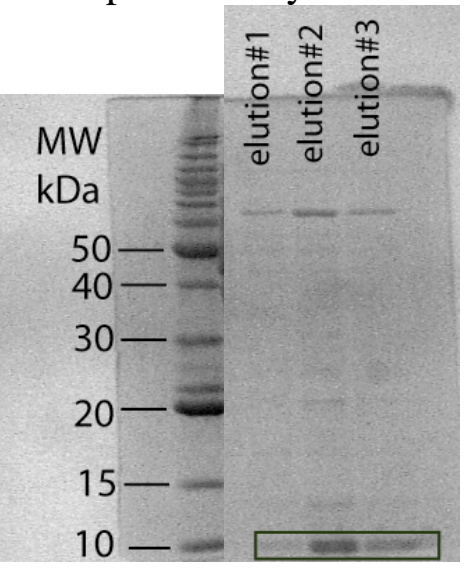

Figure 21. $12 \%(\mathrm{v} / \mathrm{v})$ polyacrylamide SDS-PAGE for the NI-NTA purification of the Munc65aa-(his) 6 protein.

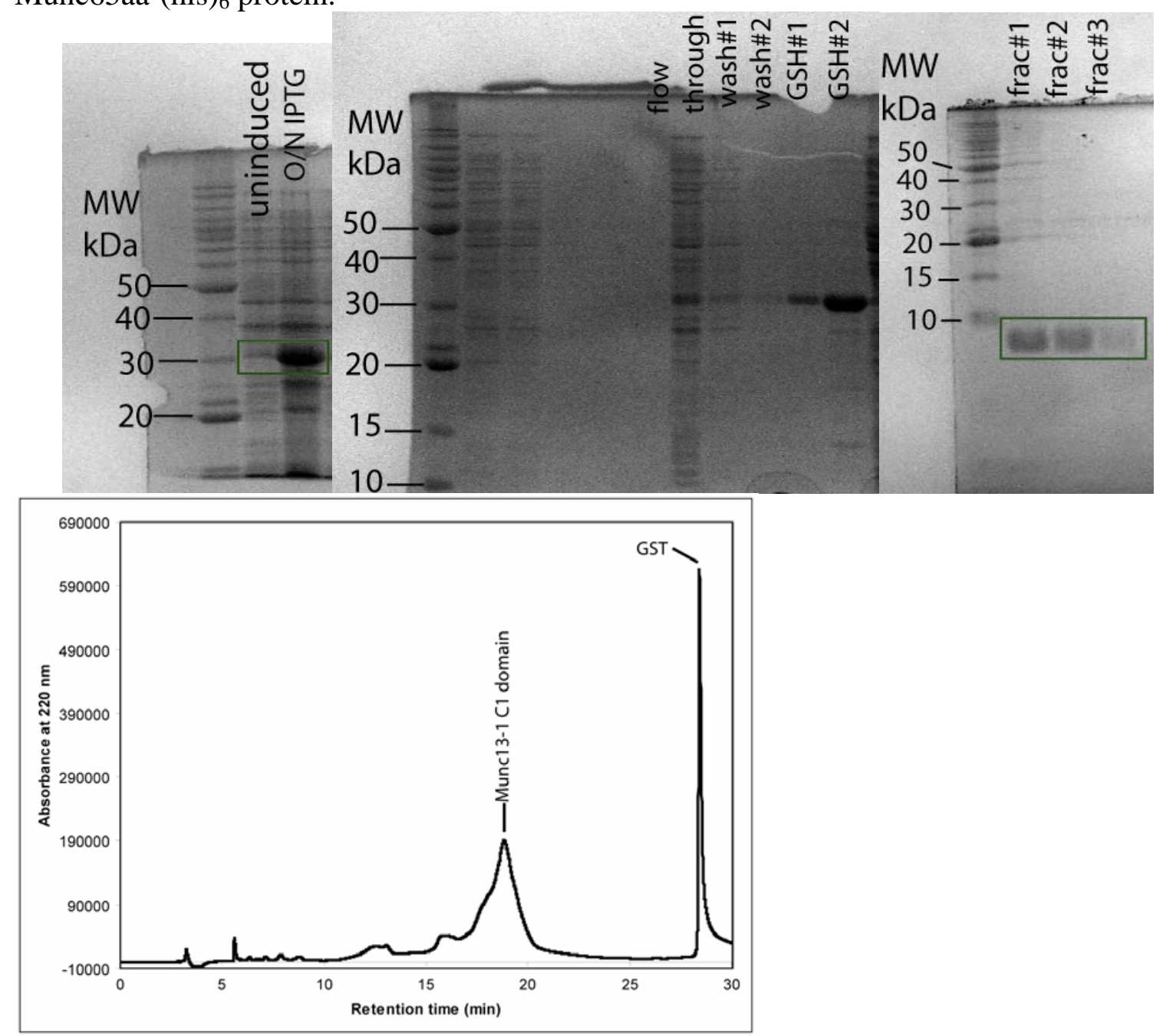

Figure 22. $12 \%(\mathrm{v} / \mathrm{v})$ Polyacrylamide SDS-PAGE for the overexpression of the GSTMunc65aa fusion protein (left); its purification with the GST-Sepharose column (middle) and after cleavage with thrombin (right). At the bottom, the chromatogram of the RP-HPLC separation of the Munc65 protein from the GST tag is shown. 


\section{g. Overexpression and purification of the Munc185 protein}

The N-terminal hexahistidine fusion protein of Munc185 could be expressed in the desired amounts. However the protein was found in the pellet fraction after the lysis, see Figure 23. For this reason a co-expression approach was chosen to increase the stability and solubility of the Munc185 protein in the presence of its interaction partner CaM (Figure 24).

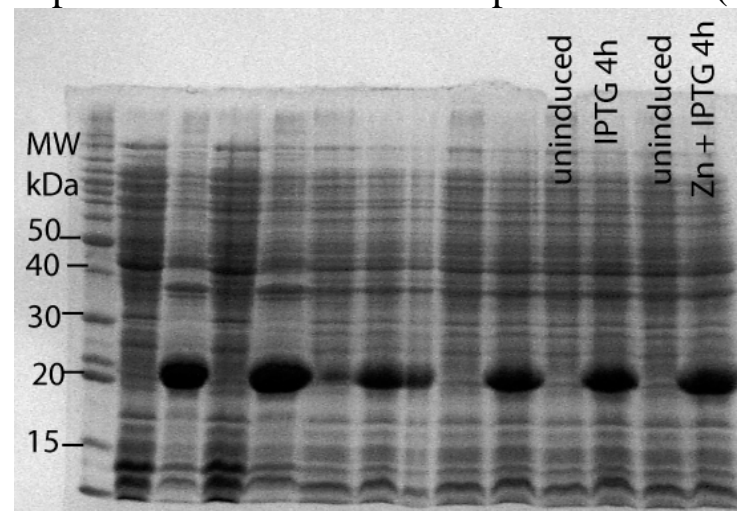

Figure 23. $12 \%(\mathrm{v} / \mathrm{v})$ polyacrylamide SDS-PAGE for the over expression of the pET16bMunc185aa construct.

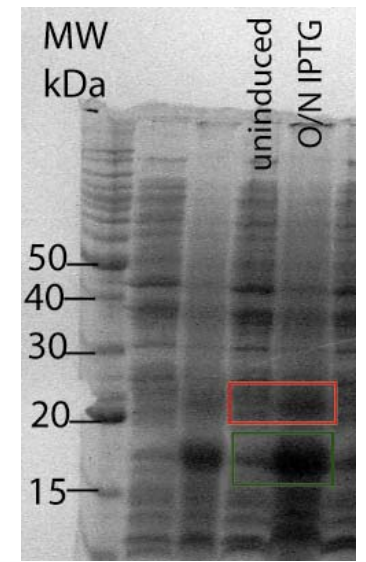

Figure 24. $12 \%(\mathrm{v} / \mathrm{v})$ polyacrylamide SDS-PAGE for the co-expression of the pET16bMunc185aa and pET28a CaM constructs in E.coli BL21 (DE3) with both ampicillin and kanamycin to achieve the co-expression.

The co-expression was done initially by co-transformation of E.coli BL21(DE3) bacteria with the pET16b-TEV Munc185 construct and the pET28a CaM construct which are resistant to two different antibiotics. Afterwards the cloning into the pETDuet- 1 vector succeeded and the co-expression could be done from a single plasmid DNA with ampicillin resistance (Figure 25).

The CaM/Munc185aa complex was highly soluble and was found mainly in the supernatant fraction after the lysis. The complex was co-purified by means of the hexahistidine affinity tag in the Munc185 protein with a Ni-NTA agarose column. The elution was done upon increasing the amount of imidazole to $300 \mathrm{mM}$. The elution fractions are shown in Figure 25. The CaM/Munc185 interaction appeared to be of high affinity since it remained as a bound complex after the Ni-NTA purification step. The (his) 6 tag was removed by cleavage with TEV protease (see Figure 26).

A further purification step with size-exclusion chromatography was done to obtain a homogenous sample. The chromatogram and SDS-PAGE analysis of the eluted fractions is shown in Figure 26. The CaM/Munc185 complex was in equilibrium between a monomeric (1:1) and a dimeric (2:2) species. To obtain an idea of the hydrodynamic radius of these two different species of the complex, a dynamic light scattering (DLS) analysis was done (see 
Figure 26). $89 \%$ of the the monomeric (1:1) species had a hydrodynamic radius of $5.7 \mathrm{~nm}$, which agreed well with the expected molecular weight of $38 \mathrm{kDa}$; whereas $11 \%$ consisted of a large oligomeric species with a hydrodynamic radius of $\sim 100 \mathrm{~nm}$. The dimeric (2:2) species had a hydrodynamic radius of $17.2 \mathrm{~nm}$; this value did not agree well with the expected molecular weight of $76 \mathrm{kDa}$, suggesting that this complex deviates from a globular shape.

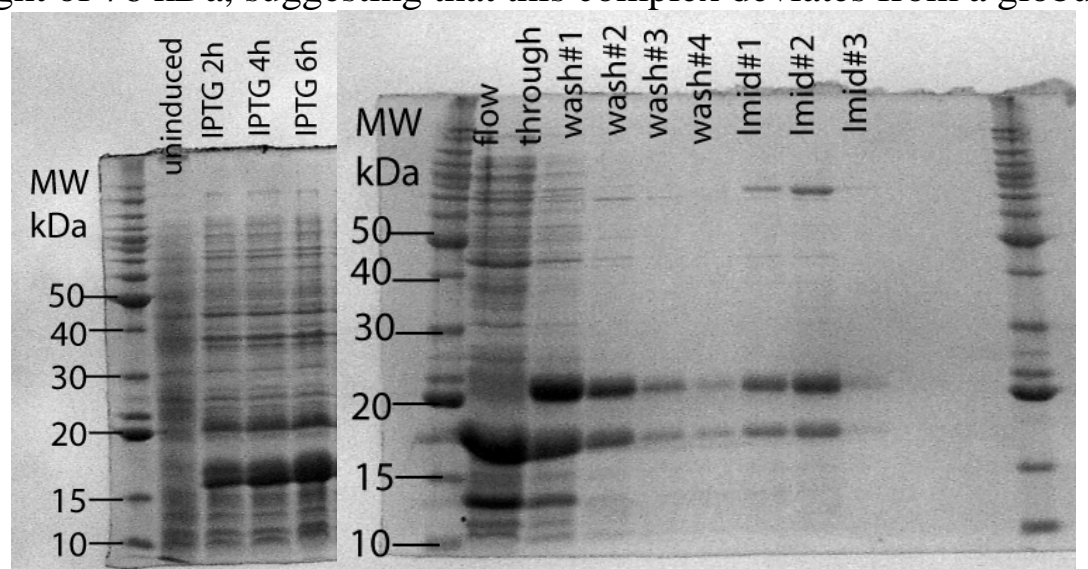

Figure 25. Overexpression of the CaM/Munc185aa complex from the pETDuet-1(CaMMunc185aa) construct (left) and purification by metal affinity chromatography with Ni-NTA agarose (right).
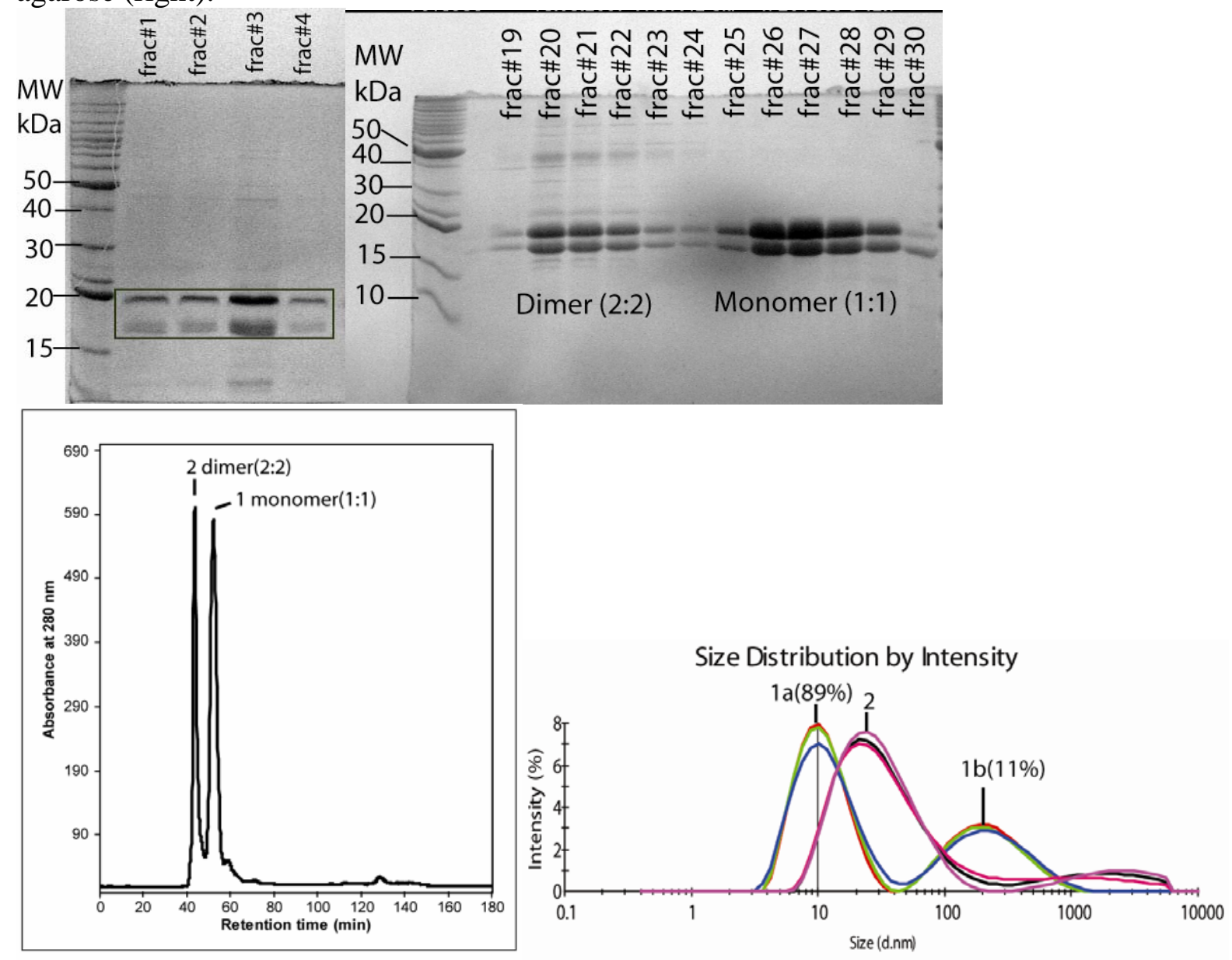

Figure 26. Purification of CaM/Munc185aa. 12\%(v/v) Polyacrylamide SDS-PAGE of the collected fractions after the TEV-cleavage of the CaM/Munc185aa complex (upper-left). Chormatogram of the size-exclusion run for the CaM/Munc185aa complex (lower-left) and $12 \%(\mathrm{v} / \mathrm{v})$ polyacrylamide SDS-PAGE of the elution fractions containing the monomeric and dimeric species of the CaM/Munc185aa complex (upper-right). On the lower-right panel, the DLS profiles for the two different CaM/Munc185aa complex species is shown. 
The N-terminal GST fusion protein of Munc185 could be expressed in high amounts. However the protein was found in the pellet fraction after the lysis. The co-expression approach with CaM was also tried in this case to increase its solubility. Indeed, the CaM/GST-Munc185 complex was highly soluble and could be co-purified with GSTSepharose beads (Figure 27).

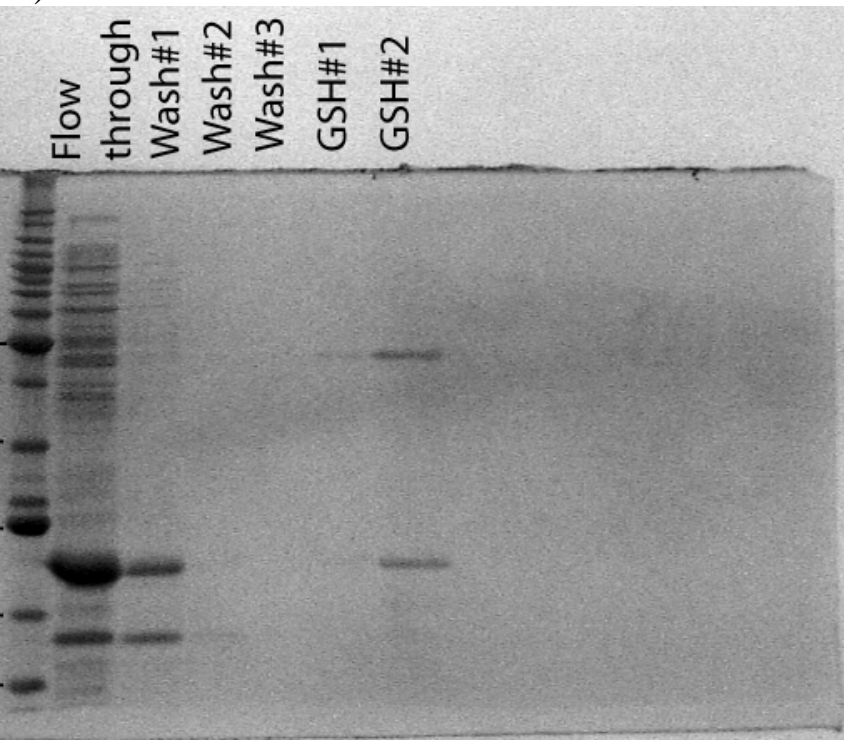

Figure 27. $12 \%(\mathrm{v} / \mathrm{v})$ polyacrylamide SDS-PAGE for the purification of the CaM/GSTMunc185aa complex.

\section{h. Overexpression and purification of calmodulin}

CaM was over expressed in high amounts and was found mainly in the supernatant fraction after the lysis (Figure 28). After a bulk purification step with Trichloroacetic acid (TCA) precipitation; CaM was purified by hydrophobic interaction chromatography on a phenyl-Sepharose column; the chromatogram and the SDS-PAGE analysis of the fractions containing $\mathrm{CaM}$ are shown in Figure 28. The elution was achieved with a buffer containing EDTA; CaM could be purified in high amounts and with high purity.

\section{i. Biochemical separation of Munc185 from calmodulin}

The Munc185 appeared to be unstable in the absence of its interaction partner CaM. However at low concentrations $(<10 \mu \mathrm{M})$ it remains soluble. It is possible to disrupt the strong interaction of the CaM/Munc185 complex with large amounts of EDTA (50mM) and a $\mathrm{pH}$ of 8.8. Afterwards, the proteins were separated by cation exchange chromatography at a $\mathrm{pH}$ of 5.5. The chromatogram is shown in Figure 29. The eluted fractions were analyzed by SDS-PAGE (Figure 29). The free protein could be added to uniformly ${ }^{15} \mathrm{~N}$-labeled CaM for NMR studies. 


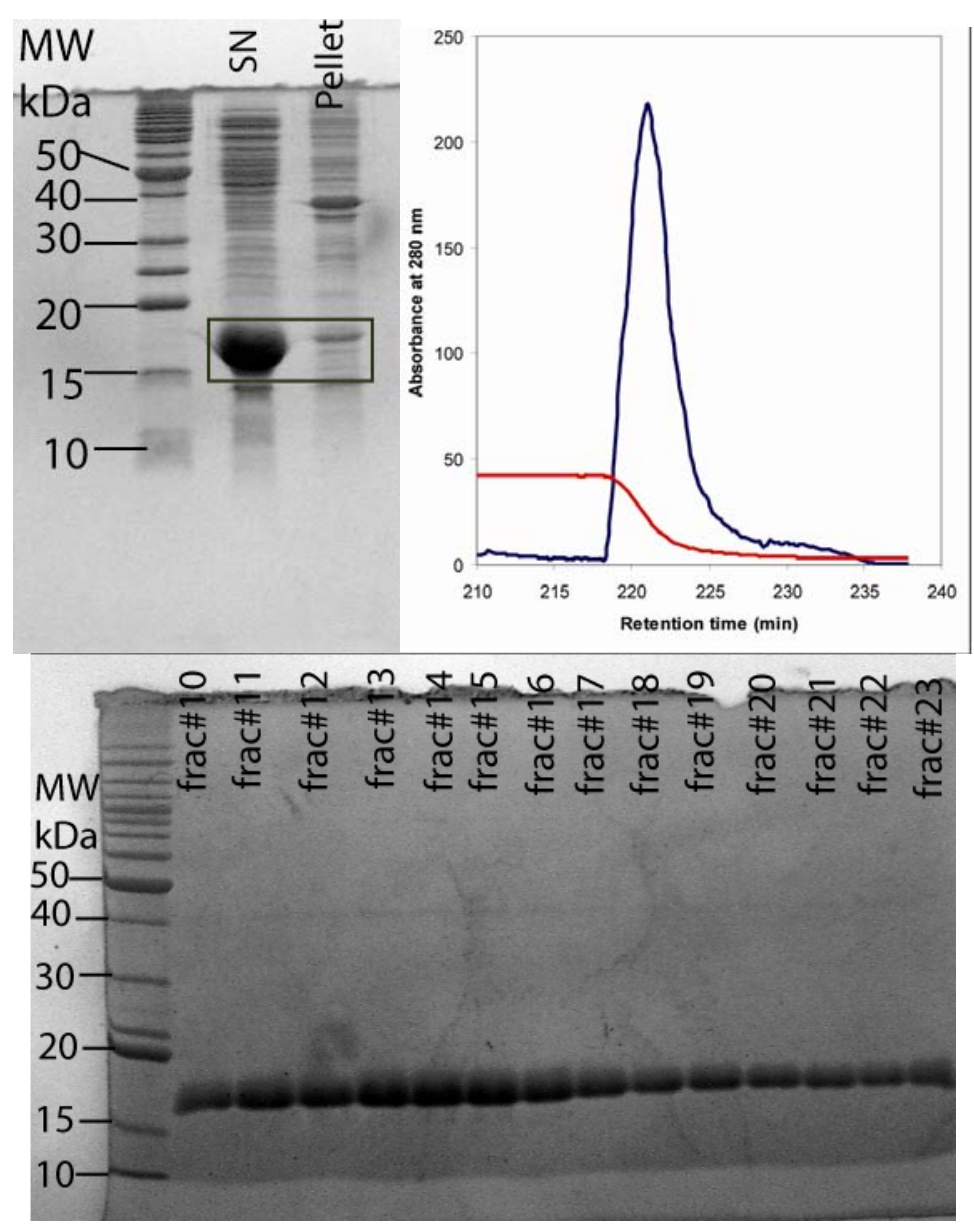

Figure 28. CaM purification. $12 \%$ (v/v) Polyacrylamide SDS-PAGE showing the supernatant and pellet fractions of the CaM cell-lysate (left). The chromatogram of the Phenyl-Sepharose column is shown on middle. On the right a $12 \%(\mathrm{v} / \mathrm{v})$ polyacrylamide SDS-PAGE shows the CaM containing fractions eluted from the Phenyl-Sepharose column.

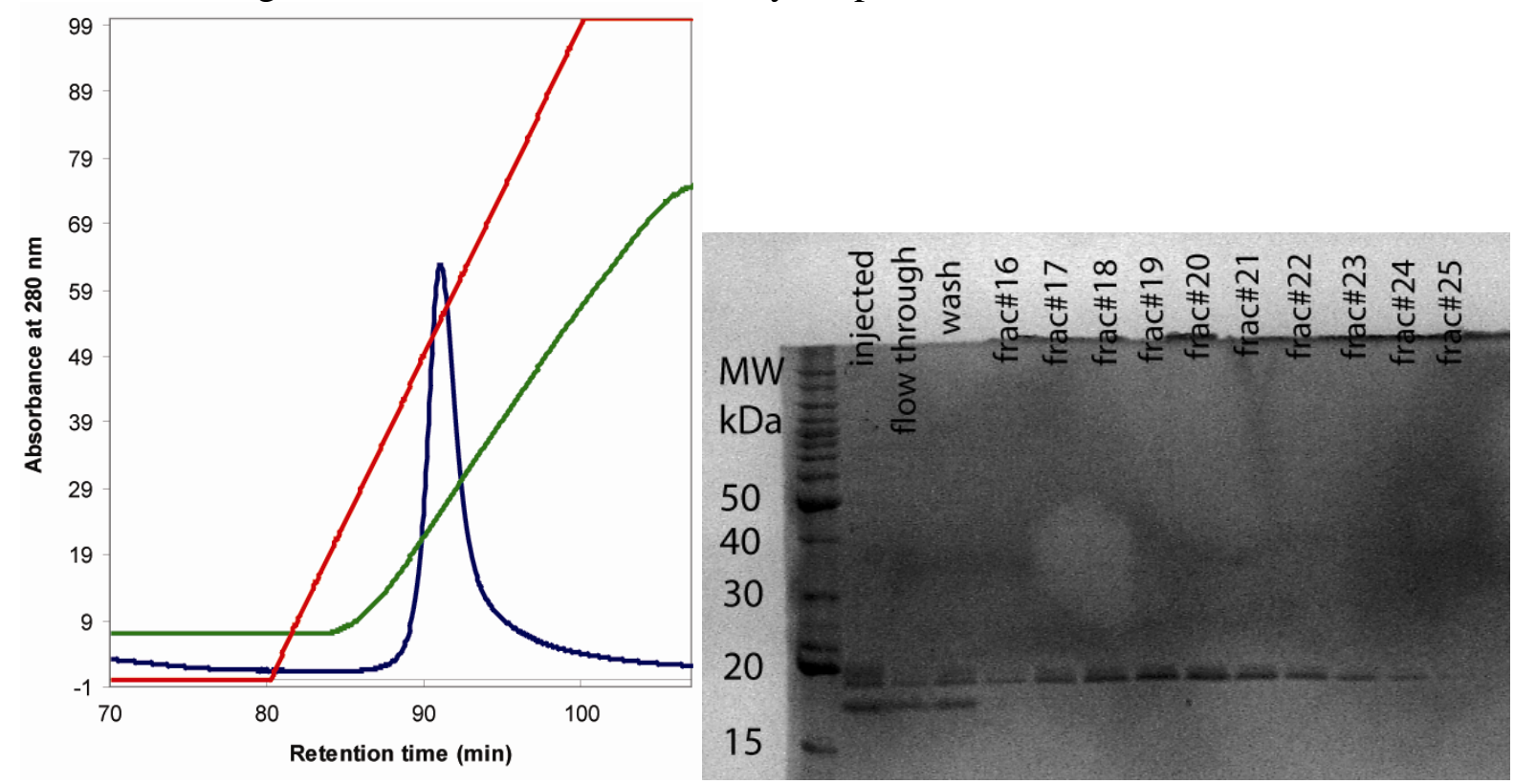

Figure 29. Biochemical separation of Munc185aa. The chromatogram of the SP-Sepharose column is shown on the left side, the blue trace represents the absorbance at $280 \mathrm{~nm}$, the red trace represents the linear $\mathrm{NaCl}$ gradient and the green trace represents the conductivity. On the right, a $12 \%(\mathrm{v} / \mathrm{v})$ polyacrylamide SDS-PAGE shows the content of the collected fractions after elution with high $\mathrm{NaCl}$ concentrations. 


\section{Biophysical characterization of CaM and Munc13-1 proteins}

a. NMR studies on the ${ }^{15} \mathrm{~N},{ }^{13} \mathrm{C}$ CaM/Munc13-1(459-479) peptide complex

The HSQC-based titration of the Munc13-1(459-479) synthetic peptide corresponding to the CaM binding site identified by Junge et al. 2004 to ${ }^{15} \mathrm{~N}$ CaM showed that the peptide binds with sub-micromolar affinity and had a slow-exchange on the NMR time scale (Figure 30).

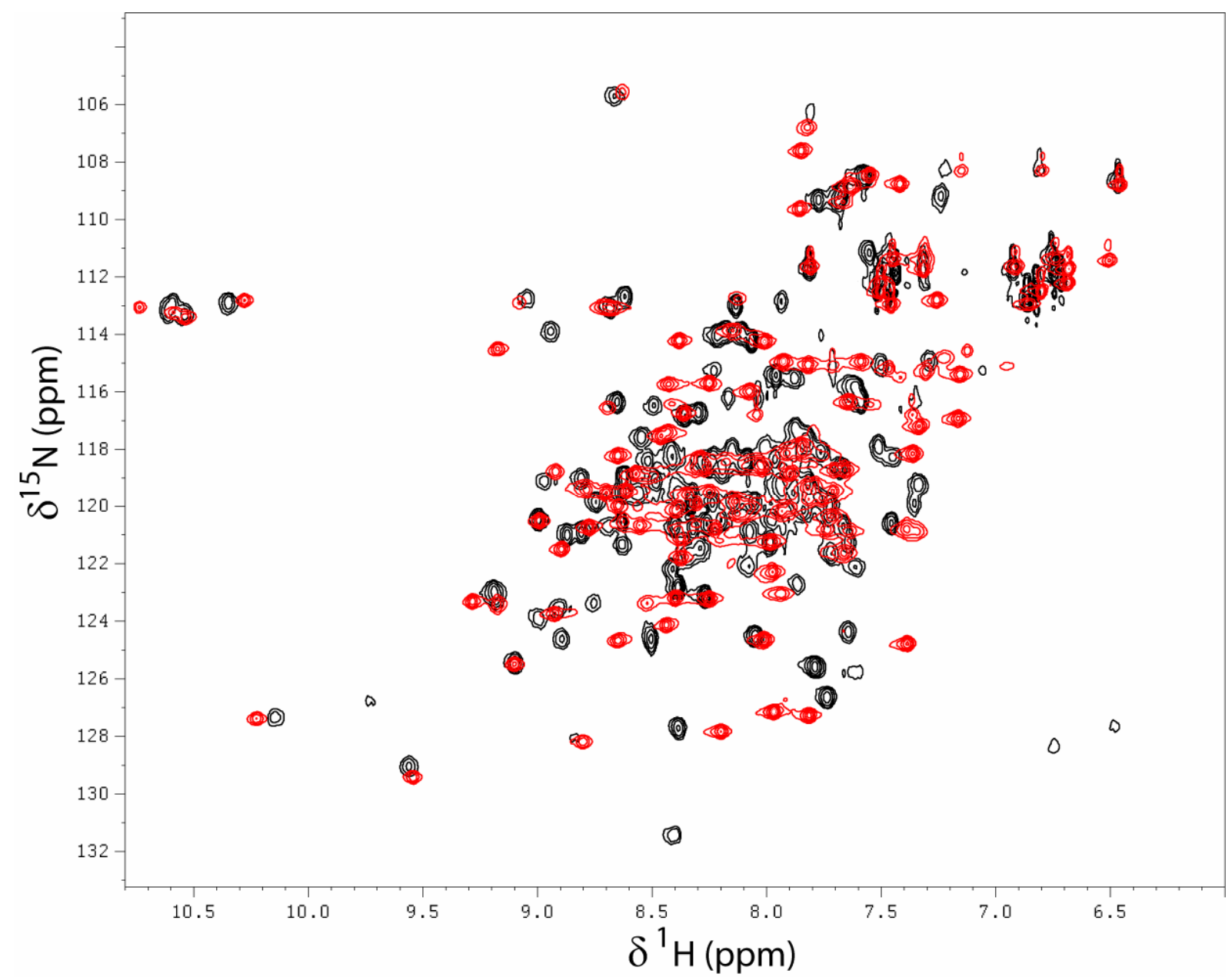

Figure 30. ${ }^{1} \mathrm{H}^{15} \mathrm{~N}$ HSQC spectrum of the uniformly labelled ${ }^{15} \mathrm{NCaM} / \mathrm{Munc} 13-1(459-479)$ peptide complex in 20mM Bis-Tris, $150 \mathrm{mM} \mathrm{KCl,} 10 \mathrm{mM} \mathrm{CaCl}_{2}$, pH 6.8 (in red) and of free CaM measured at $30^{\circ} \mathrm{C}$ (in black).

The amide resonances are a very sensitive probe for studying macromolecular interactions; the chemical shift is sensitive to both direct interaction with a binding partner and to induced conformational change in the macromolecule. To quantify the change in the HSQC spectra a chemical shift perturbation plot (see Figure 31) gave an overview of the effects upon binding of the Munc13-1(459-479) peptide to CaM. The chemical shift perturbation is a weighted average of the change in the ${ }^{1} \mathrm{H}$ resonance and the ${ }^{15} \mathrm{~N}$ resonance corrected for the difference in magnetogyric ratio. 


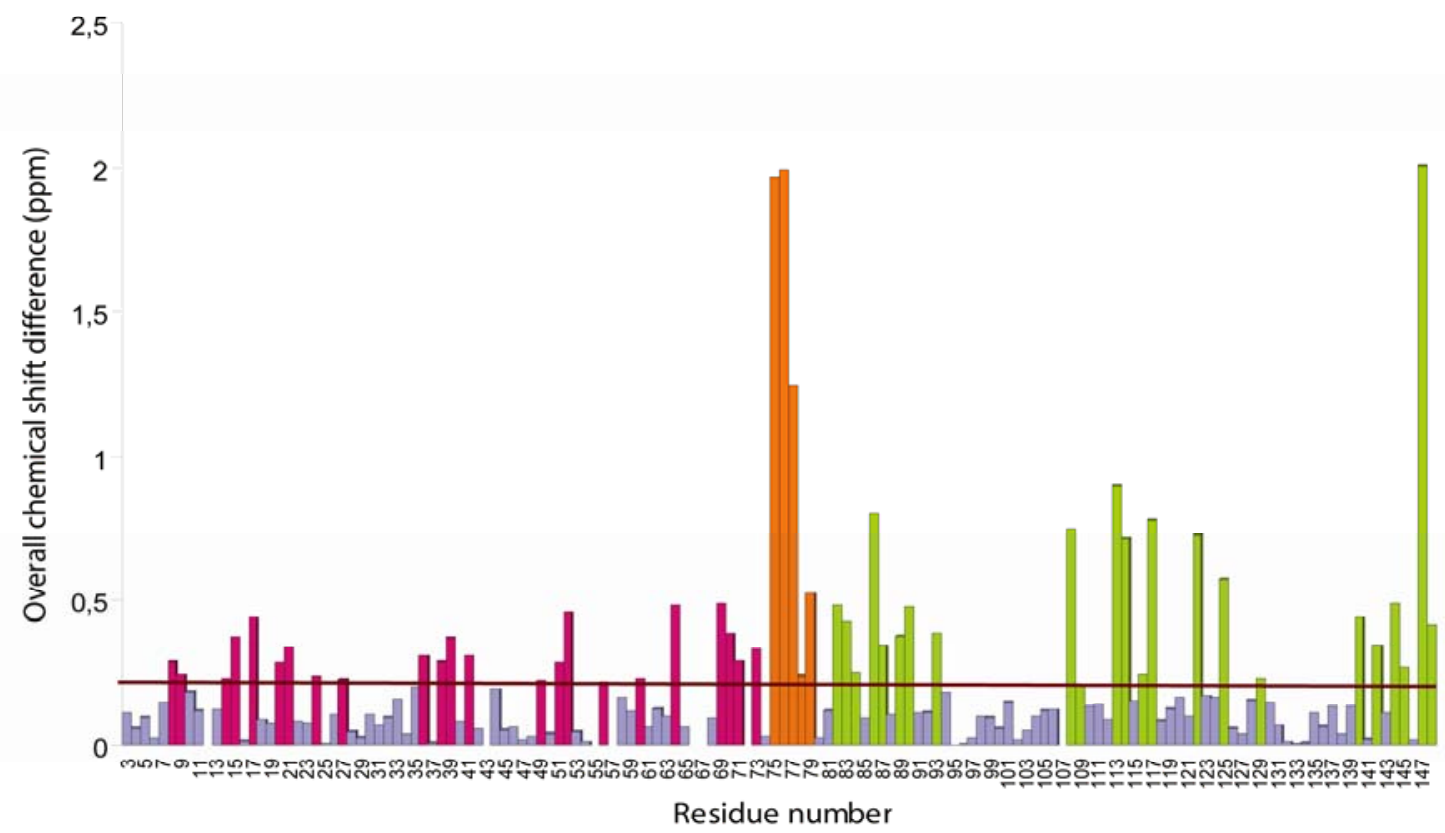

Figure 31. Chemical shift perturbation plot for CaM upon binding to the Munc13-1 (459-479) peptide. The largest perturbations are shown in green for the C-terminal domain of CaM, in orange for the linker region and in magenta for the $\mathrm{N}$-terminal domain of CaM.

The chemical shift perturbation upon binding of the Munc13-1 (459-479) peptide indicated that the interaction was stronger to the $\mathrm{C}$-terminal region of CaM; since the chemical shift changes in the N-terminal domain were rather small. It has been shown, that the binding of many of the CaM targets is antiparallel; for this reason, it was rationalized that if the peptide was extended C-terminally it could be possible to observe additional chemical shift changes in the N-terminal region of CaM. Thus, a 34 amino acids -long peptide was chemically synthesized: Munc13-1 (459-492).

\section{b. NMR studies on the ${ }^{13} \mathrm{C},{ }^{15} \mathrm{~N}$ CaM/Munc13-1(459-492) peptide complex}

Upon extending the peptide sequence towards its C-terminus an additional hydrophobic motif appeared to bind to the N-terminal domain of CaM. This was observable in the overlaid HSQC spectra (Figure 32); the changes were quantified with the aid of a chemical shift perturbation plot (Figure 33).

The hypothesis that the C-terminal extension of the Munc13-1 (459-479) peptide could interact with the $\mathrm{N}$-terminal domain appeared to be correct; since more chemical shift changes and larger in magnitude were observed in the N-terminal region of CaM for this longer peptide.

Junge et al., 2004 also reported that the Munc13-1 (459-479) peptide interacted with $\mathrm{CaM}$ in the absence of calcium. To map the region of apoCaM, which could be affected upon binding with this Munc13-1 peptide, a titration experiment was done in the presence of $5 \mathrm{mM}$ EDTA (see Figure 34).

The CaM/Munc13-1 interaction in the absence of calcium appeared to be in the intermediate- exchange regime on the NMR time scale, since many of the $\mathrm{NH}$ cross peaks broadened beyond detection. However, few peaks showed fast-exchange behaviour. This result indicated that the Munc13-1 peptide binds apoCaM with lower affinity (by several orders of magnitude) than in the presence of calcium. Since the assignment of apoCaM was available; the changes observed in the HSQC spectra upon peptide addition were mapped in 
the apoCaM structure (Figure 37). In addition, the same titration experiment was done for a synthetic peptide of the Munc13-1 homologue ubMunc13-2 (382-402), similar results were obtained; however the binding surface appeared to be smaller, i.e. less NH cross peaks were perturbed.

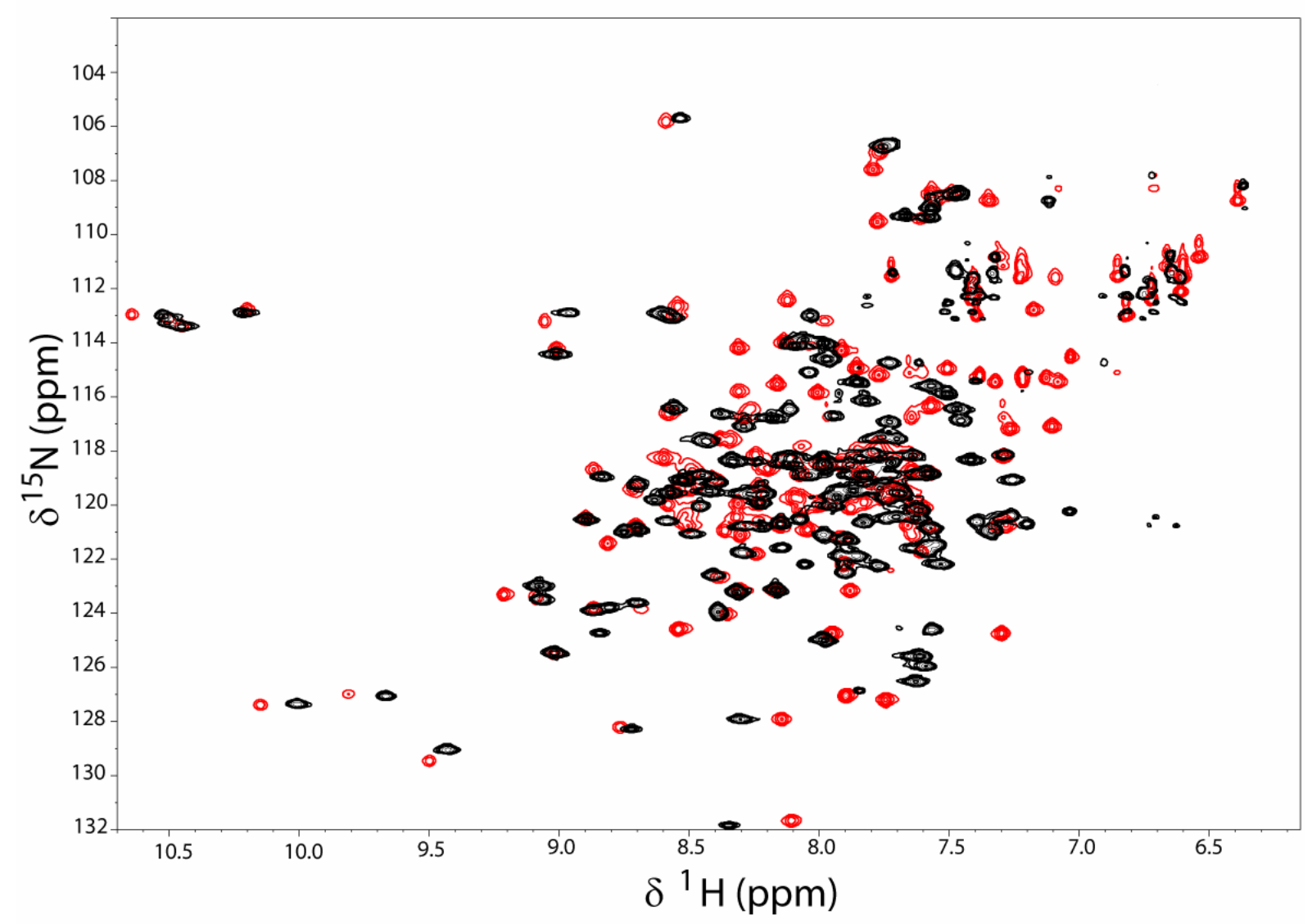

Figure 32. Overlaid ${ }^{15} \mathrm{~N}-{ }^{1} \mathrm{H}$ HSQC spectra of ${ }^{15} \mathrm{~N}$ CaM (black) and ${ }^{15} \mathrm{NCaM} /$ Munc13-1(459492) peptide complex (red).

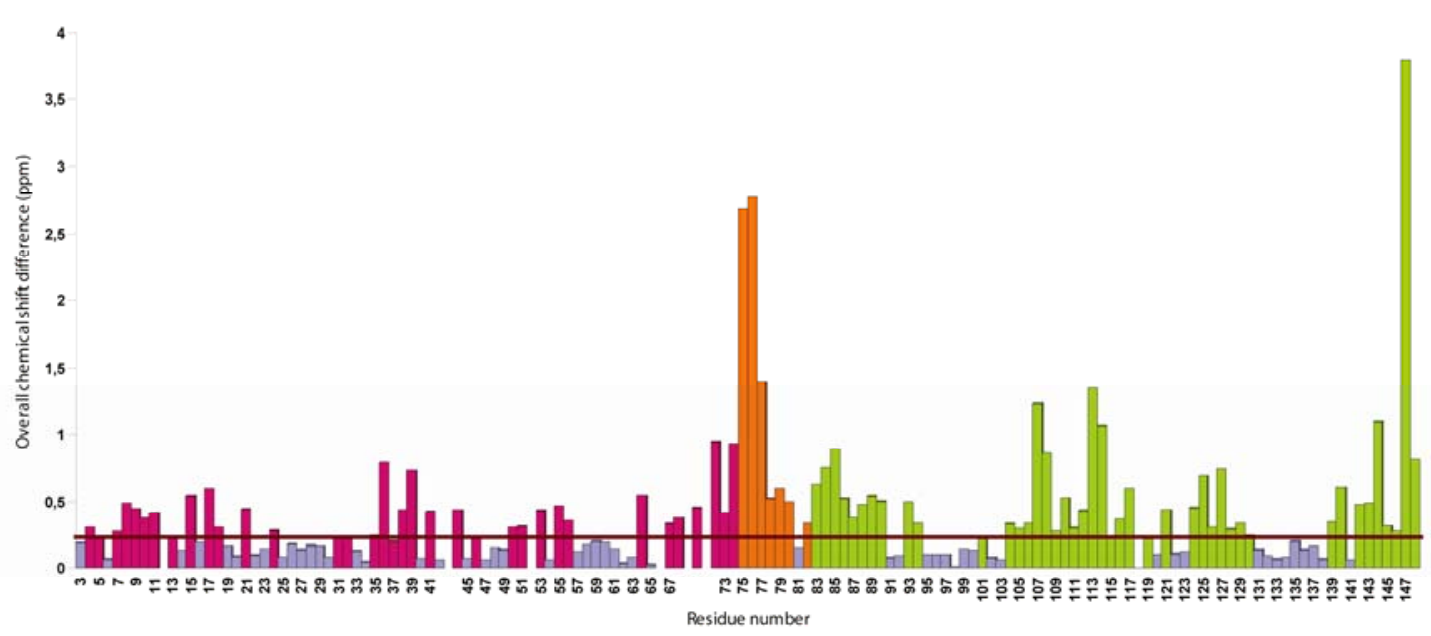

Figure 33. Chemical shift perturbation plot for the amide region in CaM upon binding to the Munc13-1 (459-492) peptide. The residues affected in the C-terminal domain, linker region and $\mathrm{N}$-terminal domain are shown in green, orange and magenta, respectively. 


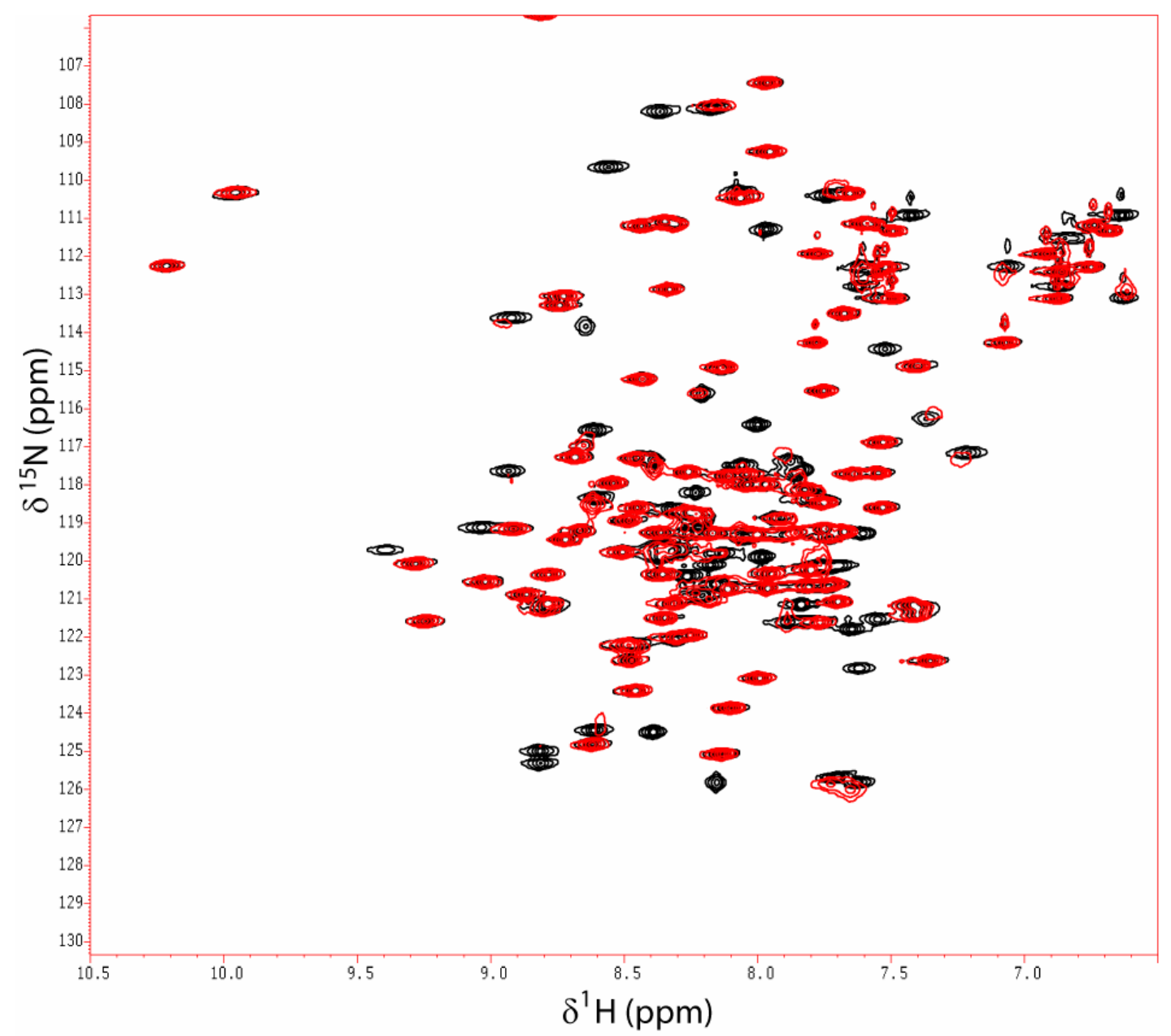

Figure 34. Overlaid ${ }^{15} \mathrm{~N}-{ }^{1} \mathrm{H}$ HSQC spectra for apoCaM in black and apoCaM/Munc13-1 (459479) peptide complex in a 1:0.5 stoichiometric ratio in red.

To get an idea of the binding affinity of this interaction; one of the few peaks that showed a fast-exchange behaviour was analyzed (see Figure 35). The peptide:apoCaM molar ratio was plotted against the chemical shift change (Figure 36). This saturation curve or isoterm was fitted with a hyperbolic function to obtain the dissociation constant $\left(k_{\mathrm{d}}\right)$ of the binding. The dissociation constant extracted from the binding isotherm was $\sim 1 \mathrm{mM}$.

To gain insight into the CaM/Munc13-1 interaction along the activation coordinate of CaM, the CaM/Munc13-1 (459-492) complex was titrated with EDTA to remove the bound calcium. Three distinct states could be defined based on the HSQC spectra: (a) The inactive state apoCaM/Munc13-1; (2) a half-activated state [2Ca $\left.{ }^{2+}\right] \mathrm{CaM} /$ munc13-1; (3) and the fully activated state holoCaM/Munc13-1. These three states could be easily recognized in the HSQC spectra focusing on the low field shifted glycines, which coordinate the calcium ion, belonging to the four different EF-hand motifs of CaM (Figure 38).

Remarkably, the chemical shift of the Glycine 134 which belongs to the EF-hand 4, did not show a chemical shift change upon calcium removal in the presence of the Munc13-1 peptide; it had a ${ }^{1} \mathrm{H}$ and ${ }^{15} \mathrm{~N}$ chemical shift of 10.32 and $112.34 \mathrm{ppm}$, respectively. This argues for a similar chemical environment and possibly similar conformation for this EF-hand in the three different activated states discussed above. The ${ }^{1} \mathrm{H}$ and ${ }^{15} \mathrm{~N}$ chemical shift for G134 in free apoCaM are 8.5 and 109.6 ppm, respectively. 


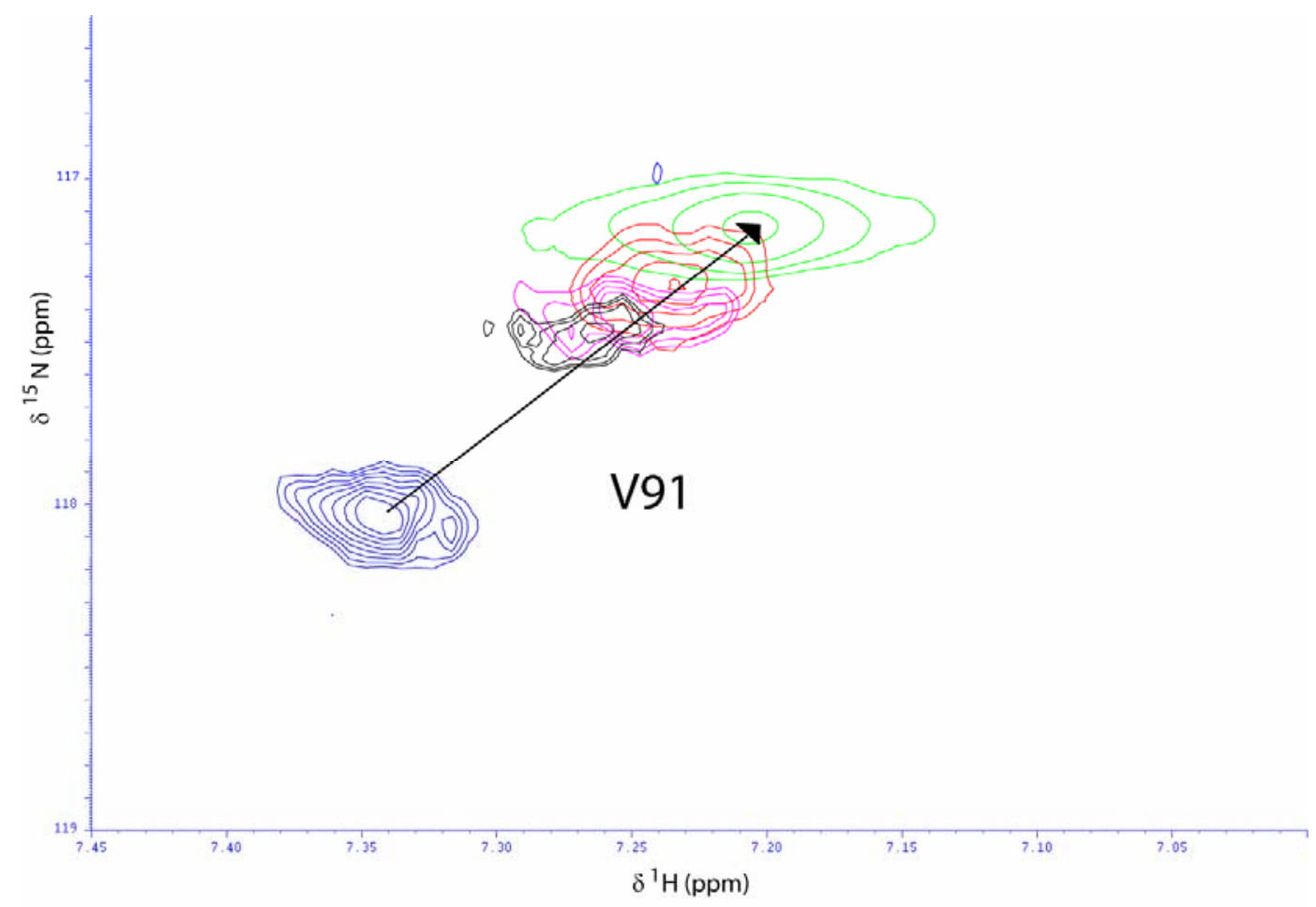

Figure 35. Overlaid ${ }^{15} \mathrm{~N}-{ }^{1} \mathrm{H}$ spectra of apoCaM with increasing amounts of Munc13-1 (459479) peptide. It shows just the progressive migration of the Valine $91 \mathrm{NH}$ cross peak; characteristic of the fast-exchange regime behaviour on the NMR time scale.

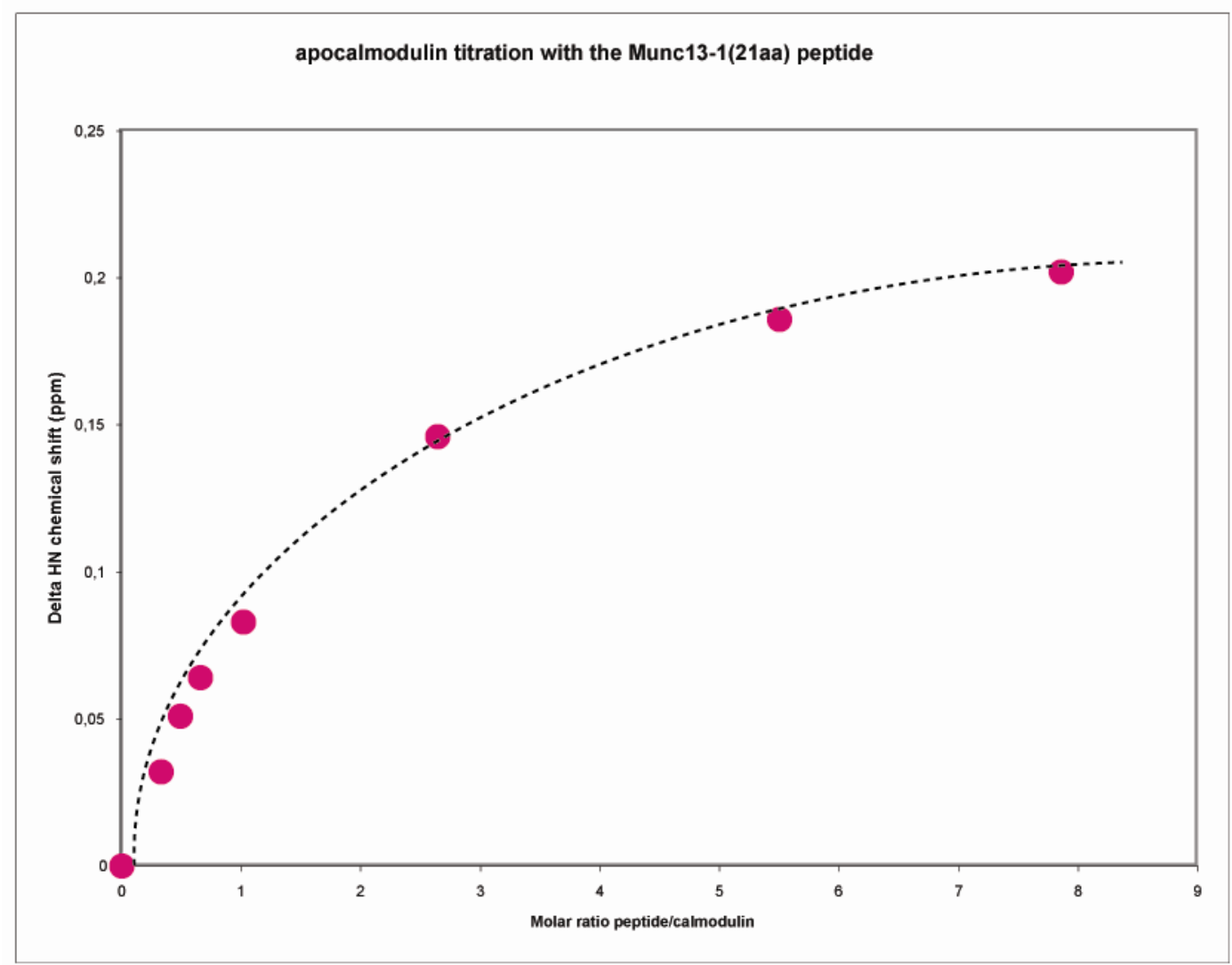

Figure 36. Saturation binding curve for the Valine $91 \mathrm{NH}$ cross peak of ${ }^{15} \mathrm{~N}$ labelled apoCaM upon increasing amounts of Munc13-1 (459-479) peptide. 

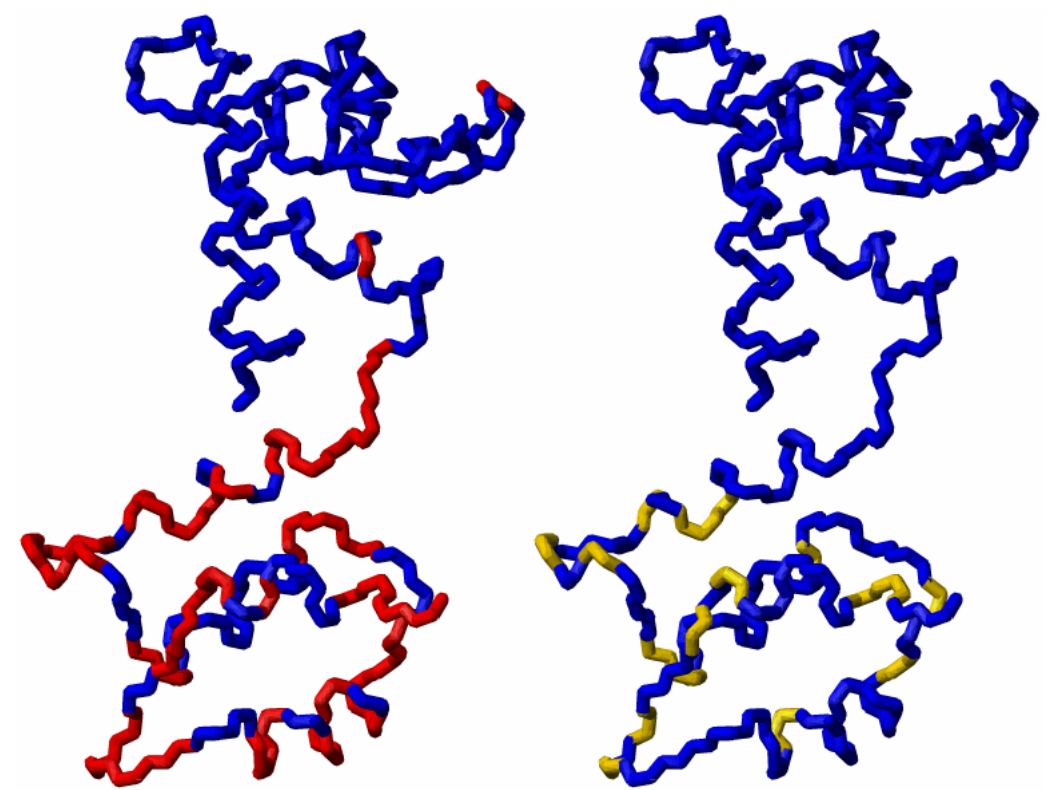

Figure 37. Mapping of the residues in apoCaM affected by the binding of the Munc13-1 (459479) peptide in red, mainly the C-terminal domain (bottom) was affected. On the right side, the residues affected by the binding of ubMunc13-2 (382-402) to apoCaM are shown in yellow; just residues in the C-terminal region were affected.

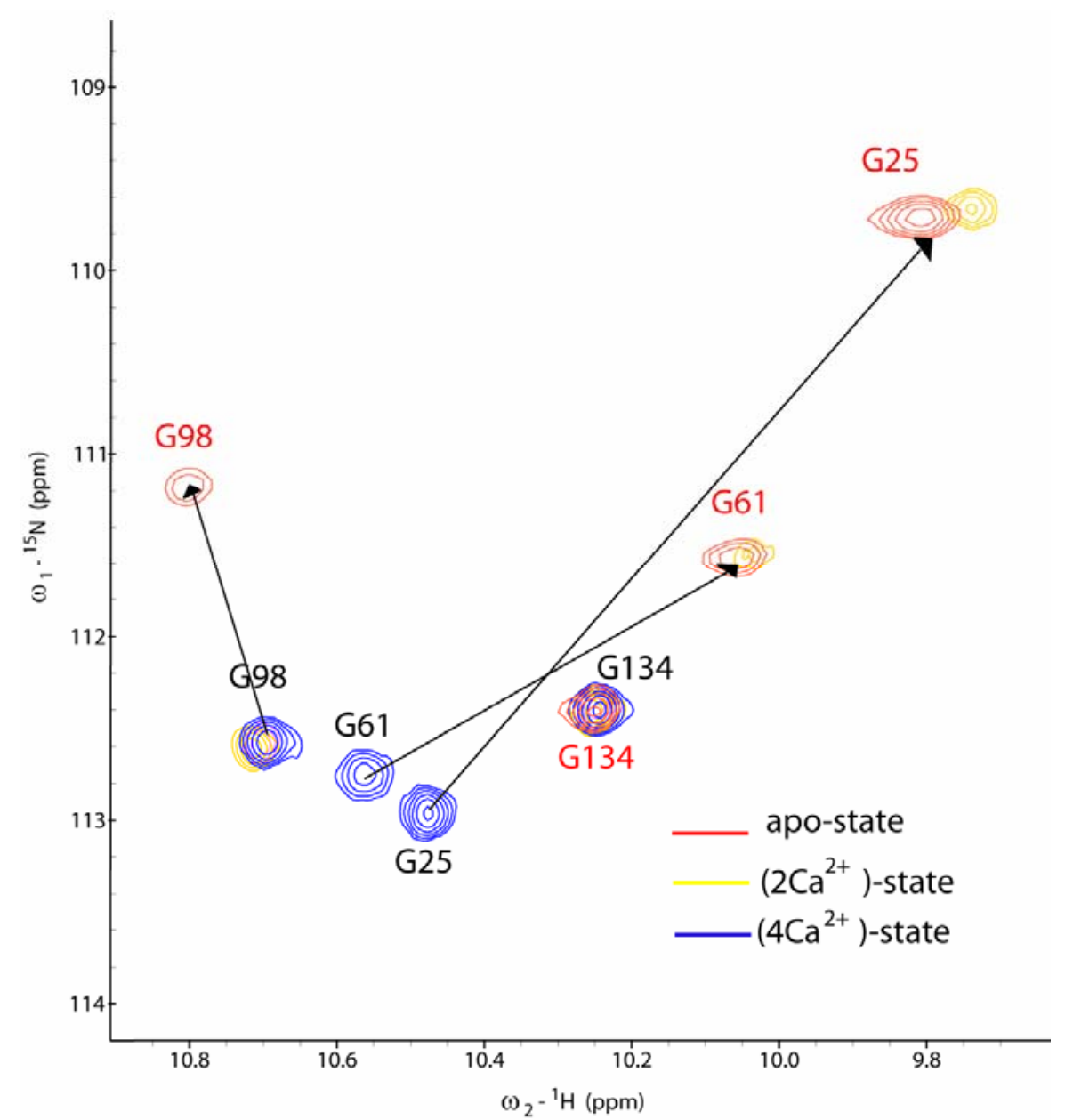

Figure 38. Overlaid ${ }^{1} \mathrm{H}^{15}{ }^{15} \mathrm{~N}$ HSQC of the CaM/Munc13-1(459-492) complex at three different activated states. The red one is in the absence of calcium; the yellow with two calcium ions bound to the C-terminal domain of CaM; and the blue one in the holo- state or $\left[4 \mathrm{Ca}^{2+}\right]$-bound state. 
To unravel the Munc13-1 conformational changes upon CaM binding, a recombinant uniformly $\left({ }^{13} \mathrm{C},{ }^{15} \mathrm{~N}\right)$-labelled peptide was studied by NMR. The first goal was to determine which conformation the free peptide had in solution. Since the peptide aggregated in solution at the concentrations needed for NMR, the sequential resonance assignment was done in the presence of urea. However, a ${ }^{1} \mathrm{H}-{ }^{15} \mathrm{~N}-\mathrm{HSQC}$ measured on a diluted sample also showed a random-coil pattern. The ${ }^{15} \mathrm{~N}-{ }^{1} \mathrm{H}$ HSQC spectrum measured in $8 \mathrm{M}$ urea is shown in Figure 39.

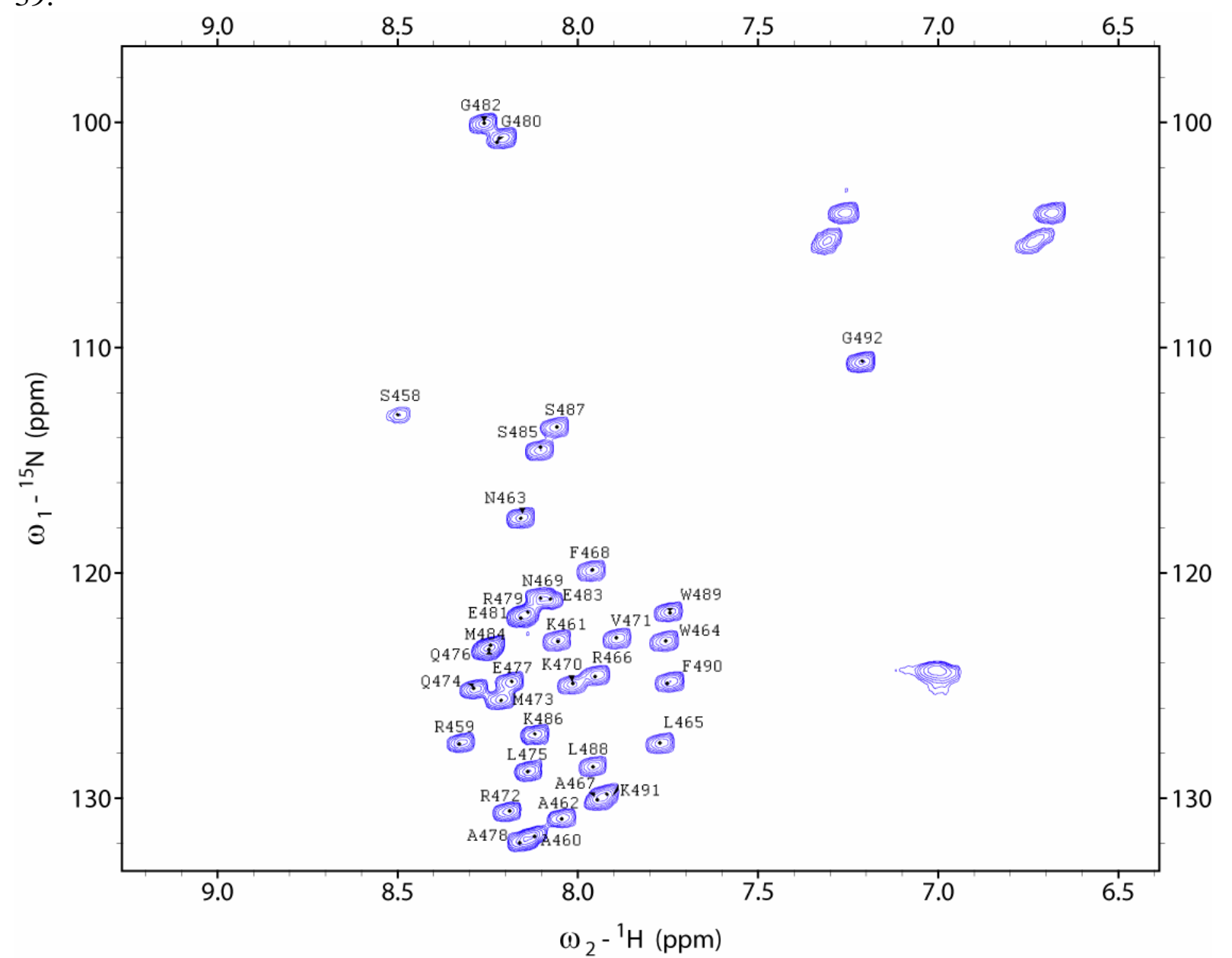

Figure 39. ${ }^{15} \mathrm{~N}-{ }^{1} \mathrm{H}$ HSQC spectrum of the ${ }^{13} \mathrm{C},{ }^{15} \mathrm{~N}$ Munc13-1(457-492) peptide in $8 \mathrm{M}$ urea at $\mathrm{pH}$ 6.8. All of the amide cross peaks were assigned with the aid of triple resonance experiments.

The sequential backbone resonance assignment of the ${ }^{13} \mathrm{C},{ }^{15} \mathrm{~N}$ Munc13-1(457-492) free peptide in $8 \mathrm{M}$ urea was done with the aid of triple resonance experiments like 3DHNCO and 3D-HN(CA)CO (see Table 3). In unfolded polypeptides there is a low dispersion in the proton and carbon chemical shifts; therefore, a full sequential assignment was only possible with the aid of the 3D-HNN experiment (see Figure 40). This experiment is popular for unfolded proteins, because it has two nitrogen evolution periods and the nitrogen chemical shift is the one with largest dispersion in unfolded polypeptides. 


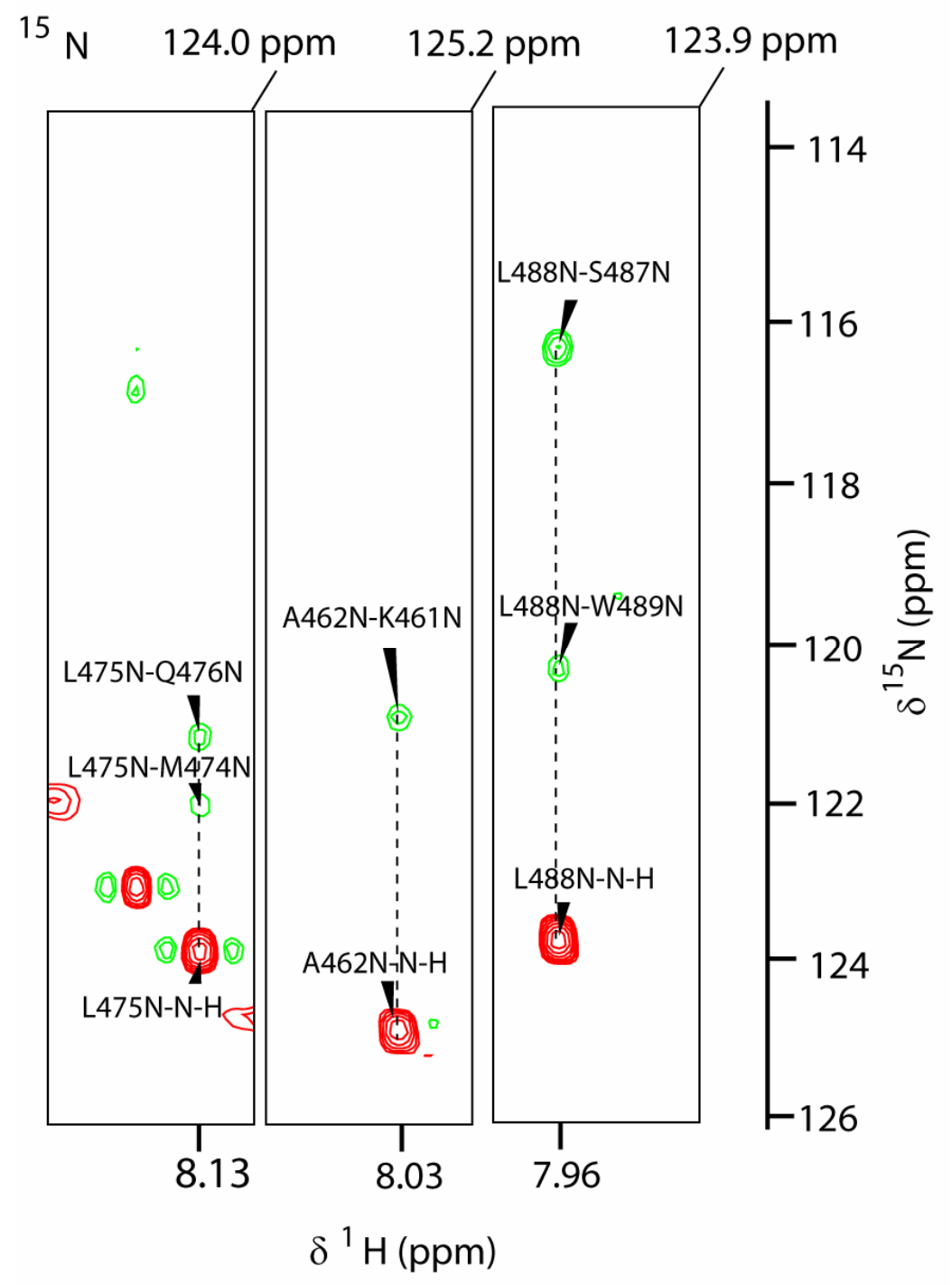

Figure 40. Strip plots of the 3D-HNN spectrum for three diagonal peaks of the ${ }^{13} \mathrm{C},{ }^{15} \mathrm{~N}$ Munc13-1(457-492) peptide in $8 \mathrm{M}$ urea. The diagonal peak had a positive phase (red) and the cross-peaks to $(i+1)$ and $(i-1)$ evolved with negative phase (green).

Afterwards, the resonances of the ${ }^{13} \mathrm{C},{ }^{15} \mathrm{~N}$-Munc13-1 (457-492) peptide were assigned in its bound state in the presence of unlabelled CaM. For this purpose, several triple resonance experiments were done to assign all the chemical shifts of the backbone and side chain resonances (see Table 3).

The ${ }^{15} \mathrm{~N}-{ }^{1} \mathrm{H}$ HSQC of the labelled Munc13-1 (457-492) peptide bound to CaM is shown in Figure 41. The ${ }^{1} \mathrm{H}$ chemical shift dispersion upon CaM binding increased significantly; it is well known that a larger dispersion for the $\mathrm{NH}$ resonances involves protein folding (to $\alpha$-helical conformation in this case).

The changes in the HSQC spectra between free Munc13-1 (457-492) peptide and the CaM-bound peptide were quantified with the aid of a chemical shift perturbation plot (Figure 42). The largest changes were observed in the N-terminal region of the peptide. Significant chemical shift perturbations were also observed in the C-terminal hydrophobic tail of the peptide. 
Table 3. NMR experiments for the sequential backbone and side chain resonance assignment of the ${ }^{13} \mathrm{C},{ }^{15} \mathrm{~N}$ Munc13-1 (457-492) peptide

\begin{tabular}{|c|c|c|}
\hline Sample & Experiment & Resonance assignment \\
\hline \multirow{3}{*}{$\begin{array}{l}\text { Free }{ }^{13} \mathrm{C},{ }^{15} \mathrm{~N} \text { Munc13- } \\
1(457-492)\end{array}$} & 3D-HNCO & $\mathrm{C}^{\prime}(i-1)$ to $\mathrm{NH}$ \\
\hline & 3D-HN(CA)CO & $\mathrm{C}^{\prime}(i-1)$ and $\mathrm{C}^{\prime}(i)$ to $\mathrm{NH}$ \\
\hline & 3D-HNN & $\mathrm{N}(i-1)$ and $\mathrm{N}(i+1)$ to $\mathrm{NH}$ \\
\hline \multirow{8}{*}{$\begin{array}{l}\text { CaM bound }{ }^{13} \mathrm{C},{ }^{15} \mathrm{~N} \\
\text { Munc13-1 }(457-492)\end{array}$} & 3D-HNCO & $\mathrm{C}^{\prime}(i-1)$ to $\mathrm{NH}$ \\
\hline & 3D-HN(CA)CO & $\mathrm{C}^{\prime}(i-1)$ and $\mathrm{C}^{\prime}(i)$ to $\mathrm{NH}$ \\
\hline & 3D-CBCA(CO)NH & $\mathrm{CB}, \mathrm{CA}(i-1)$ to $\mathrm{NH}$ \\
\hline & 3D-HNCACB & $\mathrm{CB}, \mathrm{CA}(i-1)$ and CB,CA $(i)$ to $\mathrm{NH}$ \\
\hline & $\begin{array}{l}\text { 3D-H(CC)(CO)NH- } \\
\text { TOCSY }\end{array}$ & Aliph.H $(i-1)$ to $\mathrm{NH}$ \\
\hline & $\begin{array}{l}\text { 3D- }(\mathrm{H}) \mathrm{CC}(\mathrm{CO}) \mathrm{NH}- \\
\text { TOCSY }\end{array}$ & Aliph. $\mathrm{C}(i-1)$ to $\mathrm{NH}$ \\
\hline & 3D-HCCH-TOCSY & Aliph.C and Aliph.H intra side-chain \\
\hline & $\begin{array}{l}\text { 2D-HBCB(Caro)H } \gamma \\
\text { and 2D-HBCB(Caro)H } \delta\end{array}$ & Aromatic side-chains to CB-HB \\
\hline
\end{tabular}

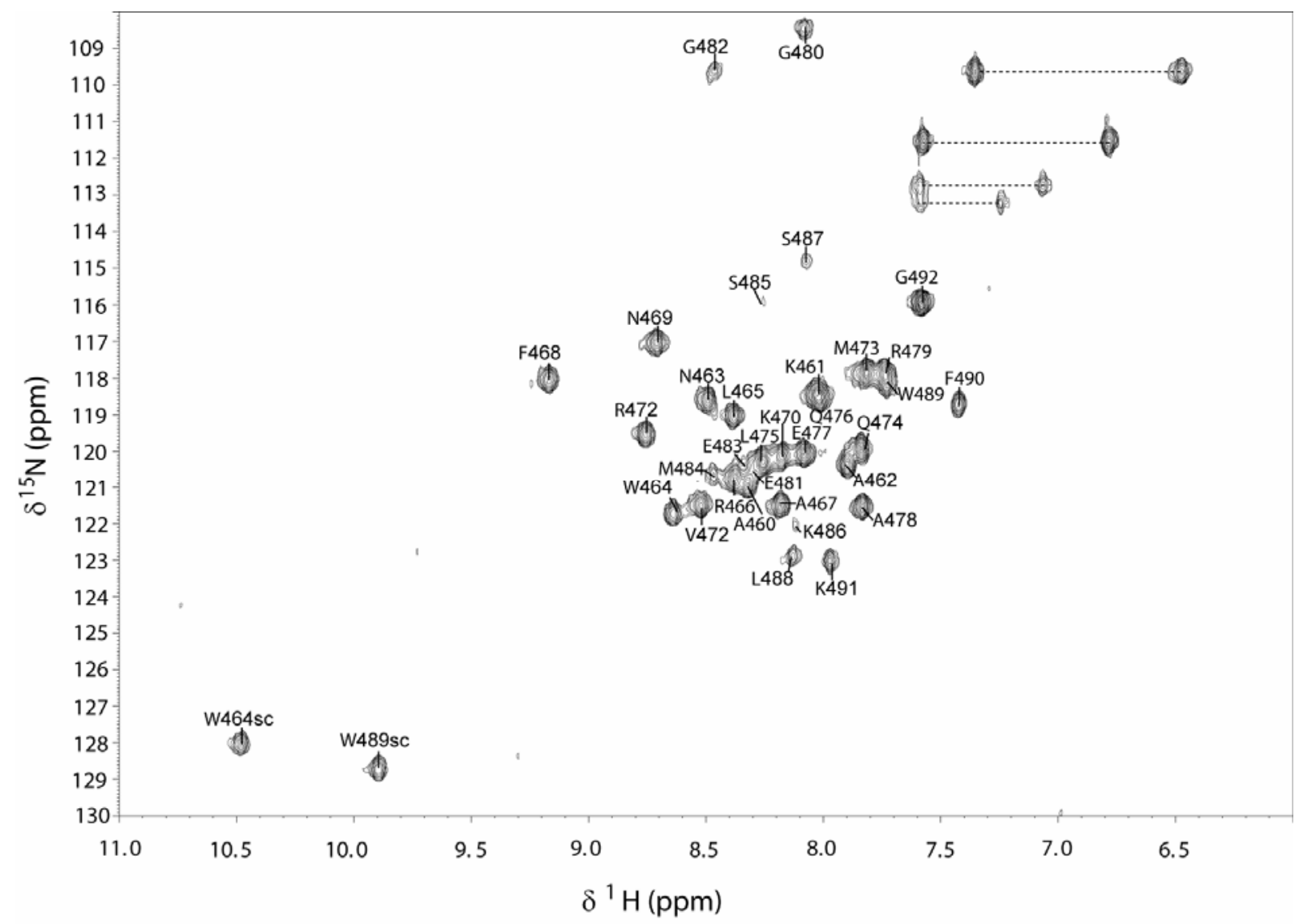

Figure $41 .{ }^{1} \mathrm{H}_{-}{ }^{15} \mathrm{~N}$ HSQC of the bound ${ }^{13} \mathrm{C},{ }^{15} \mathrm{~N}$ Munc13-1(457-492) peptide to CaM. The amide cross-peaks were assigned with the aid of various triple-resonance experiments. 


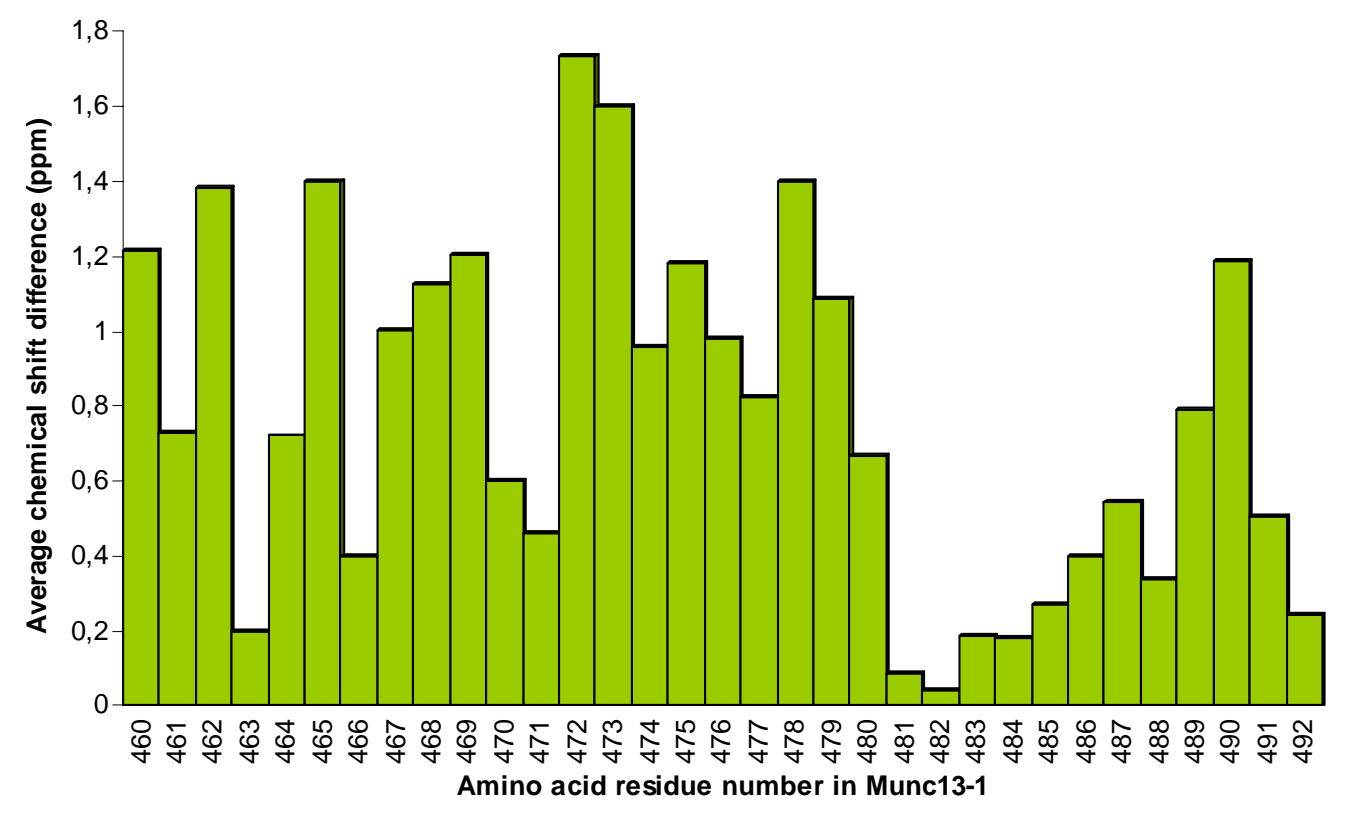

Figure 42. Chemical shift perturbation plot of the Munc13-1 (457-492) peptide upon binding to CaM. Large chemical shift changes were observed in the N-terminal part of the peptide and a second significant perturbation was observed in the C-terminal hydrophobic tail.

The Munc13-1 (457-492) peptide was also titrated with apoCaM. The ${ }^{15} \mathrm{~N}-{ }^{1} \mathrm{H}$ HSQC spectrum was almost identical to the one in the presence of holoCaM (Figure 43). Thus, it appears that the low affinity interaction between apoCaM and Munc13-1 described above in section $b$, already induces the $\alpha$-helical conformation of the Munc13-1(457-492) peptide.

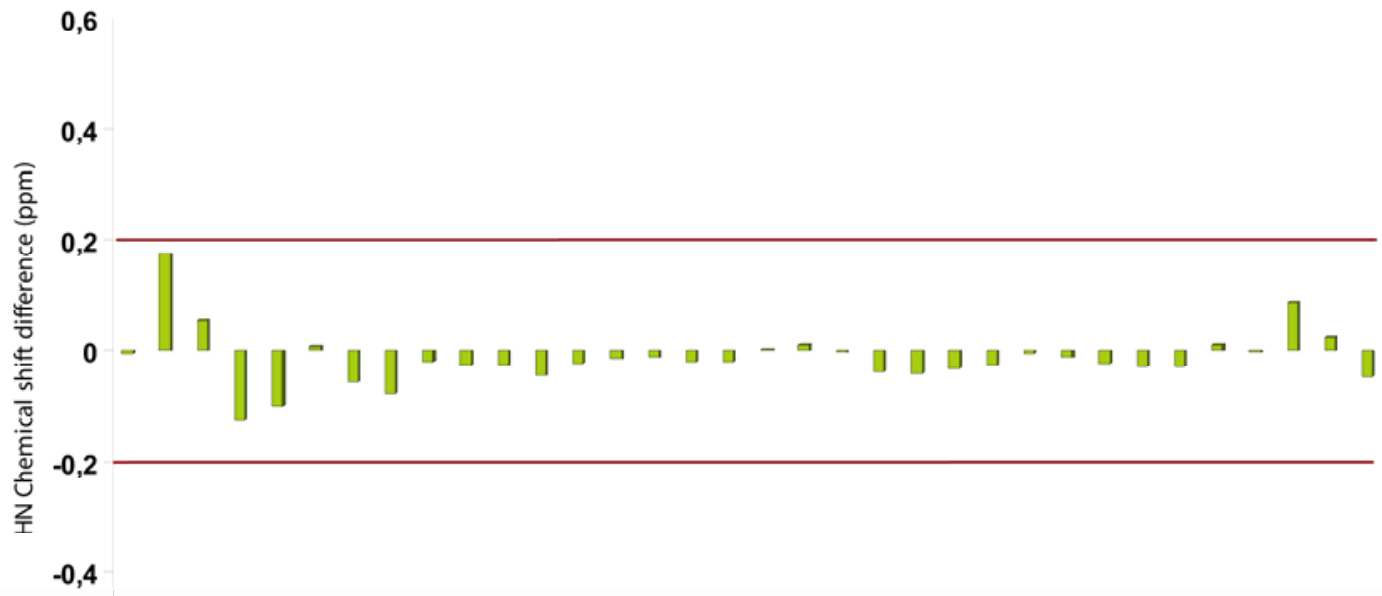

$-0,6$

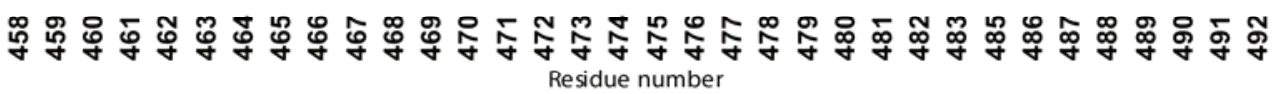

Figure 43. Chemical shift difference for the amide ${ }^{1} \mathrm{H}$ resonances between the Munc13-1 (457-492)/CaM and Munc13-1 (457-492)/apoCaM peptide complexes. The HSQC spectra were almost identical, no significant perturbations were observed. 
To derive hydrogen bond restraints and to determine the buried part of the CaM-bound Munc13-1 (457-492) peptide, a deuterium exchange experiment was done. The amide resonances that are hydrogen bonded and that are buried in the hydrophobic pocket of CaM showed a slower rate of exchange (see Figure 44).

\section{(Munc185)}

d. NMR studies of the CaM binding region and the $C_{1}$ domain of Munc13-1

There are remarkable physiological similarities between the Munc13-1 phorbol ester insensitive (H567K) mutant and the CaM insensitive (W464R) mutant. For this reason it was of interest to study the protein-protein interaction of CaM with a larger construct of Munc13-1 which included its $\mathrm{C}_{1}$ domain, which binds the phorbol ester.

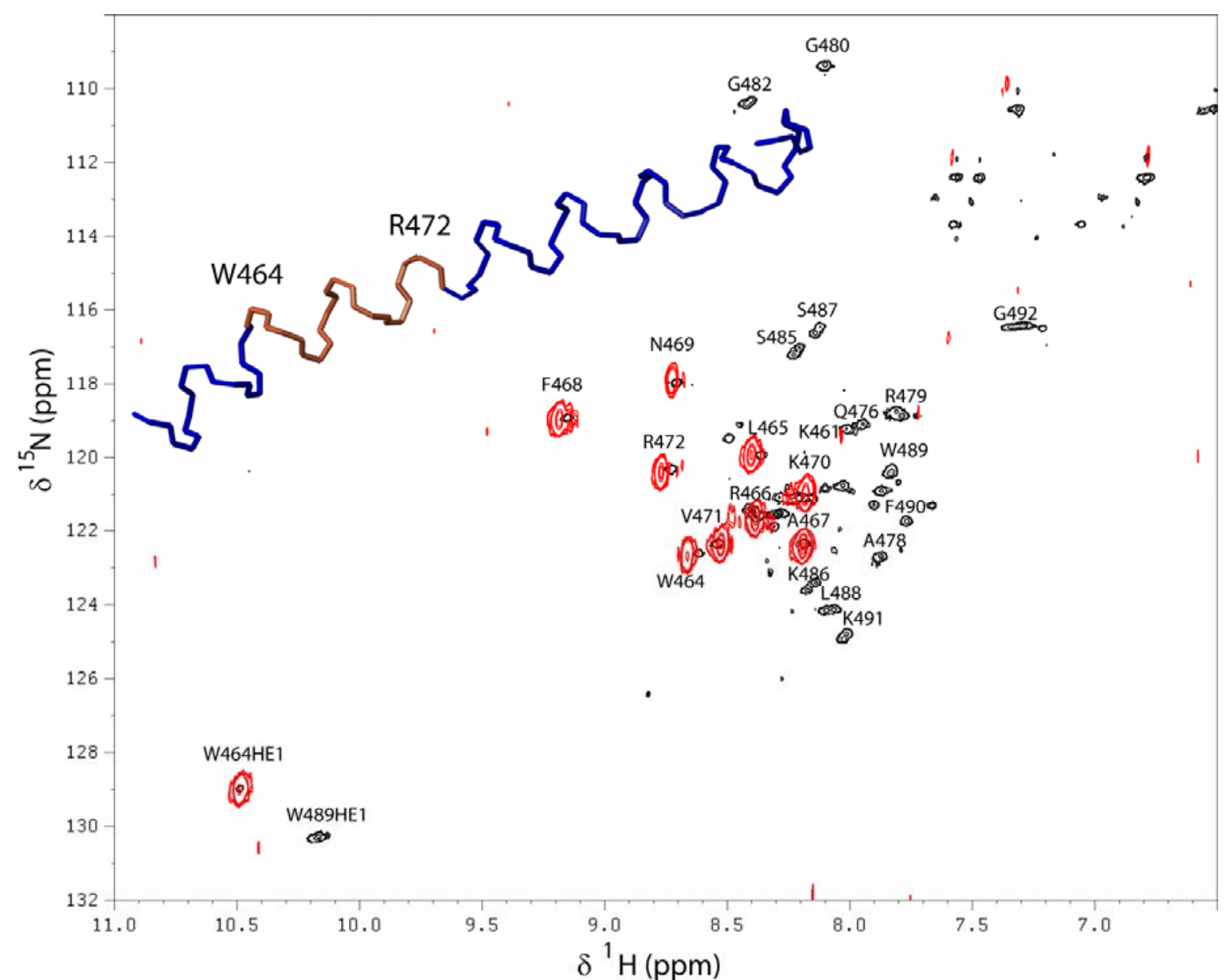

Figure 44. Overlaid ${ }^{15} \mathrm{~N}-{ }^{1} \mathrm{H}$ HSQC spectra of the bound ${ }^{15} \mathrm{~N},{ }^{13} \mathrm{C}$ Munc13-1(457-492) peptide to $\mathrm{CaM}$ in $90 \% \mathrm{H}_{2} \mathrm{O}$ and $10 \% \mathrm{D}_{2} \mathrm{O}$ (black) and in $99 \% \mathrm{D}_{2} \mathrm{O}$ (red) after 4 hours at $35^{\circ} \mathrm{C}$. In the top left corner the helical region of the peptide which was protected from exchange is shown in light brown colour.

The co-expression of the ${ }^{15} \mathrm{~N}$ CaM $/{ }^{15} \mathrm{~N}$ Munc13-1(185aa) $w t$ complex was successful in the pET Duet-1 construct, obtaining enough protein for NMR measurements. During the purification procedure using size-exclusion chromatography, monomer-dimer equilibrium was observed. The dimeric (2:2) complex was very stable and could be separated from the monomeric complex. The ${ }^{15} \mathrm{~N}-{ }^{1} \mathrm{H}$ HSQC spectrum of the monomeric sample is shown in Figure 45. The overlaid HSQC spectra of the dimeric (2:2) and monomeric (1:1) CaM/Munc13-1(185aa) wt samples is shown in Figure 46. 
Although the backbone sequential resonance assignment of this complex was not undertaken; the general appearance of the spectrum was very similar to that one of the CaM/Munc13-1 (459-492) peptide complex and numerous amide cross-peaks where at the same position and easily identified.

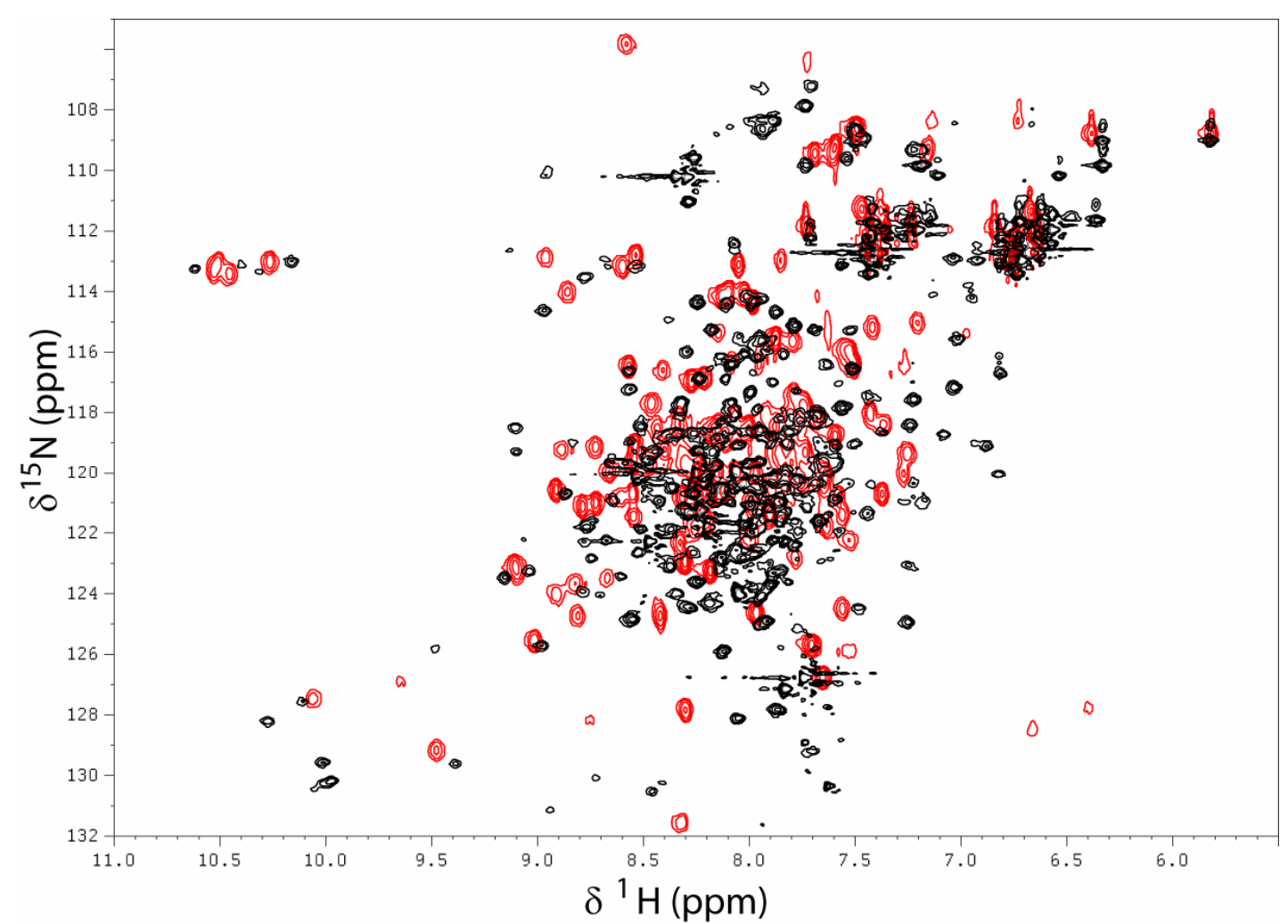

Figure 45. ${ }^{15} \mathrm{~N}-{ }^{1} \mathrm{H}$ HSQC of the ${ }^{15} \mathrm{~N}$ CaM (red) and ${ }^{15} \mathrm{~N}$ CaM $/{ }^{15} \mathrm{~N}$ Munc13-1(185aa) $w t$ monomeric (1:1) complex (black).

From the appearance of the HSQC spectrum of the dimeric (2:2) complex, it was evident that many of the amide cross-peaks broadened (i.e. the line-width increased) and some of them broadened beyond detection. To quantify these effects, the intensities for each of the peaks that overlaid well to the CaM/Munc13-1(459-492) peptide complex cross-peaks were compared between the two species and are shown in Figure 47.

The regions mostly affected in the dimer-monomer equilibrium mapped to the $\mathrm{N}$ terminal domain of CaM (see Figure 48) and the $\mathrm{C}_{1}$ domain of Munc13-1. This suggested that there is a conformational exchange equilibrium process between these two domains in the $\mu \mathrm{s}$ to ms time scale in this dimeric $(2: 2)$ species.

e. Tryptophan $\mathrm{N} \varepsilon-\mathrm{H} \varepsilon 1$ side chain heterogeneity as evidence of a switch mechanism in the calcium coupling mechanism of CaM and Munc13-1

From the studies on the CaM/Munc13-1(457-492) peptide complex it was determined that CaM had a bipartite interaction with Munc13-1, i.e. the C-terminal domain interacted with the amphiphilic $\alpha$-helix (1-5-8) motif and the N-terminal domain interacted with a hydrophobic LW motif at the C-terminal tail of the peptide. The question that arose was whether the small hydrophobic LW motif in the C-terminal tail of the peptide was also binding to the $\mathrm{N}$-terminal domain of CaM in the context of the larger Munc13-1 (185aa) construct.

The analysis of the tryptophan $\mathrm{N} \varepsilon-\mathrm{H} \varepsilon 1$ side-chain region for the CaM/Munc13-1 (457492) peptide and CaM/Munc13-1 (185aa) complexes revealed the presence of heterogeneity in both the W489 and W588 Ne-He1 side-chain resonances (Figures 49).The identity of each 
tryptophan $\mathrm{N} \varepsilon-\mathrm{H} \varepsilon 1$ side-chain was unambiguously identified with the aid of two CaM/Munc13-1(185aa) mutant samples (W489A and W588A).

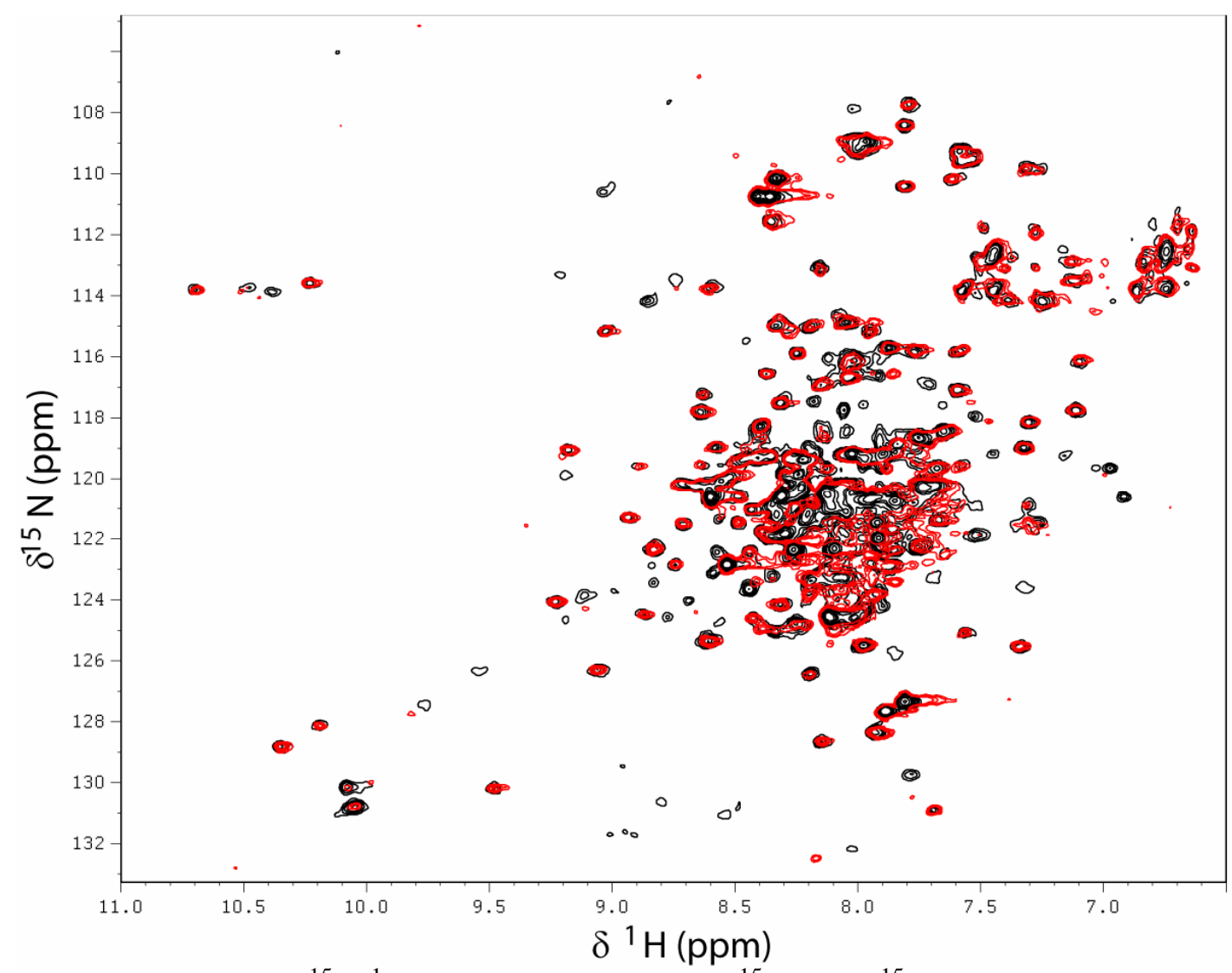

Figure 46. Overlaid ${ }^{15} \mathrm{~N}-{ }^{1} \mathrm{H}$ HSQC spectra for the ${ }^{15} \mathrm{~N}$ CaM $/{ }^{15} \mathrm{~N}$ Munc13-1(185aa) $w t$ monomeric (1:1) complex (black) and dimeric (2:2) complex (red).

Intensity ratio for the dimeric/monomeric 15NCaM/15NMunc13-1(185aa)

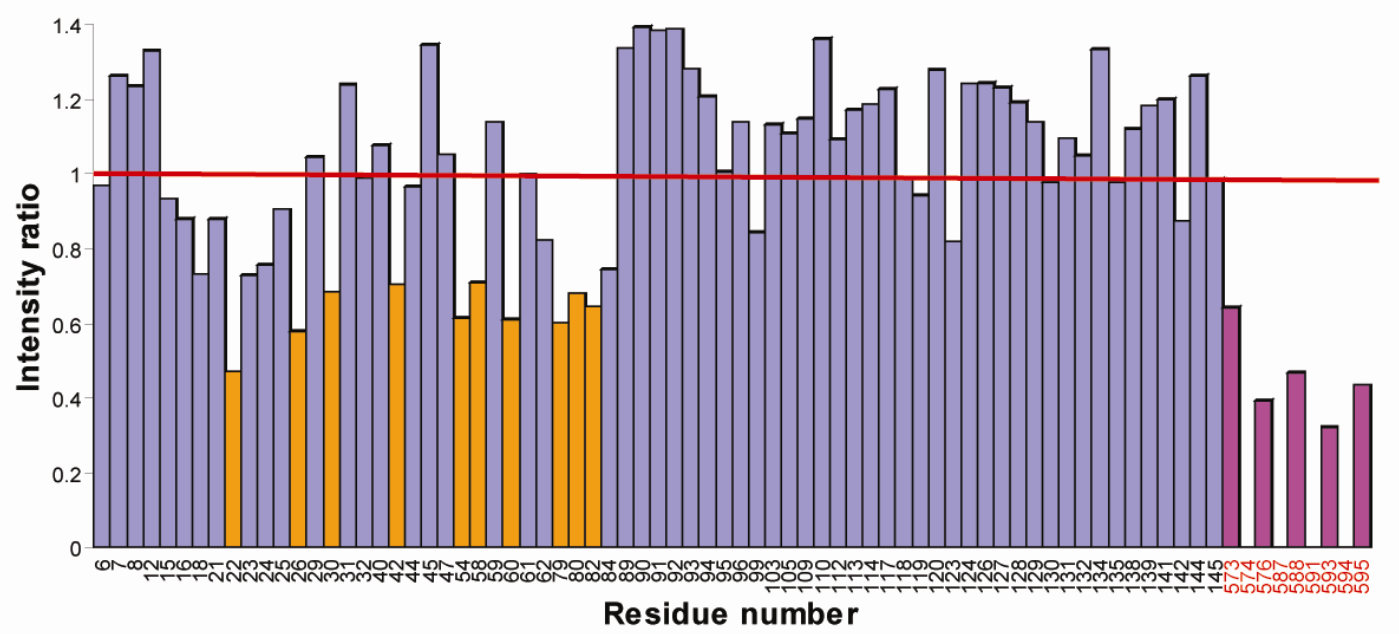

Figure 47. Intensity ratio of the amide cross-peaks between the monomeric (1:1) and dimeric $(2: 2){ }^{15} \mathrm{~N} \mathrm{CaM} /{ }^{15} \mathrm{~N}$ Munc13-1(185aa) wt complexes. The regions affected were the $\mathrm{C}_{1}$ domain of Munc13-1 (residues labelled in red) shown in magenta and some amides in the N-terminal domain of CaM shown in orange. 


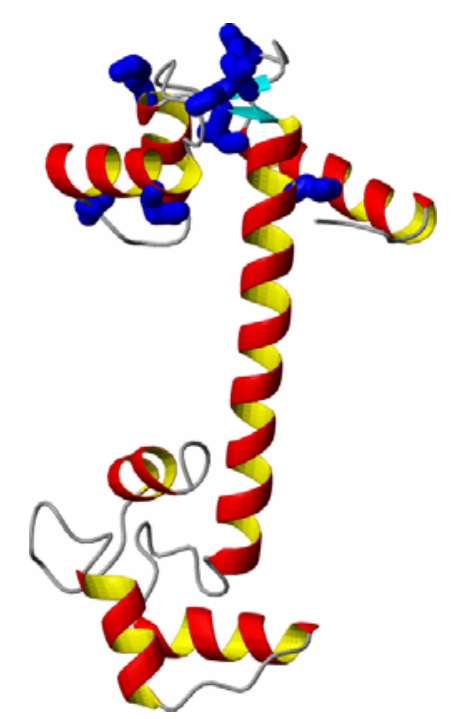

Figure 48. Residues in the N-terminal domain of CaM that showed line-broadening upon dimerization with Munc13-1 (185aa).

The heterogeneity could have arisen either from different $\chi$ rotamer populations of the tryptophan side chain with slow exchange on the NMR time scale or from two different complex states a tryptophan-bound or -free state. However, evidence coming from the assigned W588 tryptophan $\mathrm{N} \varepsilon-\mathrm{H} \varepsilon 1$ side-chain resonance in the absence of CaM discussed below in section $f$, argues for two distinct complex states and therefore are labelled as free side chain (sc) and bound side chain (bsc) in all the spectra. For W489, the bsc resonance represents $19.2 \%$ of the total volume and for W588, the bsc resonance represents $60.5 \%$ of the total volume.

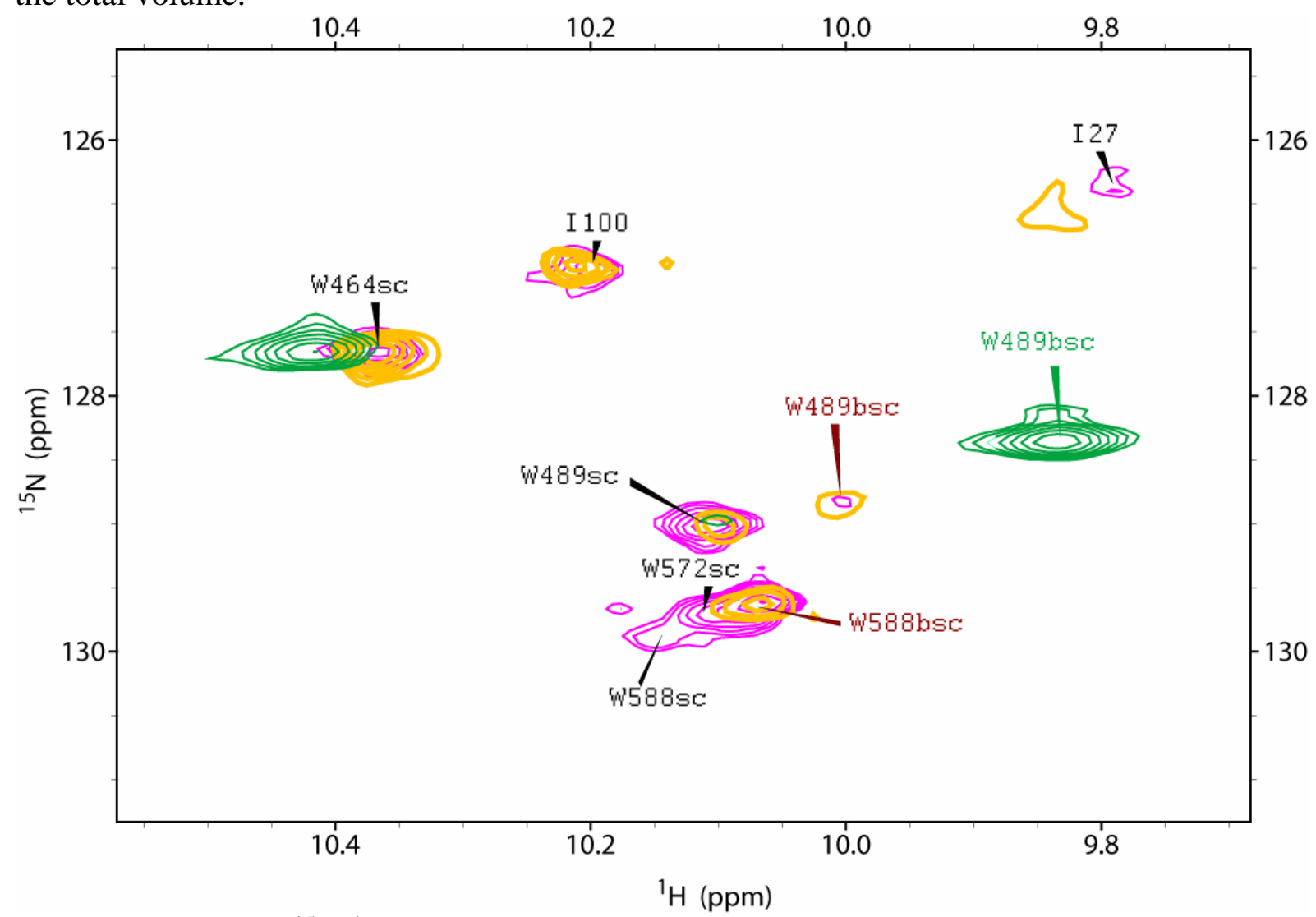

Figure 49. Overlaid ${ }^{15} \mathrm{~N}-{ }^{1} \mathrm{H}$ HSQC spectra showing the tryptophan $\mathrm{N} \varepsilon$-H $\varepsilon 1$ side-chain region for the ${ }^{13} \mathrm{C},{ }^{15} \mathrm{~N}$ Munc13-1(457-492) bound peptide (green); the ${ }^{15} \mathrm{~N} \mathrm{CaM} /{ }^{15} \mathrm{~N}$ Munc131(185aa) wt monomeric (1:1) complex (magenta); and dimeric (2:2) complex (orange). 
None of the mutations (W489A and W588A) appeared to resolve the heterogeneity observed in the tryptophan $\mathrm{N} \varepsilon-\mathrm{H} \varepsilon 1$ side-chain. Assuming that the heterogeneity arises from two different complex states, a switch calcium-gating mechanism model was proposed: the Nterminal domain of CaM could switch between the hydrophobic motif in the W489 region and the hydrophobic motif in the W588 region within the $\mathrm{C}_{1}$ domain of Munc13-1. Nonetheless, the description of a direct contact by intermolecular NOEs or pseudocontact shifts, for example, between the W588 residue and CaM would provide a definite proof. If this proof is provided, it would represent a structural correlate to the physiological observations in the functional mutant studies of the phorbol insensitive (H567K) Munc13-1 and the CaM insensitive (W464R) Munc13-1 mutants mentioned before.

\section{f. NMR studies of the $C_{1}$ domain of Munc13-1 (Munc65)}

Based on the studies done on the CaM/Munc13-1(185aa) construct it was also of interest to study the $\mathrm{C}_{1}$ domain of Munc13-1 in the absence of CaM. Although the NMR structure of this domain has been reported before by Shen et al., 2005; there is no resonance assignment available in the Biological Magnetic Resonance Data Bank (BMRB); the only assignments come from the few labels in the HSQC spectrum reported in that publication.

In order to identify the free tryptophan $\mathrm{N} \varepsilon-\mathrm{H} \varepsilon 1$ side chains of the $\mathrm{C}_{1}$ domain of Munc13-1 a 1D- ${ }^{1} \mathrm{H}$ spectrum with presaturation of the water resonance was measured (Figure 50) and compared to the published spectrum.

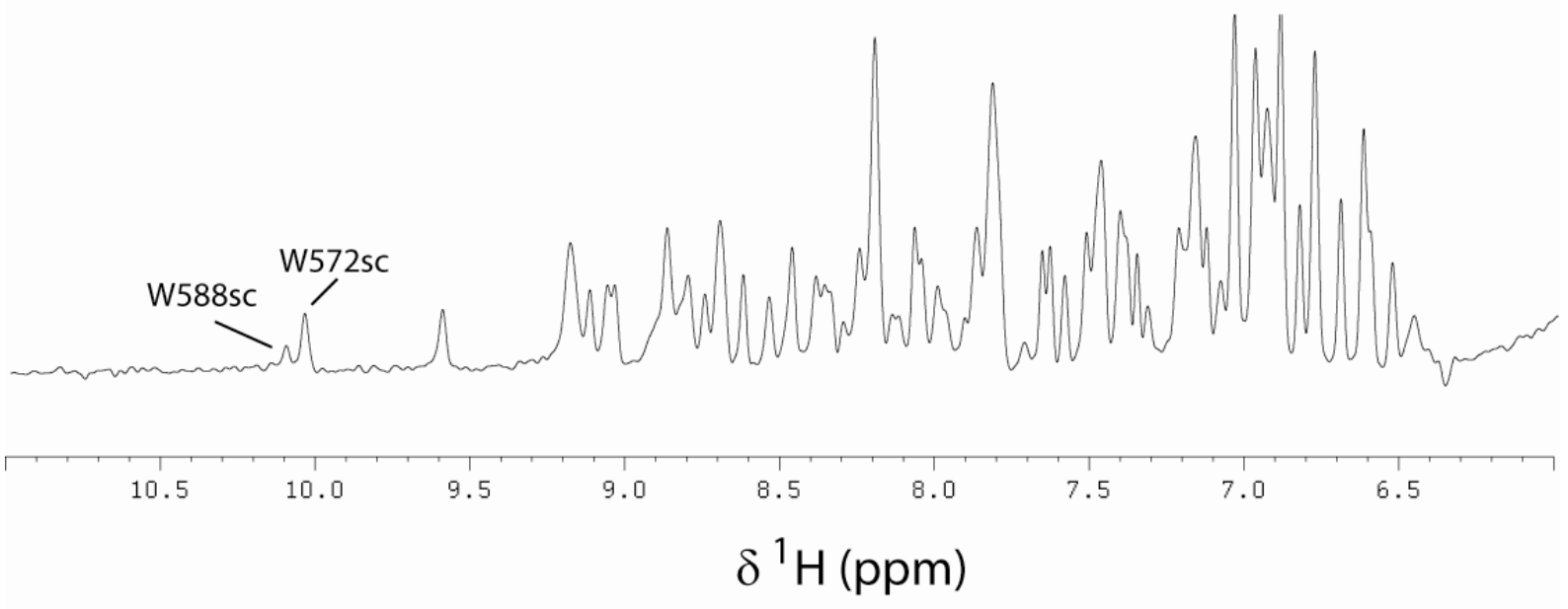

Figure 50. 1D- ${ }^{1} \mathrm{H}$ spectrum for the amide region of unlabelled Munc13-1 $\mathrm{C}_{1}$ domain (65aa) at $900 \mathrm{MHz}$.

Afterwards, with the availability of a ${ }^{15} \mathrm{~N}$ labelled sample, a ${ }^{15} \mathrm{~N}-{ }^{1} \mathrm{H}-\mathrm{HSQC}$ of the $\mathrm{C}_{1}$ domain of Munc13-1 was also recorded (Figure 51) for comparison purposes to the published spectrum mentioned above.

The tryptophan $\mathrm{N} \varepsilon-\mathrm{H} \varepsilon 1$ side-chain region of the Munc13-1 $\mathrm{C}_{1}$ domain HSQC spectrum was overlaid to that one of the ${ }^{15} \mathrm{~N} \mathrm{CaM} /{ }^{15} \mathrm{~N}$ Munc13-1 (185aa) wt complex (Figure 52). The lack of heterogeneity for the $\mathrm{W} 588 \mathrm{~N} \varepsilon-\mathrm{H} \varepsilon 1$ side-chain in the $\mathrm{C}_{1}$ domain alone, suggested that in the complex with CaM, the heterogeneity should have arisen either from the direct interaction with $\mathrm{CaM}$ or from an intramolecular interaction with an element upstream from the $\mathrm{C}_{1}$ domain of Munc13-1. 
g. NMR studies of ${ }^{15} \mathrm{~N}$ CaM/ $/{ }^{15} \mathrm{~N}$ Munc13-1(185aa) complex with its agonist: phorbol dibutyrate $(\mathrm{PDBu})$

The ${ }^{15} \mathrm{~N}$ CaM $/{ }^{15} \mathrm{~N}$ Munc13-1(185aa) wt dimeric (2:2) complex was treated with a slight excess of the $\mathrm{C}_{1}$ domain agonist phorbol dibutyrate (PDBu) to observe its effect on the ${ }^{15} \mathrm{~N}-{ }^{1} \mathrm{H}$ HSQC spectrum; two resolved areas of the spectrum are shown in Figure 53.

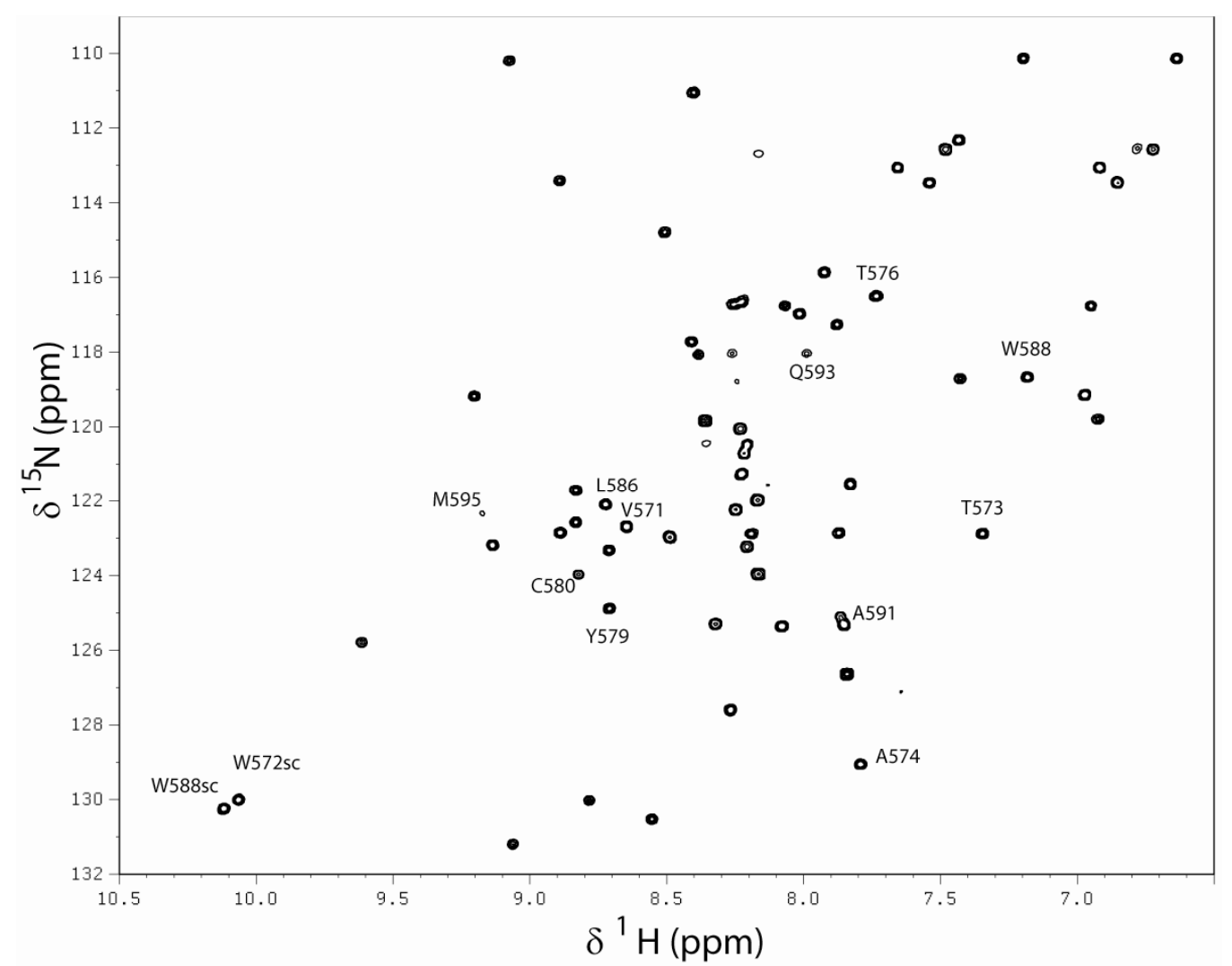

Figure $51 .{ }^{15} \mathrm{~N}-{ }^{1} \mathrm{H}$ HSQC of the $\mathrm{C}_{1}$ domain of Munc13-1 $(100 \mu \mathrm{M})$ in $20 \mathrm{mM}$ Bis-Tris, 150 $\mathrm{mM} \mathrm{KCl}, 5 \mathrm{mM} \mathrm{CaCl}_{2}$ recorded at $25^{\circ} \mathrm{C}$ with 1024 and 256 real points in the $t_{2}$ and $t_{1}$ dimensions, respectively.

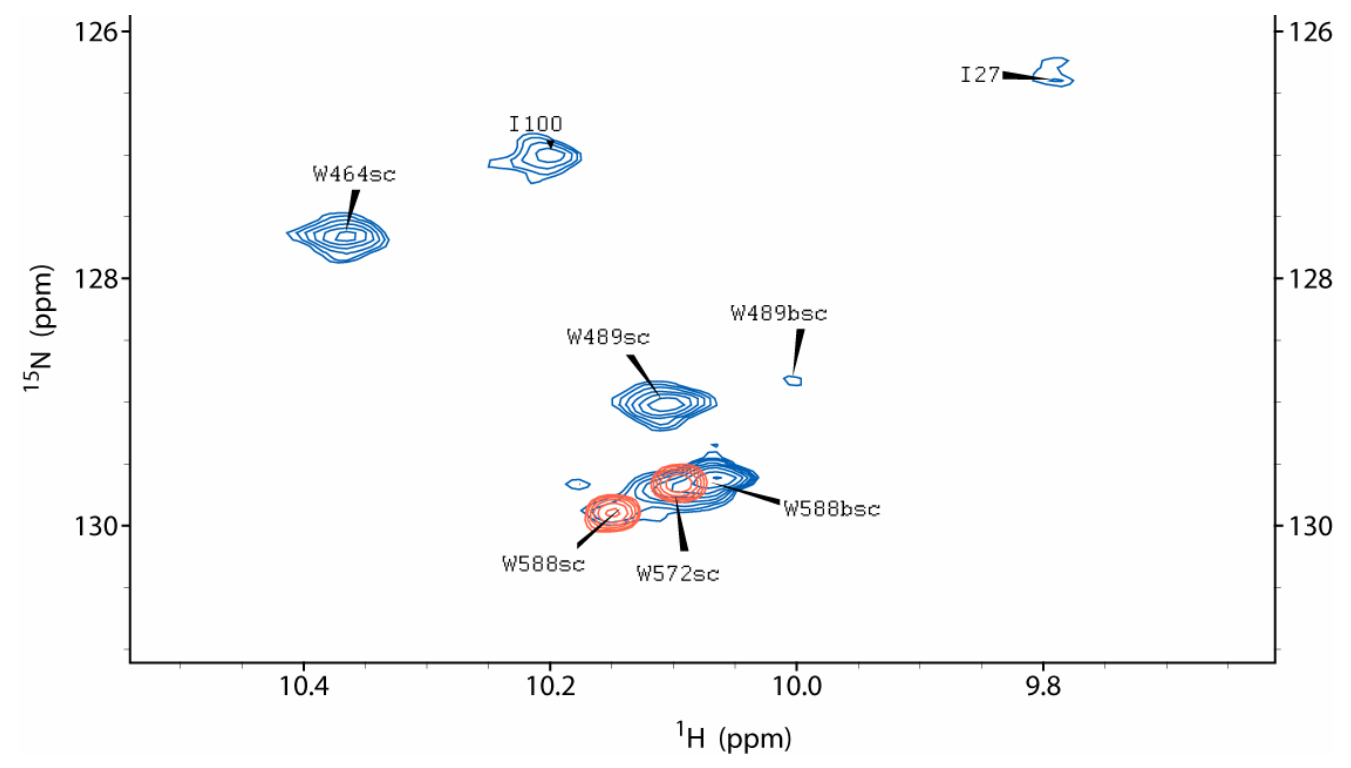

Figure 52. Overlaid ${ }^{15} \mathrm{~N}-{ }^{1} \mathrm{H}$ HSQC spectra for the ${ }^{15} \mathrm{~N}$ CaM $/{ }^{15} \mathrm{~N}$ Munc13-1(185aa) $w t$ monomeric (1:1) complex in blue and the ${ }^{15} \mathrm{~N}$ Munc13-1 $\mathrm{C}_{1}$ domain in red. 

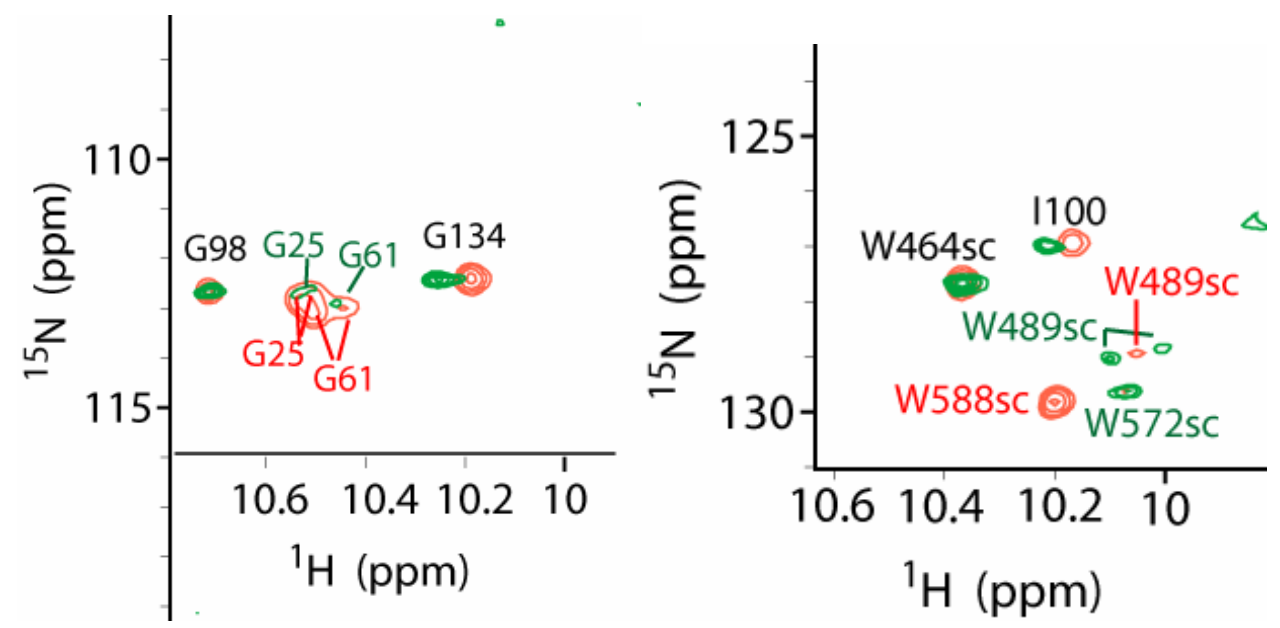

Figure 53. Overlaid ${ }^{15} \mathrm{~N}-{ }^{1} \mathrm{H}$ HSQC spectra of the ${ }^{15} \mathrm{~N}$ CaM/ $/{ }^{15} \mathrm{~N}$ Munc13-1(185aa) wt dimeric (2:2) complex in the inactive state (green) and in its active state (red) in the presence of a slight excess of the $\mathrm{C}_{1}$ domain agonist PDBu.

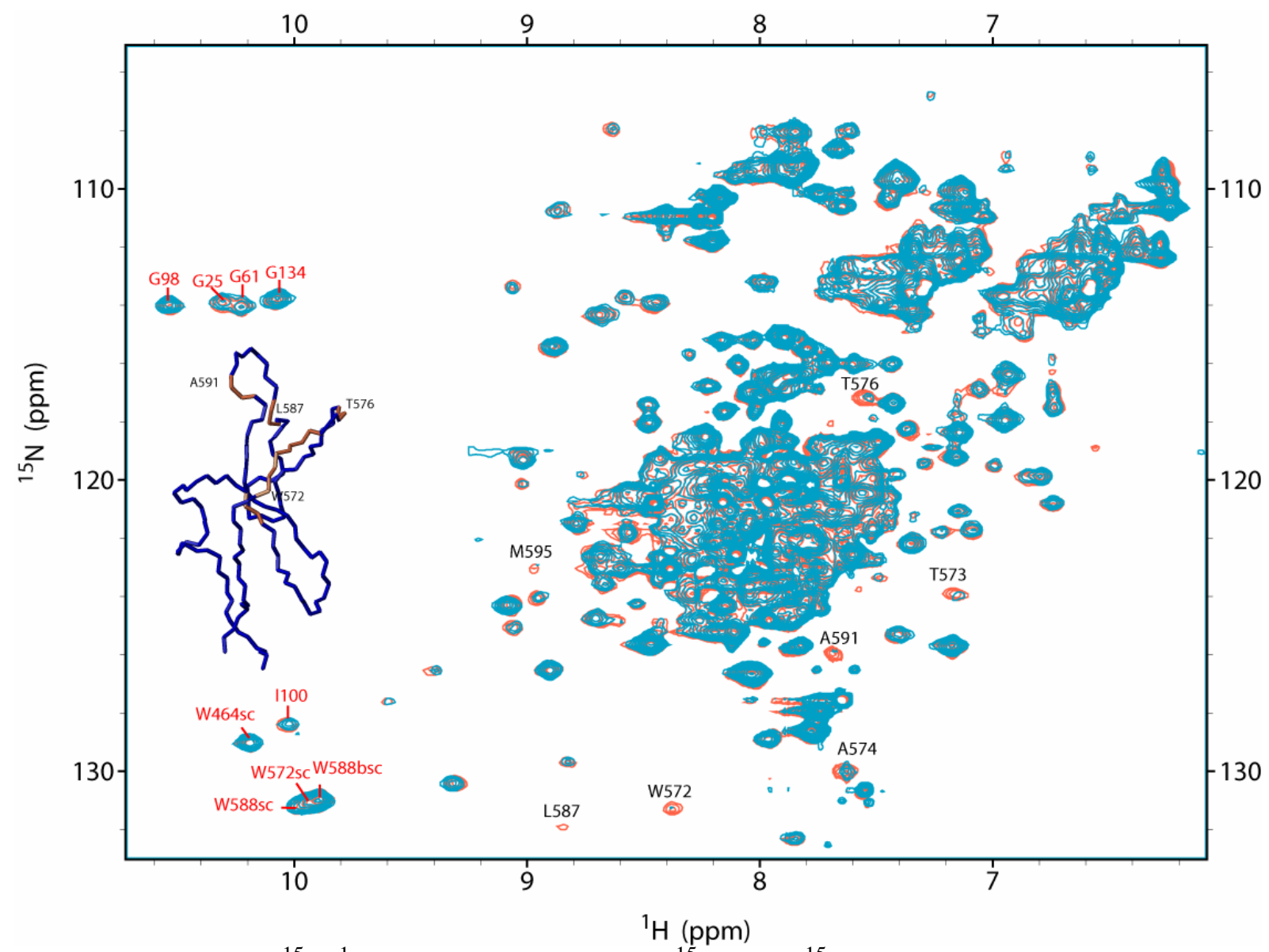

Figure 54. Overlaid ${ }^{15} \mathrm{~N}-{ }^{1} \mathrm{H}$ HSQC spectra for the ${ }^{15} \mathrm{~N}$ CaM $/{ }^{15} \mathrm{~N}$ Munc13-1(185aa) W489A mutant monomeric (1:1) complex in its inactive state (red) and in its activated state with a slight excess of the agonist PDBu (in light blue). In the left side of the spectrum the backbone structure of the $\mathrm{C}_{1}$ domain of Munc13-1 shows the regions affected by $\mathrm{PDBu}$ in light brown. The residues with red labels are the ones analyzed in the (2:2) complex; they were not affected by the PDBu addition in this sample.

The effects of the agonist on the ${ }^{15} \mathrm{~N}$ CaM $/{ }^{15} \mathrm{~N}$ Munc13-1(185aa) wt dimeric (2:2) complex were clearly visible. There were chemical shift perturbations as well as a shift in the 
conformational exchange equilibrium previously described in the $d$ section, since the line shape and $\mathrm{S} / \mathrm{N}$ ratio of several amide cross-peaks improved significantly. The broad resonances for the G25 and G61 cross-peaks sharpened and doubled in the activated state. In the tryptophan $\mathrm{N} \varepsilon-\mathrm{H} \varepsilon 1$ side-chain region, the broad W588 resonance in the inactive spectrum had a low field ${ }^{1} \mathrm{H}$ chemical shift change of $\sim 0.2 \mathrm{ppm}$ in the presence of PDBu. The heterogeneity of the W489 resonance observed in the inactive state was lost in the presence of PDBu and a single cross peak was observed with a chemical shift between the free and bound complex states. The changes in the C-terminal domain of CaM appeared to be less pronounced consistent with the bipartite interaction model of this complex.

The same agonist activation experiment was done for the ${ }^{15} \mathrm{~N}$ CaM $/{ }^{15} \mathrm{~N}$ Munc131(185aa) W489A monomeric (1:1) complex. The overlaid spectra are shown in Figure 54.

The effects of the agonist PDBu on the monomeric (1:1) state of the ${ }^{15} \mathrm{~N} \mathrm{CaM} /{ }^{15} \mathrm{~N}$ Munc13-1(185aa)W489A mutant complex were more moderate than those for the dimeric (2:2) complex. Nonetheless, there was clear binding of the ligand to the described binding pocket of the $\mathrm{C}_{1}$ domain. The binding was in the intermediate exchange regime on the NMR time-scale. The effects are shown in Figure 55.

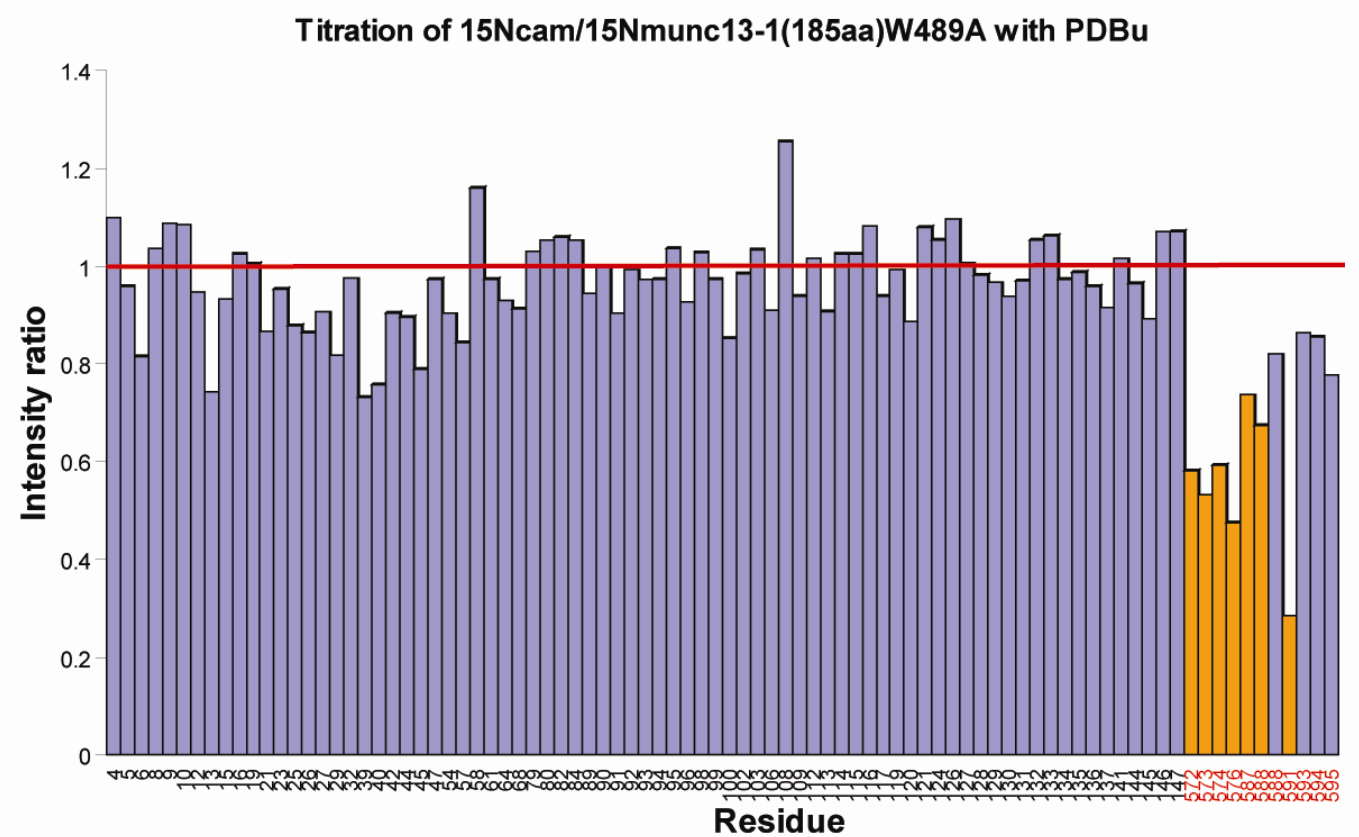

Figure 55. Intensity ratio of the amide cross-peaks for the ${ }^{15} \mathrm{~N} \mathrm{CaM} /{ }^{15} \mathrm{~N}$ Munc13-1(185aa) W489A mutant in the absence and presence of its agonist PDBu.. The CaM residues are labelled in black font and the Munc13-1 residues in red font. The amide cross-peaks greatly affected are shown in orange.

The residue most affected by the PDBu binding to the ${ }^{15} \mathrm{~N}$ CaM $/{ }^{15} \mathrm{~N}$ Munc131(185aa)W489A monomeric complex was A591 in the binding pocket of the $\mathrm{C}_{1}$ domain of Munc13-1. The intensity change upon agonist addition was followed, to observe its saturation curve (Figure 56).

The isotherm binding followed a sigmoidal curve suggesting cooperativity; the stoichiometry appeared to be (1:1) as expected. Cooperative binding of active site ligands follows the Monod-Wyman-Changeux (MWC) model of allostery, in this model, equilibrium between two conformational states, $\mathrm{T}$ and $\mathrm{R}$ are assumed. The equilibria between liganded and unliganded forms are described in terms of two microscopic binding constants, $\mathrm{k}_{\mathrm{T}}$ and $k_{\mathrm{R}}$, where $k_{\mathrm{R}}>k_{\mathrm{T}}$. An equilibrium constant $L$ connects the unliganded forms (Kensal et al. 2006). Since the CaM/Munc13-1(185aa) complex has a monomer (1:1)-dimer (2:2) equilibrium such an allosteric activation model could be possible. NMR studies done on a model enzyme 
showing allosterism (aspartate transcarbamoylase), have demonstrated that although the spectrum reveals just the T form of the enzyme, titrations with MgATP clearly perturbed the R-T equilibrium (Velyvis et al., 2007). In the system studied here, the observed cooperativity for the agonist binding is interesting from the physiological point of view, since it has been reported previously that there is an allosteric positive cooperativity between calcium and phorbol esters in electrophysiological measurements done on the Calyx of Held synapse (Lou et al., 2005). Additional experiments in the presence of phosphatidyl-serine to mimic the phospholipid bilayer of the plasma membrane would need to be done in order to confirm this model rigorously.

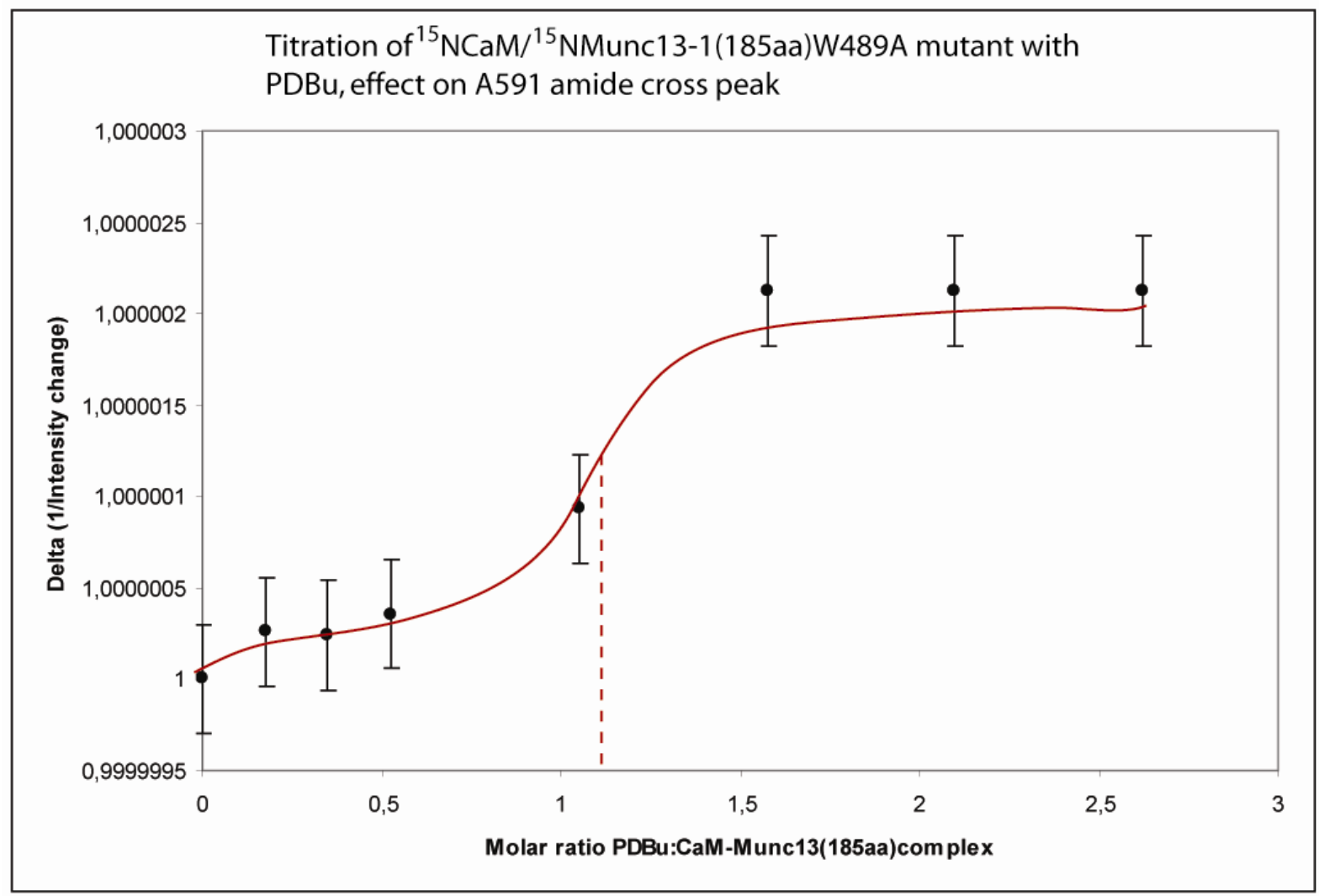

Figure 56. Binding curve of the $\mathrm{C}_{1}$ domain agonist $\mathrm{PDBu}$ to the ${ }^{15} \mathrm{~N} \mathrm{CaM} /{ }^{15} \mathrm{~N}$ Munc131(185aa) W489A monomeric (1:1) complex based on the decrease in the A591 NH cross peak intensity.

h. Sequential backbone and side-chain resonance assignment for the ${ }^{13} \mathrm{C},{ }^{15} \mathrm{~N}$ CaM/Munc131(459-492) peptide complex

For the sequential backbone and side chain resonance assignment, a series of triple resonance experiments were performed (see Table 4).

In biomolecular NMR, the ${ }^{1} \mathrm{H}-{ }^{15} \mathrm{~N}$ HSQC spectrum of the amide resonances in a polypeptide represent its fingerprint and the identification of each cross-peak is essential for various studies like protein-ligand interactions; the assigned HSQC spectrum for the ${ }^{13} \mathrm{C},{ }^{15} \mathrm{~N}$ CaM/Munc13-1(459-492) peptide complex is shown in Figure 57.

In this section, some strip plots of the various $3 \mathrm{D}$ experiments were extracted to illustrate the sequential assignment strategy. The 3D-HNCACB and the 3D-CBCA(CO)NH correlate the $\mathrm{C} \beta$ and $\mathrm{C} \alpha$ of amino acid pairs to the NH cross-peak (Figure 58). 
Table 4. NMR experiments for the sequential resonance assignment of the backbone and side chain atoms in the ${ }^{13} \mathrm{C},{ }^{15} \mathrm{~N} \mathrm{CaM} /$ Munc13-1(459-492) peptide complex

\begin{tabular}{|c|c|c|}
\hline Sample & Experiment & Resonances assigned \\
\hline \multirow[t]{6}{*}{${ }^{13} \mathrm{C},{ }^{15} \mathrm{NCaM} / \mathrm{Munc} 13-1 \mathrm{H}_{2} \mathrm{O}$} & $\mathrm{HNCO}$ & $\mathrm{C}^{\prime}(\mathrm{i}-1)$ to $\mathrm{NH}$ \\
\hline & $\mathrm{HN}(\mathrm{CA}) \mathrm{CO}$ & $\mathrm{C}^{\prime}(\mathrm{i}-1)$ and $\mathrm{C}^{\prime}(\mathrm{i})$ to $\mathrm{NH}$ \\
\hline & $\mathrm{CBCA}(\mathrm{CO}) \mathrm{NH}$ & CB,CA (i-1) to NH \\
\hline & HNCACB & $\mathrm{CB}, \mathrm{CA}(\mathrm{i}-1)$ and (i) to $\mathrm{NH}$ \\
\hline & $\mathrm{H}(\mathrm{CC})(\mathrm{CO}) \mathrm{NH}-\mathrm{TOCSY}$ & Aliph. H (i-1) to NH \\
\hline & (H)CC(CO)NH-TOCSY & Aliph. C (i-1) to NH \\
\hline \multirow[t]{3}{*}{${ }^{13} \mathrm{C},{ }^{15} \mathrm{NCaM} /$ Munc13-1 $\mathrm{D}_{2} \mathrm{O}$} & HCCH-TOCSY & Aliph. C and Aliph. H \\
\hline & $\begin{array}{l}\mathrm{HBCBCaroH} \gamma \text { and } \\
\mathrm{HBCBCaroH} \delta\end{array}$ & Aromatic $\mathrm{H}$ side-chains \\
\hline & HMBC- $\mathrm{CH}_{3} \varepsilon$ Methionine & Methionine $\mathrm{CH}_{3} \varepsilon$ side-chains \\
\hline
\end{tabular}

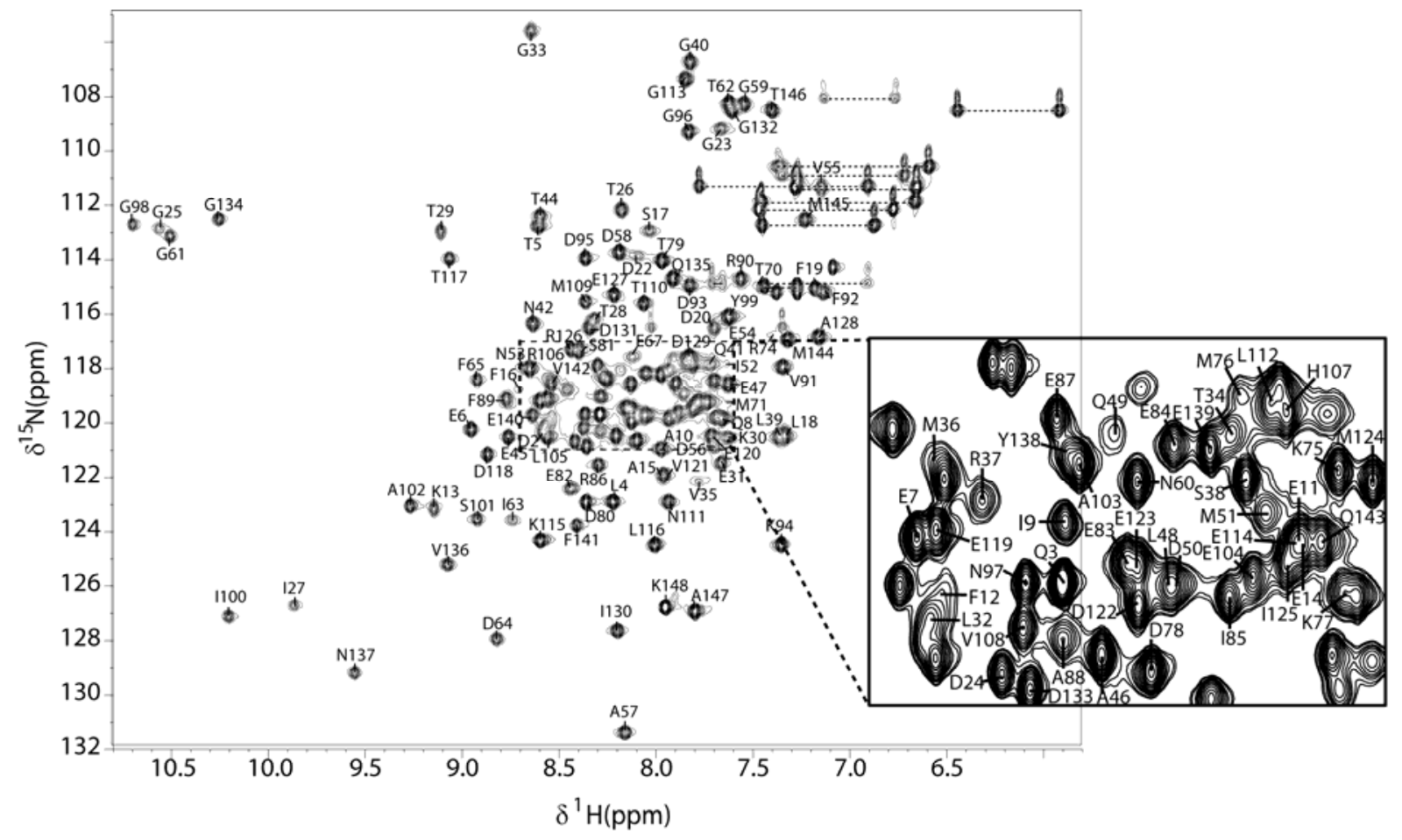

Figure 57. Assigned ${ }^{1} \mathrm{H}_{-}{ }^{15} \mathrm{~N}-\mathrm{HSQC}$ spectrum of the ${ }^{13} \mathrm{C},{ }^{15} \mathrm{NCaM} / \mathrm{Munc} 13-1(459-492)$ peptide complex measured at $308 \mathrm{~K}$. The highly overlapped central region is enlarged on the right side. 


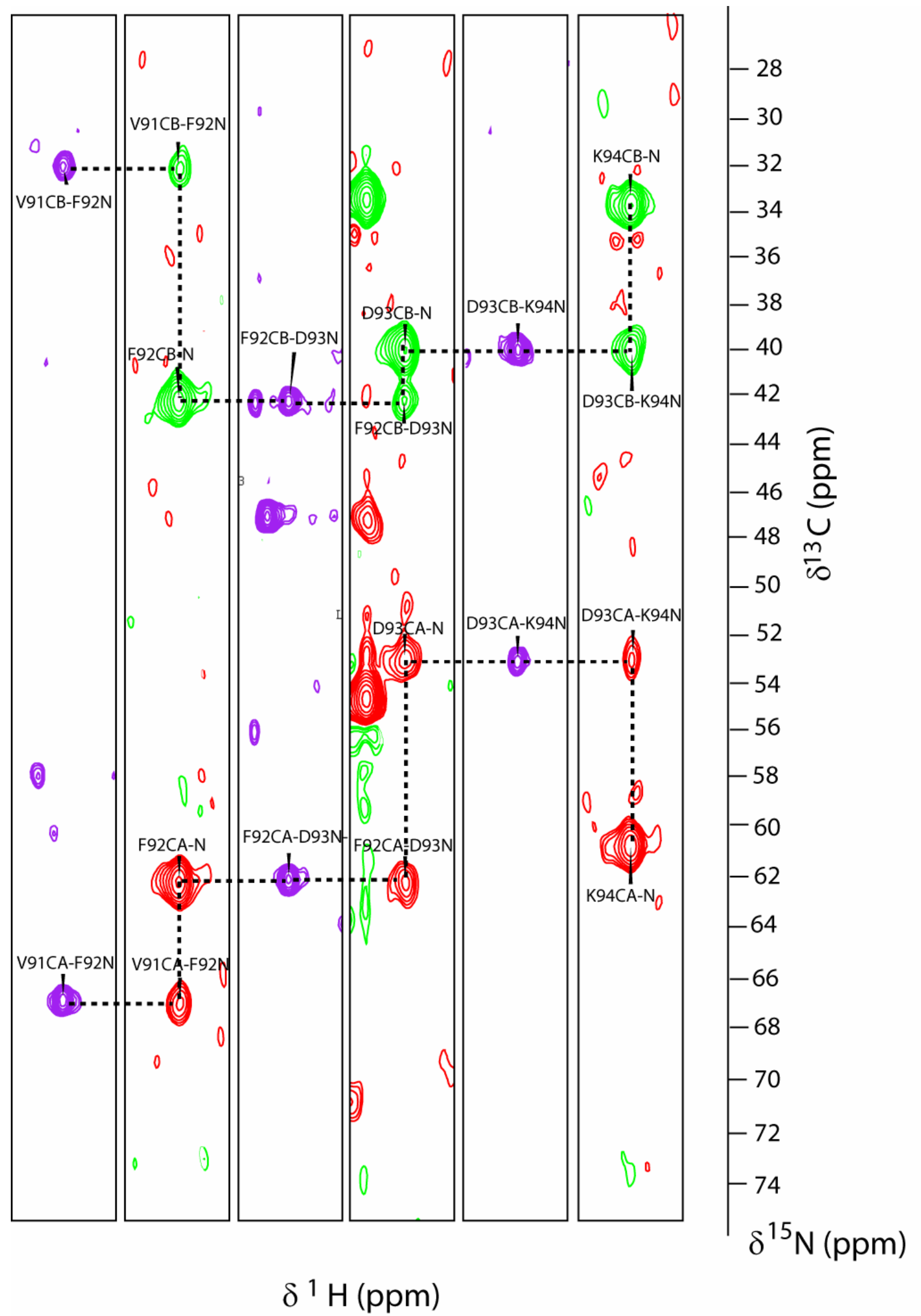

Figure 58. Strips extracted from the 3D-CBCA(CO)NH spectrum in purple and from the 3DHNCACB spectrum in red/green to illustrate the connectivity information to obtain the sequential resonance assignment of the backbone nuclei. 
The backbone assignment was also corroborated with the inter-residue carbonyl connectivity. For this purpose the 3D-HNCO and 3D-HN(CA)CO experiments were also recorded, processed and analyzed (Figure 59).

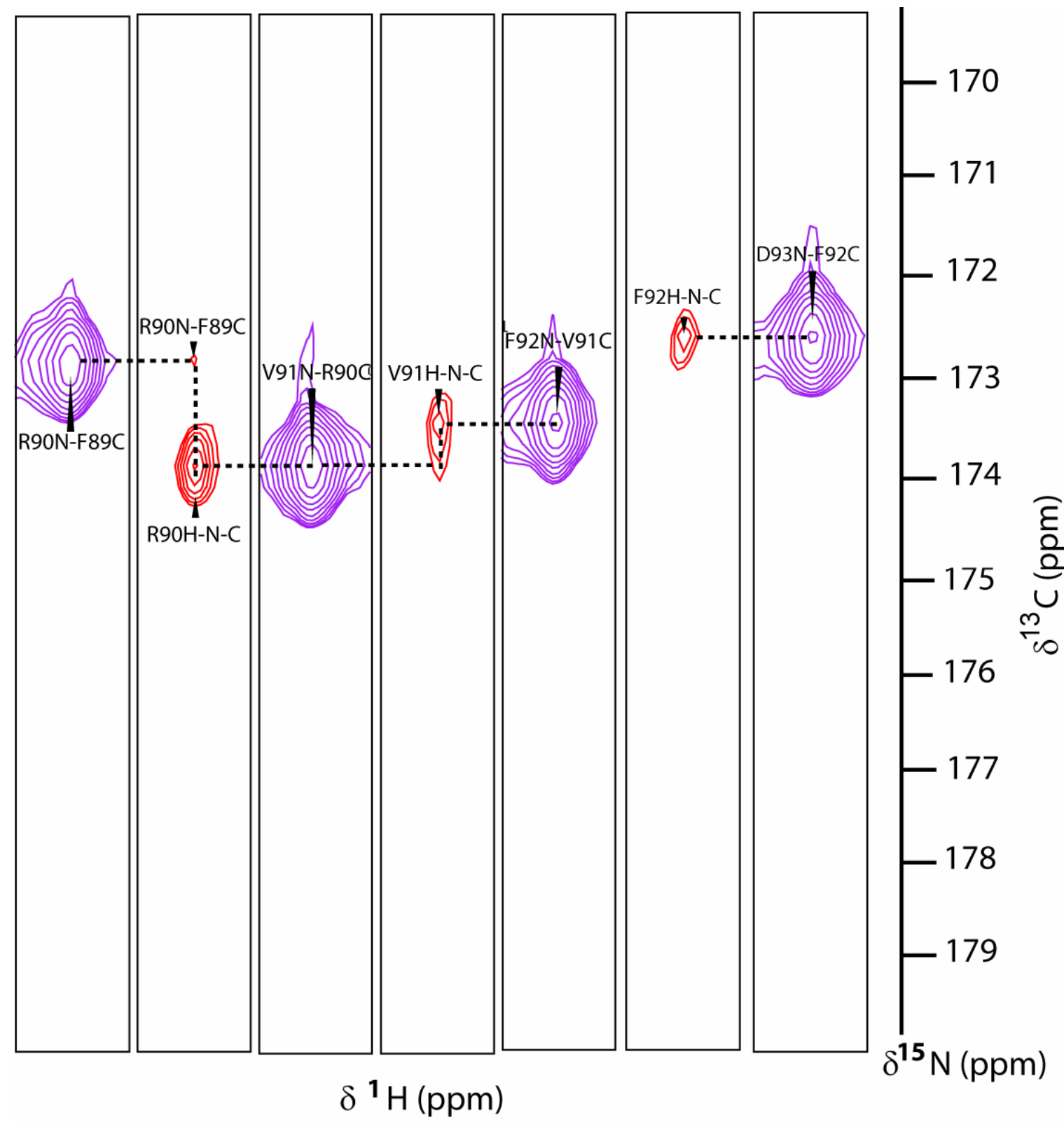

Figure 59. Strips from the 3D-HNCO spectrum (purple) and from the $3 \mathrm{D}-\mathrm{HN}(\mathrm{CA}) \mathrm{CO}$ spectrum (red) for residues F89 to D93 were extracted to illustrate the sequential connectivity information that aids in the backbone sequential resonance assignment.

As can be seen in Figure 60, the overlap of the aliphatic side-chain resonances is very large; for this reason a series of 3D experiments were done to assign the side-chain of each amino acid residue in CaM to the backbone resonances. The aliphatic Carbon resonances were initially assigned using the 3D-(H)CC(CO)NH-TOCSY experiment (Figure 61). 


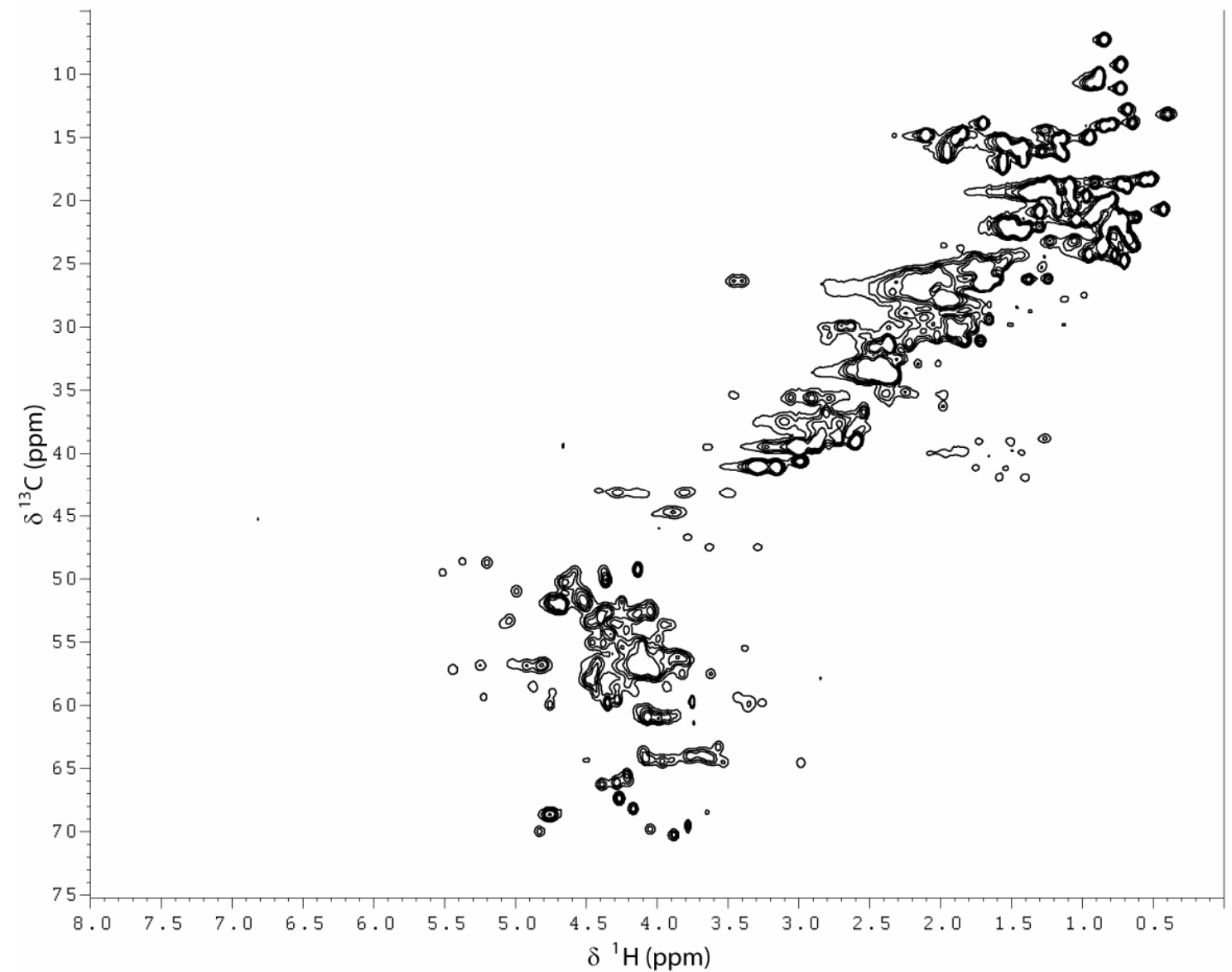

Figure 60. ${ }^{13} \mathrm{C}-{ }^{1} \mathrm{H}$ HSQC spectrum of ${ }^{13} \mathrm{C},{ }^{15} \mathrm{~N} \mathrm{CaM} /$ Munc $13-1(459-492)$ complex in $99 \% \mathrm{D}_{2} \mathrm{O}$ 


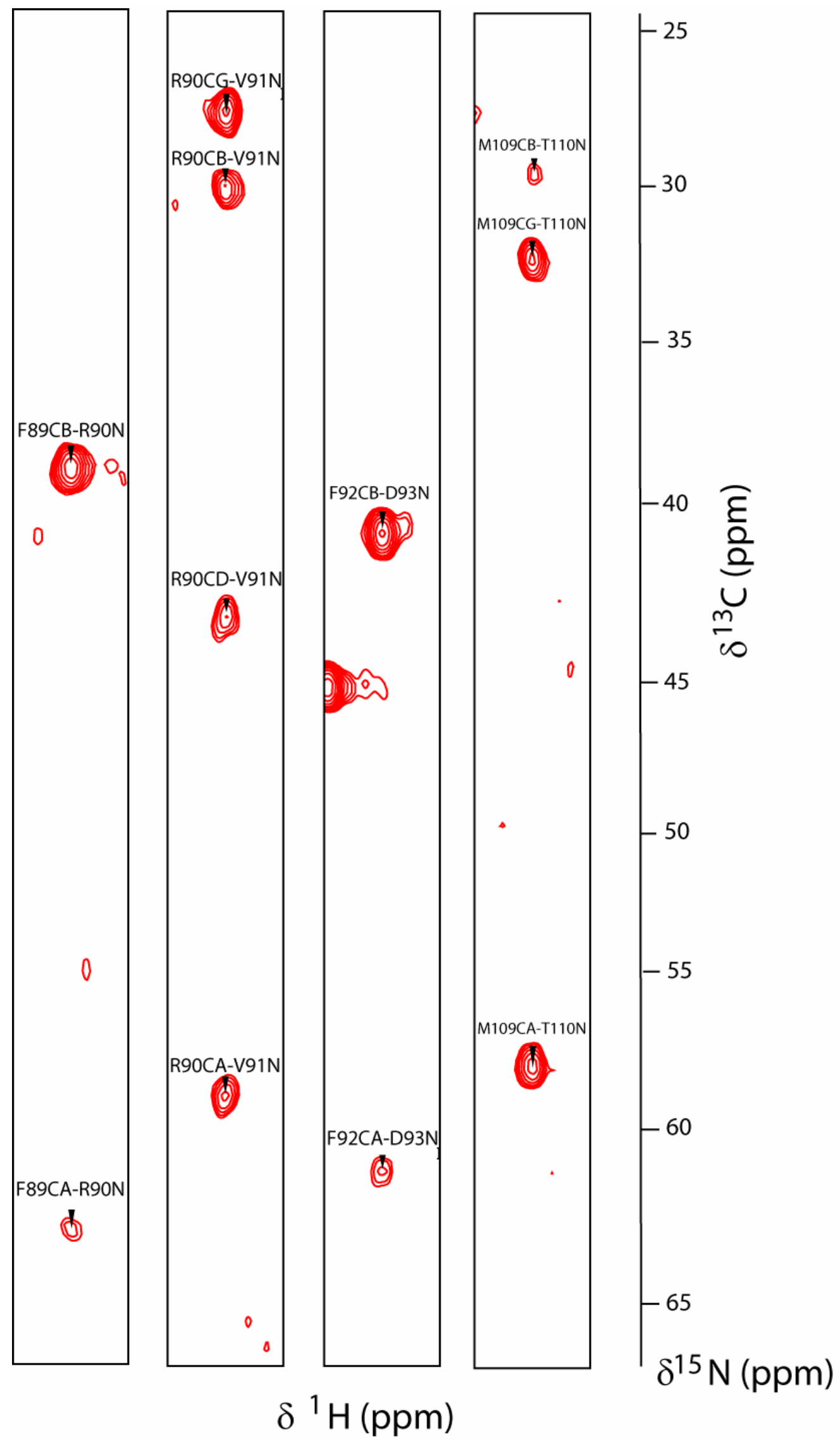

Figure 61. Strips extracted from the 3D-(H)CC(CO)NH-TOCSY spectrum to illustrate the assignment of the aliphatic carbons from different side-chains to the amide cross-peak in the backbone. 
The aliphatic proton resonance were assigned with the $3 \mathrm{D}-\mathrm{H}(\mathrm{CC})(\mathrm{CO}) \mathrm{NH}-\mathrm{TOCSY}$ experiment (Figure 62).

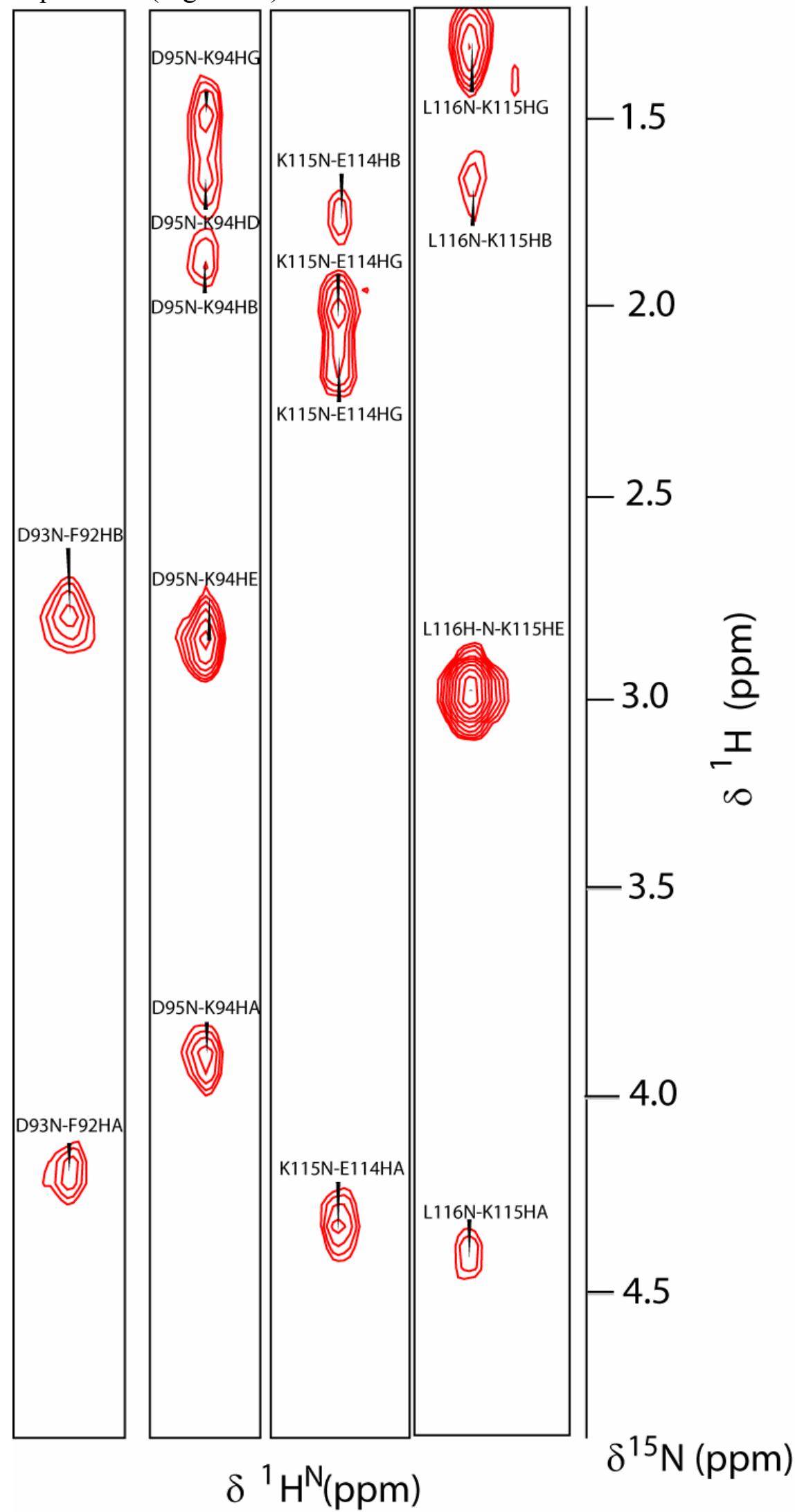

Figure 62. Strips extracted from the 3D-H(CC)(CO)NH-TOCSY spectrum to illustrate the assignment of the side-chain protons to the amide resonance in the backbone. 
To confirm and complete the side-chain resonance assignment a 3D-HCCH-TOCSY experiment was also recorded, processed and analyzed (Figure 63).

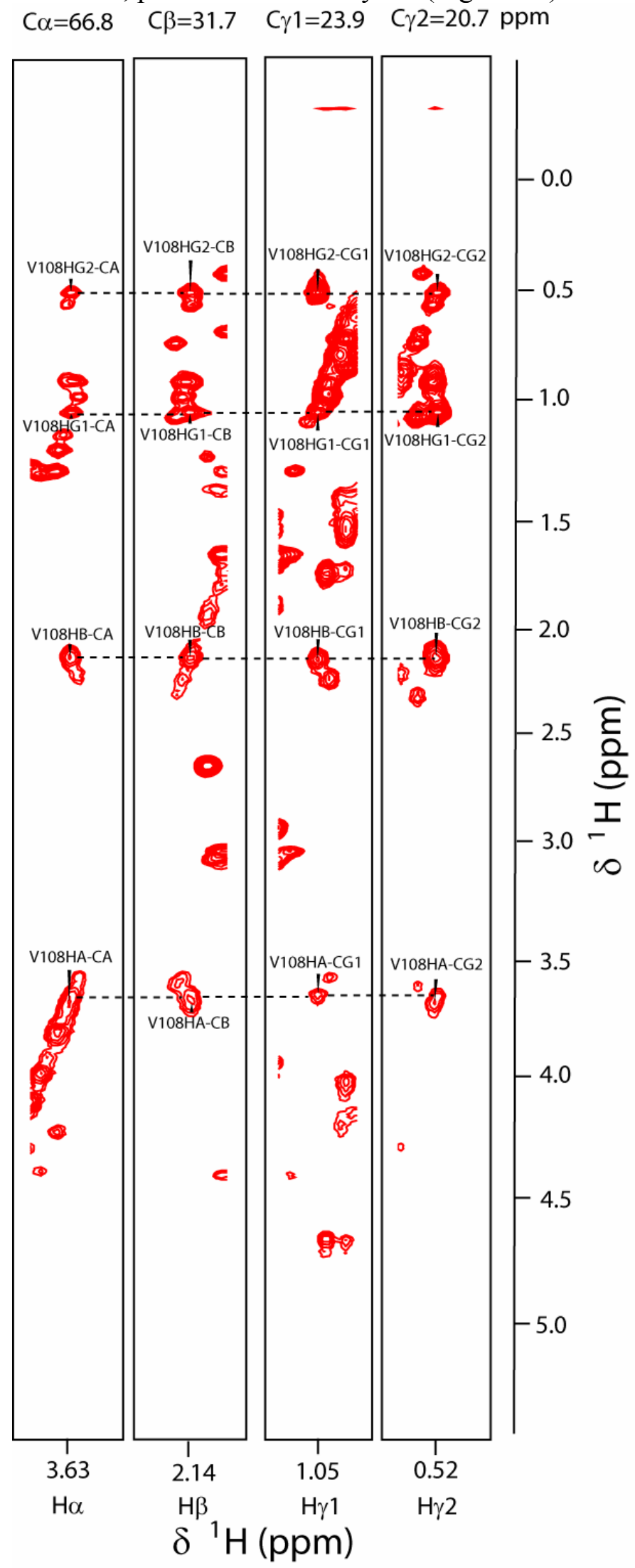

Figure 63. Strips selected from the 3D-HCCH-TOCSY spectrum to illustrate the side-chain assignment of Valine108. Unambiguous assignment can be obtained with this spectrum, since there is redundant information. 
The aromatic side-chains were assigned by the aid of two spectra named 2D$\mathrm{HBCB}(\mathrm{Caro}) \mathrm{HE}$ and 2D-HBCB(Caro)HD (Figure 64); the first one evolves the chemical shift of the $\mathrm{H} \varepsilon$ and the second one those of the $\mathrm{H} \delta$ of the aromatic side-chains; they are correlated to the $\mathrm{C} \beta-\mathrm{H} \beta$ resonance of the aromatic side-chain.

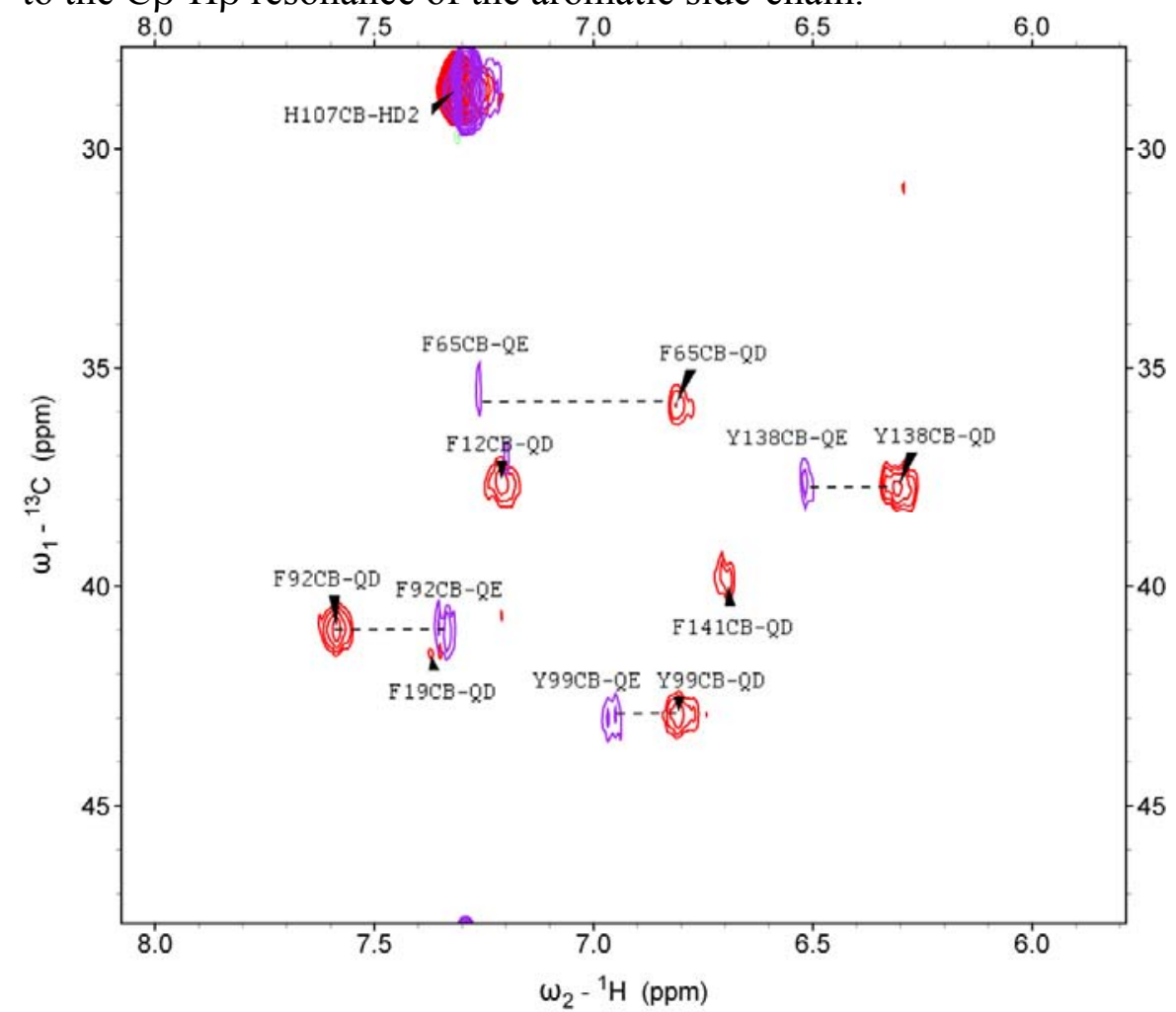

Figure 64. Overlaid 2D-HBCB(Caro) spectra for the aromatic side chain proton assignments $(\mathrm{H} \varepsilon$ and $\mathrm{H} \delta$ ). The spectrum that correlates the $\mathrm{H} \varepsilon$ proton to the $\mathrm{C} \beta-\mathrm{H} \beta$ resonance of the aromatic side-chain is shown in purple; the one that correlates the $\mathrm{H} \delta$ proton to the $\mathrm{C} \beta-\mathrm{H} \beta$ resonance is shown in red.

$\mathrm{CaM}$ has nine methionine residues in its primary sequence, well above the average for a 148 aa protein; this is because the methionine methyl side-chains are important for building plastic hydrophobic contacts with its targets. Thus, the unambiguous assignment of the $\varepsilon-$ methyl resonances of the methionines is essential for the study of protein-protein interactions involving CaM. The $\varepsilon$-methyl resonances were assigned with the aid of a HMBC type of experiment (See Figure 65) developed by Bax et al., 1994. 


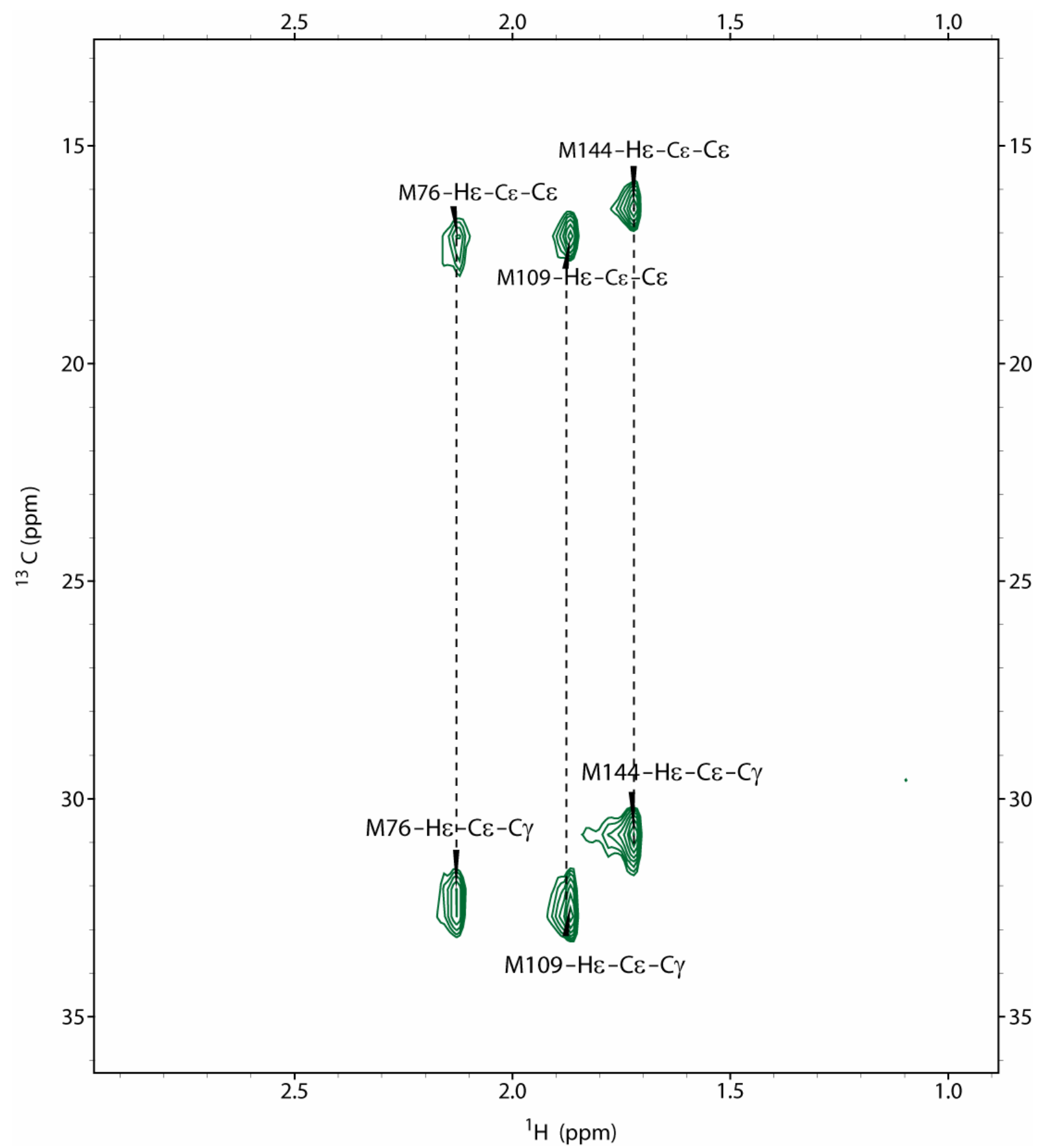

Figure 65. 3D-HMBC spectrum for methionine $\varepsilon-\mathrm{CH}_{3}$ assignment. The $\varepsilon-\mathrm{CH}_{3}$ resonance is correlated to the $\mathrm{C} \gamma$ resonance of the methionine side-chain.

i. Long-range NMR restraints for the structure determination of the CaM/Munc13-1 (457-492) peptide complex

The distance restraints for the solution structure determination of a biomolecule with NMR come primarily from NOE signals between the different protons in the biomolecule, at a distance less to $5 \AA$. Different 3D-NOESY-HSQC spectra were recorded to obtain all the restraints needed for the structure determination. 


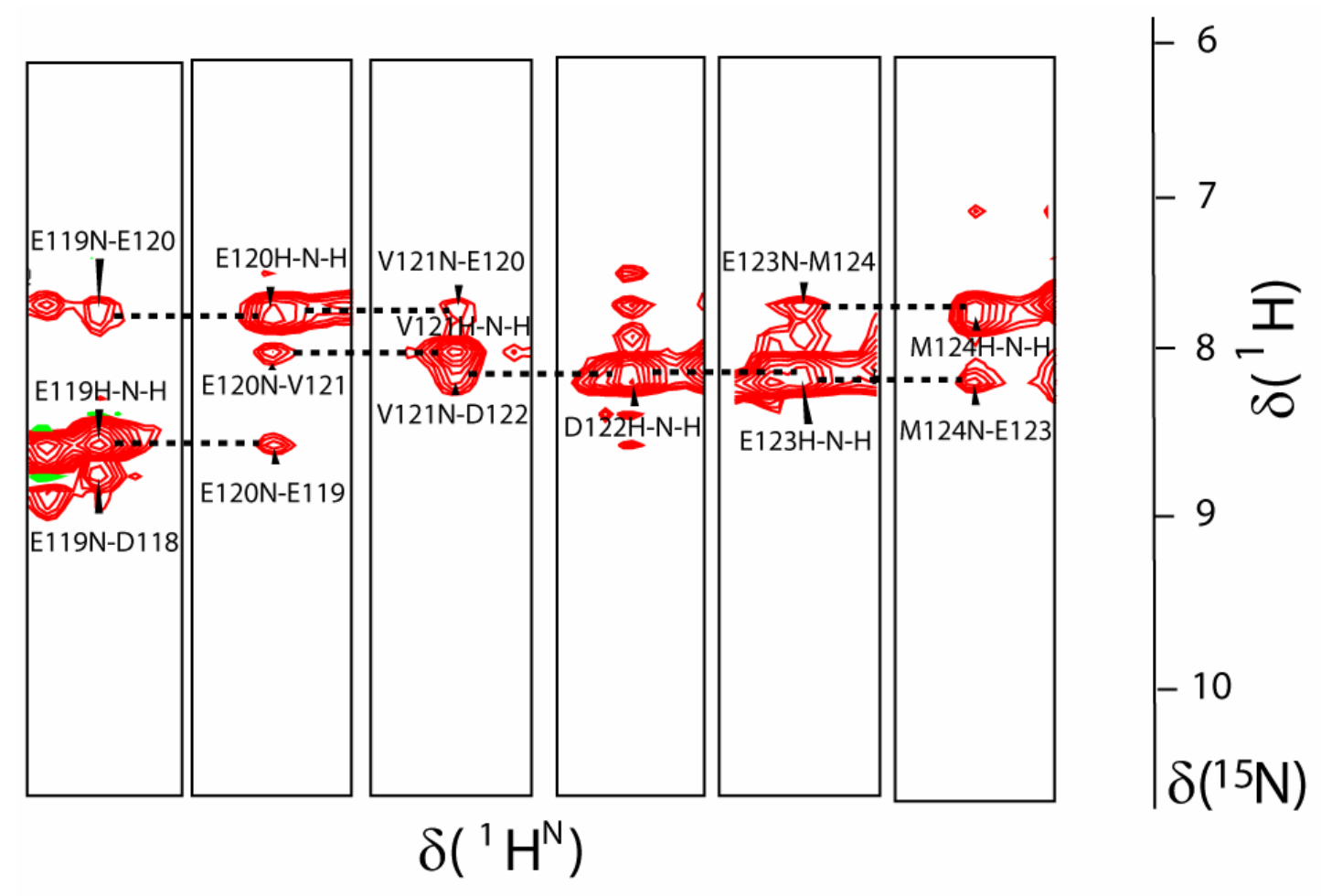

Figure 66. Selected amide cross peaks of the $3 \mathrm{D}-{ }^{15} \mathrm{~N}$ (edited) NOESY-HSQC for ${ }^{13} \mathrm{C},{ }^{15} \mathrm{~N}$ $\mathrm{CaM} / \mathrm{Munc} 13-1(459-492)$ peptide complex showing the amide sequential connectivity. 


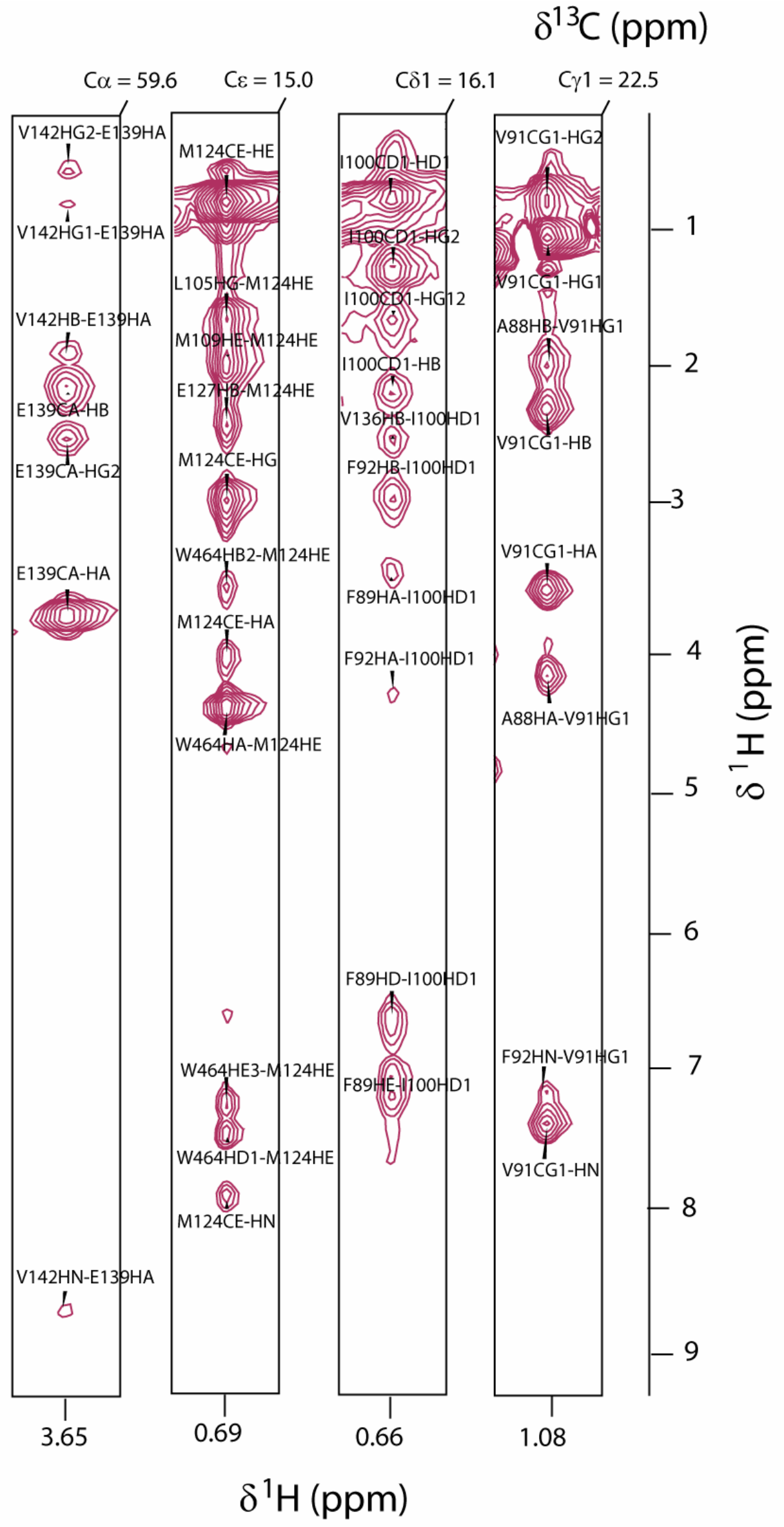

Figure 67. Selected aliphatic cross peaks of the $3 \mathrm{D}-{ }^{13} \mathrm{C}$ (edited) NOESY-HSQC for ${ }^{13} \mathrm{C},{ }^{15} \mathrm{~N}$ CaM/Munc13-1(459-492) peptide complex. 


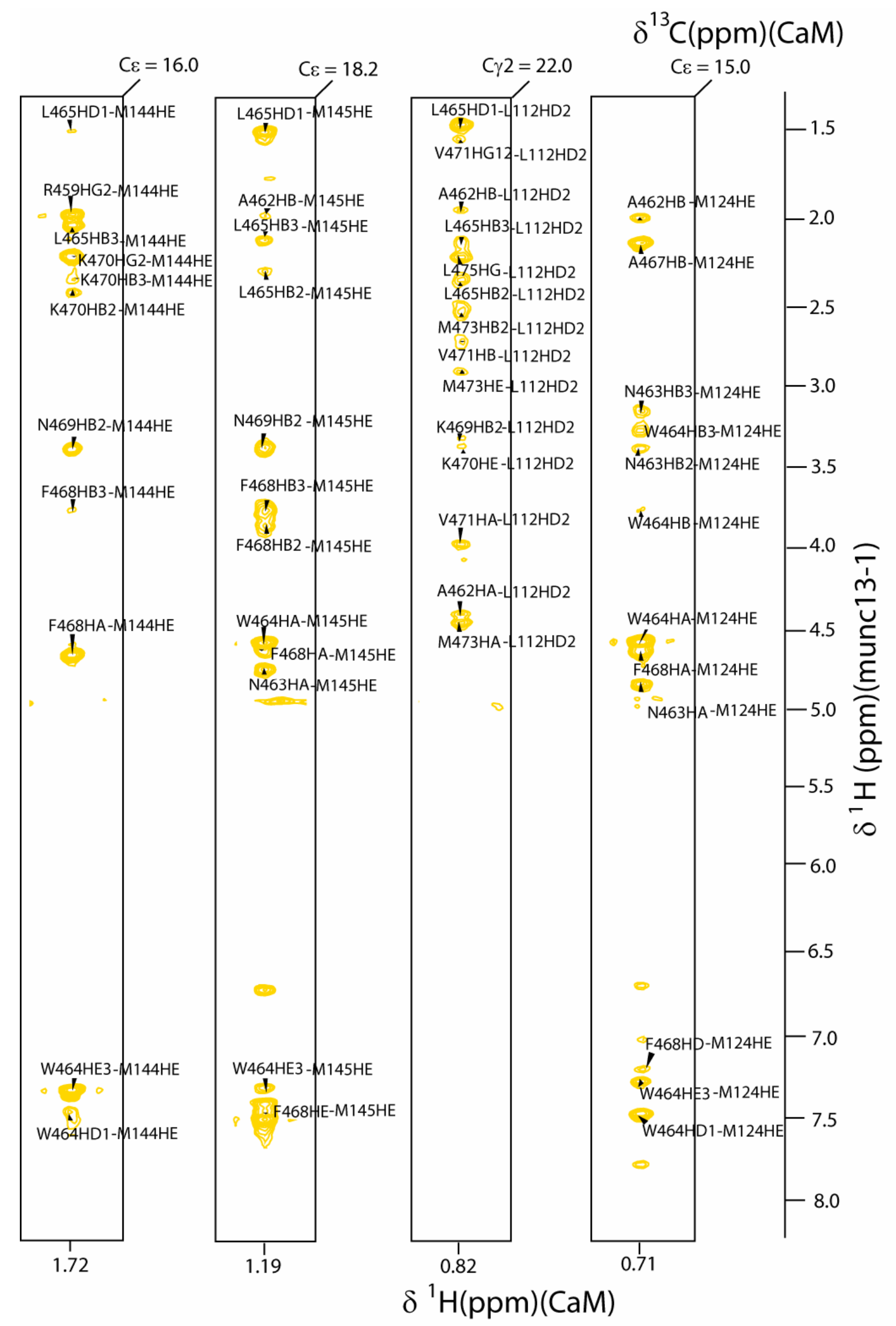

Figure 68. Selected aliphatic cross-peaks of CaM that show intermolecular NOEs to the Munc13-1(459-492) peptide in the 3D- $\left({ }^{13} \mathrm{C}-\right.$-filtered $)$-NOESY-HSQC 


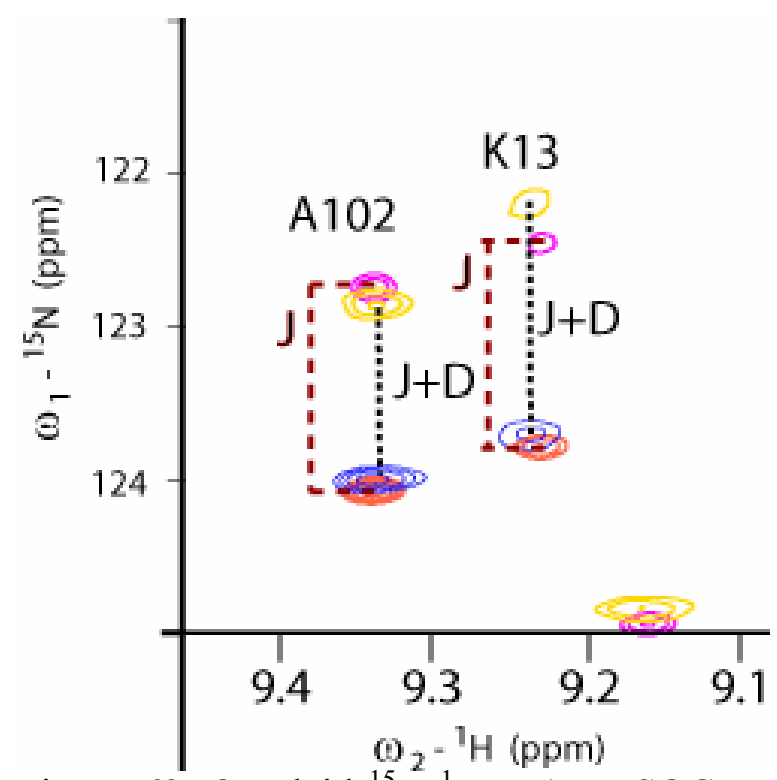

Figure 69. Overlaid ${ }^{15} \mathrm{~N}-{ }^{1} \mathrm{H}$ IPAP-HSQC spectra for ${ }^{15} \mathrm{~N}$ CaM/Munc13-1(459-492) complex (enlarged at two amide cross-peaks for illustration purposes) with bacteriophage Pf1 as cosolvent (blue and yellow) and in isotropic conditions (pink and red).

Additional long-range restraints in biomolecular NMR come from residual dipolar couplings (rDCs). To extract these restraints, a reference spectrum was measured to obtain the ${ }^{1} \mathrm{JNH}$ coupling constants and an aligned sample was used to measure the ${ }^{1} \mathrm{JNH}+{ }^{1} \mathrm{DNH}$ couplings, where the rDC information was obtained upon subtraction to the reference spectrum (see Figure 69).

Since the rDCs report on the internuclear bond orientation with respect to the static magnetic field, $\mathrm{B}_{0}$, they are very sensible to changes in the backbone conformation upon complex formation. The measured $\mathrm{NH}$ rDCs for the CaM/Munc13-1(459-492) peptide complex were compared to the simulated ones based on the refined NMR structure of free $\mathrm{CaM}$ from Chou et al., 2001. The correlation plots (Figure 70) showed that the backbone conformation of $\mathrm{CaM}$ was not perturbed much upon the binding of the peptide. This is not unexpected, since it is well known that most of the CaM-target interactions are mainly mediated by side chains.

In order to gain insight into the interdomain dynamics of the CaM/Munc13-1 (459492) peptide complex, a lanthanide-binding paramagnetic tag was attached to the C-terminal domain of $\mathrm{CaM}$ through a cysteine mutant (T146C) (Ikegami et al., 2003). The paramagnentic alignment mechanism differs from the steric one and therefore it provides nonredundant information. In the paramagnetically aligned sample, the center of alignment is localized to the unpaired electron in the lanthanide ion. For this reason, in a two-domain protein like $\mathrm{CaM}$, the alignment obtained in the domain which does not contain the lanthanide ion is reporting on the motion of this domain with respect to the statically aligned domain.

Bertini et al., 2005, reported a reduced alignment tensor by a factor of 10 (axial component) for the C-terminal domain of $\mathrm{CaM}$ when the $\mathrm{N}$-terminal domain of CaM was paramagnetically aligned using the metal binding site of $\mathrm{CaM}$ with a bound $\mathrm{Tb}^{3+}$ ion. In this study, for the CaM/Munc13-1 (459-492) peptide complex, a reduced alignment tensor by a factor of $\sim 5$ was observed (The correlation plots are shown in Figure 71). 

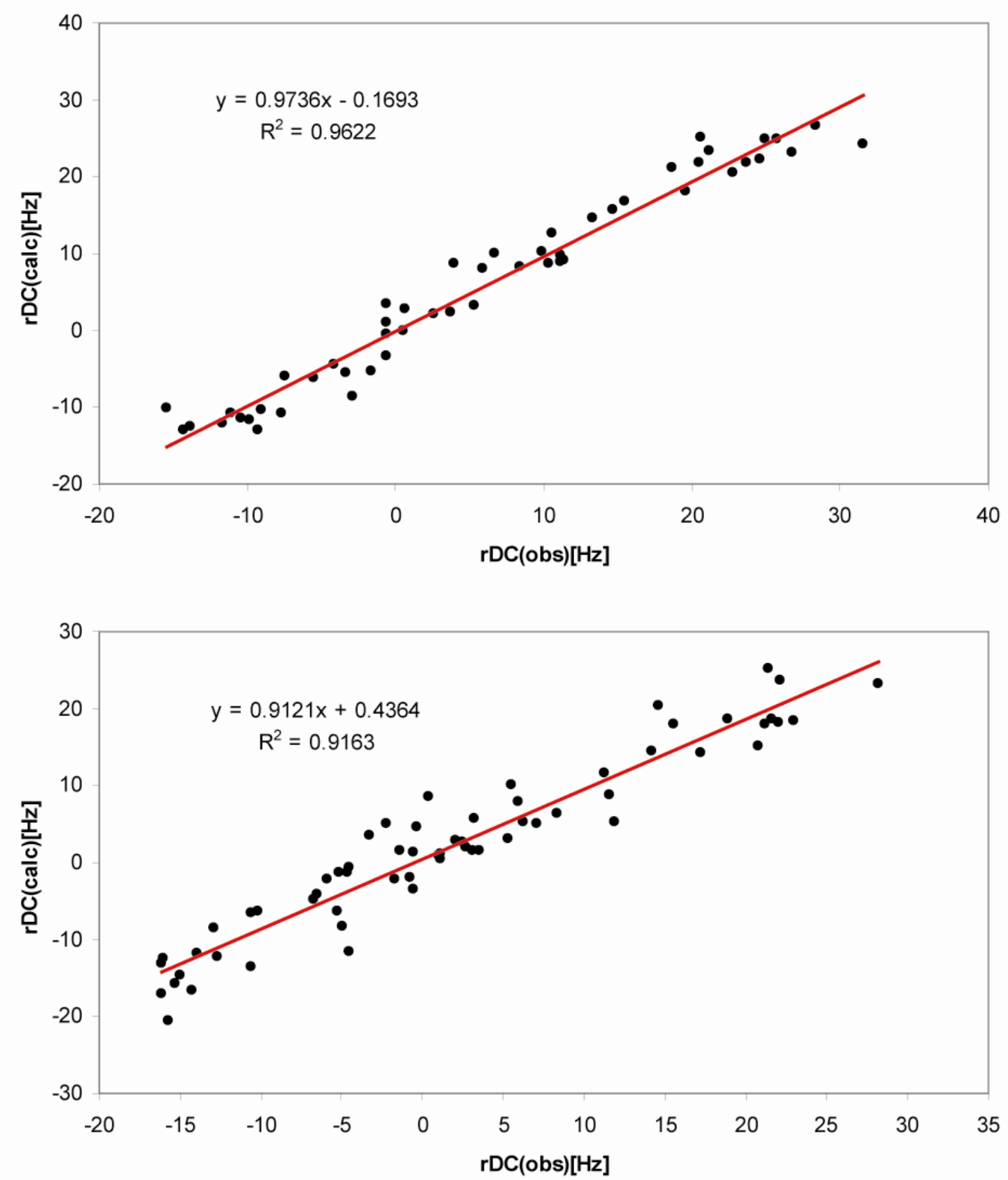

Figure 70. Correlation plots for the measured $\mathrm{NH}$ residual dipolar couplings of the ${ }^{15} \mathrm{~N}$ CaM/Munc13-1(459-492) peptide complex aligned sterically with Pf1 cosolvent against backcalculated rDCs from free CaM NMR structure. Upper plot, N-terminal domain of CaM; lower plot, C-terminal domain of CaM.

Thus, this gave an experimental proof that this complex experiences inter-domain dynamics in the sub-millisecond time scale. However, it appeared to have lesser degrees of freedom than free CaM. This is intuitive, since the complex consists of a bipartite interaction, where the Cterminal domain of $\mathrm{CaM}$ binds to the amphiphilic $\alpha$-helix of the peptide and the $\mathrm{N}$-terminal domain of $\mathrm{CaM}$ binds to the $\mathrm{C}$-terminal hydrophobic tail of the peptide. 

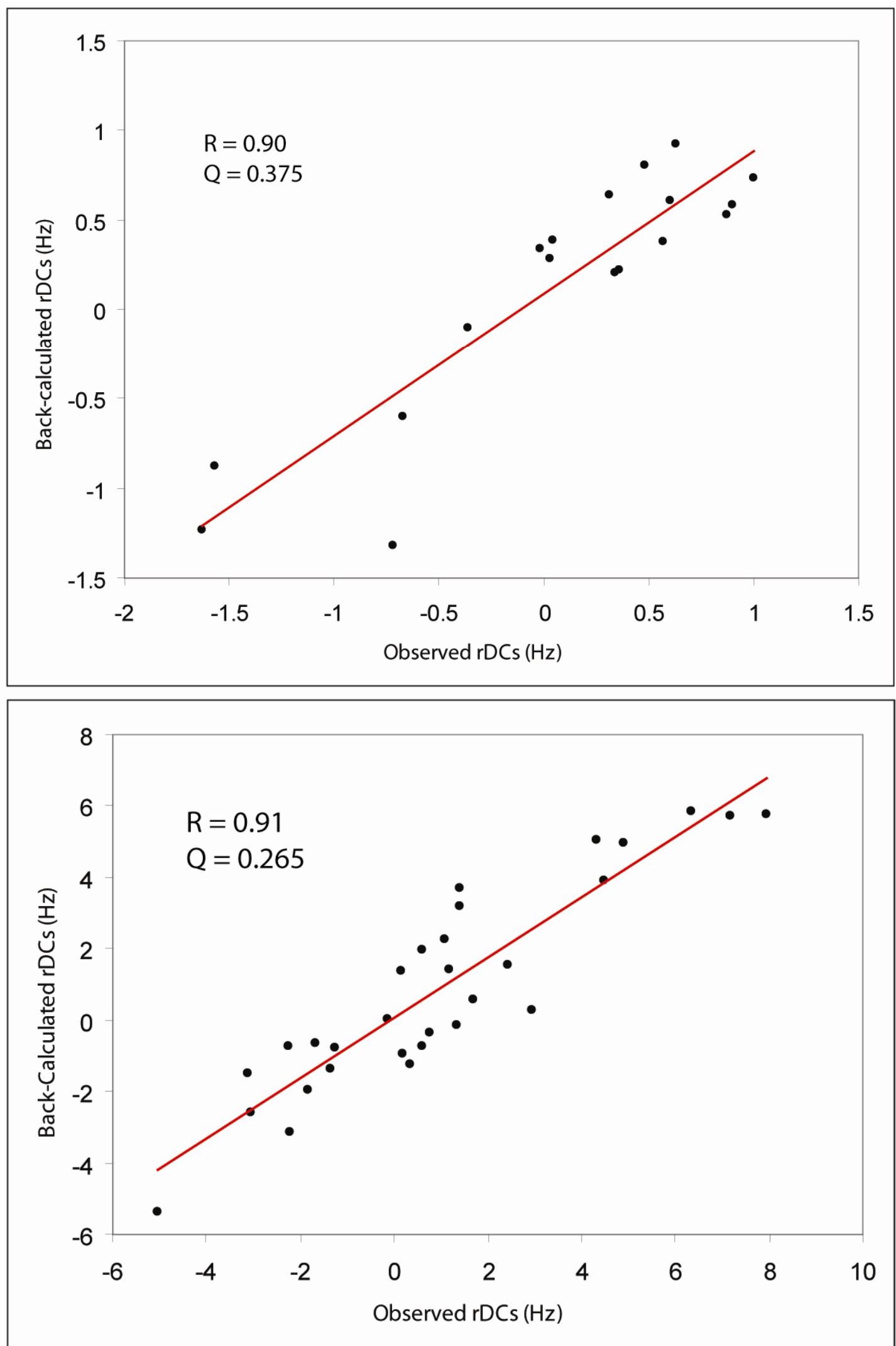

Figure 71. Paramagnetic alignment of the ${ }^{15} \mathrm{~N}$ CaM/Munc13-1(459-492) peptide complex with an EDTA-based lanthanide binding tag attached to a cysteine mutant of CaM (T146C). Upper plot: Experimental rDCs correlated against the simulated rDCc from the NMR structure of the N-terminal domain of CaM; Lower plot: Experimental rDCs correlated against the simulated rDCc from the NMR structure of the C-terminal domain of CaM. 
Since the chemical shift is sensitive to the environment of each nucleus, it was early recognized (Wishart and Sykes, 1994) that the nuclei in the different secondary structure elements experience environment effects that can be predicted. The difference of the observed chemical shifts for the $\mathrm{H} \alpha, \mathrm{C} \alpha$ and C'resonances in the structured protein to the random-coil chemical shift values are known as secondary chemical shifts. The measured secondary chemical shifts for the ${ }^{13} \mathrm{C},{ }^{15} \mathrm{~N}$ CaM/ Munc13-1 (459-492) complex predicted very well the secondary structure elements of CaM (See Figure 72). All secondary structure elements of $\mathrm{CaM}$ were preserved upon peptide binding; this is in agreement with the rDC analysis discussed above.

For the CaM bound ${ }^{13} \mathrm{C},{ }^{15} \mathrm{~N}$ Munc13-1 (457-492) peptide, the secondary chemical shift plot (Figure 73) predicted a single $\alpha$-helix in the N-terminal part of the peptide. This is in agreement with the amphiphilic $\alpha$-helix binding model of CaM targets.

The total number of NOE constraints is shown in Figure 74 and the NOE contacts along the polypeptide sequence are summarized in Figure 75. The contact map gave a picture on the long-range contacts (i.e., the tertiary structure) of this protein-peptide complex. From the diagram it was evident that both domains of $\mathrm{CaM}$ are independent and the Munc13-1 peptide had contacts mainly to the $\mathrm{C}$-terminal domain of $\mathrm{CaM}$. However there were also some contacts from the $\mathrm{C}$-terminal part of the peptide to the $\mathrm{N}$-terminal domain of $\mathrm{CaM}$

Another representation of the NOE contacts consists of horizontal bars with different thickness (Figure 76), representing the cross-peak intensity, between single amino acid residues representing the proton-proton contacts within the secondary structure elements of the polypeptide.

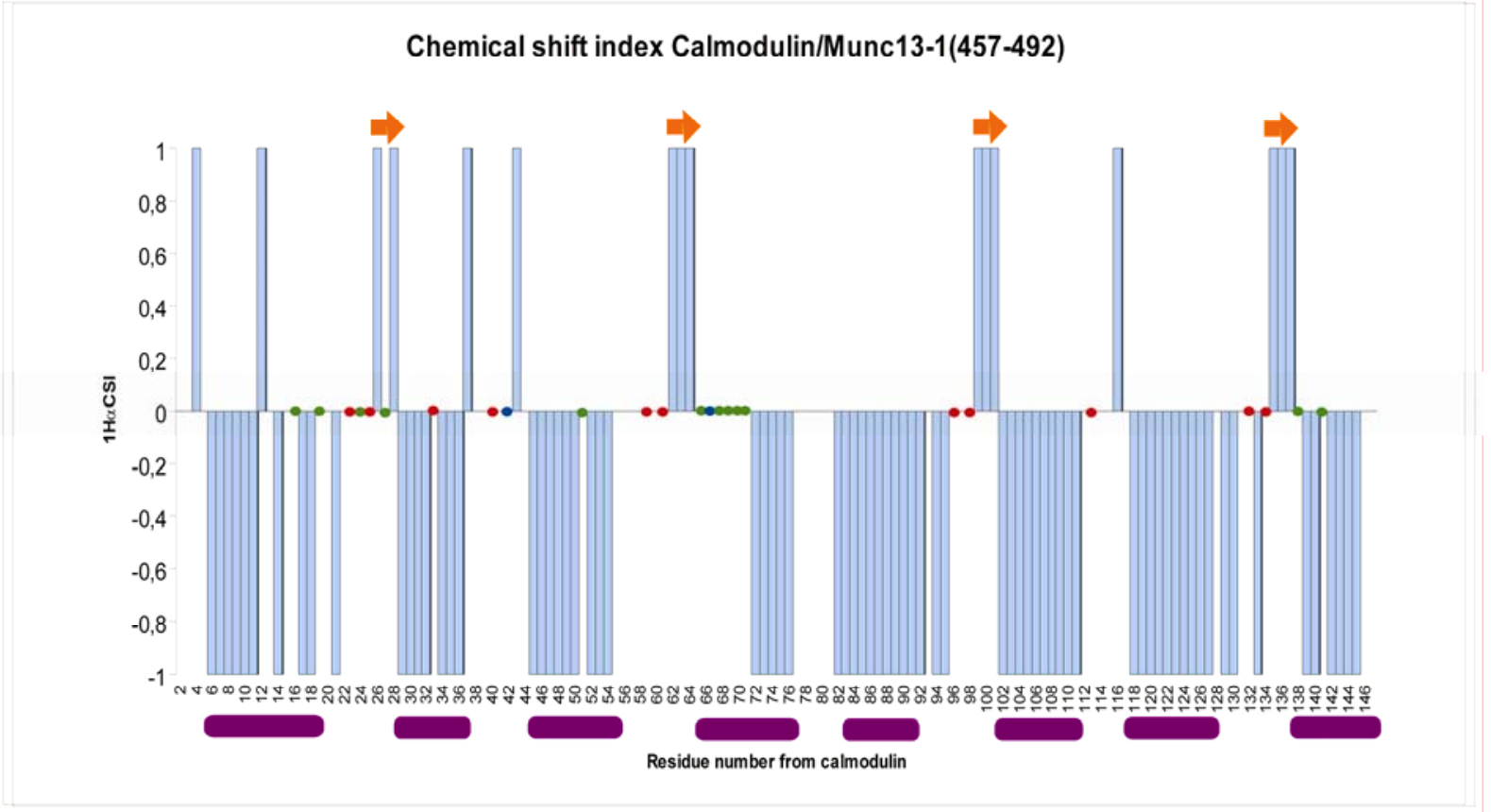

Figure 72. Secondary chemical shift for the $\mathrm{H} \alpha$ resonance of $\mathrm{CaM}$ in complex with the Munc13-1(459-492) peptide. The green dots are missing $\mathrm{H} \alpha$ assignments, the red dots are glycines and the blue dots are missing $\mathrm{H} \alpha$ in the residues preceding a proline. The $\alpha$-helices in $\mathrm{CaM}$ are represented by purple bars and the $\beta$-sheets by orange arrows. 


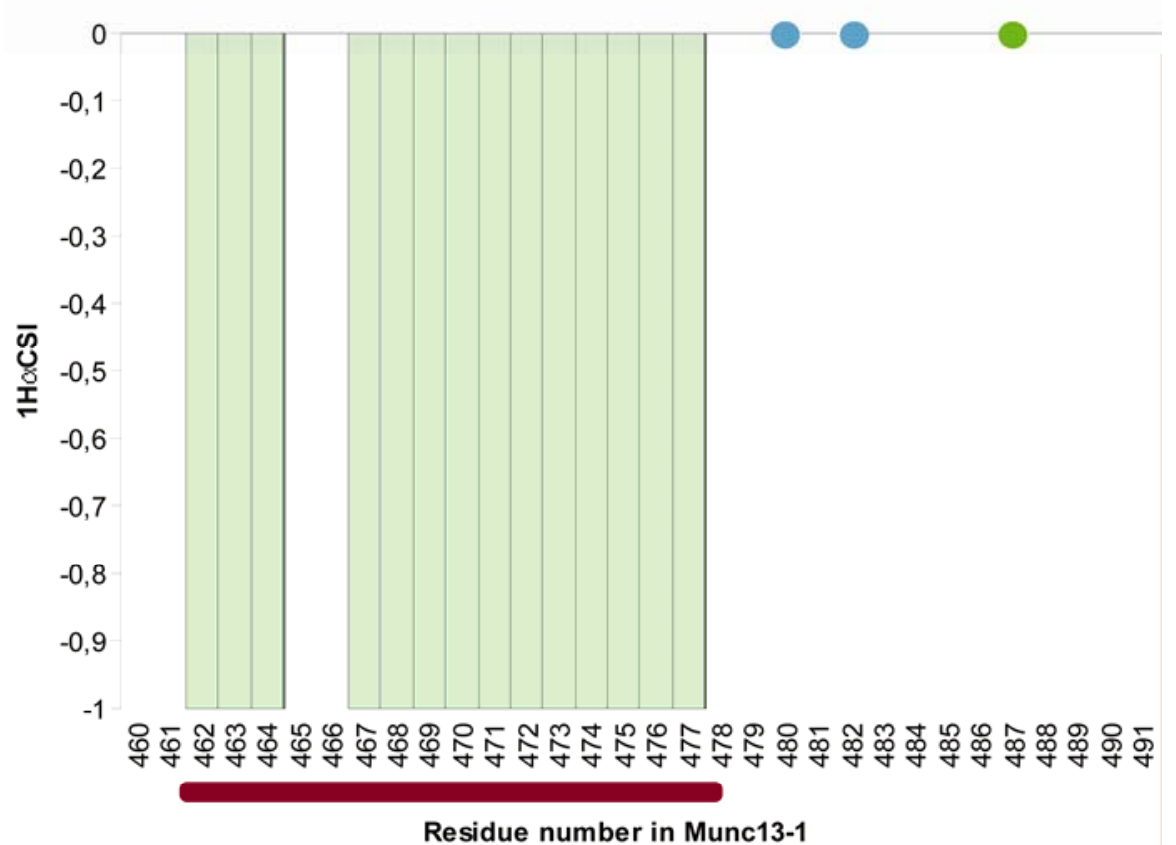

Figure 73. Secondary chemical shift for the Ho resonance of the ${ }^{13} \mathrm{C},{ }^{15} \mathrm{~N}$ Munc13-1(457-492) bound to $\mathrm{CaM}$. The blue dots are glycines and the green dot is a missing $\mathrm{H} \alpha$ resonance. The $\alpha$-helix is found between residues $462-477$.

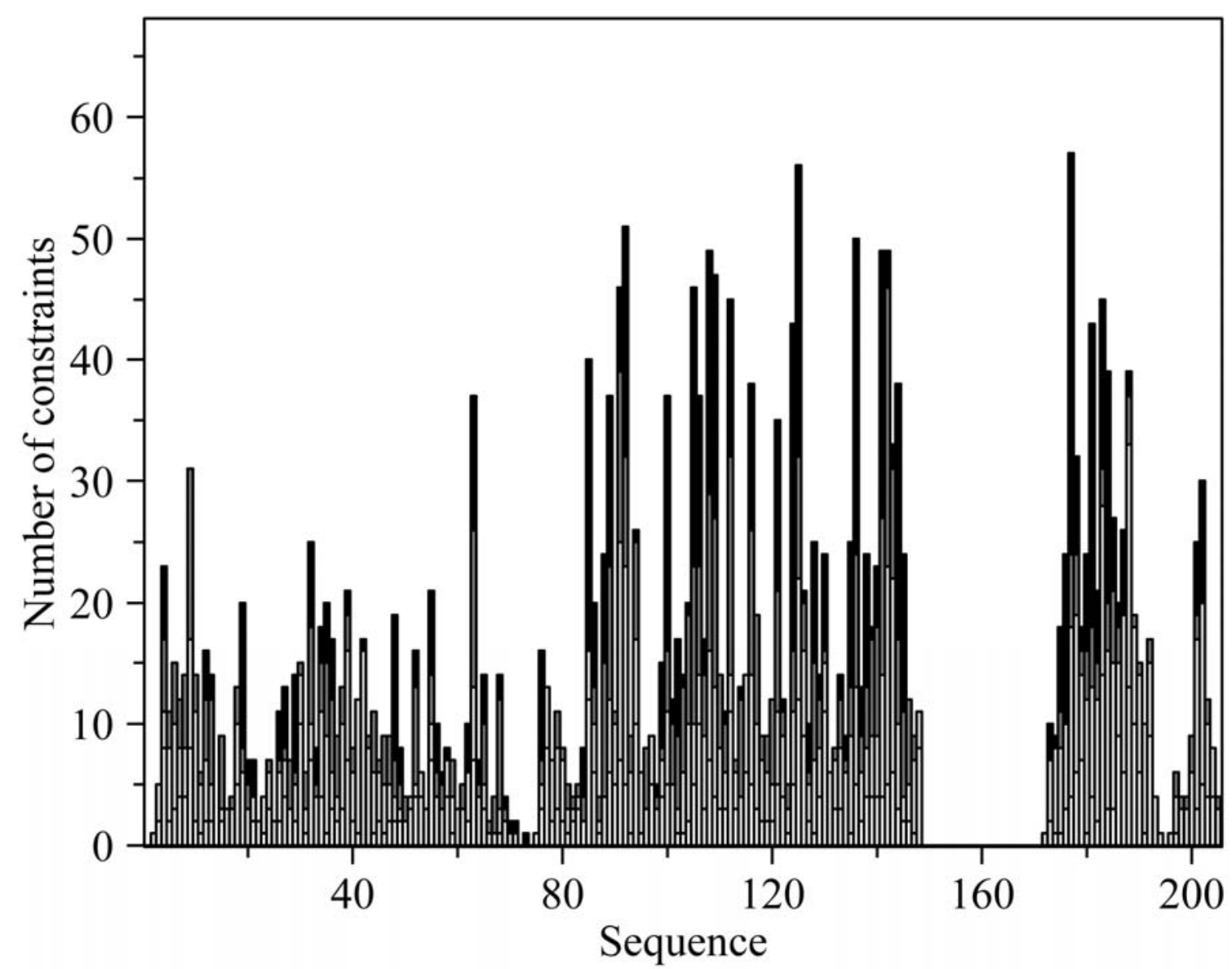

Figure 74. Number of NOE restraints per amino acid residue in CaM and the Munc13-1(457492) peptide. The white bars represent intra-residue NOEs; the light grey bars, short-range NOEs; the dark grey bars medium-range NOEs; and the black bars long-range NOEs. 


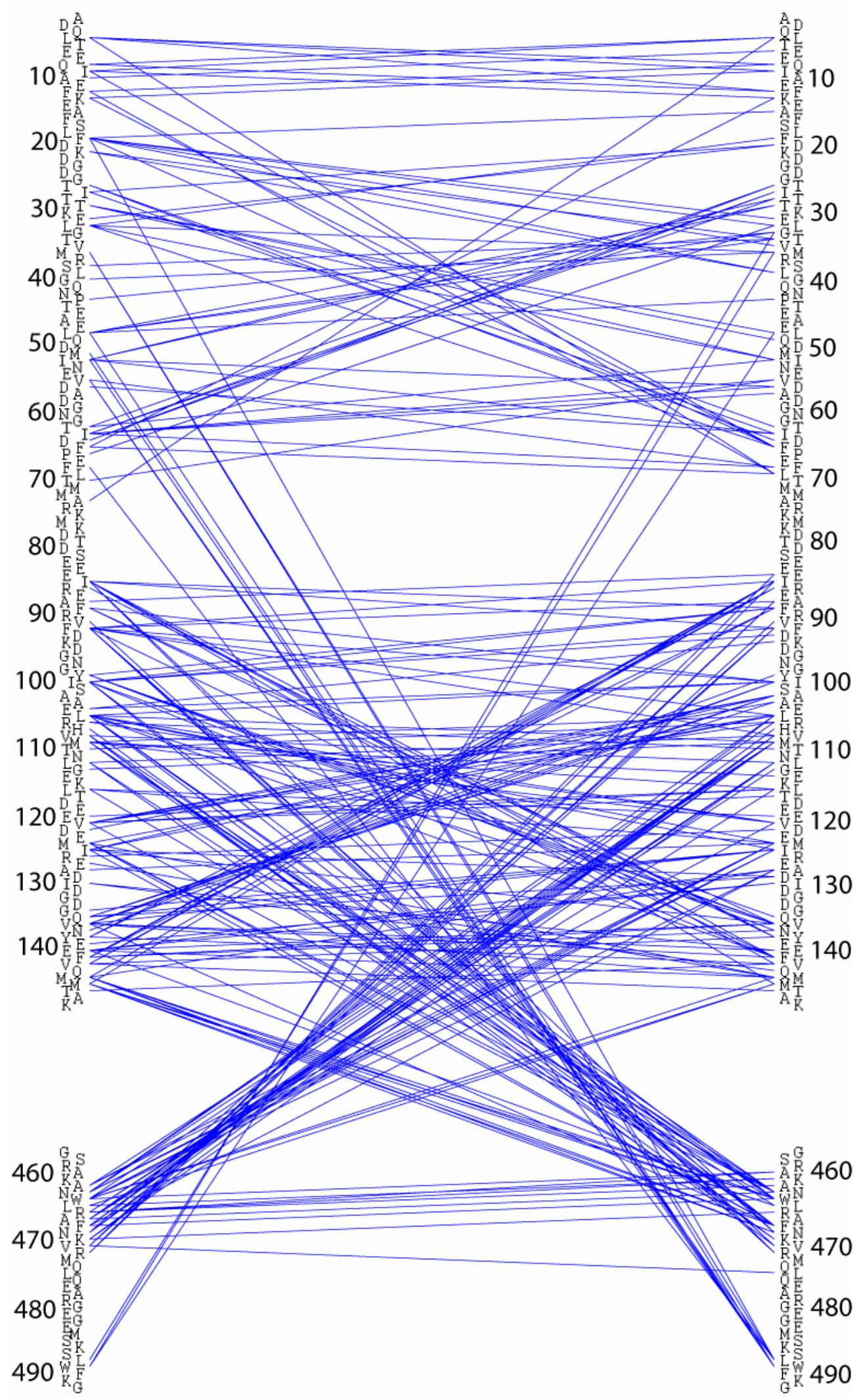

Figure 75. NOE contact map for the CaM/Munc13-1(457-492) peptide complex, these restraints were used for the NMR structure determination of the complex. 


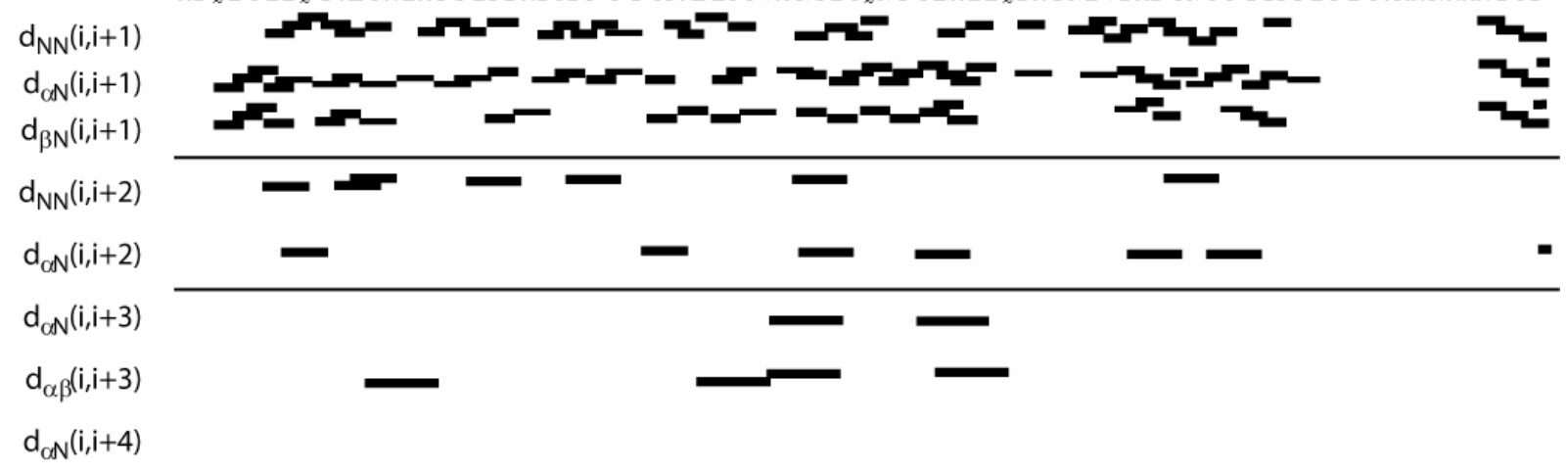

90

100

110

120

130

140

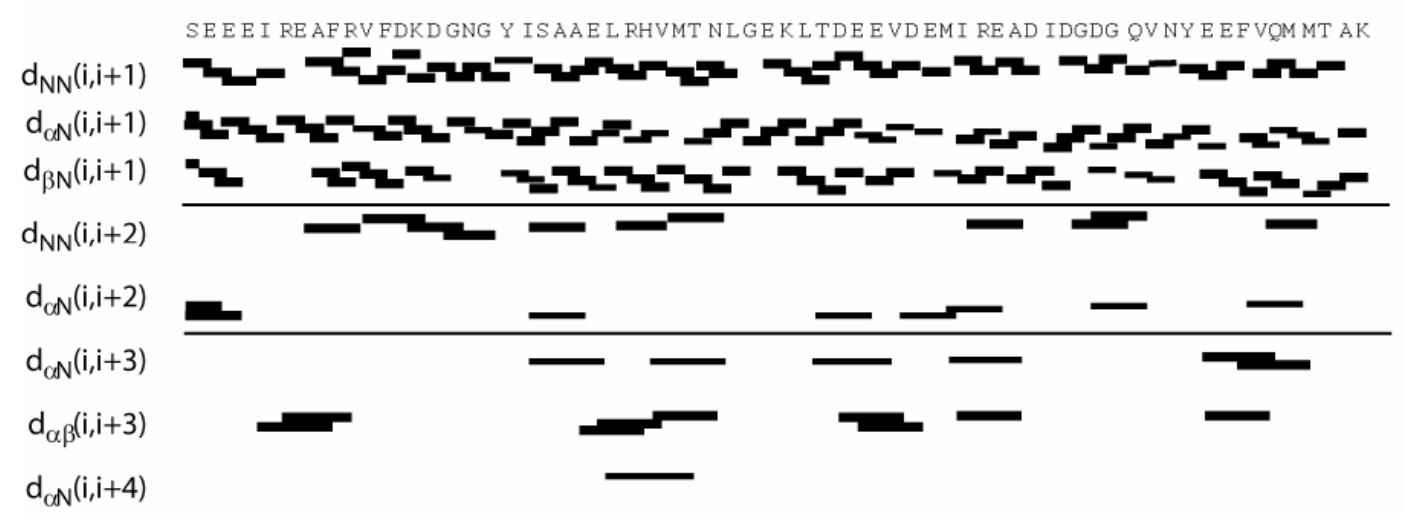

$457 \quad 467 \quad 477 \quad 487$

GSRAKANW LRA FNKVRMQLQEARGEGEM SKSLWFKG

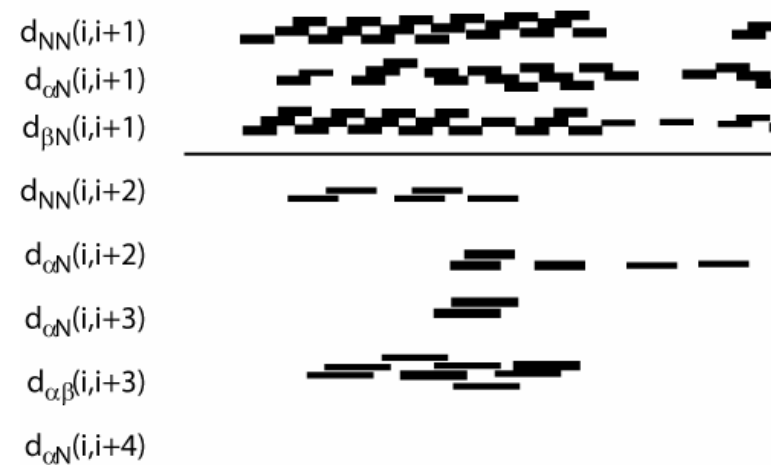

Figure 76. Survey of the sequential and medium range ${ }^{1} \mathrm{H}-{ }^{1} \mathrm{H}$ NOEs along the primary sequence of $\mathrm{CaM}$ (the first two panels) and the Munc13-1 peptide (457-492) (lowest panel) in the complex.

\section{j. NMR structure determination of the CaM/Munc13-1 (457-492) peptide complex}

The 3D structure of CaM has been extensively studied both by X-ray crystallography and NMR; there are numerous structures of the free protein as well as in complex with various interaction partners. The correlation of the $\mathrm{NH}$ residual dipolar couplings for the 
individual domains of $\mathrm{CaM}$ in the complex was not very much perturbed, i.e. there is still a high correlation to the free CaM NMR structure. Therefore, the folded individual domains were a good starting point for a structure calculation.

In the literature, no high-resolution structural information on the Munc13-1 (457-492) region was found. Based on the knowledge of the various $\mathrm{CaM}$ - peptide interactions described and the studies by Junge et al., (2004), it was predicted that the Munc13-1 (457492) could have an amphiphilic $\alpha$-helix. In addition, several lines of evidence were found for the $\alpha$-helical structure of the bound peptide: (a) the H $\alpha$ secondary chemical shift predicted an $\alpha$-helix between residues 462 and 477; (b) the phi and psi angles derived from the TALOS restraints also lied in the alpha helical part of the ramachandran plot; (c) the sequential NOEs for the $\mathrm{NH}$ and $\mathrm{H} \alpha$ have a $\alpha$-helix pattern and the rDCs also agreed well with the $\alpha$-helix model.

The next step was to generate various backbone conformation clusters for the Munc13-1 (457-492) peptide in order to use them for the docking with CaM(see Figure 77). The clusters were generated with the Rosetta program, based on the secondary structure prediction from PSI, the NH NOEs and the NH rDCs. In essence, all of the clusters predicted a $\alpha$-helical structure; they differed just in the length of the $\alpha$-helix. For the docking of the $\mathrm{CaM} /$ munc13-1(457-492) interaction based on intermolecular NOEs and rDCs, a cluster that consisted of a $\alpha$-helix going from residue 461 to residue 478 and a random-coil C-terminal tail was selected. The side-chains were modelled in the program INSIGHT II (Accelerys, Inc.) and an energy minimization with the CHARMM force field (Brooks et al., 1983) was done in order to avoid steric clashes among the side-chains.
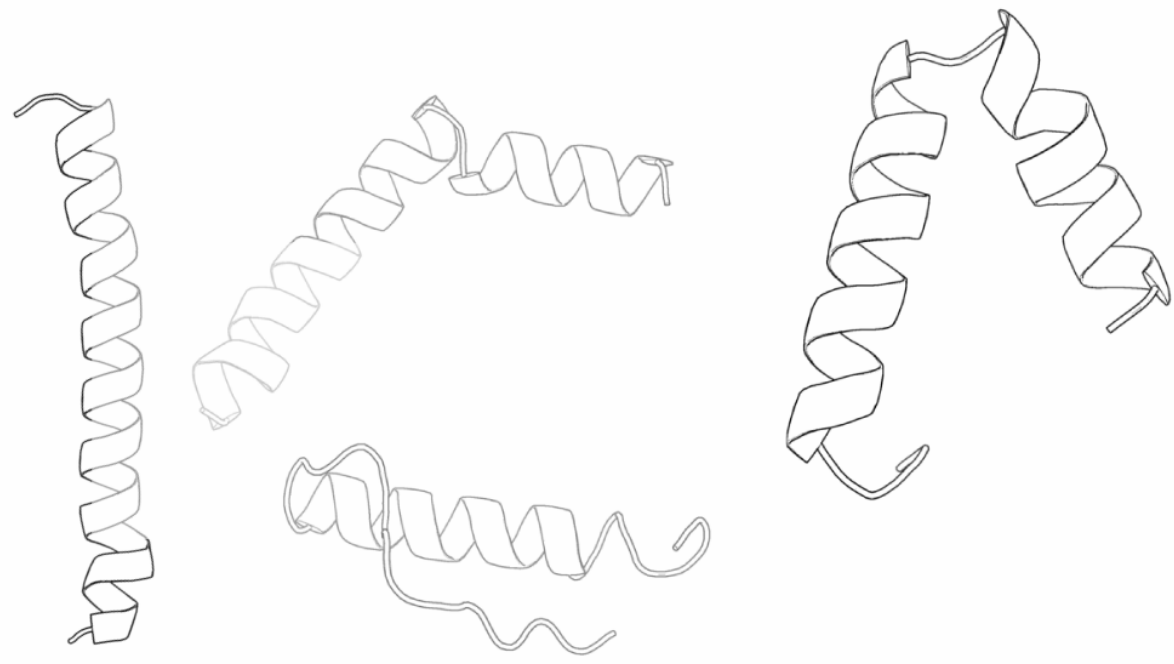

Figure 77. Different models for the backbone conformation of the Munc13-1(457-492) peptide based on NOE, NH residual dipolar couplings and chemical shift derived dihedral angles restraints from Rosetta program. The conformation on the lower part was used for the docking.

The docking protocol consisted of a rigid body minimization using a target function comprising only three terms, the experimental NOE-derived intermolecular interproton distances, the dipolar coupling restraints, and a simple intermolecular van der Waals repulsion potential (Clore, 2000). The restraints used were 35 intermolecular NOEs, and $31 \mathrm{NH}$ rDCs for C-terminal domain of CaM and 14 rDCs for the bound Munc13-1 (457-492) peptide. The docking gave a first view of the complex between CaM and Munc13-1 (457-492) (Figure 78). This docked structure was used as a starting point for the structure calculation based on CYANA. 
Automated NOESY assignment replaces the most time-consuming part of the interactive spectral analysis by a fast computational method and has thus significantly enhanced the overall efficiency of NMR structure determination (Jee and Güntert, 2003). A limiting factor for the application of these automated NOE assignment procedures is that they rely on the availability of an essentially complete list of chemical shifts from the preceding sequence-specific resonance assignment. For reliable automated NOESY assignment with the CANDID algorithm around $90 \%$ completeness of the chemical shift assignment is necessary, whereas the algorithm is remarkably tolerant with respect to incomplete NOESY peak picking. Another important pint is that the backbone root mean square deviation (RMSD) to the mean for the structure bundle of cycle 1 is below $3 \AA$.

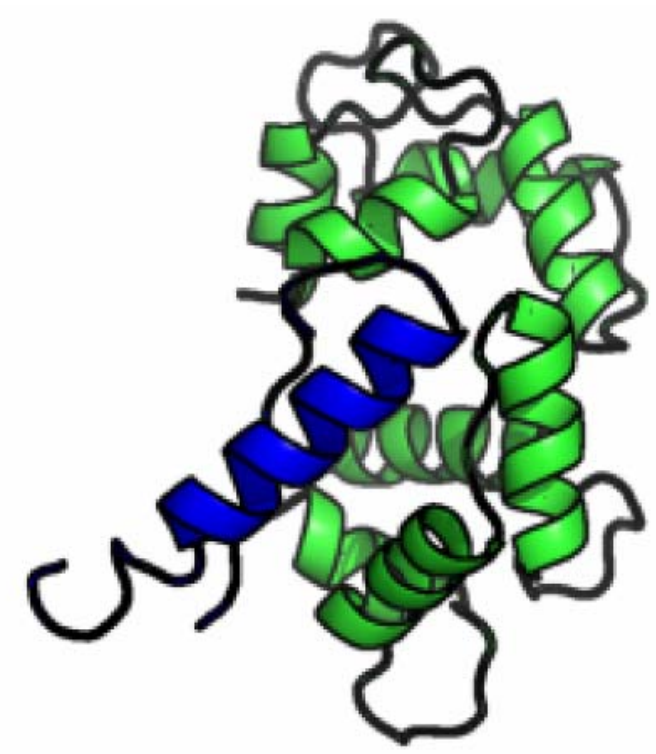

Figure 78. Model of the CaM/Munc13-1 docked structure based on intermolecular NOEs and $\mathrm{NH}$ residual dipolar couplings. CaM is shown in green and the Munc13-1(457-492) peptide in blue.

Initially it was attempted to do a structure calculation with full length $\mathrm{CaM}$ and the full length Munc13-1 (457-492) peptide; however the results were suboptimal, especially for the N-terminal domain of CaM (see Figure 79). For this reason, further calculations were done with the two domains of $\mathrm{CaM}$ as separate modules interacting with different regions in the Munc13-1 peptide; this approach was also consistent with the bipartite interaction of this complex. An ensemble of structures is shown in Figure 80.

The C-terminal domain of CaM interacts with the amphiphilic $\alpha$-helix of the Munc131 peptide and the hydrophobic anchors consist of the well studied motif 1-5-8 (W-F-V). A view into this hydrophobic pocket is shown in Figure 81.

For the N-terminal domain of CaM, the interaction with the C-terminal tail of the Munc13-1 peptide was found to be reduced to a hydrophobic motif L488 and W489 (see Figure 82). The interaction appeared to have a smaller surface area than in the case of the Cterminal domain of CaM.

After the structure calculation was optimized, the structures were refined with $\mathrm{NH}$ rDCs and their energy was minimized in explicit solvent. The ramachandran plot for the best structures is shown in Figure 83. In addition, the structure was analyzed by the PROCHECKNMR program (Laskowski et al., 1996) and the structure statistics are shown in table 5.

The EF-hand domains in CaM show the ability to occupy different conformations depending on the environment, i.e., on the presence or absence of bound calcium ions and/or target proteins. As each EF-Hand motif as a helix-loop-helix structure, the interhelical angles have been proposed as structural parameters. All structural analyses on EF-Hand domains 
suggest that a continuum of conformational states is occurring within the whole EF-Hand protein superfamily. In Table 6, the six-interhelical angles for the $\mathrm{N}$ - and $\mathrm{C}$ - terminal domains of the CaM/Munc13-1(457-492) peptide complex are compared with other ligated EF-hand states (taken from Babini et al., 2005). The model used for measuring the interhelical angles is shown in Figure 84.
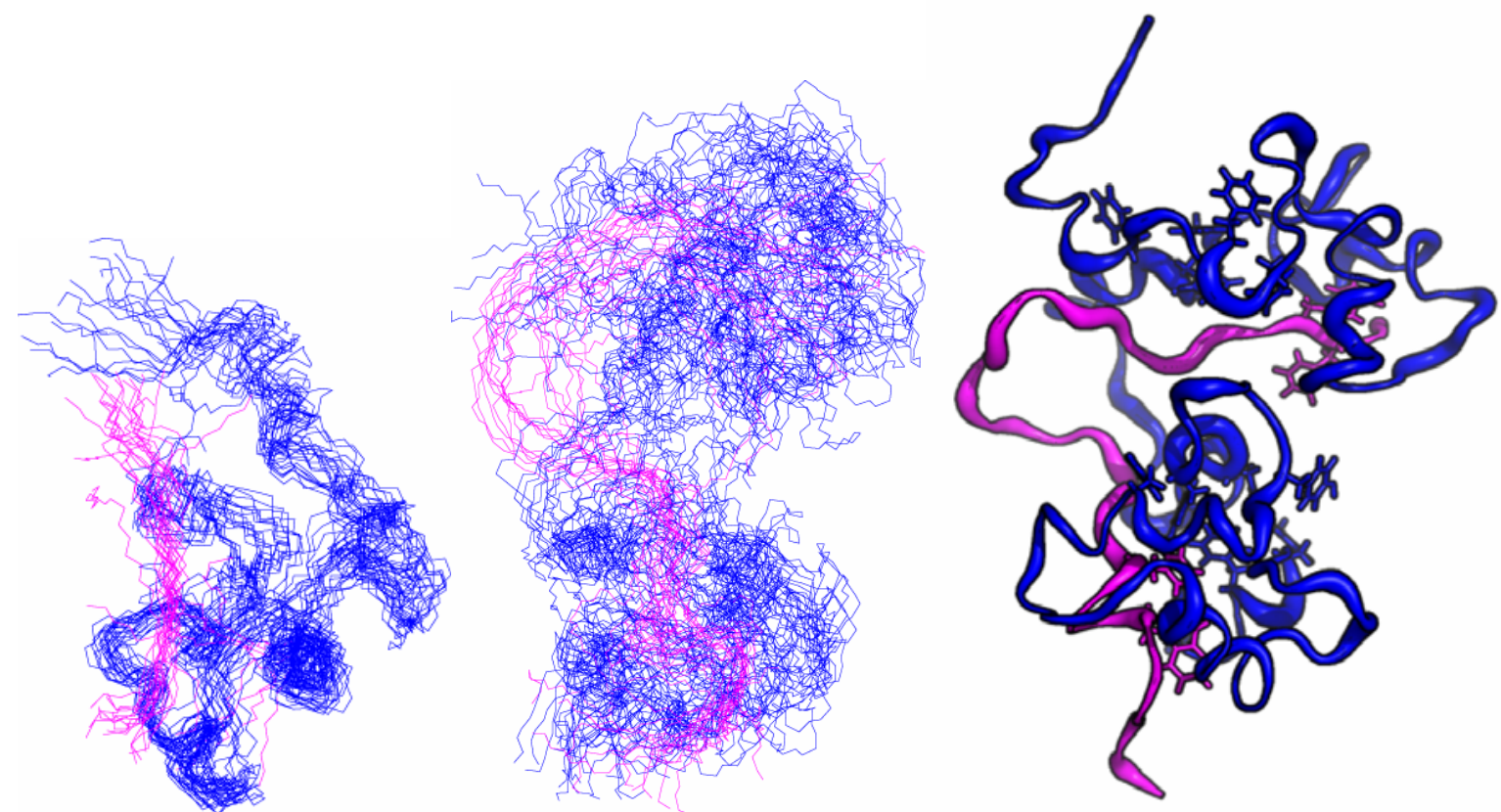

Figure 79. Ensemble of 20 structures for the CaM/Munc13-1(457-492) peptide complex calculated with CYANA using the full-length CaM and peptide restraints. On the left, the Nterminal domain was aligned. On the center, the whole complex is shown and on the right side a cartoon representation of the whole complex is shown, CaM (blue), peptide (magenta).

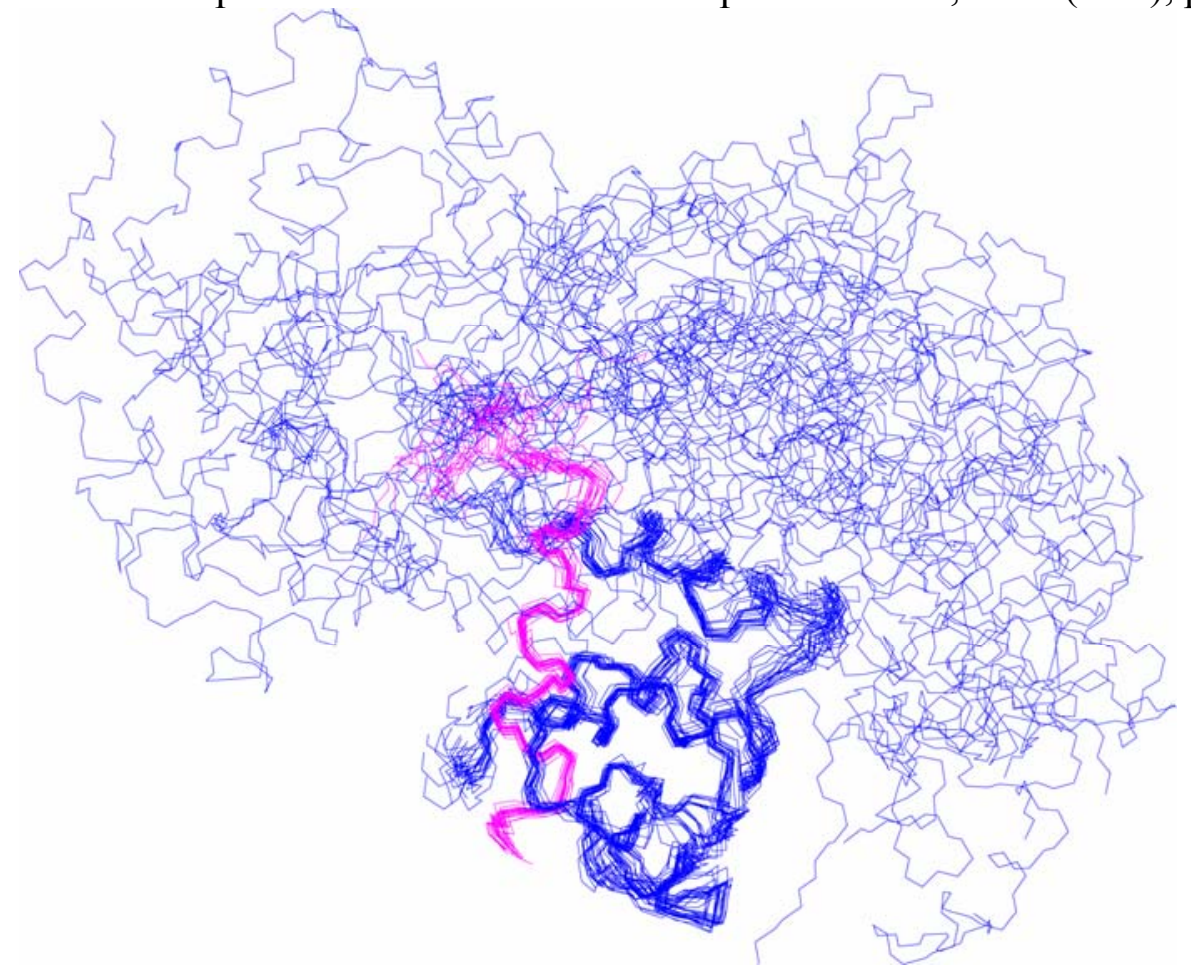

Figure 80. Ensemble of 20 structures of the CaM/Munc13-1 (457-492) peptide complex calculated with the C-terminal domain distance constraints for $\mathrm{CaM}$ and full-length distance constraints for the peptide in CYANA. CaM is shown in blue and the Munc13-1 (457-492) peptide is shown in magenta. 

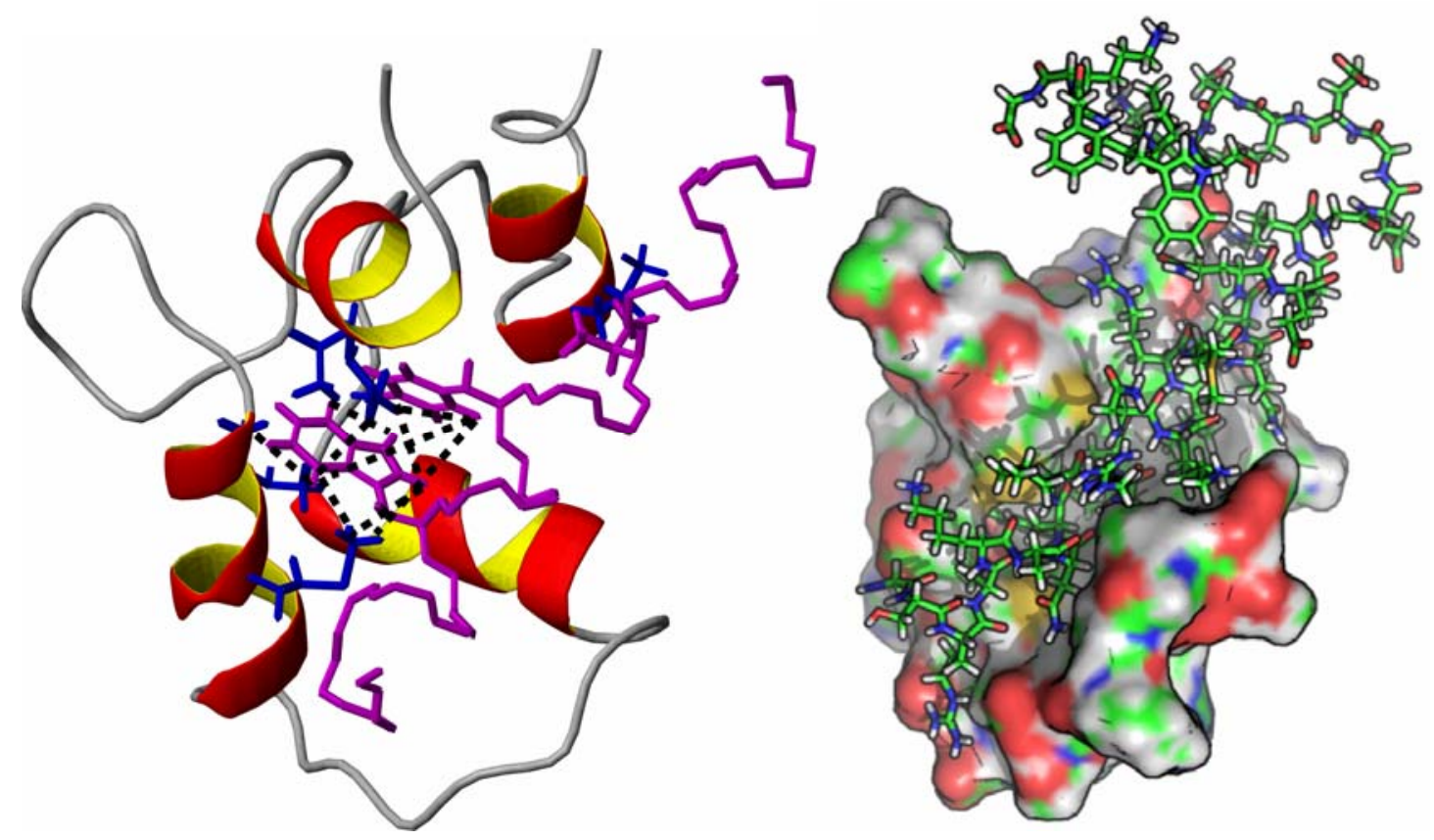

Figure 81. Ribbon (left) and surface (right) representation of the C-terminal domain of CaM bound to the N-terminal amphiphilic $\alpha$-helix of the Munc13-1 (457-492) peptide in magenta. Some of the side-chain contacts derived from intermolecular NOEs between CaM and the hydrophobic anchors W464 and F468 of Munc13-1 are shown with dotted lines
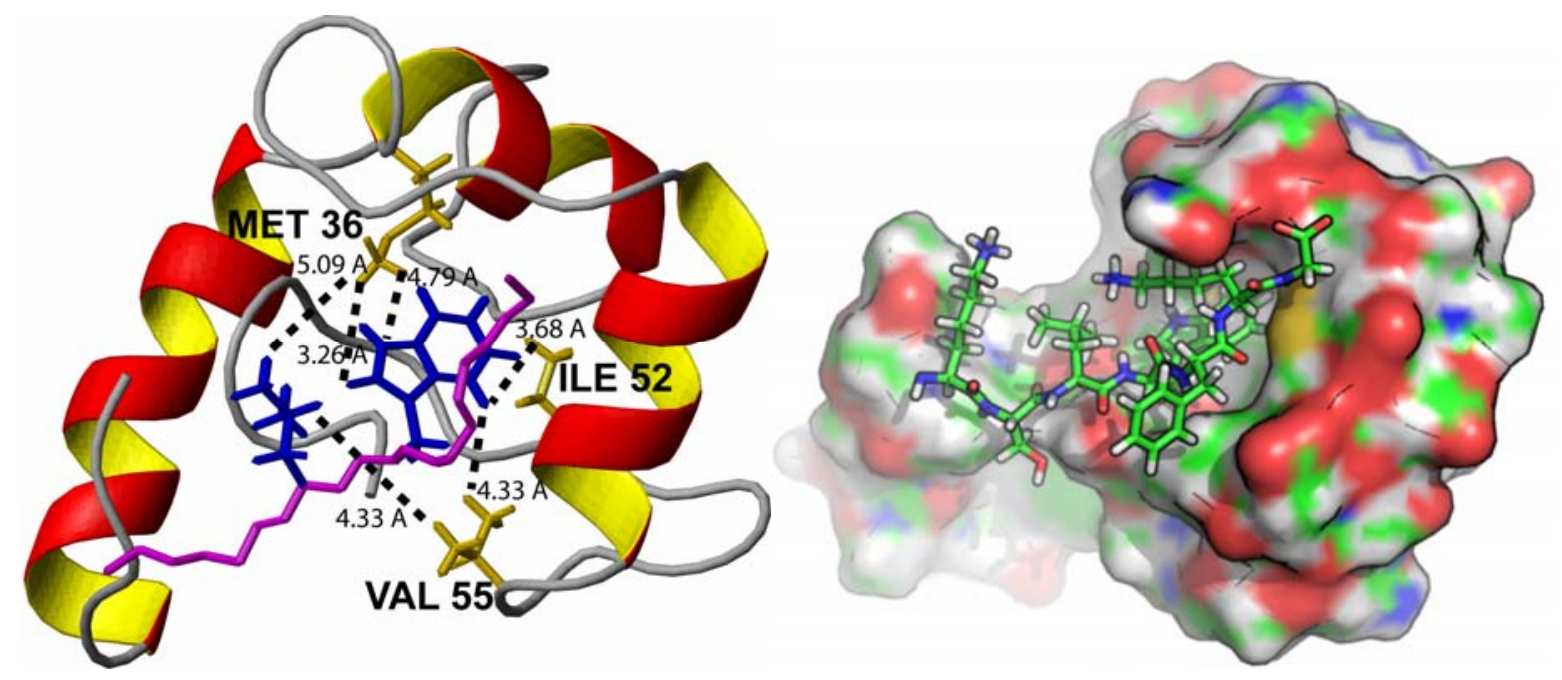

Figure 82. Ribbon (on the left) and surface (on the right) representations for the N-terminal domain of CaM bound to the C-terminal part of the Munc13-1 (457-492) peptide in magenta. Some of the side-chain contacts derived from intermolecular NOEs between CaM and the hydrophobic L488, W489 motif of Munc13-1 are shown with dotted lines and the distance in Á is also depicted. 


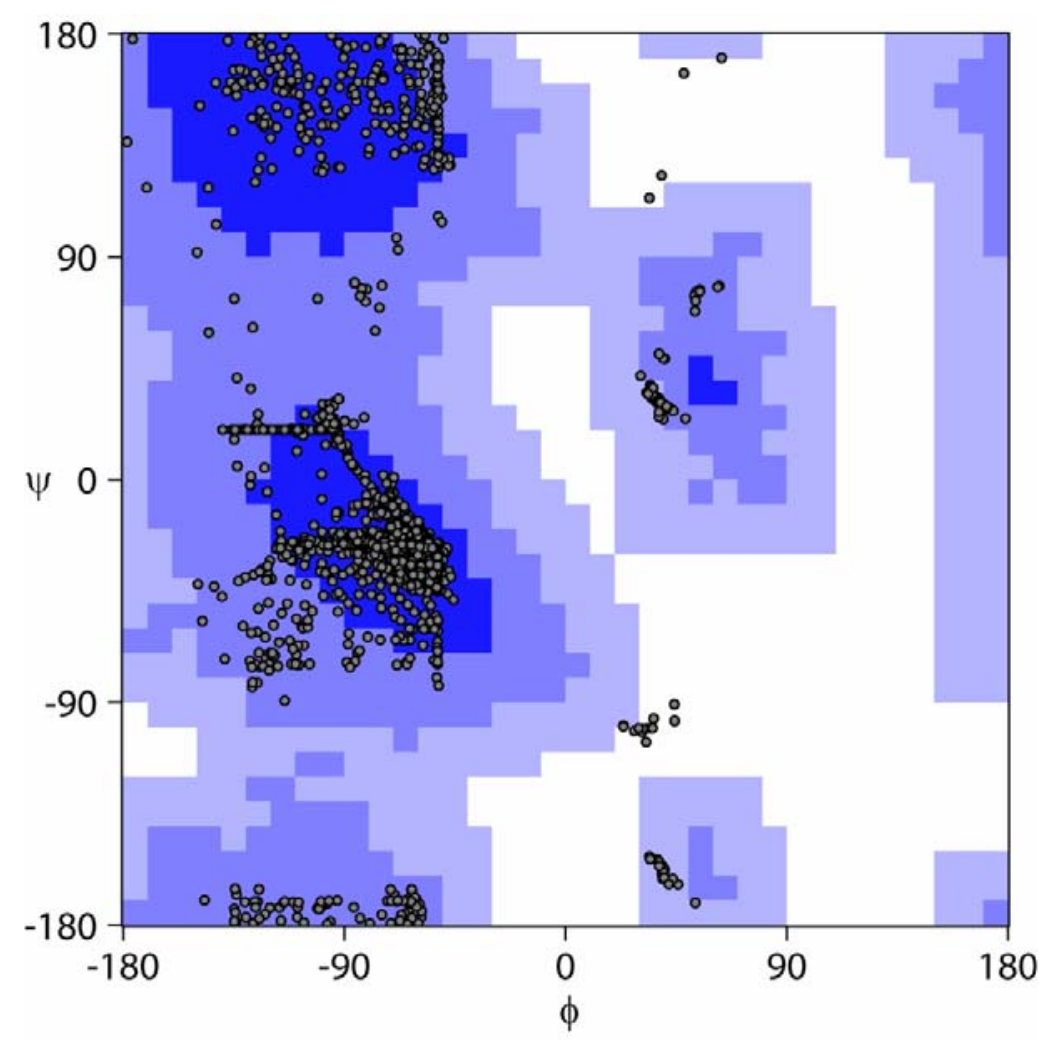

Figure 83. Ramachandran plot of the ensemble of 20 structures of the CaM/Munc13-1(457492) peptide complex.

Table 5. Summary of the structure statistics for the NMR solution structure of the CaM/Munc13-1 (457-492) peptide complex.

\begin{tabular}{|c|c|c|}
\hline Number of experimental restraints & \multicolumn{2}{|c|}{2102 (11.4 restraints/residue) } \\
\hline Distance restraints from NOEs & \multicolumn{2}{|c|}{1758 (9.6 restraints/residue) } \\
\hline $\mathrm{CaM}$ & \multicolumn{2}{|c|}{1494} \\
\hline M36 & \multicolumn{2}{|l|}{184} \\
\hline CaM-M36 & \multicolumn{2}{|l|}{80} \\
\hline Dihedral angle restraints (CaM) & \multicolumn{2}{|l|}{168} \\
\hline Hydrogen bond restraints & \multicolumn{2}{|l|}{39} \\
\hline $\mathrm{CaM}$ & \multicolumn{2}{|l|}{30} \\
\hline M36 & \multicolumn{2}{|l|}{9} \\
\hline H-N residual dipolar couplings & \multicolumn{2}{|l|}{137} \\
\hline $\mathrm{CaM}$ & \multicolumn{2}{|l|}{113} \\
\hline M36 & \multicolumn{2}{|l|}{24} \\
\hline \multicolumn{3}{|l|}{ Ramachandran analysis of CaM-M36 } \\
\hline Residues in favored regions (\%) & \multicolumn{2}{|l|}{89.4} \\
\hline Residues in additional allowed regions (\%) & \multicolumn{2}{|l|}{9.05} \\
\hline Residues in generously allowed regions (\%) & \multicolumn{2}{|l|}{1.55} \\
\hline Residues in disallowed regions (\%) & \multicolumn{2}{|l|}{0.0} \\
\hline Coordinate precision $(\AA)$ & Backbone & All heavy-atoms \\
\hline $\mathrm{CaM}$, residues 5-70, 84-146 & $0.52 \pm 0.12$ & $0.93 \pm 0.12$ \\
\hline M36, residues 4-21 & $0.41 \pm 0.52$ & $0.97 \pm 0.51$ \\
\hline CaM-M36, residues 5-70, 84-146, 4-21 & $0.46 \pm 0.32$ & $0.95 \pm 0.32$ \\
\hline
\end{tabular}


Table 6. Comparison of the interhelical angles for the CaM/Munc13-1(457-492) peptide complex and previous high resolution CaM structures with different EF-hand states

\begin{tabular}{|l|l|l|l|l|l|l|}
\hline $\begin{array}{l}\text { EF-hand } \\
\text { domain }\end{array}$ & I-II angle & I-III angle & I-IV angle & II-III angle & II-IV angle & $\begin{array}{l}\text { III-IV } \\
\text { angle }\end{array}$ \\
\hline ApoCdom & $124 / 135$ & $65 / 77$ & $140 / 160$ & $140 / 150$ & $30 / 50$ & $125 / 140$ \\
\hline $\begin{array}{l}\text { ApoCdom } \\
\text { +peptide }\end{array}$ & 99 & 113 & 153 & 141 & 64 & 90 \\
\hline holoCdom & $94 / 113$ & $130 / 150$ & $102 / 130$ & $105 / 125$ & $30 / 50$ & $83 / 104$ \\
\hline $\begin{array}{l}\text { holoCdom } \\
\text { +peptide }\end{array}$ & $83 / 112$ & $130 / 150$ & $104 / 132$ & $110 / 140$ & $34 / 81$ & $70 / 104$ \\
\hline $\begin{array}{l}\text { holoCdom } \\
\text { +munc13-1 }\end{array}$ & 99 & 156 & 119 & 102 & 43 & 84 \\
\hline ApoNdom & $132 / 145$ & $75 / 89$ & $124 / 132$ & $117 / 130$ & $50 / 60$ & $128 / 145$ \\
\hline $\begin{array}{l}\text { ApoNdom } \\
\text { +peptide }\end{array}$ & $130 / 135$ & $94 / 102$ & $130 / 138$ & $108 / 113$ & $50 / 57$ & $122 / 127$ \\
\hline holoNdom & $70 / 110$ & $130 / 155$ & $100 / 125$ & $110 / 135$ & $28 / 54$ & $80 / 100$ \\
\hline $\begin{array}{l}\text { holoNdom } \\
\text { + peptide }\end{array}$ & $73 / 102$ & $140 / 160$ & $104 / 123$ & $110 / 123$ & $38 / 72$ & $67 / 105$ \\
\hline $\begin{array}{l}\text { holoNdom } \\
\text { +munc13-1 }\end{array}$ & 85 & 157 & 120 & 112 & 42 & 83 \\
\hline
\end{tabular}
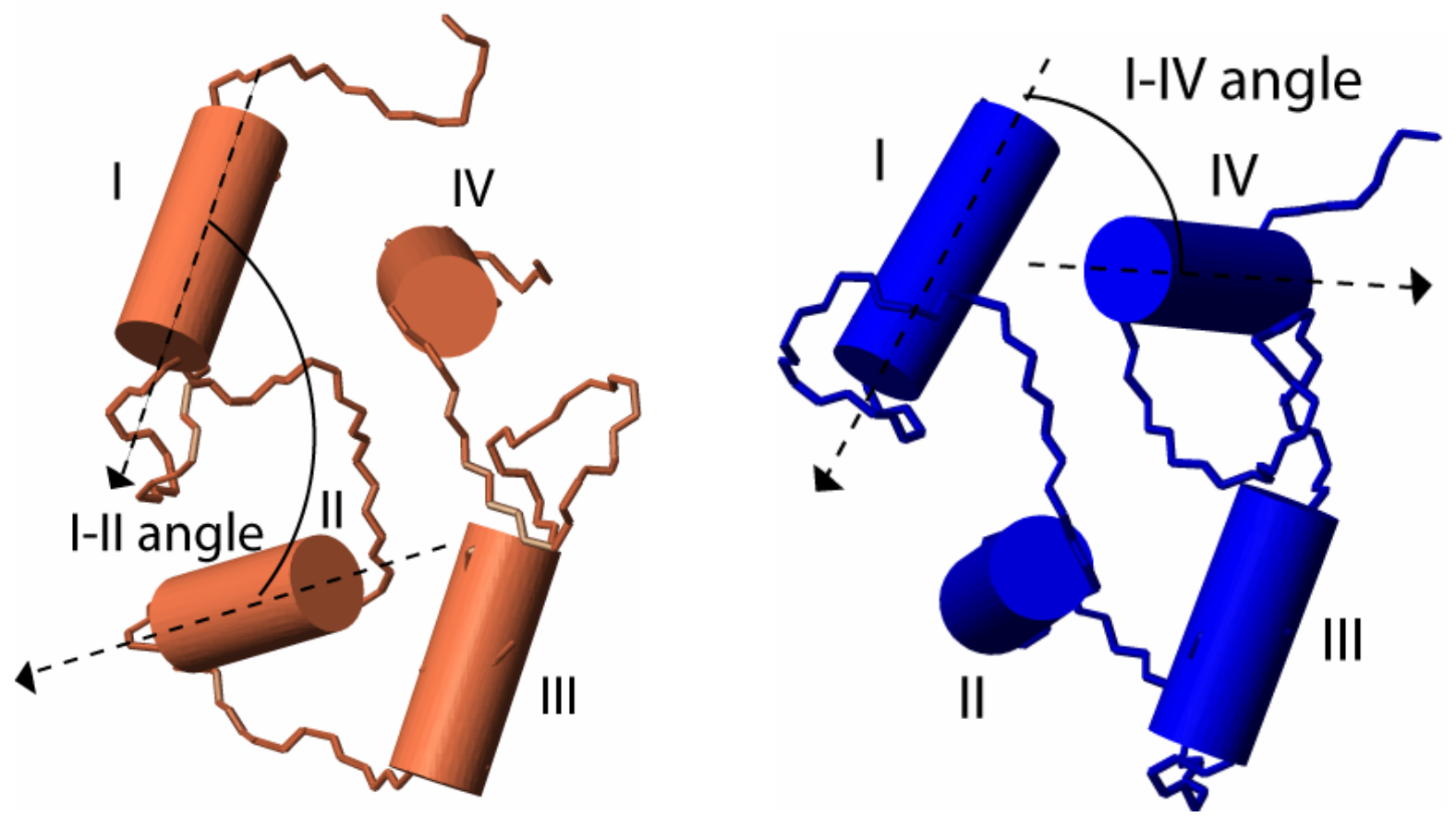

Figure 84. On the left is the N-terminal domain of CaM/Munc13-1 (457-492) peptide complex with its $\alpha$-helices represented as cylinders for measuring the interhelical angles. On the right, the $\mathrm{C}$-terminal domain of the complex is shown in blue.

The CaM/Munc13-1(457-492) peptide complex structure was overlaid with other high-resolution $\mathrm{CaM}$ structures in order to determine how well defined was the structure obtained. The backbone of the C-terminal domain region (84-146) was aligned to 10 different structures and the RMSD varied between 2.05 and $2.65 \AA$; two of the aligned structures are shown in Figure 85. 

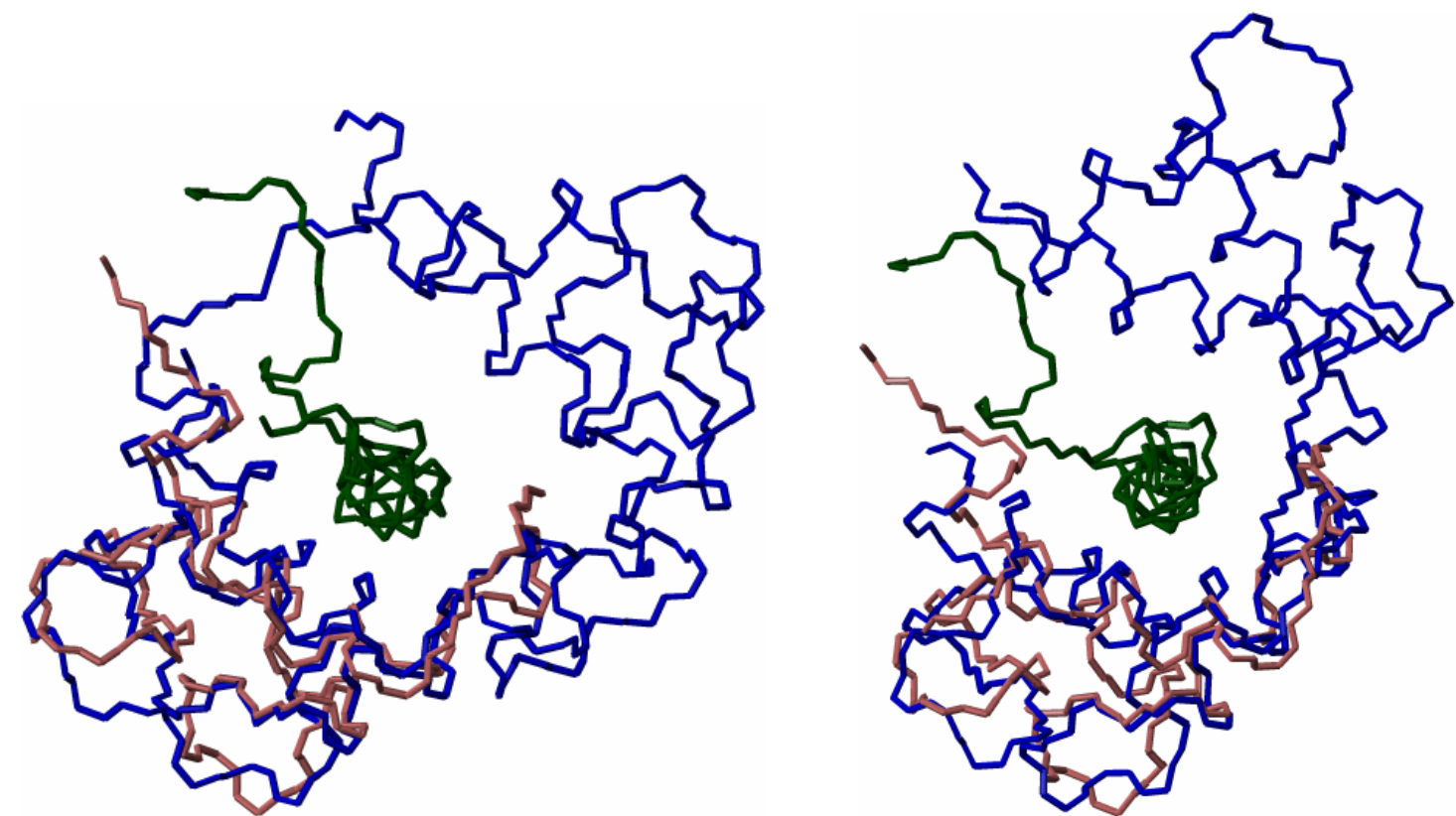

Figure 85. On the left is the overlaid C-terminal domain of CaM/Munc13-1(457-492) peptide complex structure with the CaM/smMLCK peptide complex (1CDL.pdb) and on the right with the CaM/CaMKII peptide complex (1CDM.pdb). The CaM C-domain in the $\mathrm{CaM} /$ Munc13-1 peptide complex is shown in light brown and the peptide in green. The full $\mathrm{CaM}$ backbone structure is shown for the other complexes in blue.

The backbone of the N-terminal domain region (4-70) was aligned to 9 different highresolution CaM structures and the RMSD varied between 2.40 and 3.24 Á; two of the aligned structures are shown in Figure 86.
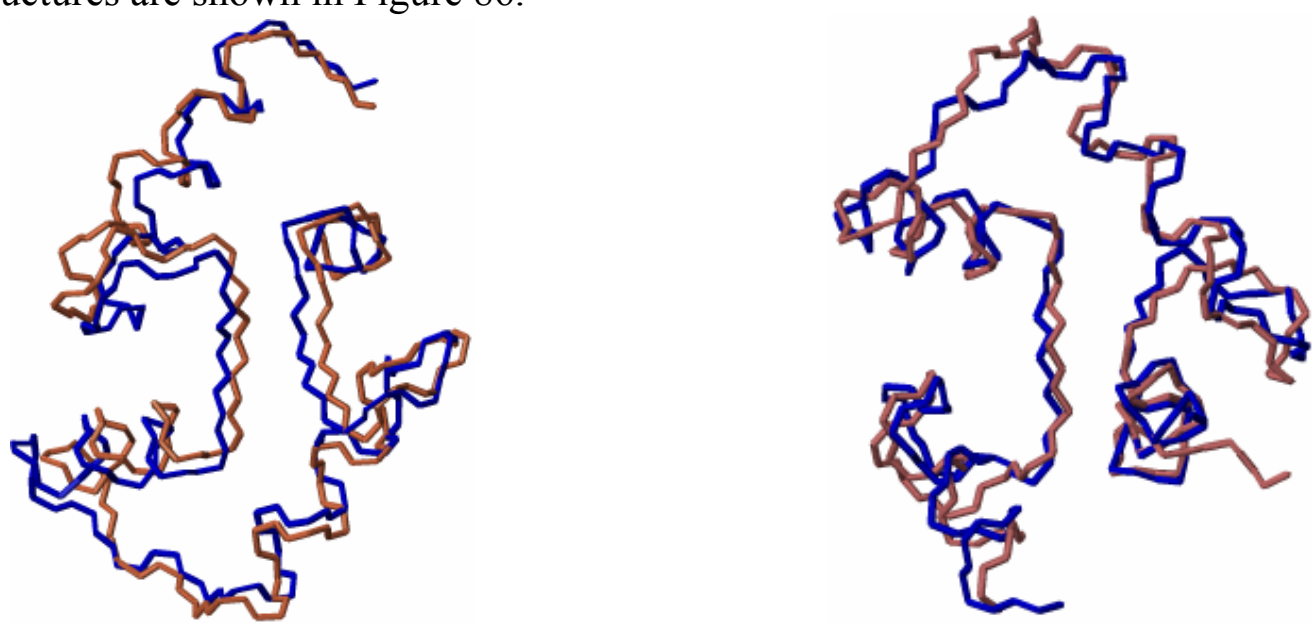

Figure 86. On the left is the overlaid N-terminal domain of the CaM/Munc13-1(457-492) peptide complex structure with the CaM/CaMKII (1CDM.pdb); and on the right overlaid with the free N-terminal domain NMR structure of CaM (1J7O.pdb). The CaM N-domain in the $\mathrm{CaM} /$ Munc13-1 peptide complex is shown in light brown and for the other models in blue.

\section{k. Dynamic studies of CaM in its various activated states with paramagnetic alignment}

$\mathrm{CaM}$ is a paradigm for multidomain proteins; it is well-recognized that interdomain orientation and dynamics play an important role in protein-protein interactions for multidomain proteins. Thus, the investigation of the interdomain dynamics at different activated states on this model system could shed light on different basic mechanisms governing macromolecular interactions of multidomain proteins. 
The advantages of studying multidomain proteins with NMR in solution is the possibility to have access both to atomic resolution information as well as dynamic information in the time-averaged ensemble of molecules studied with this spectroscopic technique. In contrast, X-ray crystallography is a very robust method for obtaining atomic resolution models of a macromolecule within a crystalline lattice, but lacks large-time scale dynamic information like interdomain motions, which are from a mechanistically point of view, of essential importance in macromolecular interactions. Other spectroscopic techniques, like fluorescence spectroscopy, lack the atomic resolution that NMR possess.

Previous studies of $\mathrm{CaM}$ in its calcium-free (apo) state have revealed a more compact structure compared to its calcium bound (holo) state. The current model is that in average, the $\mathrm{N}$ - and $\mathrm{C}$-terminal domains in apoCaM are closer to each other than in the calcium bound state. In this work, using paramagnetic alignment with an EDTA-based tag, it was possible to dissect the differences between the different activated states of CaM based on various paramagnetic restraints.<smiles>CS(=O)(=O)SC[C@H](NC(=O)[C@H](CN(CC(=O)O)CC(=O)O)N(CC(=O)O)CC(=O)O)C(=O)O</smiles><smiles>CS(=O)(=O)SC[C@H](NC(=O)[C@H](CN(CC(=O)O)CC(=O)O)N(CC(=O)O)CC(=O)O)C(=O)O</smiles>

Figure 87. On the left is the tag pk1, N-[(R)-2,3-Bis[di(carboxymethyl)amino]propionyl]-Smesyl-(R)-cysteine; on the right is the tag pk2, $\mathrm{N}-[(\mathrm{S})-2,3-\mathrm{Bis}[\mathrm{di}$ (carboxymethyl)amino] propionyl]-S-mesyl-(R)-cysteine.<smiles>CS(=O)(=O)Sc1ccc(NC(=O)[C@H](CN(CC(=O)O)CC(=O)O)N(CC(=O)O)CC(=O)O)cc1</smiles><smiles>CS(=O)(=O)Sc1ccc(NC(=O)[C@H](CN(CC(=O)O)CC(=O)O)N(CC(=O)O)CC(=O)O)cc1</smiles>

Figure 88. On the left side is the tag t43L, S-[4-[(R)-2,3-bis[di(tert-butoxycarbonylmehyl) amino]propionylamino]phenyl]ester; on the right side is the tag t43D, S-[4-[(S)-2,3-bis[di (tert-butoxycarbonylmehyl)amino]propionylamino]phenyl]ester.

The molecular structures of the EDTA-based paramagnetic tags used in this study are shown in Figures 87 and 88 . They contain a mesyl- moiety which is a good leaving group; therefore they react readily with the thiol group of solvent accessible cysteines in the target protein.

$\mathrm{CaM}$ does not have any cysteine residues in its primary sequence; therefore it has to be introduced by site-directed mutagenesis. It is preferable to substitute an amino acid with similar properties like serine or threonine to minimize the risk of changing the folding or properties of CaM. 
The studies on apoCaM were done on the S17C mutant. The N-terminal domain was paramagnetically aligned titrating $\mathrm{Tb}^{3+}$ (Figure 89) and the effects on the C-terminal domain were analyzed; however as discussed below the alignment tensor could not be measured.

The axial component of the alignment tensor of the N-terminal domain of apoCaM had a magnitude of $3.2 \mathrm{~Hz}$ at the $900 \mathrm{MHz}$ spectrometer for the alignment with t43L (see Table 7 for the tensor information on the other alignments). The alignment tensor was well defined (see Figure 90). The measured rDCs on the C-terminal domain were rather small and therefore noisy. For this reason no correlation was found between the experimental data and the simulated data based on the refined NMR structure of apoCaM. However, pseudocontact shift measurements are more precise, establishing a difference between apo- and holo-CaM based on these paramagnetic restraints (See Figure 91).

Table 7. Axial (Da-HN) and Rhombic(Rhomb.) components (apoCaMS17C) of the alignment tensors and angles between them achieved for the four paramagnetic tags bound to Terbium.

\begin{tabular}{|l|l|l|l|l|}
\hline Tag1/Tag2 & Deg angle & tag & Hz Da-HN & Rhomb. \\
\hline Pk1/pk2 & 61 & Pk1 & 6.2 & 0.5 \\
\hline Pk1/t43L & 92 & Pk2 & 2.1 & 0.43 \\
\hline Pk1/t43D & 112 & T43D & 3.4 & 0.32 \\
\hline Pk2/t43L & 115 & T43L & 3.2 & 0.48 \\
\hline Pk2/t43D & 106 & & & \\
\hline T43D/t43L & 26 & & & \\
\hline
\end{tabular}

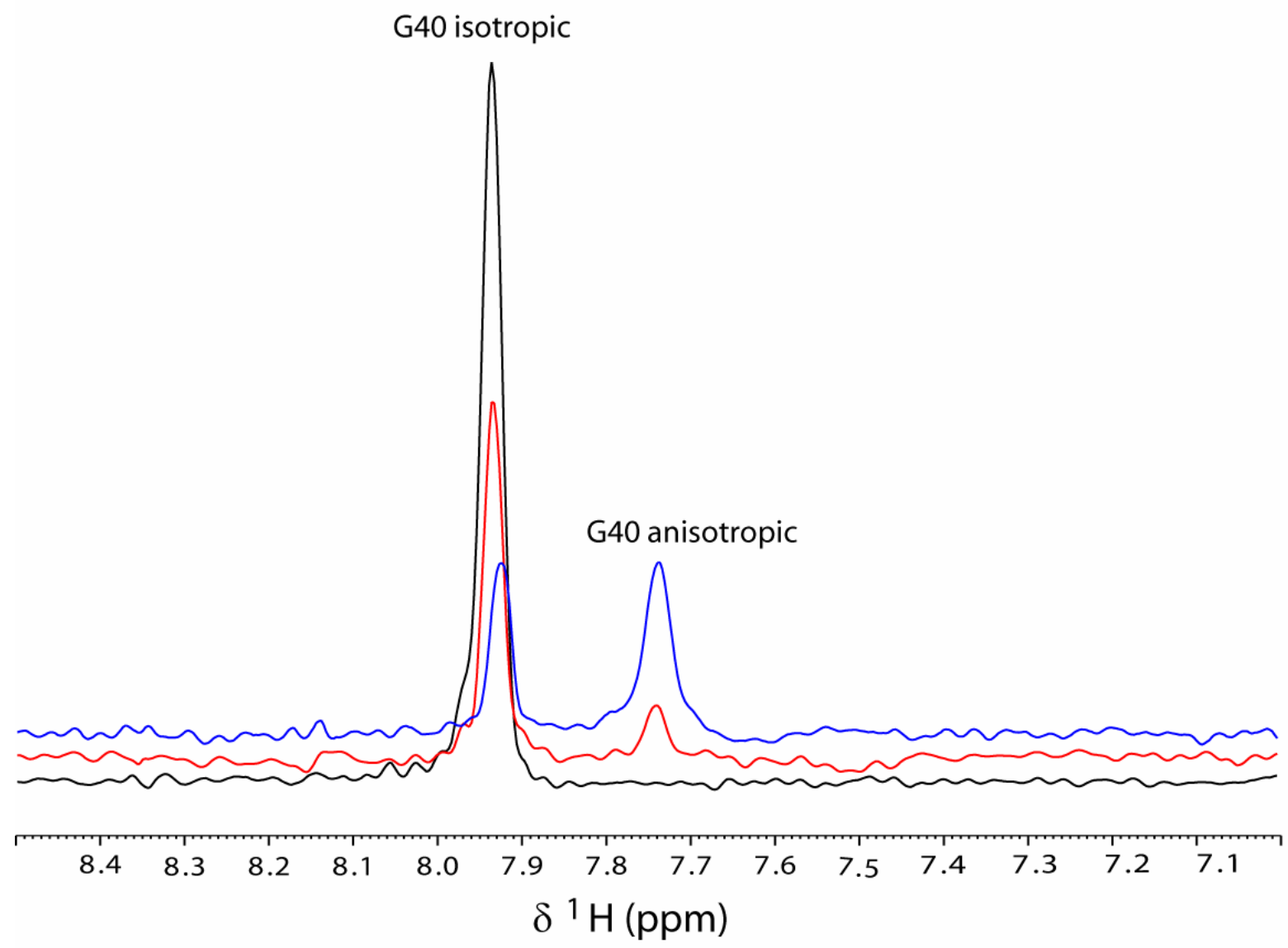

Figure 89. Titration of the apoCaMS17C-t43D with $\mathrm{Tb}^{3+}$. In black is the isotropic spectrum in the absence of lanthanide ion; in red is the protein titrated with $20 \%$ of lanthanide ion and in blue is the protein with $60 \%$ of lanthanide ion. 

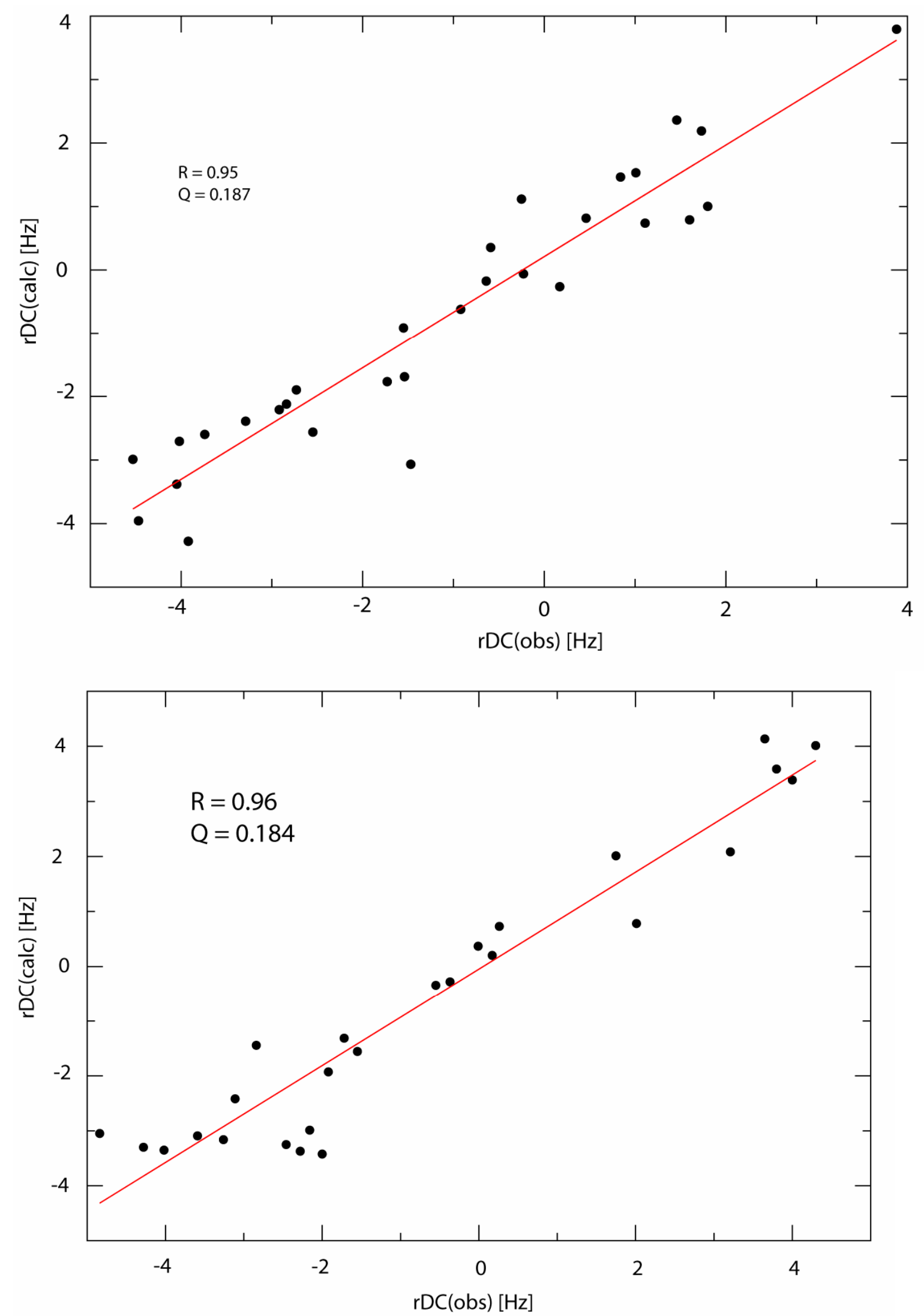

Figure 90. Correlation plot of the measured $\mathrm{rDCs}$ for the N-terminal domain of apoCaMS17C-T43D-Tb ${ }^{3+}$ (upper plot) and apoCaMS17C-T43L-Tb ${ }^{3+}$ (lower plot) against the back-calculated rDCs based on the refined NMR structure of apoCaM. 


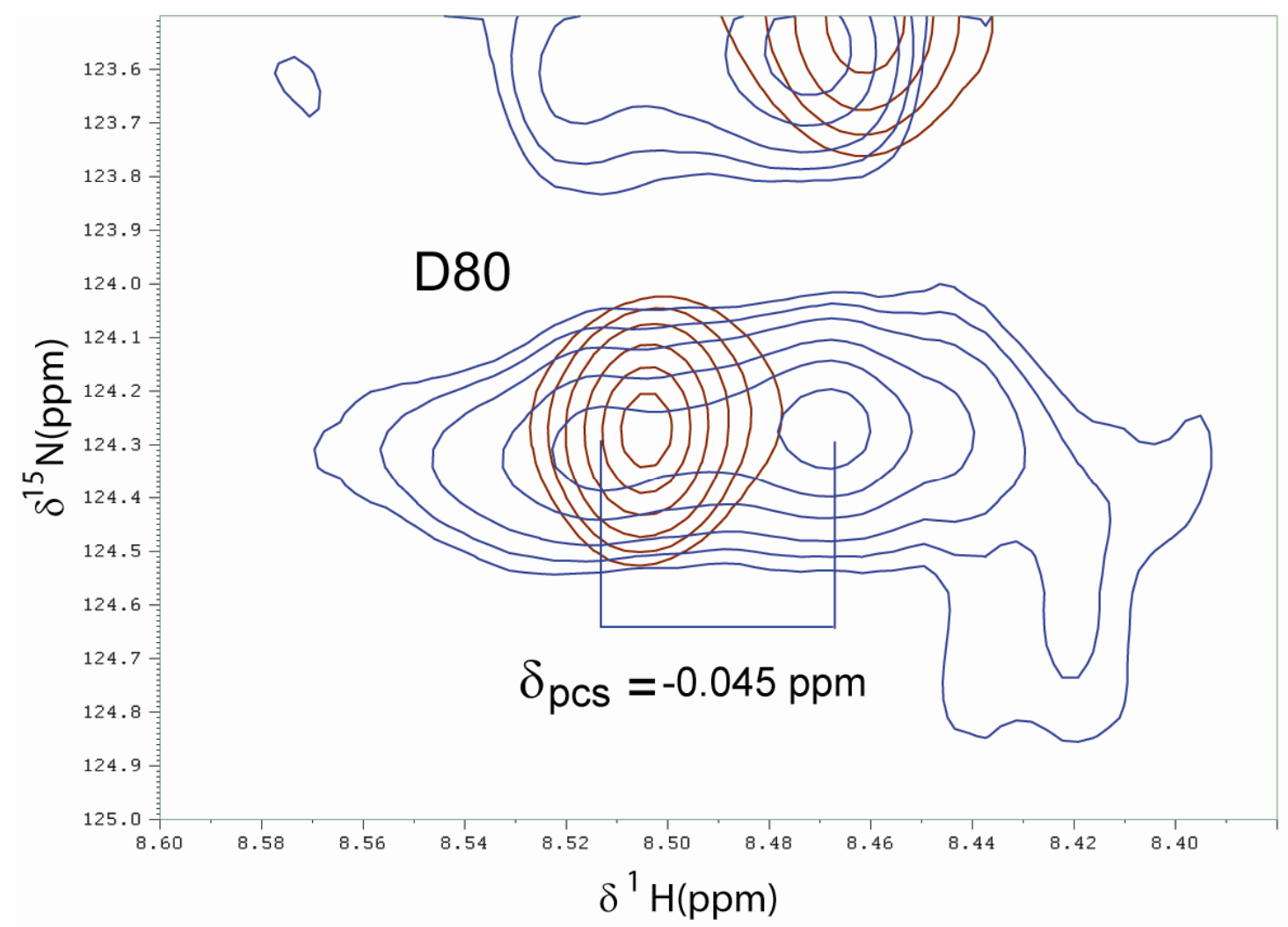

Figure 91. .Overlaid ${ }^{15} \mathrm{~N}-{ }^{1} \mathrm{H}-\mathrm{HSQC}$ spectra for ${ }^{15} \mathrm{~N}$ apoCaMS17C-t43L in brown and ${ }^{15} \mathrm{~N}$ apoCaM S17C-t43L- $\mathrm{Tb}^{3+}$ in blue. In the presence of the lanthanide ion, pseudocontact shifts were observed in the linker region (D80 NH cross-peak is shown) of apoCaM.

The pseudocontact shifts contain translational information $\left(r^{3}\right.$ term) as well as information on orientation with respect to the magnetic field $\left(\left(3 \cos ^{2} \theta-1\right)\right.$ term) for the vector between the unpaired electron of the lanthanide ion and the $\mathrm{NH}$ bond vector in this case. Thus, the coordinates of the metal ion in the molecular frame of apoCaM (Kubinowa et al., 1995) could be located. The correlation plots between the measured PCS and the backcalculated PCS based on the NMR srtructure of apoCaM for three different tags are shown in Figures 92 and 93. The location of the metal relative to apoCaM is shown in Figure 94.

In Figure 95, the relationship between the metal-amide distances and the measured PCS were analyzed; a power $\left(r^{3}\right)$ function was fitted to the data an upper bound $r^{3}$ function was constructed minimizing the values for the angular values in the PCS expression. This analysis allowed the qualitative description of a partial averaging of the PCS residues in the linker region of apoCaM; providing evidence of the existence of interdomain motions in apoCaM in the sub-millisecond time scale.

The interdomain dynamics of holoCaM have been previously studied by $\mathrm{T}_{1}$ relaxation measurements of $\mathrm{NH}$ resonances at different temperatures (Chang et al., 2003); this study provided a model of a cone motion of one domain of $\mathrm{CaM}$ relative to the other domain. However, $\mathrm{T}_{1}$ relaxation measurements have the limitation that they just sense the dynamics on ps to ns time-scale. Therefore it would be interesting to compare these results with interdomain dynamics probed by residual dipolar couplings, which sense motions up to the sub-millisecond time scale. Bertini et al., 2004 reported the paramagnetic alignment of the Nterminal domain of $\mathrm{CaM}$ and found a reduction of $\sim 10$ fold in the axial component of the alignment tensor for the $\mathrm{C}$-terminal domain of $\mathrm{CaM}$, they derived a model in which the $\mathrm{C}$ terminal domain of $\mathrm{CaM}$ describes a cone motion with a semiangle of $20^{\circ}$. 

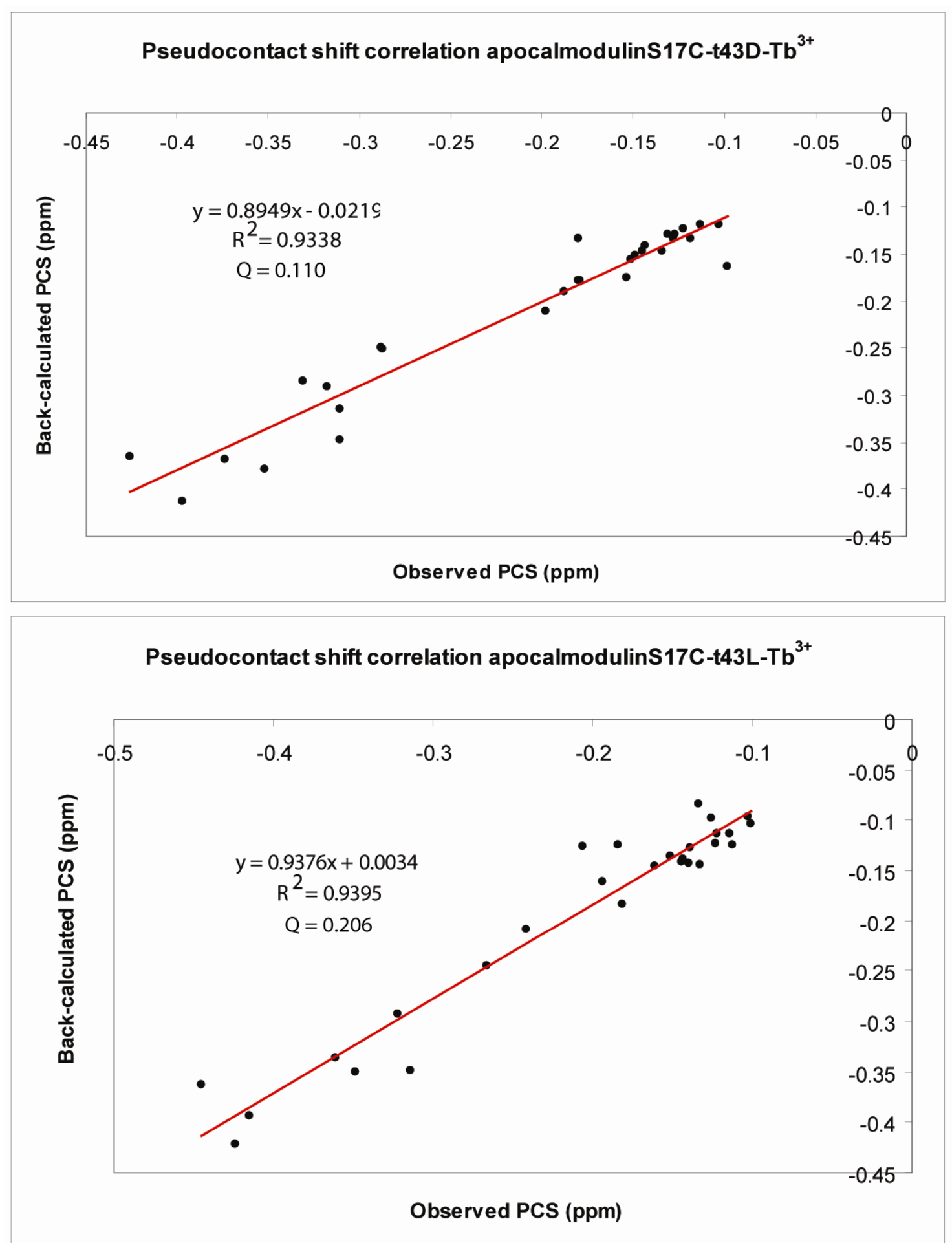

Figure 92. Correlation plot between the measured PCS for apoCaMS17C-t43D--Tb ${ }^{3+}$ (upper plot) and apoCaMS17C-t43L-Tb ${ }^{3+}$ (lower plot) against the back-calculated PCS based on the NMR structure of the N-terminal domain of apoCaM.

In this thesis, it was possible to establish a difference in the interdomain dynamics between holoCaM and the $\mathrm{CaM} / \mathrm{C} 20 \mathrm{~W}$ peptide complex from the plasma membrane calcium channel based on paramagnetic derived rDCs. A previous study based on small angle X-ray scattering measurements established a significant difference in the radius of gyration of holoCaM and the CaM/C20W complex (Kataoka et al., 1991). 


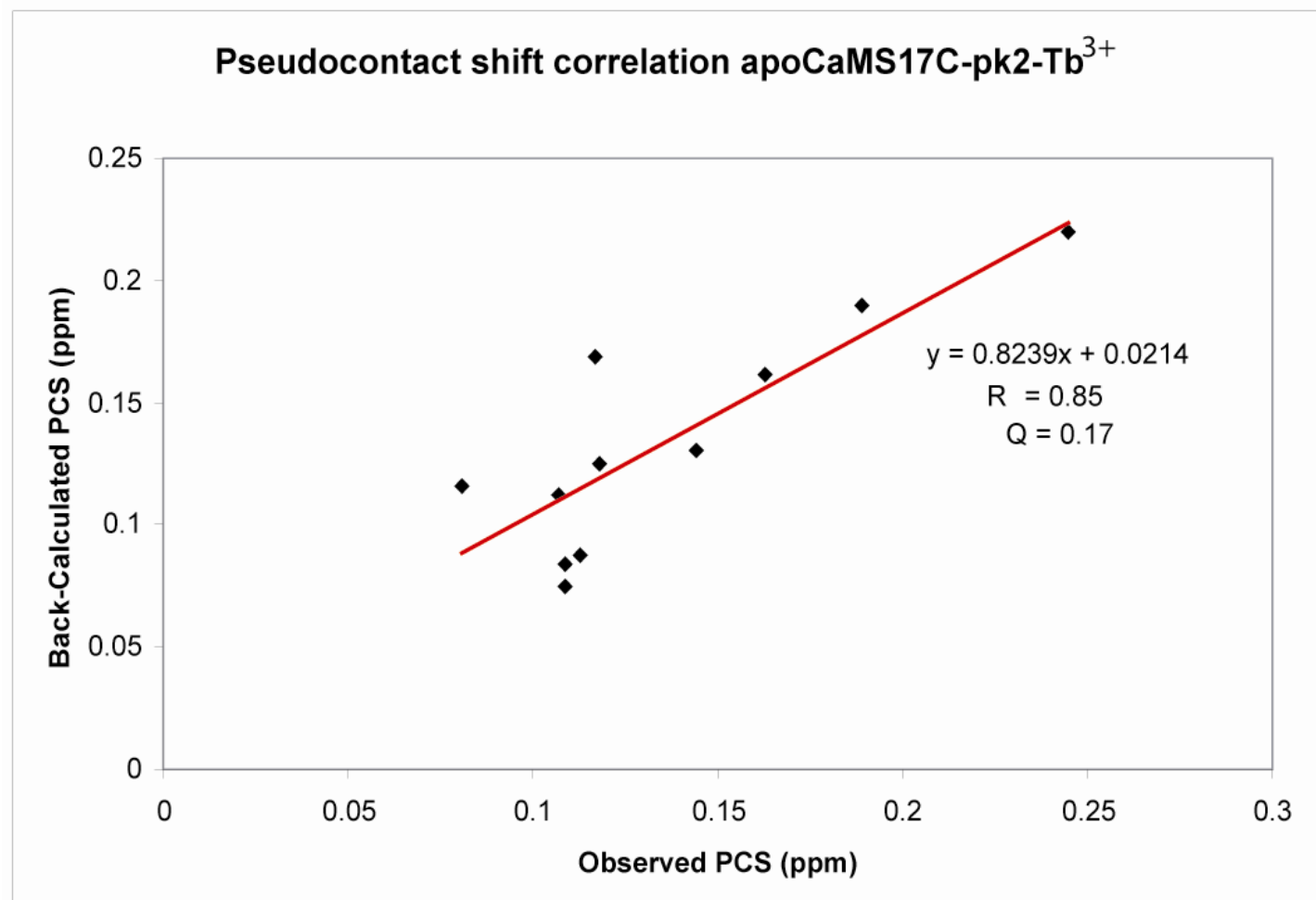

Figure 93. Correlation plot between the measured PCS for apoCaMS17C-pk2- $\mathrm{Tb}^{3+}$ and the back-calculated PCS from the NMR structure of the N-terminal domain of apoCaM.

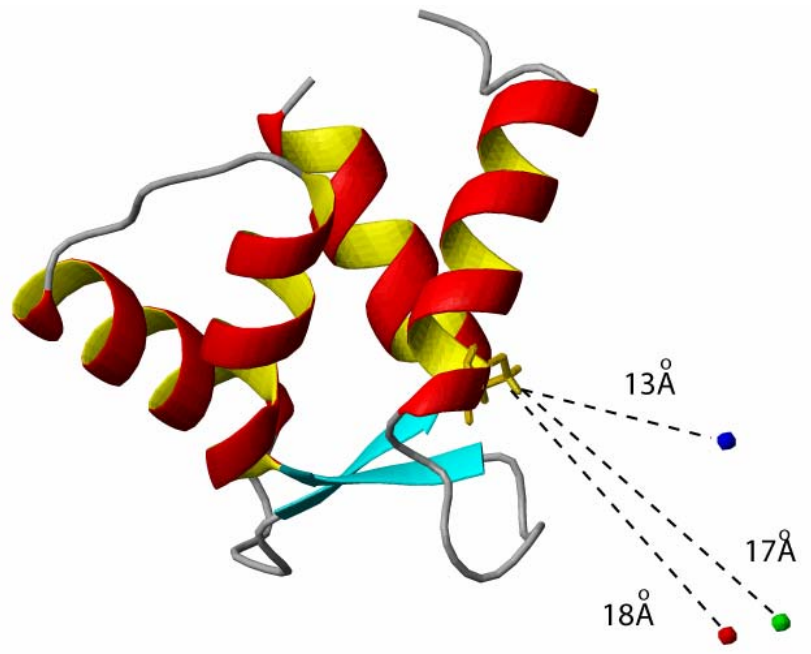

Figure 94. Location of the metal ion $\left(\mathrm{Tb}^{3+}\right)$ based on the pseudocontact shift restraints for the N-terminal domain of apoCaMS17C-t43D in red, apoCaMS17C-t43L in green and apoCaMS17C-pk2 in blue. The distance of the longest axis from the Oxygen atom of the serine in the $w t$ protein to the metal ion is also given.

In principle, it is possible to derive the elements of the alignment tensor $\left(d_{\mathrm{zz}}, d_{\mathrm{yy}}\right.$ and $d_{\mathrm{xx}}$ ) from the histogram analysis of the rDCs (Figures 96 and 97). However, since the NMR structure of the $\mathrm{N}$ - and $\mathrm{C}$-terminal domain of CaM has been refined against numerous rDCs (Chou et al., 2001), it was preferred to derive the tensor values with the singular value decomposition (SVD) algorithm from PALES (Zweckstetter and Bax, 2000). In this manner, the experimental values can be tested against the reference structural model and a correlation plot of the experimental values to the back-calculated rDCs is obtained. In addition, several other parameters are obtained, which provide an extended view of the error in the data. 

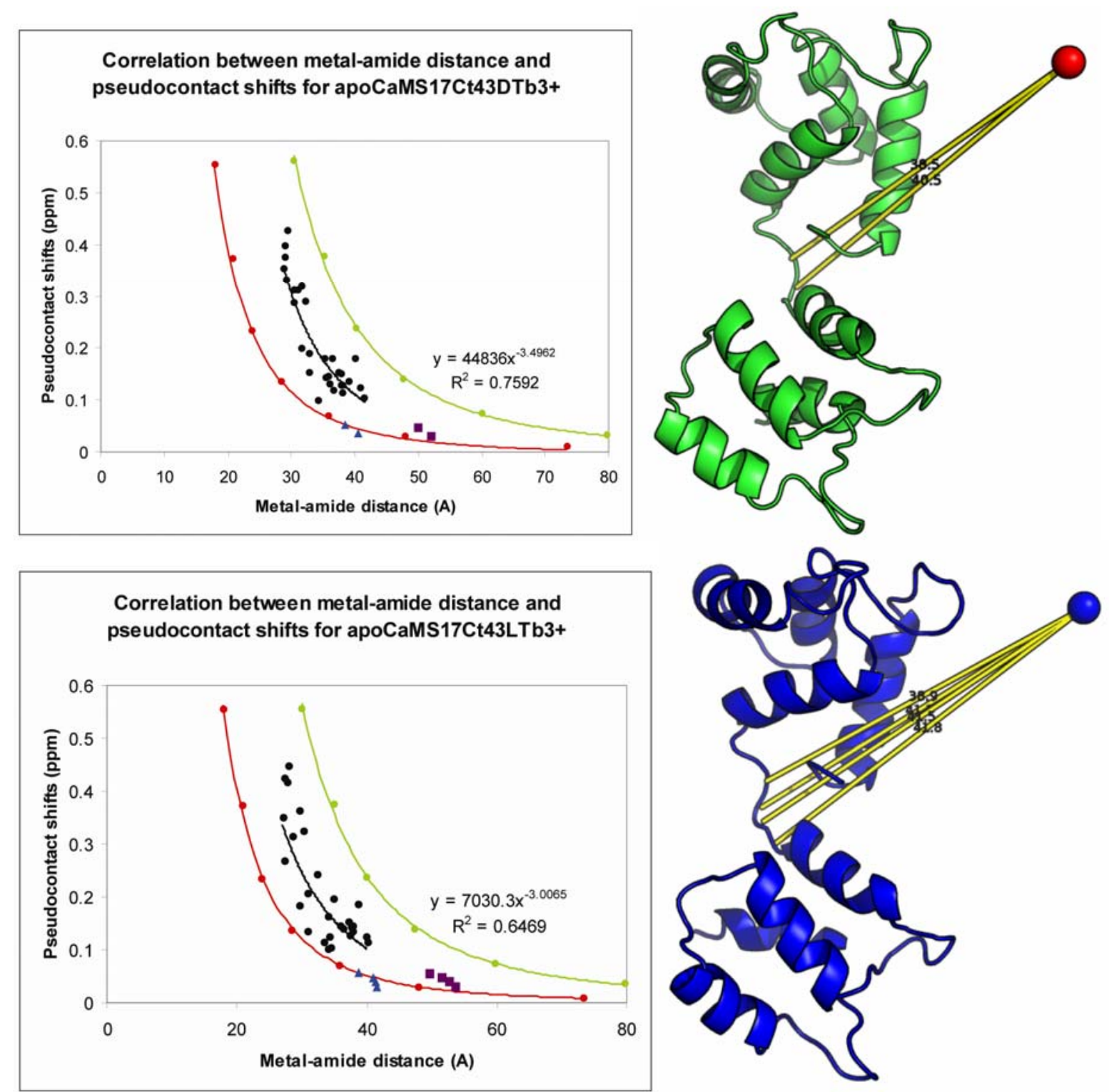

Figure 95. Relationship of the measured distances between the lanthanide ion and each amide proton and the observed PCS for apoCaMS17Ct43D- $\mathrm{Tb}^{3+}$. An upper bound $r^{3}$ plot is shown in red and a lower-bound $r^{3}$ plot is shown in green. The blue triangles are the measured metalamide distances for the linker residues T79 and D80 in the NMR structure of apoCaM. The purple squares are the predicted distances based on the $r^{3}$ function. On the right, the distances from the lanthanide ion (red) to the two amides in the linker are illustrated. The lower panel shows the same plot for apoCaMS17Ct43L-Tb ${ }^{3+}$. The blue triangles and purple squares have the same meaning and represent the T79, D80, S81 and E82 in the linker region.

Table 8. Axial (Da-HN) and Rhombic(Rhomb.) components of the alignment tensors and angles between them achieved for the holoCaM samples aligned with Terbium.

\begin{tabular}{|c|c|c|c|c|c|}
\hline Mutant & Tag1/tag2 & Deg & tag & $\mathrm{Hz}$ Da-HN & Rhomb. \\
\hline \multirow{2}{*}{$\begin{array}{l}\text { holoCaMS17C } \\
\text { (N-domain tensor) }\end{array}$} & \multirow[t]{2}{*}{$\mathrm{pk} 1 / \mathrm{t} 43 \mathrm{~L}$} & \multirow[t]{2}{*}{126} & Pk1 & 2.2 & 0.32 \\
\hline & & & T43L & 2.3 & 0.15 \\
\hline \multirow{4}{*}{$\begin{array}{l}\text { holoCaMT146C } \\
\text { (C-domain tensor) }\end{array}$} & & & $\mathrm{Pk} 2$ & 3.0 & 0.58 \\
\hline & & & Pk1 & 3.9 & 0.22 \\
\hline & & & T43L & 3.23 & 0.18 \\
\hline & & & T43L & 2.47 & 0.23 \\
\hline
\end{tabular}




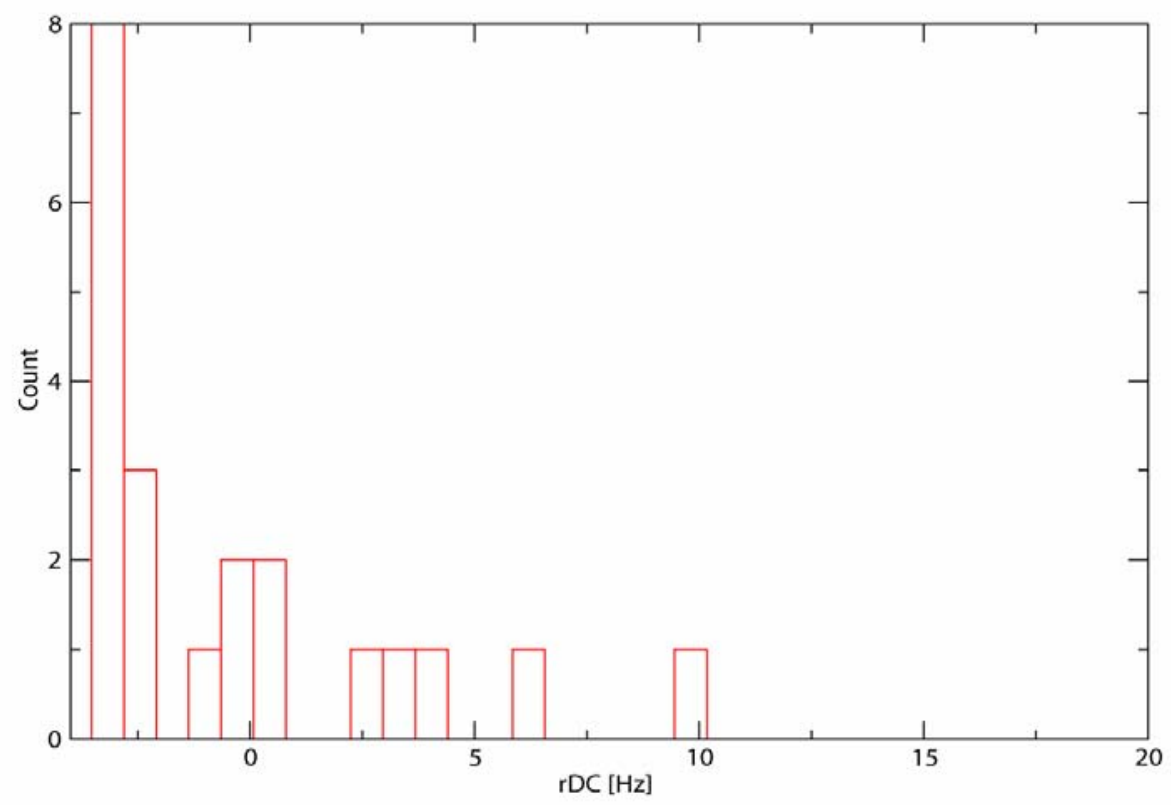

Figure 96. Histogram for the measured residual dipolar couplings of the $\mathrm{C}$-terminal domain of the CaMT146C-pk2-Tb ${ }^{3+} / \mathrm{C} 20 \mathrm{~W}$ peptide complex. From the histogram it is evident the anisotropic distribution of these couplings.

Based on numerous $\mathrm{rDC}$ analyses for the $\mathrm{CaM} / \mathrm{C} 20 \mathrm{~W}$ peptide complex see Table 9, it was possible to establish residual interdomain motions in this complex. Moreover, it appears that the motions are more restricted than in the case of holoCaM. The experiments were done with the CaM T146C mutant. Thus, the C-terminal domain was paramgnetically aligned in the static magnetic field, $\mathrm{B}_{0}$, and the effects on the $\mathrm{N}$-terminal domain were measured (See Figure 98). In average, a reduction in the magnitude of the axial component of the alignment tensor of a factor of 4 was found between the two domains.

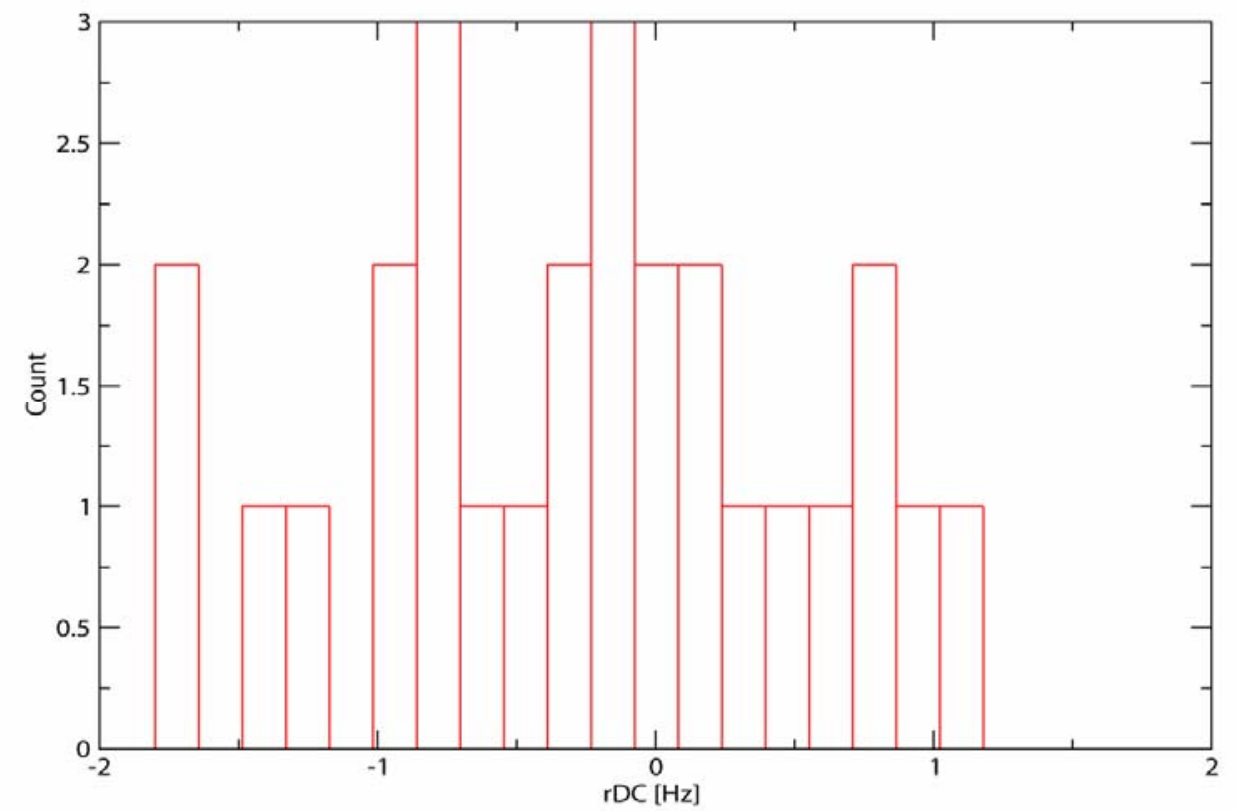

Figure 97. Histogram for the measured residual dipolar couplings of the $\mathrm{N}$-terminal domain of the CaMT146C-pk2- $\mathrm{Tb}^{3+} / \mathrm{C} 20 \mathrm{~W}$ peptide complex. In contrast to the previous histogram, the distribution of these $\mathrm{rDCs}$ is isotropic. 
Table 9. Axial (Da-HN) and Rhombic(Rhomb.) components of the alignment tensors and angles between them achieved the CaMT146C/C20W complex aligned with Terbium

\begin{tabular}{|l|l|l|l|l|l|}
\hline $\begin{array}{l}\text { Observed } \\
\text { domain }\end{array}$ & Tag1/tag2 & Deg angle & tag & Hz Da-HN & Rhomb. \\
\hline $\begin{array}{l}\text { C-domain } \\
\text { Tensor }\end{array}$ & Pk2/t43L & 26 & Pk2 & 3.47 & 0.13 \\
\cline { 4 - 6 } & & & T43L & 3.42 & 0.43 \\
\cline { 3 - 6 } & & T43D & 3.0 & 0.24 \\
\hline \multirow{2}{*}{$\begin{array}{l}\text { N-domain } \\
\text { Tensor }\end{array}$} & Pk2/t43L & 92 & Pk2 & 0.8 & 0.48 \\
\cline { 2 - 6 } & T43L/t43D & 49 & T43L & 0.9 & 0.48 \\
\cline { 3 - 6 } & & & T43D & 1.0 & 0.39 \\
\hline
\end{tabular}
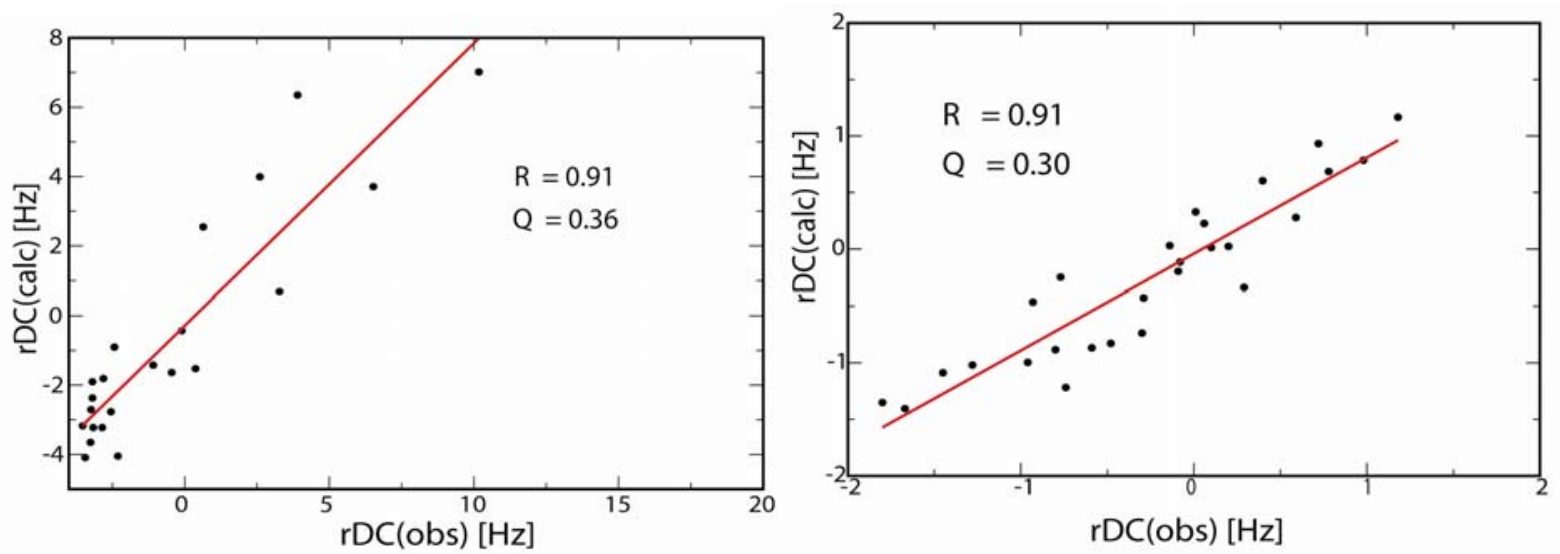

Figure 98. Correlation plots for the measured rDCs for the C-terminal domain (left side) and $\mathrm{N}$-terminal domain (right side) of the CaMT146C-pk2- $\mathrm{Tb}^{3+} / \mathrm{C} 20 \mathrm{~W}$ peptide complex

For holoCaMT146C it was not possible to measure the paramagnetic restraints on the $\mathrm{N}$-terminal domain, since the measurement error was on the same order of magnitude than for the expected rDCs based on the results obtained by Bertini et al., 2004. In order to measure the alignment tensor of the N-terminal domain of $\mathrm{CaM}$, a paramagnetic tag with a stronger alignment would be needed. However the tensor values were obtained for the C-terminal domain (Figure 100).

For $\mathrm{rDC}$ data analysis it is important to obtain various data sets with different orientation with respect to the magnetic field; the reason for this is that the rDCs suffer from orientational degeneracy, which can be resolved measuring several independent data sets. For the paramagnetic tags used in this study, this was possible by the different enantiomers of the tag. Another possibility is to complex that tag with different lanthanide ions, with different magnetic susceptibility properties. $\mathrm{Tb}^{3+}$ and $\mathrm{Dy}^{3+}$ are both similar in their alignment properties, but $\mathrm{Tm}^{3+}$ provides an alignment with a different orientation, this is illustrated in Figure 99.

In order to be confident about the statistical significance of the observed difference among the two states of CaM, the SVD algorithm was repeated 1000 times within a Monte Carlo simulation procedure and it was possible to derive some statistics about the alignment tensors. First, the distribution of the axial component (Da) for the tensors showed that the Nterminal domain in the $\mathrm{CaM} / \mathrm{C} 20 \mathrm{~W}$ peptide complex (Figure 101) has a definite value above the noise of the data $(0.8 \pm 0.2 \mathrm{~Hz})$.

The distribution in the rhombic component of the alignment tensor was larger (Figure 102), however it could be established that the $\mathrm{C}$-terminal domain of the $\mathrm{CaM} / \mathrm{C} 20 \mathrm{~W}$ complex has a low rhombic value $(0.13 \pm 0.1)$ whereas the $\mathrm{C}$-terminal domain of the free CaM has a large rhombic value $(0.58 \pm 0.15)$. On the other hand, the rhombic value for the tensor of the $\mathrm{N}$-terminal domain of the $\mathrm{CaM} / \mathrm{C} 20 \mathrm{~W}$ complex is axially symmetric $(0.48 \pm 0.15)$. 


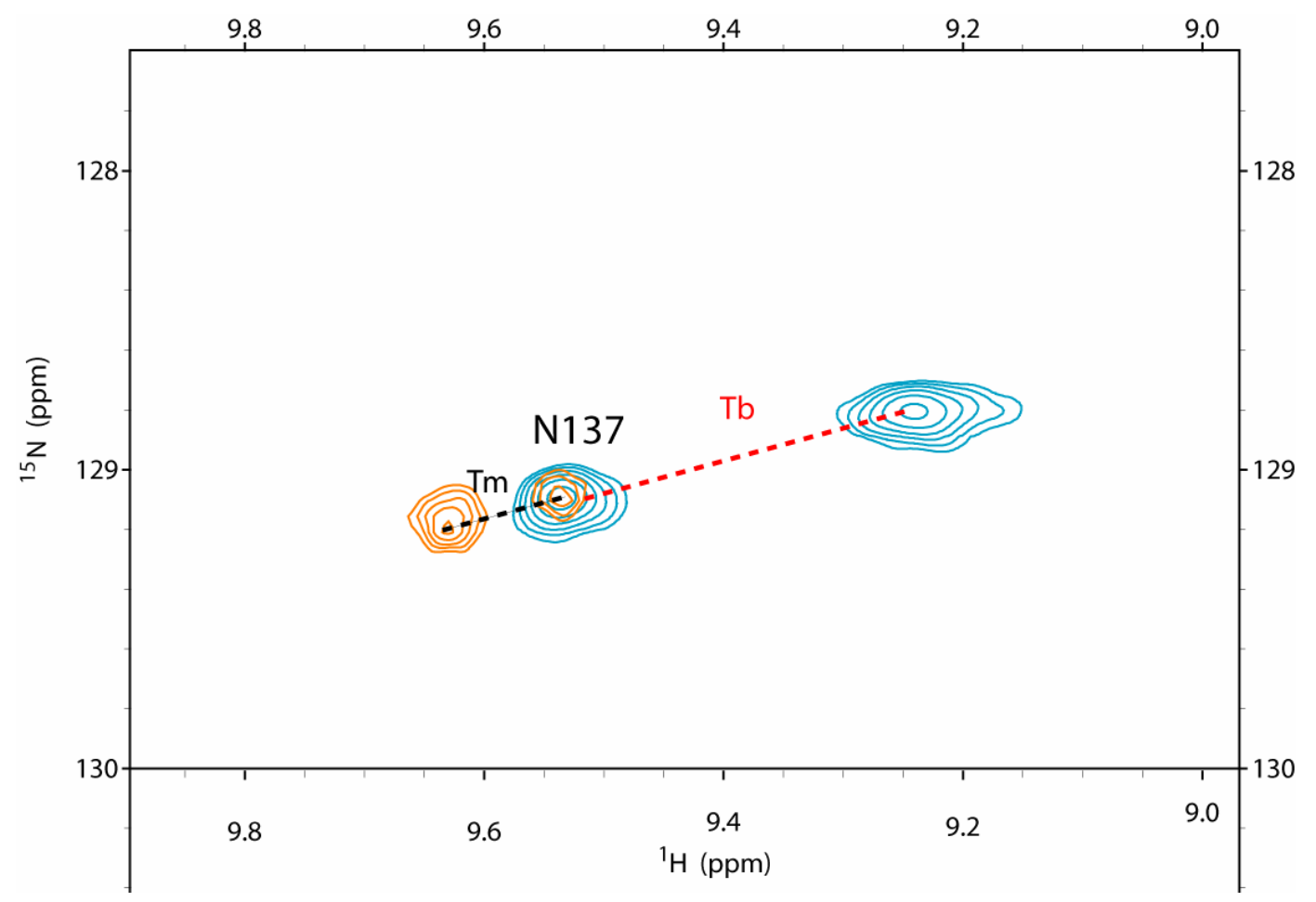

Figure 99. Overlaid ${ }^{15} \mathrm{~N}-{ }^{1} \mathrm{H}$ HSQC spectra for the holoCaMT146C-t43D aligned with $\mathrm{Tb}^{3+}$ (blue) and with $\mathrm{Tm}^{3+}$ (orange). For the $\mathrm{NH}$ of $\mathrm{N} 137 \mathrm{Tm}^{3+}$ gives a positive PCS whereas $\mathrm{Tb}^{3+}$ a negative one, therefore these two data sets give also different alignment tensors in terms of orientation.

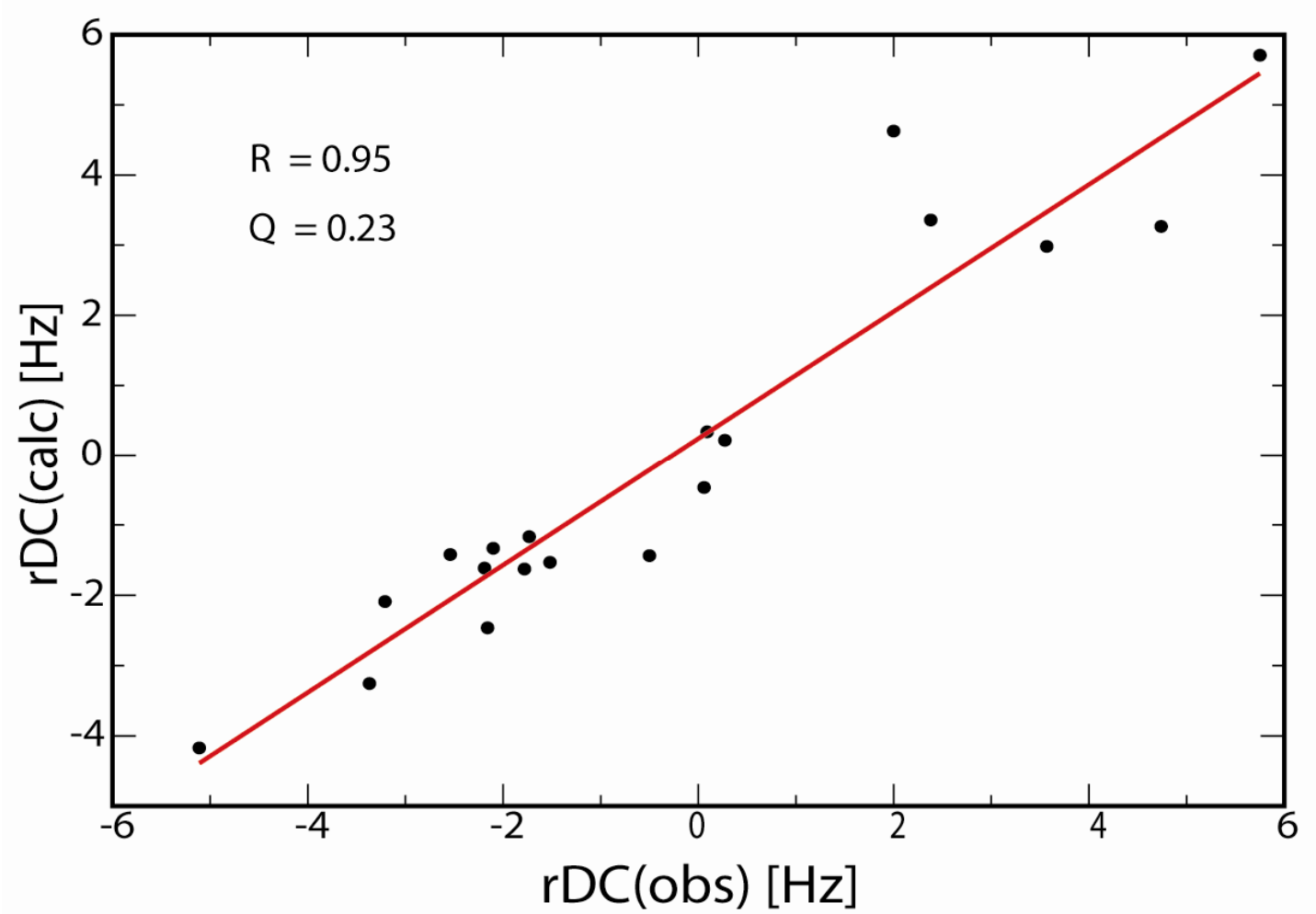

Figure 100. Correlation plot between the measured rDCs and back-calculated rDCs for the Cterminal domain of CaMT146C-pk2-Tb ${ }^{3+}$. 

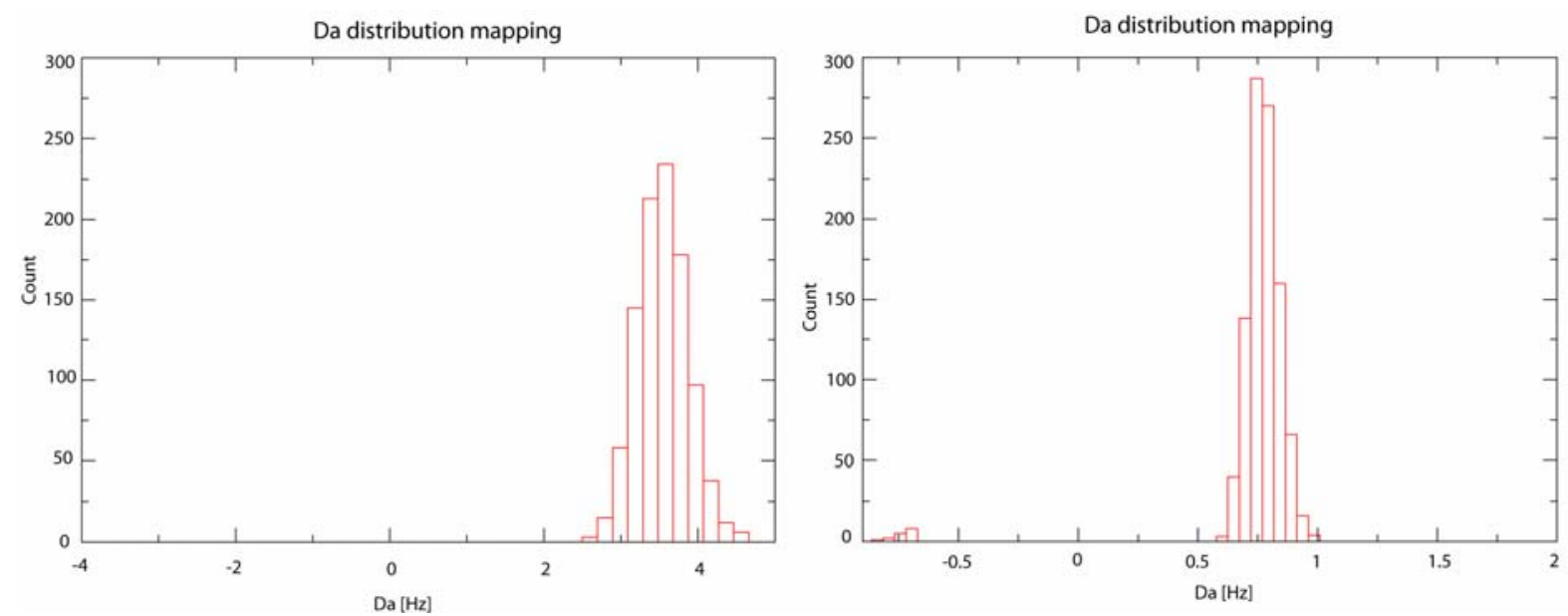

Figure 101. Distribution for the Axial component of the alignment tensor for the CaMT146C$\mathrm{pk} 2-\mathrm{Tb}^{3+} / \mathrm{C} 20 \mathrm{~W}$ peptide complex. On the left is the tensor for the C-terminal domain and on the right for the N-terminal domain.
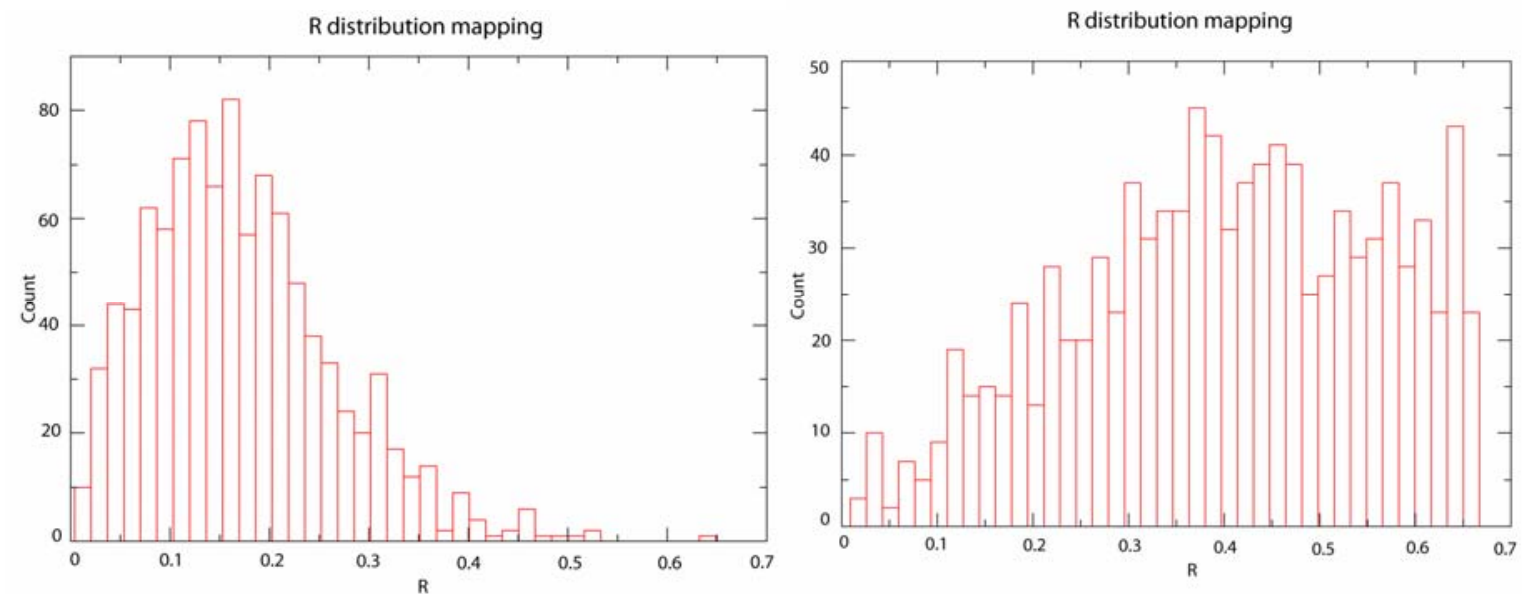

Figure 102. Distribution for the Rhombic component of the alignment tensor for the CaMT146C-pk2-Tb ${ }^{3+} / \mathrm{C} 20 \mathrm{~W}$ peptide complex. On the left is the tensor for the C-terminal domain and on the right for the $\mathrm{N}$-terminal domain.

The tensor distribution can also be visualized with the aid of a 3D plot. The plots of the tensor values for the $\mathrm{C}$ - and $\mathrm{N}$ - terminal domains of the CaM/C20W complex are shown in Figure 103. These plots show that both tensors are well defined and have a low spread around the average value.
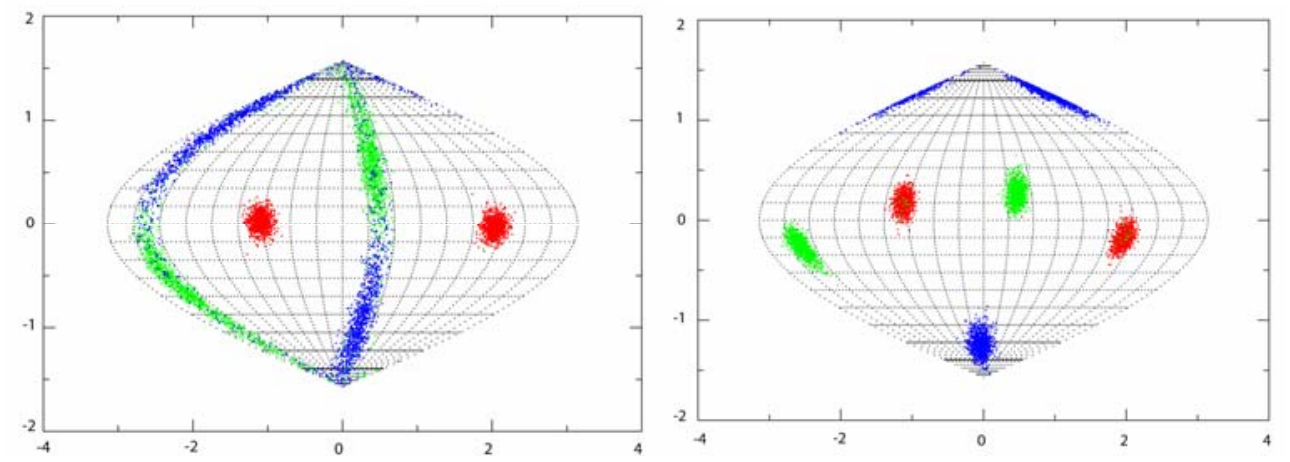

Figure 103. Mapping of the individual tensor values for the 1000 SVD calculations on a 3D plot for the CaMT146C-pk2- $\mathrm{Tb}^{3+} / \mathrm{C} 20 \mathrm{~W}$ complex. On the left is the C-terminal domain and on the right is the N-terminal domain. The blue and green dots represent the $d_{\mathrm{xx}}$ and $d_{\mathrm{yy}}$ values and the red dots represent the $d_{\mathrm{zz}}$ values. 
The distribution of the axial component $(\mathrm{Da})$ for the tensors showed that the $\mathrm{N}$ terminal domain in holoCaM is ambiguous. However the distribution of the axial component of the alignment tensor for the C-terminal domain gave a Da value of $3.1 \pm 0.7 \mathrm{~Hz}$ (Figure 104).
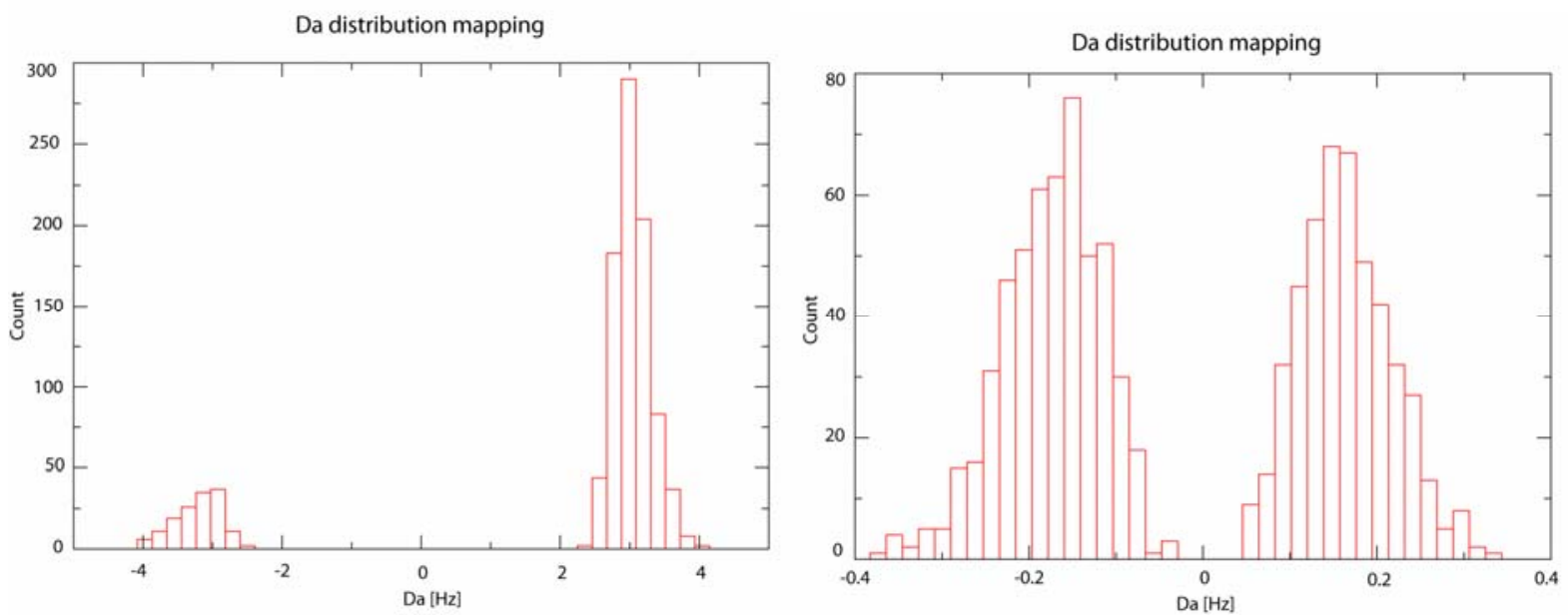

Figure 104. Distribution for the axial component (Da) of the alignment tensor for CaMT146C-pk2- $\mathrm{Tb}^{3+}$. On the left is the tensor for the C-terminal domain and on the right for the N-terminal domain.

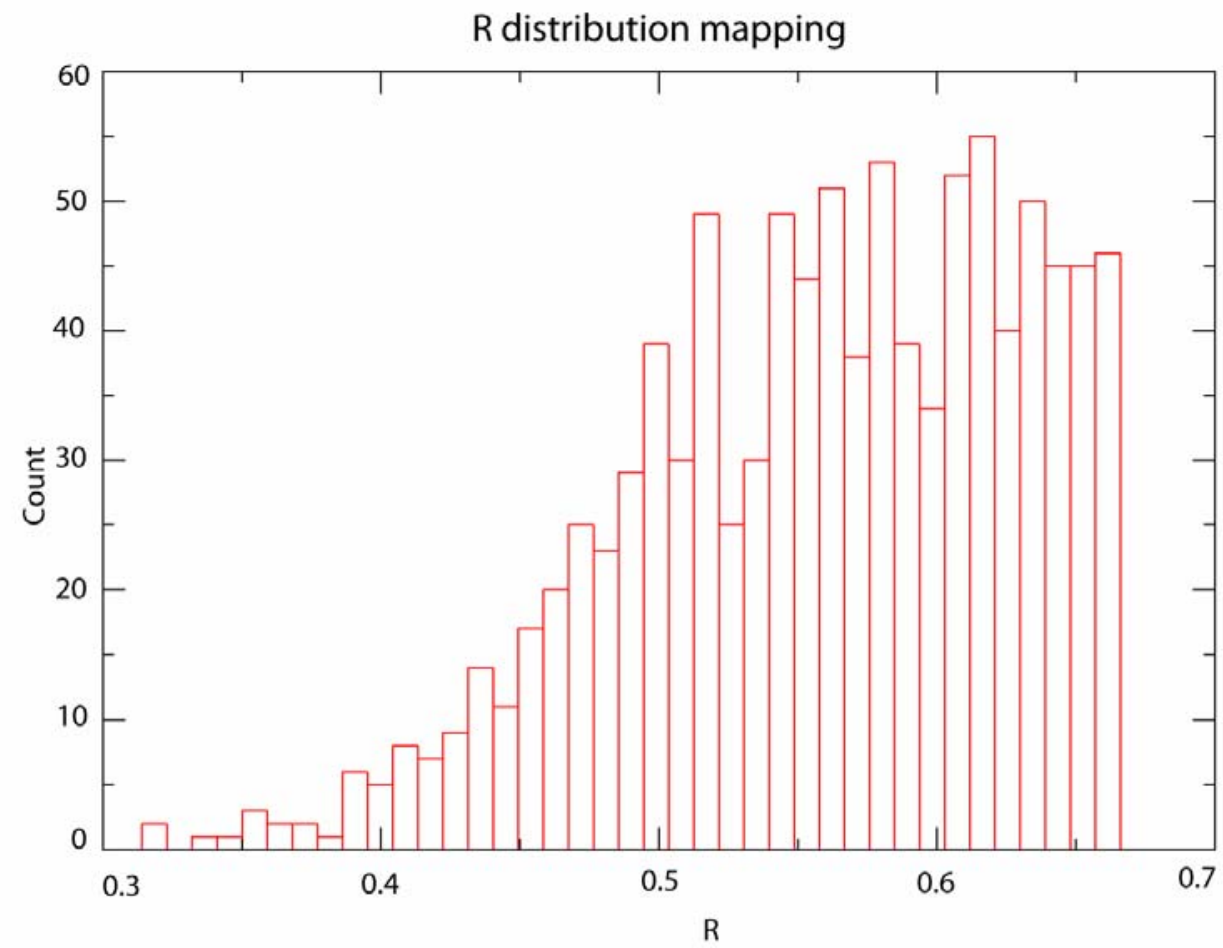

Figure 105. Distribution for the Rhombic component of the alignment tensor for the Cterminal domain of CaMT146C-pk2-Tb ${ }^{3+}$.

The plots of the tensor values for the $\mathrm{C}$ - and $\mathrm{N}$ - terminal domains of $\mathrm{CaM}$ is shown in Figure 106; these plots show that just the alignment tensor of the C-terminal domain was well defined and had a low spread around the average value. In contrast, the alignment tensor for the $\mathrm{N}$-terminal domain was randomized by the noise in the data. 

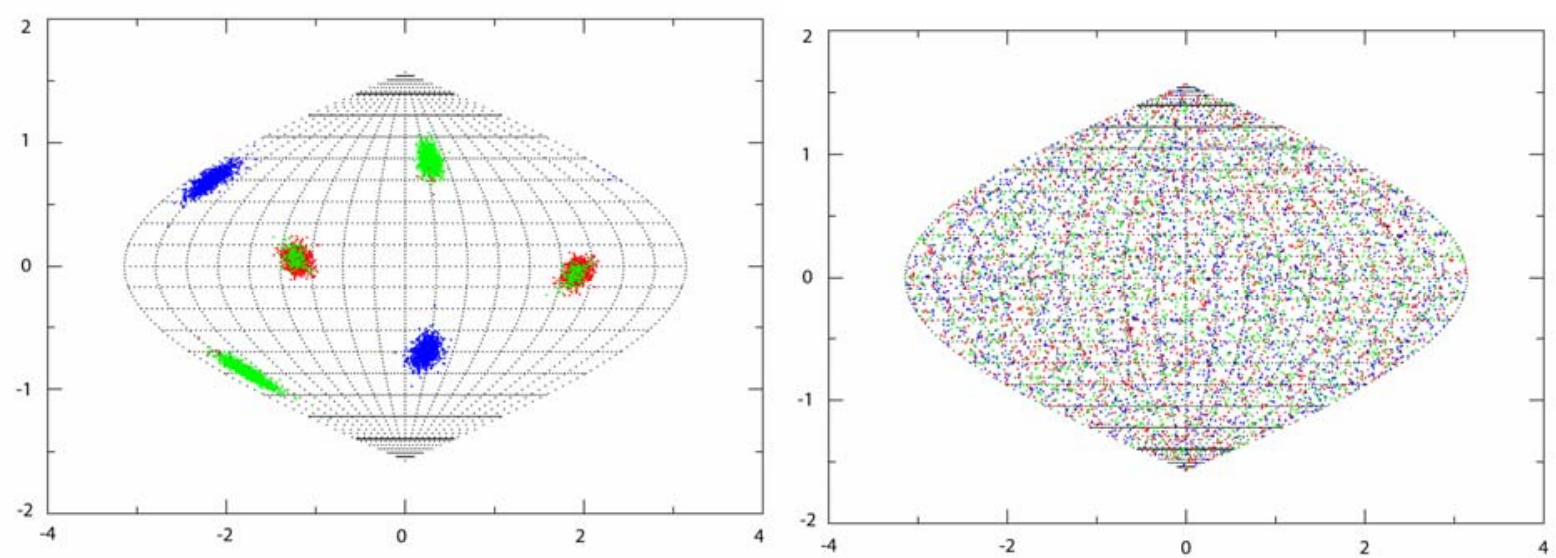

Figure 106. Mapping of the individual tensor values for the 1000 SVD calculations on a 3D plot for CaMT146C-pk2-Tb ${ }^{3+}$. On the left is the C-terminal domain and on the right is the Nterminal domain.

The position of the metal was located based on the pseudocontact shift restraints (Figure 107). It was observed that the metal ion had a different position for holoCaMT146pk2 and for the CaMT146C-pk2/C20W peptide complex. This suggested that the peptide might restrict the motion of the tag; this would also explain the larger anisotropy of the rDC distribution for this sample. In addition, the metal ion was found to be close to an acidic patch in helix E (Figure 108 and 109); it is possible that the coordination sphere of the lanthanide ion bound to the EDTA moiety could be completed by carboxylate side-chains of these residues. In a similar analysis done for apoCaM, the relationship between the metal-amide distances and the measured PCS was determined for the CaMT146C-pk2 and in its complex with C20W.

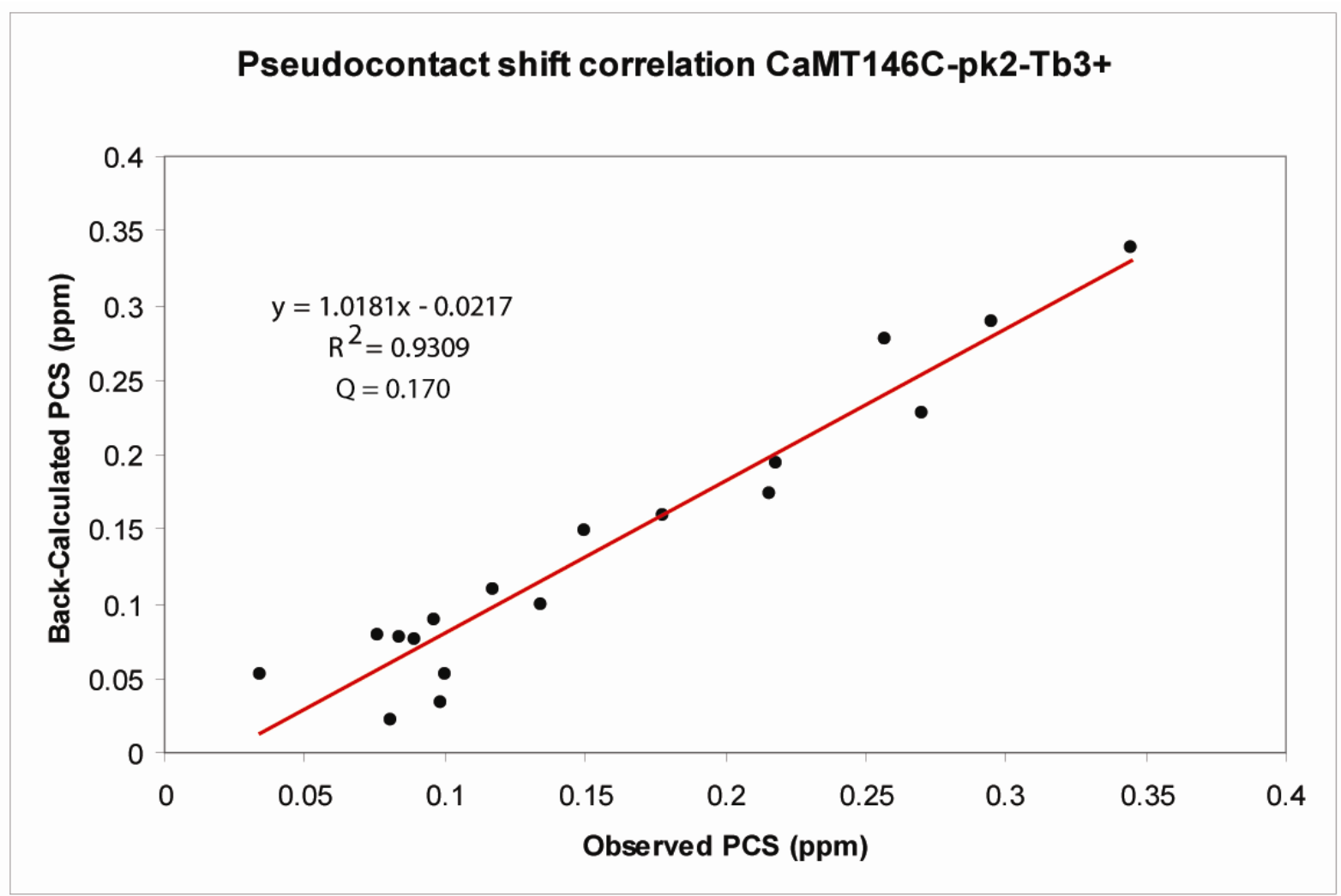




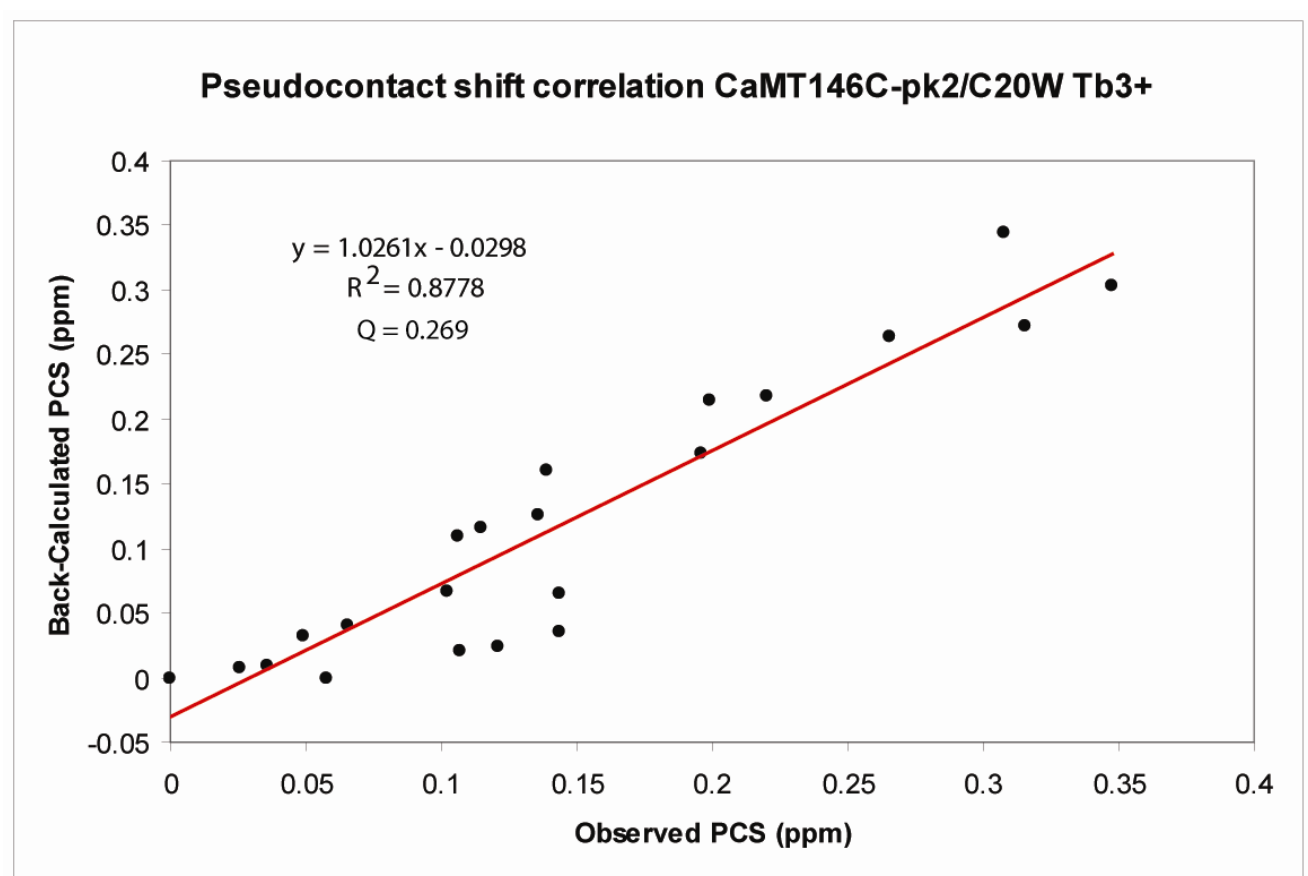

Figure 107. Correlation plot of the measured PCS for the C-terminal domain of CaMT146Cpk2- $\mathrm{Tb}^{3+}$ (upper plot) and CaMT146C-pk2- $\mathrm{Tb}^{3+} / \mathrm{C} 20 \mathrm{~W}$ peptide complex (lower plot) against the back-calculated PCS based on the NMR structure of holoCaM.

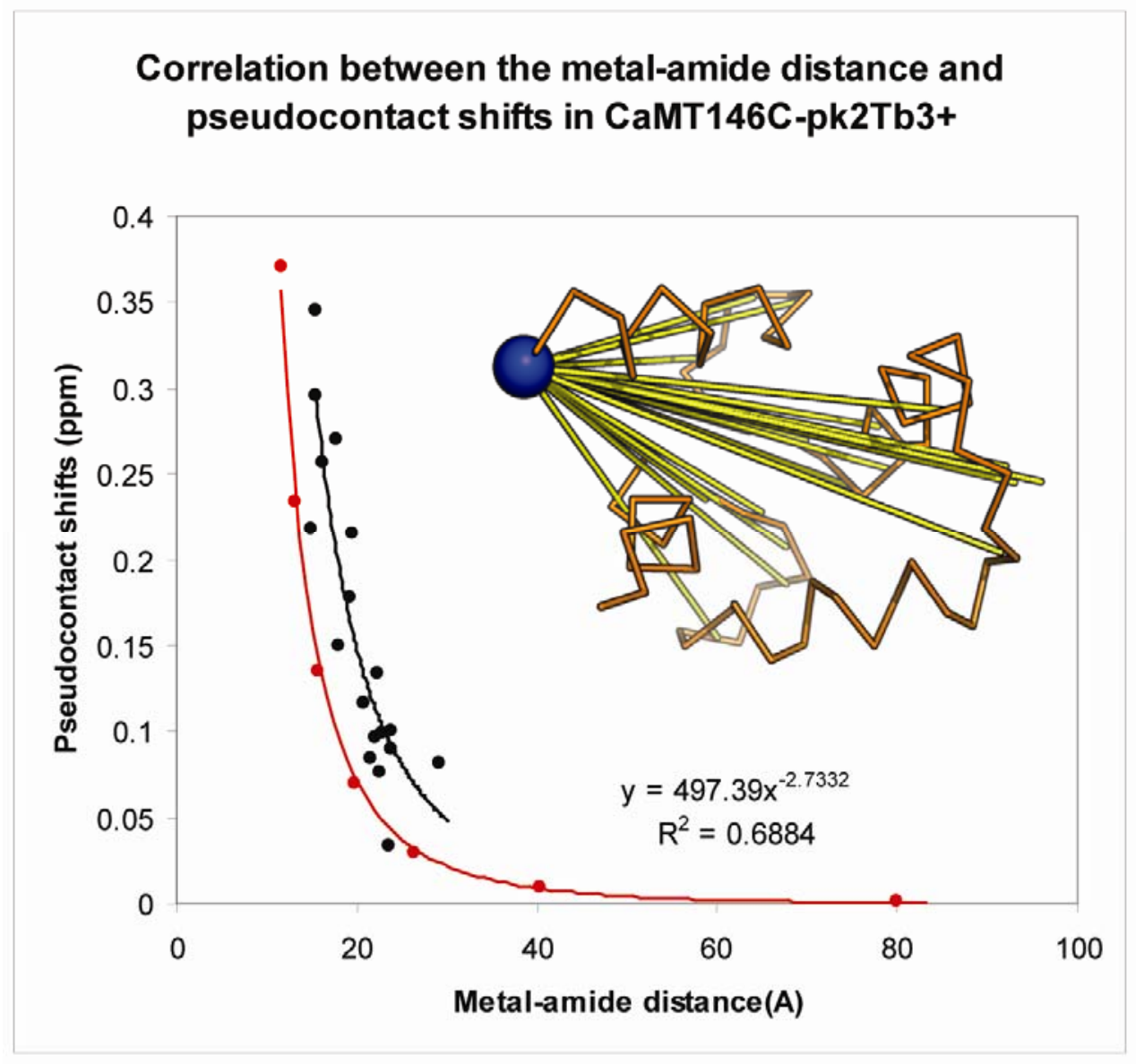

Figure 108. Correlation plot between the measured distances between the lanthanide ion and each amide proton and the observed pseudocontact shift for CaMT146C-pk2-Tb ${ }^{3+}$. The curve was fit with a power function. An upper bound $r^{3}$ plot is shown in red. 


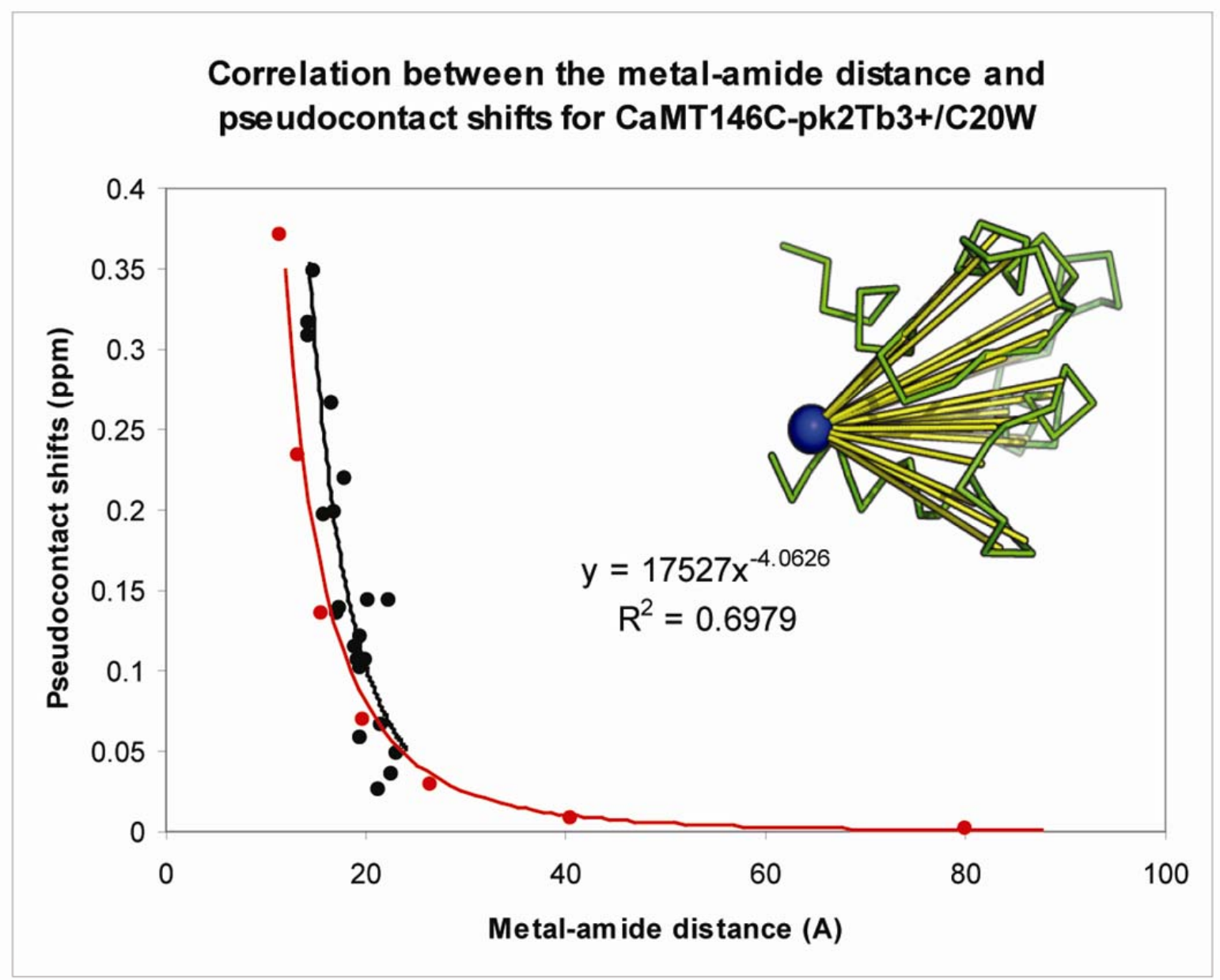

Figure 109. Correlation plot between the measured distances between the lanthanide ion and each amide proton and the observed pseudocontact shift for CaMT146C-pk2- $\mathrm{Tb}^{3+} / \mathrm{C} 20 \mathrm{~W}$ peptide complex. The curve was fit with a power function. An upper bound $r^{3}$ plot is shown in red. 


\section{DISCUSSION}

\section{Dynamic Studies on apoCaM}

The paramagnetic alignment of the apoCaMS17C mutant with three different tags loaded with Terbium ion was successful. The alignment tensor parameters could be obtained and the coordinates of the metal could be located based on pseudocontact shift data for the three cases. However, the rDCs measured on the C-terminal domain were too small in magnitude and could not be measured with enough accuracy. Therefore the interdomain dynamic studies focused on the pseudocontact shifts measured on the linker region of apoCaM, for the alignments with t43D and t43L. In both cases, a PCS could be measured for the T79 and D80 amide cross-peaks and for the alignment with t43L the PCS for S81 and E82 were also obtained. In general, PCS data pose more difficulties in their analysis, since they are dependent both on the distance $\left(r^{3}\right)$ and the orientation of the vector described by the unpaired electron of the metal ion and the spin under observation (amide in this case). In some cases, these two components can be disentangled with the aid of relaxation measurements using a high relaxing metal ion (like Gadolinium or Manganese) bound to the same paramagnetic tag; the relaxation enhancement provides upper bounds of the distance between the metal ion and the spin of interest with a $r^{6}$ dependency.

In this work, the apoCaMS17C-t43L sample was titrated with $\mathrm{MnCl}_{2}$, but the effect on the line width broadening for the amides of interest in the linker region was very small (between 2 and $5 \mathrm{~Hz}$ ), therefore, this approach was not successful. In order to obtain upper bounds for the PCS data, a $r^{3}$ function was constructed using the angles that maximized the $\left(3 \cos ^{2} \theta-1\right)$ and $\left(\sin ^{2} \theta \cos 2 \Omega\right)$ terms in the expression for the pseudocontact shift. This upper bound function was important in order to establish that the measured PCS in the linker region of apoCaM for both t43L and t43D samples deviated from the expected values based on the $r^{3}$ function. Thus, this gave experimental evidence that the residues in the liker region are not rigid and experience averaging in the submillisecond time scale due to interdomain dynamics and/or conformational exchange. It was apparent, that the averaging increases along the linker and PCS could not longer be observed in the C-terminal domain of apoCaM. Dynamic studies based on relaxation measurements on apoCaM have established that the $\mathrm{C}$-terminal domain experiences conformational exchange in the $\mu$ s time scale; it exchanges at least between a close conformation and a semi-open conformation (Tjandra et al., 1995). Chen and Hummer (2007) showed that the C-terminal domain of apoCaM populates three states in solution; a closed one, a partially unfolded one and an open one. In addition, molecular dynamic simulations have suggested that apoCaM experience large scale domain motions, and the center of mass of each domain varied from 29 to $39 \AA$ A (average distance $33 \AA$ ) during the simulation (Yang et al., 2003).

It is interesting to compare these results with those of holoCaM, since the same mutant (S17C) was also aligned with two paramagnetic tags. Although a defined tensor could be obtained for the $\mathrm{N}$-terminal domain in both samples, the alignment tensor for the C-terminal domain could not be established. In addition, no PCS were observed in the linker region. This observation is in agreement with the knowledge that in average, the domains in holoCaM are further away from each other in comparison to apoCaM. The simulation done by Yang et al., 2004, gave an average value of $38 \AA ̊$ between the centers of mass for each domain in holoCaM and the variation was from 34 to 41 Á.

\section{Dynamic Studies on holoCaM}

The studies done on the paramagnetic alignment of the T146C mutant of holoCaM could not describe the interdomain dynamics on this system; since the alignment obtained was 
too weak (rDCs up to $8 \mathrm{~Hz}$ ) in order to obtain a reliable tensor for the $\mathrm{N}$-terminal domain within the accuracy of the measurement of rDCs, based on the fact that Bertini et al., 2004 found a decrease by a factor of 10 in the axial component of the alignment tensor which did not contain the metal ion. Thus, the results obtained in this study are in nice agreement with these findings.

\section{Dynamic Studies on the CaM/C20W peptide complex}

The studies done on the paramagnetic alignment of the T146C mutant of CaM in complex with the $\mathrm{C} 20 \mathrm{~W}$ peptide showed that the mobility of this complex was more restricted than that one of holoCaM. The magnitude of the axial component for the tensor in the Nterminal domain for three different tags (pk2, t43L and t43D) complexed with Terbium got reduced in average by a factor of $\sim 4$ in comparison to that one of the paramagnetically aligned C-terminal domain. Tüchelman (1999) reported a reduced alignment tenor by a factor of two for the N-terminal domain of the $\mathrm{CaM} / \mathrm{C} 20 \mathrm{~W}$ complex aligned with DTPA-Yb in the peptide. However, the magnitude of the dipolar couplings obtained in that work were too small to give an accurate value of the tensor parameters, however qualitatively the results are in agreement with the results presented in this thesis. In addition, these data agreed well with the SAXS studies reported by Kataoka et al. 1991, where it was established that the radius of gyration for the CaM/C20W peptide complex (57.5 $\AA$ ) was significantly smaller than that one of CaM $(62.5 \AA ̊)$.

\section{Structural studies on the CaM/Munc13-1 interaction}

\section{a. apoCaM interaction with Munc13-1 and ubMunc13-2}

The observations done by Junge et al., 2004 based on intrinsic tryptophan emission fluorescence spectroscopy for the interaction of apoCaM with the Munc13-1(460-478) peptide were confirmed by recording a series of ${ }^{15} \mathrm{~N}-{ }^{1} \mathrm{H}$ HSQC spectra adding increasing amounts of the synthetic peptide to ${ }^{15} \mathrm{NapoCaM}$. The results clearly showed that the binding localizes to the $\mathrm{C}$-terminal domain of apoCaM. The studies done on the ${ }^{13} \mathrm{C},{ }^{15} \mathrm{~N}$ labeled Munc13-1(457-492) peptide showed that upon binding to apoCaM, the peptide already adopts a $\alpha$-helical conformation.

A similar HSQC-based titration experiment done for the ubMunc13-2 (382-402) synthetic peptide showed that this peptide also binds apoCaM with low affinity. The binding was also localized to the C-terminal domain of apoCaM, but it affected less NH cross peaks, suggesting that the interaction surface is smaller. Dimova et al., 2006 also probed the calcium sensitivity for the $\mathrm{CaM} / \mathrm{ubMunc} 13-2$ interaction and established that the calcium concentration needed for the complex formation is between 20 and $30 \mathrm{nM}$.

\section{b. $\left[2 \mathrm{Ca}^{2+}\right]$ CaM interaction with Munc13-1}

Upon EDTA addition to the ${ }^{15} \mathrm{NCaM} / \mathrm{Munc} 13-1(457-492)$ peptide complex it was possible to remove the two $\mathrm{Ca}^{2+}$ ions from the N-terminal domain of CaM, therefore the $\left[2 \mathrm{Ca}^{2+}\right]-{ }^{15} \mathrm{~N} \mathrm{CaM} / \mathrm{Munc} 13-1(457-492)$ peptide complex was completely populated and characterized with NMR spectroscopy. This complex state was referred as the half-activated state, since apparently, it is a high affinity $(\operatorname{sub} \mu \mathrm{M})$ interaction mediated solely by the Cterminal domain of CaM. Junge et al., 2004 found in a two-hybrid yeast screen that some clones consisting solely of the C-terminal domain of CaM were selected as Munc13-1 interaction partners, therefore the $\left[2 \mathrm{Ca}^{2+}\right] \mathrm{CaM}$ state seemed to have a strong interaction with Munc13-1 in vivo as well. This half-activated state might be of physiological relevance, since 
it would mean that at resting calcium concentrations in the neuron, the CaM/Munc13-1 interaction could be already present, increasing the kinetics of this protein-protein interaction upon full activation by eliminating the diffusion component of the reaction. Such a preformed complex has been already described in other CaM interactions like that one with the ryanodine receptor (Samsó and Wagenknecht, 2002) and with the voltage-gated $\mathrm{Ca}^{2+}$ channels (Erickson et al., 2001).

\section{c. $\left[4 \mathrm{Ca}^{2+}\right]$ CaM interaction with Munc13-1}

The initial NMR studies on the CaM/Munc13-1(459-478) peptide complex showed that this complex had a (1:1) stoichiometry as was previously described by Junge et al., 2004. With the aid of NMR experiments it was evident that this small peptide did not perturb much the $\mathrm{NH}$ cross-peaks of the N-terminal domain of CaM. For this reason, the peptide was extended towards its C-terminus and the solution structure of the CaM/Munc13-1(457-492) peptide complex was determined.

\section{d. Solution structure of the CaM/Munc13-1(457-492) peptide complex}

The solution structure revealed a novel binding mode for CaM, where a bipartite interaction is established between the N-terminal amphiphilic $\alpha$-helix of the Munc13-1 peptide and the $\mathrm{C}$-terminal domain of $\mathrm{CaM}$ via a the classical (1-5-8) hydrophobic motif; and the N-terminal domain of CaM has contacts with a hydrophobic LW motif at the C-terminal tail of the peptide. Other singular properties of this novel CaM complex include: (a) submillisecond interdomain dynamics present in the complex determined with rDC analysis from paramagnetic alignment; (b) dimerization of the complex to give a (2:2) complex favored at higher salt concentrations $(\sim 300 \mathrm{mM} \mathrm{KCl})$; and (c) heterogeneity of the $\mathrm{W} 489 \mathrm{N \varepsilon}$ $\mathrm{H} \varepsilon$ side chain of the bound LW motif; one possible source of this heterogeneity could have arisen from an unbound (8.4\%) and bound (91.6\%) LW motif; another possible explanation could be different side chain $\chi$-rotamer populations $(8.4 \%$ and $91.6 \%)$ in slow exchange on the NMR time scale, giving rise to two resonances.

\section{e. Accuracy of the solution structure of the CaM/Munc13-1(457-492) peptide complex}

The total amount of NOE restraints (1758) were enough to obtain a well defined threedimensional structure of this complex in solution. The backbone conformation converged to a RMSD of $0.56 \AA$ for the C-terminal domain of CaM and to $0.74 \AA$ for the $\mathrm{N}$-terminal domain of $\mathrm{CaM}$ to the mean structure in the ensemble. The backbone atoms of each domain were overlaid to several high-resolution structures of $\mathrm{CaM}$ in various activated states, showing that the backbone conformation was not very much changed upon binding of the Munc13-1 peptide. An average backbone RMSD of $2.4 \AA$ and $2.8 \AA$ to the analyzed structures was found for the $\mathrm{C}$ - and $\mathrm{N}$-terminal domains of $\mathrm{CaM}$, respectively. This is consistent with the observation that the C-terminal domain had a larger number of NOE restraints per residue and resonance assignments than the $\mathrm{N}$-terminal domain. Helix $\mathrm{D}$ in the $\mathrm{N}$-terminal domain of CaM posed large difficulties in the resonance assignment, because of broad resonances, pointing towards conformational averaging. The solution structure of the protein-peptide complex revealed that CaM interacts with the Munc13-1 (457-492) peptide in an extended conformation. There are numerous previous reports in the literature for CaM interacting with its binding partner in an extended conformation: (a) the crystal structure of CaM with the CaM-binding domain of a $\mathrm{Ca}^{2+}$-activated $\mathrm{K}^{+}$channel (Schumacher et al., 2001); (b) CaM complex with the Edema Factor of Bacillus anthracis (Drum et al., 2002); (c) the CaM complex with the C20W peptide of the plasma membrane $\mathrm{Ca}^{2+}$ pump (Elshorst et al., 1999); 
(d) the CaM complex with a dimeric peptide from the basic helix-loop-helix transcription factor SEF2-1/EF2-2 (Larsson et al., 2001); (e) the CaM complex with two peptides of plant glutamate decarboxilase (Yap et al., 2003): and (f) the CaM interaction with the Microtubuleassociated protein F-STOP (Bouvier et al., 2003). To our knowledge, this is the first report where an amphiphilic $\alpha$ helix motif (1-5-8) is bound to the $\mathrm{C}$-terminal domain of $\mathrm{CaM}$ and a second hydrophobic anchor $\left(\mathrm{L}^{488} \mathrm{~W}^{489}\right)$ binds to the $\mathrm{N}$-terminal domain of CaM. Therefore, the structure of the CaM/Munc13-1 (457-492) peptide complex supports the view of the studies done on the calcium-dependent inactivation mechanism of the CaM regulation of Voltage-gated calcium channels (van Petegem et al., 2005). In these studies, it has been established that the CaM-Cav1.2 IQ domain-CaM interaction has a bipartite nature, where the $\mathrm{C}$-terminal and $\mathrm{N}$-terminal domains of $\mathrm{CaM}$ bind distinct hydrophobic anchor residues in the target protein with different affinities. In the case of the Voltage-gated $\mathrm{Ca}^{2+}$ channels this differential interaction has relevance in the calcium-dependent facilitation regulation mechanism. The functional relevance for the bipartite interaction in the CaM/Munc13-1 peptide complex is not completely understood, but complementary studies done on the larger CaM/Munc13-1 (185aa) complex give hints to a switch mechanism as is discussed below.

\section{complexes \\ f. NMR studies on the CaM/Munc13-1(185aa) monomeric (1:1) and dimeric (2:2)}

The NMR studies done on the CaM/Munc13-1(185aa) complex extended the understanding of this protein-protein interaction at a molecular level. First, the heterogeneity in the W489 $\mathrm{N} \varepsilon-\mathrm{H} \varepsilon 1$ side chain described for the CaM/Munc13-1 peptide complex was also found in the NMR study of this larger complex and surprisingly, the W588 Ne-He1 side chain within the $\mathrm{C}_{1}$ domain of Munc13-1 was also heterogeneous. Second, the (2:2) complex described for the CaM/Munc13-1 (459-479) peptide interaction was also present in the CaM/Munc13-1(185) interaction. In this larger complex, the dimerization appeared to be stronger and the two complex species could be separated and studied independently. Third, experiments done on the $\mathrm{C}_{1}$ domain of Munc13-1 lacking the CaM binding site, gave a single resonance signal for the $\mathrm{W} 588 \mathrm{~N} \varepsilon-\mathrm{H} \varepsilon 1$ side-chain; therefore the heterogeneity observed in the larger CaM/Munc13-1(185aa) complex should be attributed either to its interaction with the $\mathrm{N}$-terminal domain of $\mathrm{CaM}$ or to an intramolecular interaction upstream of the $\mathrm{C}_{1}$ domain. Although the dimerization mechanism is not completely understood for this interaction, a picture starts to emerge: (a) The conformational exchange equilibrium between the N-terminal domain of $\mathrm{CaM}$ and the $\mathrm{C}_{1}$ domain of Munc13-1 described in the CaM/Munc13-1(185aa) dimeric (2:2) complex species suggest that these two domains might be involved in the dimerization process. To add more evidence on this possible novel domain-domain interaction; it would be interesting to measure the exchange contribution to the relaxation of the NH cross-peaks in these two domains, in order to determine if the exchange occurs on the same time scale. (b) The activation of the $\mathrm{C}_{1}$ domain of Munc13-1 in the CaM/Munc13-1 (185aa) dimeric (2:2) complex with its agonist, phorbol ester (PDBu), shifted the conformational exchange equilibrium towards the monomeric side of the reaction; since the line shape of the $\mathrm{NH}$ resonances improved significantly. In contrast, the same PDBu activation experiment done on the CaM/Munc13-1(185aa) monomeric (1:1) complex did not give rise to large changes in the HSQC spectrum, although clear ligand binding was observed. (c) Electrophysiology studies by Basu et al., 2007 proposed that in the basal state of Munc131 , its $\mathrm{C}_{1}$ domain appears to be inhibited by intramolecular interactions interfering with the $\mathrm{C}$ terminal MUN catalytic domain. The activation with DAG/phorbol ester induces a conformational change disrupting the intramolecular interactions releasing the catalytic domain from its inhibited state; this open configuration of Munc13-1 is also mimicked by the $\mathrm{H} 567 \mathrm{~K}$ mutation that disrupts the $\mathrm{C}_{1}$ domain fold removing one coordination site for the zinc 
metal ion in this domain. (d) In line with the structural studies by Lu et al., 2006, it can be speculated that the basal state of Munc13-1 consists of a homodimer with an inhibited Cterminal MUN catalytic domain and upon the sequential or concerted action of the RIM zincfinger domain, $\mathrm{CaM}$, and PDBu activation, the homodimer is relieved and the catalytic MUN domain gets activated for acting on its down-stream targets like syntaxin-1 and Munc18. Additional experiments need to be done to better understand the modulation on the CaM/Munc13-1(185aa) complex dimer (2:2) to monomer (1:1) equilibrium by phorbol esters and/or calcium to gain a better understanding on the regulation of the presynaptic plasticity processes in neurons. 


\section{CONCLUSIONS}

The contributions of this work to the understanding of CaM plasticity include: 1. The establishment of a difference in the dynamics of CaM in three distinct activated states based on paramagnetic restraints. Firstly, a difference between apoCaM and holoCaM dynamics could be described on the basis of pseudocontact shifts measured for the CaMS17C mutant aligned with various paramagnetic tags. Secondly a difference between holoCaM and the CaM/C20W peptide complex dynamics could be described on the basis of residual dipolar couplings measured for the CaMT146C mutant aligned with three different paramagnetic tags. In all of these three independent measurements, a residual alignment (around 25\%) of the N-terminal domain of CaM was measured for the CaM/C20W peptide complex, whereas for the free holoCaM no residual alignment could be established.

2. The determination of the NMR solution structure of CaM complexed with the Munc131(458-492) peptide belonging to the diacylglycerol-binding protein Munc13-1, an essential priming factor in neurotransmitter release. The structure describes a new binding motif for CaM consisting in a bipartite interaction, where the C-terminal domain of CaM binds the $\mathrm{N}$ terminal amphiphilic $\alpha$-helix (1-5-8) hydrophobic motif in the peptide and the N-terminal domain of CaM binds to a C-terminal LW motif in the peptide. This bipartite binding mode parallels that one on the CaM/IQ motif interaction for the regulation of the voltage-gated $\mathrm{Ca}^{2+}$-channels; however functional studies on Munc13-1 mutants should establish the physiological significance of this interaction mode.

3. The extension of the NMR studies of calmodulin with a larger fragment of Munc13-1 (447631) consisting of its CaM-binding and diacylglycerol/phorbol ester $C_{1}$ binding domain suggests that the $\mathrm{N}$-terminal domain of CaM switches between the LW motif found in the determined NMR structure of the CaM/Munc13-1 peptide complex and a second hydrophobic motif within the $\mathrm{C}_{1}$ domain of Munc13-1. A definite experimental proof (long range distance NMR restraint provided by an intermolecular NOE or pseudocontact shift) is still needed to establish a direct interaction between the $\mathrm{N}$-terminal domain of CaM and the $\mathrm{C} 1$ domain of Munc13-1, which would give a structural correlate to physiological studies suggesting interplay between these two modules in Munc13-1.

4. Strong evidence for the relevance of a dimerization mechanism on the CaM regulation of Munc13-1 could also be established, based on the NMR studies of the CaM/Munc13-1 (447631) dimeric (2:2) complex and the binding to its agonist, the phorbol ester PDBu. Additional studies are needed to fully characterize this model. 


\section{BIBLIOGRAPHY}

Babu, Y.S., Bugg, C.E., Cook, W.J. (1988) Structure of calmodulin refined at $2.2 \AA$ resolution. J. Mol. Biol. 204, 191-204.

Barbato, G., Ikura, M., Kay, L.E., Pastor, R.W., Bax, A. (1992) Backbone dynamics of calmodulin studied by ${ }^{15} \mathrm{~N}$ relaxation using inverse detected two-dimensional NMR spectroscopy: The central helix is flexible. Biochemistry. 31, 5269-5278.

Basu, J., Betz, A., Brose, N., Rosenmund, C. (2007) Munc13-1 C1 domain activation lowers the energy barrier for synaptic vesicle fusion. J. Neurosci. 27, 1200-1210.

Basu, J., Shen, N., Dulubova, I., Lu, J., Guan, R., Guryev, O., Grishin, N.V., Rosenmund, C., Rizo, J. (2005) A minimal domain responsible for Munc13 activity. Nat. Struct. Mol. Biol. 12, 1017-1018.

Bax, A., Delaglio, F., Grzesiek, S., Vuister, G.W. (1994) Resonance assignment of methionine methyl groups and $\chi^{3}$ angular information from long-range proton-carbon and carbon-carbon J correlation in a calmodulin-peptide complex. J. Biomol. NMR. 4, 787-797.

Bertini, I., Del Bianco, C., Gelis, I., Katsaros, N., Luchinat, C., Parigi, G., Peana, M., Provenzani, A., Zoroddu, M.A. (2004) Experimentally exploring the conformational space sampled by domain reorientation in calmodulin. Proc. Nat. Acad. Sci. 101, 6841-6846.

Bertini, I., Luchinat, C. (1996) “NMR of paramagnetic substances”Elsevier Science, S.A.

Bertini, I., Luchinat, C., Parigi, G. (2002) Magnetic susceptibility in paramagnetic NMR. Prog. NMR Spec. 40, 249-273.

Bonneau, R., Strauss, C.E., Rohl, C.A., Chivian, D., Bradley, P., Malmström, L., Robertson, T., Baker, D. (2002) De novo prediction of three-dimensional structures for major protein families. J. Mol. Biol. 322, 65-78.

Bouvier, D., Vanhaverbeke, C., Simorre, J-P., Arlaud, G.J., Bally, I., Forge, V., Margolis, R.L., Gans, P., Kleman, J-P. (2003) Unusual $\mathrm{Ca}^{2+}$-calmodulin binding interactions of the microtubule-associated protein F-STOP. Biochemistry. 42, 11484-11493.

Brooks, B.R., Bruccoleri, R.E., Olafson, B.D., States, D.J, Swaminathan, S., Karplus, M. (1983) CHARMM: A program for macromolecular energy, minimization, and dynamics calculations. J. Comp. Chem. 4, 187-217.

Brose, N., Rosenmund, C., (2002) Move over protein kinase C, you've company: alternative cellular effectors of diacylglycerol and phorbol esters. J. Cell. Sci. 115, 4399-4411.

Brüschweiler, R. (1994) Measurements and theoretical models of structural dynamics of biopolymers in solution. In: Tycko, R. (ed.) Nuclear Magnetic Resonance Probes of Molecular Dynamics. Kluwer Acad. Publ. 301-334.

Cavanagh, J., Fairbrother, W.J., Palmer, A.G., Skelton, N.J. (1996) Protein NMR spectroscopy: Principles and Practice. Academic Press. San Diego. 587pp. 
Chang, S-L., Szabo, A., Tjandra, N. (2003) Temperature dependence of domain motions of calmodulin probed by NMR relaxation at multiple fields. J. Am. Chem. Soc. 125, 1137911384.

Chang, S-L., Tjandra, N. (2001) Analysis of NMR relaxation data of biomolecules with slow domain motions using wobble-in-a-cone approximation. J. Am. Chem. Soc. 123, 1148411485 .

Chen, Y-G., Hummer, G. (2007) Slow conformational dynamics and unfolding of the calmodulin C-terminal domain. J. Am. Chem. Soc. 129, 2414-2415.

Chou, J.J., Li, S., Bax, A. (2000) Study of conformational rearrangement and refinement of structural homology models by the use of heteronuclear dipolar couplings. J. Biomol. NMR. $18,217-227$.

Chou, J.J., Li, S., Klee, C.B., Bax, A. (2001) Solution structure of $\mathrm{Ca}^{2+}$-calmodulin reveals flexible hand-like properties of its domains. Nature Struct. Biol. 8, 990-997.

Clore, G.M. (2000) Accurate and rapid docking of protein-protein complexes on the basis of intermolecular nuclear Overhauser enhancement data an dipolar couplings by rigid body minimization. Proc. Nat. Acad. Sci. 97, 9021-9025.

Cornilescu, G., Delaglio, F., Bax, A. (1999) Protein backbone angle restraints from searching a database for chemical shift and sequence homology. J. Biomol. NMR. 13, 289-302.

Delaglio, F., Grzesiek, S., Vuister, G.W., Zhu, G., Pfeifer, J., Bax, A. (1995) NMRPipe: a multidimensional spectral processing system based on UNIX pipes. J. Biomol. NMR. 6, 277293.

Dimova, K., Kawabe, H., Betz, A., Brose, N., Jahn, O. (2006) Characterization of the Munc13-1-calmodulin interaction by photoaffinity labeling. Biochim. Biophys. Acta. 1763, $1256-1265$.

Drum C.L., Yan, S-Z., Bard, J., Shen, Y-Q., Lu, D., Soelaiman, S., Grabarek, Z., Bohm, A., Tang, W-J. (2002) Structural basis for the activation of anthrax adenylyl cyclase exotoxin by calmodulin. Nature. 415, 396-402.

Dulubova, I., Lou, X., Lu, J., Huryeva, I., Alam, A., Schneggenburger, R., Südhof, T.C., Rizo, J. (2005) A Mucn13/RIM/Rab3 tripartite complex: from priming to plasticity? The EMBO J. 24, 2839-2850.

Elshorst, B., Hennig, M., Försterling, H., Diener, A., Maurer, M., Schulte, P., Schwalbe, H., Griesinger, C., Krebs, J., Schmid, H., Vorherr, T., Carafoli, E. (1999) NMR solution structure of a complex of calmodulin with a binding peptide of the $\mathrm{Ca}^{2+}$ pump. Biochemistry. 38 , 12320-12332.

Erickson, M.G., Alseikhan, B.A., Peterson, B.Z., Yue, D.T. (2001) Preassociation of calmodulin with voltage-gated $\mathrm{Ca}^{2+}$ channels revealed by FRET in single living cells. Neuron. 31, 973-985.

Falke, J.J. (2002) Enzymology. A moving story. Science 295, 1480-1. 
Fallon, J.L., Quiocho, F.A. (2003) A closed compact structure of native $\mathrm{Ca}^{2+}$-calmodulin. Structure. 11, 1303-1307.

Florida State University (FSU). 1999. http://fajerpc.magnet.fsu.edu.

Gaponenko, V., Sarma, S.P., Altieri, A.S., Horita, D.A., Li, J., Byrd, A. (2004) Improving the accuracy of NMR structures of large proteins using pseudocontact shifts as long-range restraints. J. Biomol. NMR. 28, 205-212.

Goddard, T.D., Kneller, D.G. (1999) SPARKY 3. University of California, San Francisco.

Grzesiek, S., Anglister, J., Ren, H., Bax, A. (1993) Carbon-13 line narrowing by deuterium decoupling in deuterium/carbon-13/nitrogen-15 enriched proteins. Application to triple resonance 4D J connectivity of sequential amides. J. Am. Chem. Soc. 115, 4369-4370.

Groffen, A.J., Friedrich, R., Brian, E.C., Ashery, U., Verhage, M. (2006) DOC2A and DOC2B are sensors for neuronal activity with unique calcium-dependent and kinetic properties. J. Neurochem. 97, 818-833.

Güntert, P., Mumenthaler, C., Wüthrich, K. (1997) Torsion Angle Dynamics for NMR structure calculation with the new program DYANA. J. Mol. Biol. 273, 283-298.

Haberz, P., Rodríguez-Castañeda, F., Junker, J., Becker, S., Leonov, A., Griesinger, C. (2006) Two new chiral EDTA-Based metal chelates for weak alignment of proteins in solution. Org. Letters. 8, 1275-1278.

Heidorn, D.B., Trewhella, J. (1988) Comparison of the crystal and solution structures of calmodulin and troponin C. Biochemistry. 27, 909-915.

Herrmann, T., Güntert, P., Wüthrich, K. (2002) Protein NMR structure determination with automated NOE assignment using the new software CANDID and the Torsion Angle Dynamics Algorithm DYANA. J. Mol. Biol. 319, 209-227.

Hoeflich, K.P., Ikura, M. (2002). Calmodulin in action: diversity in target recognition and activation mechanisms. Cell. 108, 739-742.

Holthoff, K., Tsay, D. (2002) Calcium dynamics in spines: link to synaptic plasticity. Exp. Physiol. 87, 725-731.

Igumenova, T.I., Lee, A.L., Wand, A.J. (2005) Backbone and side chain dynamics of mutant calmodulin-peptide complexes. Biochemistry. 44, 12627-12639.

Ikegami, T., Verdier, L., Sakhaii, P., Grimme, S., Pescatore, B., Saxena, K., Fiebig, K.M., Griesinger, C. (2004) Novel techniques for weak alignment of proteins in solution using chemical tags coordinating lanthanide ions. J. Biomol. NMR. 29, 339-349.

Ikura, M., Ames, J.B. (2006) Genetic polymorphism and protein conformational plasticity in the calmodulin superfamily: Two ways to promote multifunctionality. Proc. Nat. Acad. Sci. $103,1159-1164$. 
Ikura, M., Barbato, G., Klee, C.B., Bax, A. (1992) Solution structure of calmodulin and its complex with a myosin light chain kinase fragment. Cell Calcium. 13, 391-400.

Ikura, M., Clore, G.M., Gronenborn, A.M., Zhu, G., Klee, C.B., Bax, A. (1992) Solution structure of a calmodulin-target peptide complex by multidimensional NMR. Science. 256, 632-638.

Ikura, M., Kay, L.E., Bax, A. (1990) A novel approach for sequential assignment of 1H, 13C, and $15 \mathrm{~N}$ spectra of proteins: heteronuclear triple-resonance three-dimensional NMR spectroscopy. Application to calmodulin. Biochemistry. 29, 4659-4667.

Jee, J.G., Güntert, P. (2003) Influence of the completeness of chemical shift assignments on NMR structures obtained with automated NOE assignment. J. Struct. Funct. Gen. 4, 179-189.

Johnson, C.K. (2006) Calmodulin, conformational states, and calcium signaling. A singlemolecule perspective. Biochemistry. 45, 14233-14246.

Johnson, C.K., Osborn, K.D., Allen, M.W., Slaughter, B.D. (2005) Single-molecule fluorescence spectroscopy: New probes of protein function and dynamics. Physiology. 20, 1014.

Junge, H.J., Rhee, J-S., Jahn, O., Varoqueaux, F., Spiess, J., Waxham, M.N., Rosenmund, C., Brose, N. (2004) Calmodulin and Munc13 form a Ca ${ }^{2+}$ sensor/effector complex that controls short-term synaptic plasticity. Cell. 118, 389-401.

Kalla, S., Stern, M., Basu, J., Varoqueaux, F., Reim, K., Rosenmund, C., Ziv, N.E., Brose, N. (2006) Molecular dynamics of a presynaptic active zone protein studied in Munc13-1-

Enhanced Yellow Fluorescent Protein Knock-In mutant mice. J. Neurosci. 26, 13054-13066.

Kataoka, M., Head, J.F., Vorherr, T., Krebs, J., Carafoli, E. (1991) Small-angle X-ray scattering study of calmodulin bound to two peptides corresponding to parts of the calmodulin-binding domain of the plasma membrane $\mathrm{Ca}^{2+}$ pump. Biochemistry. 30, 62476251 .

Kazanietz, M.G. (2002) Novel "Nonkinase" phorbol ester receptors: The C1 domain connection. Mol. Pharmacol. 61, 759-767.

Kazanietz, M.G., Barchi, J.J., Omichinski, J.G., Blumberg, P.M. (1995) Low affinity binding of phorbol esters to protein kinase $\mathrm{C}$ and its recombinant cysteine-rich region in the absence of phospholipids. J. Biol. Chem. 270, 14679-14684.

Kensal, E., van Holde, W., Johnson, C., Ho, P-S. (2006) Principles of physical biochemistry. $2^{\text {nd }}$. Ed. Prentice Hall. New Jersey, USA. 752pp.

Kubinowa, H., Tjandra, N., Grzesiek, S., Ren, H., Klee, C.B., Bax, A. (1995) Solution structure of calcium-free calmodulin. Nat. Struct. Biol. 2, 768-776.

Larsson, G., Schleucher, J., Onions, J., Hermann, S., Grundström, T., Wijmenga, S.S. (2001) A novel target recognition revealed by calmodulin in complex with the basic helix-loop-helix transcription factor SEF2-1/E2-2. Prot. Sci. 10, 169-186. 
Larsson, G., Schleucher, J., Onions, J., Hermann, S., Grundström, T., Wijmenga, S.S. (2005) Backbone dynamics of a symmetric calmodulin dimer in complex with the calmodulinbinding domain of the basic-helix-loop-helix transcription factor SEF2-1/E2-2: A highly dynamic complex. Biophys. J. 89, 1214-1226.

Laskowski, R.A., Rullman, J.A.C., MacArthur, M.W., Kaptein, R., Thornton, J.M. (1996) AQUA and PROCHECK-NMR: Programs for checking the quality of protein structures solved by NMR. J. Biomol. NMR. 8, 477-486.

Leenders, A.G.M., Sheng, Z-H. (2005) Modulation of neurostransmitter release by the second messenger-activated protein kinases: implications for presynaptic plasticity. Pharmacol. Ther. $105,69-84$.

LeMaster, D.M. (1990) Deuterium labelling in NMR structural analysis of larger proteins. Q. Rev. Biophys. 23, 133-174.

Leonov, A., Voigt, B., Rodríguez-Castañeda, F., Sakhaii, P., Griesinger, C. (2005) Convenient syntheses of multifunctional EDTA-Based chiral metal chelates substituted with an S-mesylcysteine. Chem. Eur. J. 11, 1-8.

Li, L., Chin, L-S. (2003) The molecular machinery of synaptic vesicle exocytosis. CMLS, Cell. Mol. Life Sci. 60, 942-960.

Linge, J.P., Williams, M.A., Spronk, C.A.E.M., Bonvin, A.M.J.J., Nilges, M. (2003) Refinement of protein structures in explicit solvent. Proteins. 50, 496-506.

Lipari, G., Szabo, A. (1981) Nuclear magnetic resonance relaxation in nucleic acid fragments: models for internal motion. Biochemistry. 20, 6250-6256.

Lodish, H., Baltimore, D., Berk, A., Zipursky, S.L., Matsudaira, P., Darnell, J. (2000). Molecular Cell Biology. $4^{\text {th }}$. Ed. W.H. Freeman and Company. New York.

Lou, X., Scheuss, V., Schneggenburger, R. (2005) Allosteric modulation of the presynaptic $\mathrm{Ca}^{2+}$ sensor for vesicle fusion. Nature. 435, 497-501.

Lu, J., Machius, M., Dulubova, I., Dai, H., Südhof, T.C., Tomchick, D.R., Rizo, J. (2006) Structural basis for a Munc13-1 homodimer to munc13-1/RIM heterodimer switch. PLoS Biology. 4, e192.

Markley, J.L. and Kainosho, M. (1993) Stable isotope labeling and resonance assignments in larger proteins. In "NMR of macromolecules"edited by: Roberts, G.C.K. Oxford University Press. 399pp.

Meador, W.E., Means, A.R., Quiocho, F.A. (1993) Modulation of calmodulin plasticity in molecular recognition on the basis of X-ray structures. Science. 262, 1718-1721.

Mittermaier, A., Kay, L.E. (2006) New tools provide new insights in NMR studies of protein dynamics. Science. 312, 224-228.

Mülhardt, C. (2003) Experimentator: Molekularbiologie/Genomics. 4th. Ed. Spektrum Akademischer Verlag. 280pp. 
Nofal, S., Becherer, U., Hof, D., Matti, U., Rettig, J. (2007) Primed vesicles can be distinguished from docked vesicles by analyzing their mobility. J. Neurosci. 27, 1386-1395.

Ottiger, M., Delaglio, F., Bax, A. (1998) Measurement of $J$ and dipolar couplings from simplified two-dimensional NMR spectra. J. Magn. Res. 131, 373-378.

Panchal, S.C., Bhavesh, N.S., Hosur, R.V. (2001) Improved 3D triple resonance experiments, $\mathrm{HNN}$ and $\mathrm{HN}(\mathrm{C}) \mathrm{N}$, for $\mathrm{HN}$ and $15 \mathrm{~N}$ sequential correlations in $(13 \mathrm{C}, 15 \mathrm{~N})$ labeled proteins: application to unfolded proteins. J. Biomol. NMR. 20, 135-147.

Prestegard, J.H., Al-Hashimi, H.M., Tolman, J.R. (2000) NMR structures of biomolecules using field oriented media and residual dipolar couplings. Quarterly Rev. Biophys. 33, 371424.

Prestegard, J.H., Al-Hashimi, H.M., Tolman, J.R. (2000) NMR structures of biomolecules using field oriented media and residual dipolar couplings. Qarterly Reviews of Biophysics. $33,371-424$.

Rettig, J., Neher, E. (2002) Emerging roles of presynaptic proteins in $\mathrm{Ca}^{++}$-triggered exocytosis. Science. 298, 781-785.

Rhee, J.S., Betz, A., Pyott, S., Reim, K., Varoqueaux, F., Augustin, I., Hesse, D., Südhof, T.C., Takahashi, M., Rosenmund, C., Brose, N. (2002) Beta phorbol ester- and diacylglycerol- induced augmentation of transmitter release is mediated by Munc13s and not by PKCs. Cell. 108, 121-133.

Richmond, J. (2005) Synaptic function, Wormbook, ed. The C. elegans Research Community, Wormbook, doi/10.1895/wormbook.1.69.1, http://www.wormbook.org.

Rodríguez-Castañeda, F., Haberz, P., Leonov, A., Griesinger, C. (2006) Paramagnetic tagging of diamagnetic proteins for solution NMR. Magn. Reson. Chem. 44, S10-S16.

Sambrook, J., Russell, D.W. (2001) Molcular Cloning: A Laboratory Manual. $3^{\text {rd }}$ Ed. Cold Spring Harbor Laboratry Press. New York. 2344pp.

Samsó, M., Wagenknecht, T. (2002) Apocalmodulin and $\mathrm{Ca}^{2+}$-Calmodulin bind to neighboring locations on the ryanodine receptor. J. Biol. Chem. 277, 1349-1353.

Sartor, M. (2003) Dynamic Light Scattering. University of California San Diego. USA.

Sattler, M., Schleucher, J., Griesinger, C. (1999) Heteronuclear multidimensional NMR experiments for the structure determination of proteins in solution. Prog. NMR Spec. 34, $93 ß 158$.

Shen, N., Guryev, O., Rizo, J. (2005) Intramolecular occlusion of the diacylglycerol binding site in the $\mathrm{C}_{1}$ domain of munc13-1. Biochemistry. 44, 1089-1096.

Schumacher, M.A., Rivard, A.F., Bächinger, H.P., Adelman, J.P. (2001) Structure of the gating domain of a $\mathrm{Ca}^{2+}$-activated $\mathrm{K}^{+}$channel complexed with $\mathrm{Ca}^{2+} /$ calmodulin. Nature. 410 , 1120-1124. 
Shen, N., Guryev, O., Rizo, J. (2005) Intramolecular occlusion of the Diacylglycerol-binding site in the C1 domain of Munc13-1. Biochemistry. 44, 1089-1096.

Siivari, K., Zhang, M., Palmer III, A.G., Vogel, H.J. (1995) NMR studies of the methionine methyl groups in calmodulin. FEBS Letters. 366, 104-108.

Silinski, E.M., Searl, T.J. (2003) Phorbol esters and neurotransmitter release: more than just protein kinase C? British J. of Pharm. 138, 1191-1201.

Stevens, D.R., Wu, Z-X., Matti, U., Junge, H.J., Schirra, C., Becherer, U., Wojcik, S.M., Brose, N., Rettig, J. (2005) Identification of the minimal protein domain required for priming activity of Munc13-1. Curr. Biol. 15, 2243-2248.

Südhof, T.C. (2004) The synaptic vesicle cycle. Annu. Rev. Neurosci. 27, 509-547.

Tjandra, N., Bax, A. (1997) Direct measurement of distances and angles in biomolecules by NMR in a dilute liquid crystalline medium. Science. 278, 1111-1114.

Tjandra, N., Kuboniwa, H., Ren, H., Bax, A. (1995) Rotational dynamics of calcium-free calmodulin studied by ${ }^{15}$ N-NMR relaxation measurements. Eur. J. Biochem. 230, 1014-1024.

Tüchelman, A. (1999) Synthese paramagnetischer Tags für Biomakromoleküle und applikation elektrischer felder zur gewinnung langreichweitiger NMR-Strukturinformation. Dissertation. Frankfurt am Main. 234 pp.

Van Petegem, F., Chatelain, F.C., Minor, D.L.Jr. (2005) Insights into voltage-gated calcium channel regulation from the structure of the $\mathrm{CaV} 1.2$ IQ domain- $\mathrm{Ca}^{2+} /$ calmodulin complex. Nat. Struct. Biol. 12, 1108-1115.

Velyvis, A., Yang, Y.R., Schachman, H.K., Kay, L.E. (2007) A solution NMR study showing that active site ligands and nucleotides directly perturb the allosteric equilibrium in aspartate transcarbamoylase. Proc. Nat. Acad. Sci. 104, 8815-8820.

Vetter, S.W., Leclerc, E. (2003) Novel aspects of calmodulin target recognition and activation. Eur. J. Biochem. 270, 404-414.

Vriend, G. (1990) WHAT IF: a molecular modeling and drug design program. J. Mol. Graph. $8,52-56$.

Wang, T., Frederick, K.K., Igumenova, T.I., Wand, A.J., Zuiderweg, E.R. (2005) Changes in calmodulin main-chain dynamics upon ligand binding revealed by cross-correlated NMR relaxation measurements. J. Am. Chem. Soc. 127, 828-829.

Wand, A.J. (2001) On the dynamics origins of allosteric activation. Science. 293, 1395.

Watterson, D.M., Sharief, F., Vanaman, T.C. (1980) The complete amino acid sequence of the $\mathrm{Ca}^{2+}$-dependent modulator protein (calmodulin) of bovine brain. J. Biol. Chem. 255, 962-975. 
Wishart, D.S., Sykes, B.D. (1994) The 13C chemical-shift index: A simple method for the identification of protein secondary structure using 13C chemical-shift data. J. Biomol. NMR. 4, 171-180.

Wolff, D.J., Huebner, J.A., Siegel, F.L. (1972) Calcium-binding phosphoprotein of pig brain: effects of cations on the calcium binding. J. Neurochem. 19, 2855-2862.

Wu, X.S., Wu, L.G. (2001) Protein kinase C increases the apparent affinity of the release machinery to $\mathrm{Ca} 2+$ by enhancing the release machinery downstream of the $\mathrm{Ca} 2+$ sensor. $\mathrm{J}$. Neurosci. 21, 7928-7936.

Wühtrich, K. (1986) NMR of proteins and nucleic acids. John Wiley \& Sons, Inc. 292pp.

Xia, Z., Storm, D.R. (2005) The role of calmodulin as a signal integrator for synaptic plasticity. Nature reviews neuroscience. 6, 267-275.

Xu, X-Z. S., Wes, P.D., Chen, H., Li, H-S., Yu, M., Morgan, S., Liu, Y., Montell, C. (1998) Retinal targets for calmodulin include proteins implicated in synaptic transmission. J. Biol. Chem. 273, 31297-31307.

Yang, C., Jas, G.S., Kuczera, K. (2003) Structure, dynamics and interaction with kinase targets: computer simulations of calmodulin. Biochim. Biophys. Acta. 1697, 289-300.

Yap, K.L., Yuan, T., Mal, T.K., Vogel, H.J., Ikura, M. (2003) Structural basis for simultaneous binding of two carboxy-terminal peptides of plant glutamate decarboxylase to calmodulin. J. Mol. Biol. 328, 193-204.

Zhang, G., Kazanietz, M.G., Blumberg, P.M., Hurley, J.H. (1995) Crystal structure of the Cys2 activator-binding domain of protein knase $\mathrm{C} \delta$ in complex with phorbol ester. Cell. 81, 917-924.

Zweckstetter, M., Bax, A. (2000) Prediction of sterically induced alignment in a dilute liquid crystalline phase: aid to protein structure determination by NMR. J. Am. Chem. Soc. 122, 3791-3792. 


\section{APENDIX}

1. Magnetization pathways in the backbone nuclei of the polypeptide chain

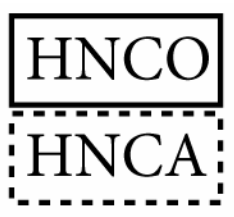

\begin{tabular}{|l|}
\hline $\mathrm{HN}(\mathrm{CO}) \mathrm{CA}$ \\
\hline $\mathrm{HN}(\mathrm{CA}) \mathrm{CO}$ \\
\hline$\ldots . . . . . . . .$.
\end{tabular}
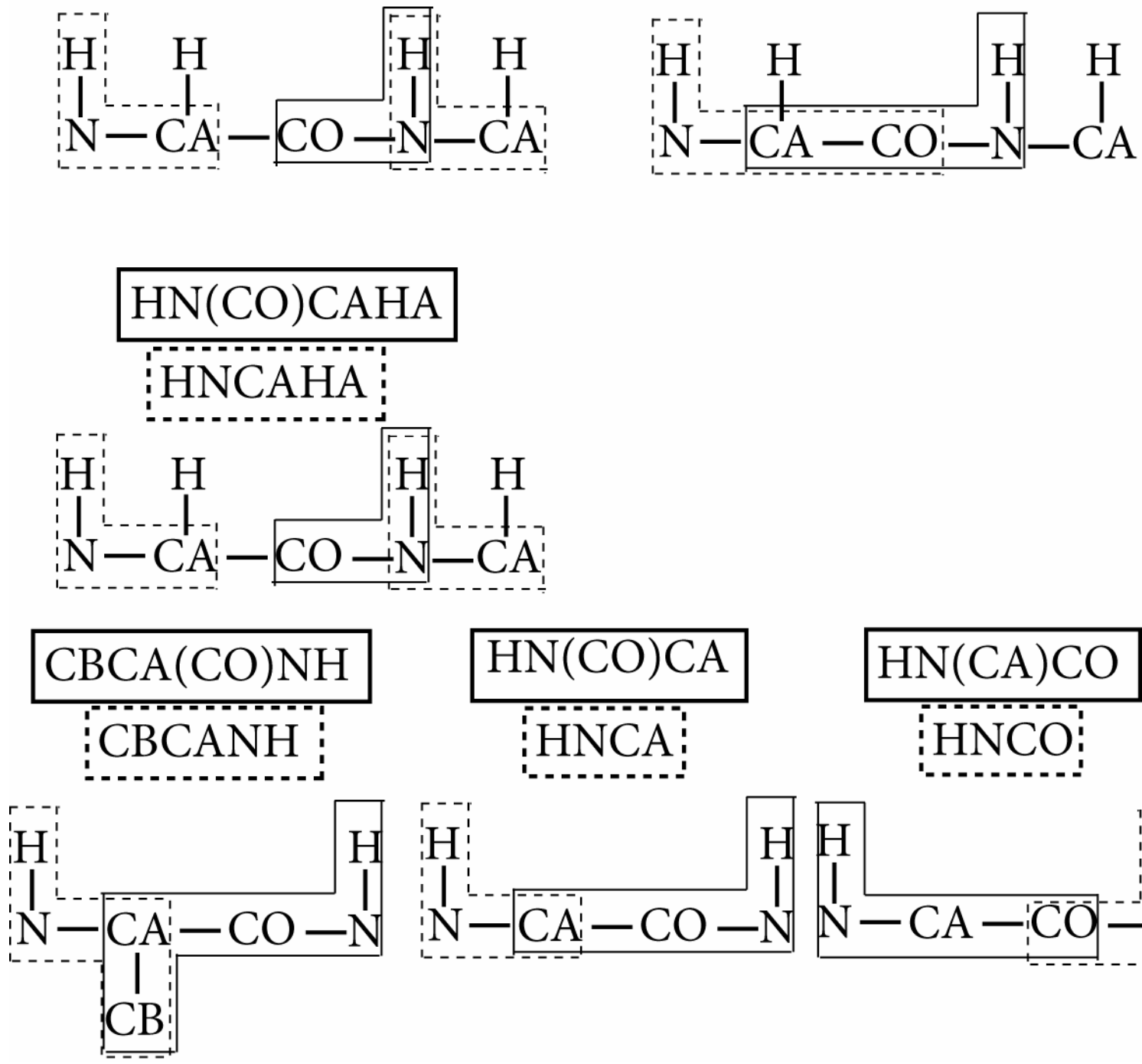

$\mathrm{HN}(\mathrm{CA}) \mathrm{CO}$

HNCO
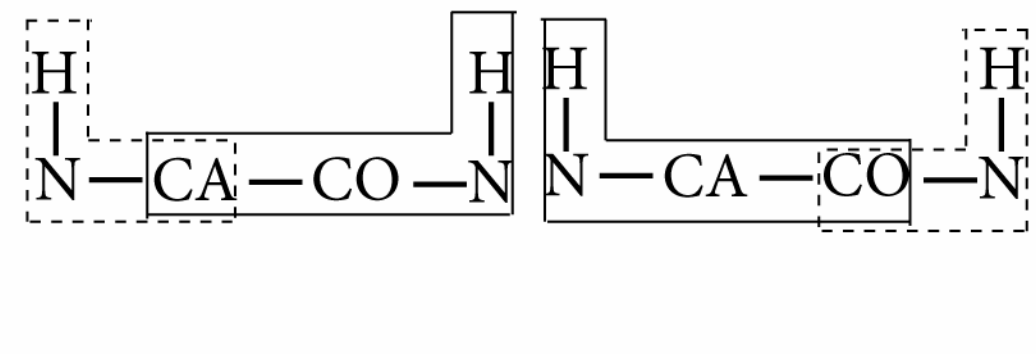
2. Pictorial view of the heteronuclear multidimensional NMR pulse sequences

a. Pulse sequence for atomatic side chain resonance assignment

$(\mathrm{H} \beta) \mathrm{C} \beta(\mathrm{C} \gamma \mathrm{C} \delta) \mathrm{H} \delta$ and $(\mathrm{H} \beta) \mathrm{C} \beta(\mathrm{C} \gamma \mathrm{C} \delta \mathrm{C} \varepsilon) \mathrm{H} \varepsilon$

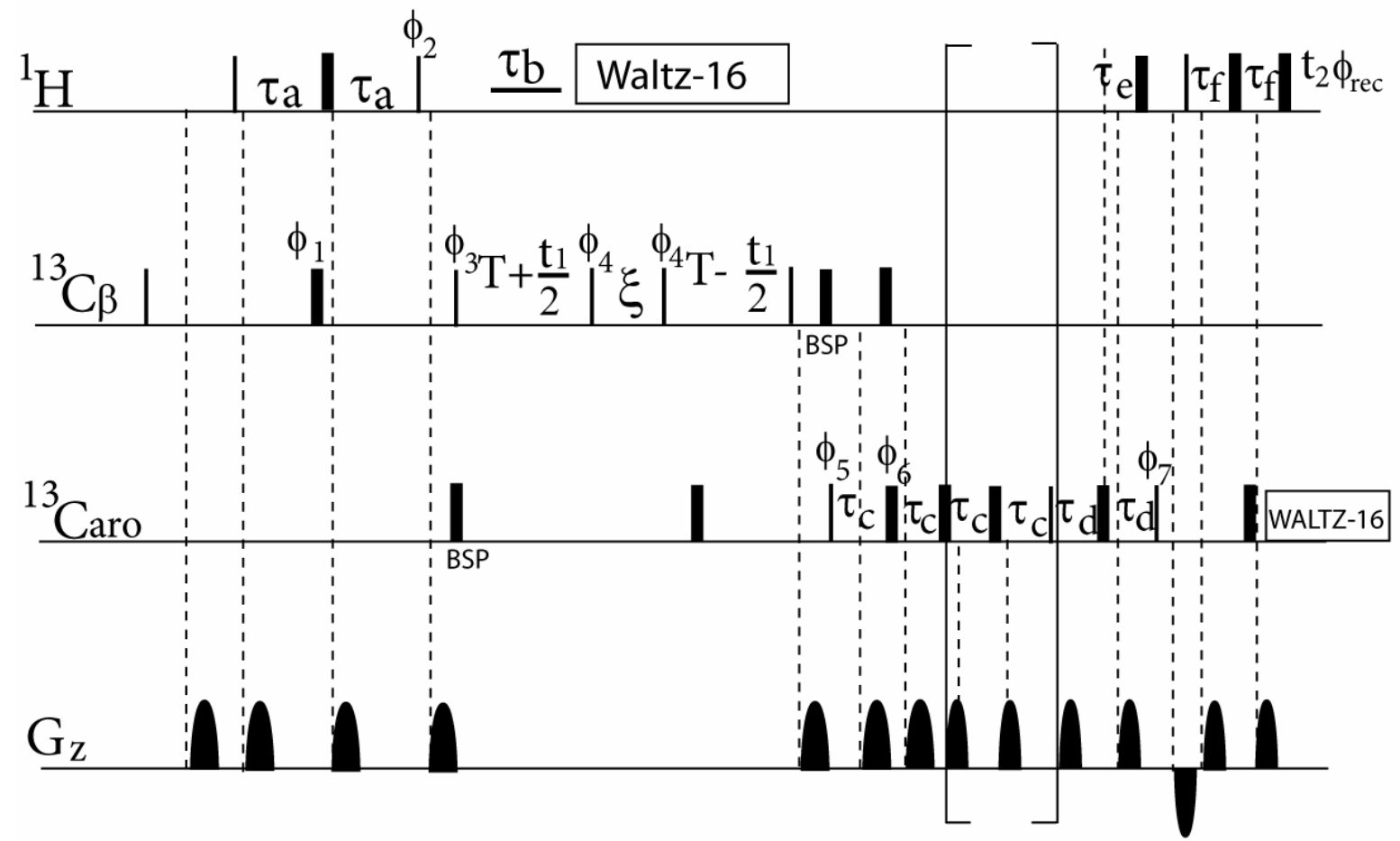

b. CBCA(CO)NH Pulse sequence for sequential backbone resonance assignment 3D CBCA(CO)NH $\mathrm{H} \beta, \mathrm{H} \alpha->\mathrm{C} \beta, \mathrm{C} \alpha\left(\mathrm{t}_{1}\right)->\mathrm{C}^{\prime}->\mathrm{N}\left(\mathrm{t}_{2}\right)->\mathrm{H}^{\mathrm{N}}\left(\mathrm{t}_{3}\right)$

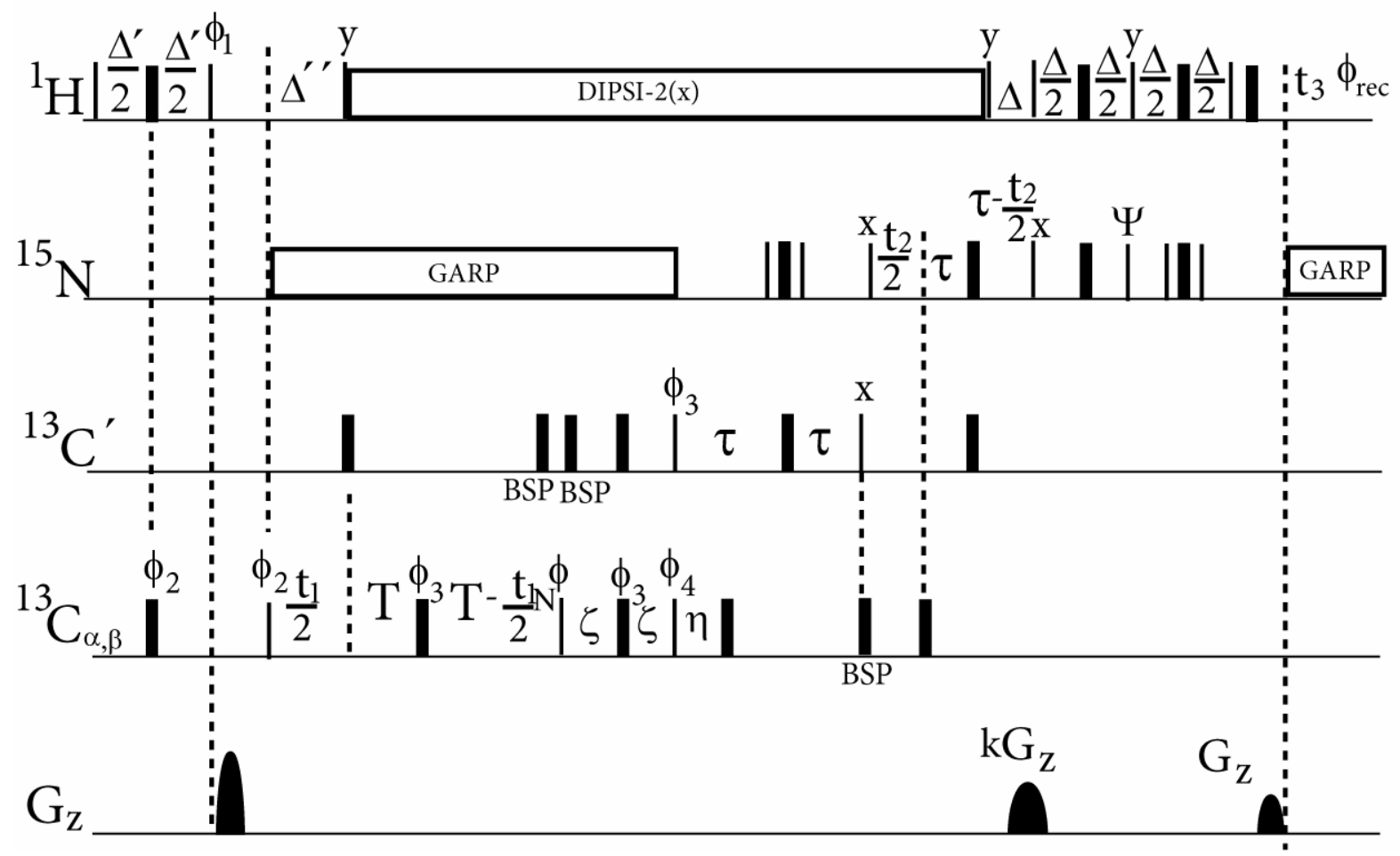


c. HNCA pulse sequence for sequential backbone resonance assignment

3D HNCA transfer: $\mathrm{H} \alpha->\mathrm{C} \alpha\left(\mathrm{t}_{1}\right)->\mathrm{N}\left(\mathrm{t}_{2}\right)->\mathrm{H}^{\mathrm{N}}\left(\mathrm{t}_{3}\right)$
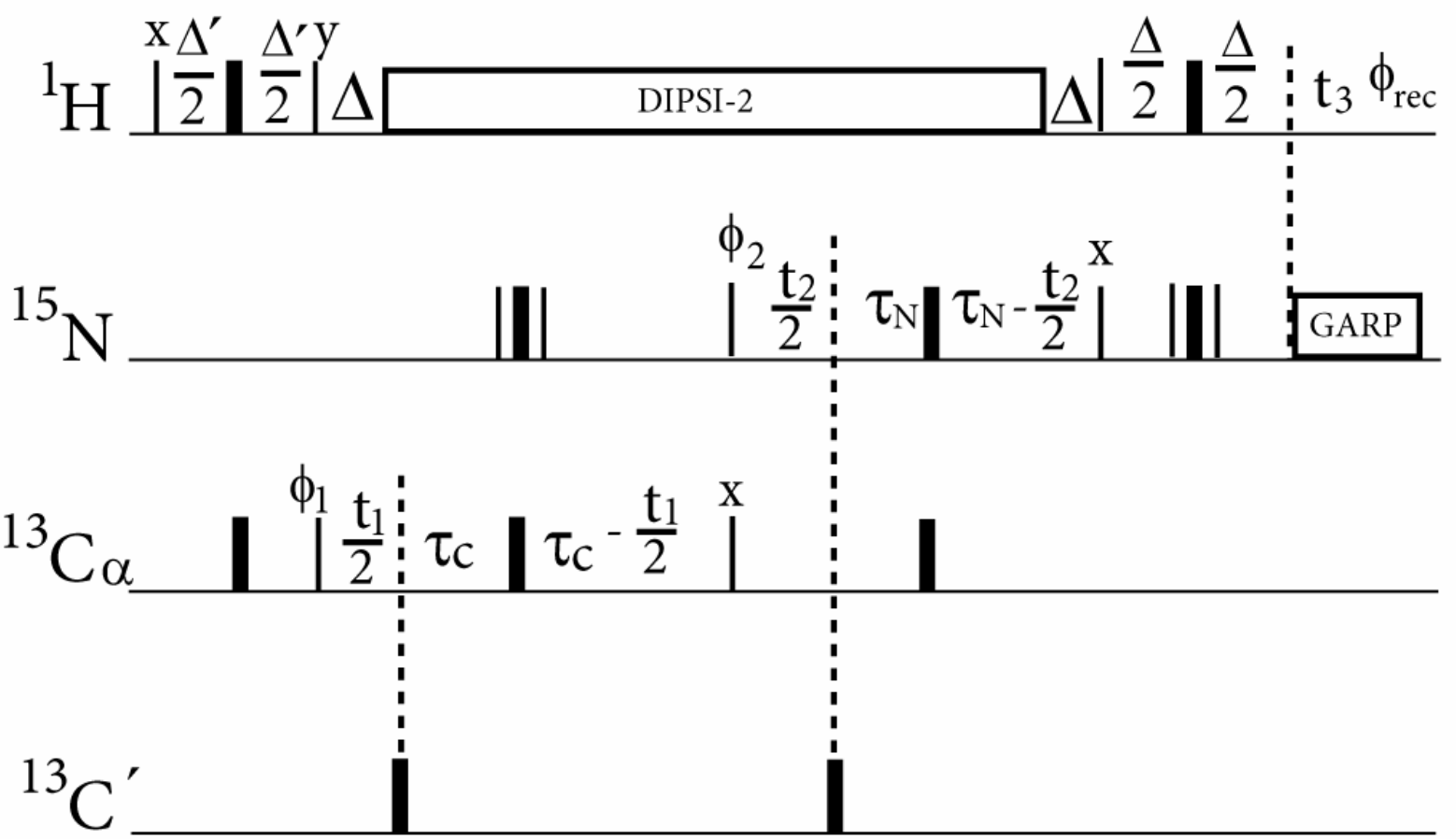

d. HNCACB pulse sequence for sequential backbone resonance assignment

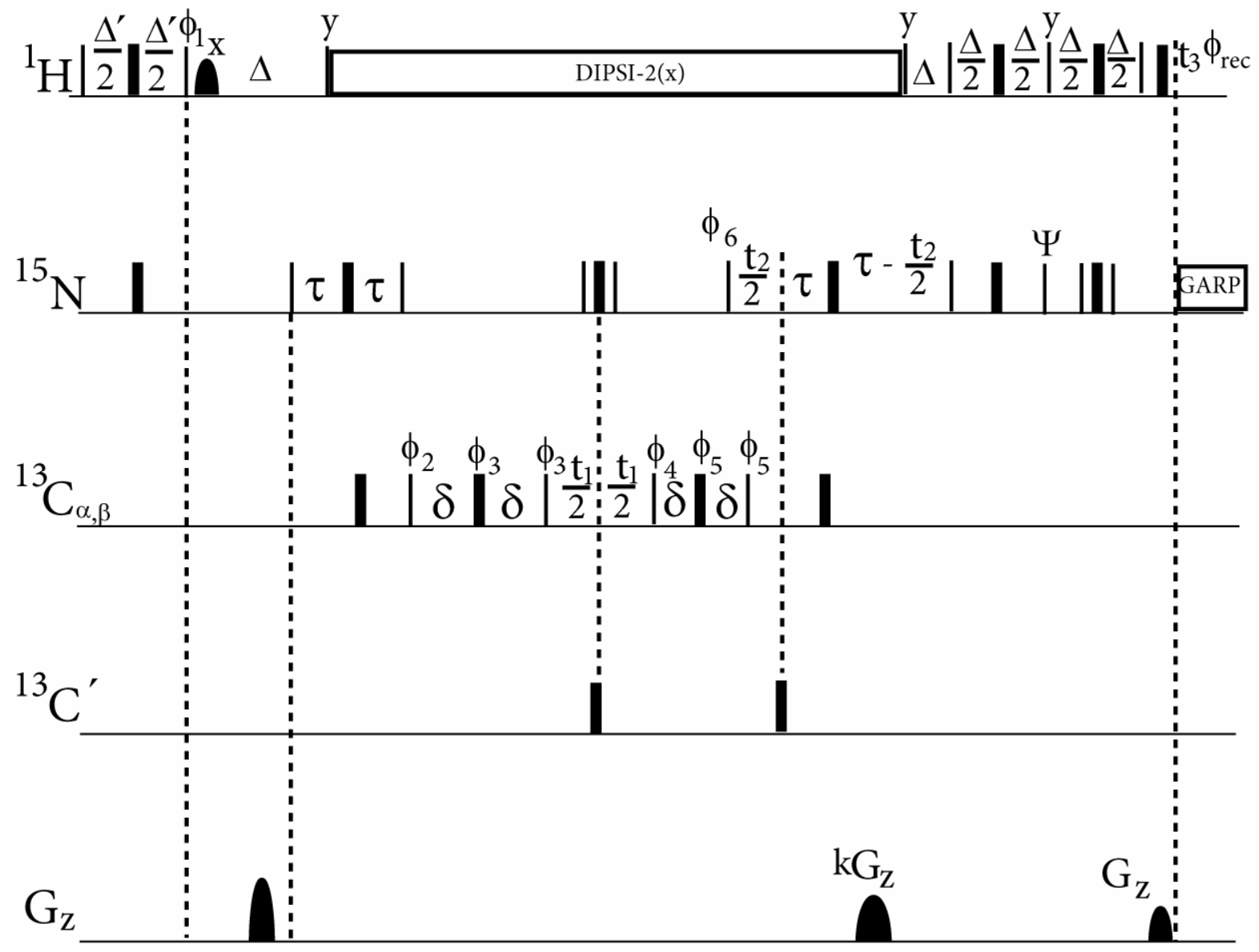


e. HNN pulse sequence for sequential backbone resonance assignment

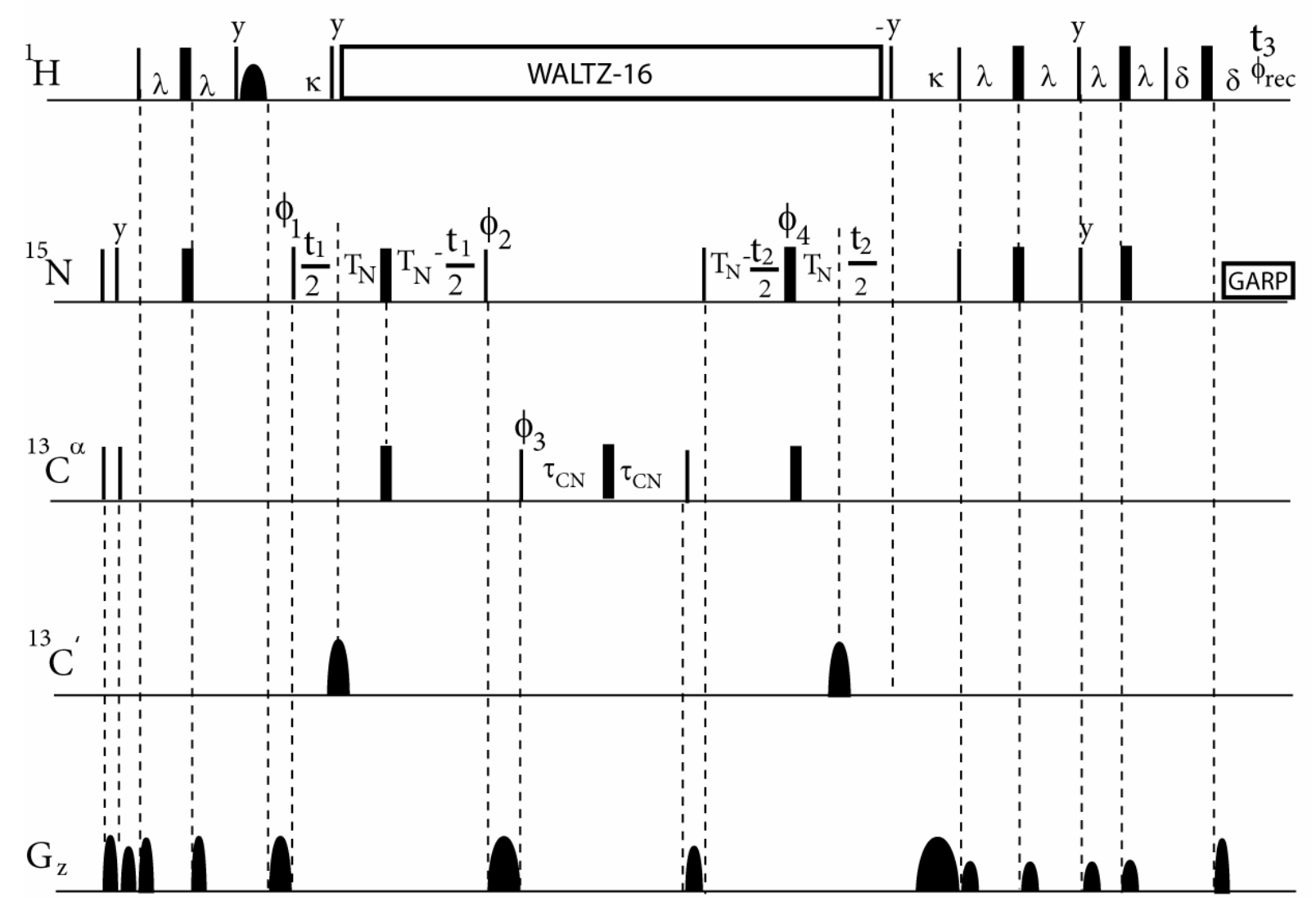

f. Pulse sequence for the resonance assignment of $\varepsilon$-methyl groups in methionines

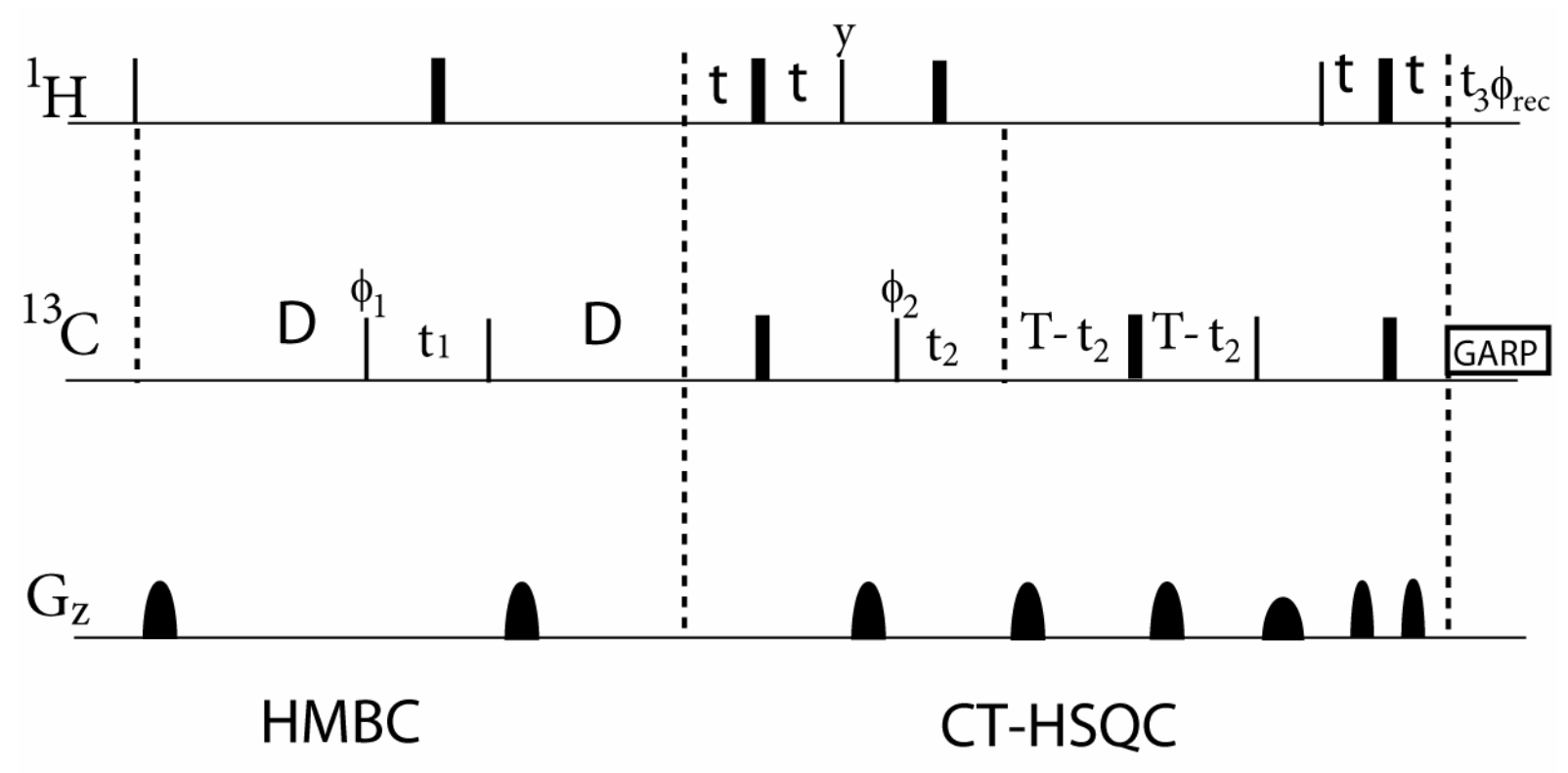


g. Pulse sequence of the $3 \mathrm{D}-{ }^{15} \mathrm{~N}$-edited-NOESY-HSQC

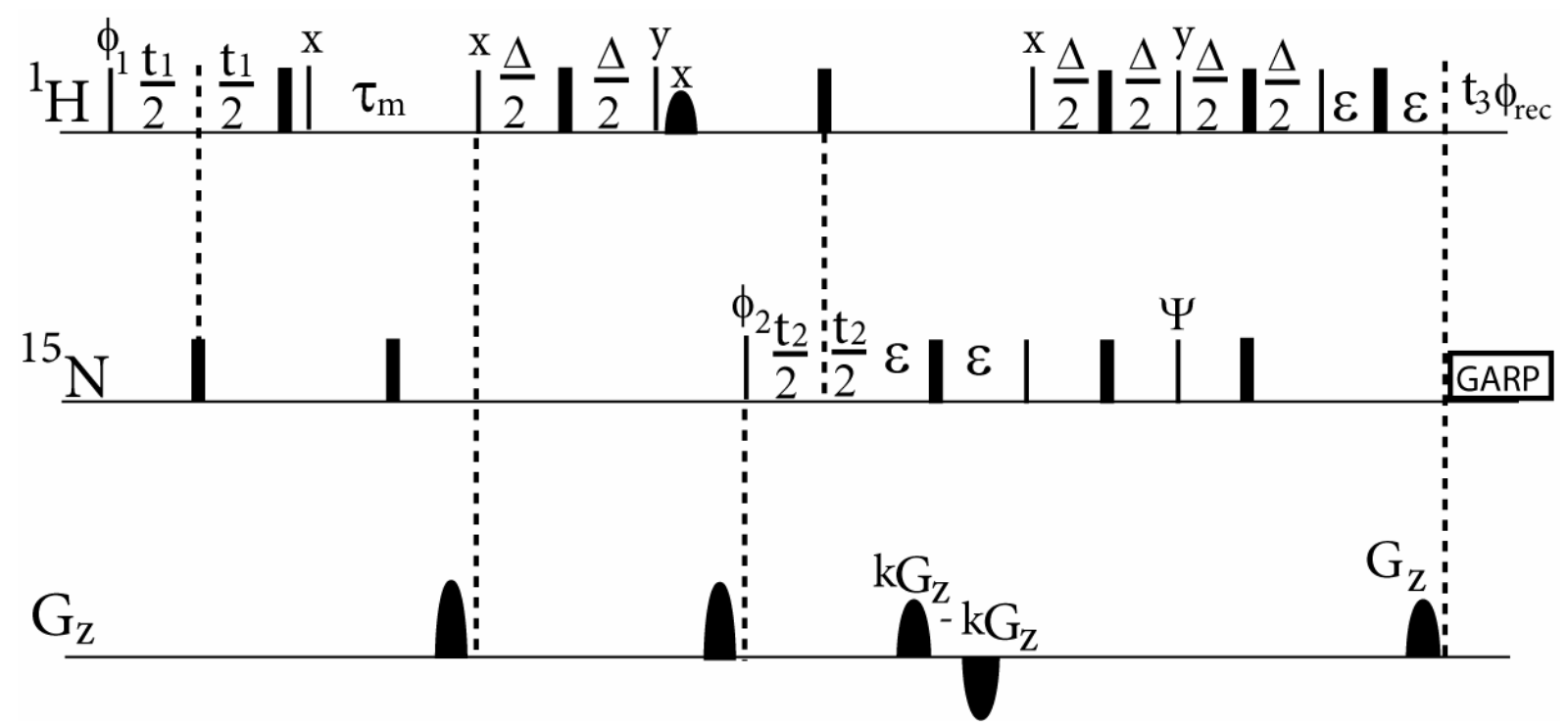

h. Pulse sequence of the isotope-filetered $3 \mathrm{D}-{ }^{15} \mathrm{~N}$-edited-NOESY-HSQC

$3 \mathrm{D} \varpi_{1}$-edit/filter -NOESY- ${ }^{15} \mathrm{~N},{ }^{1} \mathrm{H}$ - HSQC

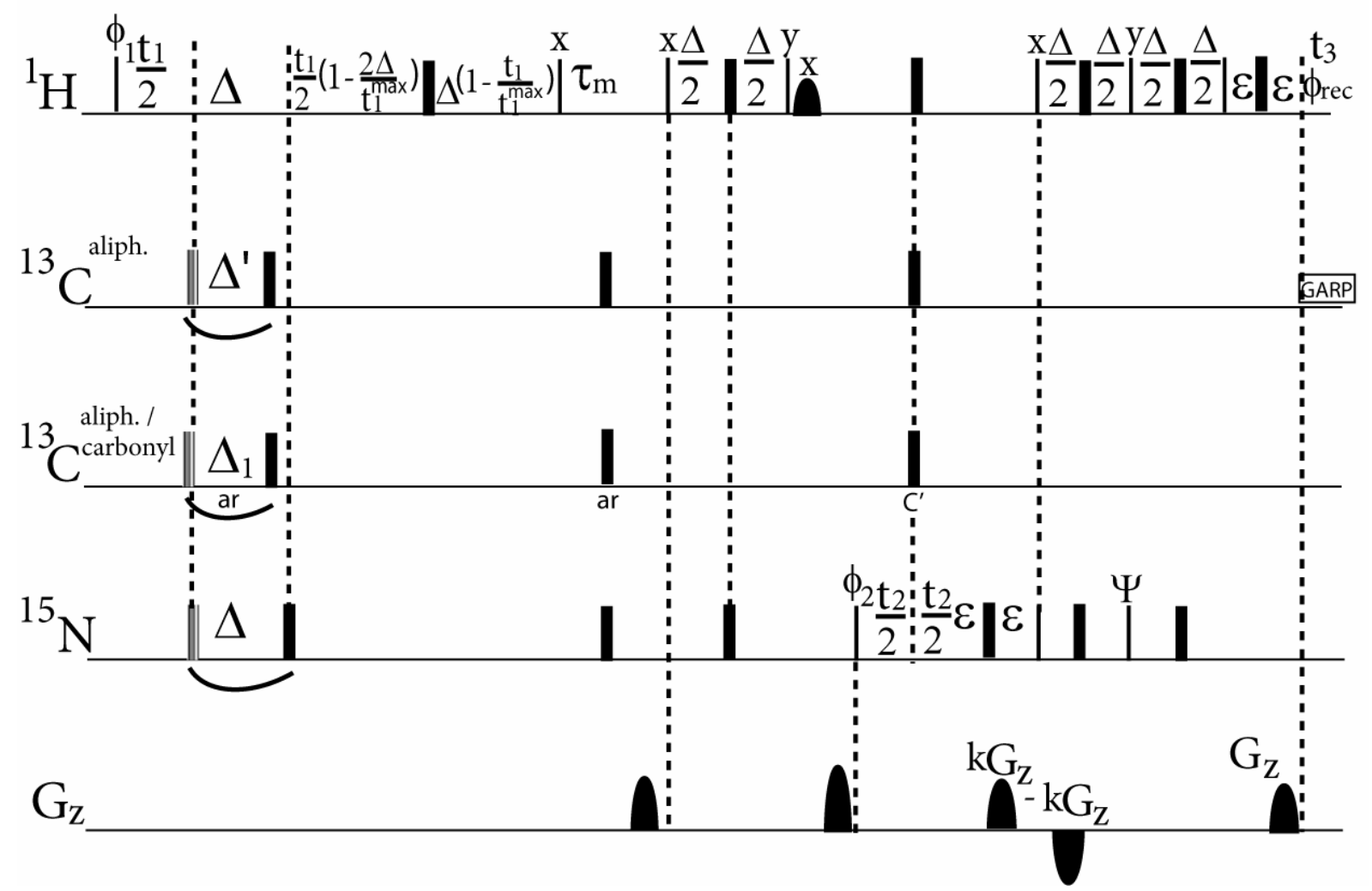


i. Pulse sequence of the IPAP-HSQC (S3E sequence)

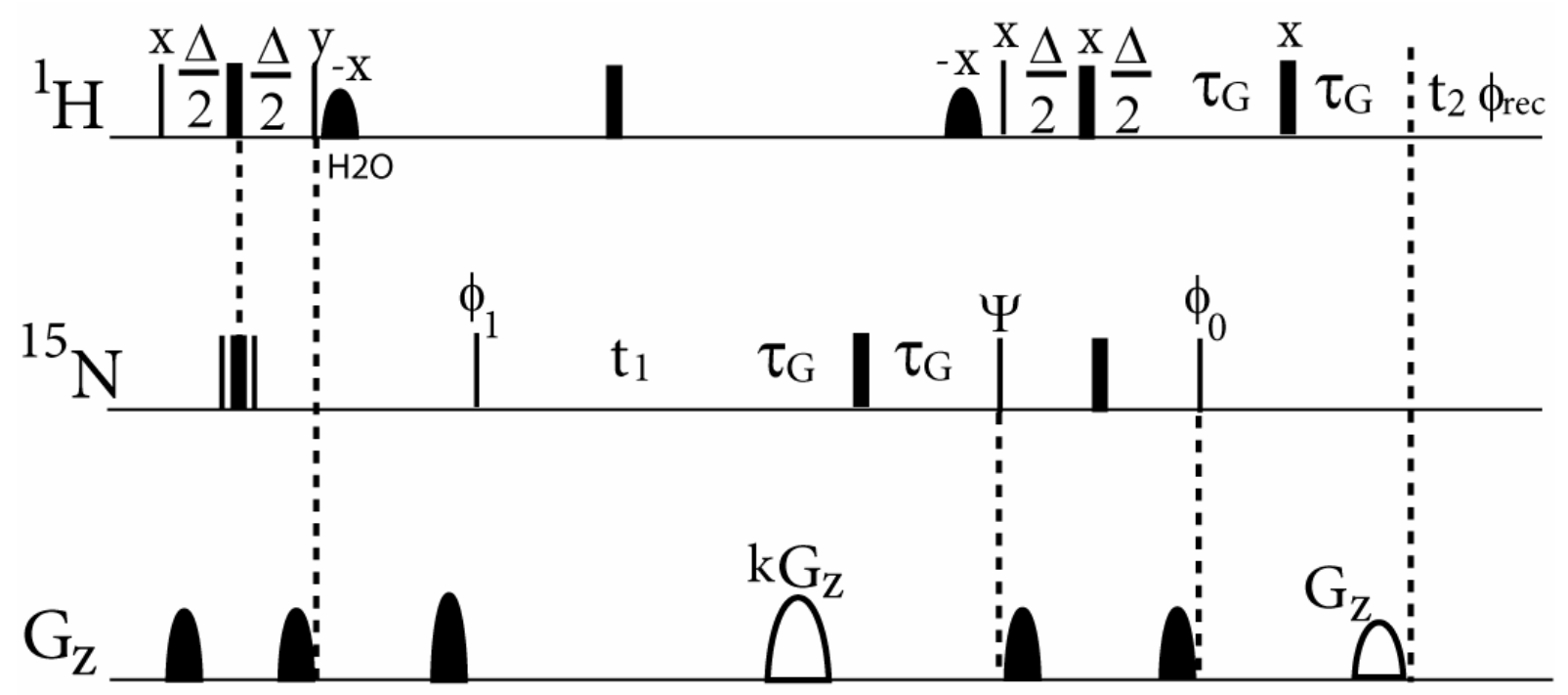

j. Pulse sequence of the TROSY-HSQC

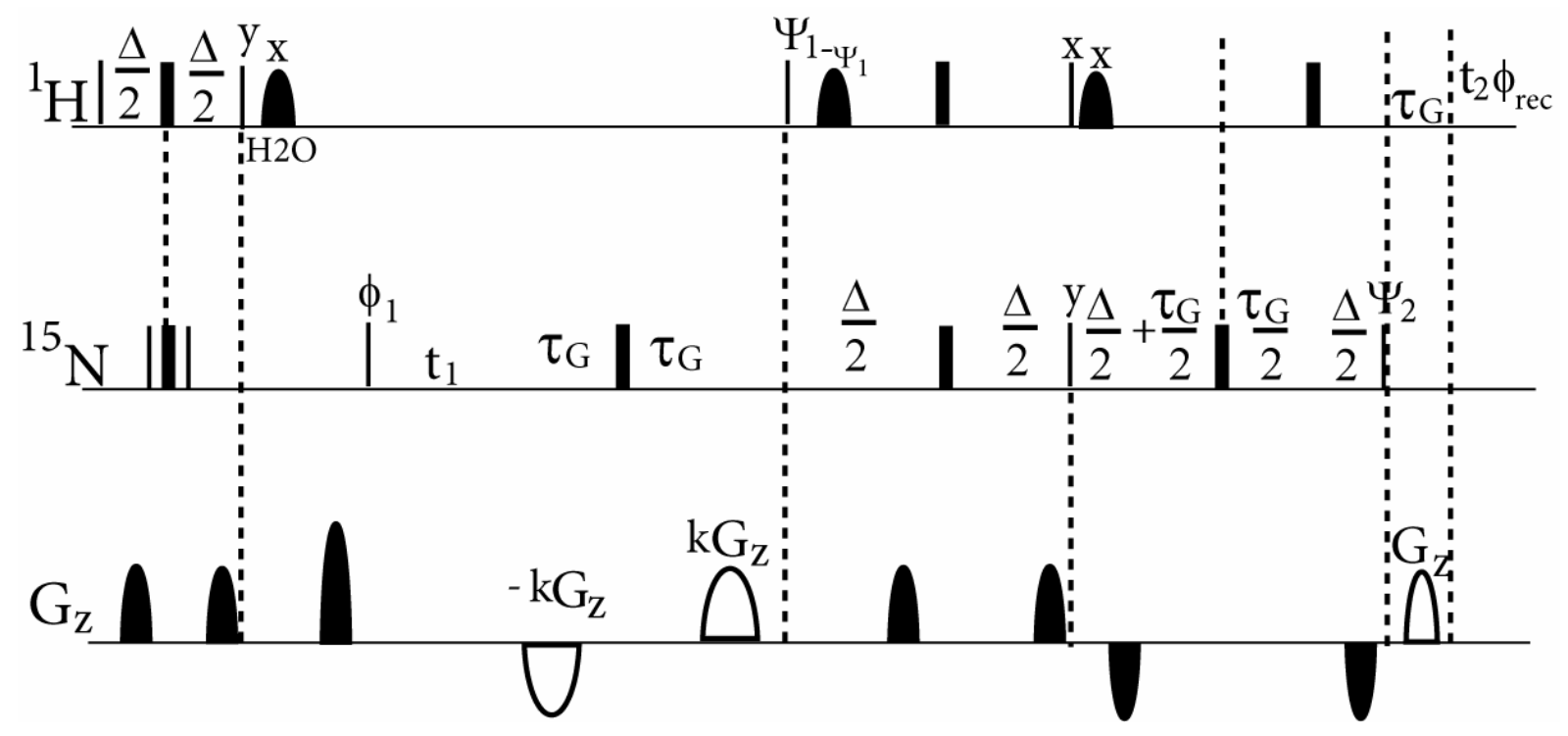


3. Chemical shift assignment list for the CaM/Munc13-1(457-492) peptide complex Calmodulin

\begin{tabular}{|c|c|c|c|c|c|c|c|c|c|c|c|}
\hline om & Seq & Res & Atom & Chem & & Atom & Seq & Res & Atom & Chem & \\
\hline & \# & Type & ID & shift & error & \# & \# & Type & ID & shift & error \\
\hline 1 & 1 & ALA & CB & 19.353 & 0.4 & 58 & 7 & GLU & HB2 & 2.112 & 0.05 \\
\hline 2 & 1 & ALA & HA & 4.626 & 0.05 & 59 & 7 & GLU & HB3 & 1.956 & 0.05 \\
\hline 3 & 1 & ALA & $\mathrm{HB}$ & 1.510 & 0.05 & 60 & 7 & GLU & $\mathrm{N}$ & 118.929 & 0.4 \\
\hline 4 & 2 & ASP & C & 172.776 & 0.4 & 61 & 7 & GLU & HG2 & 2.348 & 0.05 \\
\hline 5 & 2 & ASP & CA & 54.72 & 0.4 & 62 & 8 & GLN & C & 175.426 & 0.4 \\
\hline 6 & 2 & ASP & $\mathrm{CB}$ & 41.41 & 0.4 & 63 & 8 & GLN & CA & 59.201 & 0.4 \\
\hline 7 & 2 & ASP & $\mathrm{H}$ & 8.649 & 0.05 & 64 & 8 & GLN & CB & 29.297 & 0.4 \\
\hline 8 & 2 & ASP & $\mathrm{HA}$ & 4.623 & 0.05 & 65 & 8 & GLN & CG & 34.994 & 0.4 \\
\hline 9 & 2 & ASP & HB2 & 2.657 & 0.05 & 66 & 8 & GLN & $\mathrm{H}$ & 7.726 & 0.05 \\
\hline 10 & 2 & ASP & HB3 & 2.577 & 0.05 & 67 & 8 & GLN & $\mathrm{HA}$ & 4.016 & 0.05 \\
\hline 11 & 2 & ASP & $\mathrm{N}$ & 120.4 & 0.4 & 68 & 8 & GLN & HB2 & 2.074 & 0.05 \\
\hline 12 & 3 & GLN & C & 172.795 & 0.4 & 69 & 8 & GLN & HB3 & 1.951 & 0.05 \\
\hline 13 & 3 & GLN & CA & 55.508 & 0.4 & 70 & 8 & GLN & $\mathrm{N}$ & 119.631 & 0.4 \\
\hline 14 & 3 & GLN & CB & 29.622 & 0.4 & 71 & 8 & GLN & HG2 & 2.346 & 0.05 \\
\hline 15 & 3 & GLN & CG & 33.763 & 0.4 & 72 & 9 & ILE & C & 174.972 & 0.4 \\
\hline 16 & 3 & GLN & $\mathrm{H}$ & 8.348 & 0.05 & 73 & 9 & ILE & CA & 66.243 & 0.4 \\
\hline 17 & 3 & GLN & $\mathrm{HA}$ & 4.371 & 0.05 & 74 & 9 & ILE & CB & 37.652 & 0.4 \\
\hline 18 & 3 & GLN & HB2 & 2.099 & 0.05 & 75 & 9 & ILE & CG1 & 30.055 & 0.4 \\
\hline 19 & 3 & GLN & HB3 & 1.984 & 0.05 & 76 & 9 & ILE & CG2 & 17.330 & 0.4 \\
\hline 20 & 3 & GLN & $\mathrm{N}$ & 119.406 & 0.4 & 77 & 9 & ILE & $\mathrm{H}$ & 8.355 & 0.05 \\
\hline 21 & 3 & GLN & HG2 & 2.343 & 0.05 & 78 & 9 & ILE & $\mathrm{HA}$ & 3.667 & 0.05 \\
\hline 22 & 4 & LEU & C & 174.846 & 0.4 & 79 & 9 & ILE & $\mathrm{HB}$ & 1.946 & 0.05 \\
\hline 23 & 4 & LEU & CA & 54.428 & 0.4 & 80 & 9 & ILE & HD1 & 0.869 & 0.05 \\
\hline 24 & 4 & LEU & CB & 43.435 & 0.4 & 81 & 9 & ILE & HG12 & 1.789 & 0.05 \\
\hline 25 & 4 & LEU & CD1 & 26.518 & 0.4 & 82 & 9 & ILE & HG13 & 1.100 & 0.05 \\
\hline 26 & 4 & LEU & CD2 & 23.607 & 0.4 & 83 & 9 & ILE & HG2 & 1.108 & 0.05 \\
\hline 27 & 4 & LEU & CG & 26.711 & 0.4 & 84 & 9 & ILE & $\mathrm{N}$ & 119.286 & 0.4 \\
\hline 28 & 4 & LEU & $\mathrm{H}$ & 8.275 & 0.05 & 85 & 10 & ALA & C & 178.227 & 0.4 \\
\hline 29 & 4 & LEU & $\mathrm{HA}$ & 4.665 & 0.05 & 86 & 10 & ALA & CA & 55.338 & 0.4 \\
\hline 30 & 4 & LEU & HB2 & 1.719 & 0.05 & 87 & 10 & ALA & CB & 17.746 & 0.4 \\
\hline 31 & 4 & LEU & HB3 & 1.508 & 0.05 & 88 & 10 & ALA & $\mathrm{H}$ & 8.037 & 0.05 \\
\hline 32 & 4 & LEU & HD1 & 0.921 & 0.05 & 89 & 10 & ALA & $\mathrm{HA}$ & 4.114 & 0.05 \\
\hline 33 & 4 & LEU & HD2 & 0.913 & 0.05 & 90 & 10 & ALA & $\mathrm{HB}$ & 1.520 & 0.05 \\
\hline 34 & 4 & LEU & $\mathrm{N}$ & 122.55 & 0.4 & 91 & 10 & ALA & $\mathrm{N}$ & 120.74 & 0.4 \\
\hline 35 & 5 & THR & C & 172.634 & 0.4 & 92 & 11 & GLU & C & 177.704 & 0.4 \\
\hline 36 & 5 & THR & CA & 60.493 & 0.4 & 93 & 11 & GLU & CA & 59.453 & 0.4 \\
\hline 37 & 5 & THR & CB & 71.039 & 0.4 & 94 & 11 & GLU & CB & 28.33 & 0.4 \\
\hline 38 & 5 & THR & $\mathrm{H}$ & 8.660 & 0.05 & 95 & 11 & GLU & CG & 35.588 & 0.4 \\
\hline 39 & 5 & THR & $\mathrm{HA}$ & 4.457 & 0.05 & 96 & 11 & GLU & $\mathrm{H}$ & 7.847 & 0.05 \\
\hline 40 & 5 & THR & $\mathrm{HB}$ & 4.742 & 0.05 & 97 & 11 & GLU & $\mathrm{HA}$ & 4.162 & 0.05 \\
\hline 41 & 5 & THR & HG2 & 1.334 & 0.05 & 98 & 11 & GLU & HB2 & 2.097 & 0.05 \\
\hline 42 & 5 & THR & $\mathrm{N}$ & 112.406 & 0.4 & 99 & 11 & GLU & HB3 & 2.017 & 0.05 \\
\hline 43 & 6 & GLU & C & 176.679 & 0.4 & 100 & 11 & GLU & $\mathrm{N}$ & 118.942 & 0.4 \\
\hline 44 & 6 & GLU & CA & 60.113 & 0.4 & 101 & 11 & GLU & HG2 & 2.0148 & 0.05 \\
\hline 45 & 6 & GLU & CB & 28.877 & 0.4 & 10 & 12 & PHE & C & 175.906 & 0.4 \\
\hline 46 & 6 & GLU & CG & 36.465 & 0.4 & 103 & 12 & PHE & CA & 58.9768 & 0.4 \\
\hline 47 & 6 & GLU & $\mathrm{H}$ & 9.016 & 0.05 & 104 & 12 & PHE & CB & 37.4318 & 0.4 \\
\hline 48 & 6 & GLU & $\mathrm{HA}$ & 3.988 & 0.05 & 105 & 12 & PHE & CD1 & 28.343 & 0.4 \\
\hline 49 & 6 & GLU & $\mathrm{N}$ & 120.053 & 0.4 & 106 & 12 & PHE & CD2 & 128.343 & 0.4 \\
\hline 50 & 6 & GLU & HB2 & 2.069 & 0.05 & 107 & 12 & PHE & $\mathrm{H}$ & 8.6068 & 0.05 \\
\hline 51 & 6 & GLU & HG2 & 2.369 & 0.05 & 108 & 12 & PHE & HA & 5.0048 & 0.05 \\
\hline 52 & 7 & GLU & C & 176.396 & 0.4 & 109 & 12 & PHE & HB2 & 3.5338 & 0.05 \\
\hline 53 & 7 & GLU & $C A$ & 59.476 & 0.4 & 110 & 12 & PHE & HB3 & 3.4618 & 0.05 \\
\hline 54 & 7 & GLU & CB & 29.216 & 0.4 & 111 & 12 & PHE & $\mathrm{N}$ & 119.64 & 0.4 \\
\hline 55 & 7 & GLU & CG & 36.573 & 0.4 & 112 & 12 & PHE & HD1 & 7.1668 & 0.05 \\
\hline 56 & 7 & GLU & $\mathrm{H}$ & 8.665 & 0.05 & 113 & 12 & PHE & HD2 & 7.1668 & 0.05 \\
\hline 57 & 7 & GLU & $\mathrm{HA}$ & 4.088 & 0.05 & 114 & 12 & PHE & HE1 & 7.2128 & 0.05 \\
\hline
\end{tabular}




\begin{tabular}{|c|c|c|c|c|c|c|c|c|c|c|c|}
\hline 115 & 12 & PHE & HE2 & 7.2128 & 0.05 & 175 & 19 & PHE & $\mathrm{CA}$ & 59.102 & \\
\hline 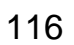 & 12 & PHE & $\mathrm{HZ}$ & 6.7078 & 0.05 & 176 & 19 & PHE & CB & 40.413 & \\
\hline 117 & 13 & LYS & C & 176.515 & 0.4 & 177 & 19 & PHE & $\mathrm{N}$ & 114.926 & \\
\hline 18 & 13 & LYS & CA & 59.815 & 0.4 & 178 & 19 & PHE & $\mathrm{HA}$ & 3.8578 & \\
\hline 119 & 13 & LYS & CB & 31.056 & 0.4 & 179 & 19 & PHE & HB2 & 2.631 & \\
\hline 20 & 13 & LYS & $\mathrm{H}$ & 9.2148 & 0.05 & 180 & 19 & PHE & $\mathrm{CB}$ & 41.4328 & \\
\hline 21 & 13 & LYS & $\mathrm{HA}$ & 4.0098 & 0.05 & 181 & 19 & PHE & CD1 & 128.595 & \\
\hline 122 & 13 & LYS & $\mathrm{N}$ & 122.92 & 0.4 & 182 & 19 & PHE & $\mathrm{CD} 2$ & 128.595 & \\
\hline 123 & 13 & LYS & HB2 & 1.9098 & 0.05 & 183 & 19 & PHE & $\mathrm{H}$ & 7.0848 & \\
\hline 124 & 13 & LYS & HG2 & 0.6978 & 0.05 & 184 & 19 & PHE & HD1 & 7.3118 & \\
\hline 125 & 13 & LYS & HD2 & 1.2578 & 0.05 & 185 & 19 & PHE & HD2 & 7.3118 & \\
\hline 126 & 13 & LYS & HD3 & 1.1078 & 0.05 & 186 & 19 & PHE & HE1 & 7.2768 & \\
\hline 127 & 13 & LYS & HE2 & 2.5478 & 0.05 & 187 & 19 & PHE & HE2 & 7.2768 & \\
\hline 128 & 14 & GLU & C & 176.562 & 0.4 & 188 & 20 & ASP & C & 174.355 & \\
\hline 129 & 14 & GLU & CA & 58.722 & 0.4 & 189 & 20 & ASP & CA & 56.149 & \\
\hline 130 & 14 & GLU & CB & 28.825 & 0.4 & 190 & 20 & ASP & CB & 39.0518 & \\
\hline 131 & 14 & GLU & $\mathrm{H}$ & 7.7328 & 0.05 & 191 & 20 & ASP & $\mathrm{H}$ & 7.7588 & \\
\hline 132 & 14 & GLU & $\mathrm{N}$ & 119.254 & 0.4 & 192 & 20 & ASP & HB2 & 2.4478 & \\
\hline 33 & 14 & GLU & $\mathrm{HA}$ & 3.8078 & 0.05 & 193 & 20 & ASP & $\mathrm{HA}$ & 4.5518 & \\
\hline 134 & 14 & GLU & HB2 & 1.8978 & 0.05 & 194 & 20 & ASP & $\mathrm{N}$ & 116.333 & \\
\hline 135 & 14 & GLU & HG2 & 2.0778 & 0.05 & 195 & 21 & LYS & $\mathrm{C}$ & 352 & \\
\hline 136 & 15 & ALA & C & 176.2 & 0.4 & 196 & 21 & LYS & CA & 58.4158 & \\
\hline 37 & 15 & ALA & CA & 55.2968 & 0.4 & 197 & 21 & LYS & CB & 32.6218 & \\
\hline 38 & 15 & ALA & CB & 18.4428 & 0.4 & 198 & 21 & LYS & $C D$ & 28.1138 & \\
\hline 139 & 15 & ALA & $\mathrm{H}$ & 8.0278 & 0.05 & 199 & 21 & LYS & CE & 41 & \\
\hline 140 & 15 & ALA & $\mathrm{HA}$ & 4.2028 & 0.05 & 200 & 21 & LYS & CG & 24.1018 & \\
\hline 141 & 15 & ALA & $\mathrm{HB}$ & 1.9258 & 0.05 & 201 & 21 & LYS & $\mathrm{H}$ & 7.6578 & \\
\hline 142 & 15 & ALA & $\mathrm{N}$ & 121.803 & 0.4 & 202 & 21 & LYS & $\mathrm{HA}$ & 3.9788 & \\
\hline 143 & 16 & PHE & C & 15 & 0.4 & 203 & 21 & LYS & $\mathrm{N}$ & 123.905 & \\
\hline 44 & 16 & PHE & CA & 61.9028 & 0.4 & 204 & 21 & LYS & HB2 & 1.8628 & \\
\hline 45 & 16 & PHE & CB & 9.7248 & 0.4 & 205 & 21 & LYS & HE2 & 2.9678 & \\
\hline 46 & 16 & PHE & CE1 & 129 & 0.4 & 206 & 21 & LYS & HG2 & 388 & \\
\hline 147 & 16 & PHE & CE2 & 129.482 & 0.4 & 207 & 21 & LYS & HD2 & 1.8878 & \\
\hline 148 & 16 & PHE & $\mathrm{H}$ & 8.7548 & 0.05 & 208 & 22 & ASP & C & 174.872 & \\
\hline 149 & 16 & PHE & $\mathrm{HA}$ & 08 & 0.05 & 2 & 22 & ASP & CA & 28 & \\
\hline 150 & 16 & PHE & $\mathrm{N}$ & 118.544 & 0.4 & 210 & 22 & ASP & CB & 39. & \\
\hline 151 & 16 & PHE & HB2 & 2.9218 & 0.05 & 211 & 22 & ASP & $\mathrm{H}$ & 8.1708 & \\
\hline 152 & 16 & PHE & HD1 & 6.5058 & 0.05 & 212 & 22 & ASP & $\mathrm{HA}$ & 4.5878 & \\
\hline 53 & 16 & PHE & HD2 & & 0.05 & 213 & 22 & ASP & HB2 & 78 & \\
\hline 154 & 16 & PHE & HE1 & 6.9738 & 0.05 & 214 & 22 & ASP & $\mathrm{HB}$ & 78 & \\
\hline 155 & 16 & PHE & HE2 & 6.9738 & 0.05 & 215 & 22 & ASP & $\mathrm{N}$ & 113.617 & \\
\hline 156 & 17 & SER & C & & 0.4 & 21 & 23 & GLY & C & & \\
\hline 157 & 17 & SER & CA & 08 & 0.4 & 217 & 23 & GLY & CA & 47. & \\
\hline 158 & 17 & SER & $\mathrm{CB}$ & 63.2668 & 0.4 & 218 & 23 & GLY & $\mathrm{H}$ & 7.7238 & \\
\hline 159 & 17 & SER & $\mathrm{H}$ & 8.0948 & 0.05 & 219 & 23 & GLY & HA2 & 3.8868 & \\
\hline 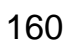 & 17 & SER & $\mathrm{HA}$ & & 0.05 & 22 & 23 & GLY & HA3 & & \\
\hline 61 & 17 & SER & HB2 & 28 & 0.05 & 22 & 23 & GLY & $N$ & 108 & \\
\hline 162 & 17 & SER & $\mathrm{N}$ & 112.66 & 0.4 & 222 & 24 & ASP & C & 174.627 & \\
\hline 163 & 18 & LEU & C & 174 & 0.4 & 223 & 24 & ASP & $\mathrm{CA}$ & 08 & \\
\hline 164 & 18 & LEU & $\mathrm{CA}$ & & 0.4 & 2 & 24 & ASP & $\mathrm{CB}$ & & \\
\hline 16 & 18 & LEU & $\mathrm{CB}$ & 38 & 0.4 & 2 & 24 & ASP & $\mathrm{H}$ & 338 & \\
\hline 166 & 18 & LEU & $\mathrm{H}$ & 7.4048 & 0.05 & 226 & 24 & ASP & $\mathrm{HA}$ & 4.4938 & \\
\hline 167 & 18 & LEU & $\mathrm{HA}$ & & 0.05 & 227 & 24 & ASP & HB2 & 3.0608 & \\
\hline 168 & 18 & LEU & $\mathrm{HB} 2$ & & 0.05 & 22 & 24 & ASP & $\mathrm{HB}$ & 2.4508 & \\
\hline 169 & 18 & LEU & HB3 & 4898 & 0.05 & 229 & 24 & ASP & $\mathrm{N}$ & 120.446 & \\
\hline 170 & 18 & LEU & HD1 & 6838 & 0.05 & 230 & 25 & GLY & C & 170.854 & \\
\hline 171 & 18 & LEU & HD2 & & 0.05 & 231 & 25 & GLY & CA & 538 & \\
\hline 172 & 18 & LEU & HG & & 0.05 & 232 & 25 & GLY & $\mathrm{H}$ & 10.6268 & \\
\hline 173 & 18 & LEU & $\mathrm{N}$ & 120.184 & 0.4 & 233 & 25 & GLY & HA2 & 4.3938 & \\
\hline 174 & 19 & PHE & C & 173.816 & 0.4 & 234 & 25 & GLY & HA3 & 3.7288 & \\
\hline
\end{tabular}




\begin{tabular}{|c|c|c|c|c|c|c|c|c|c|c|c|}
\hline 235 & 25 & GLY & $\mathrm{N}$ & 112.712 & 0.4 & 296 & 32 & LEU & $\mathrm{CB}$ & 968 & \\
\hline 236 & 26 & THR & CA & 60.71 & 0.4 & 297 & 32 & LEU & CD1 & 25.8618 & \\
\hline 237 & 26 & THR & C & 170.112 & 0.4 & 298 & 32 & LEU & CD2 & 23.5738 & \\
\hline 238 & 26 & THR & $\mathrm{CB}$ & 72.5468 & 0.4 & 299 & 32 & LEU & CG & 25.9618 & \\
\hline 239 & 26 & THR & CG2 & 21.7198 & 0.4 & 300 & 32 & LEU & $\mathrm{H}$ & 8.6388 & \\
\hline 240 & 26 & THR & $\mathrm{H}$ & 8.2408 & 0.05 & 301 & 32 & LEU & $\mathrm{HA}$ & 4.0978 & \\
\hline 241 & 26 & THR & $\mathrm{HA}$ & 5.4288 & 0.05 & 302 & 32 & LEU & HB2 & 1.8158 & \\
\hline 242 & 26 & THR & $\mathrm{HB}$ & 3.8598 & 0.05 & 303 & 32 & LEU & HB3 & 1.3948 & \\
\hline 43 & 26 & THR & HG2 & 1.0698 & 0.05 & 304 & 32 & LEU & HD1 & 0.6208 & \\
\hline 44 & 26 & THR & $\mathrm{N}$ & 111.977 & 0.4 & 305 & 32 & LEU & HD2 & 0.6018 & \\
\hline 45 & 27 & ILE & $\mathrm{H}$ & 9.9298 & 0.05 & 306 & 32 & LEU & $H G$ & 0.5868 & \\
\hline 46 & 27 & ILE & C & 173.259 & 0.4 & 307 & 32 & LEU & $\mathrm{N}$ & 119.776 & \\
\hline 47 & 27 & ILE & CA & 60.675 & 0.4 & 308 & 33 & GLY & C & 172.329 & \\
\hline 248 & 27 & ILE & CB & 40.092 & 0.4 & 309 & 33 & GLY & CA & 48.2338 & \\
\hline 249 & 27 & ILE & $\mathrm{HA}$ & 4.8338 & 0.05 & 310 & 33 & GLY & $\mathrm{H}$ & 8.7038 & \\
\hline 250 & 27 & ILE & $\mathrm{HB}$ & 1.8038 & 0.05 & 311 & 33 & GLY & HA2 & 3.9738 & \\
\hline 51 & 27 & ILE & HG12 & 1.2588 & 0.05 & 312 & 33 & GLY & $\mathrm{HA}$ & 3.5418 & \\
\hline 52 & 27 & ILE & HG13 & 0.9878 & 0.05 & 313 & 33 & GLY & $\mathrm{N}$ & 105.213 & \\
\hline 53 & 27 & ILE & HG2 & 0.3518 & 0.05 & 314 & 34 & THR & CA & 66.7648 & \\
\hline 54 & 27 & ILE & $\mathrm{N}$ & 126.544 & 0.4 & 31 & 34 & THR & $\mathrm{CE}$ & 68. & \\
\hline 255 & 28 & THR & C & 173.729 & 0.4 & 31 & 34 & THR & $\mathrm{H}$ & 7.9948 & \\
\hline 256 & 28 & THR & CA & 59.1738 & 0.4 & 317 & 34 & THR & $\mathrm{HA}$ & 3.9408 & \\
\hline 257 & 28 & THR & CB & 72.1978 & 0.4 & 318 & 34 & THR & HB & 4.3668 & \\
\hline 258 & 28 & THR & $\mathrm{H}$ & 8.3888 & 0.05 & 31 & 34 & THR & $H G$ & 1.2878 & \\
\hline 259 & 28 & THR & $\mathrm{HA}$ & 4.8678 & 0.05 & 320 & 34 & THR & $\mathrm{N}$ & 117.782 & \\
\hline 260 & 28 & THR & HB & 4.8228 & 0.05 & 321 & 35 & VAL & C & 176.416 & \\
\hline 261 & 28 & THR & HG2 & 1.2978 & 0.05 & 322 & 35 & VAL & CA & 66 & \\
\hline 262 & 28 & THR & $\mathrm{N}$ & 115.935 & 0.4 & 32 & 35 & VAL & CB & 628 & \\
\hline 263 & 29 & THR & C & 174.376 & 0.4 & 324 & 35 & VAL & CG2 & 20.5538 & \\
\hline 264 & 29 & THR & CA & 6.4158 & 0.4 & 325 & 35 & VAL & $\mathrm{H}$ & 7.8458 & \\
\hline 265 & 29 & THR & CB & 68. & 0.4 & 32 & 35 & VAL & $\mathrm{HA}$ & 148 & \\
\hline 266 & 29 & THR & CG2 & 23.4908 & 0.4 & 327 & 35 & VAL & $\mathrm{HE}$ & 388 & \\
\hline 267 & 29 & THR & $\mathrm{H}$ & 9.1708 & 0.05 & 328 & 35 & VAL & HG1 & 688 & \\
\hline 268 & 29 & THR & $\mathrm{HA}$ & 3.7388 & 0.05 & 32 & 35 & VAL & HG2 & 318 & \\
\hline 269 & 29 & THR & $\mathrm{HB}$ & 4.1838 & 0.05 & 33 & 5 & VAL & $\mathrm{N}$ & 12 & \\
\hline 270 & 29 & THR & HG2 & 1.1998 & 0.05 & 33 & 36 & MET & C & 17 & \\
\hline 271 & 29 & THR & $\mathrm{N}$ & 112.731 & 0.4 & 33 & 36 & MET & CA & & \\
\hline 272 & 30 & LYS & C & 6 & 0.4 & J & 36 & MET & $C B$ & & \\
\hline 273 & 30 & LYS & CA & 59.2648 & 0.4 & 33 & 36 & MET & CE & 17 & \\
\hline 274 & 30 & LYS & CB & 32.4218 & 0.4 & 335 & 36 & MET & CG & & \\
\hline 275 & 30 & LYS & $C D$ & & 0.4 & 33 & 36 & MET & $\mathrm{H}$ & & \\
\hline 276 & 30 & LYS & $\mathrm{CE}$ & & 0.4 & 337 & 6 & MET & $H$ & & \\
\hline 277 & 30 & LYS & CG & 24.6968 & 0.4 & 338 & 36 & MET & HB2 & 178 & \\
\hline 278 & 30 & LYS & $\mathrm{H}$ & 7.6 & 0.05 & 339 & 36 & MET & $\mathrm{HE}$ & & \\
\hline 279 & 30 & LYS & $\mathrm{HA}$ & & 0.05 & 3 & 36 & MET & $\mathrm{N}$ & & \\
\hline 280 & 30 & LYS & $\mathrm{N}$ & 120. & 0.4 & 341 & 6 & MET & $H G$ & 08 & \\
\hline 281 & 30 & LYS & HB2 & 1.8228 & 0.05 & 342 & 37 & ARG & C & $17 \varepsilon$ & \\
\hline 282 & 30 & LYS & HD2 & & 0.0 & 32 & 37 & ARG & $\mathrm{CA}$ & & \\
\hline 283 & 3 & LYS & HE2 & & 0.0 & 34 & 37 & ARG & $\mathrm{C}$ & & \\
\hline 284 & 30 & LYS & HG2 & 18 & 0.05 & 34 & 37 & ARG & $C D$ & & \\
\hline 285 & 31 & GLU & C & 176.336 & 0.4 & 346 & 37 & ARG & HG2 & 178 & \\
\hline 286 & 31 & GLU & CA & & 0.4 & 347 & 37 & ARG & CG & & \\
\hline 287 & 3 & GLU & $\mathrm{CB}$ & & 0.4 & & 37 & ARG & $\mathrm{H}$ & & \\
\hline 288 & 31 & GLU & CG & 38.8128 & 0.4 & 349 & 37 & ARG & $\mathrm{H}$ & 4.7788 & \\
\hline 289 & 31 & GLU & $\mathrm{H}$ & 68 & 0.05 & 350 & 37 & ARG & HD2 & & \\
\hline 290 & 31 & GLU & $\mathrm{HA}$ & & 0.05 & 3 & 37 & ARG & HD3 & & \\
\hline 29 & 31 & GLU & $\mathrm{N}$ & 121 & 01 & 2 & 37 & ARG & - & $11 \varepsilon$ & \\
\hline 292 & 31 & GLU & HB2 & 2.7148 & 0.05 & 353 & 37 & ARG & HB2 & 1.9028 & \\
\hline 293 & 31 & GLU & HG2 & 2.4038 & 0.05 & & 38 & SER & C & 172.053 & \\
\hline 29 & 32 & LEU & C & 176.129 & 0.4 & כנד & 38 & SER & C & 61.2398 & \\
\hline 295 & 32 & LEU & CA & 57.7408 & 0.4 & 35 & 38 & SER & $\mathrm{CB}$ & 63.1948 & \\
\hline 357 & 38 & SER & $\mathrm{H}$ & 7.9658 & 0.05 & 408 & 43 & PRO & HB2 & 1.9038 & \\
\hline
\end{tabular}




\begin{tabular}{|c|c|c|c|c|c|c|c|c|c|c|c|}
\hline 358 & 38 & SER & HA & 4.4128 & 0.05 & 418 & 45 & GLU & C & 176.176 & 0.4 \\
\hline 359 & 38 & SER & $\mathrm{N}$ & 118.269 & 0.4 & 419 & 45 & GLU & CA & 59.9988 & 0.4 \\
\hline 360 & 38 & SER & HB2 & 4.0548 & 0.05 & 420 & 45 & GLU & CB & 29.4078 & 0.4 \\
\hline 361 & 39 & LEU & C & 174.161 & 0.4 & 421 & 45 & GLU & CG & 36.3798 & 0.4 \\
\hline 362 & 39 & LEU & CA & 54.5358 & 0.4 & 422 & 45 & GLU & $\mathrm{H}$ & 8.8228 & 0.05 \\
\hline 363 & 39 & LEU & $\mathrm{CB}$ & 42.0178 & 0.4 & 423 & 45 & GLU & $\mathrm{HA}$ & 3.9828 & 0.05 \\
\hline 364 & 39 & LEU & CD1 & 25.6788 & 0.4 & 424 & 45 & GLU & $\mathrm{N}$ & 120.212 & 0.4 \\
\hline 365 & 39 & LEU & CD2 & 22.4138 & 0.4 & 425 & 45 & GLU & HB2 & 1.4588 & 0.05 \\
\hline 366 & 39 & LEU & CG & 25.9148 & 0.4 & 426 & 45 & GLU & HG2 & 2.3548 & 0.05 \\
\hline 367 & 39 & LEU & $\mathrm{H}$ & 7.4338 & 0.05 & 427 & 46 & ALA & C & 177.394 & 0.4 \\
\hline 368 & 39 & LEU & $\mathrm{HA}$ & 4.4918 & 0.05 & 428 & 46 & ALA & CA & 55.0398 & 0.4 \\
\hline 369 & 39 & LEU & HD1 & 0.8268 & 0.05 & 429 & 46 & ALA & CB & 18.1898 & 0.4 \\
\hline 370 & 39 & LEU & HD2 & 0.8108 & 0.05 & 430 & 46 & ALA & $\mathrm{H}$ & 8.2698 & 0.05 \\
\hline 371 & 39 & LEU & $H G$ & 0.8018 & 0.05 & 431 & 46 & ALA & $\mathrm{HA}$ & 4.0878 & 0.05 \\
\hline 372 & 39 & LEU & $\mathrm{N}$ & 120.296 & 0.4 & 432 & 46 & ALA & $\mathrm{HB}$ & 1.3958 & 0.05 \\
\hline 373 & 39 & LEU & HB2 & 1.8358 & 0.05 & 433 & 46 & ALA & $\mathrm{N}$ & 120.147 & 0.4 \\
\hline 374 & 40 & GLY & C & 171.564 & 0.4 & 434 & 47 & GLU & C & 177.405 & 0.4 \\
\hline 375 & 40 & GLY & CA & 45.4398 & 0.4 & 435 & 47 & GLU & CA & 58.9238 & 0.4 \\
\hline 376 & 40 & GLY & $\mathrm{H}$ & 7.8938 & 0.05 & 436 & 47 & GLU & CB & 28.2468 & 0.4 \\
\hline 377 & 40 & GLY & HA2 & 4.2518 & 0.05 & 437 & 47 & GLU & CG & 33.9888 & 0.4 \\
\hline 378 & 40 & GLY & HA3 & 3.7918 & 0.05 & 438 & 47 & GLU & $\mathrm{H}$ & 7.6948 & 0.05 \\
\hline 379 & 40 & GLY & $\mathrm{N}$ & 106.425 & 0.4 & 439 & 47 & GLU & $\mathrm{HA}$ & 3.9848 & 0.05 \\
\hline 380 & 41 & GLN & C & 171.332 & 0.4 & 440 & 47 & GLU & $\mathrm{N}$ & 118.332 & 0.4 \\
\hline 381 & 41 & GLN & CA & 54.4658 & 0.4 & 441 & 47 & GLU & HB2 & 1.8868 & 0.05 \\
\hline 382 & 41 & GLN & $\mathrm{CB}$ & 30.6968 & 0.4 & 442 & 47 & GLU & HG2 & 2.4058 & 0.05 \\
\hline 383 & 41 & GLN & CG & 33.5098 & 0.4 & 443 & 48 & LEU & C & 175.752 & 0.4 \\
\hline 384 & 41 & GLN & $\mathrm{H}$ & 7.7978 & 0.05 & 444 & 48 & LEU & CA & 57.8508 & 0.4 \\
\hline 385 & 41 & GLN & $\mathrm{HA}$ & 4.4858 & 0.05 & 445 & 48 & LEU & CB & 42.1988 & 0.4 \\
\hline 386 & 41 & GLN & HE21 & 7.4098 & 0.05 & 446 & 48 & LEU & CG & 25.8498 & 0.4 \\
\hline 387 & 41 & GLN & HE22 & 6.7788 & 0.05 & 447 & 48 & LEU & $\mathrm{H}$ & 8.1268 & 0.05 \\
\hline 388 & 41 & GLN & $\mathrm{N}$ & 117.557 & 0.4 & 448 & 48 & LEU & $\mathrm{HA}$ & 4.0138 & 0.05 \\
\hline 389 & 41 & GLN & NE2 & 110.556 & 0.4 & 449 & 48 & LEU & HD2 & 0.6978 & 0.05 \\
\hline 390 & 41 & GLN & HB2 & 1.6168 & 0.05 & 450 & 48 & LEU & HG & 1.0458 & 0.05 \\
\hline 391 & 41 & GLN & HG2 & 2.1668 & 0.05 & 451 & 48 & LEU & $\mathrm{N}$ & 119.439 & 0.4 \\
\hline 392 & 41 & GLN & HG3 & 2.1138 & 0.05 & 452 & 48 & LEU & HB2 & 2.0508 & 0.05 \\
\hline 393 & 42 & ASN & CA & 50.35 & 0.4 & 453 & 48 & LEU & HD1 & 0.7938 & 0.05 \\
\hline 394 & 42 & ASN & CB & 39.044 & 0.4 & 454 & 49 & GLN & C & 175.703 & 0.4 \\
\hline 395 & 42 & ASN & $\mathrm{H}$ & 8.7018 & 0.05 & 455 & 49 & GLN & CA & 59.1438 & 0.4 \\
\hline 396 & 42 & ASN & $\mathrm{HA}$ & 4.3298 & 0.05 & 456 & 49 & GLN & CB & 28.051 & 0.4 \\
\hline 397 & 42 & ASN & HD21 & 7.7668 & 0.05 & 457 & 49 & GLN & CG & 37.2758 & 0.4 \\
\hline 398 & 42 & ASN & HD22 & 6.9678 & 0.05 & 458 & 49 & GLN & $\mathrm{H}$ & 8.2468 & 0.05 \\
\hline 399 & 42 & ASN & $\mathrm{N}$ & 116.151 & 0.4 & 459 & 49 & GLN & $\mathrm{HA}$ & 3.8418 & 0.05 \\
\hline 400 & 42 & ASN & ND2 & 114.422 & 0.4 & 460 & 49 & GLN & HE21 & 7.5158 & 0.05 \\
\hline 401 & 42 & ASN & HB2 & 2.6418 & 0.05 & 461 & 49 & GLN & HE22 & 6.9308 & 0.05 \\
\hline 402 & 43 & PRO & C & 174.906 & 0.4 & 462 & 49 & GLN & $\mathrm{N}$ & 117.775 & 0.4 \\
\hline 403 & 43 & PRO & CA & 61.9858 & 0.4 & 463 & 49 & GLN & NE2 & 112.455 & 0.4 \\
\hline 404 & 43 & PRO & CB & 31.9148 & 0.4 & 464 & 49 & GLN & HB2 & 1.8108 & 0.05 \\
\hline 405 & 43 & PRO & $\mathrm{HA}$ & 4.7228 & 0.05 & 465 & 49 & GLN & HG2 & 2.4358 & 0.05 \\
\hline 406 & 43 & PRO & HD2 & 3.5758 & 0.05 & 466 & 50 & ASP & CA & 57.1128 & 0.4 \\
\hline 407 & 43 & PRO & HD3 & 3.2618 & 0.05 & 467 & 50 & ASP & CB & 40.0358 & 0.4 \\
\hline 408 & 43 & PRO & HB2 & 1.9038 & 0.05 & 468 & 50 & ASP & $\mathrm{H}$ & 8.1208 & 0.05 \\
\hline 409 & 43 & PRO & HG2 & 1.9418 & 0.05 & 469 & 50 & ASP & $\mathrm{HA}$ & 4.4378 & 0.05 \\
\hline 410 & 44 & THR & C & 172.336 & 0.4 & 470 & 50 & ASP & $\mathrm{N}$ & 119.405 & 0.4 \\
\hline 411 & 44 & THR & CA & 60.5658 & 0.4 & 471 & 50 & ASP & HB2 & 2.8628 & 0.05 \\
\hline 412 & 44 & THR & CB & 70.7718 & 0.4 & 472 & 51 & MET & $\mathrm{H}$ & 7.9218 & 0.05 \\
\hline 413 & 44 & THR & $\mathrm{H}$ & 8.6648 & 0.05 & 473 & 51 & MET & $\mathrm{HA}$ & 4.0798 & 0.05 \\
\hline 414 & 44 & THR & HA & 4.4338 & 0.05 & 474 & 51 & MET & $\mathrm{N}$ & 118.728 & 0.4 \\
\hline 415 & 44 & THR & $\mathrm{HB}$ & 4.7188 & 0.05 & 475 & 51 & MET & HB2 & 1.9378 & 0.05 \\
\hline 416 & 44 & THR & HG2 & 1.4328 & 0.05 & 476 & 51 & MET & HB3 & 1.6278 & 0.05 \\
\hline 417 & 44 & THR & $\mathrm{N}$ & 112.272 & 0.4 & 477 & 51 & MET & HG2 & 2.4078 & 0.05 \\
\hline
\end{tabular}




\begin{tabular}{|c|c|c|c|c|c|c|c|c|c|c|c|}
\hline 478 & 51 & MET & HG3 & 2.1978 & 0.05 & 539 & 58 & ASP & $\mathrm{CB}$ & 39.7758 & 0.4 \\
\hline 479 & 51 & MET & $\mathrm{HE}$ & 1.6978 & 0.05 & 540 & 58 & ASP & $\mathrm{H}$ & 8.2578 & 0.05 \\
\hline 480 & 52 & ILE & $\mathrm{H}$ & 7.5878 & 0.05 & 541 & 58 & ASP & $\mathrm{HA}$ & 4.6198 & 0.05 \\
\hline 481 & 52 & ILE & $\mathrm{N}$ & 117.735 & 0.4 & 542 & 58 & ASP & HB2 & 3.0668 & 0.05 \\
\hline 482 & 52 & ILE & C & 174.965 & 0.4 & 543 & 58 & ASP & HB3 & 2.6918 & 0.05 \\
\hline 483 & 52 & ILE & CA & 64.1228 & 0.4 & 544 & 58 & ASP & $\mathrm{N}$ & 113.496 & 0.4 \\
\hline 484 & 52 & ILE & CB & 36.377 & 0.4 & 545 & 59 & GLY & C & 172.107 & 0.4 \\
\hline 485 & 52 & ILE & CD1 & 11.4728 & 0.4 & 546 & 59 & GLY & CA & 46.9268 & 0.4 \\
\hline 486 & 52 & ILE & CG2 & 16.2578 & 0.4 & 547 & 59 & GLY & $\mathrm{H}$ & 7.6128 & 0.05 \\
\hline 487 & 52 & ILE & HA & 3.7538 & 0.05 & 548 & 59 & GLY & HA2 & 3.8788 & 0.05 \\
\hline 488 & 52 & ILE & $\mathrm{HB}$ & 2.0858 & 0.05 & 549 & 59 & GLY & HA3 & 3.8278 & 0.05 \\
\hline 489 & 52 & ILE & HD1 & 0.6988 & 0.05 & 550 & 59 & GLY & $\mathrm{N}$ & 108.087 & 0.4 \\
\hline 490 & 52 & ILE & HG12 & 1.5958 & 0.05 & 551 & 60 & ASN & C & 174.007 & 0.4 \\
\hline 491 & 52 & ILE & HG13 & 1.2778 & 0.05 & 552 & 60 & ASN & CA & 52.273 & 0.4 \\
\hline 492 & 52 & ILE & HG2 & 0.7698 & 0.05 & 553 & 60 & ASN & CB & 37.6628 & 0.4 \\
\hline 493 & 53 & ASN & C & 174.533 & 0.4 & 554 & 60 & ASN & $\mathrm{H}$ & 8.1948 & 0.05 \\
\hline 494 & 53 & ASN & CA & 55.729 & 0.4 & 555 & 60 & ASN & $\mathrm{HA}$ & 4.6068 & 0.05 \\
\hline 495 & 53 & ASN & $\mathrm{CB}$ & 37.9058 & 0.4 & 556 & 60 & ASN & HB2 & 3.3368 & 0.05 \\
\hline 496 & 53 & ASN & $\mathrm{H}$ & 8.7088 & 0.05 & 557 & 60 & ASN & HB3 & 2.6538 & 0.05 \\
\hline 497 & 53 & ASN & $\mathrm{HA}$ & 4.4348 & 0.05 & 558 & 60 & ASN & $\mathrm{N}$ & 118.324 & 0.4 \\
\hline 498 & 53 & ASN & HB2 & 2.9978 & 0.05 & 559 & 61 & GLY & C & 170.445 & 0.4 \\
\hline 499 & 53 & ASN & HB3 & 2.8708 & 0.05 & 560 & 61 & GLY & CA & 45.4348 & 0.4 \\
\hline 500 & 53 & ASN & HD21 & 7.8368 & 0.05 & 561 & 61 & GLY & $\mathrm{H}$ & 10.5728 & 0.05 \\
\hline 501 & 53 & ASN & HD22 & 6.9718 & 0.05 & 562 & 61 & GLY & HA2 & 4.2558 & 0.05 \\
\hline 502 & 53 & ASN & $\mathrm{N}$ & 117.691 & 0.4 & 563 & 61 & GLY & HA3 & 3.4938 & 0.05 \\
\hline 503 & 53 & ASN & ND2 & 110.953 & 0.4 & 564 & 61 & GLY & $\mathrm{N}$ & 112.871 & 0.4 \\
\hline 504 & 54 & GLU & CA & 58.6148 & 0.4 & 565 & 62 & THR & C & 170.378 & 0.4 \\
\hline 505 & 54 & GLU & CG & 35.9758 & 0.4 & 566 & 62 & THR & CA & 59.213 & 0.4 \\
\hline 506 & 54 & GLU & $\mathrm{H}$ & 7.6898 & 0.05 & 567 & 62 & THR & CB & 72.1128 & 0.4 \\
\hline 507 & 54 & GLU & $\mathrm{HA}$ & 4.0438 & 0.05 & 568 & 62 & THR & CG2 & 22.1088 & 0.4 \\
\hline 508 & 54 & GLU & $\mathrm{N}$ & 115.838 & 0.4 & 569 & 62 & THR & $\mathrm{H}$ & 7.6938 & 0.05 \\
\hline 509 & 54 & GLU & HB2 & 1.8668 & 0.05 & 570 & 62 & THR & $\mathrm{HA}$ & 4.7958 & 0.05 \\
\hline 510 & 54 & GLU & HG2 & 2.3468 & 0.05 & 571 & 62 & THR & $\mathrm{HB}$ & 4.0208 & 0.05 \\
\hline 511 & 55 & VAL & C & 172.915 & 0.4 & 572 & 62 & THR & HG2 & 1.1598 & 0.05 \\
\hline 512 & 55 & VAL & CA & 60.9138 & 0.4 & 573 & 62 & THR & $\mathrm{N}$ & 108.099 & 0.4 \\
\hline 513 & 55 & VAL & CB & 39.549 & 0.4 & 574 & 63 & ILE & C & 172.687 & 0.4 \\
\hline 514 & 55 & VAL & CG1 & 22.5128 & 0.4 & 575 & 63 & ILE & CA & 58.9958 & 0.4 \\
\hline 515 & 55 & VAL & CG2 & 20.8048 & 0.4 & 576 & 63 & ILE & CB & 39.07 & 0.4 \\
\hline 516 & 55 & VAL & $\mathrm{H}$ & 7.2148 & 0.05 & 577 & 63 & ILE & CG1 & 27.365 & 0.4 \\
\hline 517 & 55 & VAL & $\mathrm{HA}$ & 4.2588 & 0.05 & 578 & 63 & ILE & CG2 & 18.3588 & 0.4 \\
\hline 518 & 55 & VAL & $\mathrm{HB}$ & 2.1608 & 0.05 & 579 & 63 & ILE & CD1 & 15.3698 & 0.4 \\
\hline 519 & 55 & VAL & HG1 & 0.8278 & 0.05 & 580 & 63 & ILE & $\mathrm{H}$ & 8.8238 & 0.05 \\
\hline 520 & 55 & VAL & HG2 & 0.8788 & 0.05 & 581 & 63 & ILE & $\mathrm{HA}$ & 5.2228 & 0.05 \\
\hline 521 & 55 & VAL & $\mathrm{N}$ & 110.912 & 0.4 & 582 & 63 & ILE & $\mathrm{HB}$ & 2.1488 & 0.05 \\
\hline 522 & 56 & ASP & C & 173.361 & 0.4 & 583 & 63 & ILE & HD1 & 0.3718 & 0.05 \\
\hline 523 & 56 & ASP & CA & 53.8648 & 0.4 & 584 & 63 & ILE & HG13 & 0.9158 & 0.05 \\
\hline 524 & 56 & ASP & CB & 40.1588 & 0.4 & 585 & 63 & ILE & HG2 & 1.1978 & 0.05 \\
\hline 525 & 56 & ASP & $\mathrm{H}$ & 7.7608 & 0.05 & 586 & 63 & ILE & $\mathrm{N}$ & 123.323 & 0.4 \\
\hline 526 & 56 & ASP & $\mathrm{HA}$ & 4.5098 & 0.05 & 587 & 64 & ASP & C & 173.494 & 0.4 \\
\hline 527 & 56 & ASP & HB2 & 3.0068 & 0.05 & 588 & 64 & ASP & CA & 51.8118 & 0.4 \\
\hline 528 & 56 & ASP & HB3 & 2.7238 & 0.05 & 589 & 64 & ASP & CB & 42.2468 & 0.4 \\
\hline 529 & 56 & ASP & $\mathrm{N}$ & 120.629 & 0.4 & 590 & 64 & ASP & $\mathrm{H}$ & 8.8928 & 0.05 \\
\hline 530 & 57 & ALA & C & 175.842 & 0.4 & 591 & 64 & ASP & $\mathrm{HA}$ & 5.4908 & 0.05 \\
\hline 531 & 57 & ALA & CA & 54.2908 & 0.4 & 592 & 64 & ASP & HB2 & 3.1518 & 0.05 \\
\hline 532 & 57 & ALA & CB & 19.4558 & 0.4 & 593 & 64 & ASP & HB3 & 2.8708 & 0.05 \\
\hline 533 & 57 & ALA & $\mathrm{H}$ & 8.2438 & 0.05 & 594 & 64 & ASP & $\mathrm{N}$ & 127.718 & 0.4 \\
\hline 534 & 57 & ALA & $\mathrm{HA}$ & 4.2228 & 0.05 & 595 & 65 & PHE & CA & 63.193 & 0.4 \\
\hline 535 & 57 & ALA & $\mathrm{HB}$ & 1.5318 & 0.05 & 596 & 65 & PHE & CB & 35.6308 & 0.4 \\
\hline 536 & 57 & ALA & $\mathrm{N}$ & 131.226 & 0.4 & 597 & 65 & PHE & CD1 & 129.971 & 0.4 \\
\hline 537 & 58 & ASP & C & 175.471 & 0.4 & 598 & 65 & PHE & CD2 & 129.971 & 0.4 \\
\hline 538 & 58 & ASP & CA & 52.7878 & 0.4 & 599 & 65 & PHE & CE1 & 128.531 & 0.4 \\
\hline
\end{tabular}




\begin{tabular}{|c|c|c|c|c|c|c|c|c|c|c|c|}
\hline 600 & 65 & PHE & CE2 & 128.531 & 0.4 & 661 & 72 & MET & HB2 & 1.0678 & 0.05 \\
\hline 601 & 65 & PHE & $\mathrm{H}$ & 8.9878 & 0.05 & 662 & 72 & MET & HB3 & 0.9978 & 0.05 \\
\hline 602 & 65 & PHE & $\mathrm{HA}$ & 3.7838 & 0.05 & 663 & 72 & MET & HG2 & 1.4578 & 0.05 \\
\hline 603 & 65 & PHE & HB2 & 2.8208 & 0.05 & 664 & 72 & MET & HG3 & 1.3778 & 0.05 \\
\hline 604 & 65 & PHE & HB3 & 2.0848 & 0.05 & 665 & 73 & ALA & C & 177.075 & 0.4 \\
\hline 605 & 65 & PHE & $\mathrm{N}$ & 118.282 & 0.4 & 666 & 73 & ALA & CA & 54.504 & 0.4 \\
\hline 606 & 65 & PHE & HD1 & 6.7628 & 0.05 & 667 & 73 & ALA & CB & 18.2718 & 0.4 \\
\hline 607 & 65 & PHE & HD2 & 6.7628 & 0.05 & 668 & 73 & ALA & $\mathrm{H}$ & 7.8968 & 0.05 \\
\hline 608 & 65 & PHE & HE1 & 7.2108 & 0.05 & 669 & 73 & ALA & $\mathrm{HA}$ & 4.0248 & 0.05 \\
\hline 609 & 65 & PHE & HE2 & 7.2108 & 0.05 & 670 & 73 & ALA & $\mathrm{HB}$ & 1.3728 & 0.05 \\
\hline 610 & 66 & PRO & $\mathrm{HA}$ & 3.8528 & 0.05 & 671 & 73 & ALA & $\mathrm{N}$ & 120.06 & 0.4 \\
\hline 611 & 66 & PRO & HB2 & 2.1578 & 0.05 & 672 & 74 & ARG & C & 175.04 & 0.4 \\
\hline 612 & 66 & PRO & HB3 & 1.8038 & 0.05 & 673 & 74 & ARG & CA & 58.032 & 0.4 \\
\hline 613 & 66 & PRO & HG2 & 1.3648 & 0.05 & 674 & 74 & ARG & CB & 29.32 & 0.4 \\
\hline 614 & 66 & PRO & HD2 & 3.7078 & 0.05 & 675 & 74 & ARG & $\mathrm{HA}$ & 3.7378 & 0.05 \\
\hline 615 & 67 & GLU & $\mathrm{H}$ & 8.1738 & 0.05 & 651 & 71 & MET & $\mathrm{HA}$ & 3.4178 & 0.05 \\
\hline 616 & 67 & GLU & HA & 4.4498 & 0.05 & 652 & 71 & MET & HB2 & 1.7678 & 0.05 \\
\hline 617 & 67 & GLU & HB2 & 1.7678 & 0.05 & 653 & 71 & MET & HG2 & 2.0978 & 0.05 \\
\hline 18 & 67 & GLU & HG2 & 2.6078 & 0.05 & 654 & 71 & MET & $\mathrm{HE}$ & 1.3978 & 0.05 \\
\hline 619 & 67 & GLU & HG3 & 2.2878 & 0.05 & 655 & 72 & MET & $\mathrm{HA}$ & 3.6478 & 0.05 \\
\hline 620 & 67 & GLU & $\mathrm{N}$ & 116.98 & 0.4 & 656 & 72 & MET & C & 175.697 & 0.4 \\
\hline 621 & 68 & PHE & CD1 & 129.642 & 0.4 & 657 & 72 & MET & CA & 60.0268 & 0.4 \\
\hline 622 & 68 & PHE & CD2 & 129.642 & 0.4 & 658 & 72 & MET & CG & 31.9708 & 0.4 \\
\hline 623 & 68 & PHE & CE1 & 129.082 & 0.4 & 659 & 72 & MET & $\mathrm{H}$ & 8.1888 & 0.05 \\
\hline 624 & 68 & PHE & CE2 & 129.082 & 0.4 & 660 & 72 & MET & $\mathrm{N}$ & 115.879 & 0.4 \\
\hline 625 & 68 & PHE & $\mathrm{H}$ & 8.6708 & 0.05 & 661 & 72 & MET & HB2 & 1.0678 & 0.05 \\
\hline 626 & 68 & PHE & $\mathrm{N}$ & 122.872 & 0.4 & 662 & 72 & MET & HB3 & 0.9978 & 0.05 \\
\hline 627 & 68 & PHE & $\mathrm{HA}$ & 3.6278 & 0.05 & 663 & 72 & MET & HG2 & 1.4578 & 0.05 \\
\hline 628 & 68 & PHE & HB2 & 3.1278 & 0.05 & 665 & 73 & ALA & C & 177.075 & 0.4 \\
\hline 629 & 68 & PHE & HB3 & 2.8278 & 0.05 & 666 & 73 & ALA & CA & 54.504 & 0.4 \\
\hline 630 & 68 & PHE & HD1 & 6.9108 & 0.05 & 667 & 73 & ALA & CB & 18.2718 & 0.4 \\
\hline 631 & 68 & PHE & HD2 & 6.9108 & 0.05 & 668 & 73 & ALA & $\mathrm{H}$ & 7.8968 & 0.05 \\
\hline 632 & 68 & PHE & HE1 & 7.1818 & 0.05 & 669 & 73 & ALA & $\mathrm{HA}$ & 4.0248 & 0.05 \\
\hline 633 & 68 & PHE & HE2 & 7.1818 & 0.05 & 670 & 73 & ALA & $\mathrm{HB}$ & 1.3728 & 0.05 \\
\hline 634 & 69 & LEU & $\mathrm{H}$ & 8.6748 & 0.05 & 671 & 73 & ALA & $\mathrm{N}$ & 120.06 & 0.4 \\
\hline 635 & 69 & LEU & $\mathrm{N}$ & 119.155 & 0.4 & 672 & 74 & ARG & C & 175.04 & 0.4 \\
\hline 636 & 69 & LEU & CB & 38.6428 & 0.4 & 673 & 74 & ARG & CA & 58.032 & 0.4 \\
\hline 637 & 69 & LEU & CD1 & 25.5958 & 0.4 & 674 & 74 & ARG & CB & 29.32 & 0.4 \\
\hline 638 & 69 & LEU & $\mathrm{HA}$ & 3.9398 & 0.05 & 675 & 74 & ARG & $\mathrm{HA}$ & 3.7378 & 0.05 \\
\hline 639 & 69 & LEU & HD1 & 0.8388 & 0.05 & 676 & 74 & ARG & HB2 & 1.4878 & 0.05 \\
\hline 640 & 69 & LEU & HD2 & 0.0638 & 0.05 & 677 & 74 & ARG & HB3 & 1.3178 & 0.05 \\
\hline 641 & 69 & LEU & HB2 & 1.3058 & 0.05 & 678 & 74 & ARG & HG2 & 1.0778 & 0.05 \\
\hline 642 & 69 & LEU & $H G$ & 0.6778 & 0.05 & 679 & 74 & ARG & HD2 & 2.7378 & 0.05 \\
\hline 643 & 70 & THR & CB & 67.8278 & 0.4 & 680 & 74 & ARG & $C D$ & 43.7328 & 0.4 \\
\hline 644 & 70 & THR & $\mathrm{H}$ & 7.5458 & 0.05 & 681 & 74 & ARG & CG & 27.3618 & 0.4 \\
\hline 645 & 70 & THR & $\mathrm{HA}$ & 3.4478 & 0.05 & 682 & 74 & ARG & $\mathrm{H}$ & 7.4768 & 0.05 \\
\hline 646 & 70 & THR & $\mathrm{HB}$ & 4.1948 & 0.05 & 683 & 74 & ARG & $\mathrm{N}$ & 116.115 & 0.4 \\
\hline 647 & 70 & THR & HG2 & 1.2488 & 0.05 & 684 & 75 & LYS & C & 174.877 & 0.4 \\
\hline 648 & 70 & THR & $\mathrm{N}$ & 114.017 & 0.4 & 685 & 75 & LYS & CA & 57.313 & 0.4 \\
\hline 649 & 71 & MET & $\mathrm{H}$ & 7.6178 & 0.05 & 686 & 75 & LYS & CB & 31.54 & 0.4 \\
\hline 650 & 71 & MET & $\mathrm{N}$ & 119.16 & 0.4 & 687 & 75 & LYS & CE & 40.7878 & 0.4 \\
\hline 651 & 71 & MET & HA & 3.4178 & 0.05 & 688 & 75 & LYS & $\mathrm{H}$ & 7.7728 & 0.05 \\
\hline 652 & 71 & MET & HB2 & 1.7678 & 0.05 & 689 & 75 & LYS & $\mathrm{HA}$ & 3.9348 & 0.05 \\
\hline 653 & 71 & MET & HG2 & 2.0978 & 0.05 & 690 & 75 & LYS & $\mathrm{N}$ & 118.389 & 0.4 \\
\hline 654 & 71 & MET & $\mathrm{HE}$ & 1.3978 & 0.05 & 691 & 75 & LYS & HB2 & 1.8288 & 0.05 \\
\hline 655 & 72 & MET & $\mathrm{HA}$ & 3.6478 & 0.05 & 692 & 75 & LYS & HE2 & 3.5488 & 0.05 \\
\hline 656 & 72 & MET & C & 175.697 & 0.4 & 693 & 75 & LYS & HG2 & 1.4908 & 0.05 \\
\hline 657 & 72 & MET & CA & 60.0268 & 0.4 & 694 & 75 & LYS & HD2 & 1.6878 & 0.05 \\
\hline 658 & 72 & MET & CG & 31.9708 & 0.4 & 695 & 76 & MET & C & 173.683 & 0.4 \\
\hline 659 & 72 & MET & $\mathrm{H}$ & 8.1888 & 0.05 & 696 & 76 & MET & CA & 56.4598 & 0.4 \\
\hline r & 72 & MET & $\mathrm{N}$ & 115.879 & 0.4 & 697 & 76 & MET & CB & 28.262 & 0.4 \\
\hline
\end{tabular}




\begin{tabular}{|c|c|c|c|c|c|c|c|c|c|c|c|}
\hline 698 & 76 & MET & CE & 17.0608 & 0.4 & 759 & 82 & GLU & $\mathrm{N}$ & 122.235 & \\
\hline 699 & 76 & MET & CG & 32.1808 & 0.4 & 760 & 82 & GLU & HB2 & 2.1328 & \\
\hline 700 & 76 & MET & $\mathrm{H}$ & 7.9748 & 0.05 & 761 & 82 & GLU & HG2 & 2.3588 & \\
\hline 701 & 76 & MET & $\mathrm{HA}$ & 4.3438 & 0.05 & 762 & 83 & GLU & C & 176.436 & \\
\hline 702 & 76 & MET & HB2 & 2.1528 & 0.05 & 763 & 83 & GLU & CA & 59.2618 & \\
\hline 703 & 76 & MET & HB3 & 2.1098 & 0.05 & 764 & 83 & GLU & CB & 29.5468 & \\
\hline 04 & 76 & MET & $\mathrm{HE}$ & 2.0868 & 0.05 & 765 & 83 & GLU & CG & 35.8858 & \\
\hline 705 & 76 & MET & HG2 & 2.6608 & 0.05 & 766 & 83 & GLU & $\mathrm{H}$ & 8.2248 & \\
\hline 706 & 76 & MET & HG3 & 2.6188 & 0.05 & 767 & 83 & GLU & $\mathrm{HA}$ & 4.1048 & \\
\hline 707 & 76 & MET & $\mathrm{N}$ & 117.535 & 0.4 & 768 & 83 & GLU & $\mathrm{N}$ & 119.203 & \\
\hline 08 & 77 & LYS & C & 173.704 & 0.4 & 769 & 83 & GLU & HB2 & 2.0948 & \\
\hline 09 & 77 & LYS & CA & 56.8018 & 0.4 & 770 & 83 & GLU & HG2 & 2.3658 & \\
\hline 710 & 77 & LYS & CB & 32.7798 & 0.4 & 771 & 84 & GLU & C & 177.343 & \\
\hline 711 & 77 & LYS & $C D$ & 28.8688 & 0.4 & 772 & 84 & GLU & CA & 59.6828 & \\
\hline 712 & 77 & LYS & CE & 41.9038 & 0.4 & 773 & 84 & GLU & $\mathrm{CB}$ & 30.3448 & \\
\hline 713 & 77 & LYS & CG & 24.5088 & 0.4 & 774 & 84 & GLU & CG & 36.2148 & \\
\hline 714 & 77 & LYS & $\mathrm{H}$ & 7.7548 & 0.05 & 775 & 84 & GLU & $\mathrm{H}$ & 8.1158 & \\
\hline 5 & 77 & LYS & $\mathrm{HA}$ & 4.2988 & 0.05 & 776 & 84 & GLU & $\mathrm{HA}$ & 4.1448 & \\
\hline 16 & 77 & LYS & HB2 & 1.8628 & 0.05 & 777 & 84 & GLU & HG2 & 2.4798 & \\
\hline 717 & 77 & LYS & HB3 & 1.8208 & 0.05 & 778 & 84 & GLU & HG3 & 2.3118 & \\
\hline 718 & 77 & LYS & $\mathrm{N}$ & 119.562 & 0.4 & 779 & 84 & GLU & $\mathrm{N}$ & 117.902 & \\
\hline 719 & 77 & LYS & HD2 & 1.6868 & 0.05 & 780 & 84 & GLU & HB2 & 2.1508 & \\
\hline 720 & 77 & LYS & HE2 & 2.9838 & 0.05 & 781 & 85 & ILE & C & 174.656 & \\
\hline 721 & 77 & LYS & HG2 & 1.4828 & 0.05 & 782 & 85 & ILE & CA & 65.6048 & \\
\hline 722 & 78 & ASP & CA & 54.3988 & 0.4 & 783 & 85 & ILE & CB & 37 & \\
\hline 723 & 78 & ASP & C & 173.716 & 0.4 & 784 & 85 & ILE & CD1 & 13.3228 & \\
\hline 724 & 78 & ASP & CB & 40.8618 & 0.4 & 785 & 85 & ILE & CG1 & 29.7868 & \\
\hline 725 & 78 & ASP & $\mathrm{H}$ & 8.1648 & 0.05 & 786 & 85 & ILE & CG2 & 18.5638 & \\
\hline 726 & 78 & ASP & $\mathrm{HA}$ & 4.7048 & 0.05 & 787 & 35 & ILE & $\mathrm{H}$ & 18 & \\
\hline 727 & 78 & ASP & HB2 & 2.8028 & 0.05 & 788 & 85 & ILE & $\mathrm{HA}$ & 4.0898 & \\
\hline 728 & 78 & ASP & HB3 & 2.7438 & 0.05 & 789 & 85 & ILE & $\mathrm{HB}$ & 2.2168 & \\
\hline 729 & 78 & ASP & $\mathrm{N}$ & 120.459 & 0.4 & 790 & 85 & ILE & HD1 & 0.7028 & \\
\hline 730 & 79 & THR & C & 171.541 & 0.4 & 791 & 85 & ILE & HG12 & 1.9488 & \\
\hline 731 & 79 & THR & CA & 62.1658 & 0.4 & 792 & 85 & ILE & HG13 & 0.9628 & \\
\hline 732 & 79 & THR & CB & 38 & 0.4 & 79 & 85 & ILE & HG2 & 078 & \\
\hline 733 & 79 & THR & CG2 & 21.4248 & 0.4 & 794 & 5 & ILE & $\mathrm{N}$ & 58 & \\
\hline 734 & 79 & THR & $\mathrm{H}$ & 8.0398 & 0.05 & 795 & 86 & ARG & CA & 60.3318 & \\
\hline 735 & 79 & THR & $\mathrm{HA}$ & 4.3208 & 0.05 & 796 & 86 & ARG & CB & 29.9378 & \\
\hline 736 & 79 & THR & $\mathrm{HB}$ & 4.2538 & 0.05 & 797 & 86 & ARG & $C D$ & 42 & \\
\hline 737 & 79 & THR & HG2 & 1.2238 & 0.05 & 798 & 86 & ARG & CG & 27. & \\
\hline 738 & 79 & THR & $\mathrm{N}$ & 113.759 & 0.4 & 799 & 86 & ARG & $\mathrm{H}$ & 8.3748 & \\
\hline 739 & 80 & ASP & C & & 0.4 & 80 & 86 & ARG & $\mathrm{HA}$ & & \\
\hline 740 & 80 & ASP & CA & 98 & 0.4 & 801 & 86 & ARG & HB2 & 68 & \\
\hline 741 & 80 & ASP & CB & 40.9508 & 0.4 & 802 & 86 & ARG & HB3 & 1.8628 & \\
\hline 742 & 80 & ASP & $\mathrm{H}$ & 8.4268 & 0.05 & 803 & 86 & ARG & HG2 & 1.6538 & \\
\hline 743 & 80 & ASP & $\mathrm{HA}$ & & 0.05 & 8 & 86 & ARG & HG3 & 28 & \\
\hline 744 & 80 & ASP & $\mathrm{N}$ & 122.666 & 0.4 & 8 & 6 & ARG & $\mathrm{N}$ & 121 & \\
\hline 745 & 80 & ASP & HB2 & 2.7928 & 0.05 & 806 & 86 & ARG & HD2 & 2.9658 & \\
\hline 746 & 81 & SER & C & 72.788 & 0.4 & 807 & 87 & GLU & CA & 59.2028 & \\
\hline 747 & 81 & SER & CA & & 0.4 & 808 & 87 & GLU & CB & & \\
\hline 748 & 81 & SER & CB & 78 & 0.4 & 809 & 87 & GLU & CG & 36 & \\
\hline 749 & 81 & SER & $\mathrm{H}$ & 8.4558 & 0.05 & 810 & 87 & GLU & $\mathrm{H}$ & 8.3608 & 0. \\
\hline 750 & 81 & SER & $\mathrm{HA}$ & 4.4138 & 0.05 & 811 & 87 & GLU & $\mathrm{HA}$ & 4.0938 & \\
\hline 751 & 81 & SER & $\mathrm{N}$ & 117 & 0.4 & 812 & 37 & GLU & $\mathrm{N}$ & 11 & \\
\hline 752 & 81 & SER & HB2 & 3.9998 & 0.05 & 813 & 87 & GLU & HB2 & 58 & \\
\hline 753 & 82 & GLU & C & 175.133 & 0.4 & 814 & 87 & GLU & HG2 & 2.3498 & \\
\hline 754 & 82 & GLU & CA & 58.6118 & 0.4 & 815 & 88 & ALA & C & 176.595 & \\
\hline 755 & 82 & GLU & $\mathrm{CB}$ & & 0.4 & 816 & 88 & ALA & CA & 54.9348 & \\
\hline 756 & 82 & GLU & CG & 36.6058 & 0.4 & 817 & 88 & ALA & CB & 18.5808 & \\
\hline 757 & 82 & GLU & $\mathrm{H}$ & 8.5098 & 0.05 & 818 & 88 & ALA & $\mathrm{H}$ & 8.3528 & U. \\
\hline 758 & 82 & GLU & $\mathrm{HA}$ & 4.1568 & 0.05 & 819 & 88 & ALA & $\mathrm{HA}$ & 4.1738 & \\
\hline
\end{tabular}




\begin{tabular}{|c|c|c|c|c|c|c|c|c|c|c|c|}
\hline 820 & 88 & ALA & $\mathrm{HB}$ & 1.9338 & 0.05 & 881 & 93 & ASP & HB2 & 2.2178 & $0 . c^{2}-x$ \\
\hline 821 & 88 & ALA & $\mathrm{N}$ & 120.095 & 0.4 & 882 & 94 & LYS & C & 175.567 & \\
\hline 822 & 89 & PHE & C & 173.566 & 0.4 & 883 & 94 & LYS & CA & 59.3298 & \\
\hline 823 & 89 & PHE & $C A$ & 61.8048 & 0.4 & 884 & 94 & LYS & CB & 32.5108 & \\
\hline 824 & 89 & PHE & CB & 38.9878 & 0.4 & 885 & 94 & LYS & CD & 28.2778 & \\
\hline 825 & 89 & PHE & $\mathrm{H}$ & 8.8348 & 0.05 & 886 & 94 & LYS & CE & 41.5298 & \\
\hline 826 & 89 & PHE & $\mathrm{HA}$ & 3.3128 & 0.05 & 887 & 94 & LYS & CG & 23.7708 & \\
\hline 827 & 89 & PHE & $\mathrm{HZ}$ & 7.2848 & 0.05 & 888 & 94 & LYS & $\mathrm{H}$ & 7.4178 & \\
\hline 828 & 89 & PHE & $\mathrm{N}$ & 118.86 & 0.4 & 889 & 94 & LYS & $\mathrm{HA}$ & 3.8858 & \\
\hline 829 & 89 & PHE & HB2 & 3.0008 & 0.05 & 890 & 94 & LYS & HD2 & 1.6468 & \\
\hline 830 & 89 & PHE & CE1 & 126.947 & 0.4 & 891 & 94 & LYS & HD3 & 1.4968 & \\
\hline 831 & 89 & PHE & CE2 & 126.947 & 0.4 & 892 & 94 & LYS & HG2 & 1.5198 & \\
\hline 832 & 89 & PHE & HD1 & 6.5598 & 0.05 & 893 & 94 & LYS & HG3 & 1.4738 & \\
\hline 833 & 89 & PHE & HD2 & 6.5598 & 0.05 & 894 & 94 & LYS & $\mathrm{N}$ & 124.29 & \\
\hline 834 & 89 & PHE & HE1 & 7.0808 & 0.05 & 895 & 94 & LYS & HB2 & 1.8608 & \\
\hline 835 & 89 & PHE & HE2 & 7.0808 & 0.05 & 896 & 94 & LYS & HE2 & 2.8508 & \\
\hline 836 & 90 & ARG & C & 174.665 & 0.4 & 897 & 95 & ASP & CA & 53.9748 & \\
\hline 837 & 90 & ARG & CA & 58.3618 & 0.4 & 898 & 95 & ASP & C & 57 & \\
\hline 838 & 90 & ARG & CB & 30.3938 & 0.4 & 899 & 95 & ASP & CB & 39.9358 & \\
\hline 839 & 90 & ARG & $C D$ & 43.3538 & 0.4 & 900 & 95 & ASP & $\mathrm{H}$ & 8.4428 & \\
\hline 840 & 90 & ARG & CG & 27.8308 & 0.4 & 901 & 95 & ASP & HA & 4.5038 & \\
\hline 841 & 90 & ARG & $\mathrm{H}$ & 7.6278 & 0.05 & 902 & 95 & ASP & HB2 & 328 & \\
\hline 842 & 90 & ARG & $\mathrm{HA}$ & 3.9408 & 0.05 & 903 & 95 & ASP & HB3 & 2.1948 & \\
\hline 843 & 90 & ARG & HB2 & 1.9898 & 0.05 & 904 & 95 & ASP & $\mathrm{N}$ & 113.716 & \\
\hline 844 & 90 & ARG & HB3 & 1.9528 & 0.05 & 905 & 96 & GLY & C & 324 & \\
\hline 845 & 90 & ARG & HG2 & 1.9688 & 0.05 & 90 & 96 & GLY & CA & 46 & \\
\hline 846 & 90 & ARG & HG3 & 1.7988 & 0.05 & 907 & 96 & GLY & $\mathrm{H}$ & 7.9008 & \\
\hline 847 & 90 & ARG & $\mathrm{N}$ & 114.46 & 0.4 & 908 & 96 & GLY & HA2 & 3.9218 & \\
\hline 848 & 90 & ARG & HD2 & 3.2628 & 0.05 & 909 & 96 & GLY & HA3 & 578 & \\
\hline 849 & 91 & VAL & C & 174.176 & 0.4 & 910 & 96 & GLY & $\mathrm{N}$ & 038 & \\
\hline 850 & 91 & VAL & CA & 65.6048 & 0.4 & 911 & 97 & ASN & C & 173.111 & \\
\hline 851 & 91 & VAL & CB & 78 & 0.4 & 912 & 97 & ASN & CA & 18 & \\
\hline 852 & 91 & VAL & CG1 & 22.5558 & 0.4 & 913 & 97 & ASN & CB & 228 & \\
\hline 853 & 91 & VAL & CG2 & 20.9048 & 0.4 & 914 & 97 & ASN & $\mathrm{H}$ & 8.4328 & \\
\hline 854 & 91 & VAL & $\mathrm{H}$ & 7.4118 & 0.05 & 915 & 97 & ASN & $\mathrm{HA}$ & 208 & \\
\hline 855 & 91 & VAL & $\mathrm{HA}$ & 3.5488 & 0.05 & 916 & 97 & ASN & HB2 & 58 & \\
\hline 856 & 91 & VAL & HB & 2.2488 & 0.05 & 917 & 97 & ASN & HB3 & 2.6908 & \\
\hline 857 & 91 & VAL & HG1 & 1.0318 & 0.05 & 918 & 97 & ASN & HD21 & 918 & \\
\hline 858 & 91 & VAL & HG2 & 0.7098 & 0.05 & 919 & 97 & ASN & $\mathrm{HD}^{2}$ & & \\
\hline 859 & 91 & VAL & $\mathrm{N}$ & 32 & 0.4 & 920 & 97 & ASN & $\mathrm{N}$ & & \\
\hline 860 & 92 & PHE & C & 173.432 & 0.4 & 921 & 97 & ASN & ND2 & 116.123 & \\
\hline 861 & 92 & PHE & $C A$ & 0.5698 & 0.4 & 922 & 98 & GLY & C & $16 \mathrm{~s}$ & \\
\hline 862 & 92 & PHE & CB & & 0.4 & 923 & 98 & GLY & CA & & \\
\hline 863 & 92 & PHE & CD1 & 130.697 & 0.4 & 924 & 98 & GLY & $\mathrm{H}$ & 10. & \\
\hline 864 & 92 & PHE & CD2 & 130.697 & 0.4 & 925 & 98 & GLY & HA2 & 4.0968 & \\
\hline 865 & 92 & PHE & CE1 & & 0.4 & 926 & 98 & GLY & HA3 & 38 & \\
\hline 866 & 92 & PHE & CE2 & & 0.4 & 927 & 98 & GLY & $\mathrm{N}$ & & \\
\hline 867 & 92 & PHE & $\mathrm{H}$ & 7.2058 & 0.05 & 928 & 99 & TYR & C & 383 & \\
\hline 868 & 92 & PHE & $\mathrm{HA}$ & 4.1938 & 0.05 & 929 & 99 & TYR & CA & 55.7868 & \\
\hline 869 & 92 & PHE & $\mathrm{N}$ & 114 & 0.4 & 930 & 99 & TYR & CB & 42. & \\
\hline 870 & 92 & PHE & HB2 & & 0.05 & 931 & 99 & TYR & CD1 & & \\
\hline 871 & 92 & PHE & HD1 & 7.5308 & 0.05 & 932 & 99 & TYR & CD2 & 47 & \\
\hline 872 & 92 & PHE & HD2 & 7.5308 & 0.05 & 933 & 99 & TYR & CE1 & 116.395 & \\
\hline 873 & 92 & PHE & HE1 & & 0.05 & 934 & 99 & TYR & CE2 & 395 & \\
\hline 874 & 92 & PHE & HE2 & 7.2868 & 0.05 & 935 & 99 & TYR & $\mathrm{H}$ & 7.6948 & 0. \\
\hline 875 & 93 & ASP & C & 174.487 & 0.4 & 936 & 99 & TYR & $\mathrm{HA}$ & 5.0578 & \\
\hline 876 & 93 & ASP & CA & 52.4208 & 0.4 & 937 & 99 & TYR & HB2 & 2.5608 & \\
\hline 877 & 93 & ASP & CB & 38.8108 & 0.4 & 938 & 99 & TYR & HB3 & 2.5378 & \\
\hline 878 & 93 & ASP & $\mathrm{H}$ & 7.8908 & 0.05 & 939 & 99 & TYR & $\mathrm{N}$ & 115.788 & \\
\hline 879 & 93 & ASP & $\mathrm{HA}$ & 4.5588 & 0.05 & 940 & 99 & TYR & HD1 & 6.7708 & \\
\hline 880 & 93 & ASP & $\mathrm{N}$ & 114.746 & 0.4 & 941 & 99 & TYR & HD2 & 6.7708 & \\
\hline
\end{tabular}




\begin{tabular}{|c|c|c|c|c|c|c|c|c|c|c|c|}
\hline 942 & 99 & TYR & HE1 & 6.9438 & 0.05 & 1002 & 106 & ARG & $C A$ & 59.8628 & 0.4 \\
\hline 943 & 99 & TYR & HE2 & 6.9438 & 0.05 & 1003 & 106 & ARG & $C B$ & 30.0268 & 0.4 \\
\hline 944 & 100 & ILE & C & 172.814 & 0.4 & 1004 & 106 & ARG & $C D$ & 43.4158 & 04 \\
\hline 945 & 100 & ILE & CA & 61.4788 & 0.4 & 1005 & 106 & ARG & CG & 27.4768 & 0.4 \\
\hline 946 & 100 & ILE & CB & 39.6218 & 0.4 & 1006 & 106 & ARG & $\mathrm{H}$ & 8.7128 & 0.05 \\
\hline 947 & 100 & ILE & CD1 & 16.1228 & 0.4 & 1007 & 106 & ARG & $\mathrm{HA}$ & 3.8018 & 0.05 \\
\hline 948 & 100 & ILE & CG1 & 26.7908 & 0.4 & 1008 & 106 & ARG & HB2 & 1.9498 & 0.05 \\
\hline 949 & 100 & ILE & CG2 & 17.5398 & 0.4 & 1009 & 106 & ARG & HB3 & 1.7308 & 0.05 \\
\hline 950 & 100 & ILE & $\mathrm{H}$ & 10.2758 & 0.05 & 1010 & 106 & ARG & HD2 & 3.2588 & 0.05 \\
\hline 951 & 100 & ILE & $\mathrm{HA}$ & 4.7148 & 0.05 & 1011 & 106 & ARG & HD3 & 3.1358 & 0.05 \\
\hline 952 & 100 & ILE & $\mathrm{HB}$ & 1.9998 & 0.05 & 1012 & 106 & ARG & $\mathrm{N}$ & 117.74 & 0.4 \\
\hline 953 & 100 & ILE & HD1 & 0.6218 & 0.05 & 1013 & 106 & ARG & HG2 & 1.6418 & 0.05 \\
\hline 954 & 100 & ILE & HG12 & 1.5078 & 0.05 & 1014 & 107 & HIS & C & 175.029 & 0.4 \\
\hline 955 & 100 & ILE & HG13 & 0.6148 & 0.05 & 1015 & 107 & HIS & $C A$ & 59.0188 & 0.4 \\
\hline 956 & 100 & ILE & HG2 & 1.1548 & 0.05 & 1016 & 107 & HIS & CB & 28.6158 & 0.4 \\
\hline 957 & 100 & ILE & $\mathrm{N}$ & 126.892 & 0.4 & 1017 & 107 & HIS & CD2 & 118.538 & 0.4 \\
\hline 958 & 101 & SER & C & 172.611 & 0.4 & 1018 & 107 & HIS & CE1 & 134.929 & 0.4 \\
\hline 959 & 101 & SER & CA & 55.3568 & 0.4 & 1019 & 107 & HIS & $\mathrm{H}$ & 7.8868 & 0.05 \\
\hline 960 & 101 & SER & CB & 66.6608 & 0.4 & 1020 & 107 & HIS & $\mathrm{HA}$ & 4.3998 & 0.05 \\
\hline 961 & 101 & SER & $\mathrm{H}$ & 8.9958 & 0.05 & 1021 & 107 & HIS & HB2 & 3.4228 & 0.05 \\
\hline 962 & 101 & SER & $\mathrm{HA}$ & 5.0128 & 0.05 & 1022 & 107 & HIS & HB3 & 3.3728 & 0.05 \\
\hline 963 & 101 & SER & $\mathrm{N}$ & 123.234 & 0.4 & 1023 & 107 & HIS & HD2 & 7.2578 & 0.05 \\
\hline 964 & 101 & SER & HB2 & 4.0118 & 0.05 & 1024 & 107 & HIS & HE1 & 8.5098 & 0.05 \\
\hline 965 & 102 & ALA & C & 176.445 & 0.4 & 1025 & 107 & HIS & $\mathrm{N}$ & 117.72 & 0.4 \\
\hline 966 & 102 & ALA & CA & 55.9148 & 0.4 & 1026 & 108 & VAL & C & 175.248 & 0.4 \\
\hline 967 & 102 & ALA & CB & 17.7798 & 0.4 & 1027 & 108 & VAL & CA & 538 & 0.4 \\
\hline 968 & 102 & ALA & $\mathrm{H}$ & 9.3348 & 0.05 & 1028 & 108 & VAL & CB & 31.5708 & 0.4 \\
\hline 969 & 102 & ALA & $\mathrm{HA}$ & 3.9268 & 0.05 & 1029 & 108 & VAL & CG1 & 23.7288 & 0.4 \\
\hline 970 & 102 & ALA & $\mathrm{HB}$ & 1.5048 & 0.05 & 1030 & 108 & VAL & CG2 & 588 & 0.4 \\
\hline 971 & 102 & ALA & $\mathrm{N}$ & 122.755 & 0.4 & 1031 & 108 & VAL & $\mathrm{H}$ & 398 & 0.05 \\
\hline 972 & 103 & ALA & C & 178.77 & 0.4 & 1032 & 108 & VAL & HA & 3.5928 & 0.05 \\
\hline 973 & 103 & ALA & $C A$ & 54.8718 & 0.4 & 1033 & 108 & VAL & HG1 & 1.0148 & 0.05 \\
\hline 974 & 103 & ALA & CB & 18.3008 & 0.4 & 1034 & 108 & VAL & HG2 & 0.4988 & 0.05 \\
\hline 975 & 103 & ALA & $\mathrm{H}$ & 8.3158 & 0.05 & 1035 & 108 & VAL & $\mathrm{N}$ & 119.959 & 0.4 \\
\hline 976 & 103 & ALA & $\mathrm{HA}$ & 4.0298 & 0.05 & 1036 & 108 & VAL & HB & 2.0938 & 0.05 \\
\hline 977 & 103 & ALA & $\mathrm{HB}$ & 98 & 0.05 & 1037 & 109 & MET & C & 437 & 0.4 \\
\hline 978 & 103 & ALA & $\mathrm{N}$ & 118.198 & 0.4 & 1038 & 109 & MET & CA & 908 & 0.4 \\
\hline 979 & 104 & GLU & C & 176.136 & 0.4 & 1039 & 109 & MET & $C B$ & 29.5328 & 0.4 \\
\hline 980 & 104 & GLU & CA & 59.0858 & 0.4 & 1040 & 109 & MET & CE & 16.9208 & 0.4 \\
\hline 981 & 104 & GLU & CB & 28 & 0.4 & 1041 & 109 & MET & CG & 398 & 0.4 \\
\hline 982 & 104 & GLU & CG & 37.9988 & 0.4 & 1042 & 109 & MET & $\mathrm{H}$ & 8.4358 & 0.05 \\
\hline 983 & 104 & GLU & $\mathrm{H}$ & 7.9448 & 0.05 & 1043 & 109 & MET & HA & 4.2358 & 0.05 \\
\hline 984 & 104 & GLU & HA & & 0.05 & 1044 & 109 & MET & $\mathrm{HE}$ & 318 & 0.05 \\
\hline 985 & 104 & GLU & $\mathrm{N}$ & 119. & 0.4 & 1045 & 109 & MET & HG2 & 948 & 0.05 \\
\hline 986 & 104 & GLU & HB2 & 1.8718 & 0.05 & 1046 & 109 & MET & HG3 & 2.3318 & 0.05 \\
\hline 987 & 104 & GLU & HG2 & 2.6128 & 0.05 & 1047 & 109 & MET & $\mathrm{N}$ & 115.295 & 0.4 \\
\hline 988 & 105 & LEU & C & & 0.4 & 1048 & 109 & MET & HB2 & 128 & 0.05 \\
\hline 989 & 105 & LEU & CA & 58 & 0.4 & 1049 & 110 & THR & C & 497 & 0.4 \\
\hline 990 & 105 & LEU & CB & 42.1528 & 0.4 & 1050 & 110 & THR & $C A$ & 66.5808 & 0.4 \\
\hline 991 & 105 & LEU & CD1 & 26.475 & 0.4 & 1051 & 110 & THR & $C B$ & 218 & 0.4 \\
\hline 992 & 105 & LEU & CD2 & & 0.4 & 1052 & 110 & THR & CG2 & 928 & 0.4 \\
\hline 993 & 105 & LEU & CG & 26.6848 & 0.4 & 1044 & 109 & MET & $\mathrm{HE}$ & 1.8318 & 0.05 \\
\hline 994 & 105 & LEU & $\mathrm{H}$ & 8.6268 & 0.05 & 1045 & 109 & MET & HG2 & 2.7948 & 0.05 \\
\hline 995 & 105 & LEU & $\mathrm{HA}$ & 4.1598 & 0.05 & 1046 & 109 & MET & HG3 & 2.3318 & 0.05 \\
\hline 996 & 105 & LEU & HD1 & & 0.05 & 1047 & 109 & MET & $\mathrm{N}$ & 115.295 & 0.4 \\
\hline 997 & 105 & LEU & HD2 & 0.7538 & 0.05 & 1048 & 109 & MET & HB2 & 2.3128 & 0.05 \\
\hline 998 & 105 & LEU & HG & 1.5638 & 0.05 & 1049 & 110 & THR & C & 175.497 & 0.4 \\
\hline 999 & 105 & LEU & $\mathrm{N}$ & 120.144 & 0.4 & 1050 & 110 & THR & $C A$ & 66.5808 & 0.4 \\
\hline 00 & 105 & LEU & HB2 & 1.9418 & 0.05 & 1051 & 110 & THR & CB & 68.5218 & 0.4 \\
\hline 01 & 106 & ARG & C & 175.898 & 0.4 & 1052 & 110 & THR & CG2 & 21.3928 & 0.4 \\
\hline
\end{tabular}




\begin{tabular}{|c|c|c|c|c|c|c|c|c|c|c|c|}
\hline 053 & 110 & THR & $\mathrm{H}$ & 8.1258 & 0.05 & 1113 & 116 & LEU & $\mathrm{CB}$ & 44.1758 & 0.4 \\
\hline 1054 & 110 & THR & HA & 4.0568 & 0.05 & 1114 & 116 & LEU & CD1 & 24.0658 & 0.4 \\
\hline 1055 & 110 & THR & $\mathrm{HB}$ & 4.2578 & 0.05 & 1115 & 116 & LEU & CD2 & 23.7548 & 0.4 \\
\hline 1056 & 110 & THR & HG2 & 1.2748 & 0.05 & 1116 & 116 & LEU & CG & 26.9828 & 0.4 \\
\hline 1057 & 110 & THR & $\mathrm{N}$ & 115.318 & 0.4 & 1117 & 116 & LEU & 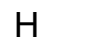 & 8.0528 & 0.05 \\
\hline 1058 & 111 & ASN & CA & 55.8368 & 0.4 & 1118 & 116 & LEU & HA & 4.6688 & 0.05 \\
\hline 1059 & 111 & ASN & CB & 37.9678 & 0.4 & 1119 & 116 & LEU & HB2 & 1.5498 & 0.05 \\
\hline 1060 & 111 & ASN & $\mathrm{H}$ & 7.9988 & 0.05 & 1120 & 116 & LEU & HB3 & 1.3748 & 0.05 \\
\hline 1061 & 111 & ASN & HA & 4.4508 & 0.05 & 1121 & 116 & LEU & HD1 & 0.6908 & 0.05 \\
\hline 1062 & 111 & ASN & HD21 & 7.5168 & 0.05 & 1122 & 116 & LEU & HD2 & 0.6898 & 0.05 \\
\hline 1063 & 111 & ASN & HD22 & 6.7198 & 0.05 & 1123 & 116 & LEU & HG & 0.6798 & 0.05 \\
\hline 1064 & 111 & ASN & $\mathrm{N}$ & 122.63 & 0.4 & 1124 & 116 & LEU & $\mathrm{N}$ & 124.274 & 1 \\
\hline 1065 & 111 & ASN & ND2 & 111.625 & 0.4 & 1125 & 117 & THR & CA & 60.9128 & 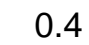 \\
\hline 1066 & 111 & ASN & HB2 & 2.7928 & 0.05 & 1126 & 117 & THR & CB & 70.9388 & 0.4 \\
\hline 1067 & 112 & LEU & C & 174.584 & 0.4 & 1127 & 117 & THR & CG2 & 21.5258 & 0.4 \\
\hline 1068 & 112 & LEU & CA & 54.8318 & 0.4 & 1128 & 117 & THR & $\mathrm{H}$ & 9.1258 & .05 \\
\hline 1069 & 112 & LEU & $\mathrm{CB}$ & 42.5438 & 0.4 & 1129 & 117 & THR & $\mathrm{HA}$ & 4.4238 & .05 \\
\hline 1070 & 112 & LEU & CD1 & 25.9018 & 0.4 & 1130 & 117 & THR & $\mathrm{HB}$ & 4.7248 & 0.05 \\
\hline 1071 & 112 & LEU & CD2 & 22.0388 & 0.4 & 1131 & 117 & THR & HG2 & 1.3388 & 0.05 \\
\hline 1072 & 112 & LEU & CG & 26.2718 & 0.4 & 1132 & 117 & THR & $\mathrm{N}$ & 113.635 & 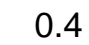 \\
\hline 1073 & 112 & LEU & $\mathrm{H}$ & 7.9058 & 0.05 & 1133 & 118 & ASP & C & 175.917 & 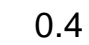 \\
\hline 1074 & 112 & LEU & HA & 4.3308 & 0.05 & 1134 & 118 & ASP & CA & 57.6808 & 0.4 \\
\hline 1075 & 112 & LEU & HB2 & 1.9378 & 0.05 & 1135 & 118 & ASP & CB & 39.5228 & 0.4 \\
\hline 1076 & 112 & LEU & HB3 & 1.6348 & 0.05 & 1136 & 118 & ASP & $\mathrm{H}$ & 8.9128 & .05 \\
\hline 1077 & 112 & LEU & HD1 & 0.8158 & 0.05 & 1137 & 118 & ASP & $\mathrm{HA}$ & 4.2238 & .05 \\
\hline 1078 & 112 & LEU & HD2 & 0.7718 & 0.05 & 1138 & 118 & ASP & HB2 & 3.0378 & 0.05 \\
\hline 1079 & 112 & LEU & HG & 1.6608 & 0.05 & 1139 & 118 & ASP & HB3 & 2.6168 & 0.05 \\
\hline 1080 & 112 & LEU & $\mathrm{N}$ & 117.455 & 0.4 & 1140 & 118 & ASP & $\mathrm{N}$ & 120.87 & 0.4 \\
\hline 1081 & 113 & GLY & C & 171.823 & 0.4 & 1141 & 119 & GLU & C & 176.438 & 4 \\
\hline 1082 & 113 & GLY & CA & 45.5118 & 0.4 & 1142 & 119 & GLU & $C A$ & 9.7978 & 0.4 \\
\hline 1083 & 113 & GLY & $\mathrm{H}$ & 7.9138 & 0.05 & 1143 & 119 & GLU & CB & 28.7848 & 0.4 \\
\hline 1084 & 113 & GLY & HA2 & 4.1398 & 0.05 & 1144 & 119 & GLU & CG & 35.8818 & 0.4 \\
\hline 1085 & 113 & GLY & HA3 & 3.7798 & 0.05 & 1145 & 119 & GLU & $\mathrm{H}$ & 8.6268 & 5 \\
\hline 1086 & 113 & GLY & $\mathrm{N}$ & 107.043 & 0.4 & 1146 & 119 & GLU & HA & 4.1258 & 0.05 \\
\hline 1087 & 114 & GLU & C & 172.814 & 0.4 & 1147 & 119 & GLU & HB2 & 2.3388 & 0.05 \\
\hline 1088 & 114 & GLU & CA & 55.1648 & 0.4 & 1148 & 119 & GLU & HB3 & 2.0548 & 0.05 \\
\hline 1089 & 114 & GLU & CB & 30.5298 & 0.4 & 1149 & 119 & GLU & $\mathrm{N}$ & 118.815 & 0.4 \\
\hline 1090 & 114 & GLU & CG & 35.2058 & 0.4 & 1150 & 119 & GLU & HG2 & 2.3498 & 0.05 \\
\hline 1091 & 114 & GLU & $\mathrm{H}$ & 7.8588 & 0.05 & 1151 & 120 & GLU & C & 176.259 & 0.4 \\
\hline 1092 & 114 & GLU & HA & 4.3238 & 0.05 & 1152 & 120 & GLU & CA & .5158 & 0.4 \\
\hline 1093 & 114 & GLU & HG2 & 2.1 & 0.05 & 1153 & 120 & GLU & CB & 08 & 0.4 \\
\hline 1094 & 114 & GLU & HG3 & 1.9848 & 0.05 & 1154 & 120 & GLU & CG & 38.2848 & 0.4 \\
\hline 1095 & 114 & GLU & $\mathrm{N}$ & 119.028 & 0.4 & 1155 & 120 & GLU & $\mathrm{H}$ & 7.7748 & 0.05 \\
\hline 1096 & 114 & GLU & HB2 & 1.7328 & 0.05 & 1156 & 120 & GLU & $\mathrm{HA}$ & 9208 & 0.05 \\
\hline 1097 & 115 & LYS & C & 172. & 0.4 & 1157 & 120 & GLU & $\mathrm{N}$ & 120.193 & 0.4 \\
\hline 1098 & 115 & LYS & CA & 55.2348 & 0.4 & 1158 & 120 & GLU & HB2 & 2.1208 & 0.05 \\
\hline 1099 & 115 & LYS & CB & 31.7618 & 0.4 & 1159 & 120 & GLU & HG2 & 2.3668 & 0.05 \\
\hline 1100 & 115 & LYS & $C D$ & 28.8418 & 0.4 & 1160 & 121 & VAL & C & 5.276 & 0.4 \\
\hline 1101 & 115 & LYS & CE & 41.4828 & 0.4 & 1161 & 121 & VAL & CA & 66.8298 & 0.4 \\
\hline 1102 & 115 & LYS & CG & 24.3778 & 0.4 & 1162 & 121 & VAL & CB & 31.1878 & 0.4 \\
\hline 1103 & 115 & LYS & $\mathrm{H}$ & 8.6538 & 0.05 & 1163 & 121 & VAL & CG1 & 23.3228 & 0.4 \\
\hline 110 & 115 & LYS & HA & 4.3778 & 0.05 & 1164 & 121 & VAL & CG2 & 21.8348 & 0.4 \\
\hline 110 & 115 & LYS & HB2 & 1.7448 & 0.05 & 1165 & 121 & VAL & $\mathrm{H}$ & 8.0268 & 0.05 \\
\hline 1106 & 115 & LYS & HB3 & 1.6408 & 0.05 & 1166 & 121 & VAL & $\mathrm{HA}$ & 3.5098 & 0.05 \\
\hline 1107 & 115 & LYS & $\mathrm{N}$ & 123.982 & 0.4 & 1167 & 121 & VAL & HB & 2.1848 & 0.05 \\
\hline 1108 & 115 & LYS & HD & 1.6378 & 0.05 & 1168 & 121 & VAL & HG & 0.8808 & 0.05 \\
\hline 1109 & 115 & LYS & HE2 & 2.9508 & 0.05 & 1169 & 121 & VAL & HG2 & 0.9418 & 0.05 \\
\hline 1110 & 115 & LYS & HG2 & 1.2958 & 0.05 & 1170 & 121 & VAL & $\mathrm{N}$ & 121.619 & 0.4 \\
\hline 1111 & 116 & LEU & C & 175.378 & 0.4 & 1171 & 122 & ASP & C & 176.327 & 0.4 \\
\hline 1112 & 116 & LEU & $C A$ & 54.1328 & 0.4 & 1172 & 122 & ASP & $C A$ & 57.5188 & 0.4 \\
\hline
\end{tabular}




\begin{tabular}{|c|c|c|c|c|c|c|c|c|c|c|c|}
\hline 173 & 122 & ASP & $\mathrm{CB}$ & 40.0848 & 0.4 & 1233 & 128 & ALA & C & 788 & 0.4 \\
\hline 1174 & 122 & ASP & $\mathrm{H}$ & 8.1848 & 0.05 & 1234 & 128 & ALA & CA & 51.6648 & 0.4 \\
\hline 1175 & 122 & ASP & $\mathrm{HA}$ & 4.3648 & 0.05 & 1235 & 128 & ALA & CB & 20.8668 & 0.4 \\
\hline 1176 & 122 & ASP & HB2 & 2.7908 & 0.05 & 1236 & 128 & ALA & $\mathrm{H}$ & 7.2248 & 0.05 \\
\hline 1177 & 122 & ASP & HB3 & 2.6998 & 0.05 & 1237 & 128 & ALA & HA & 4.3508 & 0.05 \\
\hline 1178 & 122 & ASP & $\mathrm{N}$ & 119.587 & 0.4 & 1238 & 128 & ALA & HB & 1.2118 & 05 \\
\hline 1179 & 123 & GLU & C & 175.641 & 0.4 & 1239 & 128 & ALA & $\mathrm{N}$ & 116.627 & 0.4 \\
\hline 1180 & 123 & GLU & CA & 59.3828 & 0.4 & 1240 & 129 & ASN & C & 172.792 & 0.4 \\
\hline 1181 & 123 & GLU & CB & 30.1128 & 0.4 & 1241 & 129 & ASN & $C A$ & 54.3598 & 0.4 \\
\hline 1182 & 123 & GLU & CG & 36.4218 & 0.4 & 1242 & 129 & ASN & CB & 40.3068 & 4 \\
\hline 1183 & 123 & GLU & $\mathrm{H}$ & 8.1898 & 0.05 & 1243 & 129 & ASN & $\mathrm{H}$ & 7.8978 & 0.05 \\
\hline 1184 & 123 & GLU & $\mathrm{HA}$ & 3.9668 & 0.05 & 1244 & 129 & ASN & $\mathrm{HA}$ & 4.4838 & 0.05 \\
\hline 1185 & 123 & GLU & $\mathrm{N}$ & 119.238 & 0.4 & 1245 & 129 & ASN & HB2 & 2.8958 & .05 \\
\hline 1186 & 123 & GLU & HB2 & 2.2938 & 0.05 & 1246 & 129 & ASN & HB3 & 2.5088 & 05 \\
\hline 1187 & 123 & GLU & HG2 & 2.4258 & 0.05 & 1247 & 129 & ASN & $\mathrm{N}$ & 117.321 & 0.4 \\
\hline 1188 & 124 & MET & C & 177.098 & 0.4 & 1248 & 130 & ILE & C & 175.157 & 0.4 \\
\hline 1189 & 124 & MET & CA & 60.6858 & 0.4 & 1249 & 130 & ILE & CA & 63.2378 & \\
\hline 1190 & 124 & MET & CB & 29.557 & 0.4 & 1250 & 130 & ILE & CB & 38.5768 & 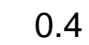 \\
\hline 1191 & 124 & MET & CG & 33.3538 & 0.4 & 1251 & 130 & ILE & CD1 & 12.3058 & 0.4 \\
\hline 1192 & 124 & MET & CE & 14.9908 & 0.4 & 1252 & 130 & ILE & CG1 & 27.6178 & 0.4 \\
\hline 1193 & 124 & MET & $\mathrm{H}$ & 7.7708 & 0.05 & 1253 & 130 & ILE & CG2 & 17.2438 & 04 \\
\hline 1194 & 124 & MET & $\mathrm{HA}$ & 3.9128 & 0.05 & 1254 & 130 & ILE & $\mathrm{H}$ & 8.2758 & 0.05 \\
\hline 1195 & 124 & MET & $\mathrm{HE}$ & 0.6608 & 0.05 & 1255 & 130 & ILE & HA & 3.8998 & 0.05 \\
\hline 1196 & 124 & MET & $\mathrm{N}$ & 118.205 & 0.4 & 1256 & 130 & ILE & HB & 1.9548 & 0.05 \\
\hline 1197 & 124 & MET & HB2 & 1.8458 & 0.05 & 1257 & 130 & ILE & HD1 & 0.8608 & .05 \\
\hline 1198 & 124 & MET & HG2 & 2.7888 & 0.05 & 1258 & 130 & ILE & HG12 & 1.6528 & 5 \\
\hline 1199 & 125 & ILE & CA & 63.0148 & 0.4 & 1259 & 130 & ILE & HG13 & 1.2608 & 0.05 \\
\hline 1200 & 125 & ILE & CB & 35.5488 & 0.4 & 1260 & 130 & ILE & HG2 & 0.9198 & 0.05 \\
\hline 1201 & 125 & ILE & CD1 & 9.4738 & 0.4 & 1261 & 130 & ILE & $\mathrm{N}$ & 127.388 & 0.4 \\
\hline 1202 & 125 & ILE & CG1 & 26.9728 & 0.4 & 1262 & 131 & ASP & C & 175.047 & 4 \\
\hline 1203 & 125 & ILE & CG2 & 16.4428 & 0.4 & 1263 & 131 & ASP & CA & 4.0058 & 0.4 \\
\hline 1204 & 125 & ILE & $\mathrm{H}$ & 7.8768 & 0.05 & 1264 & 131 & ASP & CB & 40.0758 & 0.4 \\
\hline 1205 & 125 & ILE & $\mathrm{HA}$ & 3.8498 & 0.05 & 1265 & 131 & ASP & $\mathrm{H}$ & 8.4118 & 0.05 \\
\hline 1206 & 125 & ILE & $\mathrm{HB}$ & 2.3988 & 0.05 & 1266 & 131 & ASP & HA & 4.5008 & 05 \\
\hline 1207 & 125 & ILE & HD1 & 0.8248 & 0.05 & 1267 & 131 & ASP & HB2 & 3.0488 & 0.05 \\
\hline 1208 & 125 & ILE & HG12 & 1.7318 & 0.05 & 1268 & 131 & ASP & HB3 & 2.6358 & 0.05 \\
\hline 1209 & 125 & ILE & HG13 & 1.4938 & 0.05 & 1269 & 131 & ASP & $\mathrm{N}$ & 116.226 & 0.4 \\
\hline 1210 & 125 & ILE & HG2 & 0.8108 & 0.05 & 1270 & 132 & GLY & C & 172.439 & 0.4 \\
\hline 1211 & 125 & ILE & $\mathrm{N}$ & 119.224 & 0.4 & 1271 & 132 & GLY & CA & 47.2278 & 0.4 \\
\hline 1212 & 126 & ARG & C & 176.484 & 0.4 & 1272 & 132 & GLY & $\mathrm{H}$ & 7.6768 & 0.05 \\
\hline 1213 & 126 & ARG & CA & 59.5788 & 0.4 & 1273 & 132 & GLY & HA2 & 3.9908 & 0.05 \\
\hline 1214 & 126 & ARG & CB & & 0.4 & 1274 & 132 & GLY & HA3 & 08 & 0.05 \\
\hline 1215 & 126 & ARG & $C D$ & 43.4668 & 0.4 & 1275 & 132 & GLY & $\mathrm{N}$ & 108.352 & 0.4 \\
\hline 1216 & 126 & ARG & CG & 27.9058 & 0.4 & 1276 & 133 & ASP & C & 174.74 & 0.4 \\
\hline 1217 & 126 & ARG & $\mathrm{H}$ & 8.5 & 0.05 & 1277 & 133 & ASP & $C A$ & 53.9868 & 0.4 \\
\hline 1218 & 126 & ARG & $\mathrm{HA}$ & 4.0 & 0.05 & 1278 & 133 & ASP & $C B$ & 39. & 0.4 \\
\hline 1219 & 126 & ARG & HG2 & 1.8238 & 0.05 & 1279 & 133 & ASP & $\mathrm{H}$ & 8.4278 & 0.05 \\
\hline 1220 & 126 & ARG & HG3 & 1.6708 & 0.05 & 1280 & 133 & ASP & HA & 4.4988 & 0.05 \\
\hline 1221 & 126 & ARG & $\mathrm{N}$ & 117.057 & 0.4 & 1281 & 133 & ASP & HB2 & 2.9688 & 0.05 \\
\hline 1222 & 126 & ARG & HB2 & 1.8898 & 0.05 & 1282 & 133 & ASP & HB3 & 2.5268 & 0.05 \\
\hline 1223 & 126 & ARG & HD2 & 3.2478 & 0.05 & 1283 & 133 & ASP & $\mathrm{N}$ & 120.564 & 0.4 \\
\hline 1224 & 127 & GLU & C & 174.423 & 0.4 & 1284 & 134 & GLY & C & 169.86 & 0.4 \\
\hline 1225 & 127 & GLU & CA & & 0.4 & 1285 & 134 & GLY & $C A$ & 5.5158 & 0.4 \\
\hline 1226 & 127 & GLU & CB & 30.1128 & 0.4 & 1286 & 134 & GLY & $\mathrm{H}$ & 10.3178 & 0.05 \\
\hline 1227 & 127 & GLU & CG & 36.6448 & 0.4 & 1287 & 134 & GLY & HA2 & 4.0688 & 0.05 \\
\hline 1228 & 127 & GLU & $\mathrm{H}$ & 8.2808 & 0.05 & 1288 & 134 & GLY & HA3 & 3.4518 & 0.05 \\
\hline 1229 & 127 & GLU & $\mathrm{HA}$ & 4.0608 & 0.05 & 1289 & 134 & GLY & $\mathrm{N}$ & 112.342 & 0 \\
\hline 1230 & 127 & GLU & $\mathrm{N}$ & 115.021 & 0.4 & 1290 & 135 & GLN & C & 172.149 & $0_{1}$ \\
\hline 1231 & 127 & GLU & HB2 & 2.3078 & 0.05 & 1291 & 135 & GLN & CA & 53.2138 & 0.4 \\
\hline 1232 & 127 & GLU & HG2 & 2.4958 & 0.05 & 1292 & 135 & GLN & CB & 32.3578 & 1 \\
\hline
\end{tabular}




\begin{tabular}{|c|c|c|c|c|c|c|c|c|c|c|c|}
\hline 293 & 135 & GLN & CG & 33.0208 & 0.4 & 1353 & 140 & GLU & CG & 508 & 0.4 \\
\hline 1294 & 135 & GLN & $\mathrm{H}$ & 7.9788 & 0.05 & 1354 & 140 & GLU & $\mathrm{H}$ & 8.7048 & 0.05 \\
\hline 1295 & 135 & GLN & $\mathrm{HA}$ & 4.9768 & 0.05 & 1355 & 140 & GLU & $\mathrm{HA}$ & 3.8288 & 0.05 \\
\hline 1296 & 135 & GLN & HE21 & 6.5038 & 0.05 & 1356 & 140 & GLU & HB2 & 2.1588 & 0.05 \\
\hline 1297 & 135 & GLN & HE22 & 5.9758 & 0.05 & 1357 & 140 & GLU & HB3 & 1.9918 & 0.05 \\
\hline 1298 & 135 & GLN & HG2 & 2.0118 & 0.05 & 1358 & 140 & GLU & HG2 & 2.8448 & 0.05 \\
\hline 1299 & 135 & GLN & HG3 & 1.6658 & 0.05 & 1359 & 140 & GLU & HG3 & 2.3648 & 0.05 \\
\hline 1300 & 135 & GLN & $\mathrm{N}$ & 114.483 & 0.4 & 1360 & 140 & GLU & $\mathrm{N}$ & 119.391 & 0.4 \\
\hline 1301 & 135 & GLN & NE2 & 108.133 & 0.4 & 1361 & 141 & PHE & C & 173.478 & 0.4 \\
\hline 302 & 135 & GLN & HB2 & 1.7268 & 0.05 & 1362 & 141 & PHE & CA & 1.5828 & 04 \\
\hline 303 & 136 & VAL & C & 172.968 & 0.4 & 1363 & 141 & PHE & CB & 39.8728 & 0.4 \\
\hline 1304 & 136 & VAL & CA & 61.6478 & 0.4 & 1364 & 141 & PHE & CD1 & 129.982 & 0.4 \\
\hline 305 & 136 & VAL & CB & 33.494 & 0.4 & 1365 & 141 & PHE & CD2 & 129.982 & 0.4 \\
\hline 306 & 136 & VAL & CG1 & 23.0538 & 0.4 & 1366 & 141 & PHE & CE1 & 129.459 & 01 \\
\hline 307 & 136 & VAL & CG2 & 21.0718 & 0.4 & 1367 & 141 & PHE & CE2 & 129.466 & 0.4 \\
\hline 1308 & 136 & VAL & $\mathrm{H}$ & 9.1448 & 0.05 & 1368 & 141 & PHE & $\mathrm{H}$ & 8.4738 & 0.05 \\
\hline 1309 & 136 & VAL & $\mathrm{HA}$ & 5.2068 & 0.05 & 1369 & 141 & PHE & $\mathrm{HA}$ & 3.3988 & 0.05 \\
\hline 310 & 136 & VAL & $\mathrm{HB}$ & 2.3778 & 0.05 & 1370 & 141 & PHE & HB2 & 3.2698 & 0.05 \\
\hline 1311 & 136 & VAL & HG1 & 1.1408 & 0.05 & 1371 & 141 & PHE & HB3 & 2.8878 & 0.05 \\
\hline 1312 & 136 & VAL & HG2 & 1.0668 & 0.05 & 1372 & 141 & PHE & $\mathrm{HZ}$ & 8.5208 & 0.05 \\
\hline 313 & 136 & VAL & $\mathrm{N}$ & 124.979 & 0.4 & 1373 & 141 & PHE & $\mathrm{N}$ & 123.444 & 0.4 \\
\hline 314 & 137 & ASN & C & 171.857 & 0.4 & 1374 & 141 & PHE & HD1 & 6.6488 & 0.05 \\
\hline 1315 & 137 & ASN & CA & 50.7558 & 0.4 & 1375 & 141 & PHE & HD2 & 6.6488 & 0.05 \\
\hline 1316 & 137 & ASN & CB & 38.3368 & 0.4 & 1376 & 141 & PHE & HE1 & 7.0578 & 0.05 \\
\hline 317 & 137 & ASN & $\mathrm{H}$ & 9.6288 & 0.05 & 1377 & 141 & PHE & HE2 & 7.0578 & 0.05 \\
\hline 1318 & 137 & ASN & $\mathrm{HA}$ & 5.3468 & 0.05 & 1378 & 142 & VAL & C & 176.735 & 0.4 \\
\hline 1319 & 137 & ASN & HD21 & 7.5038 & 0.05 & 1379 & 142 & VAL & $C A$ & 66.8108 & 0.4 \\
\hline 1320 & 137 & ASN & HD22 & 7.3288 & 0.05 & 1380 & 142 & VAL & $C B$ & 188 & 0.4 \\
\hline 1321 & 137 & ASN & $\mathrm{N}$ & 128.916 & 0.4 & 1381 & 142 & VAL & CG1 & 21.1198 & 0.4 \\
\hline 1322 & 137 & ASN & ND2 & 111.858 & 0.4 & 1382 & 142 & VAL & CG2 & 22.8868 & 0.4 \\
\hline 1323 & 137 & ASN & HB2 & 3.1808 & 0.05 & 1383 & 142 & VAL & $\mathrm{H}$ & 8.6118 & 0.05 \\
\hline 1324 & 138 & TYR & C & 173.214 & 0.4 & 1384 & 142 & VAL & $\mathrm{HA}$ & 2.9608 & 0.05 \\
\hline 1325 & 138 & TYR & CA & 62.0288 & 0.4 & 1385 & 142 & VAL & HB & 1.7098 & 0.05 \\
\hline 1326 & 138 & TYR & CB & 37.5368 & 0.4 & 1386 & 142 & VAL & HG1 & 598 & 0.05 \\
\hline 1327 & 138 & TYR & CD1 & 17 & 0.4 & 1387 & 142 & VAL & HG2 & 0.4038 & 0.05 \\
\hline 1328 & 138 & TYR & CD2 & 130.347 & 0.4 & 1388 & 142 & VAL & $\mathrm{N}$ & 118.366 & 0.4 \\
\hline 1329 & 138 & TYR & CE1 & 116.007 & 0.4 & 1389 & 143 & GLN & $C A$ & 58.7918 & 0.4 \\
\hline 1330 & 138 & TYR & CE2 & 116. & 0.4 & 1390 & 143 & GLN & $C B$ & 27. & 0.4 \\
\hline 1331 & 138 & TYR & $\mathrm{H}$ & 8.3 & 0.05 & 1391 & 143 & GLN & CG & 33.7228 & 0.4 \\
\hline 1332 & 138 & TYR & $\mathrm{HA}$ & 3.2258 & 0.05 & 1392 & 143 & GLN & $\mathrm{H}$ & 7.8028 & 0.05 \\
\hline 1333 & 138 & TYR & HB2 & 2.3428 & 0.05 & 1393 & 143 & GLN & $\mathrm{HA}$ & 3.7 & 0.05 \\
\hline 1334 & 138 & TYR & HB3 & & 0.05 & 1394 & 143 & GLN & $\mathrm{HF}$ & & 0.05 \\
\hline 1335 & 138 & TYR & $\mathrm{N}$ & 118.187 & 0.4 & 1395 & 143 & GLN & HE2 & 6.7088 & 0.05 \\
\hline 1336 & 138 & TYR & HD1 & 6.2598 & 0.05 & 1396 & 143 & GLN & $\mathrm{N}$ & 119.002 & 0.4 \\
\hline 1337 & 138 & TYR & HD2 & 6.2 & 0.05 & 1397 & 143 & GLN & NE2 & 111.126 & 0.4 \\
\hline & 138 & TYR & HE1 & & 0.05 & & 143 & GLN & HB2 & 18 & 0.05 \\
\hline 1339 & 138 & TYR & HE2 & 6.4808 & 0.05 & 1399 & 143 & GLN & HG2 & 2.3288 & 0.05 \\
\hline 1340 & 139 & GLU & C & 177.373 & 0.4 & 1400 & 144 & MET & C & 173.938 & 0.4 \\
\hline 1341 & 139 & GLU & CA & 59.7 & 0.4 & 1401 & 144 & MET & $\mathrm{CA}$ & 56.2 & 0.4 \\
\hline 134 & 139 & GLU & CB & 29. & 0.4 & 1402 & 144 & MET & CB & 69 & 0.4 \\
\hline 1343 & 139 & GLU & CG & 35.8908 & 0.4 & 1403 & 144 & MET & CE & 16.0918 & 0.4 \\
\hline 1344 & 139 & GLU & $\mathrm{H}$ & 8.0338 & 0.05 & 1404 & 144 & MET & CG & 30.6068 & 0.4 \\
\hline 1345 & 139 & GLU & $\mathrm{HA}$ & 3.5918 & 0.05 & 1405 & 144 & MET & $\mathrm{H}$ & 7.3888 & 0.05 \\
\hline & 139 & GLU & HG2 & 2.3258 & 0.05 & 1406 & 144 & MET & $\mathrm{H}$ & 4.1898 & 0.05 \\
\hline 1347 & 139 & GLU & HG3 & 2.2958 & 0.05 & 1407 & 144 & MET & $\mathrm{HE}$ & 1.6808 & 0.05 \\
\hline 1348 & 139 & GLU & $\mathrm{N}$ & 117.957 & 0.4 & 1408 & 144 & MET & $\mathrm{N}$ & 116.678 & 0.4 \\
\hline 134 & 139 & GLU & $\mathrm{HB}$ & 2.0058 & 0.05 & 1409 & 144 & MET & $\mathrm{HE}$ & 1.4298 & 0.05 \\
\hline 1350 & 140 & GLU & C & 176.476 & 0.4 & 1410 & 144 & MET & HG2 & 1.6998 & 0.05 \\
\hline 1351 & 140 & GLU & CA & 58.5078 & 0.4 & 1411 & 145 & MET & C & 174.791 & 0.4 \\
\hline 1352 & 140 & GLU & CB & 28.8998 & 0.4 & 1412 & 145 & MET & CA & 55.0798 & 0.4 \\
\hline
\end{tabular}




\begin{tabular}{|c|c|c|c|c|c|c|c|c|c|c|c|}
\hline 1413 & 145 & MET & $\mathrm{CB}$ & 30.904 & 0.4 & 1433 & 147 & ALA & C & 174.107 & 0.4 \\
\hline 1414 & 145 & MET & CE & 18.1958 & 0.4 & 1434 & 147 & ALA & CA & 52.4268 & 0.4 \\
\hline 1415 & 145 & MET & CG & 32.2298 & 0.4 & 1435 & 147 & ALA & CB & 19.0508 & 0.4 \\
\hline 1416 & 145 & MET & $\mathrm{H}$ & 7.3048 & 0.05 & 1436 & 147 & ALA & $\mathrm{H}$ & 7.8608 & 0.05 \\
\hline 1417 & 145 & MET & $\mathrm{HA}$ & 4.0238 & 0.05 & 1437 & 147 & ALA & $\mathrm{HA}$ & 4.3328 & 0.05 \\
\hline 1418 & 145 & MET & HB2 & 1.4938 & 0.05 & 1438 & 147 & ALA & $\mathrm{HB}$ & 1.3908 & 0.05 \\
\hline 1419 & 145 & MET & HB3 & 1.1088 & 0.05 & 1439 & 147 & ALA & $\mathrm{N}$ & 126.675 & 0.4 \\
\hline 1420 & 145 & MET & $\mathrm{HE}$ & 1.1438 & 0.05 & 1440 & 148 & LYS & CA & 57.6978 & 0.4 \\
\hline 1421 & 145 & MET & HG2 & 1.4608 & 0.05 & 1441 & 148 & LYS & CB & 33.241 & 0.4 \\
\hline 1422 & 145 & MET & HG3 & 1.3398 & 0.05 & 1442 & 148 & LYS & $C D$ & 28.8568 & 0.4 \\
\hline 1423 & 145 & MET & $\mathrm{N}$ & 112.325 & 0.4 & 1443 & 148 & LYS & CE & 41.6988 & 0.4 \\
\hline 1424 & 146 & THR & C & 171.522 & 0.4 & 1444 & 148 & LYS & CG & 24.7878 & 0.4 \\
\hline 1425 & 146 & THR & CA & 61.8998 & 0.4 & 1445 & 148 & LYS & $\mathrm{H}$ & 8.0078 & 0.05 \\
\hline 1426 & 146 & THR & CB & 70.5428 & 0.4 & 1446 & 148 & LYS & $\mathrm{HA}$ & 4.0798 & 0.05 \\
\hline 1427 & 146 & THR & CG2 & 21.1358 & 0.4 & 1447 & 148 & LYS & HB2 & 1.7998 & 0.05 \\
\hline 1428 & 146 & THR & $\mathrm{H}$ & 7.4728 & 0.05 & 1448 & 148 & LYS & HB3 & 1.7308 & 0.05 \\
\hline 1429 & 146 & THR & $\mathrm{HA}$ & 4.2518 & 0.05 & 1449 & 148 & LYS & $\mathrm{N}$ & 126.4 & 0.4 \\
\hline 1430 & 146 & THR & $\mathrm{HB}$ & 4.1468 & 0.05 & 1450 & 148 & LYS & HD2 & 1.6478 & 0.05 \\
\hline 1431 & 146 & THR & HG2 & 1.0658 & 0.05 & 1451 & 148 & LYS & HE2 & 2.9868 & 0.05 \\
\hline 1432 & 146 & THR & $\mathrm{N}$ & 108.155 & 0.4 & 1452 & 148 & LYS & HG2 & 1.3938 & 0.05 \\
\hline
\end{tabular}

\begin{tabular}{|c|c|c|c|c|c|c|c|c|c|c|c|}
\hline \multicolumn{12}{|c|}{ Munc13-1(457-492) } \\
\hline Atom & Seq & $\begin{array}{l}\text { Res } \\
\text { Type }\end{array}$ & $\begin{array}{l}\text { Atom } \\
\text { ID }\end{array}$ & $\begin{array}{r}\text { Chem } \\
\text { shift }\end{array}$ & error & Atom & Seq & $\begin{array}{l}\text { Res } \\
\text { Type }\end{array}$ & $\begin{array}{l}\text { Atom } \\
\text { ID }\end{array}$ & $\begin{array}{l}\text { Chem } \\
\text { shift }\end{array}$ & erro \\
\hline 1 & 458 & SER & CA & 55.709 & 0.5 & 38 & 463 & ASN & CA & 53.033 & 0.5 \\
\hline 2 & 458 & SER & $\mathrm{CB}$ & 61.137 & 0.5 & 39 & 463 & ASN & CB & 35.5 & 0.5 \\
\hline 3 & 458 & SER & C & 175.368 & 0.5 & 40 & 463 & ASN & $\mathrm{H}$ & 8.372 & 0.05 \\
\hline 4 & 458 & SER & $\mathrm{H}$ & 8.193 & 0.05 & 41 & 463 & ASN & $\mathrm{HA}$ & 4.459 & 0.05 \\
\hline 5 & 458 & SER & HB2 & 3.879 & 0.05 & 42 & 463 & ASN & HB2 & 2.858 & 0.05 \\
\hline 6 & 458 & SER & $\mathrm{N}$ & 120.637 & 0.5 & 43 & 463 & ASN & HB3 & 2.683 & 0.0 \\
\hline 7 & 459 & ARG & C & 173.718 & 0.5 & 44 & 463 & ASN & $\mathrm{N}$ & 118.243 & 0 \\
\hline 8 & 459 & ARG & CA & 55.993 & 0.5 & 45 & 464 & TRP & C & 72.698 & 0.5 \\
\hline 9 & 459 & ARG & $\mathrm{CB}$ & 27.411 & 0.5 & 46 & 464 & TRP & $\mathrm{CA}$ & 58.19 & 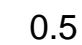 \\
\hline 10 & 459 & ARG & CG & 23.675 & 0.5 & 47 & 464 & TRP & $\mathrm{CB}$ & 26.538 & \\
\hline 11 & 459 & ARG & $\mathrm{H}$ & 8.654 & 0.05 & 48 & 464 & TRP & CD1 & 128.771 & \\
\hline 12 & 459 & ARG & HA & 3.838 & 0.05 & 49 & 464 & TRP & $\mathrm{H}$ & 8.533 & 0.0 \\
\hline 13 & 459 & ARG & HG2 & 1.546 & 0.05 & 50 & 464 & TRP & $\mathrm{HA}$ & 4.203 & 0.0 \\
\hline 14 & 459 & ARG & $\mathrm{N}$ & 125.365 & 0.5 & 51 & 464 & TRP & HB2 & 3.382 & 0.0 \\
\hline 15 & 460 & ALA & C & 171.624 & 0.5 & 52 & 464 & TRP & $\mathrm{H}$ & 2.867 & 0.0 \\
\hline 16 & 460 & ALA & CA & 52.358 & 0.5 & 53 & 464 & TRP & HD1 & 7.313 & 0.0 \\
\hline 17 & 460 & ALA & CB & 15.958 & 0.5 & 54 & 464 & TRP & HE1 & 10.381 & 0.0 \\
\hline 18 & 460 & ALA & $\mathrm{H}$ & 8.193 & 0.05 & 55 & 464 & TRP & HZ2 & 7.128 & 0.0 \\
\hline 19 & 460 & ALA & HA & 4.164 & 0.05 & 56 & 464 & TRP & $\mathrm{HH} 2$ & 6.987 & 0.0 \\
\hline 20 & 460 & ALA & $\mathrm{HB}$ & 1.353 & 0.05 & 57 & 464 & TRP & $\mathrm{N}$ & 121.805 & 0 \\
\hline 21 & 460 & ALA & $\mathrm{N}$ & 120.862 & 0.5 & 58 & 464 & TRP & NE1 & 129.285 & 0 \\
\hline 22 & 461 & LYS & C & 173.138 & 0.5 & 59 & 465 & LEU & C & 171.618 & 0 \\
\hline 23 & 461 & LYS & CA & 56.959 & 0.5 & 60 & 465 & LEU & $\mathrm{C}$ & 55. & 0. \\
\hline 24 & 461 & LYS & CB & 28.819 & 0.5 & 61 & 465 & LEU & $\mathrm{CB}$ & 38.641 & 0 \\
\hline 25 & 461 & LYS & $C D$ & 26.742 & 0.5 & 62 & 465 & LEU & CD1 & 22.725 & 0 \\
\hline 26 & 461 & LYS & $\mathrm{H}$ & 7.9 & 0.05 & 63 & 465 & LEU & CG & 23.208 & 0 \\
\hline 27 & 461 & LYS & HA & 4.185 & 0.05 & 64 & 465 & LEU & $\mathrm{H}$ & 8.264 & 0.0 \\
\hline 28 & 461 & LYS & HB2 & 1.895 & 0.05 & 65 & 465 & LEU & $\mathrm{H}$ & 4.172 & 0.0 \\
\hline 29 & 461 & LYS & HB3 & 1.863 & 0.05 & 66 & 465 & LEU & HB2 & 1.841 & 0.0 \\
\hline 30 & 461 & LYS & $\mathrm{N}$ & 118.064 & 0.5 & 67 & 465 & LEU & HB3 & 1.6 & 0.0 \\
\hline 31 & 462 & ALA & $\mathrm{CA}$ & 52.15 & 0.5 & 68 & 465 & LEU & HD1 & 0.881 & 0.0 \\
\hline 32 & 462 & ALA & CB & 14.865 & 0.5 & 69 & 465 & LEU & HD2 & 0.886 & 0.0 \\
\hline 33 & 462 & ALA & $\mathrm{H}$ & 7.778 & 0.05 & 70 & 465 & LEU & $H G$ & 1.785 & 0.0 \\
\hline 34 & 462 & ALA & HA & 4.06 & 0.05 & 71 & 465 & LEU & $\mathrm{N}$ & 118.722 & \\
\hline 35 & 462 & ALA & HB & 1.444 & 0.05 & 72 & 466 & ARG & C & 172.228 & 0 \\
\hline 36 & 462 & ALA & $\mathrm{N}$ & 20.253 & 0.5 & 73 & 466 & ARG & $\mathrm{CA}$ & 56.888 & 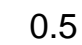 \\
\hline 37 & 463 & SN & C & 3.138 & 0.5 & 74 & 466 & ARG & CB & 27.368 & \\
\hline
\end{tabular}




\begin{tabular}{|c|c|c|c|c|c|c|c|c|c|c|c|}
\hline 75 & 466 & ARG & $\mathrm{H}$ & 8.264 & 0.05 & 137 & 472 & ARG & CA & 56.805 & \\
\hline 76 & 466 & ARG & $\mathrm{HA}$ & 4.128 & 0.05 & 138 & 472 & ARG & CB & 26.506 & \\
\hline 77 & 466 & ARG & HG2 & 0.882 & 0.05 & 139 & 472 & ARG & $C D$ & 40.184 & \\
\hline 78 & 466 & ARG & $\mathrm{N}$ & 120.684 & 0.5 & 140 & 472 & ARG & CG & 22.439 & \\
\hline 79 & 466 & ARG & HB2 & 1.927 & 0.05 & 141 & 472 & ARG & $\mathrm{H}$ & 8.639 & \\
\hline 80 & 466 & ARG & HD2 & 3.251 & 0.05 & 142 & 472 & ARG & $\mathrm{HA}$ & 3.763 & \\
\hline 81 & 467 & ALA & C & 172.178 & 0.5 & 143 & 472 & ARG & HD2 & 3.333 & \\
\hline 82 & 467 & ALA & CA & 52.558 & 0.5 & 144 & 472 & ARG & HD3 & 2.98 & \\
\hline 83 & 467 & ALA & CB & 16.041 & 0.5 & 145 & 472 & ARG & HG2 & 1.335 & \\
\hline 84 & 467 & ALA & $\mathrm{H}$ & 8.067 & 0.05 & 146 & 472 & ARG & HG3 & 1.207 & \\
\hline 85 & 467 & ALA & $\mathrm{HA}$ & 3.978 & 0.05 & 147 & 472 & ARG & $\mathrm{N}$ & 119.209 & \\
\hline 86 & 467 & ALA & $\mathrm{HB}$ & 1.584 & 0.05 & 148 & 472 & ARG & HB2 & 1.565 & \\
\hline 87 & 467 & ALA & $\mathrm{N}$ & 121.482 & 0.5 & 149 & 473 & MET & C & 172.688 & \\
\hline 88 & 468 & PHE & $c$ & 174.298 & 0.5 & 150 & 473 & MET & CA & 55.673 & \\
\hline 89 & 468 & PHE & CA & 58.326 & 0.5 & 151 & 473 & MET & CB & 29.324 & \\
\hline 90 & 468 & PHE & CB & 37.668 & 0.5 & 152 & 473 & MET & CE & 14.99 & \\
\hline 91 & 468 & PHE & CE1 & 129.841 & 0.5 & 153 & 473 & MET & 11 & 7.701 & \\
\hline 92 & 468 & PHE & CE2 & 29.841 & 0.5 & 154 & 473 & MET & $\mathrm{HA}$ & 4.105 & \\
\hline 93 & 468 & PHE & $\mathrm{H}$ & 9.052 & 0.05 & 155 & 473 & MET & HB2 & 2.115 & \\
\hline 94 & 468 & PHE & $\mathrm{HA}$ & 4.35 & 0.05 & 156 & 473 & MET & HB3 & 2.1 & \\
\hline 95 & 468 & PHE & AB2 & 3.418 & 0.05 & 157 & 473 & MET & $\mathrm{HE}$ & 2.509 & \\
\hline 96 & 468 & PHE & HB3 & 3.354 & 0.05 & 158 & 473 & MET & IV & 17.427 & \\
\hline 97 & 468 & PHE & $\mathrm{N}$ & 117.615 & 0.5 & 159 & 473 & MET & HG2 & 2.186 & \\
\hline 98 & 468 & PHE & HD1 & 7.082 & 0.05 & 160 & 474 & GLN & C & 172.068 & \\
\hline 99 & 468 & PHE & HD2 & 7.082 & 0.05 & 161 & 474 & GLN & CA & 76 & \\
\hline .00 & 468 & PHE & HE1 & 7.157 & 0.05 & 162 & 474 & GLN & CB & 24.407 & \\
\hline Q1 & 468 & PHE & HE2 & 7.157 & 0.05 & 163 & 474 & GLN & CG & 31.276 & \\
\hline $\mathrm{D} 2$ & 469 & ASN & C & 173.128 & 0.5 & 164 & 474 & GLN & $\mathrm{H}$ & 7.733 & \\
\hline 03 & 469 & ASN & CA & 09 & 0.5 & 165 & 474 & GLN & $\mathrm{HA}$ & 4.049 & \\
\hline 4 & 469 & ASN & CB & 35.325 & 0.5 & 166 & 474 & GLN & HB2 & 2.142 & \\
\hline 5 & 469 & ASN & $\mathrm{H}$ & 8.602 & 0.05 & 167 & 474 & GLN & HB3 & 2.084 & \\
\hline 06 & 469 & ASN & $\mathrm{HA}$ & 4.166 & 0.05 & 168 & 474 & $\mathrm{GL}$ & HG2 & 2.438 & \\
\hline 107 & 469 & ASN & HB2 & 2.95 & 0.05 & 169 & 474 & GLN & HG3 & 2.262 & \\
\hline 10 & 469 & ASN & HB3 & 2.703 & 0.05 & 170 & 474 & GLN & $\mathrm{N}$ & 19.752 & \\
\hline 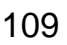 & 469 & ASN & $N$ & 25 & 0.5 & 171 & 475 & LEU & C & 558 & \\
\hline 10 & 470 & LYS & C & 71.718 & 0.5 & 172 & 475 & LEU & $\mathrm{CA}$ & 86 & \\
\hline 111 & 470 & LYS & CA & 57.174 & 0.5 & 173 & 475 & LEU & CB & 39.047 & \\
\hline & 470 & LYS & CB & & 0.5 & 174 & 475 & LEU & CD & & \\
\hline 15 & 470 & L & $C D$ & & 0.5 & 175 & 475 & LEU & CD2 & 87 & \\
\hline 4 & 470 & LYS & CG & 22.045 & 0.5 & 176 & 475 & LEU & CG & 25.779 & \\
\hline 115 & 470 & LYS & $\mathrm{H}$ & 8.076 & 0.05 & 177 & 475 & LEU & $\mathrm{H}$ & 8.151 & \\
\hline & 470 & LYS & 10 & & 0.05 & 178 & 475 & LEU & & 13 & \\
\hline 17 & 470 & LYS & AB2 & 1 & 0.05 & 179 & 475 & LEU & $\mathrm{H}$ & 1.871 & \\
\hline 118 & 470 & LYS & HB3 & 1.839 & 0.05 & 180 & 475 & LEU & HB3 & 1.371 & \\
\hline & 470 & LYS & HD2 & & 0.05 & 181 & 475 & LEU & HD & 0.733 & \\
\hline & 470 & & & & 0.05 & 182 & 475 & LEU & HD2 & 18 & \\
\hline 21 & 470 & LYS & HG2 & 1.681 & 0.05 & 183 & 475 & LEU & 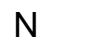 & 20.063 & \\
\hline 122 & 470 & LYS & HG3 & 1.4 & 0.05 & 184 & 475 & LEU & HG & 1.78 & \\
\hline 12 & 470 & LYS & HE2 & & 0.05 & 185 & 476 & GLN & C & 172.968 & \\
\hline & 470 & & $N$ & & 0 & 186 & 476 & $\mathrm{GL}$ & C & & \\
\hline 12 & 471 & VAL & C & 22 & 0.5 & 187 & 476 & GLN & $\mathrm{CB}$ & 25 & \\
\hline 126 & 471 & VAL & CA & & 0.5 & 188 & 476 & GLN & CG & 31.233 & \\
\hline 127 & 471 & VAL & CB & & 0.5 & 189 & 476 & GLN & 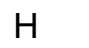 & 7.911 & \\
\hline 128 & 471 & & G 1 & & 0. & 190 & 476 & GLN & & 3.98 & \\
\hline 129 & 471 & VAL & CG2 & 18.862 & 0.5 & 191 & 476 & GLN & HB2 & 2.33 & \\
\hline 130 & 471 & VAL & $\mathrm{H}$ & 8.41 & 0.05 & 192 & 476 & GLN & HB3 & 2.078 & \\
\hline 13 & 471 & VAL & $\mathrm{HA}$ & 3.532 & 0.0 & 193 & 476 & GLN & $\mathrm{HG}^{2}$ & 2.412 & \\
\hline 13 & 471 & VA & HG1 & 0.938 & 0.05 & 194 & 476 & GLN & HG3 & 2.337 & \\
\hline 133 & 471 & VAL & HG2 & 0.839 & 0.05 & 195 & 476 & GLN & $\mathrm{N}$ & 118.054 & \\
\hline 13 & 471 & VAL & $\mathrm{N}$ & 21.488 & 0.5 & 196 & 477 & GLU & C & .73 .278 & \\
\hline 13 & 471 & VAL & $\mathrm{HB}$ & 2.218 & 0.05 & 197 & 477 & GLU & CA & 55.49 & \\
\hline 13 & 472 & ARG & C & 172.261 & 0.5 & 198 & 477 & GLU & CB & 26.666 & \\
\hline
\end{tabular}




\begin{tabular}{|c|c|c|c|c|c|c|c|c|c|c|c|}
\hline 199 & 477 & GLU & CG & 34.168 & 0.5 & 261 & 484 & MET & CG & 33.966 & \\
\hline 200 & 477 & GLU & $\mathrm{H}$ & 7.975 & 0.05 & 262 & 484 & MET & $\mathrm{H}$ & 8.348 & \\
\hline 201 & 477 & GLU & $\mathrm{HA}$ & 4.016 & 0.05 & 263 & 484 & MET & $\mathrm{HA}$ & 4.175 & \\
\hline 02 & 477 & GLU & HG2 & 2.31 & 0.05 & 264 & 484 & MET & HB2 & 2.022 & \\
\hline 03 & 477 & GLU & HG3 & 2.184 & 0.05 & 265 & 484 & MET & HB3 & 1.948 & \\
\hline 04 & 477 & GLU & $\mathrm{N}$ & 19.803 & 0.5 & 266 & 484 & MET & $\mathrm{HE}$ & 2.052 & \\
\hline 5 & 477 & GLU & HB2 & 2.012 & 0.05 & 267 & 484 & MET & $\mathrm{N}$ & 20.436 & \\
\hline 6 & 478 & ALA & C & 172.778 & 0.5 & 268 & 484 & MET & HG3 & 2.176 & \\
\hline 07 & 478 & ALA & CA & 50.517 & 0.5 & 269 & 485 & SER & C & 176.328 & \\
\hline 08 & 478 & ALA & CB & 15.961 & 0.5 & 270 & 485 & SER & CA & 56.067 & \\
\hline 99 & 478 & ALA & П & 7.726 & 0.05 & 271 & 485 & SER & CB & 60.603 & \\
\hline 10 & 478 & ALA & $\mathrm{HA}$ & 4.19 & 0.05 & 272 & 485 & SER & $\mathrm{H}$ & 8.125 & \\
\hline 11 & 478 & ALA & $\mathrm{HB}$ & 1.385 & 0.05 & 273 & 485 & SER & $\mathrm{HA}$ & 4.338 & \\
\hline 12 & 478 & ALA & $\mathrm{N}$ & 121.648 & 0.5 & 274 & 485 & SER & HB2 & 4.12 & \\
\hline 3 & 479 & ARG & C & 73.978 & 0.5 & 275 & 485 & SER & HB3 & 3.842 & \\
\hline 4 & 479 & ARG & CA & 53.836 & 0.5 & 276 & 485 & SER & $\mathrm{N}$ & 115.589 & \\
\hline 15 & 479 & ARG & CB & 27.401 & 0.5 & 277 & 486 & LYS & C & 174.458 & \\
\hline 16 & 479 & ARG & CD & 40.823 & 0.5 & 278 & 486 & LYS & $C A$ & 53.958 & \\
\hline 17 & 479 & ARG & CG & 24.527 & 0.5 & 279 & 486 & LYS & CB & 30.005 & \\
\hline 8 & 479 & ARG & $\mathrm{H}$ & 7.645 & 0.05 & 280 & 486 & LYS & $C D$ & 26.823 & \\
\hline 19 & 479 & ARG & $\mathrm{HA}$ & 4.211 & 0.05 & 281 & 486 & LYS & CE & 39.61 & \\
\hline 20 & 479 & ARG & AG2 & 1.678 & 0.05 & 282 & 486 & LYS & CG & 22.144 & \\
\hline 21 & 479 & ARG & HG3 & 1.617 & 0.05 & 283 & 486 & LYS & $\mathrm{H}$ & 7.986 & \\
\hline 22 & 479 & ARG & $\mathrm{N}$ & 117.484 & 0.5 & 284 & 486 & LYS & HA & 4.22 & \\
\hline 3 & 479 & ARG & HB2 & 1.834 & 0.05 & 285 & 486 & LYS & HB2 & 1.706 & \\
\hline 24 & 479 & ARG & HD2 & 3.135 & 0.05 & 286 & 486 & LYS & HB3 & 1.596 & \\
\hline 25 & 480 & GLY & C & 76.758 & 0.5 & 287 & 486 & LYS & HE2 & 2.837 & \\
\hline 6 & 480 & GLY & CA & 42.457 & 0.5 & 288 & 486 & LYS & HG2 & 1.275 & \\
\hline 27 & 480 & GLY & $\mathrm{H}$ & 7.961 & 0.05 & 289 & 486 & LYS & $\mathrm{N}$ & 122.255 & \\
\hline 228 & 480 & GLY & $\mathrm{N}$ & 07.622 & 0. & 290 & 487 & SER & C & 58 & \\
\hline 29 & 480 & GLY & HA2 & 3.909 & 0.05 & 291 & 487 & SER & CA & & \\
\hline 30 & 480 & GLY & HA3 & 3.909 & 0.05 & 292 & 487 & SER & CB & 61.064 & \\
\hline 31 & 481 & GLU & C & 74.088 & 0. & 293 & 487 & SER & $\mathrm{H}$ & 7.972 & \\
\hline 32 & 481 & GLU & CA & 53.78 & 0.5 & 294 & 487 & SER & $\mathrm{HA}$ & 3.676 & \\
\hline & 481 & GLU & CB & 27.272 & 0.5 & 295 & 487 & SER & HB2 & 3.806 & \\
\hline 34 & 481 & GLU & CG & 33.333 & 0.5 & 296 & 487 & SER & HB3 & 3.744 & \\
\hline 35 & 481 & GLU & $\mathrm{H}$ & 8.163 & 0.05 & 297 & 487 & SER & $N$ & 14.856 & \\
\hline 36 & 481 & GLU & Th & 4.269 & 0.0 & 298 & 488 & LEU & C & 98 & \\
\hline 37 & 481 & GLU & ID? & 2.049 & 0.05 & 299 & 488 & LEU & $\mathrm{Cr}$ & & \\
\hline 38 & 481 & GLU & IB & 39 & 0.0 & 0 & 488 & LEU & D & & \\
\hline 239 & 481 & GLU & $\Lambda$ & 20.497 & 0. & 301 & 488 & LEU & CD1 & 26 & \\
\hline 240 & 481 & GLU & HG & 2.202 & 0.05 & 302 & 488 & LEU & CD2 & 18.346 & \\
\hline 11 & 482 & GLY & C & 76.808 & 0 & 303 & 488 & LEU & $\mathrm{H}$ & 8.017 & \\
\hline 242 & 482 & GLY & CA & 42.485 & 0 & 304 & 488 & LEU & $\mathrm{HA}$ & 4.3 & \\
\hline 43 & 482 & GLY & r & 8.35 & 0.05 & 305 & 488 & LEU & HB2 & .427 & \\
\hline 244 & 482 & GLY & $\Lambda$ & 108.85 & 0.5 & 306 & 488 & LEU & HB3 & 272 & \\
\hline & 482 & GLY & 1 & & 0. & 77 & 488 & LEU & & 33 & \\
\hline 2 & 482 & GLY & A3 & 2 & 0.0 & 08 & 488 & LEU & HD2 & 89 & \\
\hline 247 & 483 & GLU & C & 74.108 & 0.5 & 309 & 488 & LEU & HG & 1.426 & \\
\hline 248 & 483 & GLU & CA & & 0 & 0 & 488 & LEU & $\mathrm{N}$ & & \\
\hline 24 & 483 & & & & 0 & 1 & 489 & TRP & 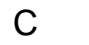 & & \\
\hline 250 & 483 & GLU & CG & 67 & 0. & 2 & 489 & TRP & C & 58 & \\
\hline 251 & 483 & GLU & $\mathrm{H}$ & 8.142 & 0.05 & 313 & 489 & TRP & $C B$ & 26.283 & \\
\hline 252 & 483 & GLU & 0 & & 0.05 & 314 & 489 & TRP & CD1 & 91 & \\
\hline & 483 & GLU & AB2 & 2.027 & 0.0 & 315 & 489 & TRP & $\mathrm{H}$ & 7.636 & \\
\hline 254 & 483 & GLU & HB3 & 911 & 0.05 & 316 & 489 & TRP & $H$ & 4.124 & \\
\hline 25 & 483 & GLU & $\Lambda$ & 20.369 & 0.5 & 317 & 489 & TRP & HB2 & 2.964 & \\
\hline 25 & 483 & GLU & 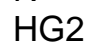 & 2.201 & 0.05 & 318 & 489 & TRP & HB3 & .925 & \\
\hline 25 & 484 & MET & C & 74.538 & 0 & 210 & 489 & TRP & HD1 & 7.073 & \\
\hline 25 & 484 & MET & CA & 53.324 & 0.5 & 320 & 489 & TRP & HE1 & 9.797 & 0 \\
\hline תГ & 484 & MET & CB & 29.849 & 0.5 & 321 & 489 & TRP & $\mathrm{HZ2}$ & 7.204 & \\
\hline 26 & 484 & MET & CE & 14.43 & 0.5 & 322 & 489 & TRP & $\mathrm{HH} 2$ & 7.075 & \\
\hline
\end{tabular}




$\begin{array}{llllrlllllrr}323 & 489 & \text { TRP } & \text { N } & 117.409 & 0.5 & 341 & 491 & \text { LYS } & \text { CB } & 30.222 & 0.5 \\ 324 & 489 & \text { TRP } & \text { NE1 } & 130.113 & 0.5 & 342 & 491 & \text { LYS } & \text { CD } & 26.208 & 0.5 \\ 325 & 490 & \text { PHE } & \text { C } & 176.158 & 0.5 & 343 & 491 & \text { LYS } & \text { CE } & 39.534 & 0.5 \\ 326 & 490 & \text { PHE } & \text { CA } & 55.014 & 0.5 & 344 & 491 & \text { LYS } & \text { CG } & 20.917 & 0.5 \\ 327 & 490 & \text { PHE } & \text { CB } & 36.68 & 0.5 & 345 & 491 & \text { LYS } & \text { H } & 7.864 & 0.05 \\ 328 & 490 & \text { PHE } & \text { CE1 } & 129.98 & 0.5 & 346 & 491 & \text { LYS } & \text { HA } & 4.195 & 0.05 \\ 329 & 490 & \text { PHE } & \text { CE2 } & 129.98 & 0.5 & 347 & 491 & \text { LYS } & \text { HB2 } & 1.759 & 0.05 \\ 330 & 490 & \text { PHE } & \text { H } & 7.377 & 0.05 & 348 & 491 & \text { LYS } & \text { HB3 } & 1.595 & 0.05 \\ 331 & 490 & \text { PHE } & \text { HA } & 4.325 & 0.05 & 349 & 491 & \text { LYS } & \text { N } & 123.484 & 0.5 \\ 332 & 490 & \text { PHE } & \text { HZ } & 7.242 & 0.05 & 350 & 491 & \text { LYS } & \text { HD2 } & 1.611 & 0.05 \\ 333 & 490 & \text { PHE } & \text { N } & 119.308 & 0.5 & 351 & 491 & \text { LYS } & \text { HE2 } & 2.902 & 0.05 \\ 334 & 490 & \text { PHE } & \text { HB2 } & 2.844 & 0.05 & 352 & 491 & \text { LYS } & \text { HG2 } & 1.297 & 0.05 \\ 335 & 490 & \text { PHE } & \text { HD1 } & 7.05 & 0.05 & 353 & 492 & \text { GLY } & \text { CA } & 43.436 & 0.5 \\ 336 & 490 & \text { PHE } & \text { HD2 } & 7.05 & 0.05 & 354 & 492 & \text { GLY } & \text { H } & 7.381 & 0.05 \\ 337 & 490 & \text { PHE } & \text { HE1 } & 6.986 & 0.05 & 355 & 492 & \text { GLY } & \text { N } & 115.197 & 0.5 \\ 338 & 490 & \text { PHE } & \text { HE2 } & 6.986 & 0.05 & 356 & 492 & \text { GLY } & \text { HA2 } & 3.647 & 0.05 \\ 339 & 491 & \text { LYS } & \text { C } & 175.708 & 0.5 & 357 & 492 & \text { GLY } & \text { HA3 } & 3.647 & 0.05 \\ 340 & 491 & \text { LYS } & \text { CA } & 53.521 & 0.5 & & & & & & \end{array}$




\title{
Curriculum Vitae
}

\author{
Personal data \\ Name: $\quad$ Fernando Rodríguez Castañeda \\ Birth date: $\quad 23.08 .1978$ \\ Place of Birth: Guatemala City, Guatemala \\ Education
}

1986-1991 Primary school “Liceo Javier”

1992-1994 Secondary school “Liceo Javier”

1995-1996 High school "Liceo Javier”

1997-2002 Biochemistry studies at the "Universidad del Valle de Guatemala"

07/2001: Bachelor of Science degree (Major in biochemistry)

04/2002: "Licenciatura" degree in biochemistry (Cum laude)

2003-2007 M.Sc./Ph.D. studies in Molecular Biology at Georg August Universität Göttingen.

03/2004: M.Sc. degree in molecular biology

\section{Working experience}

June-July 1999: Summer internship at the pharmaceutical company Frycia Centro America, S.A. February-August 2002: Quality control laboratory assistant at the pharmaceutical company Frycia Centro America, S.A.

\section{Research projects}

January -may 1999: Organic chemistry 3 research project entitled "Organic synthesis of a NADH analogue and its redox potential"

June-July 2000: Enzymology research project entitled "Over expression and purification of the recombinant protein Taq DNA polymerase"

August-November 2000: Natural products chemistry research project entitled "Phytochemical screening of the fruits, leaves and flowers of the elderberry (Caprifoliaceae: Sambucus mexicana)"

October 2000 - October 2001: Licenciatura degree thesis entitled "Genetic population of the malaria vector, the mosquito Anopheles albimanus, based on microsatellite DNA in Central America and the Caribbean"

January -march 2003: Laboratory rotation in the department of molecular genetics of the Max Planck Institute for Biophysical Chemistry. "Study of deletion mutants of the human deoxycytidine kinase".

March - may 2003: Laboratory rotation in the department of biochemistry, medical faculty University of Goettingen. "Characterization of the formylglycine formation in the Caulobacter crescentus arylsulfatase" 
May - July 2003: Laboratory rotation in the department of NMR based structural biology of the Max Planck Institute for Biophysical Chemistry. "Alignment of protein induced by a chemical coordinating lanthanide ion tag".

October 2003-March 2004: M.Sc. thesis in the department of NMR based structural biology of the Max Planck Institute for Biophysical Chemistry. "Study of the denatured state of ubiquitin by NMR"

Since April 2004: Ph.D. thesis entitled "Study of protein dynamics by NMR"

\section{Publications}

Molina-Cruz, A., de Merida, AM., Mills, K., Rodriguez, F., Schoua, C., Yurrita, M.M., Molina, E., Palmieri, M and Black, W.C. $4^{\text {th }}$. 2004. Gene flow among Anopheles albimanus populations in Central America, South America and the Caribbean assessed by microsatellites and mitochondrial DNA. Am. J. Trop. Med. Hyg., 71(3): 350-9.

Leonov, A., Voigt, B., Rodriguez-Castaneda, F., Sakhaii, P. and Griesinger, C. 2005. Convenient syntheses of multifunctional EDTA-based chiral metal chelates substituted with an S-

mesylcysteine. Chem. Eur. J., 11: 1-8.

Haberz, P., Rodriguez-Castaneda, F., Junker, J., Becker, S., Leonov, A. and Griesinger, C. 2006. Two new chiral EDTA-based metal chelates for weak alignment of proteins in solution. Organic Letters, 8(7): 1275-1278.

Rodríguez-Castañeda, F., Haberz, P., Leonov, A. and Griesinger, C. 2006. Paramagnetic tagging of diamagnetic proteins for solution NMR. Magnetic resonance in chemistry, 44: S10-S16.

Rodríguez-Castañeda, F., Brose, N., Carlomagno, T., Griesinger, C. Resonance assignment of the calmodulin-Munc13-1 peptide complex. J. Biomol. Assignments. To be submitted. 